\title{
VENTILATION DESIGN HANDBOOK ON \\ Animal Research Facilities Using Static Microisolators
}

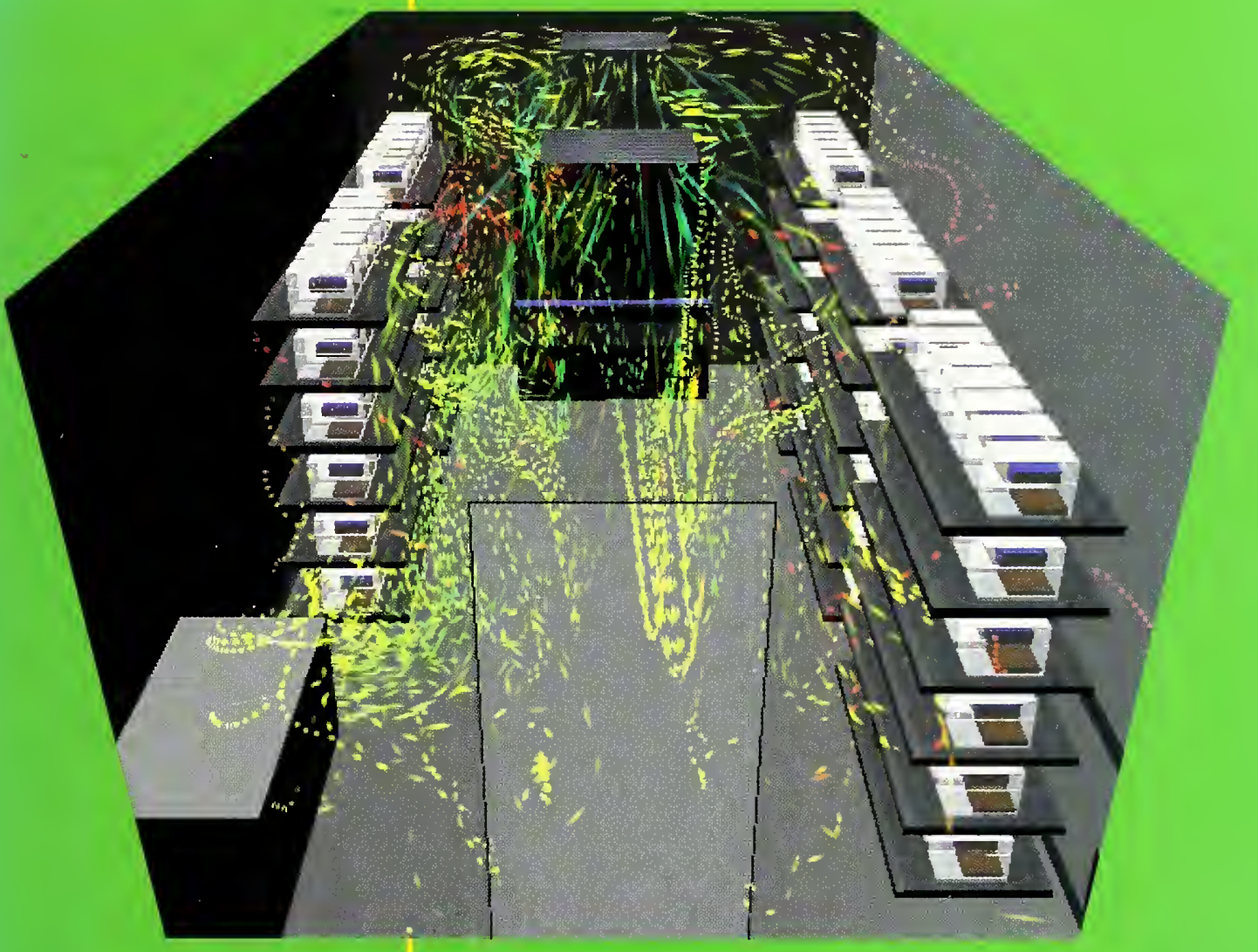

VOLUME

OF 2

ATIONAL INSTITUTES OF HEALTH

OFFICE OF THE DIRECTOR

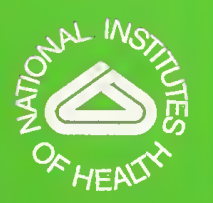





\section{National Institutes of Health}

\section{Ventilation Design Handbook on Animal Research Facilities Using Static Microisolators}

\section{VOLUME II}

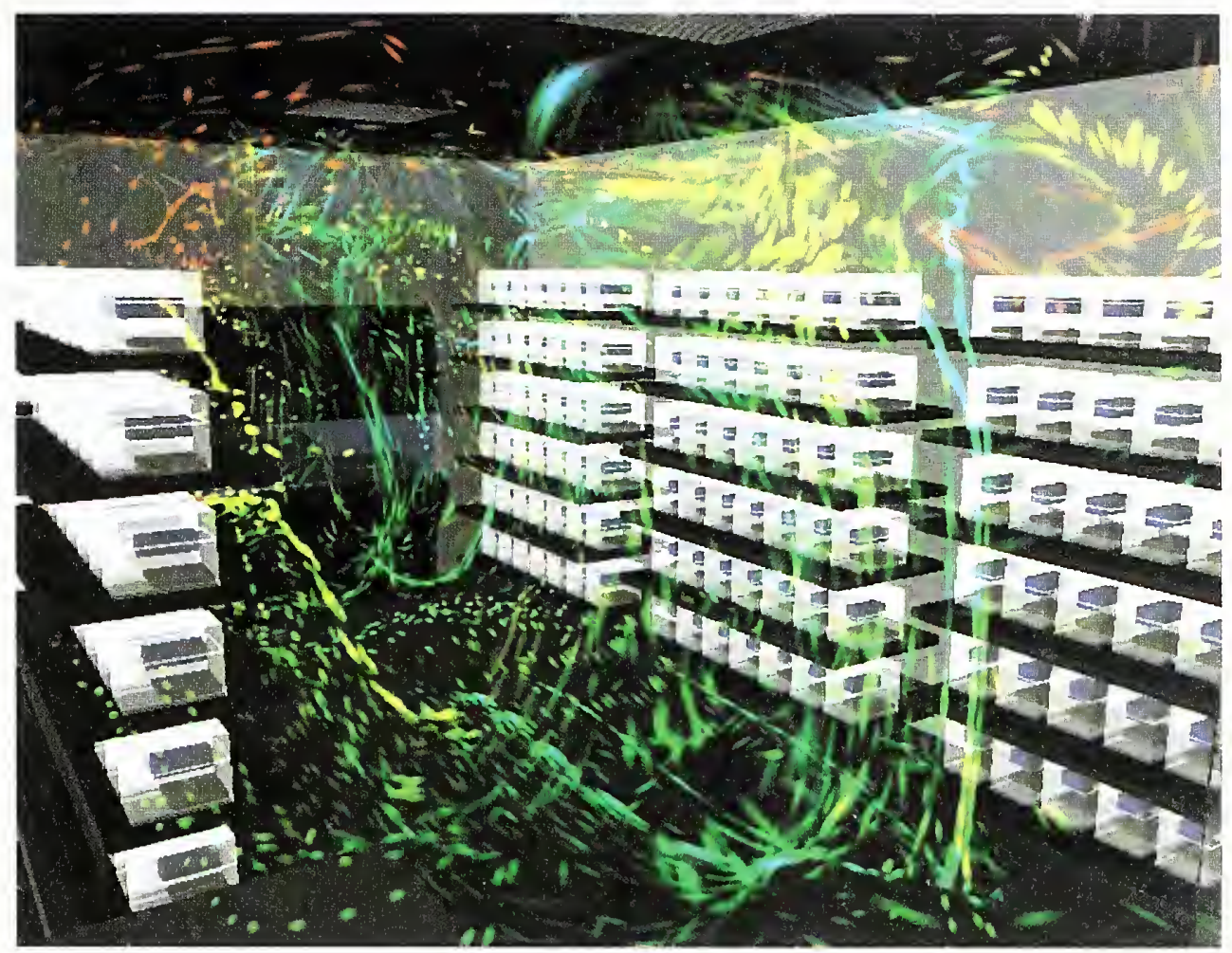

Farhad Memarzadeh Principal Investigator

Division of Engineering Services

Office of Research Services

Office of the Director

National Institutes of Health

Bethesda, Maryland

September 1998 



\section{VOLUME II: TABLE OF CONTENTS}

\section{INTRODUCTION}

II - 1

1.1 Outline of CFD Baseline Model ……………………................................ - 1

1.2 Whole Room Configurations ……………………………………………………...II - 8

2. SUMMARY OF RESULTS OF WHOLE ROOM RUNS...........II - 29

3. RANKINGS OF RESULTS OF WHOLE ROOM RUNS ..........II - 37

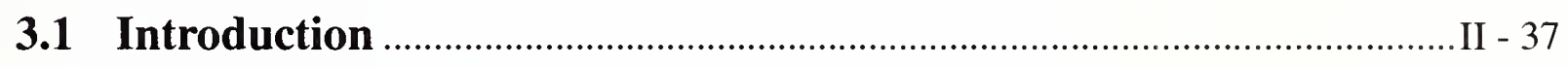

4. WHOLE ROOM SIMULATION DATABASE ...............................II - 55

4.1 Description of Simulation Data Entry........................................................II - 55

4.2 Simulation Data Entries....................................................................................II - 57 
4

1. $\angle 63$

1 60

19 


\section{INTRODUCTION}

This volume contains a database of the results of the computational fluid dynamics (CFD) simulations for the research done on the National Institutes of Health Ventilation Design Handbook on Animal Research Facilities using Static Microisolators.

Section 1 contains an overview of the basic room configuration studied and the variations considered. It is impossible to present all the data generated by the CFD simulations. The analysis of all the results produces an enormous quantity of data that would be difficult to present and interpret in its entirety. This volume contains summaries of the data in several forms.

Section 2 contains histograms of mean values for each room considered, as they apply to both the scientists' breathing zone and the cages, for temperature, relative humidity, and $\mathrm{CO}_{2}$ and $\mathrm{NH}_{3}$ concentrations.

Section 3 contains rankings of the rooms based on different variables, for example, the selection of the room configuration with the lowest $\mathrm{NH}_{3}$ concentrations in the cages.

Section 4 contains a two page summary of each room simulation, including a description of the room, mean values and standard deviations for all parameters, and histograms showing the distribution of values in the cages.

\section{$1.1 \quad$ Outline of CFD Baseline Model}

A typical animal research facility of average size, air change rate, rack layout, mouse population, pressurization, and so on, was modeled as the baseline model for the CFD simulations. The general features of the room are shown in figure 1.01 and are listed below. 


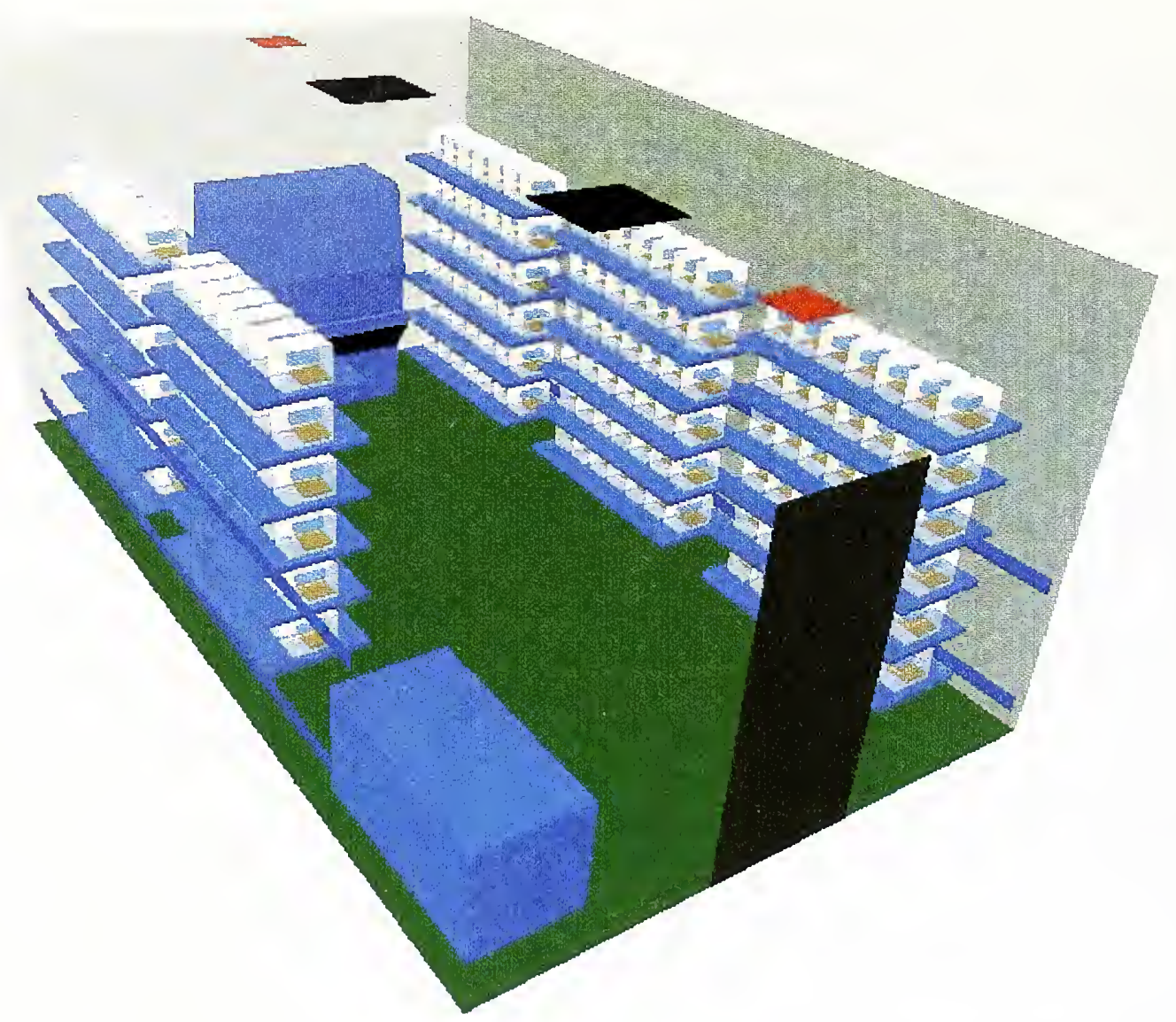

Figure 1.01 Overall Layout of Animal Room Basecase

Description in brief

The general features of the basecase room model were:

Room: $6.10 \mathrm{~m} \times 3.60 \mathrm{~m} \times 4.22 \mathrm{~m}$ (20' 0" x 12' 0" x 9' 0")

Door in short wall

Sink in corner

Laminar flow change station

5 racks

Cages: Microisolator (with filter top) mouse cage 5 mice per cage

Rack: Static system

6 shelves per rack

7 cages per shelf ( 42 cages per rack) 
Supply: 2 radial supplies each providing $270 \mathrm{cfm}\left(0.13 \mathrm{~m}^{3} / \mathrm{s}\right)$ for a total of $15 \mathrm{ACH}$

Supply discharge temperature of $18.8{ }^{\circ} \mathrm{C}\left(66^{\circ} \mathrm{F}\right)$, set such that the exhaust air temperature was $22.2^{\circ} \mathrm{C}\left(72.0^{\circ} \mathrm{F}\right)$

61 percent relative humidity (to provide 50 percent $\mathrm{RH}$ at $22.2{ }^{\circ} \mathrm{C}\left(72.0^{\circ} \mathrm{F}\right.$ )

Exhausts: 2 ceiling level exhausts removing $220 \mathrm{cfm}\left(0.1 \mathrm{~m}^{3} / \mathrm{s}\right)$ each

Makeup Air: $100 \mathrm{cfm}\left(0.047 \mathrm{~m}^{3} / \mathrm{s}\right)$ coming from around the door

\section{Overall Geometry}

In the majority of the cases considered, the animal room occupied a floor area of $6.10 \mathrm{~m}\left(20^{\prime} 0^{\prime \prime}\right)$ $x 3.66 \mathrm{~m}$ (12' 0 '). In some cases that were considered, the width of the room was increased from $3.66 \mathrm{~m}$ to $4.22 \mathrm{~m}$ ( $\left.14^{\prime} 0^{\prime \prime}\right)$. The ceiling height in all cases was $2.74 \mathrm{~m}\left(9^{\prime} 0^{\prime \prime}\right)$. There was only one door in the room, mounted centrally on one of the short walls.

In all the displacement ventilation systems considered in this project, air was introduced through ceiling mounted diffusers. All devices were mounted flush with the ceiling surface; there was no ductwork present within the upper room volume. The various diffuser types considered in this project were all modeled using a combination of several boundary conditions that were validated previously (see volume I, section 4.2.2). All the air exited through general exhausts. The number and locations of the exhausts were varied. In line with common practice, there was an imbalance between the amount of air supplied to the room and exhausted from the room. This leads to an overall pressurization of the room relative to the rooms or corridors surrounding the room. The relative level of pressurization was a parameter considered in this study. The makeup air to compensate for the supply/exhaust imbalance was allowed to enter or leave the room through $6.35 \mathrm{e}-3 \mathrm{~m}(0.25$ ") gaps on three sides of the door.

The rooms considered in this project all contained five animal cage racks. The rooms also contained one of two alternative design change stations. A fuller description of these items is given below. The only other item within the room was a sink of $0.61 \mathrm{~m}(24$ ") x $0.61 \mathrm{~m}(24$ ") $\mathrm{x}$ $0.81 \mathrm{~m}$ (32") that was situated in one of the corners to the room.

In all cases, the room was considered under dark period conditions, i.e., the lights were off and produced no additional heat load to the room. Dark period conditions were chosen because early experimental work had indicated that heat, $\mathrm{CO}_{2}$, and $\mathrm{NH}_{3}$ generation were higher in the dark period compared with periods in which the lights were on.

\section{Rack Model}

The overall dimensions of the rack were $1.52 \mathrm{~m}(60 ") \times 0.61 \mathrm{~m}(24 ") \times 1.83 \mathrm{~m}$ (72") high, as shown in figure 1.02. There were six shelves in the rack. The spacing of the shelves was $0.32 \mathrm{~m}$ (12.75") from top surface to top surface, with the lowest shelf at a height of $0.21 \mathrm{~m}(8.25$ ") above the floor. The shelves were modeled as thin rectangular blocks. Details, such as the connecting ties between the shelves and the rollers on which the racks move, were not modeled because their effect on the overall flow field and gas concentration distributions was considered insignificant. 


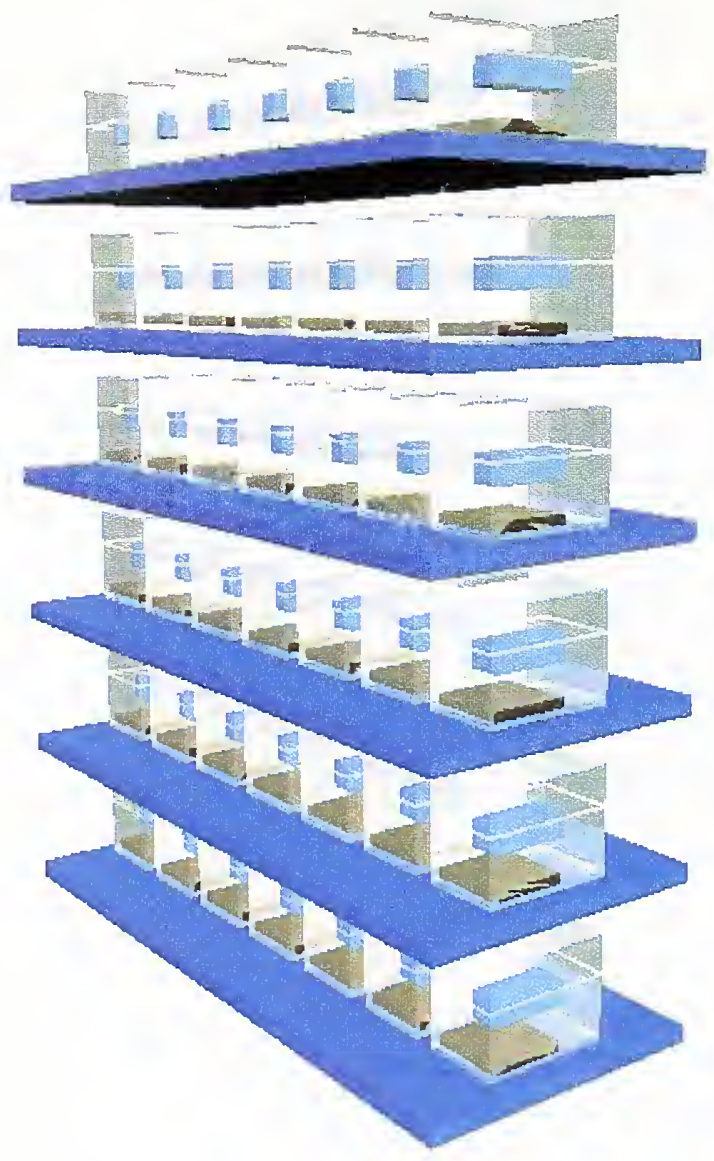

Figure 1.02 CFD Model of Cage Rack

Located on the shelves of the racks were representations of the animal cages, shown in figure 1.02. The dimensions of the cage were $0.27 \mathrm{~m}$ (10.7") x $0.16 \mathrm{~m}(6.38$ ") $\times 0.21 \mathrm{~m}$ (8.39") high, which maintained the volume of the original cage that had sloped sides. The sides of the cage were modeled as thin plates, with the thickness and conductivity of the plates set to those of the physical cage polycarbonate. The water bottle and food normally found in a cage were modeled as a single block in order to reduce the computational overhead. The volume of the block was the same as that of the bottle and food combined. The bedding of the cage was included as a rectangular block of $0.27 \mathrm{~m}(10.7 ")$ x $0.16 \mathrm{~m}(6.38 ”) \times 1.27 \mathrm{e}-2 \mathrm{~m}(0.5 ”)$.

The mice were modeled as a block of $0.11 \mathrm{~m}(4.25 ") \times 8.57 \mathrm{e}-2 \mathrm{~m}\left(3.38\right.$ ") $\times 0.22 \mathrm{~m}\left(0.88^{\prime \prime}\right)$, the same representation that was used in the experimental cage wind tunnel tests (see volume I, section 4.1.1). This simulated the effect of 'huddling' by the mice. The surface temperature of the block was fixed at $30.0^{\circ} \mathrm{C}\left(86.0^{\circ} \mathrm{F}\right)$, which is agreed to be a typical mouse body surface temperature.

Surrounding this block, a source of $\mathrm{CO}_{2}$ was defined at $2.12 \mathrm{e}-7 \mathrm{~kg} / \mathrm{s}(0.76 \mathrm{~g} / \mathrm{hr})$, which was based on the generation rate indicated for the dark period in the early tests on the effect of the 
photoperiod on the mice. The supply air was assumed to have a zero concentration of $\mathrm{CO}_{2}$. This source allowed the additional concentration of $\mathrm{CO}_{2}$ in the air to be calculated in the simulation. It also allowed the concentration of $\mathrm{NH}_{3}$, among other things, to be calculated by scaling even though it has a different molecular weight from both air and $\mathrm{CO}_{2}$. This was possible as the magnitude of the source was very small and the resulting concentrations were so low as to have a negligible effect on the density of the mixture of air, $\mathrm{CO}_{2}$, and $\mathrm{NH}_{3}$. In effect, the $\mathrm{CO}_{2}$ and $\mathrm{NH}_{3}$ are intimately mixed with, and flow with, the air.

Experimental data later showed the generation rate of $\mathrm{CO}_{2}$ was actually higher than the source used in the CFD simulations at $0.90 \mathrm{~g} / \mathrm{hr} / 100 \mathrm{~g}$ mouse body weight. This means the concentration of $\mathrm{CO}_{2}$ in the room and cages was derived from the simulated concentration multiplied by a scaling factor $(0.90 / 0.76)$. The concentrations of $\mathrm{NH}_{3}$ in both the cages and the room were also derived by scaling the concentration with a factor specified in the post-processing of the data. This factor was assumed to vary according to the number of days that passed since the bedding in the cage was changed, along with the average relative humidity in the cages. See volume I, section 4.2.1.2 for the experimental determination of the factors.

Background levels of $\mathrm{CO}_{2}$ and $\mathrm{NH}_{3}$ were assumed to be zero. This means that all values quoted in the CFD section of the report are relative to the background level. If an absolute value for $\mathrm{CO}_{2}$ is required, an additional amount in the range of $300 \mathrm{ppm}$ to $700 \mathrm{ppm}$ should be added for most locations.

The remaining cage boundary conditions are associated with the transfer mechanisms for air/gases to enter/leave the cage. The cracks at the side of the cage were modeled as $6.35 \mathrm{e}-3 \mathrm{~m}$ $(0.25$ ") high planar resistances, with the loss coefficient for these resistances having been determined from the cage wind tunnel CFD simulations (see volume I section 4.2.1.2). The top of the cage, which was filtered, was defined as a combination of a planar resistance and a planar source. The determination of the loss coefficient for the resistance, and the coefficient for the source has been outlined (see volume I, section 4.2.1.1).

The spacing of the cages on the shelves was dependent on whether the racks were single density ( 7 cages per shelf), or double density ( 14 cages per shelf). In the single density cases, the cages were centrally located in the short dimension, and equally spaced in the long dimension. The spacing was $4.88 \mathrm{e}-2 \mathrm{~m}$ (1.92") from corner of cage to corner of adjacent cage. In the double density racks, the cages were equally spaced in both the long and short dimensions. The spacing was $2.20 \mathrm{e}-2 \mathrm{~m}\left(0.87^{\prime \prime}\right)$ and $4.88 \mathrm{e}-2 \mathrm{~m}(1.92 ")$, respectively.

\section{Change Station Model}

Two alternative change stations were considered in this project. Both stations were constructed primarily from rectangular blocks and triangular prisms. The internal structure and flow field were of no concern in this project. It was only the effect of the station on the room airflow that is of importance.

The first design is shown in figure 1.03 . The station had overall dimensions of $1.32 \mathrm{~m}(52$ ") $\mathrm{x}$ $0.86 \mathrm{~m}$ (34") x $1.83 \mathrm{~m}$ (72"). This design was effectively passive in terms of direct flow field interaction. In particular, the station internally recirculated a flow of $350 \mathrm{cfm}\left(1.65 \mathrm{e}-1 \mathrm{~m}^{3} / \mathrm{s}\right)$, 
with only 10 percent leakage defined at the sash opening. The makeup air intake for this leakage was mounted at the side of the station. The station dissipated heat that was expected to affect the room's overall flow field. In particular, the station contributed a load of $720 \mathrm{~W}$ to the room. This heat was mostly confined to the lower portion of the station where the motor was located.

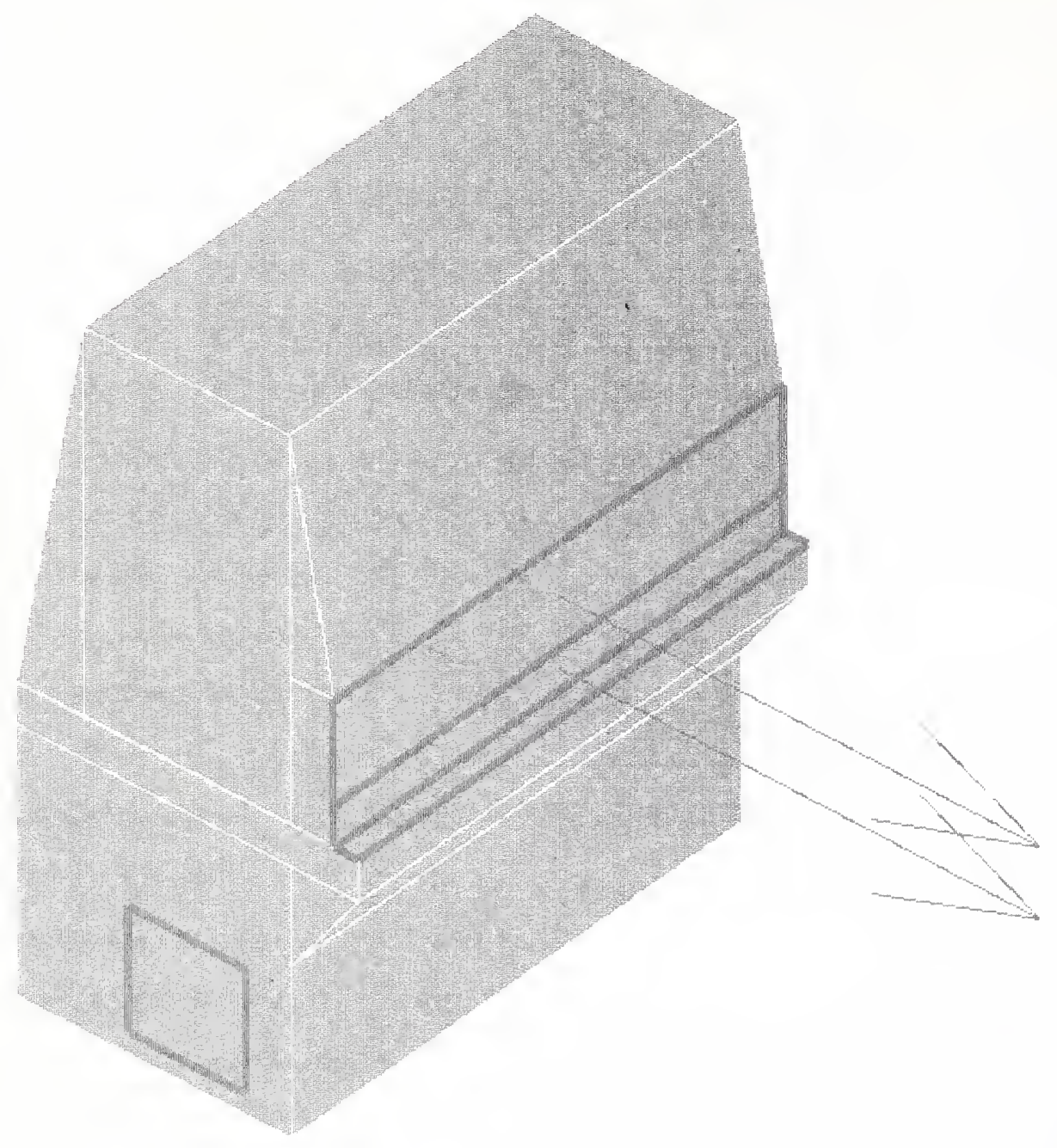

Figure 1.03 Original Change Station Design

Due to concerns raised over the relative passiveness of the first station design, a second design was considered, as is displayed in figure 1.04 . The station had overall dimensions of $1.50 \mathrm{~m}$ (59") x $0.86 \mathrm{~m}(34 ") \times 1.93 \mathrm{~m}(76 ")$ high. This station also recirculated air, at $300 \mathrm{cfm}\left(1.42 \mathrm{e}-1 \mathrm{~m}^{3} / \mathrm{s}\right)$, but discharged a much higher percentage than the first design. In particular, $200 \mathrm{cfm}(9.44 \mathrm{e}-2$ $\mathrm{m}^{3} / \mathrm{s}$ ) was discharged through grilles at the top of the station. The air makeup to compensate for this discharge was mounted at the front sill at the opening to the station. The station dissipated $660 \mathrm{~W}$, and this heat was considered to be added to the air discharge at the top of the station. 


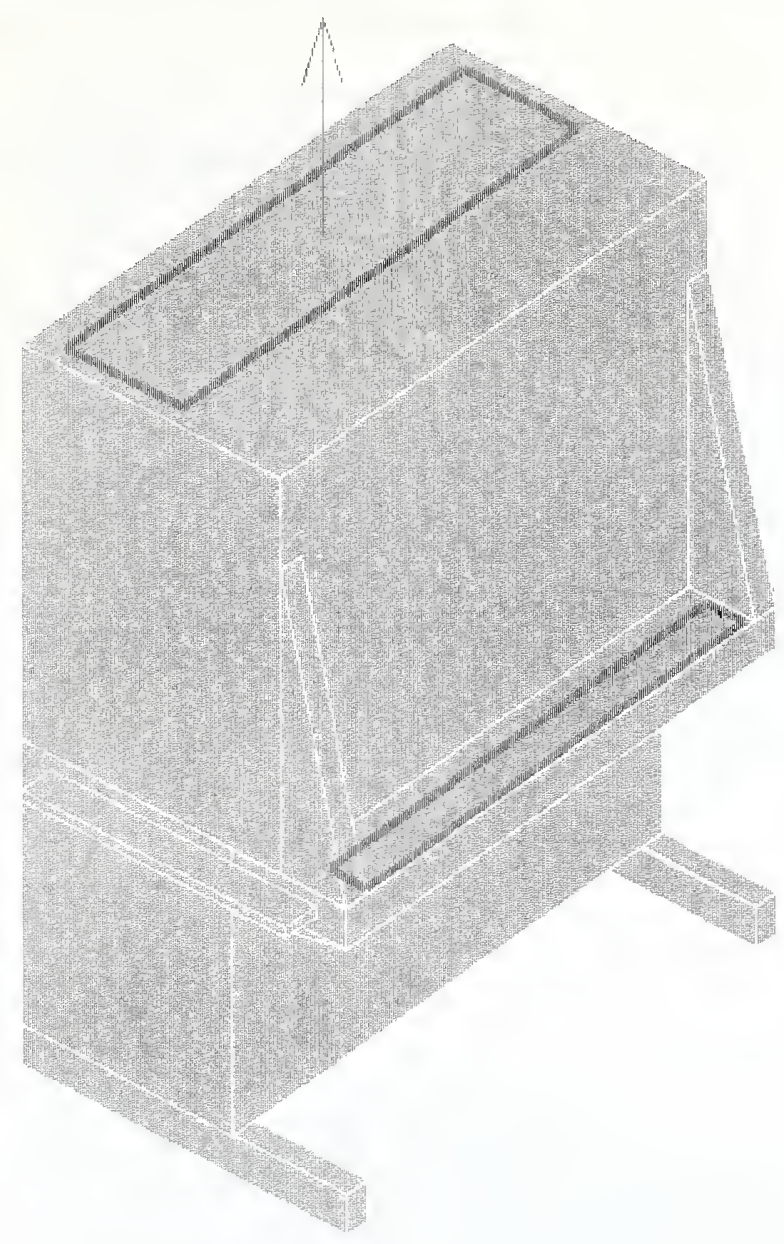

Figure 1.04 Alternative Change Station Design

\section{Modeling Assumptions}

- Solar load was not modeled through the walls of the room.

- The floor, ceiling, and walls were assumed to have no heat transfer, i.e., the surrounding areas were assumed to be at the same temperatures.

- All surfaces were treated as smooth when calculating surface friction.

- The sink in the animal room was modeled as a single rectangular block. The recess formed by the shape of the sink was not modeled because the effect of the recess would be negligible on the overall flow field within the room.

- No lighting was assumed in the animal room simulations. The reason for this was that higher $\mathrm{CO}_{2}$ and $\mathrm{NH}_{3}$ emissions occur during dark (scotophase) conditions (see volume I, section 4.1.2.8.1 and appendix I, sections 3.4.1.9 and 3.4.1.10). 
- The animal room was intended to be kept at $22.2^{\circ} \mathrm{C}\left(72.0^{\circ} \mathrm{F}\right)$, with temperature control performed on the exhaust air temperature.

- No leakage occurred into or out of the animal room other than that specified though the cracks around the door.

- Air density variations due to temperature were negligible. Density variation was therefore ignored in all terms apart from in the momentum term for the vertical velocity component. This is known as the Boussinesq approximation.

- The levels of $\mathrm{CO}_{2}$ and $\mathrm{NH}_{3}$ were so diluted in the whole room simulations, even at their source, that the variation of the mixture density due to differing molecular weights was negligible.

\section{Whole Room Configurations}

To investigate a range of parameters, the basic model described in section 1.1 was modified. Wherever possible, only one parameter was varied at a time to fully assess the effect.

The list of room runs as they were considered is given in table 1.01. Parameters considered in this report are as follows:

- Supply Diffuser Type: Three different diffuser types were considered in this project: radial diffusers, low induction diffusers, and slot diffusers. While all these diffuser types are ceiling mounted diffusers, the flow patterns resulting from them are fundamentally different. See volume I, section 4.2.2 and figures 1.05 to 1.07 ). 


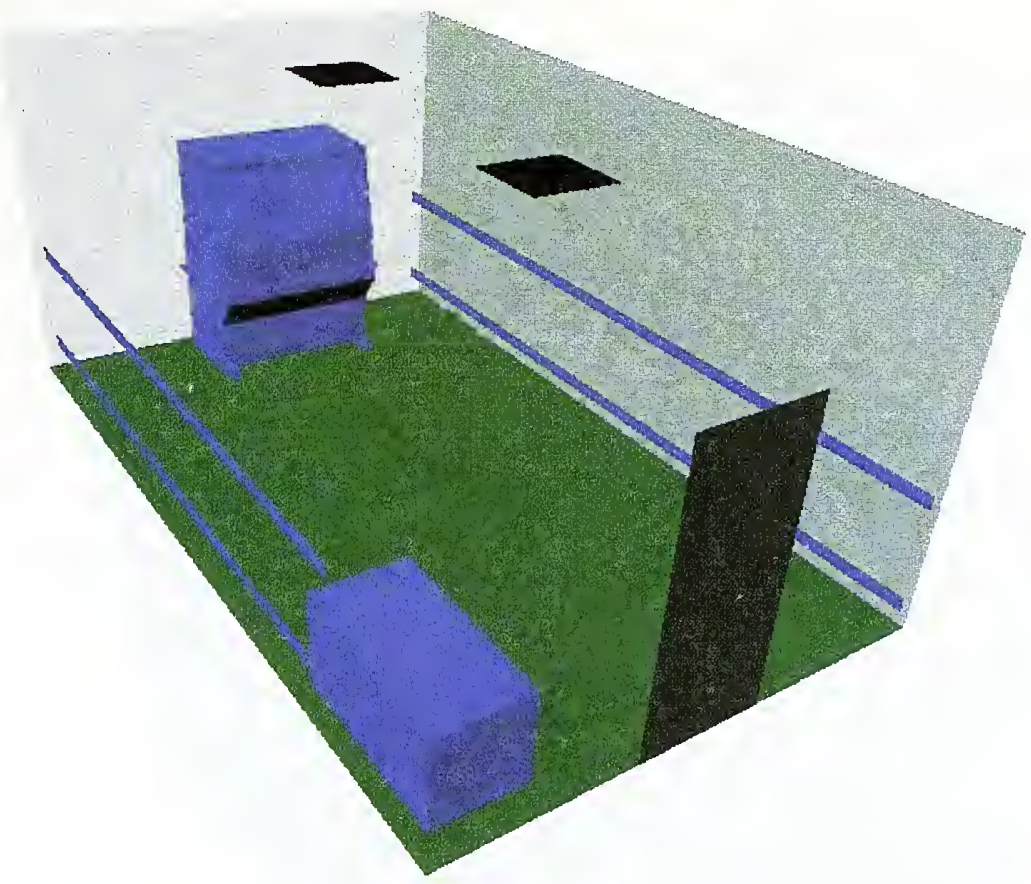

Figure 1.05 Radial Diffuser

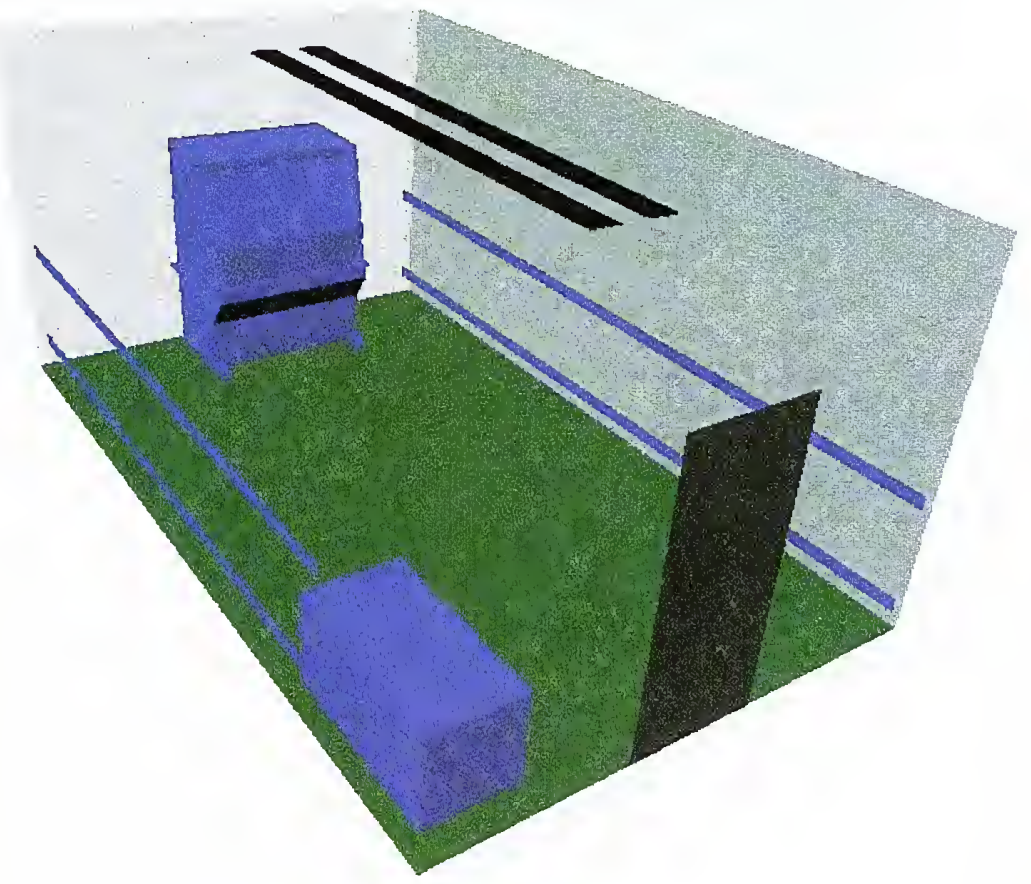

Figure 1.06 Slot Diffuser 


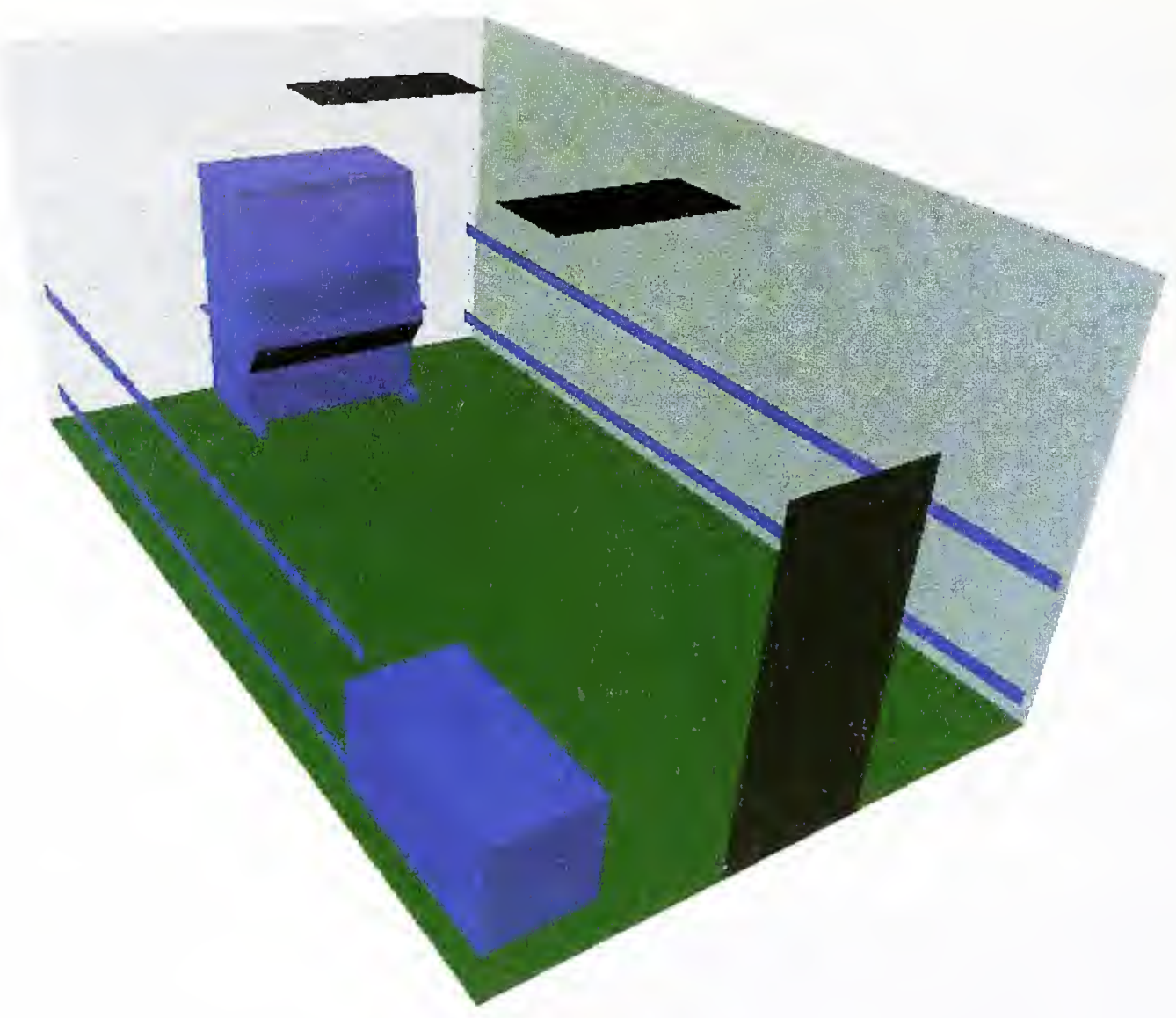

Figure 1.07 Low Induction Diffuser

- Exhaust Location and Number: Four different exhaust locations were considered: ceiling; high level, low level on the long walls, and low level on the door wall. The number of exhausts used in each of these locations was also considered as a parameter. See figures 1.08 to 1.11 . 


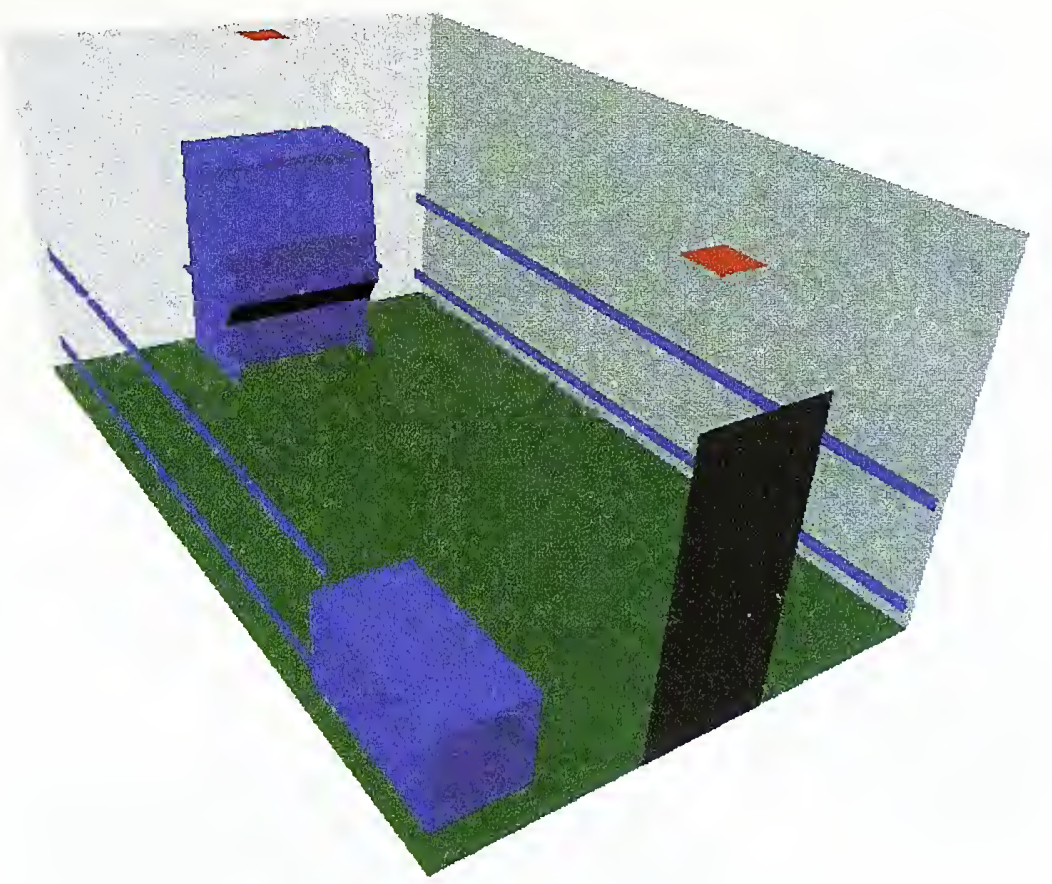

Figure 1.08 Ceiling Level Exhausts

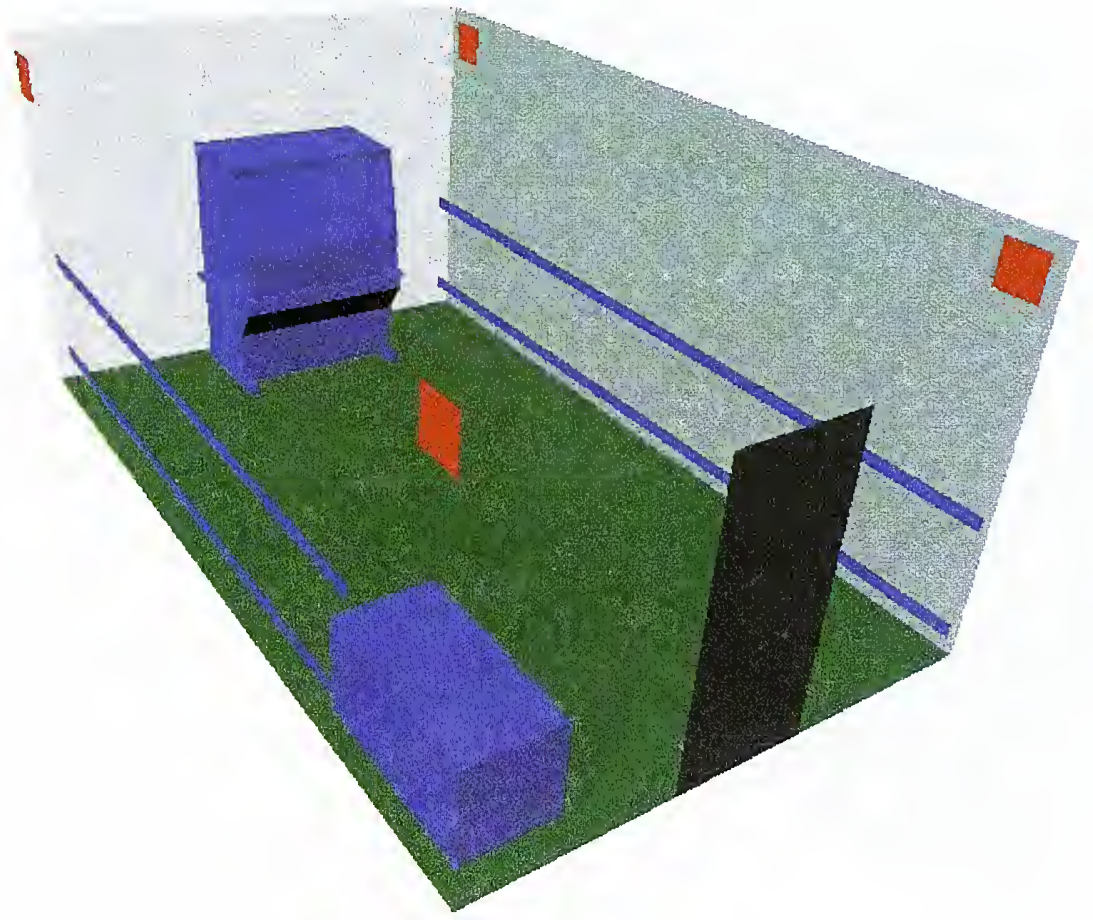

Figure 1.09 High Level Exhausts 


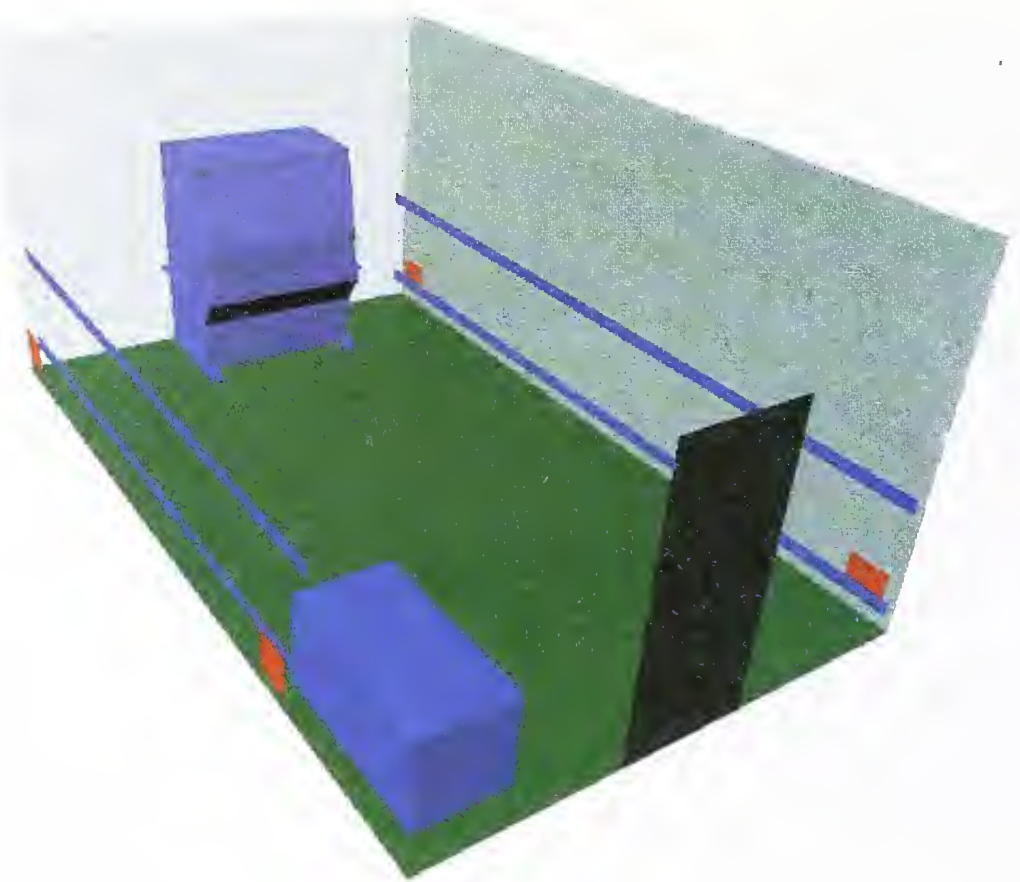

Figure 1.10 Low Level Exhausts

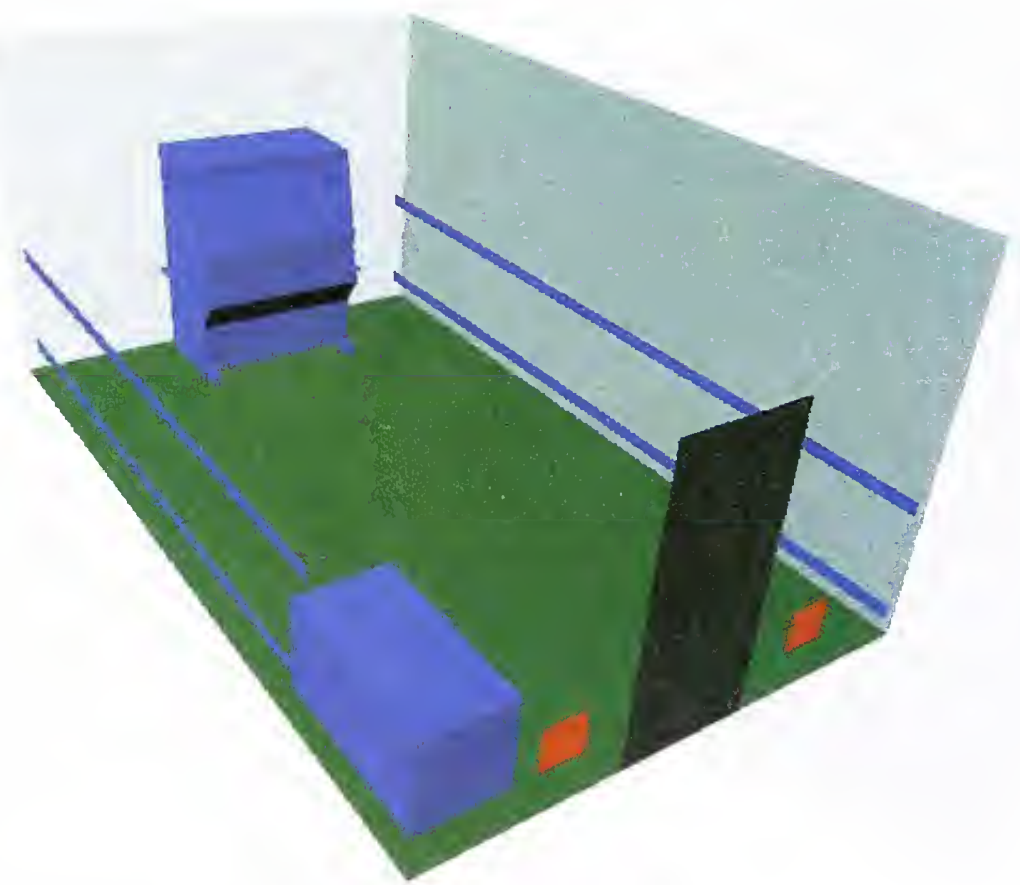

Figure 1.11 Low Level Exhausts on the Door Wall

- Room Air Change Rate: In addition to the basecase $\mathrm{ACH}$ of 15 , the following three other $\mathrm{ACH}$ values were considered: 5,10 , and $20 \mathrm{ACH}$. 
- Change Station Design and Status: Two different change station designs were considered, as outlined above. The two designs were intended to present both a passive and intrusive influence on the room volume. In the case of the former design, the station was also considered switched off.

- Pressurization of Room Relative to Corridor: The pressurization of a room is dependent on the difference between the supply and exhaust flow rates. A higher exhaust rate than supply leads to negative pressurization of the room; hence, makeup air is supplied to the room via available transfer mechanisms, namely door cracks. In this project, the amount of makeup air allowed through the door cracks was varied between $100 \mathrm{cfm}\left(4.72 \mathrm{e}-1 \mathrm{~m}^{3} / \mathrm{s}\right)$ into the room to $100 \mathrm{cfm}\left(4.72 \mathrm{e}-1 \mathrm{~m}^{3} / \mathrm{s}\right)$ out of the room.

- Orientation of Cage Racks in Room: The racks were considered both parallel to the long walls, and perpendicular to them. See figures 1.12 and 1.13

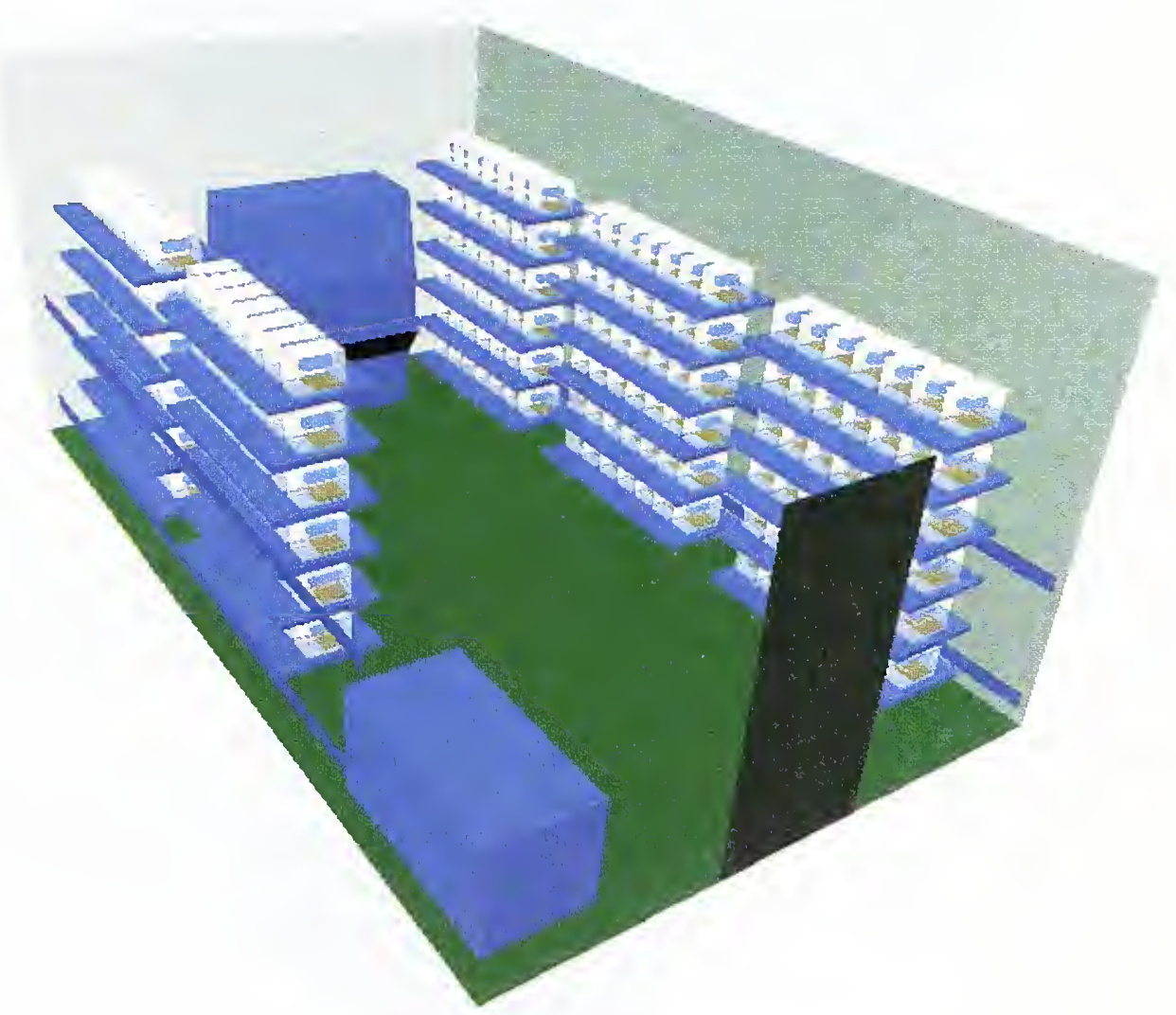

Figure 1.12 Racks Parallel to Side Walls 


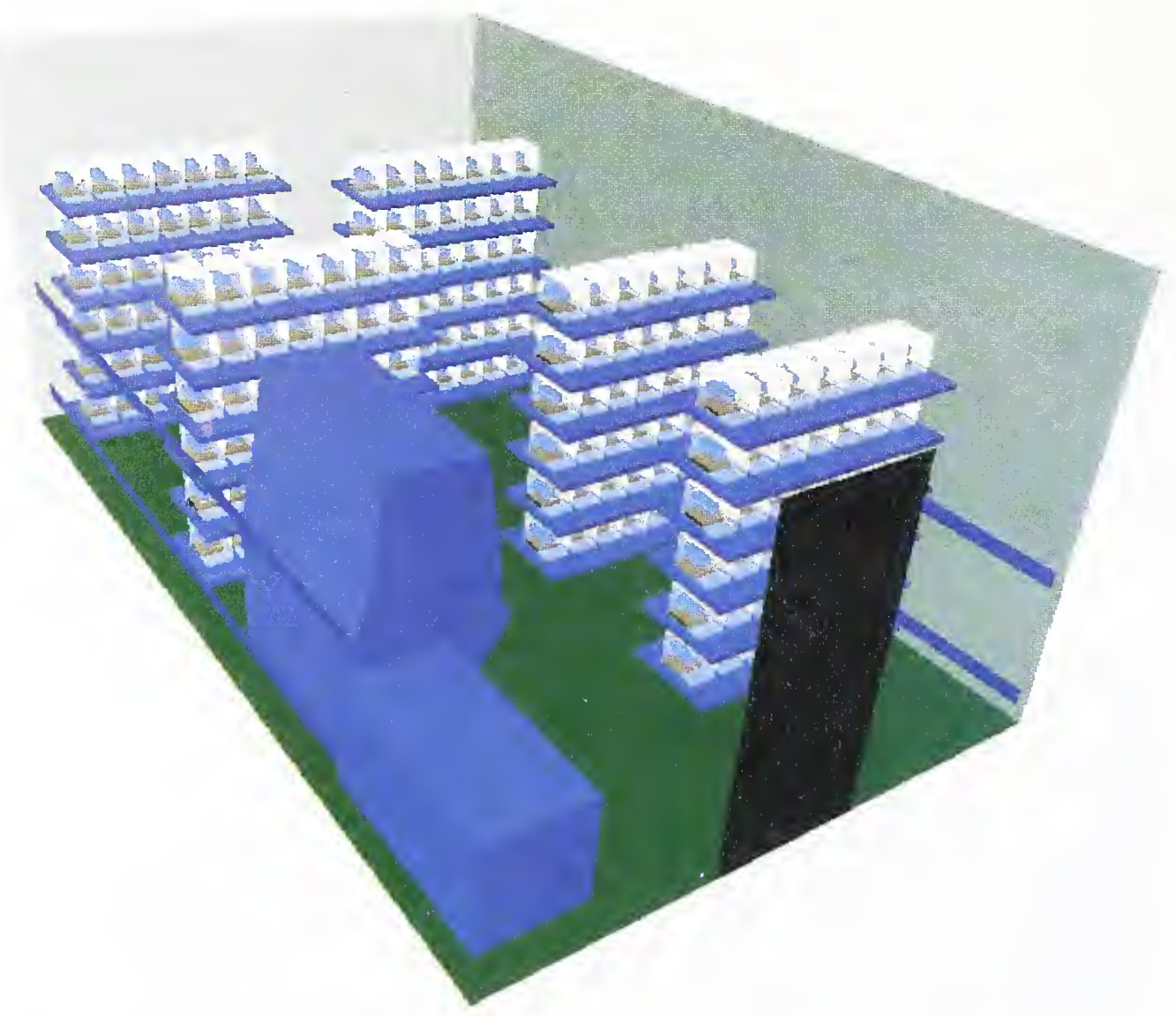

Figure 1.13 Racks Perpendicular to Side Walls

- Side Cracks of Cage Sealed Instead of Open: A series of cases was considered in which the side cracks of the cage were sealed, leaving the cage filter top as the only transfer mechanism. The main objective was to establish the relative increase in cage $\mathrm{NH}_{3}$ and $\mathrm{CO}_{2}$ levels when the secondary transfer mechanism was removed.

- Density of Cages: The number of cages per rack was considered as a parameter. In addition to the basecase value of 42 cages per rack, a double density 84 cages per rack was also considered, as was a reduced density of 28 cages per rack. See figures 1.14 and 1.15. 


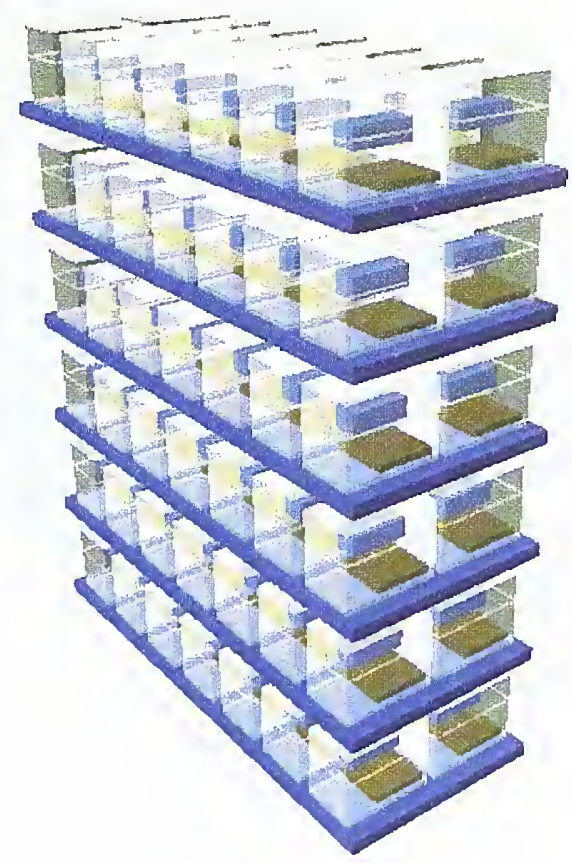

Figure 1.14 Double Density Rack

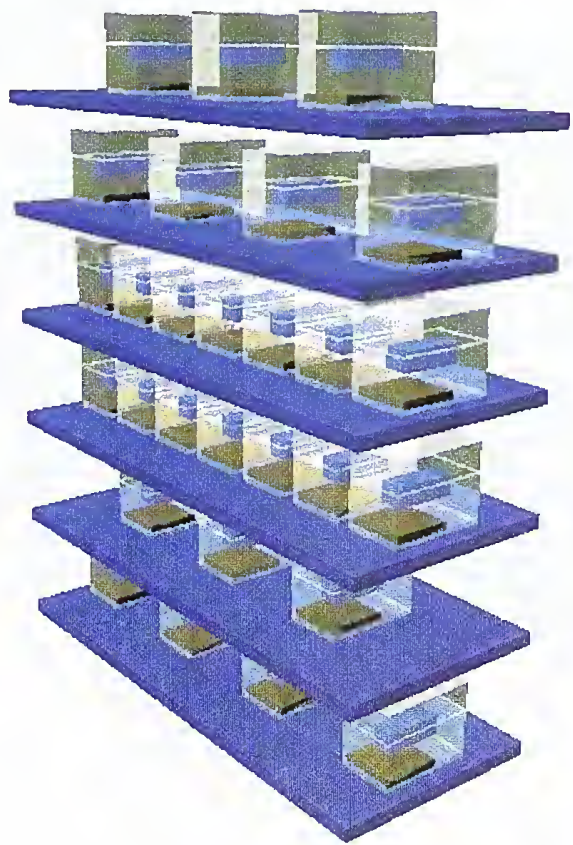

Figure 1.15 Reduced Density Rack

- Location of Change Station: The location of the change station was swapped with each of the racks in the room. See figures 1.16 to 1.20 . 


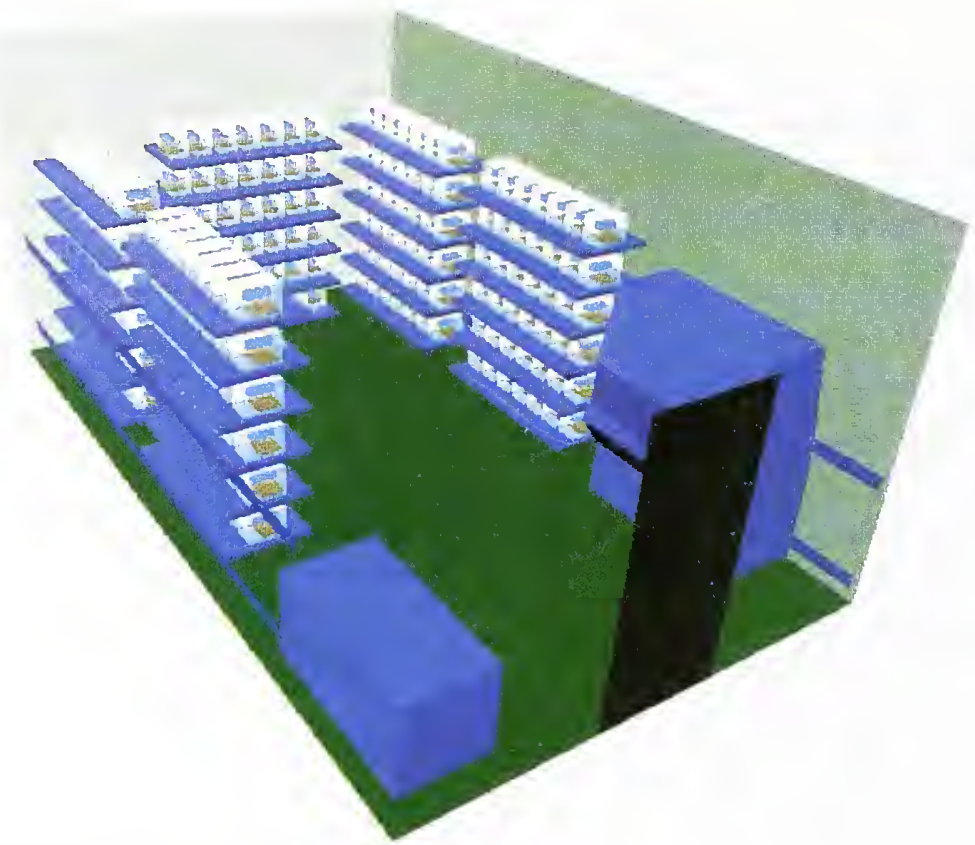

Figure 1.16 Change Station Swapped with Rack 1

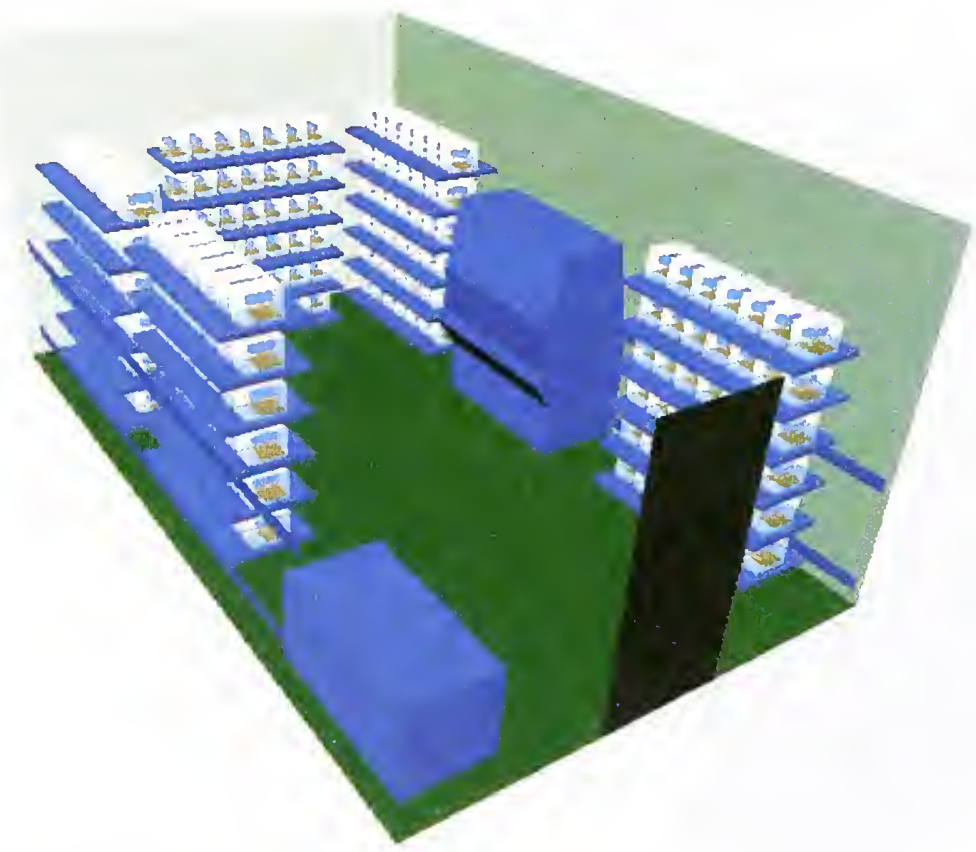

Figure 1.17 Change Station Swapped with Rack 2 


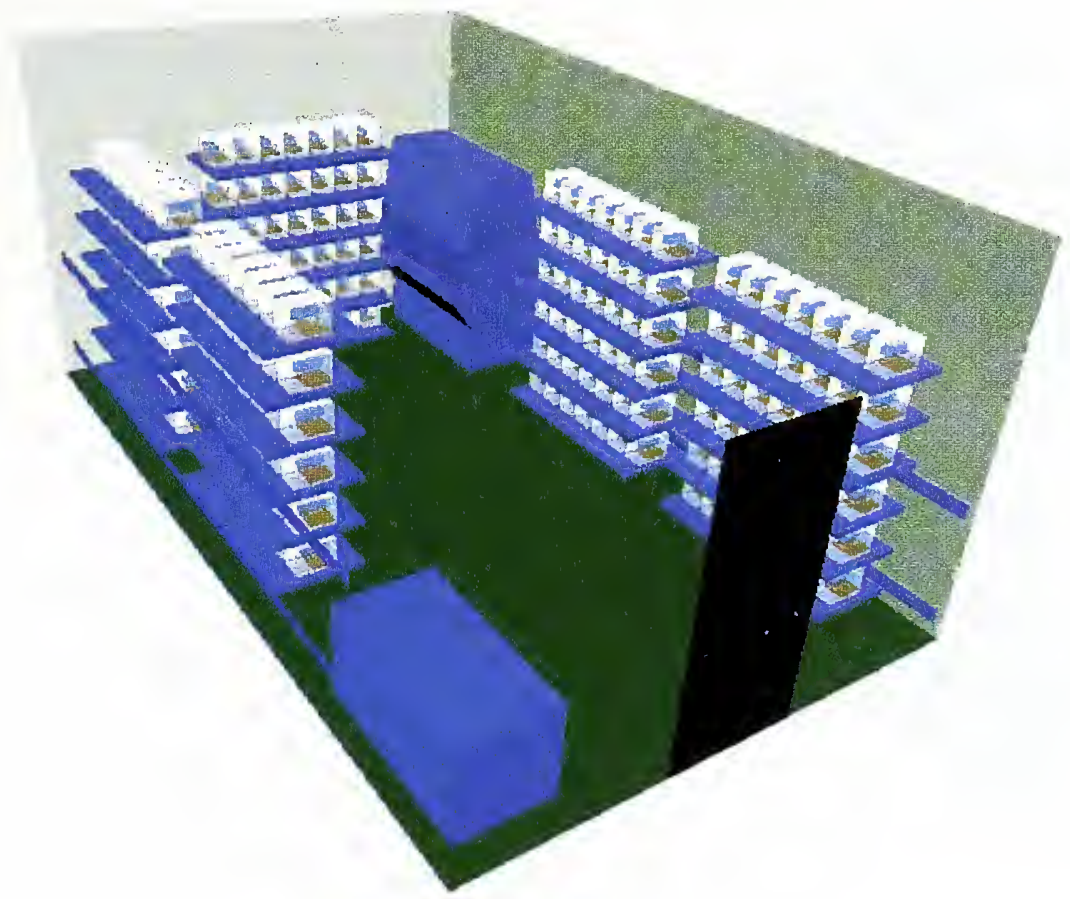

Figure 1.18 Change Station Swapped with Rack 3

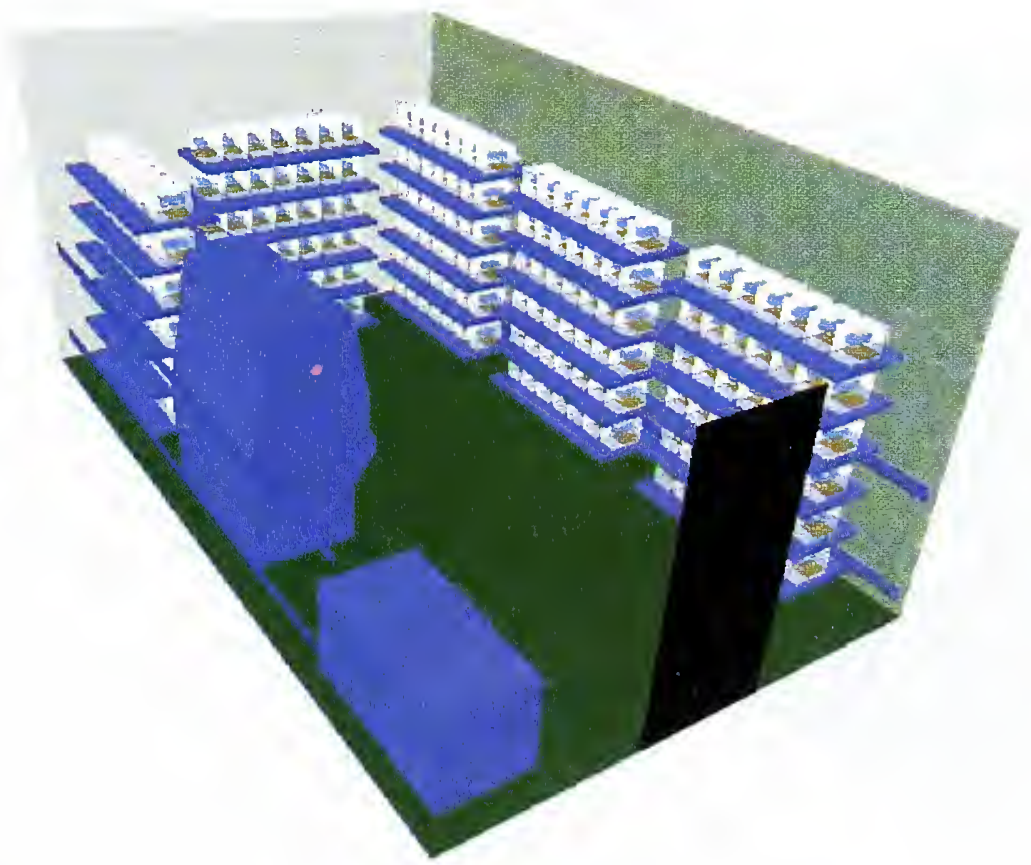

Figure 1.19 Change Station Swapped with Rack 4 


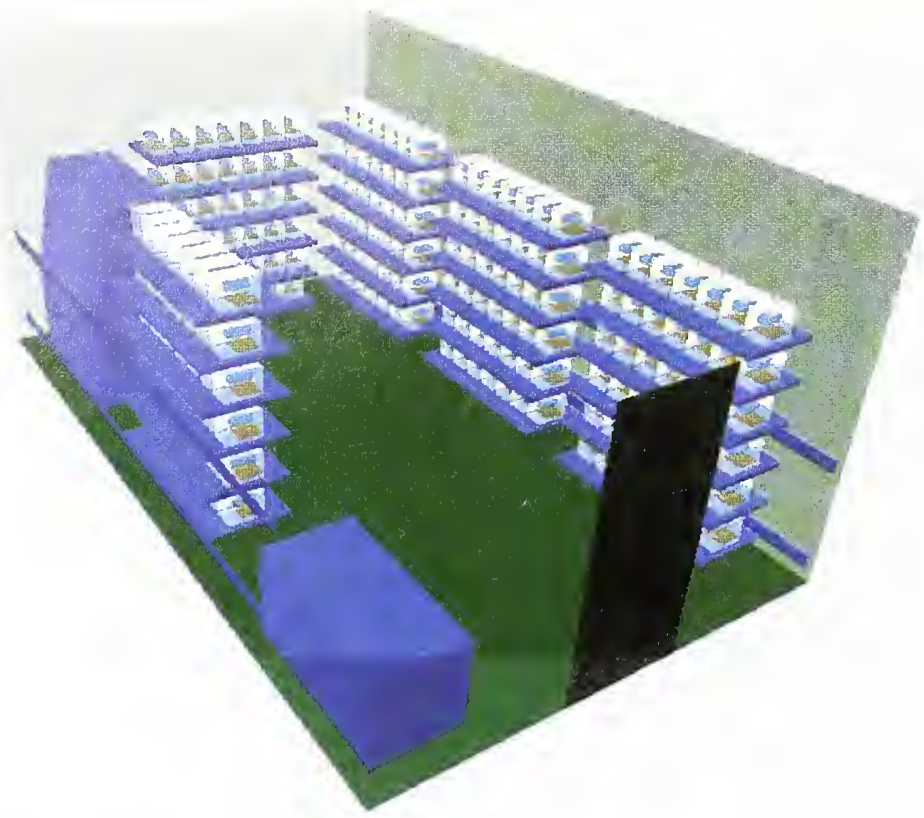

Figure 1.20 Change Station swapped with Rack 5

- Rack Grouping: In some series, the racks were considered to be along one wall only, rather than spread out through the room. See figure 1.21 .

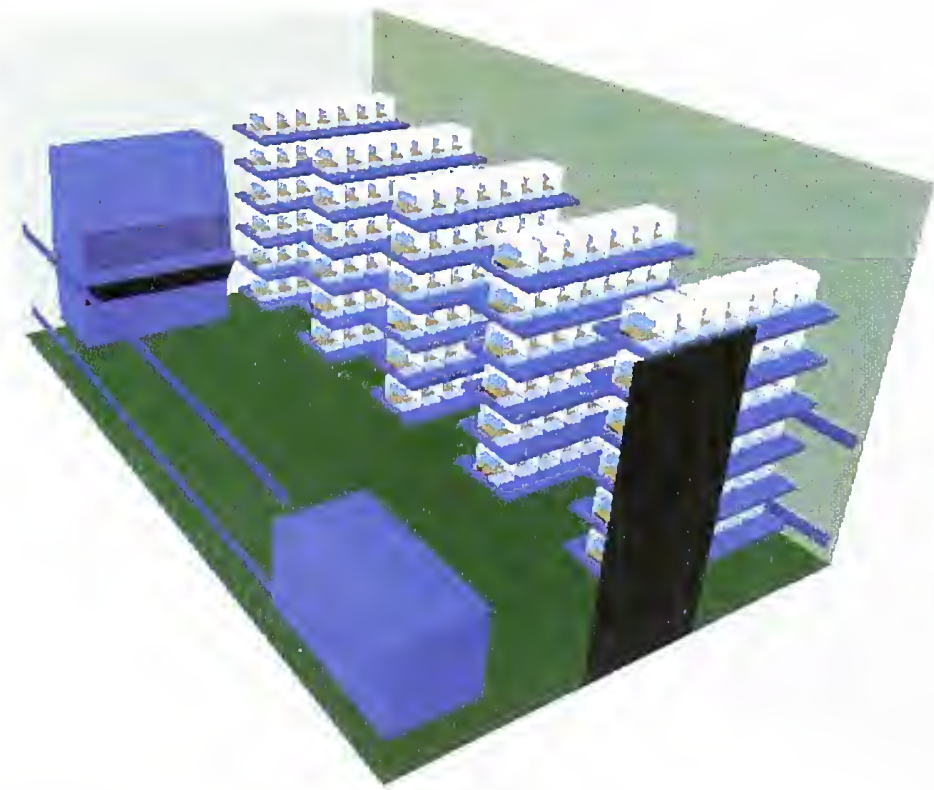

Figure 1.21 Racks on One Wall Only

- Room Width: Three runs were performed with the room width increased to 4.26m (14'0"). 
- Supply Air Temperature: In a few runs the supply air temperature was raised to $22.2^{\circ} \mathrm{C}$ $\left(72.0^{\circ} \mathrm{F}\right)$, at 49 percent $\mathrm{RH}$ which would cause the room and exhaust temperatures to be higher than the design value of the baseline case. 
Table 1.01 List of All Whole Room Runs

\begin{tabular}{|c|c|c|c|c|c|c|c|c|}
\hline Case Name & $\begin{array}{c}\text { Supply } \\
\text { Diffuser Type }\end{array}$ & $\begin{array}{c}\text { Exhaust } \\
\text { Location and } \\
\text { Number }\end{array}$ & $\begin{array}{c}\text { Change } \\
\text { Station } \\
\text { (Design/ } \\
\text { Status) } \\
\end{array}$ & $\begin{array}{c}\text { Rack } \\
\text { orientation }\end{array}$ & Rack density & $\begin{array}{l}\text { Pressure of } \\
\text { Room to } \\
\text { Corridor }\end{array}$ & $\begin{array}{c}\text { Supply } \\
\text { Temperature } \\
{ }^{\circ} \mathrm{C}\left({ }^{\circ} \mathrm{F}\right)\end{array}$ & Supply ACH \\
\hline Basecase & Radial & Ceiling (x2) & Thoren/ ON & Parallel & Single & Neg. $100 \mathrm{cfm}$ & $18.8(65.8)$ & 15 \\
\hline Case 02 & Radial & High (x4) & Thoren/ ON & Parallel & Single & Neg. $100 \mathrm{cfm}$ & $18.8(65.8)$ & 15 \\
\hline Case 03 & Radial & Low $(x 4)$ & Thoren/ ON & Parallel & Single & Neg. $100 \mathrm{cfm}$ & $18.8(65.8)$ & 15 \\
\hline Case 04 & Slot & Ceiling $(x 2)$ & Thoren/ ON & Parallel & Single & Neg. $100 \mathrm{cfm}$ & $18.8(65.8)$ & 15 \\
\hline Case 05 & Slot & High (x4) & Thoren/ ON & Parallel & Single & Neg. $100 \mathrm{cfm}$ & $18.8(65.8)$ & 15 \\
\hline Case 06 & Slot & Low $(x 4)$ & Thoren/ ON & Parallel & Single & Neg. $100 \mathrm{cfm}$ & $18.8(65.8)$ & 15 \\
\hline Case 07 & Low lnd & Ceiling $(x 2)$ & Thoren/ ON & Parallel & Single & Neg. $100 \mathrm{cfm}$ & $18.8(65.8)$ & 15 \\
\hline Case 08 & Low Ind & High $(x 4)$ & Thoren/ ON & Parallel & Single & Neg. 100cfm & $18.8(65.8)$ & 15 \\
\hline Case 09 & Low Ind & Low $(x 4)$ & Thoren/ ON & Parallel & Single & Neg. $100 \mathrm{cfm}$ & $18.8(65.8)$ & 15 \\
\hline Case 10 & Radial & Ceiling (x2) & Thoren/ OFF & Parallel & Single & Neg. $100 \mathrm{cfm}$ & $20.7(69.3)$ & 15 \\
\hline Case 11 & Radial & Low $(x 4)$ & Thoren/ OFF & Parallel & Single & Neg. $100 \mathrm{cfm}$ & $20.7(69.3)$ & 15 \\
\hline Case 12 & Slot & Ceiling (x2) & Thoren/ OFF & Parallel & Single & Neg. $100 \mathrm{cfm}$ & $20.7(69.3)$ & 15 \\
\hline Case 13 & Slot & Low $(x 4)$ & Thoren/ OFF & Parallel & Single & Neg. $100 \mathrm{cfm}$ & $20.7(69.3)$ & 15 \\
\hline Case 14 & Low Ind & Ceiling (x2) & Thoren/ OFF & Parallel & Single & Neg. 100cfm & $20.7(69.3)$ & 15 \\
\hline Case 15 & Low lnd & Low $(x 4)$ & Thoren/ OFF & Parallel & Single & Neg. 100cfm & $20.7(69.3)$ & 15 \\
\hline Case 16 & Radial & Ceiling (x2) & Thoren/ ON & Parallel & Single & Pos. $100 \mathrm{cfm}$ & $18.8(65.8)$ & 15 \\
\hline Case 17 & Slot & Ceiling (x2) & Thoren/ ON & Parallel & Single & Pos. $100 \mathrm{cfm}$ & $18.8(65.8)$ & 15 \\
\hline Case 18 & Low Ind & Ceiling (x2) & Thoren/ ON & Parallel & Single & Pos. $100 \mathrm{cfm}$ & $18.8(65.8)$ & 15 \\
\hline Case 19 & Radial & Ceiling $(\mathrm{x} 2)$ & Thoren/ ON & Perpendicular & Single & Neg. $100 \mathrm{cfm}$ & $18.8(65.8)$ & 15 \\
\hline Case 20 & Slot & Ceiling (x2) & Thoren/ ON & Perpendicular & Single & Neg. $100 \mathrm{cfm}$ & $18.8(65.8)$ & 15 \\
\hline Case 21 & Low Ind & Ceiling (x2) & Thoren/ ON & Perpendicular & Single & Neg. 100cfm & $18.8(65.8)$ & 15 \\
\hline Case 22 & Radial & Low $(x 4)$ & Thoren/ ON & Perpendicular & Single & Neg. $100 \mathrm{cfm}$ & $18.8(65.8)$ & 15 \\
\hline Case 23 & Slot & Low $(x 4)$ & Thoren/ ON & Perpendicular & Single & Neg. $100 \mathrm{cfm}$ & $18.8(65.8)$ & 15 \\
\hline Case 24 & Low Ind & Low $(x 4)$ & Thoren/ ON & Perpendicular & Single & Neg. 100cfm & $18.8(65.8)$ & 15 \\
\hline Case $25 *$ & Radial & Ceiling $(\mathrm{x} 2)$ & Thoren/ ON & Parallel & Single & Neg. $100 \mathrm{cfm}$ & $18.8(65.8)$ & 15 \\
\hline Case 26 * & Slot & Ceiling (x2) & Thoren/ ON & Parallel & Single & Neg. $100 \mathrm{cfm}$ & $18.8(65.8)$ & 15 \\
\hline Case $27 *$ & Low Ind & Ceiling (x2) & Thoren/ ON & Parallel & Single & Neg. 100cfm & $18.8(65.8)$ & 15 \\
\hline Case 28 & Low Ind & Low $(x 4)$ & Thoren/ ON & Parallel & Single & Neg. $100 \mathrm{cfm}$ & $16.8(62.2)$ & 10 \\
\hline Case 29 & Low lnd & Low $(x 4)$ & Thoren/ ON & Parallel & Single & Neg. 100cfm & $11.0(51.8)$ & 5 \\
\hline Case 30 & Low lnd & Low $(x 4)$ & Thoren/ ON & Parallel & Single & Neg. $100 \mathrm{cfm}$ & $19.8(67.6)$ & 20 \\
\hline Case 31 & Radial & Low $(x 4)$ & Thoren/ ON & Perpendicular & Double & Neg. $100 \mathrm{cfm}$ & $17.5(63.5)$ & 15 \\
\hline Case 32 & Slot & Low $(x 4)$ & Thoren/ ON & Perpendicular & Double & Neg. $100 \mathrm{cfm}$ & $17.5(63.5)$ & 15 \\
\hline Case 33 & Low Ind & Low $(x 4)$ & Thoren/ ON & Perpendicular & Double & Neg. $100 \mathrm{cfm}$ & $17.5(63.5)$ & 15 \\
\hline Case 34 & Radial & Ceiling (x2) & Thoren/ON & Perpendicular & Double & Neg. $100 \mathrm{cfm}$ & $17.5(63.5)$ & 15 \\
\hline Case 35 & Slot & Ceiling $(x 2)$ & Thoren/ ON & Perpendicular & Double & Neg. 100cfm & $17.5(63.5)$ & 15 \\
\hline Case 36 & Low Ind & Ceiling (x2) & Thoren/ ON & Perpendicular & Double & Neg. $100 \mathrm{cfm}$ & $17.5(63.5)$ & 15 \\
\hline Case 37 & Radial & $\begin{array}{c}\text { Ceiling (x } 1) \\
/ \text { Low (x4) } \\
\text { (Mass flow in } \\
50 / 50 \text { split) } \\
\end{array}$ & Thoren/ ON & Parallel & Single & Neg. $100 \mathrm{cfm}$ & $18.8(65.8)$ & 15 \\
\hline Case 38 & Slot & $\begin{array}{l}\text { Ceiling (x1) } \\
/ \text { Low (x4) } \\
\text { (Mass flow in } \\
50 / 50 \text { split) }\end{array}$ & Thoren/ ON & Parallel & Single & Neg. $100 \mathrm{cfm}$ & $18.8(65.8)$ & 15 \\
\hline Case 39 & Low Ind & $\begin{array}{l}\text { Ceiling (x I) } \\
\text { / Low (x4) } \\
\text { (Mass flow in } \\
50 / 50 \text { split) } \\
\end{array}$ & Thoren/ ON & Parallel & Single & Neg. $100 \mathrm{cfm}$ & $18.8(65.8)$ & 15 \\
\hline Case 40 & Radial & Ceiling (x4) & Thoren/ ON & Parallel & Single & Neg. $100 \mathrm{cfm}$ & $18.8(65.8)$ & 15 \\
\hline Case 41 & Slot & Ceiling $(x 4)$ & Thoren/ ON & Parallel & Single & Neg. $100 \mathrm{cfm}$ & $18.8(65.8)$ & 15 \\
\hline Case 42 & Low Ind & Ceiling (x4) & Thoren/ ON & Parallel & Single & Neg. $100 \mathrm{cfm}$ & $18.8(65.8)$ & 15 \\
\hline
\end{tabular}


Volume II - Section I - Introduction

Page II - 21

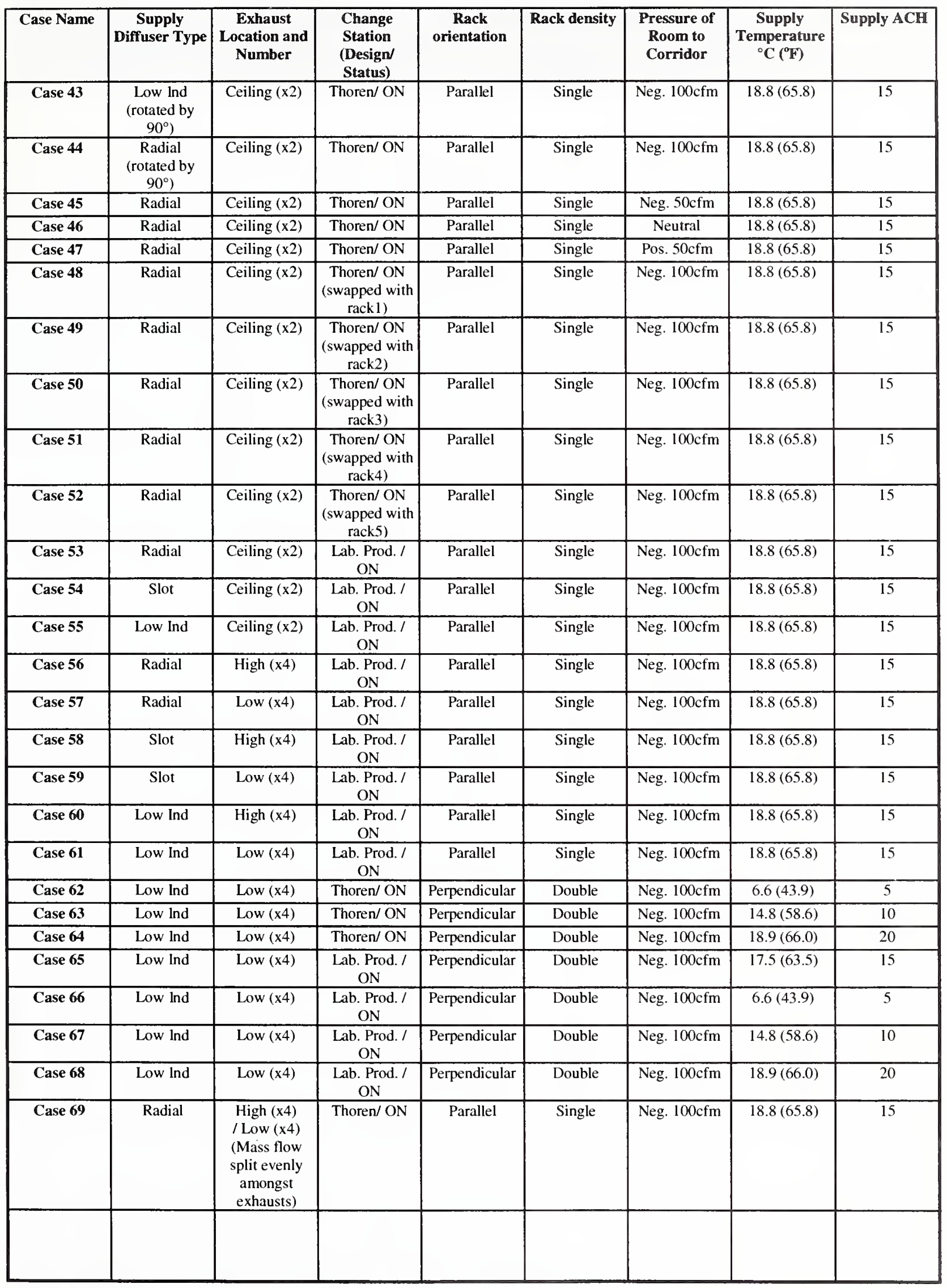




\begin{tabular}{|c|c|c|c|c|c|c|c|c|}
\hline Case Name & \begin{tabular}{c|} 
Supply \\
Diffuser Type
\end{tabular} & $\begin{array}{c}\text { Exhaust } \\
\text { Location and } \\
\text { Number }\end{array}$ & $\begin{array}{l}\text { Change } \\
\text { Station } \\
\text { (Design/ } \\
\text { Status) } \\
\end{array}$ & $\begin{array}{c}\text { Rack } \\
\text { orientation }\end{array}$ & Rack density & $\begin{array}{l}\text { Pressure of } \\
\text { Room to } \\
\text { Corridor }\end{array}$ & $\begin{array}{c}\text { Supply } \\
\text { Temperature } \\
{ }^{\circ} \mathrm{C}\left({ }^{\circ} \mathrm{F}\right)\end{array}$ & Supply ACH \\
\hline Case 70 & Slot & $\begin{array}{l}\text { High }(x 4) \\
\text { / Low }(x 4) \\
\text { (Mass flow } \\
\text { split evenly } \\
\text { amongst } \\
\text { exhausts) }\end{array}$ & Thoren/ ON & Parallel & Single & Neg. $100 \mathrm{cfm}$ & $18.8(65.8)$ & 15 \\
\hline Case 71 & Low lnd & $\begin{array}{l}\text { High }(x 4) \\
\text { / Low }(x 4) \\
\text { (Mass flow } \\
\text { split evenly } \\
\text { amongst } \\
\text { exhausts) }\end{array}$ & Thoren/ ON & Parallel & Single & Neg. $100 \mathrm{cfm}$ & $18.8(65.8)$ & 15 \\
\hline Case 72 & Radial & $\begin{array}{l}\text { High }(\times 4) \\
\text { / Low }(\times 2) \\
\text { (Mass flow } \\
\text { split evenly } \\
\text { amongst } \\
\text { exhausts) } \\
\end{array}$ & Thoren/ ON & Parallel & Single & Neg. $100 \mathrm{cfm}$ & $18.8(65.8)$ & 15 \\
\hline Case 73 & Slot & $\begin{array}{l}\text { High }(x 4) \\
\text { / Low }(\times 2) \\
\text { (Mass flow } \\
\text { split evenly } \\
\text { amongst } \\
\text { exhausts) } \\
\end{array}$ & Thoren/ ON & Parallel & Single & Neg. $100 \mathrm{cfm}$ & $18.8(65.8)$ & 15 \\
\hline Case 74 & Low Ind & $\begin{array}{l}\text { High (x4) } \\
\text { / Low (x2) } \\
\text { (Mass flow } \\
\text { split evenly } \\
\text { amongst } \\
\text { exhausts) } \\
\end{array}$ & Thoren/ ON & Parallel & Single & Neg. $100 \mathrm{cfm}$ & $18.8(65.8)$ & 15 \\
\hline Case 75 & Radial & $\begin{array}{l}\text { Ceiling }(\mathrm{x} 1) \\
/ \text { Low }(\mathrm{x} 4) \\
\text { (Mass flow in } \\
50 / 50 \text { split) }\end{array}$ & $\begin{array}{l}\text { Lab. Prod. I } \\
\text { ON }\end{array}$ & Parallel & Single & Neg. $100 \mathrm{cfm}$ & $18.8(65.8)$ & 15 \\
\hline Case $76 * *$ & Radial & Ceiling (x2) & Thoren/ ON & Parallel & Single & Neg. $100 \mathrm{cfm}$ & $19.2(66.6)$ & 15 \\
\hline Case $77 * *$ & Slot & Ceiling $(\times 2)$ & Thoren/ ON & Parallel & Single & Neg. $100 \mathrm{cfm}$ & $19.2(66.5)$ & 15 \\
\hline Case $78 * *$ & Low Ind & Ceiling $(\mathrm{x} 2)$ & Thoren/ ON & Parallel & Single & Neg. 100cfm & $19.2(66.6)$ & 15 \\
\hline Case 79 & Radial & $\begin{array}{c}2 \text { Door } \\
\text { exhausts }\end{array}$ & Thoren/ ON & Parallel & Single & Neg. $100 \mathrm{cfm}$ & $18.8(65.8)$ & 15 \\
\hline Case 80 & Slot & $\begin{array}{c}2 \text { Door } \\
\text { exhausts }\end{array}$ & Thoren/ ON & Parallel & Single & Neg. $100 \mathrm{cfm}$ & $18.8(65.8)$ & 15 \\
\hline Case 81 & Low lnd & $\begin{array}{l}2 \text { Door } \\
\text { exhausts }\end{array}$ & Thoren/ ON & Parallel & Single & Neg. I00cfm & $18.8(65.8)$ & 15 \\
\hline Case 82 & Radial & $\begin{array}{c}2 \text { Door } \\
\text { exhausts }\end{array}$ & Thoren/ ON & Parallel & Single & Neg. $100 \mathrm{cfm}$ & $18.8(65.8)$ & 15 \\
\hline Case 83 & Slot & $\begin{array}{c}2 \text { Door } \\
\text { exhausts }\end{array}$ & Thoren/ ON & Perpendicular & Double & Neg. $100 \mathrm{cfm}$ & $17.5(63.5)$ & 15 \\
\hline Case 84 & Low Ind & $\begin{array}{c}2 \text { Door } \\
\text { exhausts }\end{array}$ & Thoren/ ON & Perpendicular & Double & Neg. $100 \mathrm{cfm}$ & $17.5(63.5)$ & 15 \\
\hline Case 85 & Radial & Low $(x 4)$ & $\begin{array}{c}\text { Lab. Prod. / } \\
\text { ON }\end{array}$ & Perpendicular & Double & Neg. $100 \mathrm{cfm}$ & $17.5(63.5)$ & 15 \\
\hline Case 86 & Slot & Low $(x 4)$ & $\begin{array}{l}\text { Lab. Prod. I } \\
\text { ON }\end{array}$ & Perpendicular & Double & Neg. $100 \mathrm{cfm}$ & $17.5(63.5)$ & 15 \\
\hline Case 88 & Radial & Low $(x 4)$ & Thoren/ ON & Parallel & Reduced & Neg. $100 \mathrm{cfm}$ & $19.2(66.6)$ & 15 \\
\hline Case 88 & Slot & Low $(x 4)$ & Thoren/ ON & Parallel & Reduced & Neg. 100cfm & $19.2(66.6)$ & 15 \\
\hline Case 89 & Low Ind & Low $(x 4)$ & Thoren/ ON & Parallel & Reduced & Neg. $100 \mathrm{cfm}$ & $19.2(66.6)$ & 15 \\
\hline Case 90 & Radial & Ceiling (x2) & Thoren/ ON & $\begin{array}{l}\text { Perpendicular } \\
\text { all } 5 \text { on } 1 \text { wall }\end{array}$ & Single & Neg. $100 \mathrm{cfm}$ & $18.8(65.8)$ & 15 \\
\hline Case 91 & Slot & Ceiling (x2) & Thoren/ ON & $\begin{array}{l}\text { Perpendicular } \\
\text { all } 5 \text { on } 1 \text { wall }\end{array}$ & Single & Neg. 100cfm & $18.8(65.8)$ & 15 \\
\hline Case 92 & Low Ind & Ceiling (x2) & Thoren/ ON & $\begin{array}{l}\text { Perpendicular } \\
\text { all } 5 \text { on } 1 \text { wall } \\
\end{array}$ & Single & Neg. $100 \mathrm{cfm}$ & $18.8(65.8)$ & 15 \\
\hline & & & & & & & & \\
\hline
\end{tabular}




\begin{tabular}{|c|c|c|c|c|c|c|c|c|}
\hline Case Name & \begin{tabular}{|c|} 
Supply \\
Diffuser Type
\end{tabular} & $\begin{array}{c}\text { Exhaust } \\
\text { Location and } \\
\text { Number }\end{array}$ & $\begin{array}{c}\text { Change } \\
\text { Station } \\
\text { (Design/ } \\
\text { Status) }\end{array}$ & $\begin{array}{c}\text { Rack } \\
\text { orientation }\end{array}$ & Rack density & $\begin{array}{l}\text { Pressure of } \\
\text { Room to } \\
\text { Corridor }\end{array}$ & $\begin{array}{c}\text { Supply } \\
\text { Temperature } \\
{ }^{\circ} \mathrm{C}\left({ }^{\circ} \mathrm{F}\right)\end{array}$ & Supply ACH \\
\hline Case 93 & Radial & Ceiling (x2) & Thoren/ON & $\begin{array}{l}\text { Perpendicular } \\
\text { all } 5 \text { on } 1 \text { wall }\end{array}$ & Double & Neg. $100 \mathrm{cfm}$ & $17.5(63.5)$ & 15 \\
\hline Case 94 & Slot & Ceiling (x2) & Thoren/ ON & $\begin{array}{l}\text { Perpendicular } \\
\text { all } 5 \text { on } 1 \text { wall }\end{array}$ & Double & Neg. $100 \mathrm{cfm}$ & $17.5(63.5)$ & 15 \\
\hline Case 95 & Low Ind & Ceiling (x2) & Thoren/ ON & $\begin{array}{l}\text { Perpendicular } \\
\text { all } 5 \text { on } 1 \text { wall }\end{array}$ & Double & Neg. $100 \mathrm{cfm}$ & $17.5(63.5)$ & 15 \\
\hline Case 96 & Radial & Ceiling (x2) & $\begin{array}{l}\text { Lab. Prod. I } \\
\text { ON }\end{array}$ & $\begin{array}{l}\text { Perpendicular } \\
\text { all } 5 \text { on } 1 \text { wall }\end{array}$ & Double & Neg. $100 \mathrm{cfm}$ & $17.5(63.5)$ & 15 \\
\hline Case 97 & Slot & Ceiling (x2) & $\begin{array}{c}\text { Lab. Prod. I } \\
\text { ON }\end{array}$ & $\begin{array}{l}\text { Perpendicular } \\
\text { all } 5 \text { on } 1 \text { wall }\end{array}$ & Double & Neg. $100 \mathrm{cfm}$ & $17.5(63.5)$ & 15 \\
\hline Case 98 & Low Ind & Ceiling (x2) & $\begin{array}{c}\text { Lab. Prod. I } \\
\text { ON }\end{array}$ & $\begin{array}{l}\text { Perpendicular } \\
\text { all } 5 \text { on } 1 \text { wall }\end{array}$ & Double & Neg. $100 \mathrm{cfm}$ & $17.5(63.5)$ & 15 \\
\hline Case $99 * * *$ & Radial & Ceiling (x2) & Thoren/ ON & Parallel & Single & Neg. $100 \mathrm{cfm}$ & $22.2(72.0)$ & 15 \\
\hline Case $100 * * *$ & Radial & Low $(x 4)$ & Thoren/ ON & Parallel & Single & Neg. $100 \mathrm{cfm}$ & $22.2(72.0)$ & 15 \\
\hline Case $101 * * *$ & Low Ind & Low $(x 4)$ & $\begin{array}{l}\text { Lab. Prod. I } \\
\text { ON }\end{array}$ & Perpendicular & Double & Neg. $100 \mathrm{cfm}$ & $22.2(72.0)$ & 5 \\
\hline
\end{tabular}

* Sealed cages instead of open cages

** Room 4.26m (14'0") wide instead of 3.66m (12'0”)

*** Supply air temperature fixed at $22.2^{\circ} \mathrm{C}\left(72.0^{\circ} \mathrm{F}\right)$. In all other cases, the exhaust temperature was set to be $22.2^{\circ} \mathrm{C}\left(72.0^{\circ} \mathrm{F}\right)$ by setting the supply air temperature appropriately. 
Table 1.02 Cases Cross-Referenced Against Supply Diffuser Type

\begin{tabular}{|c|c|}
\hline Supply Diffuser Type & Case Number \\
\hline Radial & Basecase, $02-03,10-11,16,19,22,25,31$, \\
& $34,37,40,44 *, 45-53,56-57,69,72,75-$ \\
& $76,79,82,85,87,90,93,96,99-100$ \\
\hline Slot & $04-06,12-13,17,20,23,26,32,35,38,41$, \\
& $54,58-59,70,73,77,80,83,86,88,91$, \\
& 94,97 \\
\hline Low Induction & $07-09,14-15,18,21,24,27-30,33,36,39$, \\
& $42,43^{*}, 55,60-68,71,74,78,81,84,89$, \\
\hline
\end{tabular}

* Indicates that diffuser rotated by $90^{\circ}$

Table 1.03 Cases Cross-Referenced Against Exhaust Location and Number

\begin{tabular}{|c|c|}
\hline Exhaust Location and Number & Case Number \\
\hline Ceiling (x2) & $\begin{array}{c}\text { Basecase, 04, 07, 10, 12,14, 16-21, 25-27, } \\
\text { (x-36, 43-55, 76-78,90-99 }\end{array}$ \\
\hline High (x4) & $02,05,08,56,58,60$ \\
\hline Low (x4) & $03,06,09,11,13,15,22-24,28-33,57,59$, \\
& $61-68,85-89,100-101$ \\
\hline $\begin{array}{c}\text { Ceiling (x1) / Low (x4) } \\
\text { (Mass flow in 50/50 split) }\end{array}$ & $37-39,75$ \\
\hline Ceiling (x4) & $40-42$ \\
\hline $\begin{array}{c}\text { High (x4)/ Low (x4) } \\
\text { (Mass flow split evenly amongst } \\
\text { exhausts) }\end{array}$ & $69-71$ \\
\hline $\begin{array}{c}\text { High (x4)/ Low (x2) } \\
\text { (Mass flow split evenly amongst } \\
\text { exhausts) }\end{array}$ & $72-74$ \\
\hline 2 Door Exhausts & \\
\hline
\end{tabular}


Table 1.04 Cases Cross-Referenced Against Change Station Design and Status

\begin{tabular}{|c|c|}
\hline Change Station & Case Number \\
\hline ON* $^{*}$ & Basecase $-09,16-101$ \\
\hline OFF $^{*}$ & $10-15$ \\
\hline Thoren Design & Basecase $-52 * *, 62-64,69-74,76-84,87-$ \\
& $95,99-100$ \\
\hline Laboratory Products Design & $53-61,65-68,75,85-86,96-98,101$ \\
\hline
\end{tabular}

* Change in status was only considered for Thoren design change station.

**Cases 10 to 15 had Thoren design change station switched off.

Table 1.05 Cases Cross-Referenced Against Pressurization of Room Relative to Corridor

\begin{tabular}{|c|c|}
\hline Pressurization of Room to Corridor & Case Number \\
\hline Neg. $\mathbf{1 0 0} \mathbf{c f m}$ & basecase $-15,19-44,48-101$ \\
\hline Pos. 100 cfm & $16-18$ \\
\hline Neg. 50cfm & 45 \\
\hline Neutral & 46 \\
\hline Pos. 50 cfm & 47 \\
\hline
\end{tabular}

Table 1.06 Cases Cross-Referenced Against Pressurization of Room Relative to Corridor

\begin{tabular}{|c|c|}
\hline Rack Orientation & Case Number \\
\hline Parallel & basecase $-18,25-30,37-61^{* * *}, 69-82,87-$ \\
& $89,99-100$ \\
\hline Perpendicular & $19-24,31-36,62-68,83-86,101$ \\
\hline Perpendicular All 5 on 1 Wall & $90-98$ \\
\hline
\end{tabular}

*** Cases 48 to 52 had change station swapped with each of five racks.

Table 1.07 Cases Cross-Referenced Against Status of Cage Side Cracks

\begin{tabular}{|c|c|}
\hline Status of Cage Side Cracks & Case Number \\
\hline Open & basecase $-24,28-101$ \\
\hline Sealed & $25-27$ \\
\hline
\end{tabular}


Table 1.08 Cases Cross-Referenced Against Density of Cages in Rack

\begin{tabular}{|c|c|}
\hline Density of Cages in Rack & Case Number \\
\hline Single & basecase $-30,37-61,69-82,90-92,99-100$ \\
\hline Double & $31-36,62-68,83-86,93-98,101$ \\
\hline Reduced & $87-89$ \\
\hline
\end{tabular}

Table 1.09 Cases Cross-Referenced Against Room Air Change Rate

\begin{tabular}{|c|c|}
\hline Room Air Change Rate (ACH) & Case Number \\
\hline $\mathbf{5}$ & $29,62,66,101$ \\
\hline $\mathbf{1 0}$ & $28,63,67$ \\
\hline $\mathbf{1 5}$ & basecase $-27,31-61,65,69-100$ \\
\hline $\mathbf{2 0}$ & $30,64,68$ \\
\hline
\end{tabular}

Table 1.10 Cases Cross-referenced Against Room Width

\begin{tabular}{|c|c|}
\hline Room Width (ft) & Case Number \\
\hline $\mathbf{1 2}$ & basecase $-75,79-101$ \\
\hline $\mathbf{1 4}$ & $76-78$ \\
\hline
\end{tabular}

Table 1.11 Cases Cross-Referenced Against Supply Temperature (1)

\begin{tabular}{|c|c|}
\hline Supply Temperature & Case Number \\
\hline $\begin{array}{c}\text { Supply Temperature set such that air is } \\
\text { at } \mathbf{2 2 . 2 2}^{\circ} \mathbf{C ~}\left(\mathbf{7 2}^{\circ} \mathbf{F}\right) \text { at Exhaust }\end{array}$ & basecase -98 \\
\hline $\mathbf{2 2 . 2 2}^{\circ} \mathbf{C}$ & $99-101$ \\
\hline
\end{tabular}


Table 1.12 Cases Cross-Referenced Against Supply Temperature (2)

\begin{tabular}{|c|c|}
\hline Supply Temperature & Case Number \\
\hline $\mathbf{6 . 6 0}$ & 62,66 \\
\hline $\mathbf{1 1 . 0 0}$ & 29 \\
\hline $\mathbf{1 4 . 8 0}$ & 63,67 \\
\hline $\mathbf{1 6 . 8 0}$ & 28 \\
\hline $\mathbf{1 7 . 5 0}$ & $31-36,65,83-86,93-98$ \\
\hline $\mathbf{1 8 . 8 0}$ & basecase $-09,16-27,37-61,69-75,79-82$, \\
& $90-92$ \\
\hline $\mathbf{1 8 . 9 0}$ & 64,68 \\
\hline $\mathbf{1 9 . 2 0}$ & $76-78,87-89$ \\
\hline $\mathbf{1 9 . 8 0}$ & 30 \\
\hline $\mathbf{2 0 . 7 0}$ & $10-15$ \\
\hline $\mathbf{2 2 . 2 2} * * * *$ & $99-101$ \\
\hline
\end{tabular}

**** In these cases, the supply air temperature was set to $22.22^{\circ} \mathrm{C}\left(72^{\circ} \mathrm{F}\right)$. In all other cases, the exhaust air temperature was set to be $22.22{ }^{\circ} \mathrm{C}\left(72{ }^{\circ} \mathrm{F}\right)$ by setting the supply air temperature appropriately. 
2. SUMMARY OF RESULTS OF WHOLE ROOM RUNS

Discussion of these figures and tables can be found in volume I, section 3.1.

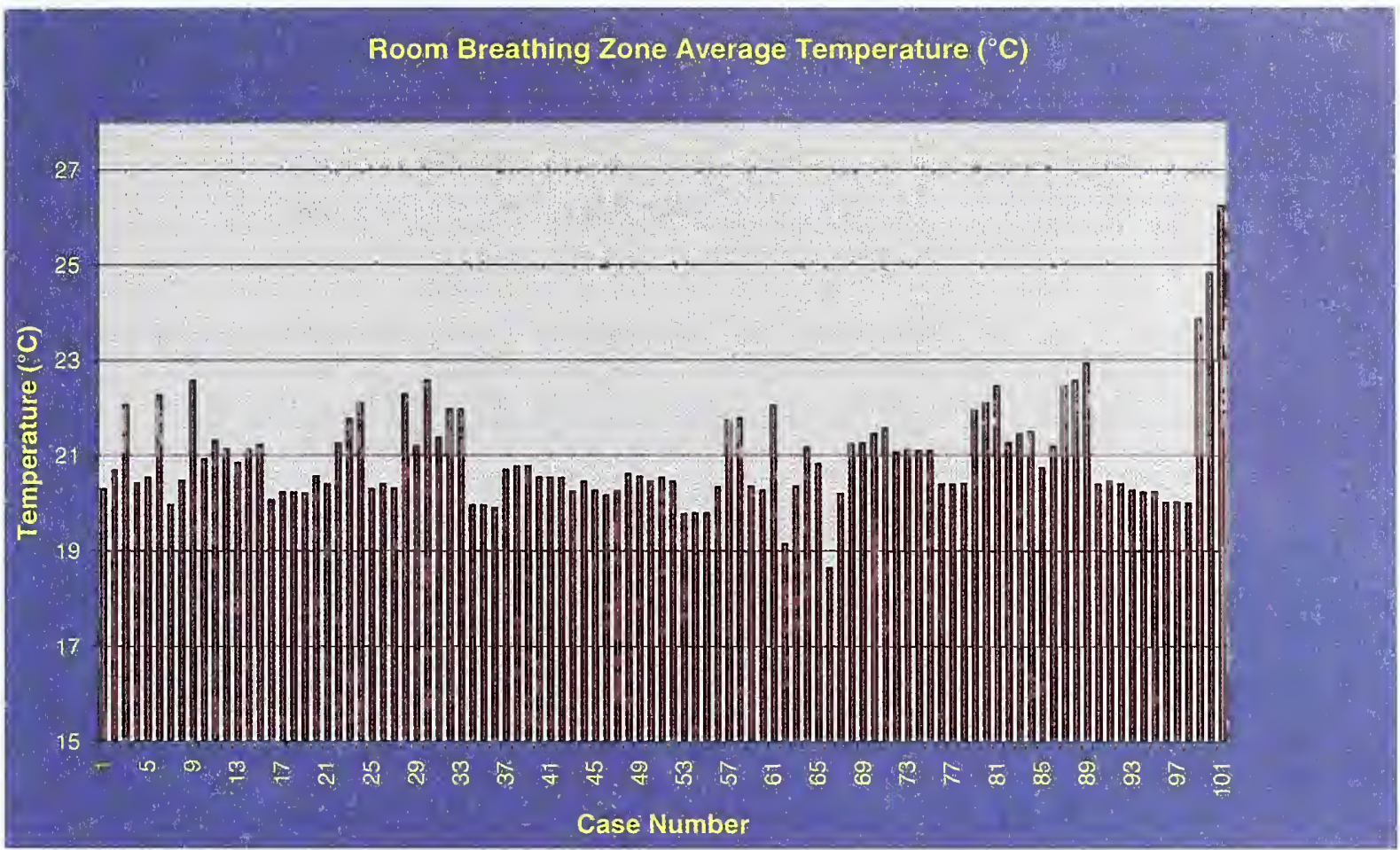

Figure 2.01 Mean Room Breathing Zone Temperature

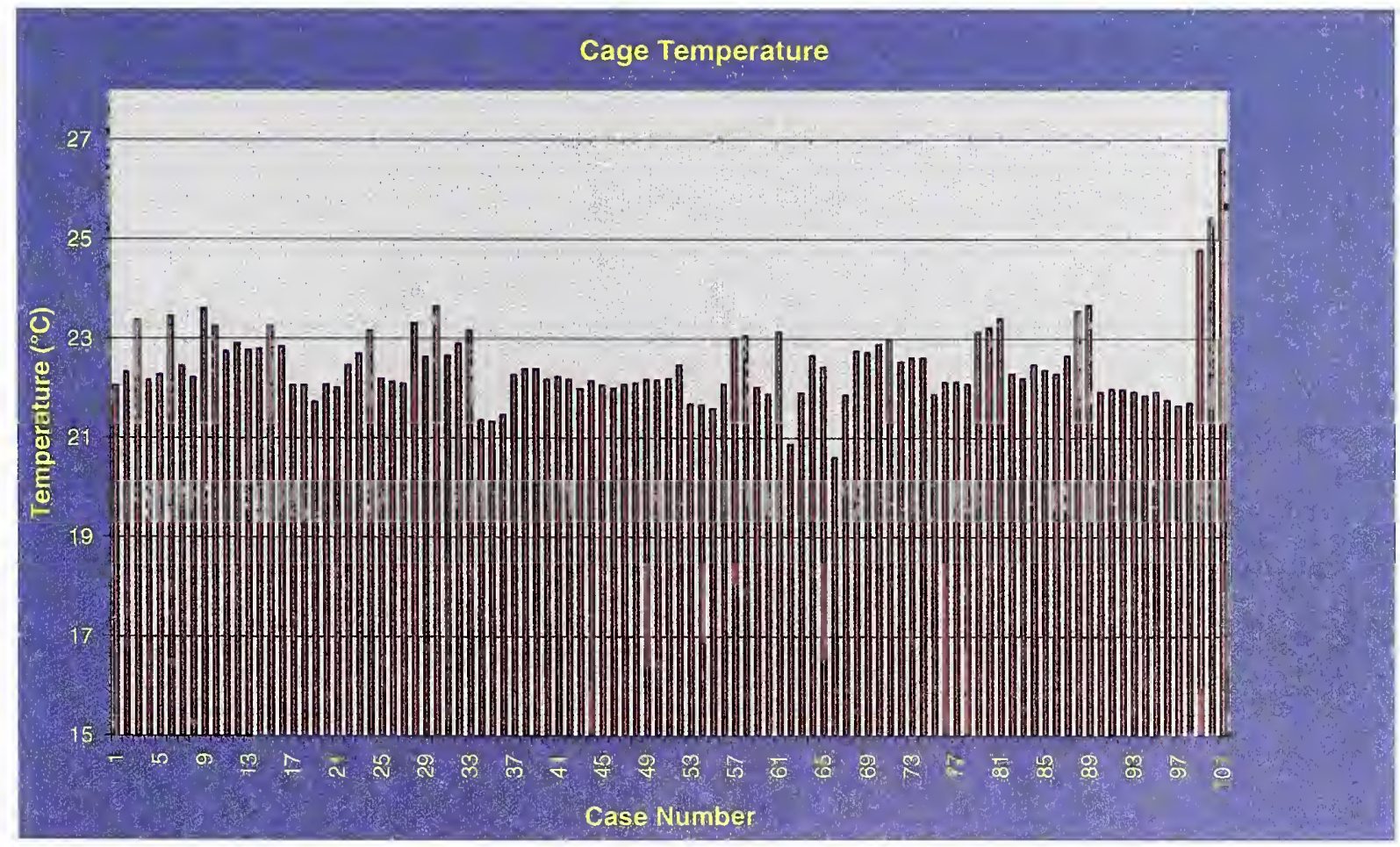

Figure 2.02 Mean Cage Temperatures 


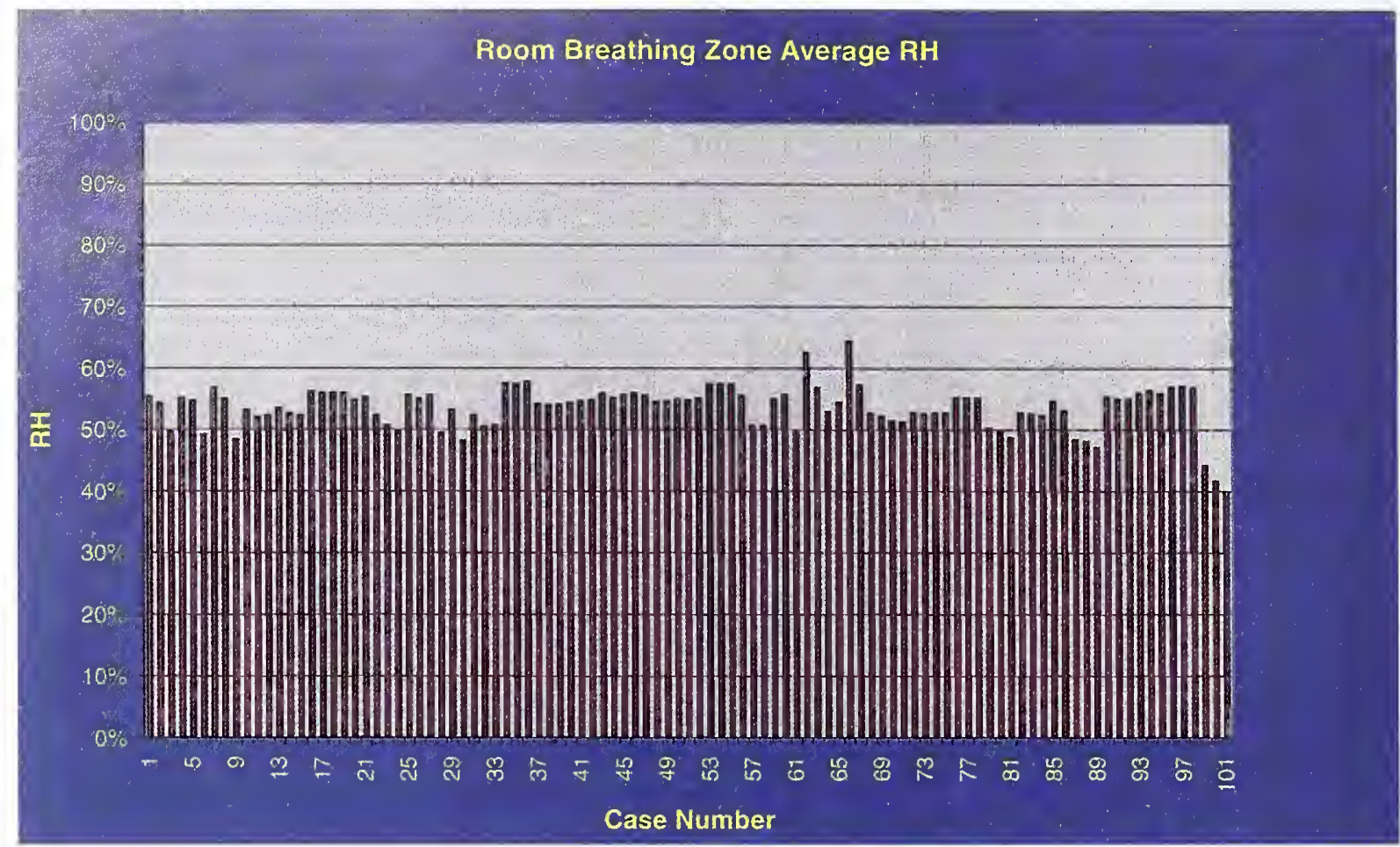

Figure 2.03 Mean Room Breathing Zone Relative Humidity (RH)

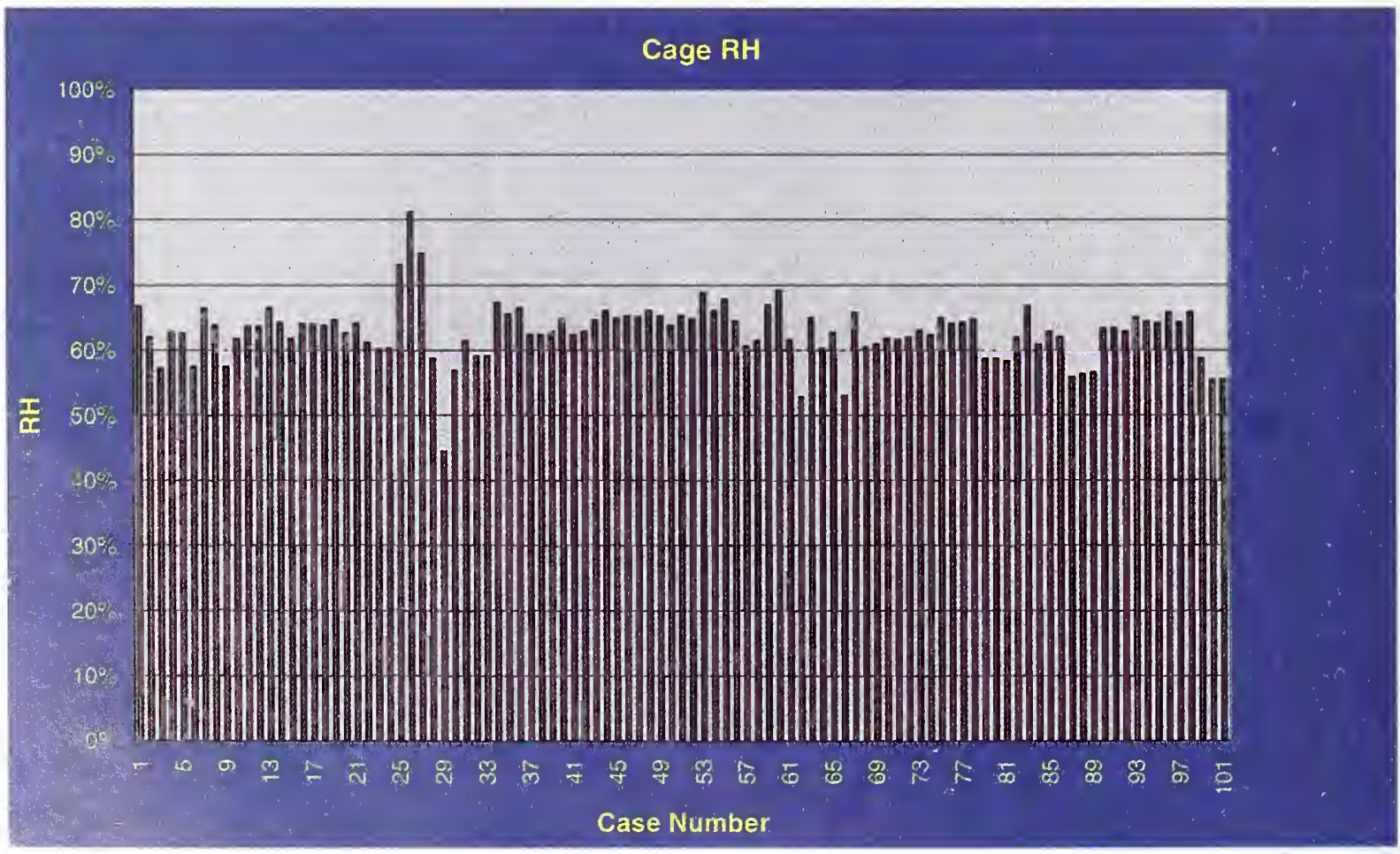

Figure 2.04 Mean Cage Relative Humidity $(R H)$ 


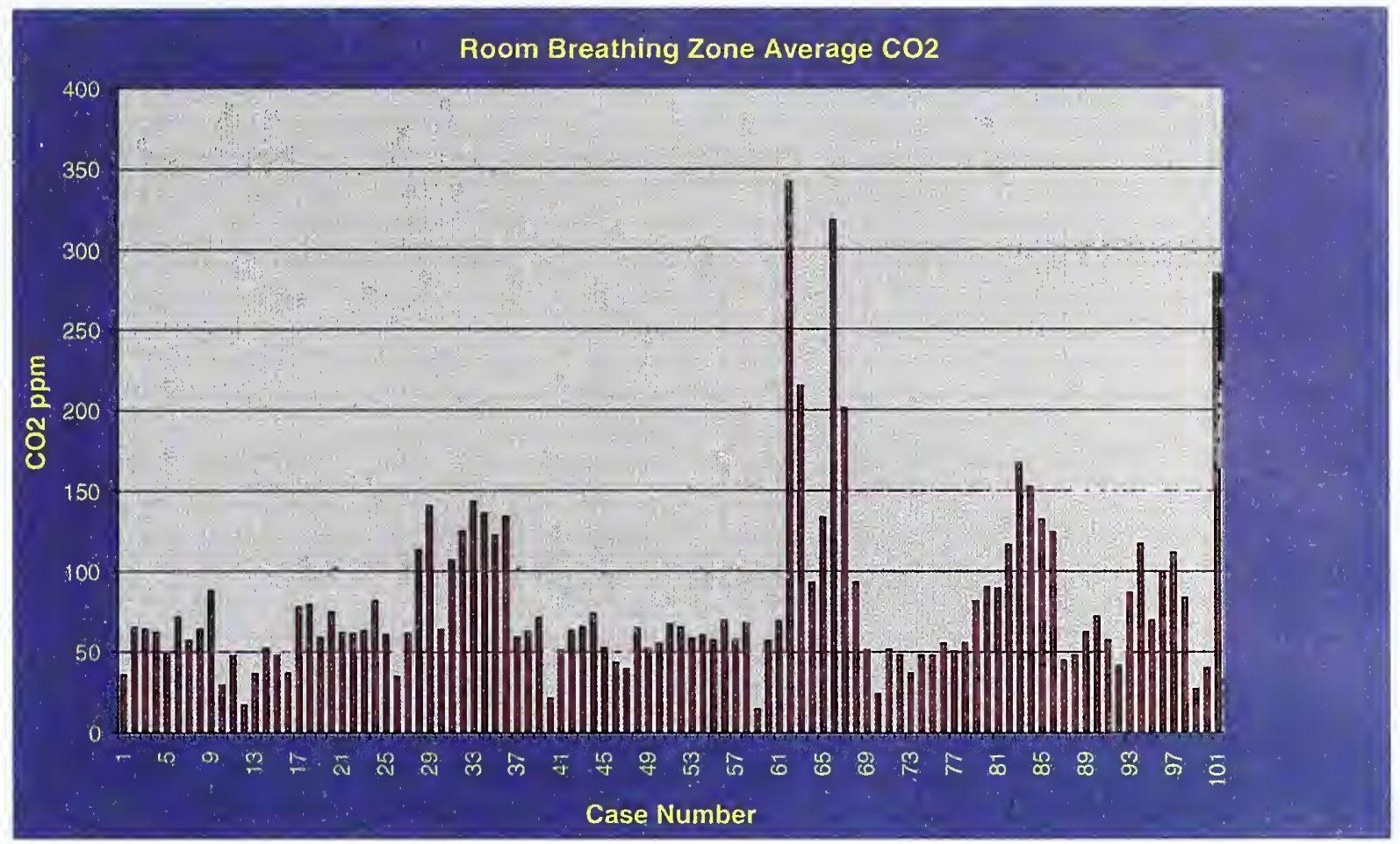

Figure 2.05 Mean Room Breathing Zone $\mathrm{CO}_{2}$ Concentration

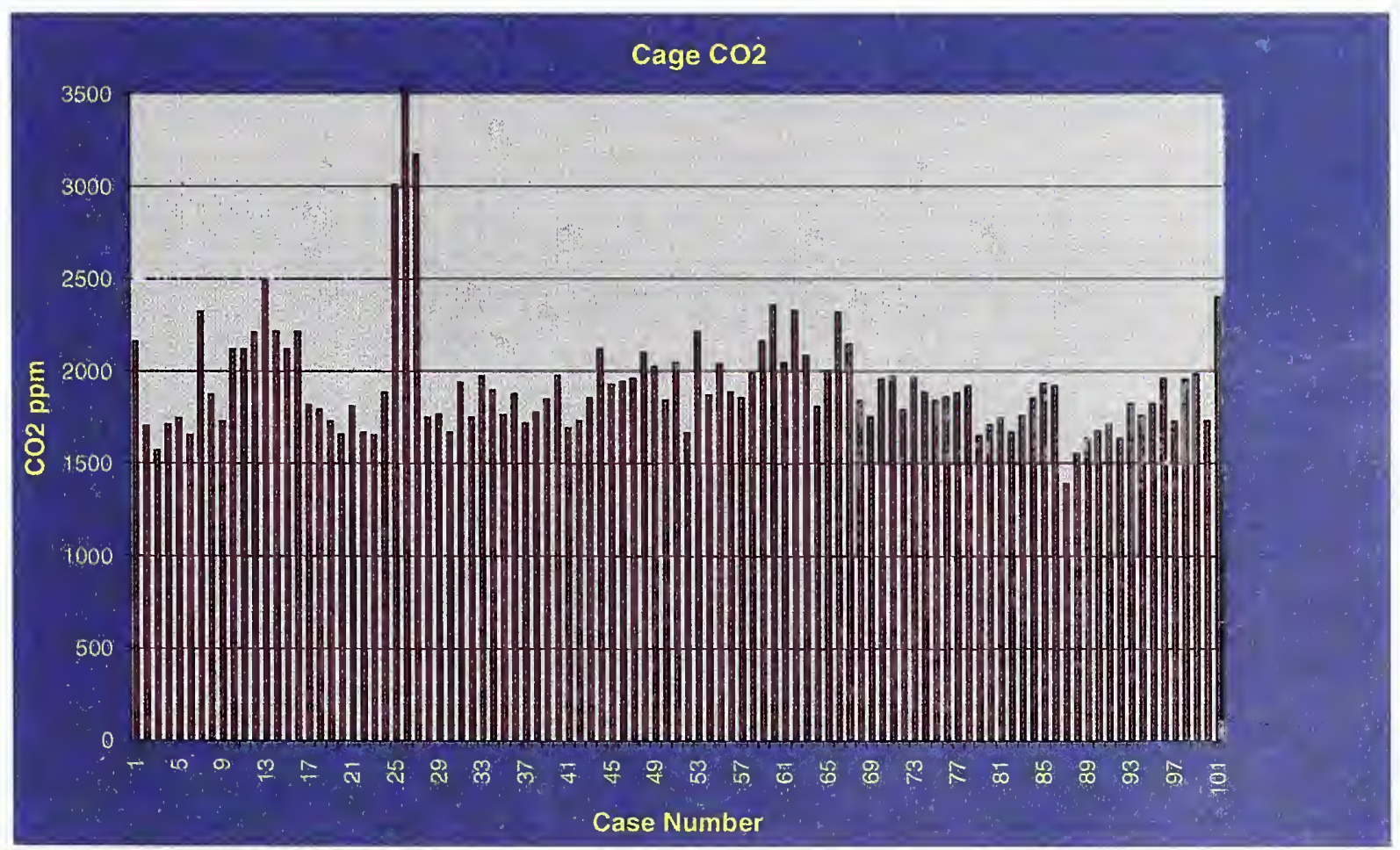

Figure 2.06 Mean Cage $\mathrm{CO}_{2}$ Concentrations 


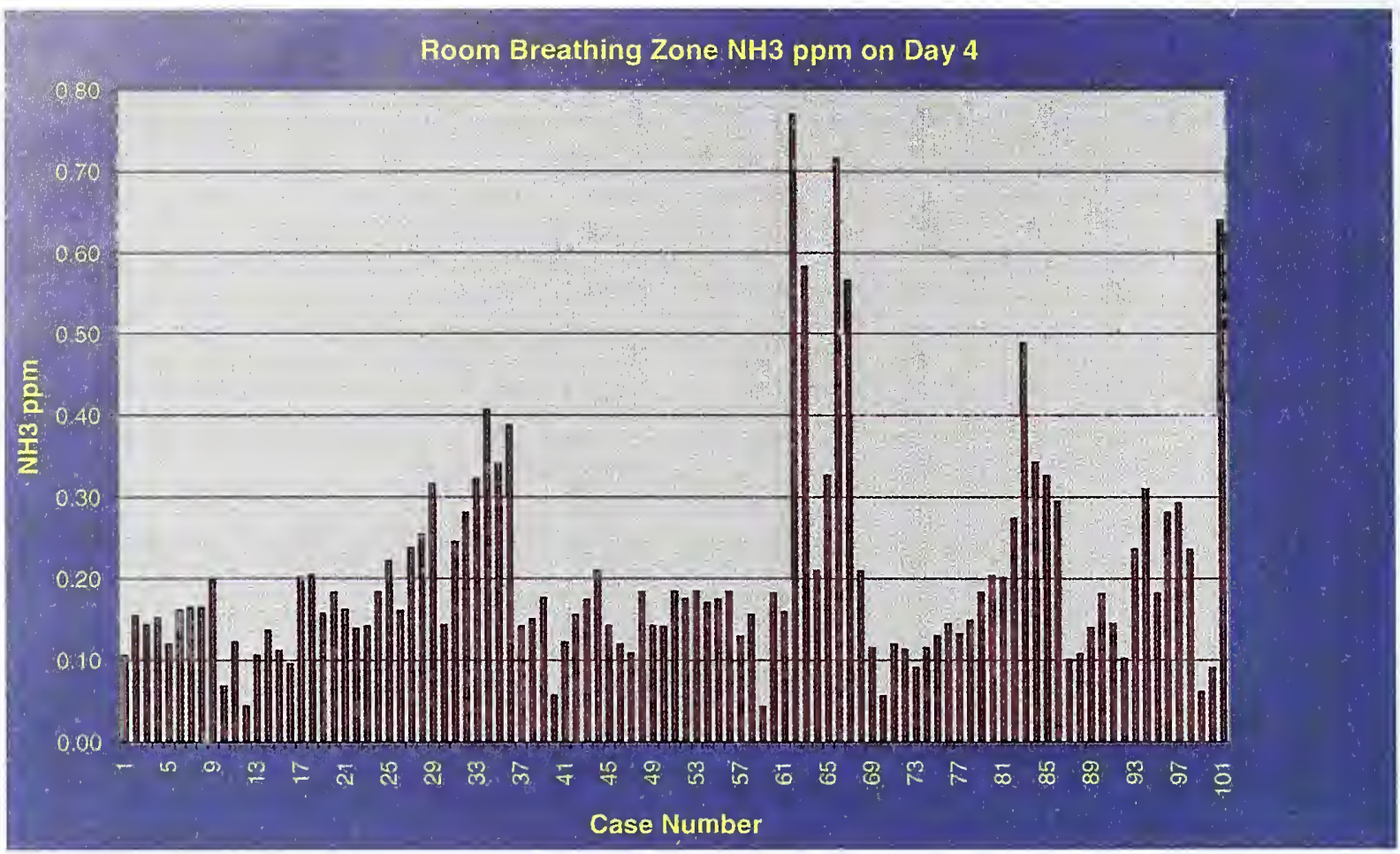

Figure 2.07 Mean Room Breathing Zone $\mathrm{NH}_{3}$ Concentration on Day 4

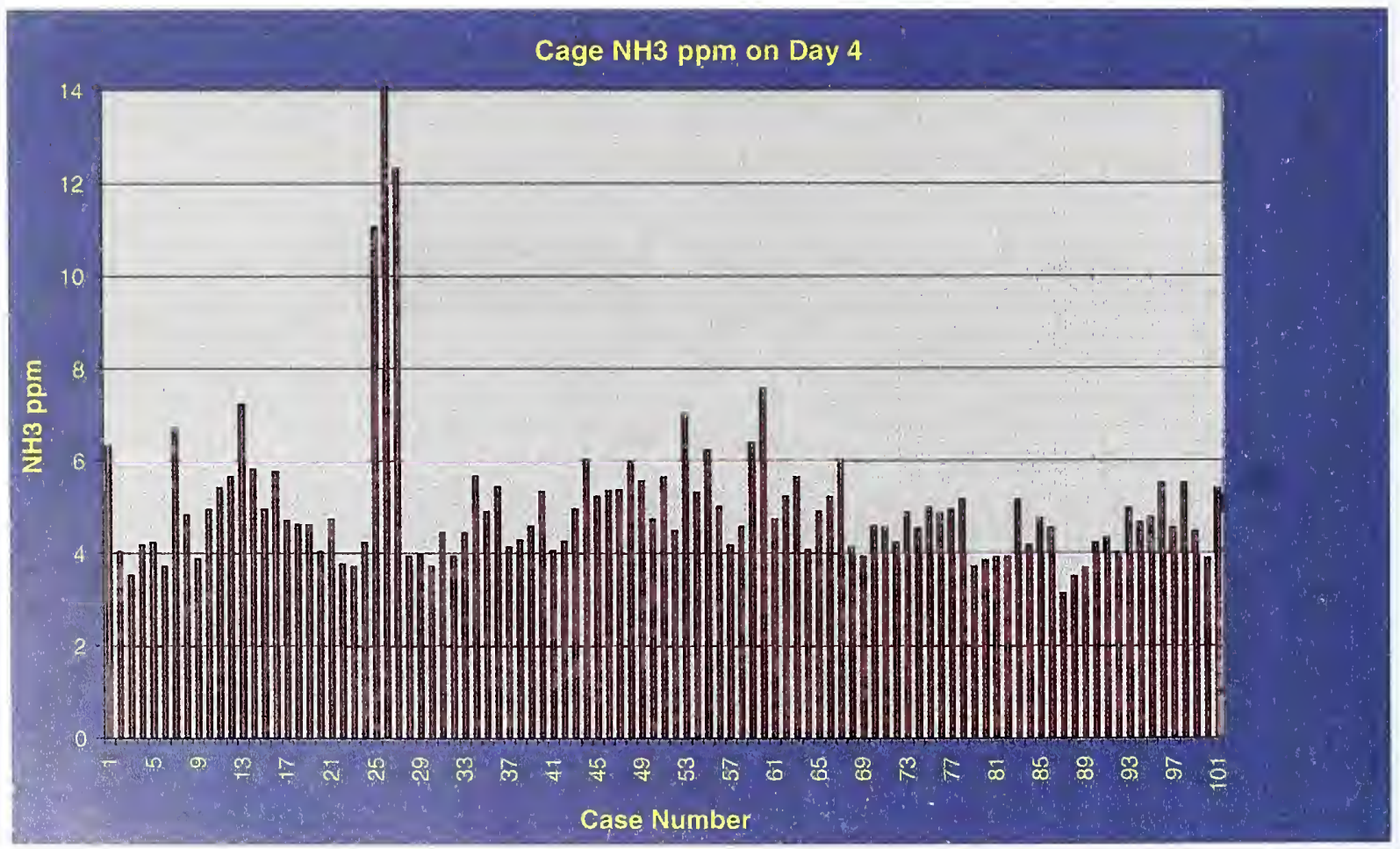

Figure 2.08 Mean Cage $\mathrm{NH}_{3}$ Concentration on Day 4 


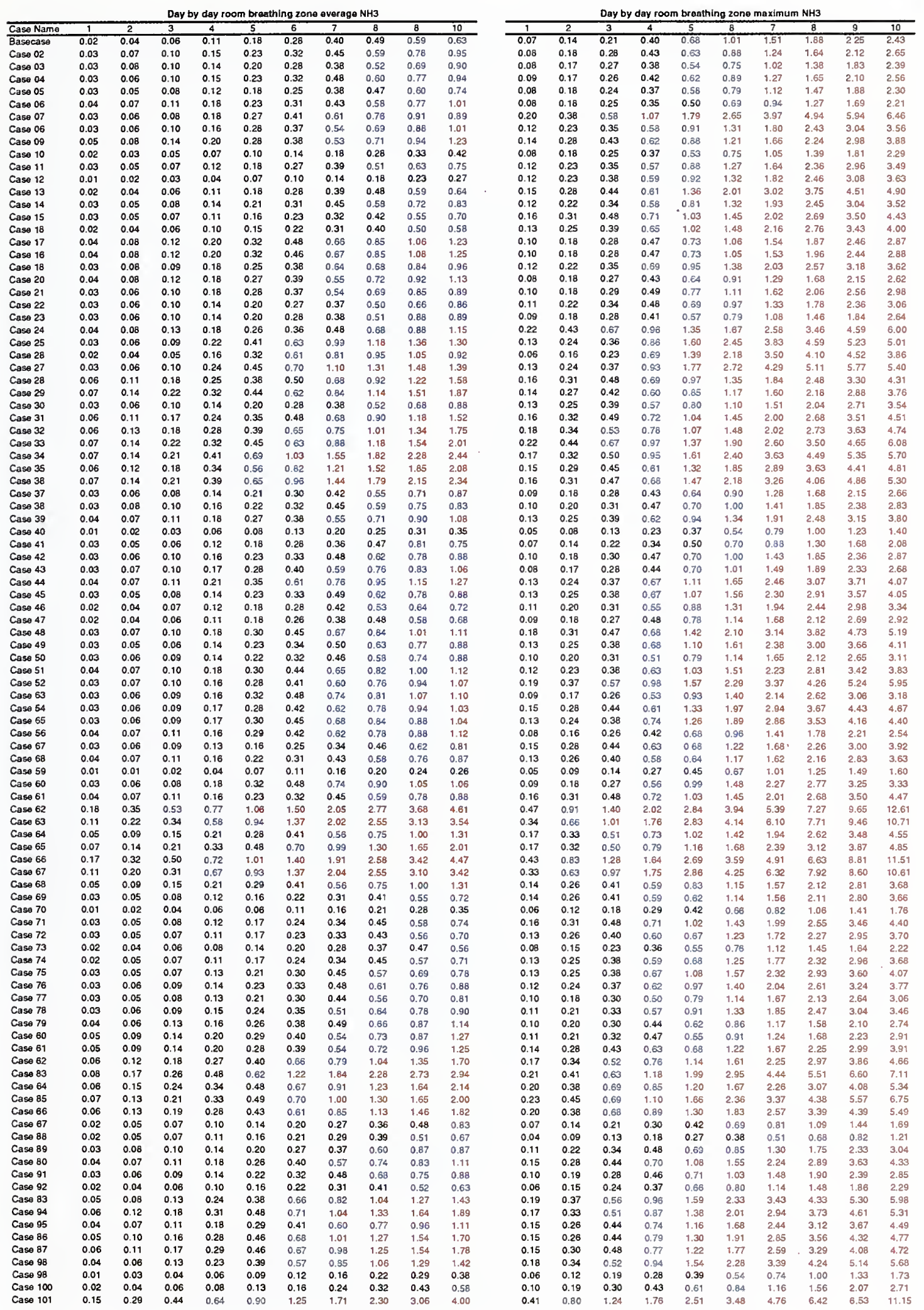

Table 2.01 Room Breathing Zone $\mathrm{NH}_{3}$ Mean and Maximum Concentrations for 10 Days 


\section{Volume II -34.Ventilation Design Handbook on Animal Research Facilities Using Static Microisolators}

Day by day cage avorage NH3

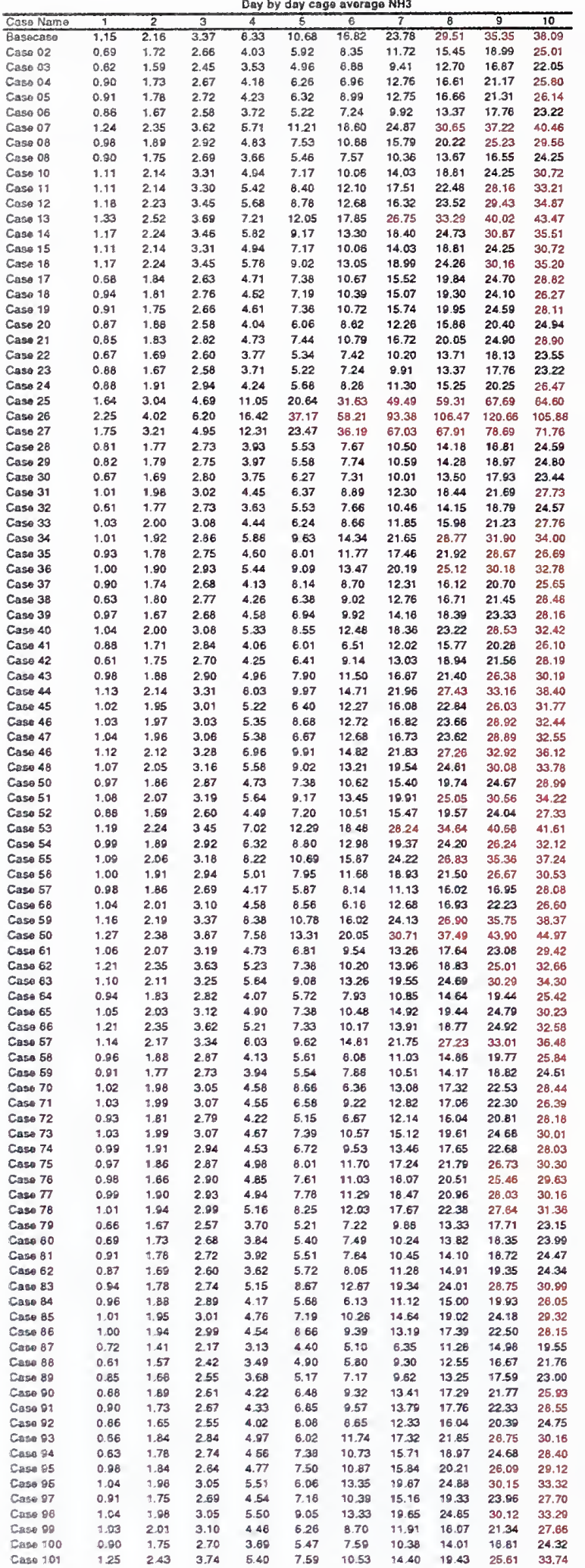

Day by day cage maximum $\mathrm{NH}_{3}$

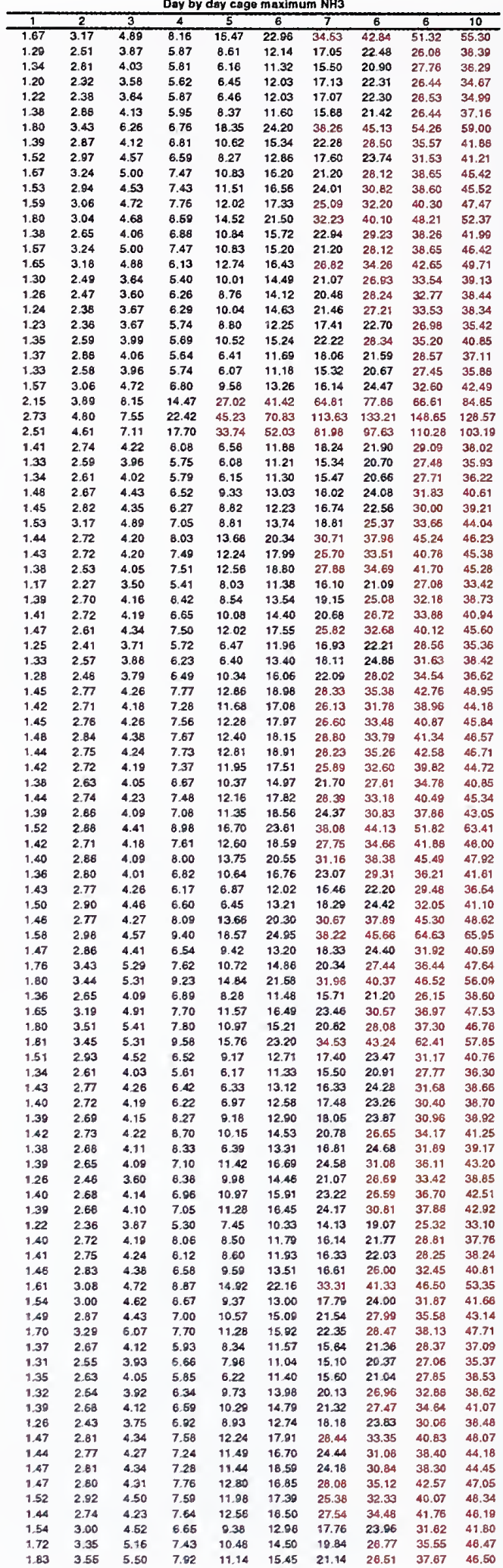

Table 2.02 Cage $\mathrm{NH}_{3}$ Mean and Maximum Concentrations for 10 Days 


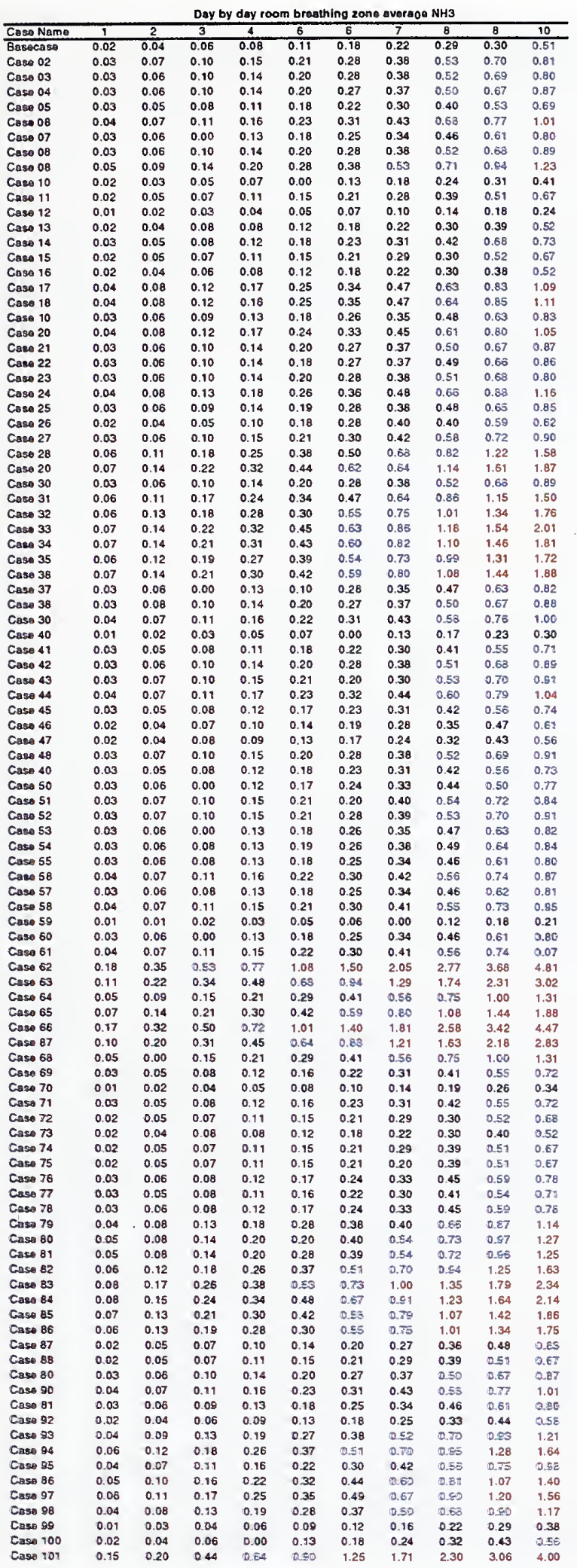

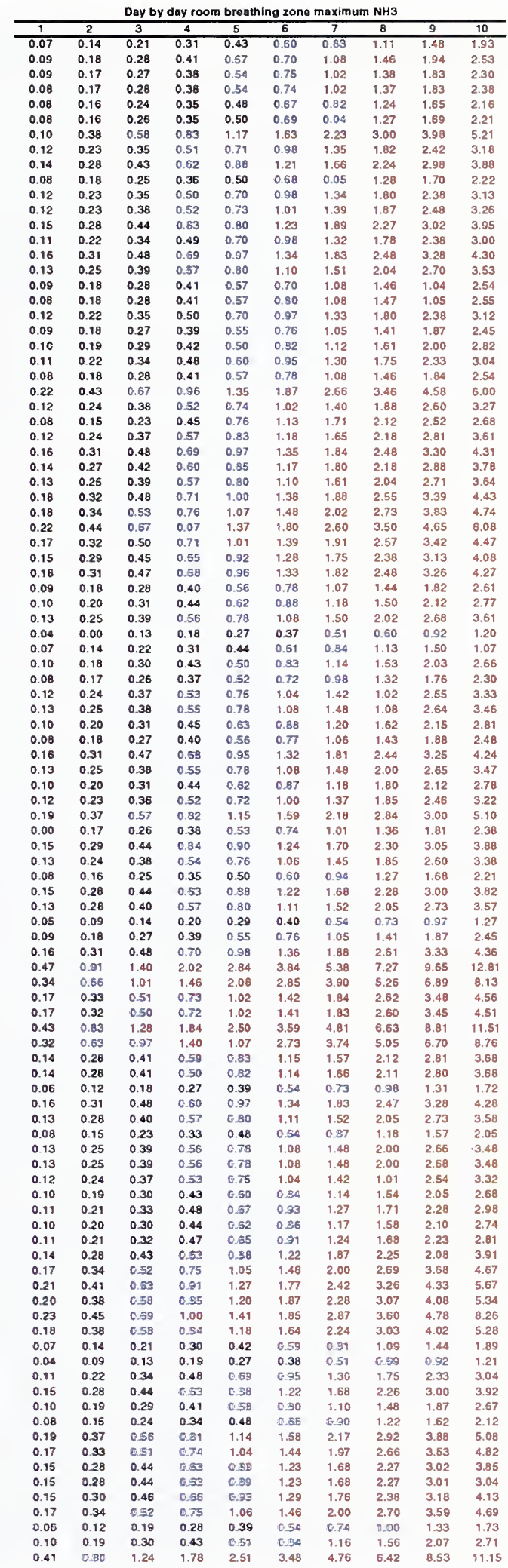

Table 2.03 Room $\mathrm{NH}_{3}$ Concentrations for 10 Days with Increased Cage Temperature 
Day by day cage avarage $\mathrm{NH} 3$

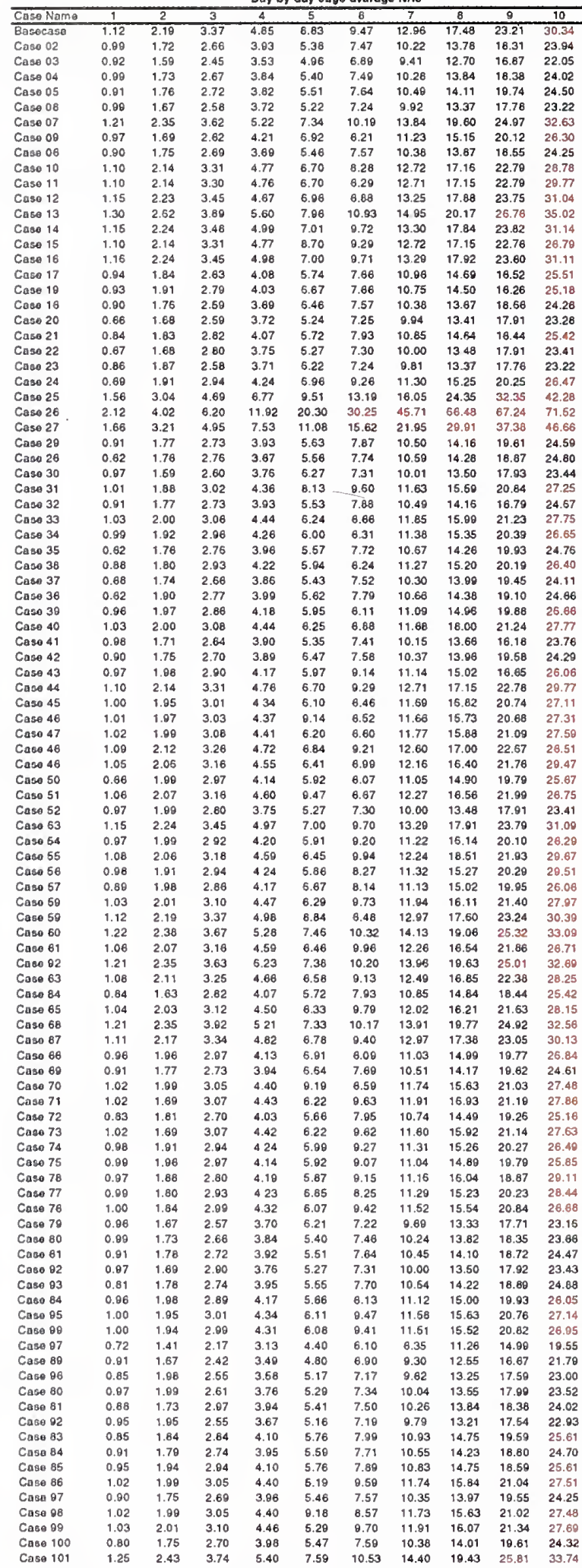

Day by day caga maximum $\mathrm{NH}$

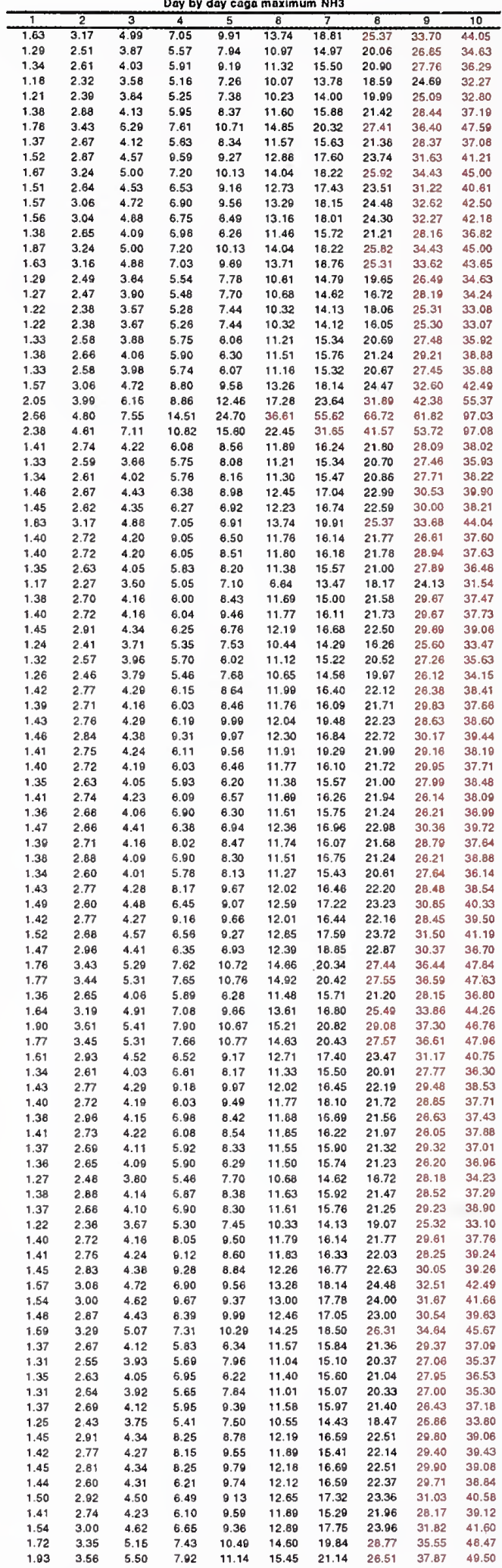

Table 2.04 Cage $\mathrm{NH}_{3}$ Concentrations for 10 Days with Increased Cage Temperature 


\section{RANKINGS OF RESULTS OF WHOLE ROOM RUNS}

\subsection{Introduction}

The following tables present rankings of the results of the CFD room simulations based on Room (scientists' breathing zone) and cages mean values for $\mathrm{CO}_{2}$ and $\mathrm{NH}_{3}$ (day 4) concentrations, temperature, and relative humidity. The tables start with the case with the lowest value and list the rest in ascending order. The case number should be used to identify all details of the case. The supply diffuser type, exhaust location, supply flow rate, and supply discharge temperature are given to provide a quick overview of the case.

Discussion of these tables can be found in volume I, section 3.3. 
Table 3.01 Ranking of Cases with respect to Room Temperature

\begin{tabular}{|c|c|c|c|c|c|c|c|}
\hline Case Name & $\begin{array}{c}\text { Supply Diffuser } \\
\text { Type }\end{array}$ & $\begin{array}{c}\text { Exhaust Location } \\
\text { and Number }\end{array}$ & Supply ACH & $\begin{array}{c}\text { Supply } \\
\text { Temperature }\left({ }^{\circ} \mathrm{C}\right)\end{array}$ & $\begin{array}{c}\text { Supply } \\
\text { Temperature }\left({ }^{\circ} \mathbf{F}\right)\end{array}$ & \begin{tabular}{|c|} 
Room Temp \\
$\left({ }^{\circ} \mathrm{C}\right)$
\end{tabular} & $\begin{array}{c}\text { Room Temp } \\
\left({ }^{\circ} \mathbf{F}\right)\end{array}$ \\
\hline Case 66 & Low Ind & Low & 5 & 6.6 & 43.9 & \begin{tabular}{|c|}
18.6 \\
\end{tabular} & 65.6 \\
\hline Case 62 & Low Ind & Low & 5 & 6.6 & 43.9 & 19.1 & 66.5 \\
\hline Case 53 & Radial & Ceiling & 15 & 18.8 & 65.8 & 19.8 & 67.6 \\
\hline Case 55 & Low Ind & Ceiling & 15 & 18.8 & 65.8 & 19.8 & 67.6 \\
\hline Case 54 & Slot & Ceiling & 15 & 18.8 & 65.8 & 19.8 & 67.6 \\
\hline Case 36 & Low Ind & Ceiling & 15 & 17.5 & 63.5 & 19.9 & 67.8 \\
\hline Case 35 & Slot & Ceiling & 15 & 17.5 & 63.5 & 20.0 & 67.9 \\
\hline Case 34 & Radial & Ceiling & 15 & 17.5 & 63.5 & 20.0 & 67.9 \\
\hline Case 07 & Low Ind & Ceiling & 15 & 18.8 & 65.8 & 20.0 & 67.9 \\
\hline Case 98 & Low ind & Ceiling & 15 & 17.5 & 63.5 & 20.0 & 68.0 \\
\hline Case 96 & Radial & Ceiling & 15 & 17.5 & 63.5 & 20.0 & 68.0 \\
\hline Case 97 & Slot & Ceiling & 15 & 17.5 & 63.5 & 20.0 & 68.0 \\
\hline Case 16 & Radial & Ceiling & 15 & 18.8 & 65.8 & 20.1 & 68.1 \\
\hline Case 46 & Radial & Ceiling & 15 & 18.8 & 65.8 & 20.2 & 68.3 \\
\hline Case 67 & Low Ind & Low & 10 & 14.8 & 58.6 & 20.2 & 68.3 \\
\hline Case 19 & Radial & Ceiling & 15 & 18.8 & 65.8 & 20.2 & 68.4 \\
\hline Case 18 & Low Ind & Ceiling & 15 & 18.8 & 65.8 & 20.2 & 68.4 \\
\hline Case 17 & Slot & Ceiling & 15 & 18.8 & 65.8 & 20.2 & 68.4 \\
\hline Case 94 & Slot & Ceiling & 15 & 17.5 & 63.5 & 20.2 & 68.4 \\
\hline Case 95 & Low Ind & Ceiling & 15 & 17.5 & 63.5 & 20.2 & 68.4 \\
\hline Case 43 & Low Ind (rot $\left.90^{\circ}\right)$ & Ceiling & 15 & 18.8 & 65.8 & 20.2 & 68.4 \\
\hline Case 47 & Radial & Ceiling & 15 & 18.8 & 65.8 & 20.3 & 68.5 \\
\hline Case 93 & Radial & Ceiling & 15 & 17.5 & 63.5 & 20.3 & 68.5 \\
\hline Case 45 & Radial & Ceiling & 15 & 18.8 & 65.8 & 20.3 & 68.5 \\
\hline Case 60 & Low Ind & High & 15 & 18.8 & 65.8 & 20.3 & 68.5 \\
\hline Basecase & Radial & Ceiling & 15 & 18.8 & 65.8 & 20.3 & 68.5 \\
\hline Case 25 & Radial & Ceiling & 15 & 18.8 & 65.8 & 20.3 & 68.5 \\
\hline Case 27 & Low Ind & Ceiling & 15 & 18.8 & 65.8 & 20.3 & 68.6 \\
\hline Case 56 & Radial & High & 15 & 18.8 & 65.8 & 20.3 & 68.6 \\
\hline Case 59 & Slot & Low & 15 & 18.8 & 65.8 & 20.3 & 68.6 \\
\hline Case 63 & Low Ind & Low & 10 & 14.8 & 58.6 & 20.3 & 68.6 \\
\hline Case 90 & Radial & Ceiling & 15 & 18.8 & 65.8 & 20.4 & 68.7 \\
\hline Case 77 & Slot & Ceiling & 15 & 18.8 & 65.8 & 20.4 & 68.7 \\
\hline Case 76 & Radial & Ceiling & 15 & 18.8 & 65.8 & 20.4 & 68.7 \\
\hline Case 21 & Low lnd & Ceiling & 15 & 18.8 & 65.8 & 20.4 & 68.7 \\
\hline Case 92 & Low Ind & Ceiling & 15 & 18.8 & 65.8 & 20.4 & 68.7 \\
\hline Case 26 & Slot & Ceiling & 15 & 18.8 & 65.8 & 20.4 & 68.7 \\
\hline Case 78 & Low Ind & Ceiling & 15 & 18.8 & 65.8 & 20.4 & 68.7 \\
\hline Case 04 & Slot & Ceiling & 15 & 18.8 & 65.8 & 20.4 & 68.8 \\
\hline Case 44 & Radial $\left(\operatorname{rot} 90^{\circ}\right)$ & Ceiling & 15 & 18.8 & 65.8 & 20.4 & 68.8 \\
\hline Case 91 & Slot & Ceiling & 15 & 18.8 & 65.8 & 20.5 & 68.8 \\
\hline Case 50 & Radial & Ceiling & 15 & 18.8 & 65.8 & 20.5 & 68.8 \\
\hline Case 52 & Radial & Ceiling & 15 & 18.8 & 65.8 & 20.5 & 68.8 \\
\hline Case 08 & Low Ind & High & 15 & 18.8 & 65.8 & 20.5 & 68.9 \\
\hline Case 42 & Low Ind & Ceiling $x 4$ & 15 & 18.8 & 65.8 & 20.5 & 68.9 \\
\hline Case 51 & Radial & Ceiling & 15 & 18.8 & 65.8 & 20.5 & 69.0 \\
\hline Case 05 & Slot & High & 15 & 18.8 & 65.8 & 20.5 & 69.0 \\
\hline Case 41 & Slot & Ceiling $x 4$ & 15 & 18.8 & 65.8 & 20.5 & 69.0 \\
\hline Case 40 & Radial & Ceiling $x 4$ & 15 & 18.8 & 65.8 & 20.5 & 69.0 \\
\hline Case 49 & Radial & Ceiling & 15 & 18.8 & 65.8 & 20.5 & 69.0 \\
\hline Case 20 & Slot & Ceiling & 15 & 18.8 & 65.8 & 20.6 & 69.0 \\
\hline Case 48 & Radial & Ceiling & 15 & 18.8 & 65.8 & 20.6 & 69.1 \\
\hline Case 02 & Radial & High & 15 & 18.8 & 65.8 & 20.7 & 69.2 \\
\hline Case 37 & Radial & Ceiling/Low 50/50 & 15 & 18.8 & 65.8 & 20.7 & 69.3 \\
\hline
\end{tabular}




\begin{tabular}{|c|c|c|c|c|c|c|c|}
\hline Case Name & $\begin{array}{c}\text { Supply Diffuser } \\
\text { Type } \\
\end{array}$ & $\begin{array}{c}\text { Exhaust Location } \\
\text { and Number }\end{array}$ & Supply ACH & $\begin{array}{c}\text { Supply } \\
\text { Temperature }\left({ }^{\circ} \mathrm{C}\right) \\
\end{array}$ & $\begin{array}{c}\text { Supply } \\
\text { Temperature }\left({ }^{\circ} \mathrm{F}\right) \\
\end{array}$ & $\begin{array}{c}\text { Room Temp } \\
\left({ }^{\circ} \mathrm{C}\right)\end{array}$ & $\begin{array}{c}\text { Room Temp } \\
\left({ }^{\circ} \mathrm{F}\right)\end{array}$ \\
\hline Case 85 & Radial & Low & 15 & 17.5 & 63.5 & 20.7 & \begin{tabular}{|l}
69.3 \\
\end{tabular} \\
\hline Case 38 & Slot & Ceiling/Low 50/50 & 15 & 18.8 & 65.8 & 20.8 & 69.4 \\
\hline Case 39 & Low Ind & Ceiling/Low 50/50 & 15 & 18.8 & 65.8 & 20.8 & 69.4 \\
\hline Case 65 & Low Ind & Low & 15 & 17.5 & 63.5 & 20.8 & 69.5 \\
\hline Case 13 & Slot & Low & 15 & 20.7 & 69.3 & 20.8 & 69.5 \\
\hline Case 10 & Radial & Ceiling & 15 & 20.7 & 69.3 & 20.9 & 69.7 \\
\hline Case 72 & Radial & High $\times 4$ / Low $x 2$ & 15 & 18.8 & 65.8 & 21.1 & 69.9 \\
\hline Case 74 & Low Ind & High $\times 4$ / Low $\times 2$ & 15 & 18.8 & 65.8 & 21.1 & 70.0 \\
\hline Case 75 & Radial & Ceiling/Low 50/50 & 15 & 18.8 & 65.8 & 21.1 & 70.0 \\
\hline Case 73 & Slot & High $\times 4$ / Low $\times 2$ & 15 & 18.8 & 65.8 & 21.1 & 70.0 \\
\hline Case 14 & Low Ind & Ceiling & 15 & 20.7 & 69.3 & 21.1 & 70.0 \\
\hline Case 12 & Slot & Ceiling & 15 & 20.7 & 69.3 & 21.1 & 70.0 \\
\hline Case 64 & Low Ind & Low & 20 & 18.9 & 66.0 & 21.2 & 70.1 \\
\hline Case 86 & Slot & Low & 15 & 17.5 & 63.5 & 21.2 & 70.1 \\
\hline Case 29 & Low Ind & Low & 5 & 11 & 51.8 & 21.2 & 70.1 \\
\hline Case 15 & Low Ind & Low & 15 & 20.7 & 69.3 & 21.2 & 70.2 \\
\hline Case 68 & Low Ind & Low & 20 & 18.9 & 66.0 & 21.2 & 70.2 \\
\hline Case 22 & Radial & Low & 15 & 18.8 & 65.8 & 21.2 & 70.2 \\
\hline Case 69 & Radial & High $\times 4$ / Low $x 4$ & 15 & 18.8 & 65.8 & 21.3 & 70.3 \\
\hline Case 82 & Radial & 2 Door exhausts & 15 & 18.8 & 65.8 & 21.3 & 70.3 \\
\hline Case 11 & Radial & Low & 15 & 20.7 & 69.3 & 21.3 & 70.4 \\
\hline Case 31 & Radial & Low & 15 & 17.5 & 63.5 & 21.4 & 70.5 \\
\hline Case 83 & Slot & 2 Door exhausts & 15 & 17.5 & 63.5 & 21.4 & 70.6 \\
\hline Case 70 & Slot & High $x 4$ / Low $x 4$ & 15 & 18.8 & 65.8 & 21.4 & 70.6 \\
\hline Case 84 & Low Ind & 2 Door exhausts & 15 & 17.5 & 63.5 & 21.5 & 70.7 \\
\hline Case 71 & Low Ind & High $\mathrm{x} 4$ / Low $\mathrm{x} 4$ & 15 & 18.8 & 65.8 & 21.6 & 70.8 \\
\hline Case 57 & Radial & Low & 15 & 18.8 & 65.8 & 21.7 & 71.1 \\
\hline Case 23 & Slot & Low & 15 & 18.8 & 65.8 & 21.8 & 71.2 \\
\hline Case 58 & Slot & High & 15 & 18.8 & 65.8 & 21.8 & 71.2 \\
\hline Case 79 & Radial & 2 Door exhausts & 15 & 18.8 & 65.8 & 21.9 & 71.5 \\
\hline Case 32 & Slot & Low & 15 & 17.5 & 63.5 & 22.0 & 71.5 \\
\hline Case 33 & Low Ind & Low & 15 & 17.5 & 63.5 & 22.0 & 71.5 \\
\hline Case 61 & Low Ind & Low & 15 & 18.8 & 65.8 & 22.0 & 71.7 \\
\hline Case 03 & Radial & Low & 15 & 18.8 & 65.8 & 22.1 & 71.7 \\
\hline Case 80 & Slot & 2 Door exhausts & 15 & 18.8 & 65.8 & 22.1 & 71.8 \\
\hline Case 24 & Low Ind & Low & 15 & 18.8 & 65.8 & 22.1 & 71.8 \\
\hline Case 06 & Slot & Low & 15 & 18.8 & 65.8 & 22.3 & 72.1 \\
\hline Case 28 & Low Ind & Low & 10 & 16.8 & 62.2 & 22.3 & 72.1 \\
\hline Case 81 & Low Ind & 2 Door exhausts & 15 & 18.8 & 65.8 & 22.5 & 72.4 \\
\hline Case 87 & Radial & Low & 15 & 19.2 & 66.6 & 22.5 & 72.4 \\
\hline Case 88 & Slot & Low & 15 & 19.2 & 66.6 & 22.6 & 72.6 \\
\hline Case 09 & Low Ind & Low & 15 & 18.8 & 65.8 & 22.6 & 72.6 \\
\hline Case 30 & Low Ind & Low & 20 & 19.8 & 67.6 & 22.6 & 72.6 \\
\hline Case 89 & Low Ind & Low & 15 & 19.2 & 66.6 & 22.9 & 73.3 \\
\hline
\end{tabular}


Table 3.02 Ranking of Cases with respect to Cage Temperature

\begin{tabular}{|c|c|c|c|c|c|c|c|}
\hline Case Name & $\begin{array}{c}\text { Supply Diffuser } \\
\text { Type } \\
\end{array}$ & $\begin{array}{l}\text { Exhaust Location } \\
\text { and Number }\end{array}$ & Supply ACH & $\begin{array}{c}\text { Supply } \\
\text { Temperature }\left({ }^{\circ} \mathrm{C}\right) \\
\end{array}$ & $\begin{array}{c}\text { Supply } \\
\text { Temperature }\left({ }^{\circ} \mathbf{F}\right)\end{array}$ & $\begin{array}{c}\text { Cage Temp } \\
\left({ }^{\circ} \mathrm{C}\right)\end{array}$ & $\begin{array}{c}\text { Cage Temp } \\
\left({ }^{\circ} \mathbf{F}\right)\end{array}$ \\
\hline Case 66 & Low Ind & Low & 5 & 6.6 & 43.9 & 20.6 & 69.1 \\
\hline Case 62 & Low Ind & Low & 5 & 6.6 & 43.9 & 20.9 & 69.6 \\
\hline Case 35 & Slot & Ceiling & 15 & 17.5 & 63.5 & 21.3 & 70.4 \\
\hline Case 34 & Radial & Ceiling & 15 & 17.5 & 63.5 & 21.4 & 70.4 \\
\hline Case 36 & Low Ind & Ceiling & 15 & 17.5 & 63.5 & 21.5 & 70.6 \\
\hline Case 55 & Low Ind & Ceiling & 15 & 18.8 & 65.8 & 21.6 & 70.9 \\
\hline Case 97 & Slot & Ceiling & 15 & 17.5 & 63.5 & 21.6 & 71.0 \\
\hline Case 54 & Slot & Ceiling & 15 & 18.8 & 65.8 & 21.7 & 71.0 \\
\hline Case 53 & Radial & Ceiling & 15 & 18.8 & 65.8 & 21.7 & 71.0 \\
\hline Case 98 & Low ind & Ceiling & 15 & 17.5 & 63.5 & 21.7 & 71.1 \\
\hline Case 19 & Radial & Ceiling & 15 & 18.8 & 65.8 & 21.7 & 71.1 \\
\hline Case 96 & Radial & Ceiling & 15 & 17.5 & 63.5 & 21.8 & 71.2 \\
\hline Case 94 & Slot & Ceiling & 15 & 17.5 & 63.5 & 21.9 & 71.3 \\
\hline Case 67 & Low Ind & Low & 10 & 14.8 & 58.6 & 21.9 & 71.4 \\
\hline Case 75 & Radial & Ceiling/Low 50/50 & 15 & 18.8 & 65.8 & 21.9 & 71.4 \\
\hline Case 60 & Low Ind & High & 15 & 18.8 & 65.8 & 21.9 & 71.4 \\
\hline Case 63 & Low Ind & Low & 10 & 14.8 & 58.6 & 21.9 & 71.4 \\
\hline Case 90 & Radial & Ceiling & 15 & 18.8 & 65.8 & 21.9 & 71.5 \\
\hline Case 95 & Low Ind & Ceiling & 15 & 17.5 & 63.5 & 21.9 & 71.5 \\
\hline Case 93 & Radial & Ceiling & 15 & 17.5 & 63.5 & 21.9 & 71.5 \\
\hline Case 92 & Low Ind & Ceiling & 15 & 18.8 & 65.8 & 22.0 & 71.6 \\
\hline Case 91 & Slot & Ceiling & 15 & 18.8 & 65.8 & 22.0 & 71.6 \\
\hline Case 43 & Low Ind (rot $90^{\circ}$ ) & Ceiling & 15 & 18.8 & 65.8 & 22.0 & 71.6 \\
\hline Case 46 & Radial & Ceiling & 15 & 18.8 & 65.8 & 22.0 & 71.6 \\
\hline Case 21 & Low Ind & Ceiling & 15 & 18.8 & 65.8 & 22.0 & 71.6 \\
\hline Case 59 & Slot & Low & 15 & 18.8 & 65.8 & 22.0 & 71.6 \\
\hline Basecase & Radial & Ceiling & 15 & 18.8 & 65.8 & 22.1 & 71.7 \\
\hline Case 18 & Low Ind & Ceiling & 15 & 18.8 & 65.8 & 22.1 & 71.7 \\
\hline Case 45 & Radial & Ceiling & 15 & 18.8 & 65.8 & 22.1 & 71.7 \\
\hline Case 17 & Slot & Ceiling & 15 & 18.8 & 65.8 & 22.1 & 71.7 \\
\hline Case 20 & Slot & Ceiling & 15 & 18.8 & 65.8 & 22.1 & 71.7 \\
\hline Case 47 & Radial & Ceiling & 15 & 18.8 & 65.8 & 22.1 & 71.7 \\
\hline Case 78 & Low lnd & Ceiling & 15 & 18.8 & 65.8 & 22.1 & 71.8 \\
\hline Case 56 & Radial & High & 15 & 18.8 & 65.8 & 22.1 & 71.8 \\
\hline Case 27 & Low lnd & Ceiling & 15 & 18.8 & 65.8 & 22.1 & 71.8 \\
\hline Case 48 & Radial & Ceiling & 15 & 18.8 & 65.8 & 22.1 & 71.8 \\
\hline Case 76 & Radial & Ceiling & 15 & 18.8 & 65.8 & 22.1 & 71.8 \\
\hline Case 26 & Slot & Ceiling & 15 & 18.8 & 65.8 & 22.1 & 71.8 \\
\hline Case 77 & Slot & Ceiling & 15 & 18.8 & 65.8 & 22.1 & 71.9 \\
\hline Case 44 & Radial (rot $90^{\circ}$ ) & Ceiling & 15 & 18.8 & 65.8 & 22.2 & 71.9 \\
\hline Case 04 & Slot & Ceiling & 15 & 18.8 & 65.8 & 22.2 & 71.9 \\
\hline Case 50 & Radial & Ceiling & 15 & 18.8 & 65.8 & 22.2 & 71.9 \\
\hline Case 49 & Radial & Ceiling & 15 & 18.8 & 65.8 & 22.2 & 71.9 \\
\hline Case 40 & Radial & Ceiling $\mathrm{x} 4$ & 15 & 18.8 & 65.8 & 22.2 & 71.9 \\
\hline Case 42 & Low Ind & Ceiling $\mathrm{x} 4$ & 15 & 18.8 & 65.8 & 22.2 & 71.9 \\
\hline Case 51 & Radial & Ceiling & 15 & 18.8 & 65.8 & 22.2 & 71.9 \\
\hline Case 25 & Radial & Ceiling & 15 & 18.8 & 65.8 & 22.2 & 72.0 \\
\hline Case 83 & Slot & 2 Door exhausts & 15 & 17.5 & 63.5 & 22.2 & 72.0 \\
\hline Case 08 & Low Ind & High & 15 & 18.8 & 65.8 & 22.2 & 72.0 \\
\hline Case 41 & Slot & Ceiling $x 4$ & 15 & 18.8 & 65.8 & 22.2 & 72.0 \\
\hline Case 37 & Radial & Ceiling/Low 50/50 & 15 & 18.8 & 65.8 & 22.3 & 72.1 \\
\hline Case 05 & Slot & High & 15 & 18.8 & 65.8 & 22.3 & 72.1 \\
\hline Case 82 & Radial & 2 Door exhausts & 15 & 18.8 & 65.8 & 22.3 & 72.1 \\
\hline Case 86 & Slot & Low & 15 & 17.5 & 63.5 & 22.3 & 72.1 \\
\hline
\end{tabular}


Volume II - Section III - Rankings of Results of Whole Room Runs

Page II - 41

\begin{tabular}{|c|c|c|c|c|c|c|c|}
\hline Case Name & $\begin{array}{c}\text { Supply Diffuser } \\
\text { Type }\end{array}$ & $\begin{array}{c}\text { Exhaust Location } \\
\text { and Number }\end{array}$ & Supply ACH & $\begin{array}{c}\text { Supply } \\
\text { Temperature }\left({ }^{\circ} \mathrm{C}\right) \\
\end{array}$ & $\begin{array}{c}\text { Supply } \\
\text { Temperature }\left({ }^{\circ} \mathbf{F}\right) \\
\end{array}$ & $\begin{array}{c}\text { Cage Temp } \\
\left({ }^{\circ} \mathrm{C}\right)\end{array}$ & $\begin{array}{c}\text { Cage Temp } \\
\left({ }^{\circ} \mathbf{F}\right)\end{array}$ \\
\hline Case 02 & Radial & High & 15 & 18.8 & 65.9 & 22.3 & 72.2 \\
\hline Case 85 & Radial & Low & 15 & 17.5 & 63.5 & 22.4 & 72.3 \\
\hline Case 38 & Slot & Ceiling/Low 50/50 & 15 & I8.8 & 65.8 & 22.4 & 72.3 \\
\hline Case 39 & Low Ind & Ceiling/Low 50/50 & 15 & 18.8 & 65.8 & 22.4 & 72.3 \\
\hline Case 65 & Low Ind & Low & 15 & 17.5 & 63.5 & 22.4 & 72.4 \\
\hline Case 07 & Low Ind & Ceiling & 15 & 18.8 & 65.8 & 22.5 & 72.4 \\
\hline Case 52 & Radial & Ceiling & 15 & 18.8 & 65.8 & 22.5 & 72.5 \\
\hline Case 22 & Radial & Low & 15 & 18.8 & 65.8 & 22.5 & 72.5 \\
\hline Case 84 & Low Ind & 2 Door exhausts & 15 & 17.5 & 63.5 & 22.5 & 72.5 \\
\hline Case 72 & Radial & High $\times 4 /$ Low $\times 2$ & 15 & 18.8 & 65.8 & 22.5 & 72.6 \\
\hline Case 73 & Slot & High $\times 4$ / Low $x 2$ & 15 & 18.8 & 65.8 & 22.6 & 72.7 \\
\hline Case 74 & Low Ind & High $x 4$ / Low $x 2$ & 15 & 18.8 & 65.8 & 22.6 & 72.7 \\
\hline Case 29 & Low Ind & Low & 5 & 11 & 51.8 & 22.6 & 72.8 \\
\hline Case 87 & Radial & Low & I5 & I9.2 & 66.6 & 22.7 & 72.8 \\
\hline Case 64 & Low Ind & Low & 20 & I8.9 & 66.0 & 22.7 & 72.8 \\
\hline Case 31 & Radial & Low & 15 & 17.5 & 63.5 & 22.7 & 72.8 \\
\hline Case 23 & Slot & Low & 15 & 18.8 & 65.8 & 22.7 & 72.9 \\
\hline Case 69 & Radial & High $x 4 /$ Low $x 4$ & 15 & 18.8 & 65.8 & 22.7 & 72.9 \\
\hline Case 11 & Radial & Low & 15 & 20.7 & 69.3 & 22.7 & 72.9 \\
\hline Case 68 & Low Ind & Low & 20 & 18.9 & 66.0 & 22.8 & 73.0 \\
\hline Case 13 & Slot & Low & 15 & 20.7 & 69.3 & 22.8 & 73.0 \\
\hline Case 14 & Low Ind & Ceiling & 15 & 20.7 & 69.3 & 22.8 & 73.0 \\
\hline Case 16 & Radial & Ceiling & 15 & 18.8 & 65.8 & 22.9 & 73.1 \\
\hline Case 70 & Slot & High $x 4$ / Low $x 4$ & 15 & 18.8 & 65.8 & 22.9 & 73.2 \\
\hline Case 32 & Slot & Low & 15 & 17.5 & 63.5 & 22.9 & 73.2 \\
\hline Case 12 & Slot & Ceiling & 15 & 20.7 & 69.3 & 22.9 & 73.2 \\
\hline Case 71 & Low Ind & High $\mathrm{x} 4$ / Low $\mathrm{x} 4$ & 15 & 18.8 & 65.8 & 23.0 & 73.3 \\
\hline Case 57 & Radial & Low & 15 & 18.8 & 65.8 & 23.0 & 73.4 \\
\hline Case 58 & Slot & High & 15 & 18.8 & 65.8 & 23.1 & 73.5 \\
\hline Case 79 & Radial & 2 Door exhausts & 15 & 18.8 & 65.8 & $23 . \overline{1}$ & $73 . \overline{6}$ \\
\hline Case 61 & Low lnd & Low & 15 & 18.8 & 65.8 & 23.1 & 73.6 \\
\hline Case 33 & Low Ind & Low & 15 & 17.5 & 63.5 & 23.2 & 73.7 \\
\hline Case 24 & Low Ind & Low & 15 & 18.8 & 65.8 & 23.2 & 73.7 \\
\hline Case 80 & Slot & 2 Door exhausts & 15 & 18.8 & 65.8 & 23.2 & 73.8 \\
\hline Case 10 & Radial & Ceiling & 15 & 20.7 & 69.3 & 23.2 & 73.8 \\
\hline Case 15 & Low Ind & Low & 15 & 20.7 & 69.3 & 23.2 & 73.8 \\
\hline Case 28 & Low Ind & Low & 10 & 16.8 & 62.2 & 23.3 & 74.0 \\
\hline Case 03 & Radial & Low & 15 & 18.8 & 65.8 & 23.4 & 74.0 \\
\hline Case 81 & Low Ind & 2 Door exhausts & 15 & 18.8 & 65.8 & 23.4 & 74.1 \\
\hline Case 06 & Slot & Low & 15 & 18.8 & 65.8 & 23.4 & 74.2 \\
\hline Case 88 & Slot & Low & 15 & 19.2 & 66.6 & 23.5 & 74.4 \\
\hline Case 09 & Low Ind & Low & 15 & 18.8 & 65.8 & 23.6 & 74.5 \\
\hline Case 30 & Low Ind & Low & 20 & 19.8 & 67.6 & 23.6 & 74.6 \\
\hline Case 89 & Low Ind & Low & 15 & 19.2 & 66.6 & 23.7 & 74.6 \\
\hline
\end{tabular}


Volume II -42 Ventilation Design Handbook on Animal Research Facilities Using Static Microisolators

Table 3.03 Ranking of Cases with respect to Room Relative Humidity

\begin{tabular}{|c|c|c|c|c|c|c|}
\hline Case Name & $\begin{array}{c}\text { Supply Diffuser } \\
\text { Type }\end{array}$ & $\begin{array}{l}\text { Exhaust Location } \\
\text { and Number }\end{array}$ & Supply ACH & $\begin{array}{c}\text { Supply } \\
\text { Temperature }\left({ }^{\circ} \mathrm{C}\right) \\
\end{array}$ & $\begin{array}{c}\text { Supply } \\
\text { Temperature }\left({ }^{\circ} \mathbf{F}\right) \\
\end{array}$ & Room RH ( percent) \\
\hline Case 89 & Low Ind & Low & 15 & 19.2 & 66.6 & 47.1 \\
\hline Case 88 & Slot & Low & 15 & 19.2 & 66.6 & 48.1 \\
\hline Case 30 & Low Ind & Low & 20 & 19.8 & 67.6 & 48.2 \\
\hline Case 09 & Low Ind & Low & 15 & 18.8 & 65.8 & 48.4 \\
\hline Case 87 & Radial & Low & 15 & 19.2 & 66.6 & 48.4 \\
\hline Case 81 & Low Ind & 2 Door exhausts & 15 & 18.8 & 65.8 & 48.7 \\
\hline Case 06 & Slot & Low & 15 & 18.8 & 65.8 & 49.2 \\
\hline Case 28 & Low Ind & Low & 10 & 16.8 & 62.2 & 49.5 \\
\hline Case 03 & Radial & Low & 15 & 18.8 & 65.8 & 49.7 \\
\hline Case 24 & Low Ind & Low & 15 & 18.8 & 65.8 & 49.8 \\
\hline Case 80 & Slot & 2 Door exhausts & 15 & 18.8 & 65.8 & 49.8 \\
\hline Case 61 & Low Ind & Low & 15 & 18.8 & 65.8 & 49.8 \\
\hline Case 79 & Radial & 2 Door exhausts & 15 & 18.8 & 65.8 & 50.3 \\
\hline Case 32 & Slot & Low & 15 & 17.5 & 63.5 & 50.5 \\
\hline Case 58 & Slot & High & 15 & 18.8 & 65.8 & 50.6 \\
\hline Case 33 & Low Ind & Low & 15 & 17.5 & 63.5 & 50.7 \\
\hline Case 23 & Slot & Low & 15 & 18.8 & 65.8 & 50.7 \\
\hline Case 57 & Radial & Low & 15 & 18.8 & 65.8 & 50.7 \\
\hline Case 71 & Low Ind & High $\times 4$ / Low $x 4$ & 15 & 18.8 & 65.8 & 51.2 \\
\hline Case 70 & Slot & High $\times 4 /$ Low $x 4$ & 15 & 18.8 & 65.8 & 51.4 \\
\hline Case 11 & Radial & Low & 15 & 20.7 & 69.3 & 52.0 \\
\hline Case 69 & Radial & High $\times 4$ / Low $x 4$ & 15 & 18.8 & 65.8 & 52.2 \\
\hline Case 31 & Radial & Low & 15 & 17.5 & 63.5 & 52.3 \\
\hline Case 15 & Low Ind & Low & 15 & 20.7 & 69.3 & 52.3 \\
\hline Case 84 & Low Ind & 2 Door exhausts & 15 & 17.5 & 63.5 & 52.3 \\
\hline Case 22 & Radial & Low & 15 & 18.8 & 65.8 & 52.3 \\
\hline Case 12 & Slot & Ceiling & 15 & 20.7 & 69.3 & 52.3 \\
\hline Case 73 & Slot & High $\times 4$ / Low $\times 2$ & 15 & 18.8 & 65.8 & 52.6 \\
\hline Case 83 & Slot & 2 Door exhausts & 15 & 17.5 & 63.5 & 52.6 \\
\hline Case 68 & Low Ind & Low & 20 & 18.9 & 66.0 & 52.7 \\
\hline Case 14 & Low Ind & Ceiling & 15 & 20.7 & 69.3 & 52.7 \\
\hline Case 74 & Low lnd & High $\times 4$ / Low $\times 2$ & 15 & 18.8 & 65.8 & 52.7 \\
\hline Case 75 & Radial & Ceiling/Low 50/50 & 15 & 18.8 & 65.8 & 52.7 \\
\hline Case 82 & Radial & 2 Door exhausts & 15 & 18.8 & 65.8 & 52.8 \\
\hline Case 72 & Radial & High $x 4$ / Low $x 2$ & 15 & 18.8 & 65.8 & 52.8 \\
\hline Case 64 & Low Ind & Low & 20 & 18.9 & 66.0 & 52.9 \\
\hline Case 86 & Slot & Low & 15 & 17.5 & 63.5 & 53.1 \\
\hline Case 10 & Radial & Ceiling & 15 & 20.7 & 69.3 & 53.1 \\
\hline Case 29 & Low Ind & Low & 5 & 11 & 51.8 & 53.2 \\
\hline Case 13 & Slot & Low & 15 & 20.7 & 69.3 & 53.5 \\
\hline Case 38 & Slot & Ceiling/Low 50/50 & 15 & 18.8 & 65.8 & 53.9 \\
\hline Case 39 & Low Ind & Ceiling/Low 50/50 & 15 & 18.8 & 65.8 & 54.0 \\
\hline Case 37 & Radial & Ceiling/Low 50/50 & 15 & 18.8 & 65.8 & 54.2 \\
\hline Case 02 & Radial & High & 15 & 18.8 & 65.8 & 54.3 \\
\hline Case 40 & Radial & Ceiling $\times 4$ & 15 & 18.8 & 65.8 & 54.4 \\
\hline Case 65 & Low Ind & Low & 15 & 17.5 & 63.5 & 54.4 \\
\hline Case 48 & Radial & Ceiling & 15 & 18.8 & 65.8 & 54.5 \\
\hline Case 49 & Radial & Ceiling & 15 & 18.8 & 65.8 & 54.6 \\
\hline Case 05 & Slot & High & 15 & 18.8 & 65.8 & 54.6 \\
\hline Case 41 & Slot & Ceiling $x 4$ & 15 & 18.8 & 65.8 & 54.6 \\
\hline Case 85 & Radial & Low & 15 & 17.5 & 63.5 & 54.7 \\
\hline Case 20 & Slot & Ceiling & 15 & 18.8 & 65.8 & 54.8 \\
\hline Case 42 & Low Ind & Ceiling $x 4$ & 15 & 18.8 & 65.8 & 54.8 \\
\hline Case 51 & Radial & Ceiling & 15 & 18.8 & 65.8 & 54.8 \\
\hline
\end{tabular}




\begin{tabular}{|c|c|c|c|c|c|c|}
\hline Case Name & $\begin{array}{c}\text { Supply Diffuser } \\
\text { Type }\end{array}$ & $\begin{array}{c}\text { Exhaust Location } \\
\text { and Number }\end{array}$ & Supply ACH & $\begin{array}{c}\text { Supply } \\
\text { Temperature }\left({ }^{\circ} \mathrm{C}\right) \\
\end{array}$ & $\begin{array}{c}\text { Supply } \\
\text { Temperature }\left({ }^{\circ} \mathbf{F}\right)\end{array}$ & Room RH ( percent) \\
\hline Case 50 & Radial & Ceiling & 15 & 18.8 & 65.8 & 54.9 \\
\hline Case 91 & Slot & Ceiling & 15 & 18.8 & 65.8 & 55.0 \\
\hline Case 08 & Low Ind & High & 15 & 18.8 & 65.8 & 55.0 \\
\hline Case 26 & Slot & Ceiling & 15 & 18.8 & 65.8 & 55.0 \\
\hline Case 59 & Slot & Low & 15 & 18.8 & 65.8 & 55.0 \\
\hline Case 52 & Radial & Ceiling & 15 & 18.8 & 65.8 & 55.0 \\
\hline Case 92 & Low Ind & Ceiling & 15 & 18.8 & 65.8 & 55.0 \\
\hline Case 78 & Low Ind & Ceiling & 15 & 18.8 & 65.8 & 55.1 \\
\hline Case 04 & Slot & Ceiling & 15 & 18.8 & 65.8 & 55.1 \\
\hline Case 77 & Slot & Ceiling & 15 & 18.8 & 65.8 & 55.2 \\
\hline Case 44 & Radial $\left(\operatorname{rot} 90^{\circ}\right)$ & Ceiling & 15 & 18.8 & 65.8 & 55.2 \\
\hline Case 76 & Radial & Ceiling & 15 & 18.8 & 65.8 & 55.2 \\
\hline Case 21 & Low Ind & Ceiling & 15 & 18.8 & 65.8 & 55.2 \\
\hline Case 90 & Radial & Ceiling & 15 & 18.8 & 65.8 & 55.4 \\
\hline Basecase & Radial & Ceiling & 15 & 18.8 & 65.8 & 55.4 \\
\hline Case 56 & Radial & High & 15 & 18.8 & 65.8 & 55.5 \\
\hline Case 47 & Radial & Ceiling & 15 & 18.8 & 65.8 & 55.5 \\
\hline Case 27 & Low Ind & Ceiling & 15 & 18.8 & 65.8 & 55.5 \\
\hline Case 25 & Radial & Ceiling & 15 & 18.8 & 65.8 & 55.6 \\
\hline Case 45 & Radial & Ceiling & 15 & 18.8 & 65.8 & 55.6 \\
\hline Case 60 & Low Ind & High & 15 & 18.8 & 65.8 & 55.6 \\
\hline Case 43 & Low Ind (rot $\left.90^{\circ}\right)$ & Ceiling & 15 & 18.8 & 65.8 & 55.8 \\
\hline Case 95 & Low Ind & Ceiling & 15 & $17 . \overline{5}$ & 63.5 & 55.9 \\
\hline Case 46 & Radial & Ceiling & 15 & 18.8 & 65.8 & 55.9 \\
\hline Case 19 & Radial & Ceiling & 15 & 18.8 & 65.8 & 55.9 \\
\hline Case 93 & Radial & Ceiling & 15 & 17.5 & 63.5 & 55.9 \\
\hline Case 17 & Slot & Ceiling & 15 & 18.8 & 65.8 & 56.0 \\
\hline Case 18 & Low Ind & Ceiling & 15 & 18.8 & 65.8 & 56.0 \\
\hline Case 16 & Radial & Ceiling & 15 & 18.8 & 65.8 & 56.2 \\
\hline Case 94 & Slot & Ceiling & 15 & 17.5 & 63.5 & 56.3 \\
\hline Case 07 & Low Ind & Ceiling & 15 & 18.8 & 65.8 & 56.7 \\
\hline Case 63 & Low Ind & Low & 10 & 14.8 & 58.6 & 56.8 \\
\hline Case 98 & Low ind & Ceiling & 15 & 17.5 & 63.5 & 56.9 \\
\hline Case 96 & Radial & Ceiling & 15 & 17.5 & 63.5 & 57.0 \\
\hline Case 97 & Slot & Ceiling & 15 & 17.5 & 63.5 & 57.0 \\
\hline Case 67 & Low Ind & Low & 10 & 14.8 & 58.6 & 57.2 \\
\hline Case 35 & Slot & Ceiling & 15 & 17.5 & 63.5 & 57.4 \\
\hline Case 55 & Low Ind & Ceiling & 15 & 18.8 & 65.8 & 57.4 \\
\hline Case 54 & Slot & Ceiling & 15 & 18.8 & 65.8 & 57.4 \\
\hline Case 53 & Radial & Ceiling & 15 & 18.8 & 65.8 & 57.5 \\
\hline Case 34 & Radial & Ceiling & 15 & 17.5 & 63.5 & 57.5 \\
\hline Case 36 & Low Ind & Ceiling & 15 & 17.5 & 63.5 & 57.7 \\
\hline Case 62 & Low Ind & Low & 5 & 6.6 & 43.9 & 62.6 \\
\hline Case 66 & Low Ind & Low & 5 & 6.6 & 43.9 & 64.4 \\
\hline
\end{tabular}


Table 3.04 Ranking of Cases with respect to Cage RH

\begin{tabular}{|c|c|c|c|c|c|c|}
\hline Case Narne & $\begin{array}{l}\text { Supply Diffuser } \\
\text { Type }\end{array}$ & $\begin{array}{l}\text { Exhaust Location } \\
\text { and Number }\end{array}$ & Supply ACH & $\begin{array}{c}\text { Supply } \\
\text { Temperature }\left({ }^{\circ} \mathrm{C}\right)\end{array}$ & $\begin{array}{c}\text { Supply } \\
\text { Temperature }\left({ }^{\circ} \mathrm{F}\right)\end{array}$ & Cage RH ( percent) \\
\hline Case 62 & Low Ind & Low & 5 & 6.6 & 43.9 & 52.8 \\
\hline Case 66 & Low Ind & Low & 5 & 6.6 & 43.9 & 53.0 \\
\hline Case 84 & Low Ind & 2 Door exhausts & 15 & 17.5 & 63.5 & 53.0 \\
\hline Case 29 & Low Ind & Low & 5 & 11 & 51.8 & 55.8 \\
\hline Case 87 & Radial & Low & 15 & 19.2 & 66.6 & 56.3 \\
\hline Case 64 & Low Ind & Low & 20 & 18.9 & 66.0 & 56.5 \\
\hline Case 23 & Slot & Low & 15 & 18.8 & 65.8 & 56.9 \\
\hline Case 69 & Radial & High $\times 4$ / Low $x 4$ & 15 & 18.8 & 65.8 & 57.1 \\
\hline Case 68 & Low Ind & Low & 20 & 18.9 & 66.0 & 57.5 \\
\hline Case 32 & Slot & Low & 15 & 17.5 & 63.5 & 57.5 \\
\hline Case 57 & Radial & Low & 15 & 18.8 & 65.8 & 58.3 \\
\hline Case 79 & Radial & 2 Door exhausts & 15 & 18.8 & 65.8 & 58.6 \\
\hline Case 33 & Low Ind & Low & 15 & 17.5 & 63.5 & 58.7 \\
\hline Case 24 & Low Ind & Low & 15 & 18.8 & 65.8 & 58.7 \\
\hline Case 80 & Slot & 2 Door exhausts & 15 & 18.8 & 65.8 & 59.1 \\
\hline Case 28 & Low lnd & Low & 10 & 16.8 & 62.2 & 59.1 \\
\hline Case 03 & Radial & Low & 15 & 18.8 & 65.8 & 60.2 \\
\hline Case 81 & Low Ind & 2 Door exhausts & 15 & 18.8 & 65.8 & 60.2 \\
\hline Case 06 & Slot & Low & 15 & 18.8 & 65.8 & 60.2 \\
\hline Case 88 & Slot & Low & 15 & 19.2 & 66.6 & 60.4 \\
\hline Case 09 & Low Ind & Low & 15 & 18.8 & 65.8 & 60.6 \\
\hline Case 30 & Low Ind & Low & 20 & 19.8 & 67.6 & 60.9 \\
\hline Case 89 & Low Ind & Low & 15 & 19.2 & 66.6 & 60.9 \\
\hline Case 22 & Radial & Low & 15 & 18.8 & 65.8 & 61.1 \\
\hline Case 31 & Radial & Low & 15 & 17.5 & 63.5 & 61.4 \\
\hline Case 58 & Slot & High & 15 & 18.8 & 65.8 & 61.4 \\
\hline Case 61 & Low Ind & Low & 15 & 18.8 & 65.8 & 61.6 \\
\hline Case 71 & Low Ind & High $x 4$ / Low $x 4$ & 15 & 18.8 & 65.8 & 61.6 \\
\hline Case 10 & Radial & Ceiling & 15 & 20.7 & 69.3 & 61.7 \\
\hline Case 15 & Low Ind & Low & 15 & 20.7 & 69.3 & 61.7 \\
\hline Case 70 & Slot & High $\mathrm{x} 4$ / Low $\mathrm{x} 4$ & 15 & 18.8 & 65.8 & 61.8 \\
\hline Case 82 & Radial & 2 Door exhausts & 15 & 18.8 & 65.8 & 61.9 \\
\hline Case 72 & Radial & High $x 4$ / Low $x 2$ & 15 & 18.8 & 65.8 & 61.9 \\
\hline Case 86 & Slot & Low & 15 & 17.5 & 63.5 & 62.0 \\
\hline Case 02 & Radial & High & 15 & 18.8 & 65.8 & 62.0 \\
\hline Case 41 & Slot & Ceiling $x 4$ & 15 & 18.8 & 65.8 & 62.3 \\
\hline Case 74 & Low Ind & High $x 4$ / Low $x 2$ & 15 & 18.8 & 65.8 & 62.3 \\
\hline Case 37 & Radial & Ceiling/Low 50/50 & 15 & 18.8 & 65.8 & 62.4 \\
\hline Case 38 & Slot & Ceiling/Low 50/50 & 15 & 18.8 & 65.8 & 62.4 \\
\hline Case 05 & Slot & High & 15 & 18.8 & 65.8 & 62.5 \\
\hline Case 20 & Slot & Ceiling & 15 & 18.8 & 65.8 & 62.6 \\
\hline Case 65 & Low Ind & Low & 15 & 17.5 & 63.5 & 62.7 \\
\hline Case 04 & Slot & Ceiling & 15 & 18.8 & 65.8 & 62.7 \\
\hline Case 42 & Low Ind & Ceiling $\times 4$ & 15 & 18.8 & 65.8 & 62.8 \\
\hline Case 92 & Low Ind & Ceiling & 15 & 18.8 & 65.8 & 62.8 \\
\hline Case 85 & Radial & Low & 15 & 17.5 & 63.5 & 62.8 \\
\hline Case 39 & Low Ind & Ceiling/Low 50/50 & 15 & 18.8 & 65.8 & 62.9 \\
\hline Case 73 & Slot & High $x 4$ / Low $x 2$ & 15 & 18.8 & 65.8 & 62.9 \\
\hline Case 90 & Radial & Ceiling & 15 & 18.8 & 65.8 & 63.3 \\
\hline Case 91 & Slot & Ceiling & 15 & 18.8 & 65.8 & 63.4 \\
\hline Case 11 & Radial & Low & 15 & 20.7 & 69.3 & 63.6 \\
\hline Case 12 & Slot & Ceiling & 15 & 20.7 & 69.3 & 63.6 \\
\hline Case 50 & Radial & Ceiling & 15 & 18.8 & 65.8 & 63.7 \\
\hline Case 18 & Low Ind & Ceiling & 15 & 18.8 & 65.8 & 63.8 \\
\hline
\end{tabular}


Volume II - Section III - Rankings of Results of Whole Room Runs

Page II - 45

\begin{tabular}{|c|c|c|c|c|c|c|}
\hline Case Name & $\begin{array}{c}\text { Supply Diffuser } \\
\text { Type }\end{array}$ & $\begin{array}{l}\text { Exhaust Location } \\
\text { and Number }\end{array}$ & Supply ACH & $\begin{array}{c}\text { Supply } \\
\text { Temperature }\left({ }^{\circ} \mathrm{C}\right)\end{array}$ & $\begin{array}{c}\text { Supply } \\
\text { Temperature }\left({ }^{\circ} \mathbf{F}\right) \\
\end{array}$ & Cage RHI ( percent) \\
\hline Case 08 & Low Ind & High & 15 & 18.8 & 65.8 & 63.8 \\
\hline Case 17 & Slot & Ceiling & 15 & 18.8 & 65.8 & 63.9 \\
\hline Case 16 & Radial & Ceiling & 15 & $18 . \overline{8}$ & 65.8 & 64.0 \\
\hline Case 76 & Radial & Ceiling & 15 & 18.8 & 65.8 & 64.1 \\
\hline Case 21 & Low Ind & Ceiling & 15 & 18.8 & 65.8 & $6 \overline{4.1}$ \\
\hline Case 95 & Low Ind & Ceiling & 15 & 17.5 & 63.5 & 64.1 \\
\hline Case 77 & Slot & Ceiling & 15 & 18.8 & 65.8 & 64.2 \\
\hline Case 14 & Low Ind & Ceiling & 15 & 20.7 & 69.3 & 64.2 \\
\hline Case 97 & Slot & Ceiling & 15 & 17.5 & 63.5 & 64.2 \\
\hline Case 94 & Slot & Ceiling & 15 & 17.5 & 63.5 & 64.4 \\
\hline Case 56 & Radial & High & 15 & 18.8 & 65.8 & 64.4 \\
\hline Case 43 & Low Ind (rot $\left.90^{\circ}\right)$ & Ceiling & 15 & 18.8 & 65.8 & 64.6 \\
\hline Case 19 & Radial & Ceiling & 15 & 18.8 & 65.8 & 64.6 \\
\hline Case 78 & Low Ind & Ceiling & 15 & 18.8 & 65.8 & 64.7 \\
\hline Case 52 & Radial & Ceiling & 15 & 18.8 & 65.8 & 64.8 \\
\hline Case 40 & Radial & Ceiling $\times 4$ & 15 & 18.8 & 65.8 & 64.8 \\
\hline Case 45 & Radial & Ceiling & 15 & 18.8 & 65.8 & 64.9 \\
\hline Case 75 & Radial & Ceiling/Low 50/50 & 15 & 18.8 & 65.8 & 64.9 \\
\hline Case 63 & Low Ind & Low & 10 & 14.8 & 58.6 & 64.9 \\
\hline Case 93 & Radial & Ceiling & 15 & 17.5 & 63.5 & 65.0 \\
\hline Case 47 & Radial & Ceiling & 15 & 18.8 & 65.8 & 65.1 \\
\hline Case 49 & Radial & Ceiling & 15 & 18.8 & 65.8 & 65.2 \\
\hline Case 46 & Radial & Ceiling & 15 & 18.8 & 65.8 & 65.2 \\
\hline Case 51 & Radial & Ceiling & 15 & 18.8 & 65.8 & 65.3 \\
\hline Case 35 & Slot & Ceiling & 15 & 17.5 & 63.5 & 65.5 \\
\hline Case 67 & Low Ind & Low & 10 & 14.8 & 58.6 & 65.8 \\
\hline Case 98 & Low ind & Ceiling & 15 & 17.5 & 63.5 & 65.8 \\
\hline Case 96 & Radial & Ceiling & 15 & 17.5 & 63.5 & 65.8 \\
\hline Case 54 & Slot & Ceiling & 15 & 18.8 & 65.8 & 66.0 \\
\hline Case 44 & Radial (rot $90^{\circ}$ ) & Ceiling & 15 & 18.8 & 65.8 & 66.0 \\
\hline Case 48 & Radial & Ceiling & 15 & 18.8 & 65.8 & 66.1 \\
\hline Case 07 & Low ind & Ceiling & 15 & 18.8 & 65.8 & 66.4 \\
\hline Case 13 & Slot & Low & 15 & 20.7 & 69.3 & 66.5 \\
\hline Case 36 & Low Ind & Ceiling & 15 & 17.5 & 63.5 & 66.5 \\
\hline Basecase & Radial & Ceiling & 15 & 18.8 & 65.8 & 66.8 \\
\hline Case 83 & Slot & 2 Door exhausts & 15 & 17.5 & 63.5 & 66.8 \\
\hline Case 59 & Slot & Low & 15 & 18.8 & 65.8 & 66.9 \\
\hline Case 34 & Radial & Ceiling & 15 & 17.5 & 63.5 & 67.2 \\
\hline Case 55 & Low Ind & Ceiling & 15 & 18.8 & 65.8 & 67.8 \\
\hline Case 53 & Radial & Ceiling & 15 & 18.8 & 65.8 & 68.8 \\
\hline Case 60 & Low Ind & High & 15 & 18.8 & 65.8 & 69.1 \\
\hline Case 25 & Radial & Ceiling & 15 & 18.8 & 65.8 & 73.0 \\
\hline Case 27 & Low lnd & Ceiling & 15 & 18.8 & 65.8 & 74.8 \\
\hline Case 26 & Slot & Ceiling & 15 & 18.8 & 65.8 & 81.2 \\
\hline
\end{tabular}


Table 3.05 Ranking of Cases with respect to Room $\mathrm{CO}_{2}$

\begin{tabular}{|c|c|c|c|c|c|c|}
\hline Case Name & $\begin{array}{c}\text { Supply Diffuser } \\
\text { Type }\end{array}$ & $\begin{array}{c}\text { Exhaust Location } \\
\text { and Number }\end{array}$ & Supply ACH & $\begin{array}{c}\text { Supply } \\
\text { Temperature }\left({ }^{\circ} \mathrm{C}\right)\end{array}$ & $\begin{array}{c}\text { Supply } \\
\text { Temperature }\left({ }^{\circ} \mathbf{F}\right) \\
\end{array}$ & Room $\mathrm{CO}_{2}(\mathrm{ppm})$ \\
\hline Case 59 & Slot & Low & 15 & 18.8 & 65.8 & 15 \\
\hline Case 12 & Slot & Ceiling & 15 & 20.7 & 69.3 & 17 \\
\hline Case 40 & Radial & Ceiling $\times 4$ & 15 & 18.8 & 65.8 & 21 \\
\hline Case 70 & Slot & High $\mathrm{x} 4$ / Low $\mathrm{x} 4$ & 15 & 18.8 & 65.8 & 24 \\
\hline Case 10 & Radial & Ceiling & 15 & 20.7 & 69.3 & 29 \\
\hline Case 26 & Slot & Ceiling & 15 & 18.8 & 65.8 & 35 \\
\hline Basecase & Radial & Ceiling & 15 & 18.8 & 65.8 & 36 \\
\hline Case 13 & Slot & Low & 15 & 20.7 & 69.3 & 37 \\
\hline Case 16 & Radial & Ceiling & 15 & 18.8 & 65.8 & 37 \\
\hline Case 73 & Slot & High $\times 4$ / Low $x 2$ & 15 & 18.8 & 65.8 & 37 \\
\hline Case 47 & Radial & Ceiling & 15 & 18.8 & 65.8 & 40 \\
\hline Case 92 & Low Ind & Ceiling & 15 & 18.8 & 65.8 & 41 \\
\hline Case 46 & Radial & Ceiling & 15 & 18.8 & 65.8 & 43 \\
\hline Case 87 & Radial & Low & 15 & 19.2 & 66.6 & 45 \\
\hline Case 11 & Radial & Low & 15 & 20.7 & 69.3 & 48 \\
\hline Case 74 & Low Ind & High $\times 4$ / Low $\times 2$ & 15 & 18.8 & 65.8 & 48 \\
\hline Case 75 & Radial & Ceiling/Low 50/50 & 15 & 18.8 & 65.8 & 48 \\
\hline Case 88 & Slot & Low & 15 & 19.2 & 66.6 & 48 \\
\hline Case 15 & Low Ind & Low & 15 & 20.7 & 69.3 & 48 \\
\hline Case 72 & Radial & High $\times 4$ / Low $\times 2$ & 15 & 18.8 & 65.8 & 48 \\
\hline Case 05 & Slot & High & 15 & 18.8 & 65.8 & 49 \\
\hline Case 77 & Slot & Ceiling & 15 & 18.8 & 65.8 & 50 \\
\hline Case 41 & Slot & Ceiling $x 4$ & 15 & 18.8 & 65.8 & 51 \\
\hline Case 69 & Radial & High $x 4$ / Low $x 4$ & 15 & 18.8 & 65.8 & 51 \\
\hline Case 71 & Low Ind & High $\times 4 /$ Low $x 4$ & 15 & 18.8 & 65.8 & 51 \\
\hline Case 49 & Radial & Ceiling & 15 & 18.8 & 65.8 & 52 \\
\hline Case 14 & Low lnd & Ceiling & 15 & 20.7 & 69.3 & 52 \\
\hline Case 45 & Radial & Ceiling & 15 & 18.8 & 65.8 & 52 \\
\hline Case 50 & Radial & Ceiling & 15 & 18.8 & 65.8 & 55 \\
\hline Case 76 & Radial & Ceiling & 15 & 18.8 & 65.8 & 55 \\
\hline Case 78 & Low Ind & Ceiling & 15 & 18.8 & 65.8 & 55 \\
\hline Case 60 & Low Ind & High & 15 & 18.8 & 65.8 & 57 \\
\hline Case 07 & Low Ind & Cẹiling & 15 & 18.8 & 65.8 & 57 \\
\hline Case 55 & Low lnd & Ceiling & 15 & 18.8 & 65.8 & 57 \\
\hline Case 91 & Slot & Ceiling & 15 & 18.8 & 65.8 & 57 \\
\hline Case 57 & Radial & Low & 15 & 18.8 & 65.8 & 57 \\
\hline Case 53 & Radial & Ceiling & 15 & 18.8 & 65.8 & 58 \\
\hline Case 37 & Radial & Ceiling/Low 50/50 & 15 & 18.8 & 65.8 & 59 \\
\hline Case 19 & Radial & Ceiling & 15 & 18.8 & 65.8 & 59 \\
\hline Case 54 & Slot & Ceiling & 15 & 18.8 & 65.8 & 60 \\
\hline Case 25 & Radial & Ceiling & 15 & 18.8 & 65.8 & 60 \\
\hline Case 22 & Radial & Low & 15 & 18.8 & 65.8 & 61 \\
\hline Case 27 & Low Ind & Ceiling & 15 & 18.8 & 65.8 & 61 \\
\hline Case 21 & Low Ind & Ceiling & 15 & 18.8 & 65.8 & 62 \\
\hline Case 04 & Slot & Ceiling & 15 & 18.8 & 65.8 & 62 \\
\hline Case 89 & Low Ind & Low & 15 & 19.2 & 66.6 & 62 \\
\hline Case 38 & Slot & Ceiling/Low 50/50 & 15 & 18.8 & 65.8 & 62 \\
\hline Case 23 & Slot & Low & 15 & 18.8 & 65.8 & 63 \\
\hline Case 42 & Low Ind & Ceiling $x 4$ & 15 & 18.8 & 65.8 & 63 \\
\hline Case 30 & Low Ind & Low & 20 & 19.8 & 67.6 & 64 \\
\hline Case 08 & Low Ind & High & 15 & 18.8 & 65.8 & 64 \\
\hline Case 03 & Radial & Low & 15 & 18.8 & 65.8 & 64 \\
\hline Case 48 & Radial & Ceiling & 15 & 18.8 & 65.8 & 65 \\
\hline Case 02 & Radial & High & 15 & 18.8 & 65.8 & 65 \\
\hline
\end{tabular}


Volume II - Section III - Rankings of Results of Whole Room Runs

Page II - 47

\begin{tabular}{|c|c|c|c|c|c|c|}
\hline Case Name & $\begin{array}{c}\text { Supply Diffuser } \\
\text { Type }\end{array}$ & $\begin{array}{l}\text { Exhaust Location } \\
\text { and Number }\end{array}$ & Supply ACH & $\begin{array}{c}\text { Supply } \\
\text { Temperature }\left({ }^{\circ} \mathrm{C}\right)\end{array}$ & $\begin{array}{c}\text { Supply } \\
\text { Temperature }\left({ }^{\circ} \mathrm{F}\right) \\
\end{array}$ & Room $\mathrm{CO}_{2}(\mathrm{ppm})$ \\
\hline Case 52 & Radial & Ceiling & 15 & 18.8 & 65.8 & 65 \\
\hline Case 43 & Low Ind (rot $90^{\circ}$ ) & Ceiling & 15 & 18.8 & 65.8 & 65 \\
\hline Case 51 & Radial & Ceiling & 15 & 18.8 & 65.8 & 67 \\
\hline Case 58 & Slot & High & 15 & 18.8 & 65.8 & 68 \\
\hline Case 61 & Low Ind & Low & 15 & 18.8 & 65.8 & 69 \\
\hline Case 56 & Radial & High & 15 & 18.8 & 65.8 & 69 \\
\hline Case 95 & Low Ind & Ceiling & 15 & 17.5 & 63.5 & 69 \\
\hline Case 39 & Low Ind & Ceiling/Low 50/50 & 15 & 18.8 & $\overline{65.8}$ & 71 \\
\hline Case 06 & Slot & Low & 15 & 18.8 & 65.8 & 72 \\
\hline Case 90 & Radial & Ceiling & 15 & 18.8 & 65.8 & 72 \\
\hline Case 44 & Radial (rot $90^{\circ}$ ) & Ceiling & 15 & 18.8 & 65.8 & 74 \\
\hline Case 20 & Slot & Ceiling & 15 & 18.8 & 65.8 & 75 \\
\hline Case 17 & Slot & Ceiling & 15 & 18.8 & 65.8 & 78 \\
\hline Case 18 & Low Ind & Ceiling & 15 & 18.8 & 65.8 & 79 \\
\hline Case 79 & Radial & 2 Door exhausts & 15 & 18.8 & 65.8 & 81 \\
\hline Case 24 & Low lnd & Low & 15 & 18.8 & 65.8 & 81 \\
\hline Case 98 & Low ind & Ceiling & 15 & 17.5 & 63.5 & 83 \\
\hline Case 93 & Radial & Ceiling & 15 & 17.5 & 63.5 & 86 \\
\hline Case 09 & Low Ind & Low & 15 & 18.8 & 65.8 & 88 \\
\hline Case 81 & Low Ind & 2 Door exhausts & 15 & 18.8 & 65.8 & 89 \\
\hline Case 80 & Slot & 2 Door exhausts & 15 & 18.8 & 65.8 & 90 \\
\hline Case 68 & Low Ind & Low & 20 & 18.9 & 66.0 & 93 \\
\hline Case 64 & Low lnd & Low & 20 & 18.9 & 66.0 & 93 \\
\hline Case 96 & Radial & Ceiling & 15 & 17.5 & 63.5 & 100 \\
\hline Case 31 & Radial & Low & 15 & 17.5 & 63.5 & 107 \\
\hline Case 97 & Slot & Ceiling & 15 & 17.5 & 63.5 & 111 \\
\hline Case 28 & Low lnd & Low & 10 & 16.8 & 62.2 & 113 \\
\hline Case 82 & Radial & 2 Door exhausts & 15 & 18.8 & 65.8 & 116 \\
\hline Case 94 & Slot & Ceiling & 15 & 17.5 & 63.5 & 117 \\
\hline Case 35 & Slot & Ceiling & 15 & 17.5 & 63.5 & 122 \\
\hline Case 86 & Slot & Low & 15 & 17.5 & 63.5 & 124 \\
\hline Case 32 & Slot & Low & 15 & 17.5 & 63.5 & 125 \\
\hline Case 85 & Radial & Low & 15 & 17.5 & 63.5 & 132 \\
\hline Case 65 & Low Ind & Low & 15 & 17.5 & 63.5 & 133 \\
\hline Case 36 & Low Ind & Ceiling & 15 & 17.5 & 63.5 & 134 \\
\hline Case 34 & Radial & Ceiling & 15 & 17.5 & 63.5 & 136 \\
\hline Case 29 & Low Ind & Low & 5 & 11 & 51.8 & 140 \\
\hline Case 33 & Low Ind & Low & 15 & 17.5 & 63.5 & 143 \\
\hline Case 84 & Low Ind & 2 Door exhausts & 15 & 17.5 & 63.5 & 152 \\
\hline Case 83 & Slot & 2 Door exhausts & 15 & 17.5 & 63.5 & 167 \\
\hline Case 67 & Low Ind & Low & 10 & 14.8 & 58.6 & 201 \\
\hline Case 63 & Low Ind & Low & 10 & 14.8 & 58.6 & 215 \\
\hline Case 66 & Low Ind & Low & 5 & 6.6 & 43.9 & 318 \\
\hline Case 62 & Low Ind & Low & 5 & 6.6 & 43.9 & 342 \\
\hline
\end{tabular}


Table 3.06 Ranking of Cases with respect to Cage $\mathrm{CO}_{2}$

\begin{tabular}{|c|c|c|c|c|c|c|}
\hline Case Name & $\begin{array}{l}\text { Supply Diffuser } \\
\text { Type }\end{array}$ & $\begin{array}{l}\text { Exhaust Location } \\
\text { and Number }\end{array}$ & Supply ACH & $\begin{array}{c}\text { Supply } \\
\text { Temperature }\left({ }^{\circ} \mathrm{C}\right)\end{array}$ & $\begin{array}{c}\text { Supply } \\
\text { Temperature }\left({ }^{\circ} \mathbf{F}\right)\end{array}$ & $\underset{(\mathrm{ppm})}{\text { Cage } \mathrm{CO}_{2}}$ \\
\hline Case 87 & Radial & Low & 15 & 19.2 & 66.6 & 1391 \\
\hline Case 88 & Slot & Low & 15 & 19.2 & 66.6 & 1550 \\
\hline Case 03 & Radial & Low & 15 & 18.8 & 65.8 & 1568 \\
\hline Case 92 & Low Ind & Ceiling & 15 & 18.8 & 65.8 & 1631 \\
\hline Case 89 & Low Ind & Low & 15 & 19.2 & 66.6 & 1636 \\
\hline Case 79 & Radial & 2 Door exhausts & 15 & 18.8 & 65.8 & 1647 \\
\hline Case 23 & Slot & Low & 15 & 18.8 & 65.8 & 1652 \\
\hline Case 06 & Slot & Low & 15 & 18.8 & 65.8 & 1652 \\
\hline Case 20 & Slot & Ceiling & 15 & 18.8 & 65.8 & 1656 \\
\hline Case 52 & Radial & Ceiling & 15 & 18.8 & 65.8 & 1665 \\
\hline Case 22 & Radial & Low & 15 & 18.8 & 65.8 & 1665 \\
\hline Case 82 & Radial & 2 Door exhausts & $\cdot 15$ & 18.8 & 65.8 & 1667 \\
\hline Case 30 & Low Ind & Low & 20 & 19.8 & 67.6 & 1667 \\
\hline Case 90 & Radial & Ceiling & 15 & 18.8 & 65.8 & 1673 \\
\hline Case 41 & Slot & Ceiling $x 4$ & 15 & 18.8 & 65.8 & 1690 \\
\hline Case 02 & Radial & High & 15 & 18.8 & 65.8 & 1703 \\
\hline Case 80 & Slot & 2 Door exhausts & 15 & 18.8 & 65.8 & 1707 \\
\hline Case 04 & Slot & Ceiling & 15 & 18.8 & 65.8 & 1709 \\
\hline Case 91 & Slot & Ceiling & 15 & 18.8 & 65.8 & 1709 \\
\hline Case 37 & Radial & Ceiling/Low 50/50 & 15 & 18.8 & 65.8 & 1715 \\
\hline Case 97 & Slot & Ceiling & 15 & 17.5 & 63.5 & 1725 \\
\hline Case 09 & Low Ind & Low & 15 & 18.8 & 65.8 & 1725 \\
\hline Case 19 & Radial & Ceiling & 15 & 18.8 & 65.8 & 1725 \\
\hline Case 42 & Low Ind & Ceiling $x 4$ & 15 & 18.8 & 65.8 & 1728 \\
\hline Case 81 & Low Ind & 2 Door exhausts & 15 & 18.8 & 65.8 & 1741 \\
\hline Case 05 & Slot & High & 15 & 18.8 & 65.8 & 1742 \\
\hline Case 32 & Slot & Low & 15 & 17.5 & 63.5 & 1747 \\
\hline Case 28 & Low Ind & Low & 10 & 16.8 & 62.2 & 1749 \\
\hline Case 69 & Radial & High $\times 4$ / Low $x 4$ & 15 & 18.8 & 65.8 & 1750 \\
\hline Case 83 & Slot & 2 Door exhausts & 15 & 17.5 & 63.5 & 1756 \\
\hline Case 94 & Slot & Ceiling & 15 & 17.5 & 63.5 & 1757 \\
\hline Case 35 & Slot & Ceiling & 15 & 17.5 & 63.5 & 1761 \\
\hline Case 29 & Low Ind & Low & 5 & 11 & 51.8 & 1764 \\
\hline Case 38 & Slot & Ceiling/Low 50/50 & 15 & 18.8 & 65.8 & 1776 \\
\hline Case 72 & Radial & High $x 4$ / Low $x 2$ & 15 & 18.8 & 65.8 & 1790 \\
\hline Case 18 & Low Ind & Ceiling & 15 & 18.8 & 65.8 & 1791 \\
\hline Case 64 & Low Ind & Low & 20 & 18.9 & 66.0 & 1808 \\
\hline Case 21 & Low Ind & Ceiling & 15 & 18.8 & 65.8 & 1808 \\
\hline Case 17 & Slot & Ceiling & 15 & 18.8 & 65.8 & 1815 \\
\hline Case 95 & Low Ind & Ceiling & 15 & 17.5 & 63.5 & 1822 \\
\hline Case 93 & Radial & Ceiling & 15 & 17.5 & 63.5 & 1822 \\
\hline Case 68 & Low Ind & Low & 20 & 18.9 & 66.0 & 1838 \\
\hline Case 75 & Radial & Ceiling/Low 50/50 & 15 & 18.8 & 65.8 & 1839 \\
\hline Case 50 & Radial & Ceiling & 15 & 18.8 & 65.8 & 1840 \\
\hline Case 39 & Low Ind & Ceiling/Low 50/50 & 15 & 18.8 & 65.8 & 1848 \\
\hline Case 84 & Low Ind & 2 Door exhausts & 15 & 17.5 & 63.5 & 1853 \\
\hline Case 57 & Radial & Low & 15 & 18.8 & 65.8 & 1855 \\
\hline Case 43 & Low Ind (rot $90^{\circ}$ ) & Ceiling & 15 & 18.8 & 65.8 & 1855 \\
\hline Case 76 & Radial & Ceiling & 15 & 18.8 & 65.8 & 1857 \\
\hline Case 54 & Slot & Ceiling & 15 & 18.8 & 65.8 & 1869 \\
\hline Case 08 & Low Ind & High & 15 & 18.8 & 65.8 & 1871 \\
\hline Case 36 & Low Ind & Ceiling & 15 & 17.5 & 63.5 & 1878 \\
\hline Case 77 & Slot & Ceiling & 15 & 18.8 & 65.8 & 1881 \\
\hline Case 24 & Low Ind & Low & 15 & 18.8 & 65.8 & 1883 \\
\hline
\end{tabular}




\begin{tabular}{|c|c|c|c|c|c|c|}
\hline Case Name & $\begin{array}{c}\text { Supply Diffuser } \\
\text { Type }\end{array}$ & $\begin{array}{c}\text { Exhaust Location } \\
\text { and Number }\end{array}$ & Supply ACH & $\begin{array}{c}\text { Supply } \\
\text { Temperature }\left({ }^{\circ} \mathrm{C}\right)\end{array}$ & $\begin{array}{c}\text { Supply } \\
\text { Temperature }\left({ }^{\circ} \mathrm{F}\right)\end{array}$ & $\begin{array}{c}\text { Cage } \mathrm{CO}_{2} \\
\text { (ppm) }\end{array}$ \\
\hline Case 74 & Low Ind & High $\times 4$ / Low $\times 2$ & 15 & 18.8 & 65.8 & 1884 \\
\hline Case 56 & Radial & High & 15 & 18.8 & 65.8 & 1886 \\
\hline Case 34 & Radial & Ceiling & 15 & 17.5 & 63.5 & 1896 \\
\hline Case 86 & Slot & Low & 15 & 17.5 & 63.5 & 1917 \\
\hline Case 78 & Low Ind & Ceiling & 15 & 18.8 & 65.8 & 1919 \\
\hline Case 45 & Radial & Ceiling & 15 & 18.8 & 65.8 & 1929 \\
\hline Case 85 & Radial & Low & 15 & 17.5 & 63.5 & 1930 \\
\hline Case 31 & Radial & Low & 15 & 17.5 & 63.5 & 1938 \\
\hline Case 46 & Radial & Ceiling & 15 & 18.8 & 65.8 & 1943 \\
\hline Case 98 & Low ind & Ceiling & 15 & 17.5 & 63.5 & 1955 \\
\hline Case 70 & Slot & High $\times 4$ / Low $x 4$ & 15 & 18.8 & 65.8 & 1955 \\
\hline Case 96 & Radial & Ceiling & 15 & 17.5 & 63.5 & 1957 \\
\hline Case 47 & Radial & Ceiling & 15 & 18.8 & 65.8 & 1961 \\
\hline Case 73 & Slot & High $\times 4$ / Low $\times 2$ & 15 & 18.8 & 65.8 & 1966 \\
\hline Case 71 & Low Ind & High $\times 4$ / Low $x 4$ & 15 & 18.8 & 65.8 & 1968 \\
\hline Case 33 & Low Ind & Low & 15 & 17.5 & 63.5 & 1974 \\
\hline Case 40 & Radial & Ceiling $\times 4$ & 15 & 18.8 & 65.8 & 1975 \\
\hline Case 58 & Slot & High & 15 & 18.8 & 65.8 & 1990 \\
\hline Case 65 & Low Ind & Low & 15 & 17.5 & 63.5 & 2002 \\
\hline Case 49 & Radial & Ceiling & 15 & 18.8 & 65.8 & 2025 \\
\hline Case 55 & Low Ind & Ceiling & 15 & 18.8 & 65.8 & 2039 \\
\hline Case 61 & Low lnd & Low & 15 & 18.8 & 65.8 & 2042 \\
\hline Case 51 & Radial & Ceiling & 15 & 18.8 & 65.8 & 2045 \\
\hline Case 63 & Low lnd & Low & 10 & 14.8 & 58.6 & 2081 \\
\hline Case 48 & Radial & Ceiling & 15 & 18.8 & 65.8 & 2099 \\
\hline Case 11 & Radial & Low & 15 & 20.7 & 69.3 & 2118 \\
\hline Case 44 & Radial $\left(\operatorname{rot} 90^{\circ}\right)$ & Ceiling & 15 & 18.8 & 65.8 & 2118 \\
\hline Case 10 & Radial & Ceiling & 15 & 20.7 & 69.3 & 2119 \\
\hline Case 15 & Low Ind & Low & 15 & 20.7 & 69.3 & 2119 \\
\hline Case 67 & Low Ind & Low & 10 & 14.8 & 58.6 & 2144 \\
\hline Basecase & Radial & Ceiling & 15 & 18.8 & 65.8 & 2158 \\
\hline Case 59 & Slot & Low & 15 & 18.8 & 65.8 & 2161 \\
\hline Case 12 & Slot & Ceiling & 15 & 20.7 & 69.3 & 2208 \\
\hline Case 53 & Radial & Ceiling & 15 & 18.8 & 65.8 & 2212 \\
\hline Case 16 & Radial & Ceiling & 15 & 18.8 & 65.8 & 2213 \\
\hline Case 14 & Low Ind & Ceiling & 15 & 20.7 & 69.3 & 2215 \\
\hline Case 66 & Low Ind & Low & 5 & 6.6 & 43.9 & 2318 \\
\hline Case 07 & Low Ind & Ceiling & 15 & 18.8 & 65.8 & 2321 \\
\hline Case 62 & Low Ind & Low & 5 & 6.6 & 43.9 & 2325 \\
\hline Case 60 & Low Ind & High & 15 & 18.8 & 65.8 & 2354 \\
\hline Case 13 & Slot & Low & 15 & 20.7 & 69.3 & 2491 \\
\hline Case 25 & Radial & Ceiling & 15 & 18.8 & 65.8 & 3008 \\
\hline Case 27 & Low Ind & Ceiling & 15 & 18.8 & 65.8 & 3171 \\
\hline Case 26 & Slot & Ceiling & 15 & 18.8 & 65.8 & 3975 \\
\hline
\end{tabular}


Table 3.07 Ranking of Cases with respect to Room $\mathrm{NH}_{3}$

\begin{tabular}{|c|c|c|c|c|c|c|}
\hline Case Name & $\begin{array}{c}\text { Supply Diffuser } \\
\text { Type }\end{array}$ & $\begin{array}{l}\text { Exhaust Location } \\
\text { and Number }\end{array}$ & Supply ACH & $\begin{array}{c}\text { Supply } \\
\text { Temperature }\left({ }^{\circ} \mathrm{C}\right) \\
\end{array}$ & $\begin{array}{c}\text { Supply } \\
\text { Temperature }\left({ }^{\circ} \mathbf{F}\right) \\
\end{array}$ & $\begin{array}{c}\text { Room } \mathrm{NH}_{3} \text { (day 4) } \\
\text { (ppm) }\end{array}$ \\
\hline Case 59 & Slot & Low & 15 & 18.8 & 65.8 & 0.04 \\
\hline Case 12 & Slot & Ceiling & 15 & 20.7 & 69.3 & 0.04 \\
\hline Case 70 & Slot & High $\times 4$ / Low $x 4$ & 15 & 18.8 & 65.8 & 0.06 \\
\hline Case 40 & Radial & Ceiling $x 4$ & 15 & 18.8 & 65.8 & 0.06 \\
\hline Case 10 & Radial & Ceiling & 15 & 20.7 & 69.3 & 0.07 \\
\hline Case 73 & Slot & High $\times 4$ / Low $x 2$ & 15 & 18.8 & 65.8 & 0.09 \\
\hline Case 16 & Radial & Ceiling & 15 & 18.8 & 65.8 & 0.10 \\
\hline Case 87 & Radial & Low & 15 & 19.2 & 66.6 & 0.10 \\
\hline Case 92 & Low Ind & Ceiling & 15 & 18.8 & 65.8 & 0.10 \\
\hline Basecase & Radial & Ceiling & 15 & 18.8 & 65.8 & 0.11 \\
\hline Case 13 & Slot & Low & 15 & 20.7 & 69.3 & 0.11 \\
\hline Case 88 & Slot & Low & 15 & 19.2 & 66.6 & 0.11 \\
\hline Case 47 & Radial & Ceiling & 15 & 18.8 & 65.8 & 0.11 \\
\hline Case 15 & Low lnd & Low & 15 & 20.7 & 69.3 & 0.11 \\
\hline Case 72 & Radial & High $\times 4$ / Low $\times 2$ & 15 & 18.8 & 65.8 & 0.11 \\
\hline Case 74 & Low Ind & High $\times 4$ / Low $x 2$ & 15 & 18.8 & 65.8 & 0.11 \\
\hline Case 69 & Radial & High $\times 4$ / Low $\times 4$ & 15 & 18.8 & 65.8 & 0.12 \\
\hline Case 46 & Radial & Ceiling & 15 & 18.8 & 65.8 & 0.12 \\
\hline Case 71 & Low Ind & High $x 4$ / Low $x 4$ & 15 & 18.8 & 65.8 & 0.12 \\
\hline Case 05 & Slot & High & 15 & 18.8 & 65.8 & 0.12 \\
\hline Case 41 & Slot & Ceiling $x 4$ & 15 & 18.8 & 65.8 & 0.12 \\
\hline Case 11 & Radial & Low & 15 & 20.7 & 69.3 & 0.12 \\
\hline Case 57 & Radial & Low & 15 & 18.8 & 65.8 & 0.13 \\
\hline Case 75 & Radial & Ceiling/Low 50/50 & 15 & 18.8 & 65.8 & 0.13 \\
\hline Case 77 & Slot & Ceiling & 15 & 18.8 & 65.8 & 0.13 \\
\hline Case 14 & Low Ind & Ceiling & 15 & 20.7 & 69.3 & 0.14 \\
\hline Case 22 & Radial & Low & 15 & 18.8 & 65.8 & 0.14 \\
\hline Case 89 & Low Ind & Low & 15 & 19.2 & 66.6 & 0.14 \\
\hline Case 50 & Radial & Ceiling & 15 & 18.8 & 65.8 & 0.14 \\
\hline Case 37 & Radial & Ceiling/Low 50/50 & 15 & 18.8 & 65.8 & 0.14 \\
\hline Case 23 & Slot & Low & 15 & 18.8 & 65.8 & 0.14 \\
\hline Case 45 & Radial & Ceiling & 15 & 18.8 & 65.8 & 0.14 \\
\hline Case 49 & Radial & Ceiling & 15 & 18.8 & 65.8 & 0.14 \\
\hline Case 30 & Low Ind & Low & 20 & 19.8 & 67.6 & 0.14 \\
\hline Case 03 & Radial & Low & 15 & 18.8 & 65.8 & 0.14 \\
\hline Case 76 & Radial & Ceiling & 15 & 18.8 & 65.8 & 0.14 \\
\hline Case 91 & Slot & Ceiling & 15 & 18.8 & 65.8 & 0.14 \\
\hline Case 78 & Low Ind & Ceiling & 15 & 18.8 & 65.8 & 0.15 \\
\hline Case 38 & Slot & Ceiling/Low 50/50 & 15 & 18.8 & 65.8 & 0.15 \\
\hline Case 04 & Slot & Ceiling & 15 & 18.8 & 65.8 & 0.15 \\
\hline Case 02 & Radial & High & 15 & 18.8 & 65.8 & 0.15 \\
\hline Case 42 & Low Ind & Ceiling $x 4$ & 15 & 18.8 & 65.8 & 0.16 \\
\hline Case 58 & Slot & High & 15 & 18.8 & 65.8 & 0.16 \\
\hline Case 19 & Radial & Ceiling & 15 & 18.8 & 65.8 & 0.16 \\
\hline Case 61 & Low Ind & Low & 15 & 18.8 & 65.8 & 0.16 \\
\hline Case 26 & Slot & Ceiling & 15 & 18.8 & 65.8 & 0.16 \\
\hline Case 06 & Slot & Low & 15 & 18.8 & 65.8 & 0.16 \\
\hline Case 21 & Low Ind & Ceiling & 15 & 18.8 & 65.8 & 0.16 \\
\hline Case 07 & Low Ind & Ceiling & 15 & 18.8 & 65.8 & 0.16 \\
\hline Case 08 & Low Ind & High & 15 & 18.8 & 65.8 & 0.16 \\
\hline Case 54 & SIot & Ceiling & 15 & 18.8 & 65.8 & 0.17 \\
\hline Case 43 & Low Ind (rot $\left.90^{\circ}\right)$ & Ceiling & 15 & 18.8 & 65.8 & 0.17 \\
\hline Case 55 & Low Ind & Ceiling & 15 & 18.8 & 65.8 & 0.17 \\
\hline Case 52 & Radial & Ceiling & 15 & 18.8 & 65.8 & 0.18 \\
\hline
\end{tabular}


Volume II - Section III - Rankings of Results of Whole Room Runs

Page II - 51

\begin{tabular}{|c|c|c|c|c|c|c|}
\hline Case Name & $\begin{array}{c}\text { Supply Diffuser } \\
\text { Type }\end{array}$ & $\begin{array}{c}\text { Exhaust Location } \\
\text { and Number }\end{array}$ & Supply ACH & $\begin{array}{c}\text { Supply } \\
\text { Temperature }\left({ }^{\circ} \mathrm{C}\right)\end{array}$ & $\begin{array}{c}\text { Supply } \\
\text { Temperature }\left({ }^{\circ} \mathbf{F}\right)\end{array}$ & $\begin{array}{c}\text { Room } \mathrm{NH}_{3} \text { (day 4) } \\
(\mathrm{ppm})\end{array}$ \\
\hline Case 39 & Low Ind & Ceiling/Low 50/50 & 15 & 18.8 & 65.8 & 0.18 \\
\hline Case 90 & Radial & Ceiling & 15 & 18.8 & 65.8 & 0.18 \\
\hline Case 60 & Low Ind & High & 15 & 18.8 & 65.8 & 0.18 \\
\hline Case 95 & Low Ind & Ceiling & 15 & 17.5 & 63.5 & 0.18 \\
\hline Case 20 & Slot & Ceiling & 15 & 18.8 & 65.8 & 0.18 \\
\hline Case 79 & Radia! & 2 Door exhausts & 15 & 18.8 & 65.8 & 0.18 \\
\hline Case 24 & Low Ind & Low & 15 & 18.8 & 65.8 & 0.18 \\
\hline Case 56 & Radial & High & 15 & 18.8 & 65.8 & 0.18 \\
\hline Case 48 & Radial & Ceiling & 15 & 18.8 & 65.8 & 0.18 \\
\hline Case 51 & Radial & Ceiling & 15 & 18.8 & 65.8 & 0.18 \\
\hline Case 53 & Radial & Ceiling & 15 & 18.8 & 65.8 & $0 . \overline{18}$ \\
\hline Case 09 & Low Ind & Low & 15 & 18.8 & 65.8 & 0.20 \\
\hline Case 81 & Low Ind & 2 Door exhausts & 15 & 18.8 & 65.8 & 0.20 \\
\hline Case 17 & Slot & Ceiling & 15 & 18.8 & 65.8 & 0.20 \\
\hline Case 80 & Slot & 2 Door exhausts & 15 & 18.8 & 65.8 & 0.20 \\
\hline Case 18 & Low lnd & Ceiling & 15 & 18.8 & 65.8 & 0.20 \\
\hline Case 68 & Low lnd & Low & 20 & 18.9 & 66.0 & 0.21 \\
\hline Case 64 & Low Ind & Low & 20 & 18.9 & 66.0 & 0.21 \\
\hline Case 44 & Radial $\left(\operatorname{rot} 90^{\circ}\right)$ & Ceiling & 15 & 18.8 & 65.8 & 0.21 \\
\hline Case 25 & Radial & Ceiling & 15 & 18.8 & 65.8 & 0.22 \\
\hline Case 98 & Low ind & Ceiling & 15 & 17.5 & 63.5 & 0.23 \\
\hline Case 93 & Radial & Ceiling & 15 & 17.5 & 63.5 & 0.24 \\
\hline Case 27 & Low Ind & Ceiling & 15 & 18.8 & 65.8 & 0.24 \\
\hline Case 31 & Radial & Low & 15 & 17.5 & 63.5 & 0.24 \\
\hline Case 28 & Low Ind & Low & 10 & 16.8 & 62.2 & 0.25 \\
\hline Case 82 & Radial & 2 Door exhausts & 15 & 18.8 & 65.8 & 0.27 \\
\hline Case 32 & Slot & Low & 15 & 17.5 & 63.5 & 0.28 \\
\hline Case 96 & Radial & Ceiling & 15 & 17.5 & 63.5 & 0.28 \\
\hline Case 97 & Slot & Ceiling & 15 & 17.5 & 63.5 & 0.29 \\
\hline Case 86 & Slot & Low & 15 & 17.5 & 63.5 & 0.29 \\
\hline Case 94 & Slot & Ceiling & 15 & 17.5 & 63.5 & 0.31 \\
\hline Case 29 & Low Ind & Low & 5 & 11 & 51.8 & 0.32 \\
\hline Case 33 & Low Ind & Low & 15 & 17.5 & 63.5 & 0.32 \\
\hline Case 85 & Radial & Low & 15 & 17.5 & 63.5 & 0.33 \\
\hline Case 65 & Low Ind & Low & 15 & 17.5 & 63.5 & 0.33 \\
\hline Case 35 & Slot & Ceiling & 15 & 17.5 & 63.5 & 0.34 \\
\hline Case 84 & Low Ind & 2 Door exhausts & 15 & 17.5 & 63.5 & 0.34 \\
\hline Case 36 & Low Ind & Ceiling & 15 & 17.5 & 63.5 & 0.39 \\
\hline Case 34 & Radial & Ceiling & 15 & 17.5 & 63.5 & 0.41 \\
\hline Case 83 & Slot & 2 Door exhausts & 15 & 17.5 & 63.5 & 0.49 \\
\hline Case 67 & Low Ind & Low & 10 & 14.8 & 58.6 & 0.57 \\
\hline Case 63 & Low Ind & Low & 10 & 14.8 & 58.6 & 0.58 \\
\hline Case 66 & Low Ind & Low & 5 & 6.6 & 43.9 & 0.72 \\
\hline Case 62 & Low Ind & Low & 5 & 6.6 & 43.9 & 0.77 \\
\hline
\end{tabular}


Table 3.08 Ranking of Cases with respect to Cage $\mathrm{NH}_{3}$ (Day 4)

\begin{tabular}{|c|c|c|c|c|c|c|}
\hline Case Name & $\begin{array}{c}\text { Supply Diffuser } \\
\text { Type }\end{array}$ & $\begin{array}{l}\text { Exhaust Location } \\
\text { and Number }\end{array}$ & $\begin{array}{l}\text { Supply } \\
\text { ACH }\end{array}$ & $\begin{array}{c}\text { Supply } \\
\text { Temperature }\left({ }^{\circ} \mathrm{C}\right)\end{array}$ & $\begin{array}{c}\text { Supply } \\
\text { Temperature } \\
\left({ }^{\circ} \mathbf{F}\right) \\
\end{array}$ & $\begin{array}{c}\text { Cage } \mathrm{NH}_{3} \\
(\text { day 4) }(\mathrm{ppm})\end{array}$ \\
\hline Case 87 & Radial & Low & 15 & 19.2 & 66.6 & 3.13 \\
\hline Case 88 & Slot & Low & 15 & 19.2 & 66.6 & 3.49 \\
\hline Case 03 & Radial & Low & 15 & 18.8 & 65.8 & 3.53 \\
\hline Case 89 & Low Ind & Low & 15 & 19.2 & 66.6 & 3.68 \\
\hline Case 79 & Radial & 2 Door exhausts & 15 & 18.8 & 65.8 & 3.70 \\
\hline Case 23 & Slot & Low & 15 & 18.8 & 65.8 & 3.71 \\
\hline Case 06 & Slot & Low & 15 & 18.8 & 65.8 & 3.72 \\
\hline Case 30 & Low Ind & Low & 20 & 19.8 & 67.6 & 3.75 \\
\hline Case 22 & Radial & Low & 15 & 18.8 & 65.8 & 3.77 \\
\hline Case 80 & Slot & 2 Door exhausts & 15 & 18.8 & 65.8 & 3.84 \\
\hline Case 09 & Low Ind & Low & 15 & 18.8 & 65.8 & 3.88 \\
\hline Case 81 & Low Ind & 2 Door exhausts & 15 & 18.8 & 65.8 & 3.92 \\
\hline Case 82 & Radial & 2 Door exhausts & 15 & 18.8 & 65.8 & 3.92 \\
\hline Case 32 & Slot & Low & 15 & 17.5 & 63.5 & 3.93 \\
\hline Case 28 & Low lnd & Low & 10 & 16.8 & 62.2 & 3.93 \\
\hline Case 69 & Radial & High $\mathrm{x} 4$ / Low $\mathrm{x} 4$ & 15 & 18.8 & 65.8 & 3.94 \\
\hline Case 29 & Low Ind & Low & 5 & 11 & 51.8 & 3.97 \\
\hline Case 92 & Low Ind & Ceiling & 15 & 18.8 & 65.8 & 4.02 \\
\hline Case 02 & Radial & High & 15 & 18.8 & 65.8 & 4.03 \\
\hline Case 20 & Slot & Ceiling & 15 & 18.8 & 65.8 & 4.04 \\
\hline Case 41 & Slot & Ceiling x4 & 15 & 18.8 & 65.8 & 4.06 \\
\hline Case 64 & Low Ind & Low & 20 & 18.9 & 66.0 & 4.07 \\
\hline Case 37 & Radial & Ceiling/Low 50/50 & 15 & 18.8 & 65.8 & 4.13 \\
\hline Case 68 & Low Ind & Low & 20 & 18.9 & 66.0 & 4.13 \\
\hline Case 84 & Low lnd & 2 Door exhausts & 15 & 17.5 & 63.5 & 4.17 \\
\hline Case 57 & Radial & Low & 15 & 18.8 & 65.8 & 4.17 \\
\hline Case 04 & Slot & Ceiling & 15 & 18.8 & 65.8 & 4.18 \\
\hline Case 72 & Radial & High $\times 4$ / Low $\times 2$ & 15 & 18.8 & 65.8 & 4.22 \\
\hline Case 90 & Radial & Ceiling & 15 & 18.8 & 65.8 & 4.22 \\
\hline Case 05 & Slot & High & 15 & 18.8 & 65.8 & 4.23 \\
\hline Case 24 & Low Ind & Low & 15 & 18.8 & 65.8 & 4.24 \\
\hline Case 42 & Low Ind & Ceiling $x 4$ & 15 & 18.8 & 65.8 & 4.25 \\
\hline Case 38 & Slot & Ceiling/Low 50/50 & 15 & 18.8 & 65.8 & 4.28 \\
\hline Case 91 & Slot & Ceiling & 15 & 18.8 & 65.8 & 4.33 \\
\hline Case 33 & Low Ind & Low & 15 & 17.5 & 63.5 & 4.44 \\
\hline Case 31 & Radial & Low & 15 & 17.5 & 63.5 & 4.45 \\
\hline Case 52 & Radial & Ceiling & 15 & 18.8 & 65.8 & 4.49 \\
\hline Case 74 & Low lnd & High $\times 4$ / Low $x 2$ & 15 & 18.8 & 65.8 & 4.53 \\
\hline Case 97 & Slot & Ceiling & 15 & 17.5 & 63.5 & 4.54 \\
\hline Case 86 & Slot & Low & 15 & 17.5 & 63.5 & 4.54 \\
\hline Case 71 & Low lnd & High $\times 4 /$ Low $\times 4$ & 15 & 18.8 & 65.8 & 4.56 \\
\hline Case 58 & Slot & High & 15 & 18.8 & 65.8 & 4.58 \\
\hline Case 39 & Low Ind & Ceiling/Low 50/50 & 15 & 18.8 & 65.8 & 4.58 \\
\hline Case 70 & Slot & High $\times 4$ / Low $x 4$ & 15 & 18.8 & 65.8 & 4.58 \\
\hline Case 19 & Radial & Ceiling & 15 & 18.8 & 65.8 & 4.61 \\
\hline Case 18 & Low lnd & Ceiling & 15 & 18.8 & 65.8 & 4.62 \\
\hline Case 94 & Slot & Ceiling & 15 & 17.5 & 63.5 & 4.66 \\
\hline Case 17 & Slot & Ceiling & 15 & 18.8 & 65.8 & 4.71 \\
\hline Case 61 & Low lnd & Low & 15 & 18.8 & 65.8 & 4.73 \\
\hline Case 21 & Low Ind & Ceiling & 15 & 18.8 & 65.8 & 4.73 \\
\hline Case 50 & Radial & Ceiling & 15 & 18.8 & 65.8 & 4.73 \\
\hline Case 85 & Radial & Low & 15 & 17.5 & 63.5 & 4.76 \\
\hline Case 95 & Low Ind & Ceiling & 15 & 17.5 & 63.5 & 4.77 \\
\hline
\end{tabular}


Volume II - Section III - Rankings of Results of Whole Room Runs

Page II - 53

\begin{tabular}{|c|c|c|c|c|c|c|}
\hline Case Name & $\begin{array}{c}\text { Supply Diffuser } \\
\text { Type }\end{array}$ & $\begin{array}{l}\text { Exhaust Location } \\
\text { and Number }\end{array}$ & $\begin{array}{l}\text { Supply } \\
\text { ACH }\end{array}$ & $\begin{array}{c}\text { Supply } \\
\text { Temperature }\left({ }^{\circ} \mathrm{C}\right)\end{array}$ & $\begin{array}{c}\text { Supply } \\
\text { Temperature } \\
\left({ }^{\circ} \mathrm{F}\right)\end{array}$ & $\begin{array}{c}\text { Cage } \mathrm{NH}_{3} \\
\text { (day 4) (ppm) }\end{array}$ \\
\hline Case 08 & Low lnd & High & 15 & 18.8 & 65.8 & 4.83 \\
\hline Case 76 & Radial & Ceiling & 15 & 18.8 & 65.8 & 4.85 \\
\hline Case 73 & Slot & High $\times 4$ / Low $\times 2$ & 15 & 18.8 & 65.8 & 4.87 \\
\hline Case 65 & Low lnd & Low & 15 & 17.5 & 63.5 & 4.90 \\
\hline Case 35 & Slot & Ceiling & 15 & 17.5 & 63.5 & 4.90 \\
\hline Case 77 & Slot & Ceiling & 15 & 18.8 & 65.8 & 4.94 \\
\hline Case 10 & Radial & Ceiling & 15 & 20.7 & 69.3 & 4.94 \\
\hline Case 15 & Low Ind & Low & 15 & 20.7 & 69.3 & 4.94 \\
\hline Case 43 & Low Ind (rot $\left.90^{\circ}\right)$ & Ceiling & 15 & 18.8 & 65.8 & 4.96 \\
\hline Case 93 & Radial & Ceiling & 15 & 17.5 & 63.5 & 4.97 \\
\hline Case 75 & Radial & Ceiling/Low 50/50 & 15 & 18.8 & 65.8 & 4.98 \\
\hline Case 56 & Radial & High & 15 & 18.8 & 65.8 & 5.01 \\
\hline Case 83 & Slot & 2 Door exhausts & 15 & 17.5 & 63.5 & 5.15 \\
\hline Case 78 & Low Ind & Ceiling & 15 & 18.8 & 65.8 & 5.16 \\
\hline Case 66 & Low lnd & Low & 5 & 6.6 & 43.9 & 5.21 \\
\hline Case 45 & Radial & Ceiling & 15 & 18.8 & 65.8 & 5.22 \\
\hline Case 62 & Low Ind & Low & 5 & 6.6 & 43.9 & 5.23 \\
\hline Case 54 & Slot & Ceiling & 15 & 18.8 & 65.8 & 5.32 \\
\hline Case 40 & Radial & Ceiling $x 4$ & 15 & 18.8 & 65.8 & 5.33 \\
\hline Case 46 & Radial & Ceiling & 15 & 18.8 & 65.8 & 5.35 \\
\hline Case 47 & Radial & Ceiling & 15 & 18.8 & 65.8 & 5.36 \\
\hline Case 11 & Radial & Low & 15 & 20.7 & 69.3 & 5.42 \\
\hline Case 36 & Low Ind & Ceiling & 15 & 17.5 & 63.5 & 5.44 \\
\hline Case 98 & Low ind & Ceiling & 15 & 17.5 & 63.5 & 5.50 \\
\hline Case 96 & Radial & Ceiling & 15 & 17.5 & 63.5 & 5.51 \\
\hline Case 49 & Radial & Ceiling & 15 & 18.8 & 65.8 & 5.56 \\
\hline Case 51 & Radial & Ceiling & 15 & 18.8 & $\overline{65.8}$ & 5.64 \\
\hline Case 63 & Low Ind & Low & 10 & 14.8 & 58.6 & 5.64 \\
\hline Case 12 & Slot & Ceiling & 15 & 20.7 & 69.3 & $5 . \overline{66}$ \\
\hline Case 34 & Radial & Ceiling & 15 & 17.5 & 63.5 & 5.66 \\
\hline Case 16 & Radial & Ceiling & 15 & 18.8 & 65.8 & $5 . \overline{76}$ \\
\hline Case 14 & Low Ind & Ceiling & 15 & 20.7 & 69.3 & 5.82 \\
\hline Case 48 & Radial & Ceiling & 15 & 18.8 & 65.8 & 5.98 \\
\hline Case 44 & Radial $\left(\operatorname{rot} 90^{\circ}\right)$ & Ceiling & 15 & 18.8 & 65.8 & 6.03 \\
\hline Case 67 & Low Ind & Low & 10 & 14.8 & 58.6 & 6.03 \\
\hline Case 55 & Low Ind & Ceiling & 15 & 18.8 & 65.8 & 6.22 \\
\hline Basecase & Radial & Ceiling & 15 & 18.8 & 65.8 & 6.33 \\
\hline Case 59 & Slot & Low & 15 & 18.8 & 65.8 & 6.38 \\
\hline Case 07 & Low Ind & Ceiling & 15 & 18.8 & 65.8 & 6.71 \\
\hline Case 53 & Radial & Ceiling & 15 & 18.8 & 65.8 & 7.02 \\
\hline Case 13 & Slot & Low & 15 & 20.7 & 69.3 & 7.21 \\
\hline Case 60 & Low Ind & High & 15 & 18.8 & 65.8 & 7.56 \\
\hline Case 25 & Radial & Ceiling & 15 & 18.8 & 65.8 & 11.05 \\
\hline Case 27 & Low Ind & Ceiling & 15 & 18.8 & 65.8 & 12.31 \\
\hline Case 26 & Slot & Ceiling & 15 & 18.8 & 65.8 & 18.42 \\
\hline
\end{tabular}




\section{WHOLE ROOM SIMULATION DATABASE}

\subsection{Description of Simulation Data Entry}

The first page contains a listing of the room geometry and ventilation configuration together with a picture. Summary result data is then presented for both average rack and room breathing zones. Temperature and $\mathrm{CO}_{2}$ are presented as single figures whilst $\mathrm{NH}_{3}$ values are shown over a ten day period. Please refer to Volume I: Section 4.1.2.8.2 for an explanation of the relationship between $\mathrm{NH}_{3}$ generation rates and cage relative humidity.

The second page shows three histogram population distributions of temperature, contamination (concentration) and relative humidity in the cage occupied zone of all cages in the room. The histograms show the number of cages (frequency) which have values that fall in the range between two values marked on the horizontal axis with the upper-value indicated. For example, in the sample histogram on the next page the column labeled 22.2 indicates that just over 40 cages have temperatures between $22^{\circ} \mathrm{C}$ and $22.2^{\circ} \mathrm{C}$. 


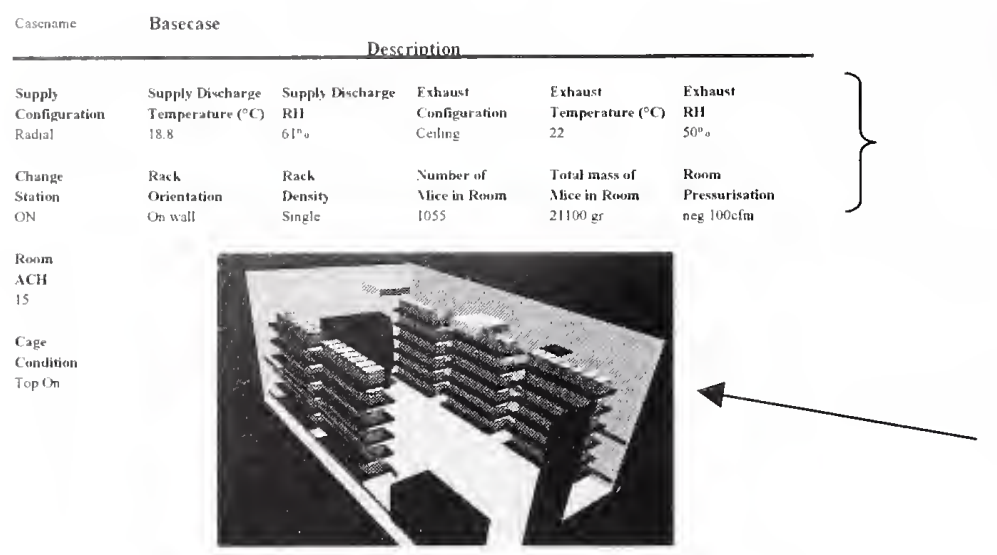

Room cage and ventilation arrangement

Room geometry

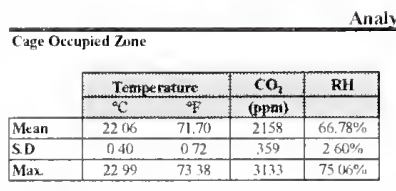

Analysis Results

\section{Cage Occupied Zune NH, (prm)}

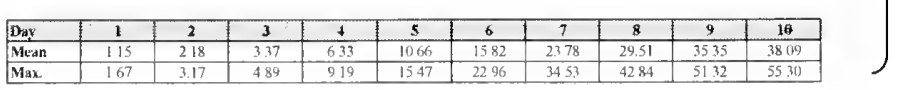

Cage occupied zone summary result data

Rousm Breathing Zune

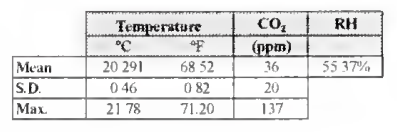

Retrom Breathing Zone $\mathrm{NH}_{3}(\mathrm{ppm})$

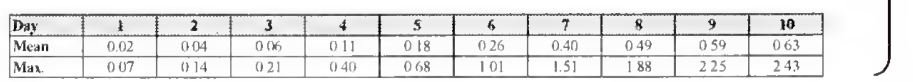

Room breathing zone

summary result data

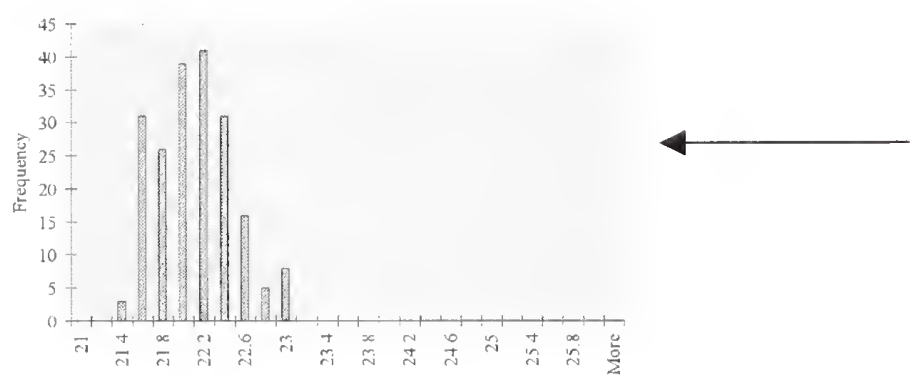

Sample histrogram population distribution 


\subsection{Simulation Data Entries}

The following section presents data entries for each of the CFD animal research facility simulations. 


\section{Casename Basecase}

\section{Description}

\begin{tabular}{|c|c|c|c|c|c|}
\hline Supply & Supply Discharge & Supply Discharge & Exhaust & Exhaust & Exhaust \\
\hline Configuration & Temperature $\left({ }^{\circ} \mathrm{C}\right)$ & RH & Configuration & Temperature $\left({ }^{\circ} \mathrm{C}\right)$ & RH \\
\hline Radial & 18.8 & $61 \%$ & Ceiling & 22 & $50 \%$ \\
\hline Change & Rack & Rack & Number of & Total mass of & Room \\
\hline Station & Orientation & Density & Mice in Room & Mice in Room & Pressurisation \\
\hline ON & On wall & Single & 1050 & $21000 \mathrm{gr}$ & neg $100 \mathrm{cfm}$ \\
\hline
\end{tabular}

Room

$\mathrm{ACH}$

15

Cage

Condition

Top On

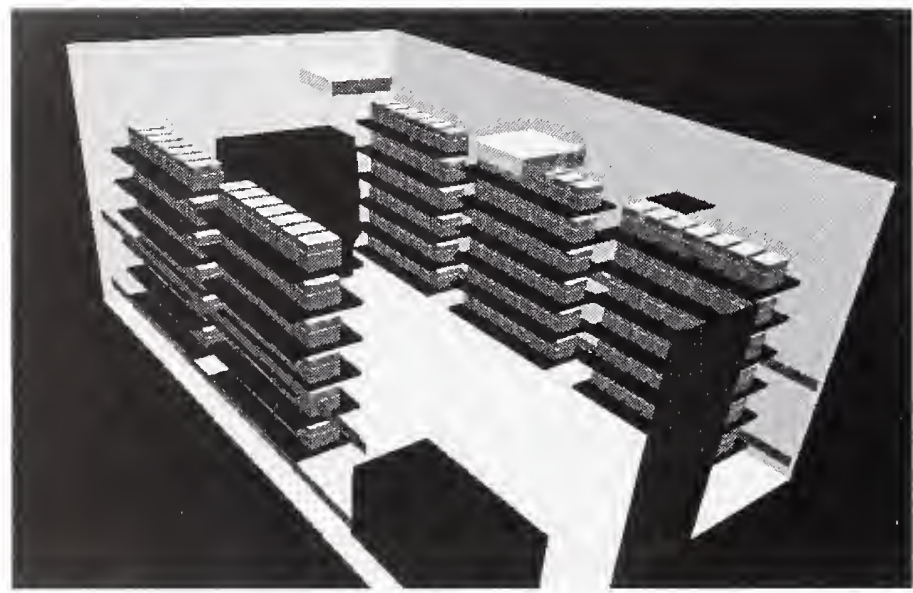

Analysis Results

Cage Occupied Zone

\begin{tabular}{|l|cc|c|c|}
\cline { 2 - 5 } \multicolumn{1}{c|}{} & \multicolumn{2}{c|}{ Temperature } & $\mathrm{CO}_{2}$ & RH \\
\cline { 2 - 5 } \multicolumn{1}{c|}{} & ${ }^{\circ} \mathrm{C}$ & ${ }^{\circ} \mathrm{F}$ & $(\mathrm{ppm})$ & \\
\hline Mean & 22.06 & 71.70 & 2158 & $66.78 \%$ \\
\hline S.D. & 0.40 & 0.72 & 359 & $2.60 \%$ \\
\hline Max. & 22.99 & 73.38 & 3133 & $75.06 \%$ \\
\hline
\end{tabular}

Cage Occupied Zone $\mathrm{NH}_{3}(\mathrm{ppm})$

\begin{tabular}{|l|c|c|c|c|c|c|c|c|c|c|}
\hline Day & $\mathbf{1}$ & $\mathbf{2}$ & $\mathbf{3}$ & $\mathbf{4}$ & $\mathbf{5}$ & $\mathbf{6}$ & $\mathbf{7}$ & $\mathbf{8}$ & $\mathbf{9}$ & $\mathbf{1 0}$ \\
\hline Mean & 1.15 & 2.18 & 3.37 & 6.33 & 10.66 & 15.82 & 23.78 & 29.51 & 35.35 & 38.09 \\
\hline Max. & 1.67 & 3.17 & 4.89 & 9.19 & 15.47 & 22.96 & 34.53 & 42.84 & 51.32 & 55.30 \\
\hline
\end{tabular}

Room Breathing Zone

\begin{tabular}{|l|cc|c|c|}
\cline { 2 - 5 } \multicolumn{1}{c|}{} & \multicolumn{2}{c|}{ Temperature } & $\mathrm{CO}_{2}$ & RH \\
\cline { 2 - 5 } \multicolumn{1}{c|}{} & ${ }^{\circ} \mathrm{C}$ & ${ }^{\circ}$ & $(\mathbf{p p m})$ & \\
\hline Mean & 20.291 & 68.52 & 36 & $55.37 \%$ \\
\hline S.D. & 0.46 & 0.82 & 20 & \multicolumn{1}{|}{} \\
\hline Max. & 21.78 & 71.20 & 137 & \multicolumn{1}{|c|}{} \\
\hline
\end{tabular}

Room Breathing Zone $\mathrm{NH}_{3}$ (ppm)

\begin{tabular}{|l|c|c|c|c|c|c|c|c|c|c|}
\hline Day & 1 & 2 & 3 & 4 & 5 & 6 & 7 & 8 & 9 & 10 \\
\hline Mean & 0.02 & 0.04 & 0.06 & 0.11 & 0.18 & 0.26 & 0.40 & 0.49 & 0.59 & 0.63 \\
\hline Max. & 0.07 & 0.14 & 0.21 & 0.40 & 0.68 & 1.01 & 1.51 & 1.88 & 2.25 & 2.43 \\
\hline
\end{tabular}




\section{Histogram Distributions}

Cage occupied zone average temperature $\left({ }^{\circ} \mathrm{C}\right)$ distribution

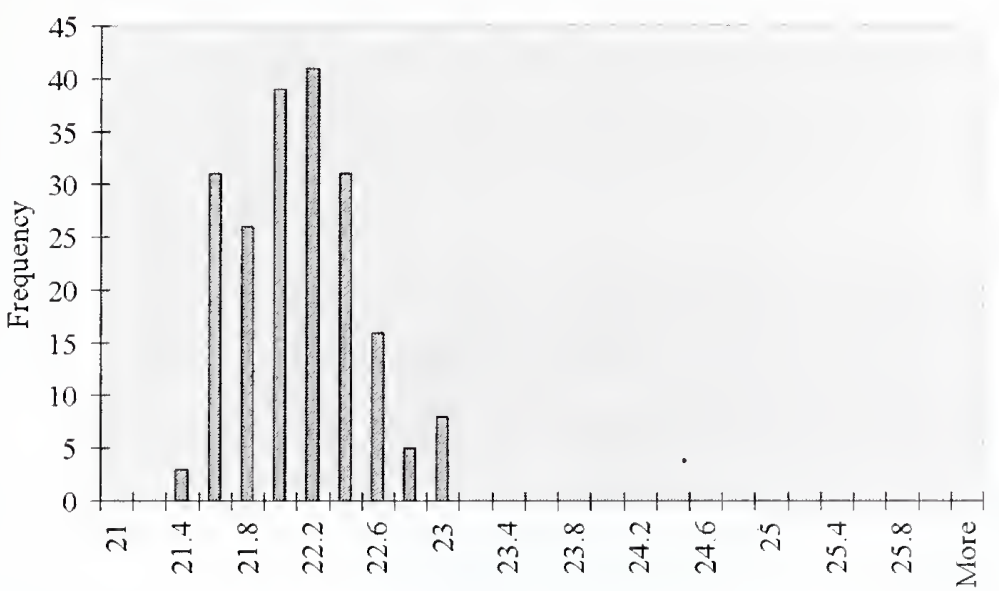

Cage occupied zone average contamination $(\mathrm{kg} / \mathrm{kg})$ distribution

Contamination conversion factors $(\mathrm{kg} / \mathrm{kg} \rightarrow \mathrm{ppm})$

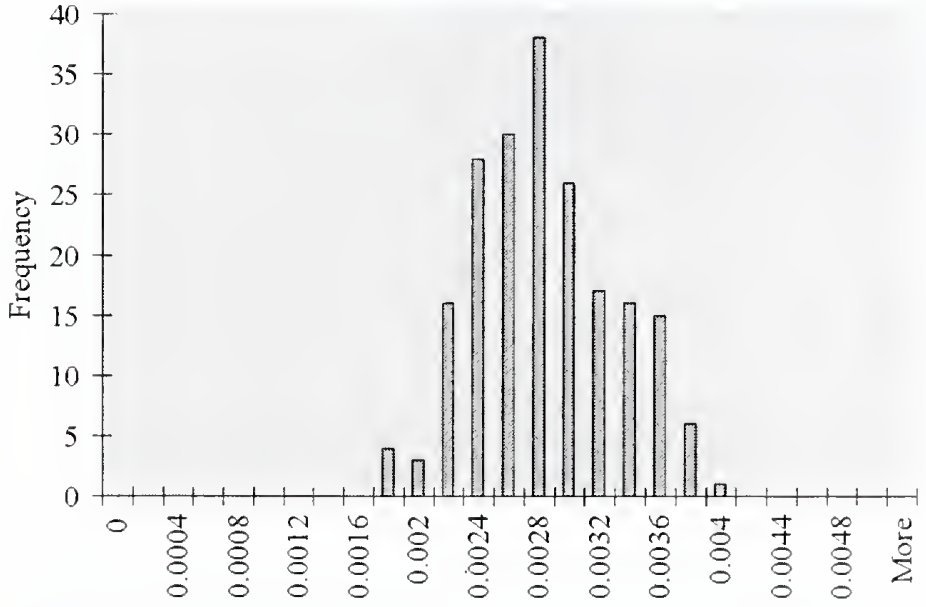

\begin{tabular}{|c|c|c|}
\hline Day & $\mathrm{CO}_{2}$ & $\mathrm{NH}_{3}$ \\
\hline $\mathbf{1}$ & 785000 & 418 \\
\hline $\mathbf{2}$ & 785000 & 795 \\
\hline $\mathbf{3}$ & 785000 & 1225 \\
\hline $\mathbf{4}$ & 785000 & 1922 \\
\hline $\mathbf{5}$ & 785000 & 2848 \\
\hline $\mathbf{6}$ & 785000 & 3443 \\
\hline $\mathbf{7}$ & 785000 & 8409 \\
\hline $\mathbf{8}$ & 785000 & 8483 \\
\hline $\mathbf{9}$ & 785000 & 8442 \\
\hline $\mathbf{1 0}$ & 785000 & 11384 \\
\hline
\end{tabular}

Cage occupied zone average relative humidity (\%) distribution

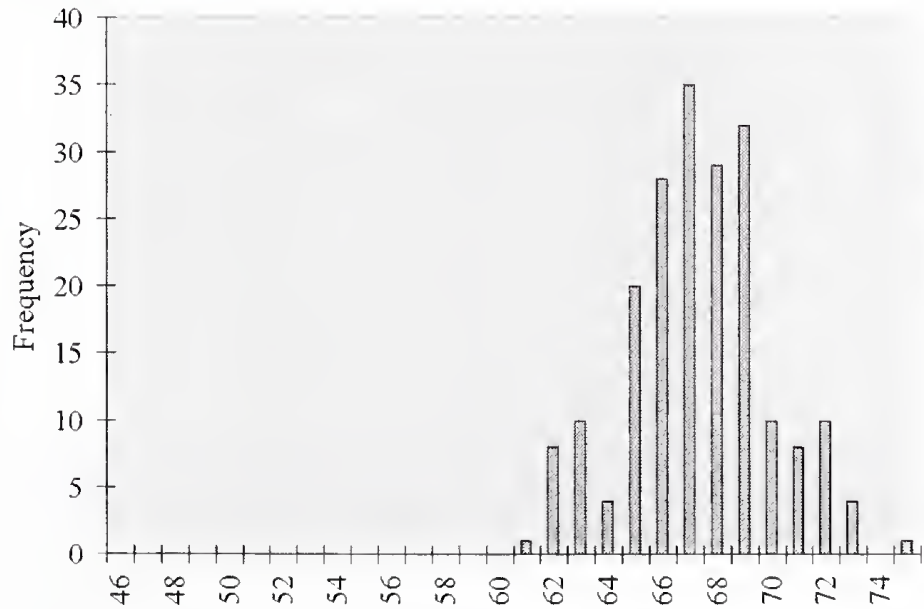




\section{Description}

\begin{tabular}{|c|c|c|c|c|c|}
\hline Supply & Supply Discharge & Supply Discharge & Exhaust & Exhaust & Exhaust \\
\hline Configuration & Temperature $\left({ }^{\circ} \mathrm{C}\right)$ & RH & Configuration & Temperature $\left({ }^{\circ} \mathrm{C}\right)$ & $\mathbf{R H}$ \\
\hline Radial & 18.8 & $61 \%$ & High & 22 & $50 \%$ \\
\hline Change & Rack & Rack & Number of & Total mass of & Room \\
\hline Station & Orientation & Density & Mice in Room & Mice in Room & Pressurisation \\
\hline $\mathrm{ON}$ & On wall & Single & 1050 & $21000 \mathrm{gr}$ & neg $100 \mathrm{cfm}$ \\
\hline
\end{tabular}

\section{Room}

ACH

15

Cage

Condition

Top On

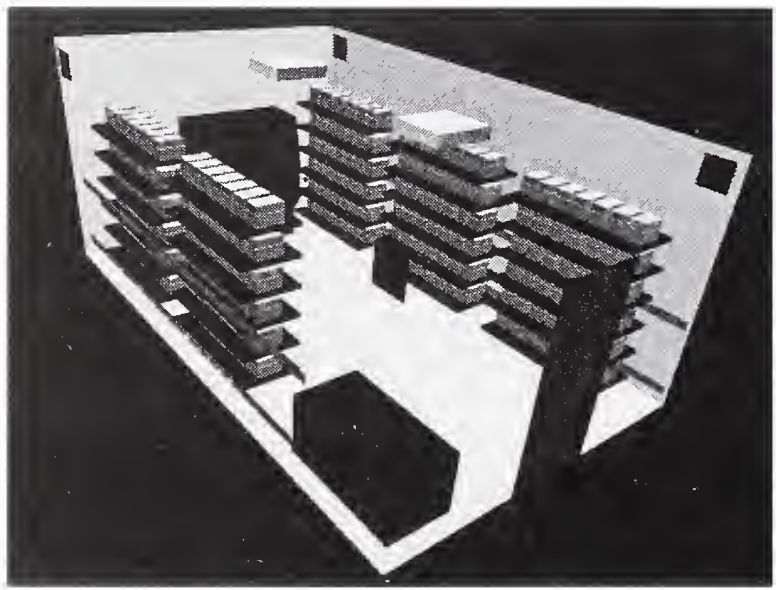

Analysis Results

Cage Occupied Zone

\begin{tabular}{|l|cc|c|c|}
\cline { 2 - 5 } \multicolumn{1}{c|}{} & \multicolumn{2}{c|}{ Temperature } & $\mathrm{CO}_{2}$ & RH \\
\cline { 2 - 5 } \multicolumn{1}{c|}{} & ${ }^{\circ} \mathrm{C}$ & ${ }^{\circ} \mathrm{F}$ & $(\mathbf{p p m})$ & \\
\hline Mean & 22.33 & 72.20 & 1703 & $62.01 \%$ \\
\hline S.D. & 0.27 & 0.48 & 321 & $2.82 \%$ \\
\hline Max. & 23.23 & 73.82 & 2478 & $68.60 \%$ \\
\hline
\end{tabular}

Cage Occupied Zone NH3 (ppm)

\begin{tabular}{|l|c|c|c|c|c|c|c|c|c|c|}
\hline Day & $\mathbf{1}$ & $\mathbf{2}$ & $\mathbf{3}$ & $\mathbf{4}$ & $\mathbf{5}$ & $\mathbf{6}$ & $\mathbf{7}$ & $\mathbf{8}$ & $\mathbf{9}$ & $\mathbf{1 0}$ \\
\hline Mean & 0.89 & 1.72 & 2.66 & 4.03 & 5.92 & 8.35 & 11.72 & 15.45 & 19.99 & 25.01 \\
\hline Max. & 1.29 & 2.51 & 3.87 & 5.87 & 8.61 & 12.14 & 17.05 & 22.48 & 29.08 & 36.39 \\
\hline
\end{tabular}

Room Breathing Zone

\begin{tabular}{|l|cc|c|c|}
\cline { 2 - 5 } \multicolumn{1}{c|}{} & \multicolumn{2}{c|}{ Temperature } & $\mathbf{C O}_{2}$ & RH \\
\cline { 2 - 5 } \multicolumn{1}{c|}{} & ${ }^{\circ} \mathrm{C}$ & ${ }^{\circ} \mathrm{F}$ & $(\mathbf{p p m})$ & \\
\hline Mean & 20.69 & 69.23 & 65 & $54.25 \%$ \\
\hline S.D. & 0.25 & 0.44 & 22 & \\
\hline Max. & 21.60 & 70.89 & 180 & \\
\hline
\end{tabular}

Room Breathing Zone NH3 (ppm)

\begin{tabular}{|l|c|c|c|c|c|c|c|c|c|c|}
\hline Day & $\mathbf{1}$ & $\mathbf{2}$ & $\mathbf{3}$ & $\mathbf{4}$ & $\mathbf{5}$ & $\mathbf{6}$ & $\mathbf{7}$ & $\mathbf{8}$ & $\mathbf{9}$ & $\mathbf{1 0}$ \\
\hline Mean & 0.03 & 0.07 & 0.10 & 0.15 & 0.23 & 0.32 & 0.45 & 0.59 & 0.76 & 0.95 \\
\hline Max. & 0.09 & 0.18 & 0.28 & 0.43 & 0.63 & 0.88 & 1.24 & 1.64 & 2.12 & 2.65 \\
\hline
\end{tabular}




\section{Histogram Distributions}

Cage occupied zone average temperature $\left({ }^{\circ} \mathrm{C}\right)$ distribution

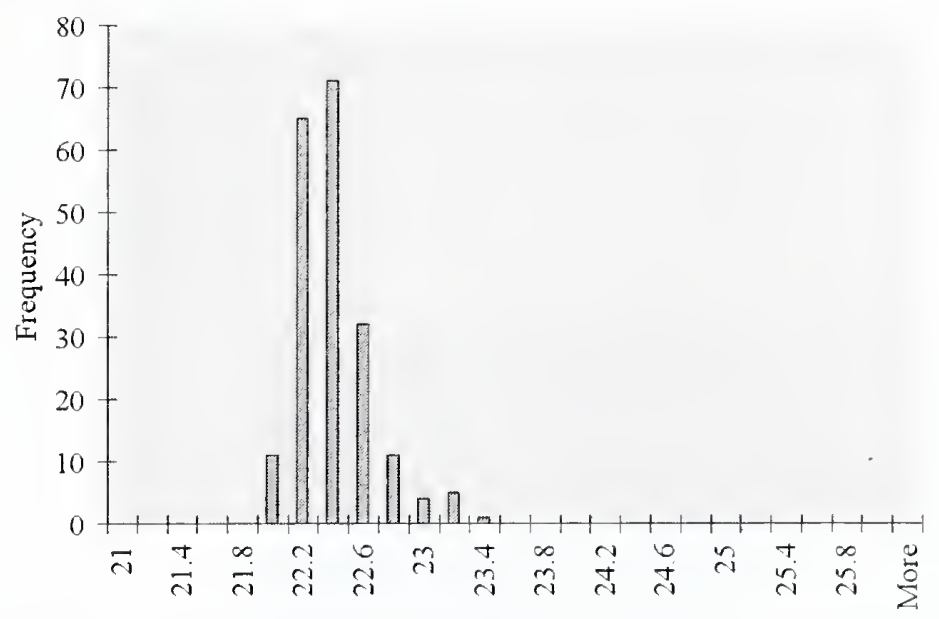

Cage occupied zone average contamination $(\mathrm{kg} / \mathrm{kg})$ distribution

Contamination conversion factors $(\mathrm{kg} / \mathrm{kg} \rightarrow \mathrm{ppm})$

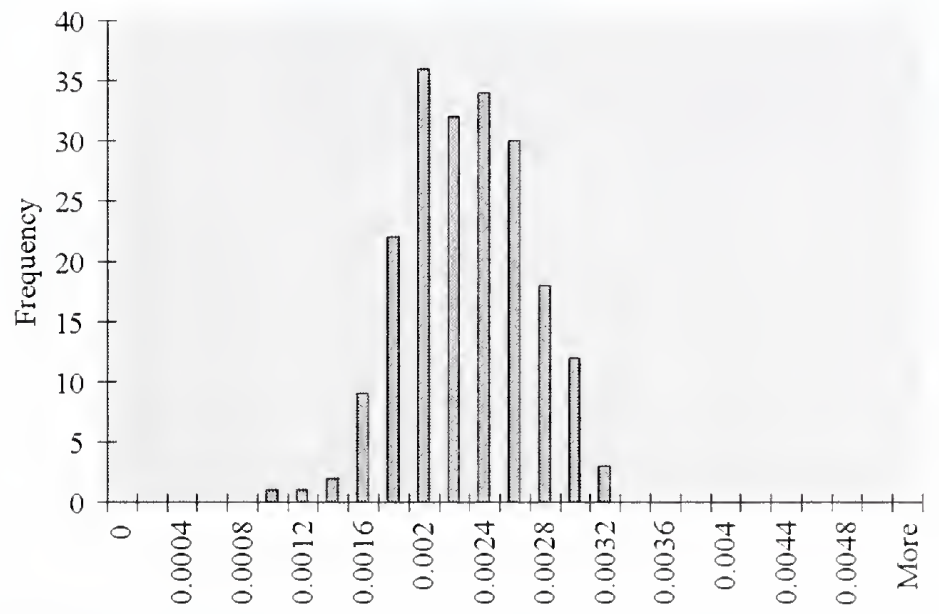

\begin{tabular}{|c|c|c|}
\hline Day & $\mathrm{CO}_{2}$ & $\mathrm{NH}_{3}$ \\
\hline $\mathbf{1}$ & 785000 & 410 \\
\hline $\mathbf{2}$ & 785000 & 795 \\
\hline $\mathbf{3}$ & 785000 & 1225 \\
\hline $\mathbf{4}$ & 785000 & 1860 \\
\hline $\mathbf{5}$ & 785000 & 2727 \\
\hline $\mathbf{6}$ & 785000 & 3847 \\
\hline 7 & 785000 & 5401 \\
\hline $\mathbf{8}$ & 785000 & 7122 \\
\hline $\mathbf{9}$ & 785000 & 9214 \\
\hline 10 & 785000 & 11384 \\
\hline
\end{tabular}

Cage occupied zone average relative humidity (\%) distribution

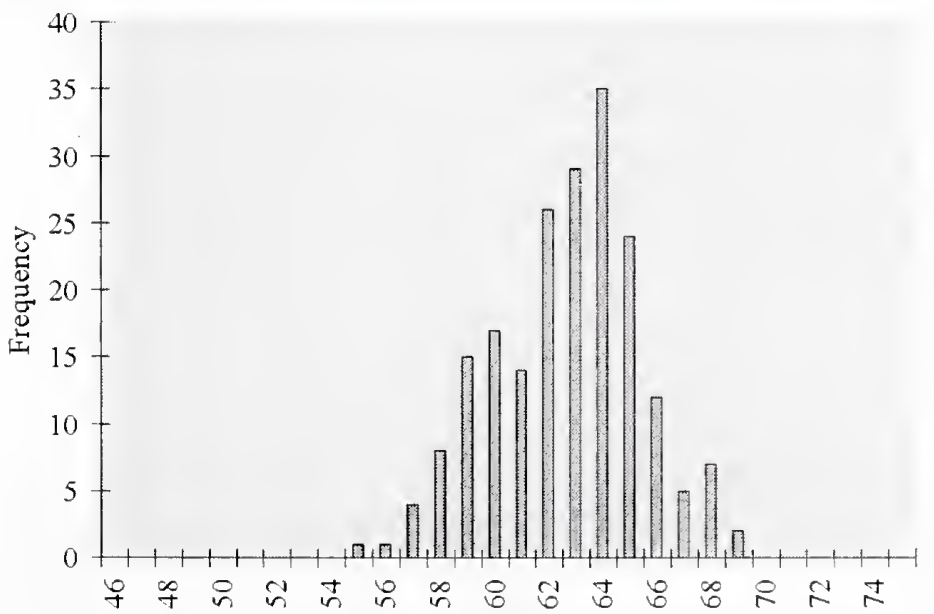




\section{Description}

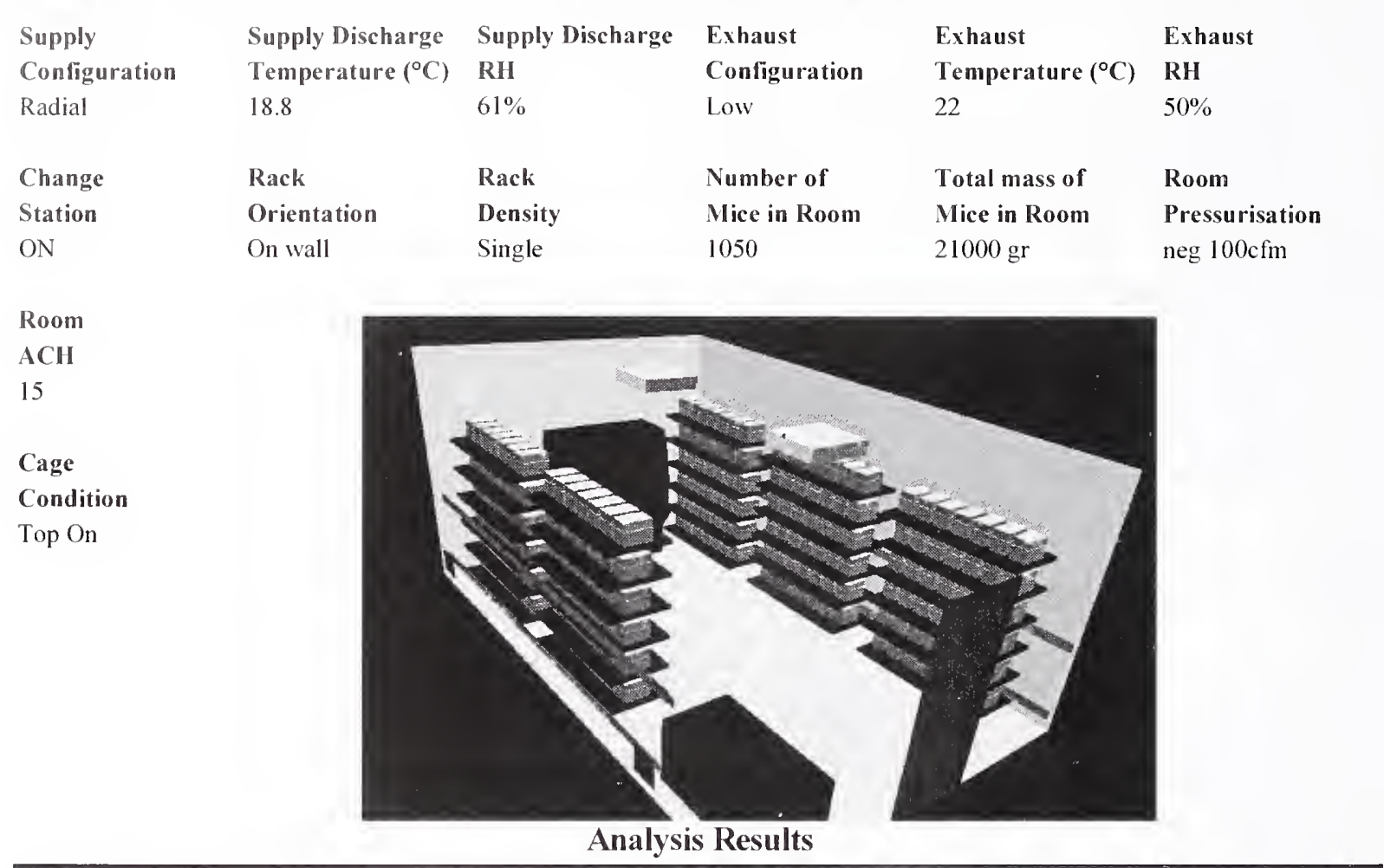

Cage Occupied Zone

\begin{tabular}{|l|cc|c|c|}
\cline { 2 - 5 } \multicolumn{1}{c|}{} & \multicolumn{2}{c|}{ Temperature } & $\mathrm{CO}_{2}$ & RH \\
\cline { 2 - 5 } \multicolumn{1}{c|}{} & ${ }^{\circ} \mathrm{C}$ & ${ }^{\circ} \mathrm{F}$ & $(\mathrm{ppm})$ & \\
\hline Mean & 23.36 & 74.05 & 1568 & $57.15 \%$ \\
\hline S.D. & 0.48 & 0.87 & 343 & $3.00 \%$ \\
\hline Max. & 24.58 & 76.25 & 2581 & $64.65 \%$ \\
\hline
\end{tabular}

Cage Occupied Zone NH3 (ppm)

\begin{tabular}{|l|c|c|c|c|c|c|c|c|c|c|}
\hline Day & $\mathbf{1}$ & $\mathbf{2}$ & $\mathbf{3}$ & $\mathbf{4}$ & $\mathbf{5}$ & $\mathbf{6}$ & $\mathbf{7}$ & $\mathbf{8}$ & $\mathbf{9}$ & $\mathbf{1 0}$ \\
\hline Mean & 0.82 & 1.59 & 2.45 & 3.53 & 4.96 & 6.88 & 9.41 & 12.70 & 16.87 & 22.05 \\
\hline Max. & 1.34 & 2.61 & 4.03 & 5.81 & 8.16 & 11.32 & 15.50 & 20.90 & 27.76 & 36.29 \\
\hline
\end{tabular}

Room Breathing Zone

\begin{tabular}{|l|cc|c|c|}
\cline { 2 - 5 } \multicolumn{1}{c|}{} & \multicolumn{2}{c|}{ Temperature } & $\mathbf{C O}_{2}$ & RH \\
\cline { 2 - 5 } \multicolumn{1}{c|}{} & ${ }^{\circ} \mathrm{C}$ & ${ }^{\circ} \mathrm{F}$ & $(\mathrm{ppm})$ & \\
\hline Mean & 22.06 & 71.71 & 64 & $49.73 \%$ \\
\hline S.D. & 0.37 & 0.66 & 25 & \\
\hline Max. & 23.37 & 74.07 & 170 & \\
\hline
\end{tabular}

Room Breathing Zone NH3 (ppm)

\begin{tabular}{|l|c|c|c|c|c|c|c|c|c|c|}
\hline Day & $\mathbf{1}$ & $\mathbf{2}$ & $\mathbf{3}$ & $\mathbf{4}$ & $\mathbf{5}$ & $\mathbf{6}$ & $\mathbf{7}$ & $\mathbf{8}$ & $\mathbf{9}$ & $\mathbf{1 0}$ \\
\hline Mean & 0.03 & 0.06 & 0.10 & 0.14 & 0.20 & 0.28 & 0.38 & 0.52 & 0.69 & 0.90 \\
\hline Max. & 0.09 & 0.17 & 0.27 & 0.38 & 0.54 & 0.75 & 1.02 & 1.38 & 1.83 & 2.39 \\
\hline
\end{tabular}




\section{Histogram Distributions}

Cage occupied zone average temperature $\left({ }^{\circ} \mathrm{C}\right)$ distribution

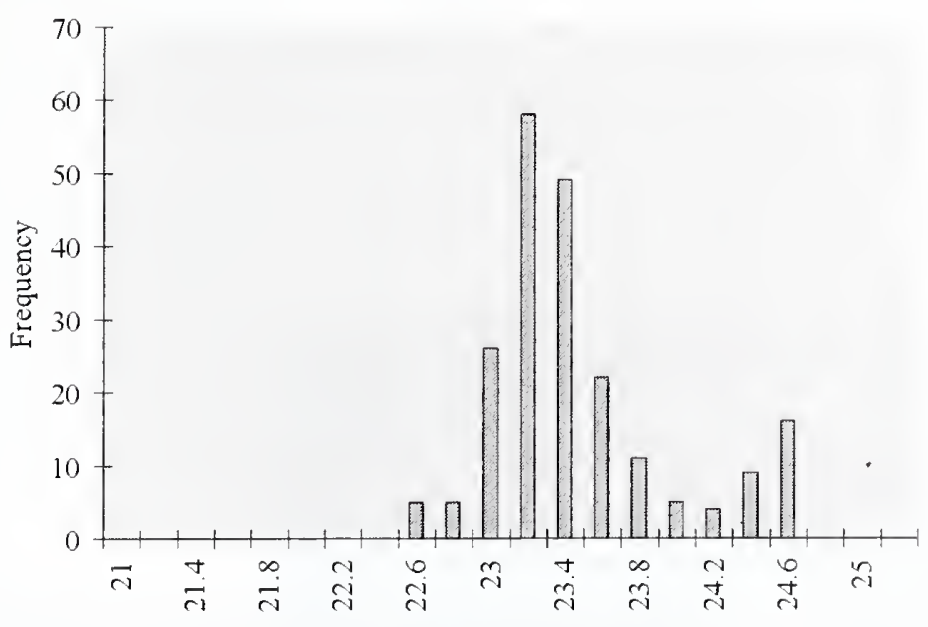

Cage occupied zone average contamination $(\mathrm{kg} / \mathrm{kg})$ distribution

Contamination conversion factors $(\mathrm{kg} / \mathrm{kg} \rightarrow \mathrm{ppm})$

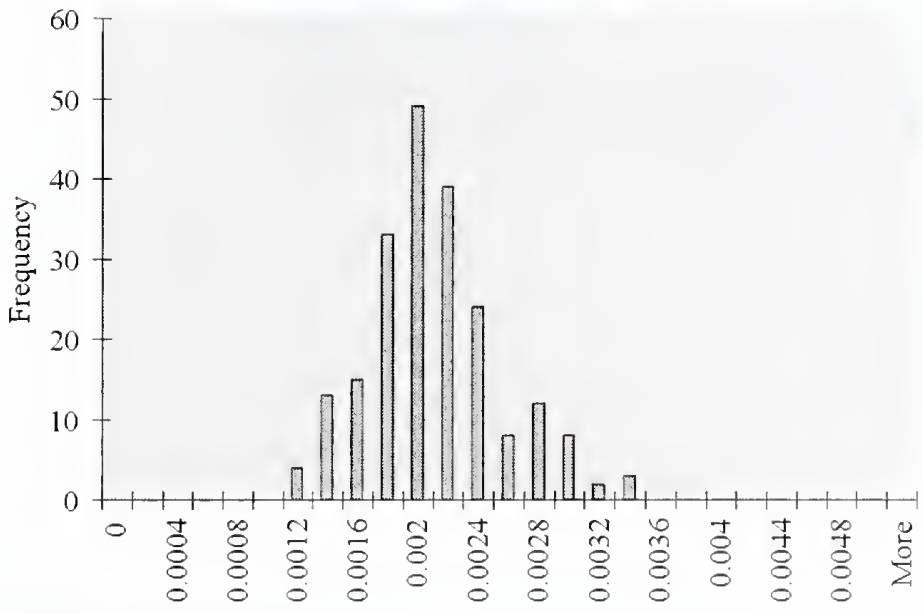

\begin{tabular}{|c|c|c|}
\hline Day & $\mathbf{C O}_{2}$ & $\mathbf{N I I}_{3}$ \\
\hline $\mathbf{1}$ & 785000 & 408 \\
\hline $\mathbf{2}$ & 785000 & 795 \\
\hline $\mathbf{3}$ & 785000 & 1225 \\
\hline $\mathbf{4}$ & 785000 & 1766 \\
\hline $\mathbf{5}$ & 785000 & 2483 \\
\hline $\mathbf{6}$ & 785000 & 3443 \\
\hline $\mathbf{7}$ & 785000 & 4712 \\
\hline $\mathbf{8}$ & 785000 & 6356 \\
\hline $\mathbf{9}$ & 785000 & 8442 \\
\hline $\mathbf{1 0}$ & 785000 & 11384 \\
\hline
\end{tabular}

Cage occupied zone average relative humidity (\%) distribution

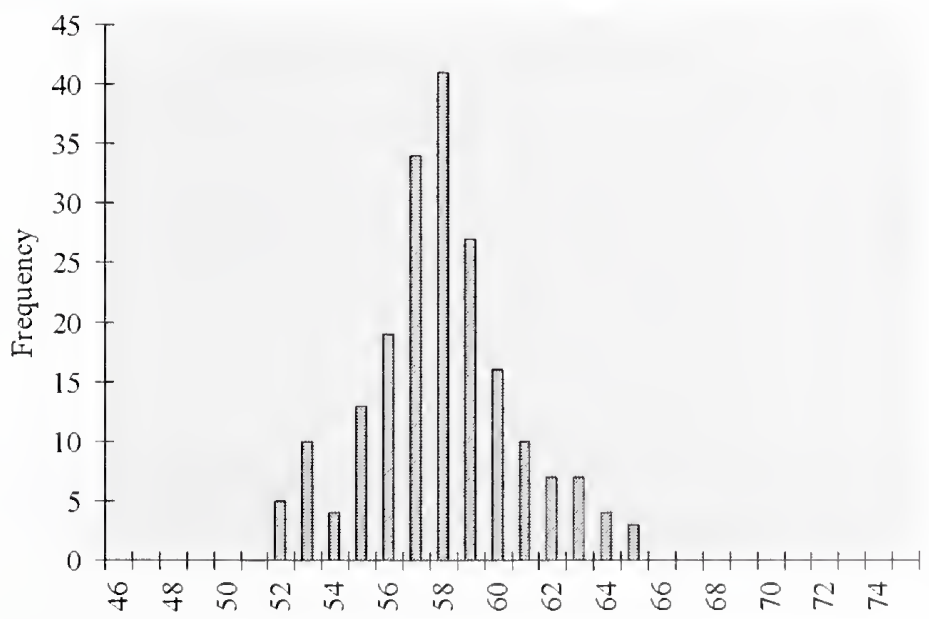




\section{Description}

\begin{tabular}{|c|c|c|c|c|c|}
\hline Supply & Supply Discharge & Supply Discharge & Exhaust & Exhaust & Exhaust \\
\hline Configuration & Temperature $\left({ }^{\circ} \mathrm{C}\right)$ & $\mathrm{RH}$ & Configuration & Temperature $\left({ }^{\circ} \mathrm{C}\right)$ & RH \\
\hline Slot & 18.8 & $61 \%$ & Ceiling & 22 & $50 \%$ \\
\hline Change & Rack & Rack & Number of & Total mass of & Room \\
\hline Station & Orientation & Density & Mice in Room & Mice in Room & Pressurisation \\
\hline ON & On wall & Single & 1050 & $21000 \mathrm{gr}$ & neg $100 \mathrm{cfm}$ \\
\hline
\end{tabular}

Room
ACH
15
Cage
Condition
Top On

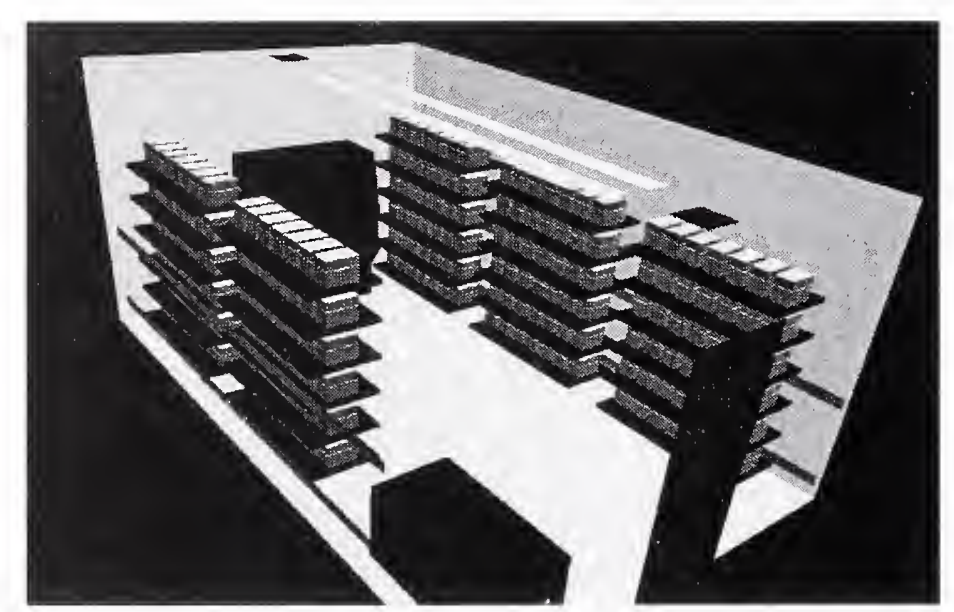

Analysis Results

Cage Occupied Zone

\begin{tabular}{|l|cc|c|c|}
\cline { 2 - 5 } \multicolumn{1}{c|}{} & \multicolumn{2}{c|}{ Temperature } & $\mathbf{C O}_{2}$ & RH \\
\cline { 2 - 5 } \multicolumn{1}{c|}{} & ${ }^{\circ} \mathrm{C}$ & ${ }^{\circ} \mathrm{F}$ & $(\mathbf{p p m})$ & \\
\hline Mean & 22.17 & 71.91 & 1709 & $62.68 \%$ \\
\hline S.D. & 0.29 & 0.52 & 286 & $2.29 \%$ \\
\hline Max. & 22.95 & 73.31 & 2296 & $67.45 \%$ \\
\hline
\end{tabular}

Cage Occupied Zone NH3 (ppm)

\begin{tabular}{|l|c|c|c|c|c|c|c|c|c|c|}
\hline Day & $\mathbf{1}$ & $\mathbf{2}$ & $\mathbf{3}$ & $\mathbf{4}$ & $\mathbf{5}$ & $\mathbf{6}$ & $\mathbf{7}$ & $\mathbf{8}$ & $\mathbf{9}$ & $\mathbf{1 0}$ \\
\hline Mean & 0.90 & 1.73 & 2.67 & 4.18 & 6.29 & $\mathbf{8 . 9 6}$ & $\mathbf{1 2 . 7 5}$ & $\mathbf{1 6 . 6 1}$ & 21.17 & 25.80 \\
\hline Max. & 1.20 & 2.32 & 3.58 & 5.62 & 8.45 & 12.03 & 17.13 & 22.31 & 28.44 & 34.67 \\
\hline
\end{tabular}

Room Breathing Zone

\begin{tabular}{|l|cc|c|c|}
\cline { 2 - 5 } \multicolumn{1}{c|}{} & \multicolumn{2}{c|}{ Temperature } & $\mathrm{CO}_{2}$ & RH \\
\cline { 2 - 5 } \multicolumn{1}{c|}{} & ${ }^{\circ} \mathrm{C}$ & ${ }^{\circ} \mathrm{F}$ & $(\mathbf{p p m})$ & \\
\hline Mean & 20.42 & 68.76 & 62 & $55.14 \%$ \\
\hline S.D. & 0.17 & 0.31 & 26 & \\
\hline Max. & 21.18 & 70.12 & 170 & \\
\hline
\end{tabular}

Room Breathing Zone NH3 (ppm)

\begin{tabular}{|l|c|c|c|c|c|c|c|c|c|c|}
\hline Day & $\mathbf{2}$ & $\mathbf{2}$ & $\mathbf{3}$ & $\mathbf{4}$ & $\mathbf{5}$ & $\mathbf{6}$ & $\mathbf{7}$ & $\mathbf{8}$ & $\mathbf{9}$ & $\mathbf{1 0}$ \\
\hline Mean & 0.03 & 0.06 & 0.10 & 0.15 & 0.23 & 0.32 & 0.46 & 0.60 & 0.77 & 0.94 \\
\hline Max. & 0.09 & 0.17 & 0.26 & 0.42 & 0.62 & 0.89 & 1.27 & 1.65 & 2.10 & 2.56 \\
\hline
\end{tabular}




\section{Histogram Distributions}

Cage occupied zone average temperature $\left({ }^{\circ} \mathrm{C}\right)$ distribution

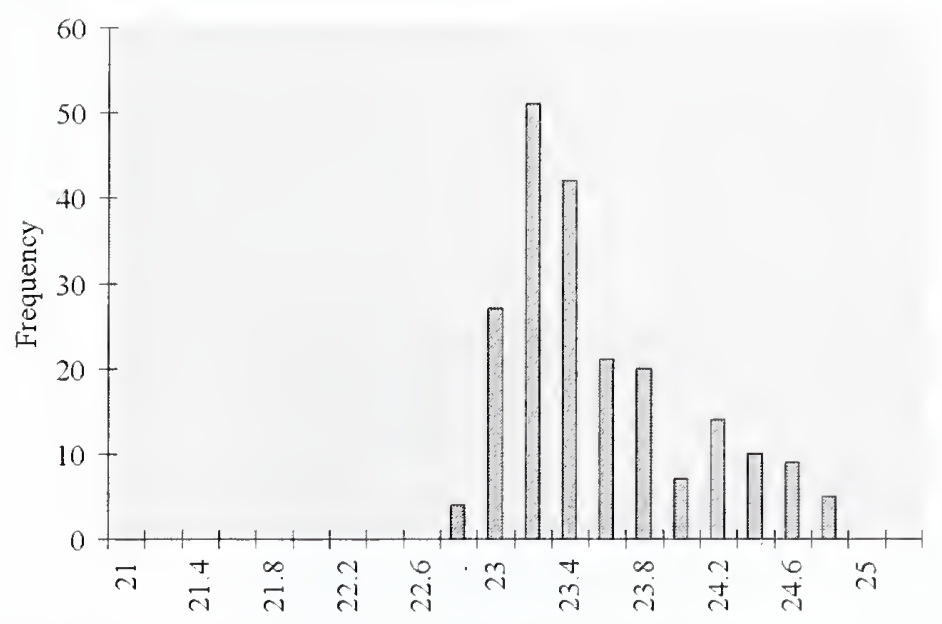

Cage occupied zone average contamination $(\mathrm{kg} / \mathrm{kg})$ distribution

Contamination conversion factors $(\mathrm{kg} / \mathrm{kg} \rightarrow \mathrm{ppm})$

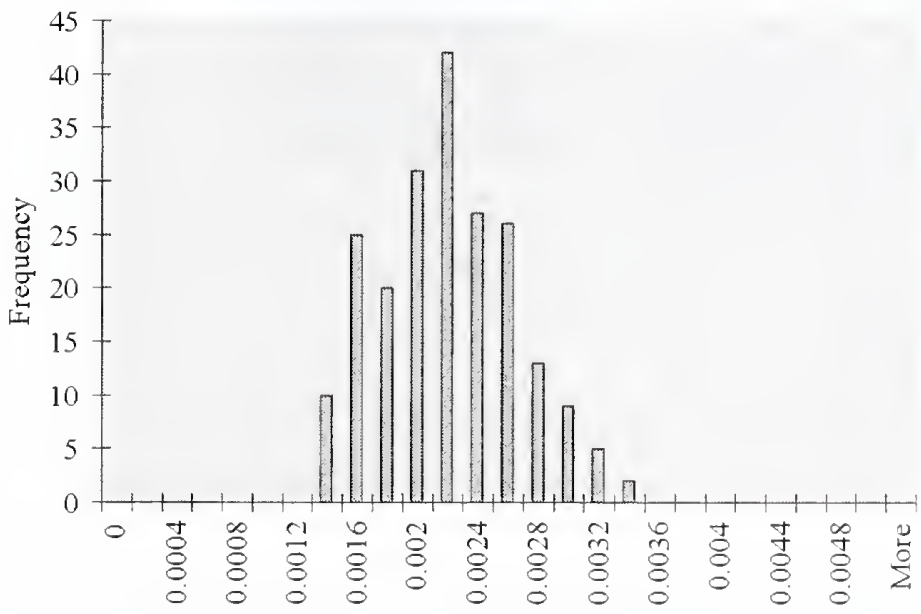

\begin{tabular}{|c|c|c|}
\hline Day & $\mathrm{CO}_{2}$ & $\mathrm{NH}_{3}$ \\
\hline $\mathbf{1}$ & 785000 & 411 \\
\hline $\mathbf{2}$ & 785000 & 795 \\
\hline $\mathbf{3}$ & 785000 & 1225 \\
\hline $\mathbf{4}$ & 785000 & 1922 \\
\hline $\mathbf{5}$ & 785000 & 2888 \\
\hline $\mathbf{6}$ & 785000 & 4114 \\
\hline 7 & 785000 & 5857 \\
\hline $\mathbf{8}$ & 785000 & 7629 \\
\hline $\mathbf{9}$ & 785000 & 9725 \\
\hline 10 & 785000 & 11384 \\
\hline
\end{tabular}

Cage occupied zone average relative humidity (\%) distribution

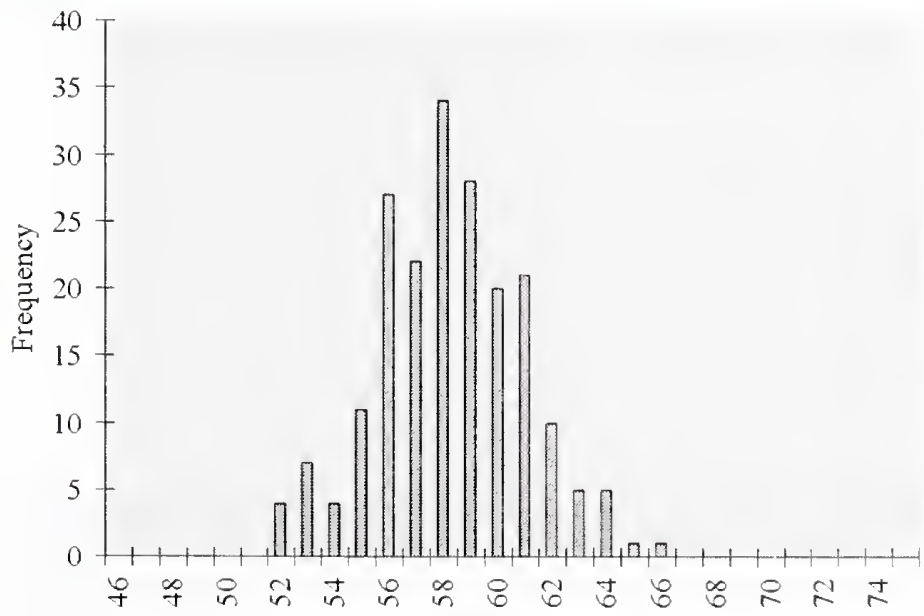


Casename

Case 05

Description

\begin{tabular}{|c|c|c|c|c|c|}
\hline Supply & Supply Discharge & Supply Discharge & Exhaust & Exhaust & Exhaust \\
\hline Configuration & Temperature $\left({ }^{\circ} \mathrm{C}\right)$ & RH & Configuration & Temperature $\left({ }^{\circ} \mathrm{C}\right)$ & RH \\
\hline Slot & 188 & $61 \%$ & High & 22 & $50 \%$ \\
\hline Change & Rack & $\begin{array}{l}\text { Rack } \\
\text { Density }\end{array}$ & Number of & $\begin{array}{l}\text { Total mass of } \\
\text { Nlice in Room }\end{array}$ & $\begin{array}{l}\text { Room } \\
\text { Pressurisation }\end{array}$ \\
\hline $\begin{array}{l}\text { Station } \\
\text { ON }\end{array}$ & $\begin{array}{l}\text { Orientation } \\
\text { On wall }\end{array}$ & $\begin{array}{l}\text { Density } \\
\text { Single }\end{array}$ & $\begin{array}{l}\text { Mice in Room } \\
1050\end{array}$ & $\begin{array}{l}\text { Mice in Room } \\
21000 \mathrm{gr}\end{array}$ & $\begin{array}{l}\text { Pressurisation } \\
\text { neg } 100 \mathrm{cfm}\end{array}$ \\
\hline
\end{tabular}

Room

ACH

15

Cage

Condition

Top On

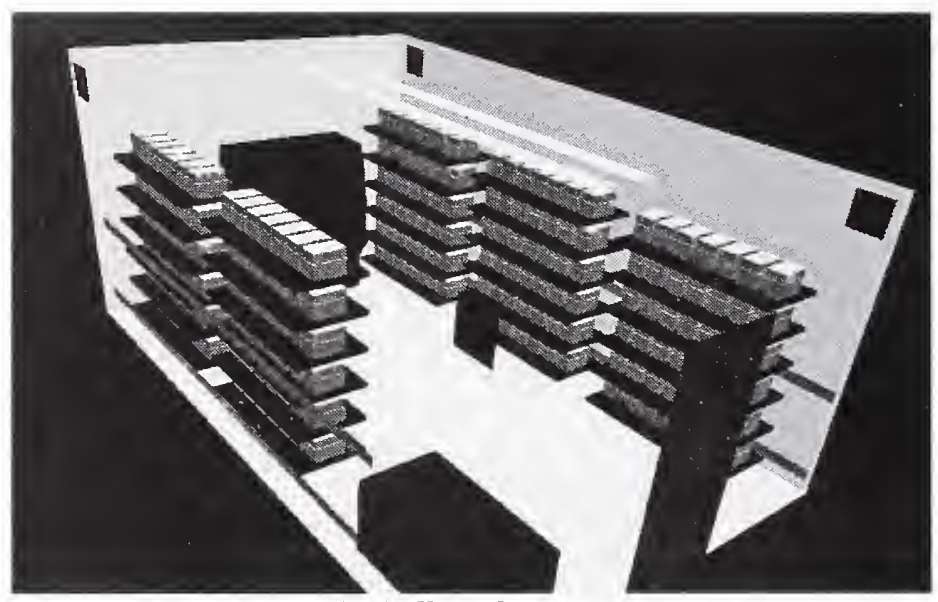

Analysis Results

Cage Occupied Zone

\begin{tabular}{|l|cc|c|c|}
\cline { 2 - 5 } \multicolumn{1}{c|}{} & \multicolumn{2}{c|}{ Temperature } & $\mathrm{CO}_{2}$ & $\mathrm{RH}$ \\
\cline { 2 - 5 } \multicolumn{1}{c|}{} & ${ }^{\circ} \mathrm{C}$ & ${ }^{\circ} \mathrm{F}$ & $(\mathrm{ppm})$ & \\
\hline Mean & 22.28 & 72.10 & 1742 & $62.51 \%$ \\
\hline S.D. & 0.25 & 0.45 & 276 & $2.09 \%$ \\
\hline Max. & 22.86 & 73.15 & 2333 & $67.21 \%$ \\
\hline
\end{tabular}

Cage Occupied Zone NH3 (ppm)

\begin{tabular}{|l|c|c|c|c|c|c|c|c|c|c|}
\hline Day & $\mathbf{1}$ & $\mathbf{2}$ & $\mathbf{3}$ & $\mathbf{4}$ & $\mathbf{5}$ & $\mathbf{6}$ & $\mathbf{7}$ & $\mathbf{8}$ & $\mathbf{9}$ & $\mathbf{1 0}$ \\
\hline Mean & 0.91 & 1.76 & 2.72 & 4.23 & 6.32 & 8.99 & 12.75 & 16.66 & 21.31 & 26.14 \\
\hline Max. & 1.22 & 2.36 & 3.64 & 5.67 & 8.46 & 12.03 & 17.07 & 22.30 & 28.53 & 34.99 \\
\hline
\end{tabular}

Room Breathing Zone

\begin{tabular}{|l|cc|c|c|}
\cline { 2 - 5 } \multicolumn{1}{c|}{} & \multicolumn{2}{c|}{ Temperature } & $\mathrm{CO}_{2}$ & RH \\
\cline { 2 - 5 } \multicolumn{1}{c|}{} & ${ }^{\circ} \mathrm{C}$ & ${ }^{\circ} \mathrm{F}$ & $(\mathrm{ppm})$ & \\
\hline Mean & 20.54 & 68.96 & 49 & $54.63 \%$ \\
\hline S.D. & 0.19 & 0.34 & 24 & \\
\hline Max. & 21.94 & 71.48 & 153 & \\
\hline
\end{tabular}

Room Breathing Zone NH3 (ppm)

\begin{tabular}{|l|c|c|c|c|c|c|c|c|c|c|}
\hline Day & $\mathbf{1}$ & $\mathbf{2}$ & $\mathbf{3}$ & $\mathbf{4}$ & $\mathbf{5}$ & $\mathbf{6}$ & $\mathbf{7}$ & $\mathbf{8}$ & $\mathbf{9}$ & $\mathbf{1 0}$ \\
\hline Mean & 0.03 & 0.05 & 0.08 & 0.12 & 0.18 & 0.25 & 0.36 & 0.47 & 0.60 & 0.74 \\
\hline Max. & 0.08 & 0.16 & 0.24 & 0.37 & 0.56 & 0.79 & 1.12 & 1.47 & 1.88 & 2.30 \\
\hline
\end{tabular}




\section{Histogram Distributions}

Cage occupied zone average temperature $\left({ }^{\circ} \mathrm{C}\right)$ distribution

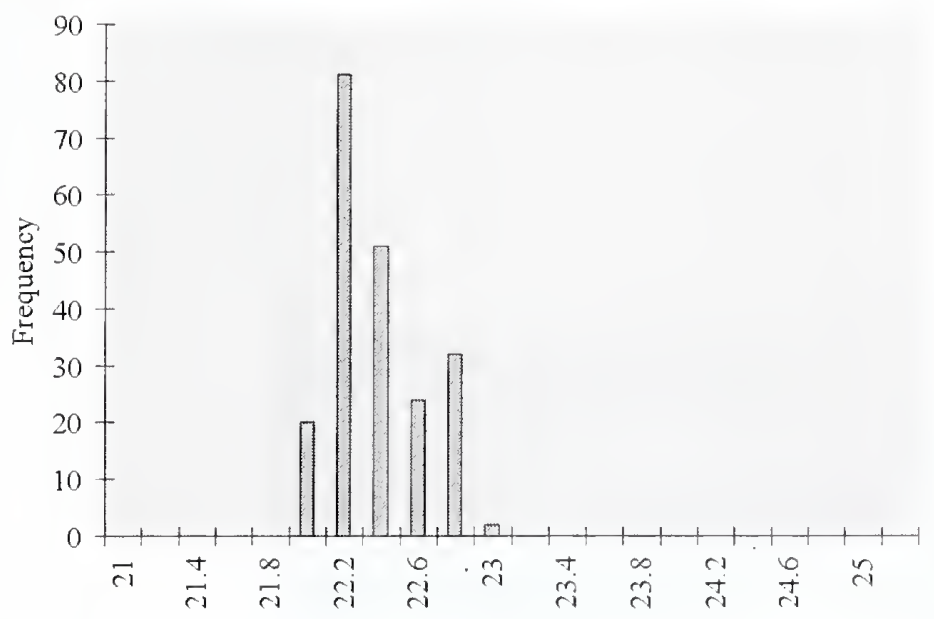

Cage occupied zone average contamination $(\mathrm{kg} / \mathrm{kg})$ distribution

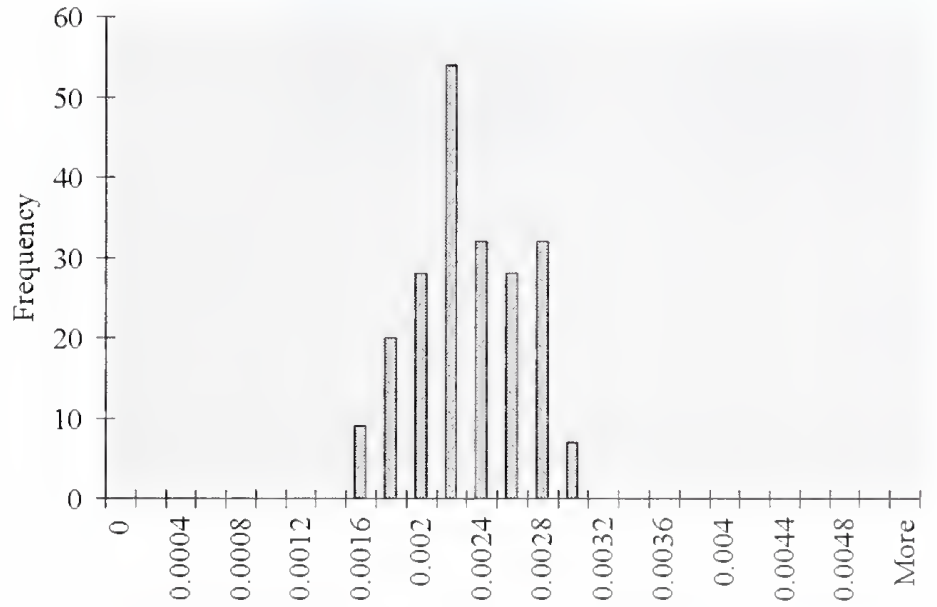

Cage occupied zone average relative humidity (\%) distribution

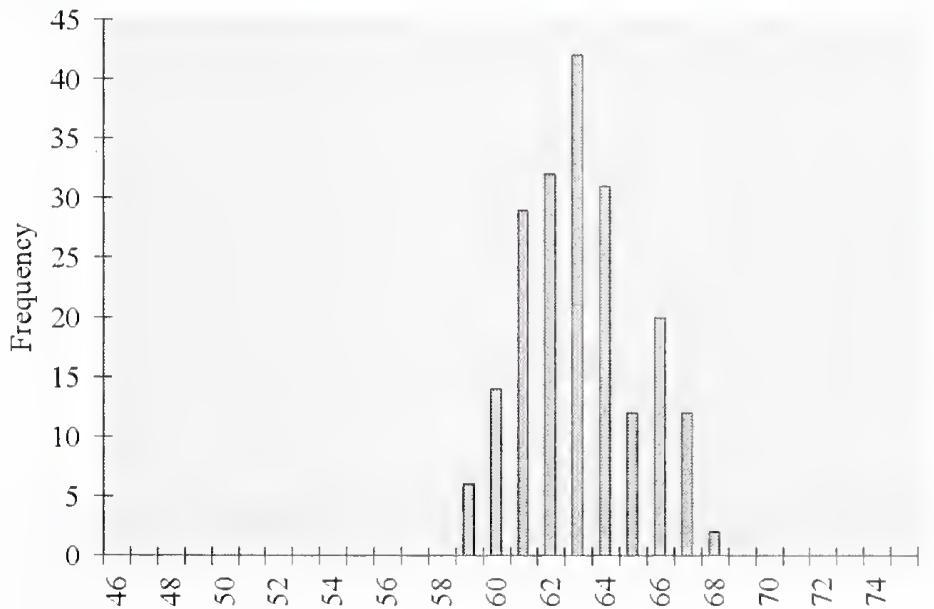

Contamination conversion factors $(\mathrm{kg} / \mathrm{kg} \rightarrow \mathrm{ppm})$

\begin{tabular}{|c|c|c|}
\hline Day & $\mathrm{CO}_{2}$ & $\mathrm{NH}_{3}$ \\
\hline $\mathbf{1}$ & 785000 & 411 \\
\hline $\mathbf{2}$ & 785000 & 795 \\
\hline $\mathbf{3}$ & 785000 & 1225 \\
\hline $\mathbf{4}$ & 785000 & 1906 \\
\hline $\mathbf{5}$ & 785000 & 2848 \\
\hline $\mathbf{6}$ & 785000 & 4048 \\
\hline 7 & 785000 & 5744 \\
\hline $\mathbf{8}$ & 785000 & 7504 \\
\hline $\mathbf{9}$ & 785000 & 9599 \\
\hline $\mathbf{1 0}$ & 785000 & 11384 \\
\hline
\end{tabular}


Casename

Case 06

\section{Description}

\begin{tabular}{|c|c|c|c|c|c|}
\hline Supply & Supply Discharge & Supply Discharge & Exhaust & Exhaust & Exhaust \\
\hline Configuration & Temperature $\left({ }^{\circ} \mathrm{C}\right)$ & RH & Configuration & Temperature $\left({ }^{\circ} \mathrm{C}\right)$ & RH \\
\hline Slot & 18.8 & $61 \%$ & Low & 22 & $50 \%$ \\
\hline Change & $\begin{array}{l}\text { Rack } \\
\text { Orientation }\end{array}$ & $\begin{array}{l}\text { Rack } \\
\text { Density }\end{array}$ & $\begin{array}{l}\text { Number of } \\
\text { Mice in Room }\end{array}$ & $\begin{array}{l}\text { Total mass of } \\
\text { Mice in Room }\end{array}$ & $\begin{array}{l}\text { Room } \\
\text { Pressurisation }\end{array}$ \\
\hline ON & On wall & Single & 1050 & $21000 \mathrm{gr}$ & neg $100 \mathrm{cfm}$ \\
\hline
\end{tabular}

Room

$\mathrm{ACH}$

15

Cage

Condition

Top On

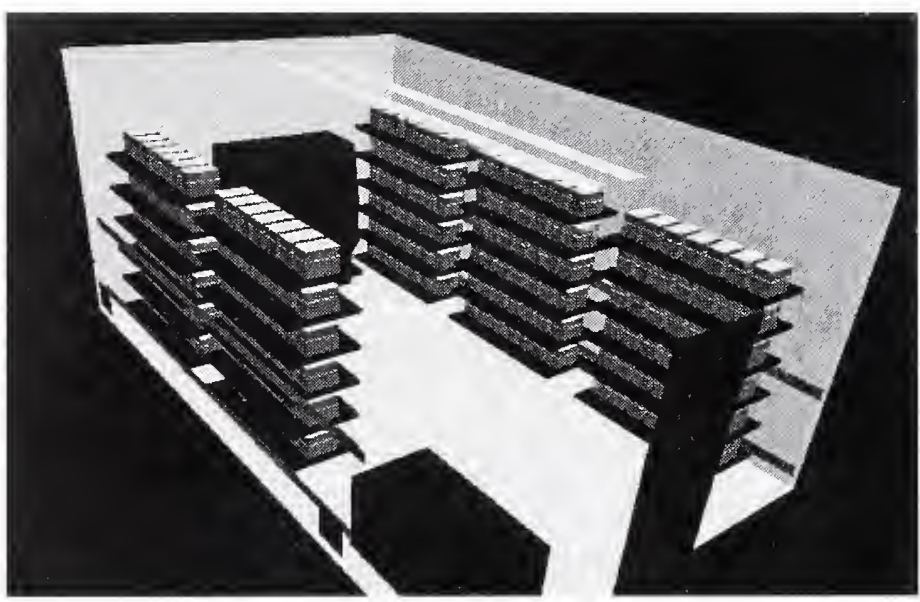

Analysis Results

Cage Occupied Zone

\begin{tabular}{|l|cc|c|c|}
\cline { 2 - 5 } \multicolumn{1}{c|}{} & \multicolumn{2}{c|}{ Temperature } & $\mathrm{CO}_{2}$ & RH \\
\cline { 2 - 5 } \multicolumn{1}{c|}{} & ${ }^{\circ} \mathrm{C}$ & ${ }^{\circ} \mathrm{F}$ & $(\mathrm{ppm})$ & \\
\hline Mean & 23.45 & 74.21 & 1652 & $57.45 \%$ \\
\hline S.D. & 0.49 & 0.88 & 353 & $3.04 \%$ \\
\hline Max. & 24.70 & 76.46 & 2645 & $65.27 \%$ \\
\hline
\end{tabular}

Cage Occupied Zone NH3 (ppm)

\begin{tabular}{|l|c|c|c|c|c|c|c|c|c|c|}
\hline Day & $\mathbf{1}$ & $\mathbf{2}$ & $\mathbf{3}$ & $\mathbf{4}$ & $\mathbf{5}$ & $\mathbf{6}$ & $\mathbf{7}$ & $\mathbf{8}$ & $\mathbf{9}$ & $\mathbf{1 0}$ \\
\hline Mean & 0.86 & 1.67 & 2.58 & 3.72 & 5.22 & 7.24 & 9.92 & 13.37 & 17.76 & 23.22 \\
\hline Max. & 1.38 & 2.68 & 4.13 & 5.95 & 8.37 & 11.60 & 15.88 & 21.42 & 28.44 & 37.18 \\
\hline
\end{tabular}

Room Breathing Zone

\begin{tabular}{|l|cc|c|c|}
\cline { 2 - 5 } \multicolumn{1}{c|}{} & \multicolumn{2}{c|}{ Temperature } & $\mathrm{CO}_{\mathbf{2}}$ & $\mathrm{RH}$ \\
\cline { 2 - 5 } \multicolumn{1}{c|}{} & ${ }^{\circ} \mathrm{C}$ & ${ }^{\circ} \mathrm{F}$ & $(\mathrm{ppm})$ & \\
\hline Mean & 22.26 & 72.07 & 72 & $49.16 \%$ \\
\hline S.D. & 0.44 & 0.80 & 17 & \\
\hline Max. & 23.47 & 74.25 & 157 & \\
\hline
\end{tabular}

Room Breathing Zone NH3 (ppm)

\begin{tabular}{|l|c|c|c|c|c|c|c|c|c|c|}
\hline Day & $\mathbf{1}$ & $\mathbf{2}$ & $\mathbf{3}$ & $\mathbf{4}$ & $\mathbf{5}$ & $\mathbf{6}$ & $\mathbf{7}$ & $\mathbf{8}$ & $\mathbf{9}$ & $\mathbf{1 0}$ \\
\hline Mean & 0.04 & 0.07 & 0.11 & 0.16 & 0.23 & 0.31 & 0.43 & 0.58 & 0.77 & 1.01 \\
\hline Max. & 0.08 & 0.16 & 0.25 & 0.35 & 0.50 & 0.69 & 0.94 & 1.27 & 1.69 & 2.21 \\
\hline
\end{tabular}




\section{Histogram Distributions}

Cage occupied zone average temperature $\left({ }^{\circ} \mathrm{C}\right)$ distribution

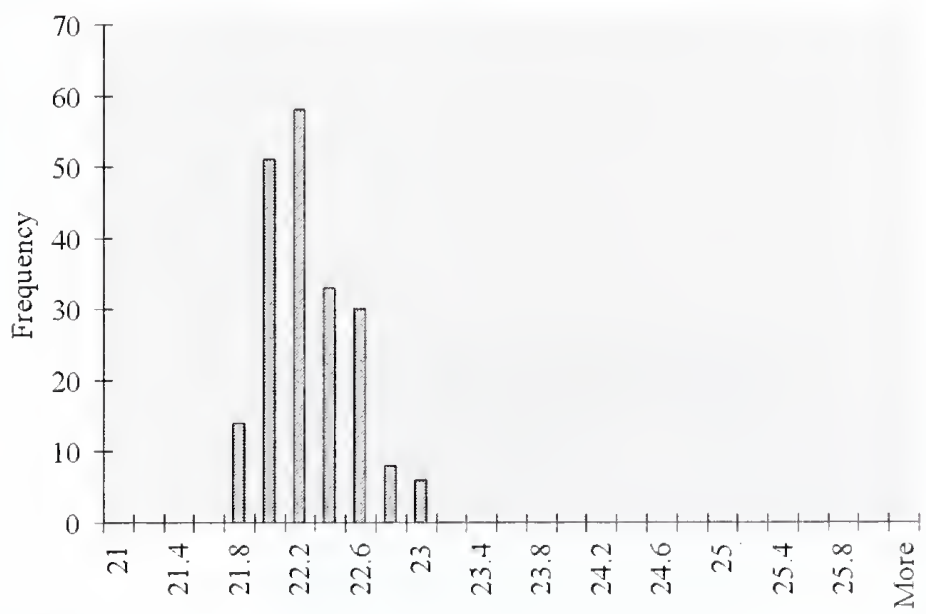

Cage occupied zone average contamination $(\mathrm{kg} / \mathrm{kg})$ distribution

Contamination conversion factors $(\mathrm{kg} / \mathrm{kg} \rightarrow \mathrm{ppm})$

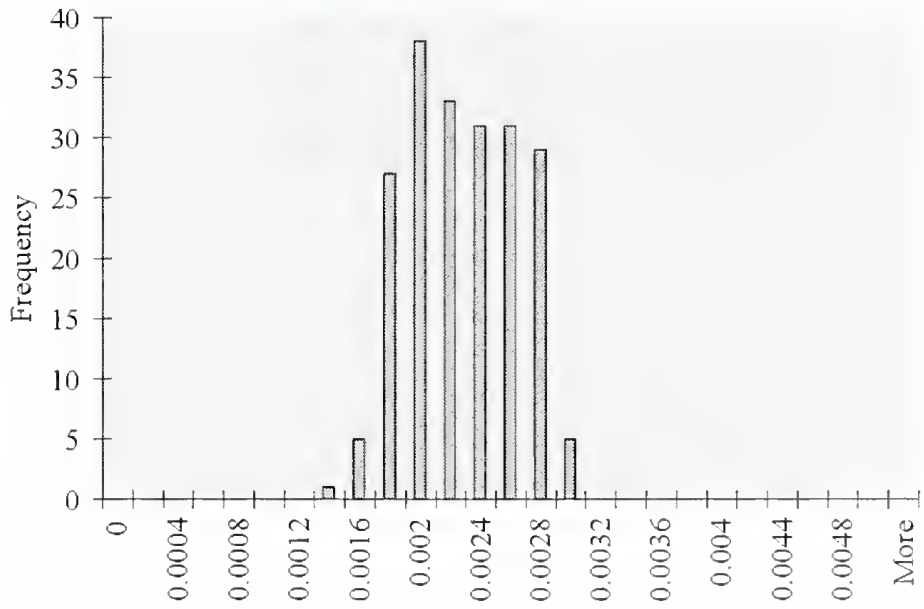

\begin{tabular}{|c|c|c|}
\hline Day & $\mathrm{CO}_{2}$ & $\mathrm{NH}_{3}$ \\
\hline $\mathbf{1}$ & 785000 & 408 \\
\hline $\mathbf{2}$ & 785000 & 795 \\
\hline 3 & 785000 & 1225 \\
\hline 4 & 785000 & 1766 \\
\hline $\mathbf{5}$ & 785000 & 2483 \\
\hline $\mathbf{6}$ & 785000 & 3443 \\
\hline 7 & 785000 & 4712 \\
\hline $\mathbf{8}$ & 785000 & 6356 \\
\hline $\mathbf{9}$ & 785000 & 8442 \\
\hline $\mathbf{1 0}$ & 785000 & 11384 \\
\hline
\end{tabular}

Cage occupied zone average relative humidity (\%) distribution

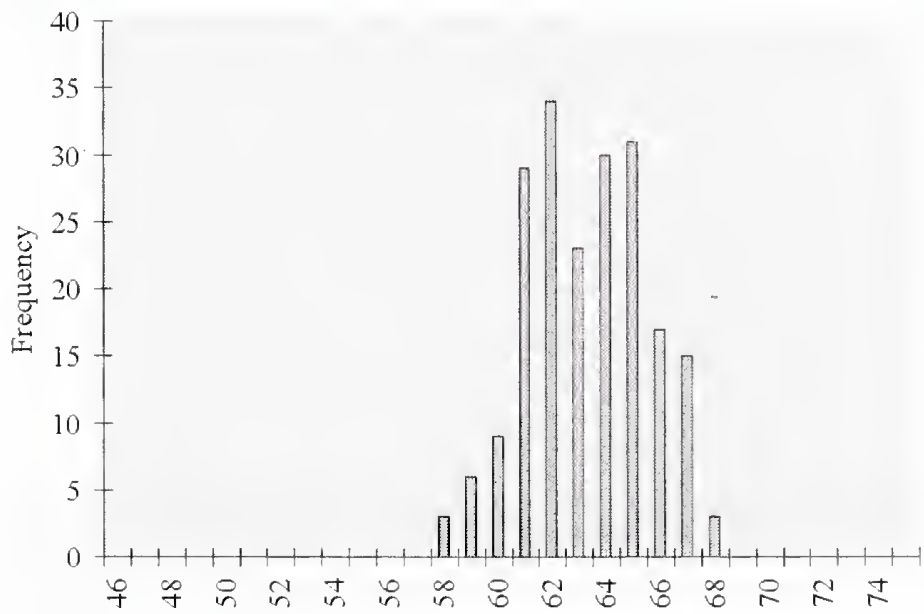




\section{Description}

\begin{tabular}{|c|c|c|c|c|c|}
\hline Supply & Supply Discharge & Supply Discharge & Exhaust & Exhaust & Exhaust \\
\hline Configuration & Temperature $\left({ }^{\circ} \mathrm{C}\right)$ & RH & Configuration & Temperature $\left({ }^{\circ} \mathrm{C}\right)$ & RH \\
\hline Low lnd & 18.8 & $61 \%$ & Ceiling & 22 & $50 \%$ \\
\hline Change & Rack & Rack & Number of & Total mass of & Room \\
\hline Station & Orientation & Density & Mice in Room & Mice in Room & Pressurisation \\
\hline ON & On wall & Single & 1050 & $21000 \mathrm{gr}$ & neg $100 \mathrm{cfm}$ \\
\hline
\end{tabular}

\section{Room}

ACH

15

Cage

Condition

Top On

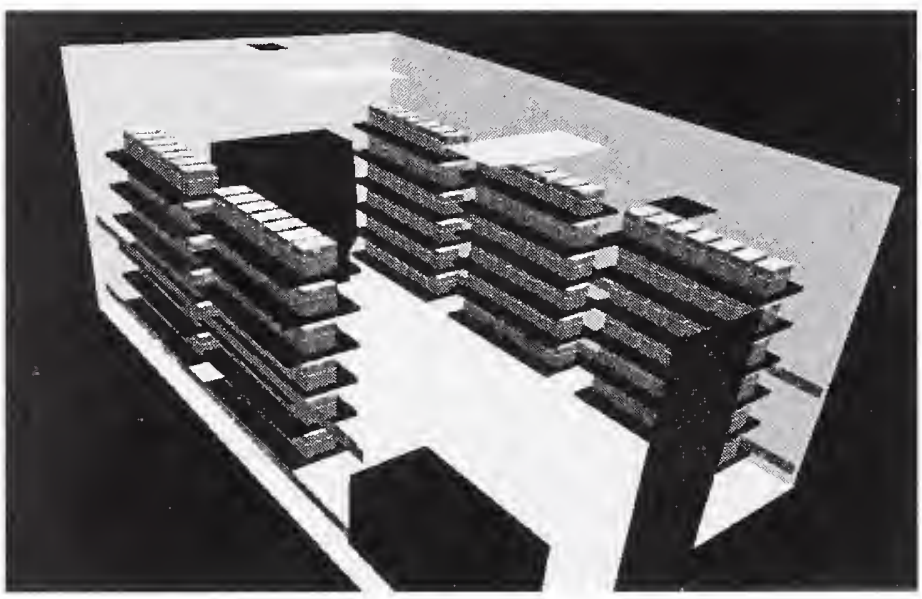

Analysis Results

Cage Occupied Zone

\begin{tabular}{|l|cc|c|c|}
\cline { 2 - 5 } \multicolumn{1}{c|}{} & \multicolumn{2}{c|}{ Temperature } & $\mathrm{CO}_{2}$ & RH \\
\cline { 2 - 5 } \multicolumn{1}{c|}{} & ${ }^{\circ} \mathrm{C}$ & ${ }^{\circ} \mathrm{F}$ & $(\mathrm{ppm})$ & \\
\hline Mean & 22.45 & 72.42 & 2321 & $66.43 \%$ \\
\hline S.D. & 0.66 & 1.19 & 488 & $3.53 \%$ \\
\hline Max. & 23.71 & 74.68 & 3385 & $74.89 \%$ \\
\hline
\end{tabular}

Cage Occupied Zone NH3 (ppm)

\begin{tabular}{|l|c|c|c|c|c|c|c|c|c|c|}
\hline Day & $\mathbf{1}$ & $\mathbf{2}$ & $\mathbf{3}$ & $\mathbf{4}$ & $\mathbf{5}$ & $\mathbf{6}$ & $\mathbf{7}$ & $\mathbf{8}$ & $\mathbf{9}$ & $\mathbf{1 0}$ \\
\hline Mean & 1.24 & 2.35 & 3.62 & 6.71 & 11.21 & 16.60 & 24.87 & 30.95 & 37.22 & 40.46 \\
\hline Max. & 1.80 & 3.43 & 5.28 & 9.79 & 16.35 & 24.20 & 36.26 & 45.13 & 54.28 & 59.00 \\
\hline
\end{tabular}

Room Breathing Zone

\begin{tabular}{|l|cc|c|c|}
\cline { 2 - 5 } \multicolumn{1}{c|}{} & \multicolumn{2}{c|}{ Temperature } & $\mathrm{CO}_{2}$ & RH \\
\cline { 2 - 5 } \multicolumn{1}{c|}{} & ${ }^{\circ} \mathrm{C}$ & ${ }^{\circ}$ & $(\mathbf{p p m})$ & \\
\hline Mean & 19.96 & 67.93 & 57 & $56.73 \%$ \\
\hline S.D. & 0.68 & 1.22 & 82 & \\
\hline Max. & 22.20 & 71.96 & 371 & \\
\hline
\end{tabular}

Room Breathing Zone NH3 (ppm)

\begin{tabular}{|l|c|c|c|c|c|c|c|c|c|c|}
\hline Day & $\mathbf{1}$ & $\mathbf{2}$ & $\mathbf{3}$ & $\mathbf{4}$ & $\mathbf{5}$ & $\mathbf{6}$ & $\mathbf{7}$ & $\mathbf{8}$ & $\mathbf{9}$ & $\mathbf{1 0}$ \\
\hline Mean & 0.03 & 0.06 & 0.09 & 0.16 & 0.27 & 0.41 & 0.61 & 0.76 & 0.91 & 0.99 \\
\hline Max. & 0.20 & 0.38 & 0.58 & 1.07 & 1.79 & 2.65 & 3.97 & 4.94 & 5.94 & 6.46 \\
\hline
\end{tabular}




\section{Histogram Distributions}

Cage occupied zone average temperature $\left({ }^{\circ} \mathrm{C}\right)$ distribution

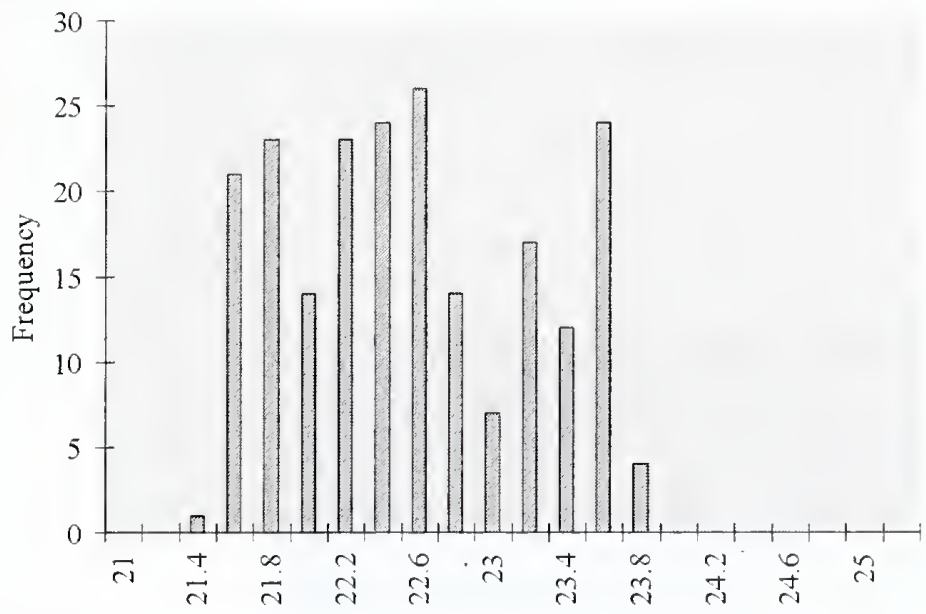

Cage occupied zone average contamination $(\mathrm{kg} / \mathrm{kg})$ distribution

Contamination conversion factors $(\mathrm{kg} / \mathrm{kg} \rightarrow \mathrm{ppm})$

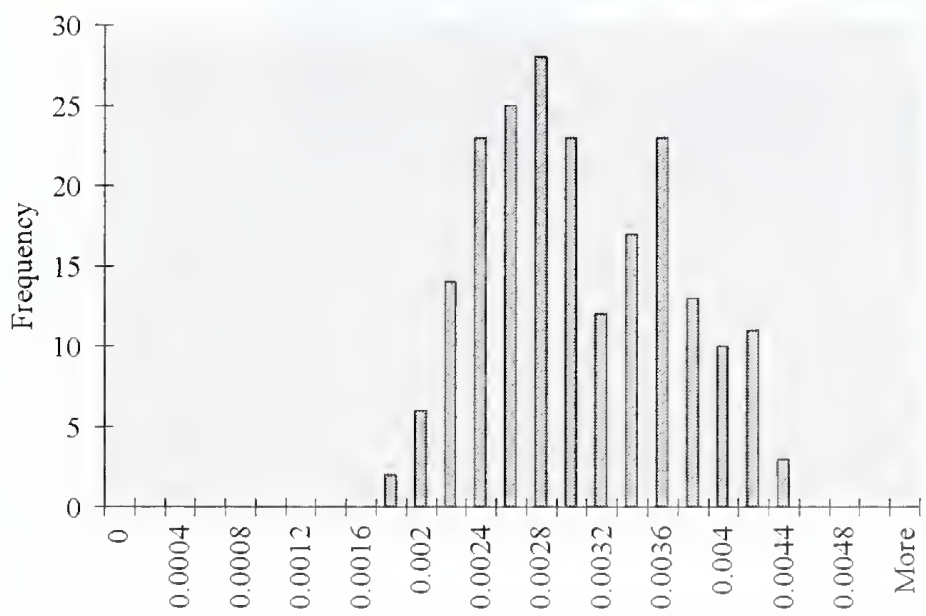

\begin{tabular}{|c|c|c|}
\hline Day & $\mathrm{CO}_{2}$ & $\mathrm{NII}_{3}$ \\
\hline $\mathbf{1}$ & 785000 & 418 \\
\hline $\mathbf{2}$ & 785000 & 795 \\
\hline $\mathbf{3}$ & 785000 & 1225 \\
\hline $\mathbf{4}$ & 785000 & 2270 \\
\hline $\mathbf{5}$ & 785000 & 3791 \\
\hline $\mathbf{6}$ & 785000 & 5612 \\
\hline 7 & 785000 & 8409 \\
\hline $\mathbf{8}$ & 785000 & 10466 \\
\hline $\mathbf{9}$ & 785000 & 12587 \\
\hline $\mathbf{1 0}$ & 785000 & 11384 \\
\hline
\end{tabular}

Cage occupied zone average relative humidity (\%) distribution

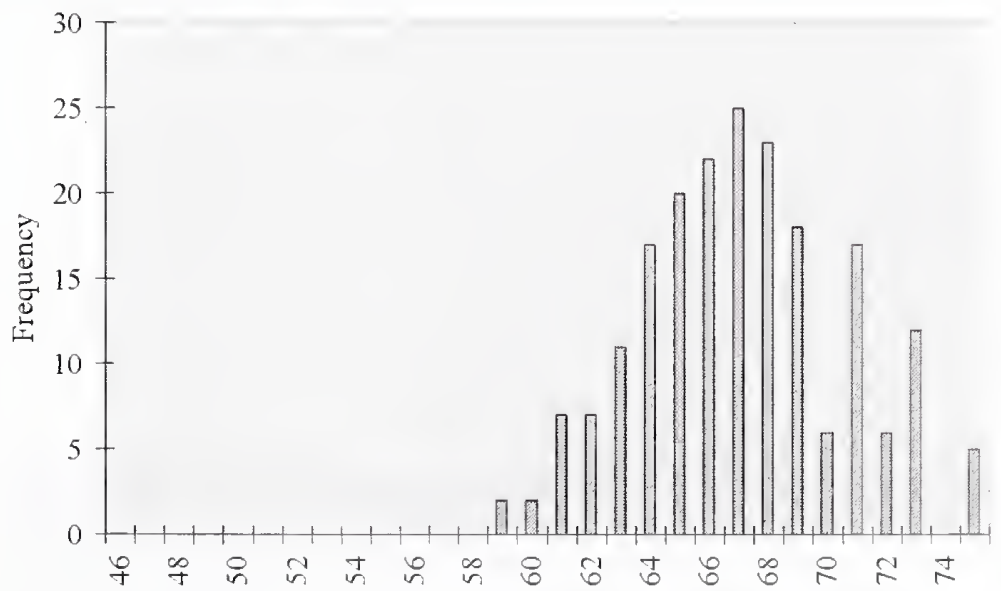


Description

$\begin{array}{llllll}\begin{array}{l}\text { Supply } \\ \text { Configuration }\end{array} & \begin{array}{l}\text { Supply Discharge } \\ \text { Low lnd }\end{array} & \begin{array}{l}\text { Supply Discharge } \\ \text { Temperature }\left({ }^{\circ} \mathrm{C}\right)\end{array} & \begin{array}{l}\text { RH } \\ \text { Exhaust }\end{array} & \begin{array}{l}\text { Exhaust } \\ \text { Configuration }\end{array} & \begin{array}{l}\text { Temperature }\left({ }^{\circ} \mathrm{C}\right) \\ \text { RH }\end{array} \\ \text { Change } & 18.8 & & \text { High } & 22 & 50 \% \\ \text { Station } & \text { Rack } & \text { Rack } & \text { Number of } & \text { Total mass of } & \text { Room } \\ \text { ON } & \text { Orientation } & \text { Density } & \text { Mice in Room } & \text { Mice in Room } & \text { Pressurisation } \\ & \text { On wall } & \text { Single } & 1050 & 21000 \text { gr } & \text { neg } 100 \mathrm{cfm}\end{array}$

Room

ACH

15

Cage

Condition

Top On

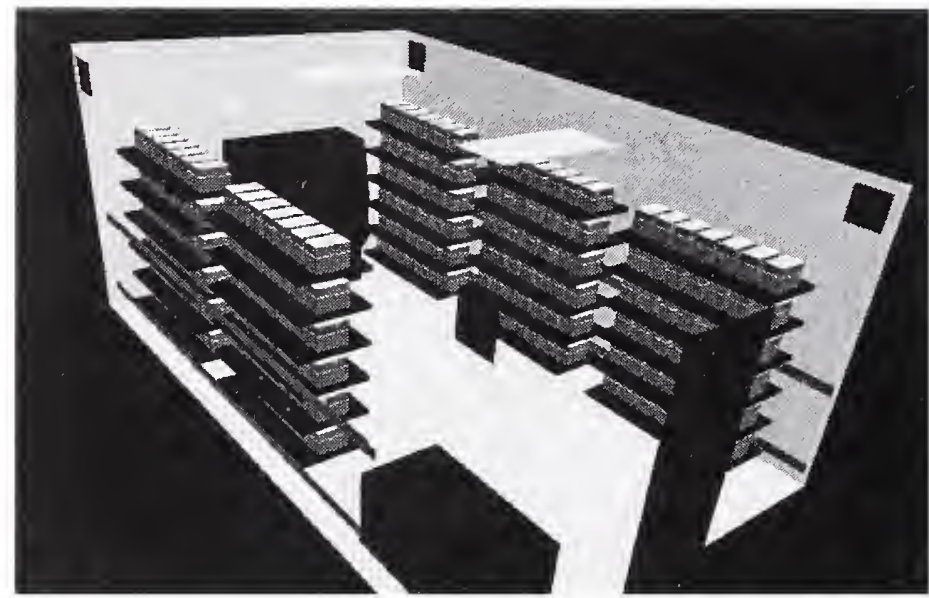

Analysis Results

Cage Occupied Zone

\begin{tabular}{|l|cc|c|c|}
\cline { 2 - 5 } \multicolumn{1}{c|}{} & \multicolumn{2}{c|}{ Temperature } & $\mathrm{CO}_{2}$ & RH \\
\cline { 2 - 5 } \multicolumn{1}{c|}{} & ${ }^{\mathrm{C}}$ & $\mathrm{F}$ & $(\mathrm{ppm})$ & \\
\hline Mean & 22.22 & 71.99 & 1871 & $63.81 \%$ \\
\hline S.D. & 0.25 & 0.45 & 328 & $2.73 \%$ \\
\hline Max. & 22.83 & 73.10 & 2638 & $69.33 \%$ \\
\hline
\end{tabular}

Cage Occupied Zone NH3 (ppm)

\begin{tabular}{|l|c|c|c|c|c|c|c|c|c|c|}
\hline Day & $\mathbf{1}$ & $\mathbf{2}$ & $\mathbf{3}$ & $\mathbf{4}$ & $\mathbf{5}$ & $\mathbf{6}$ & $\mathbf{7}$ & $\mathbf{8}$ & $\mathbf{9}$ & $\mathbf{1 0}$ \\
\hline Mean & 0.98 & 1.89 & 2.92 & 4.83 & 7.53 & 10.88 & 15.79 & 20.22 & 25.23 & 29.56 \\
\hline Max. & 1.39 & 2.67 & 4.12 & 6.81 & 10.62 & 15.34 & 22.26 & 28.50 & 35.57 & 41.68 \\
\hline
\end{tabular}

Room Breathing Zone

\begin{tabular}{|l|cc|c|c|}
\cline { 2 - 5 } \multicolumn{1}{c|}{} & \multicolumn{2}{c|}{ Temperature } & $\mathrm{CO}_{2}$ & RH \\
\cline { 2 - 5 } \multicolumn{1}{c|}{} & ${ }^{\circ} \mathrm{C}$ & ${ }^{\circ} \mathrm{F}$ & $(\mathrm{ppm})$ & \\
\hline Mean & 20.48 & 68.86 & 64 & $54.97 \%$ \\
\hline S.D. & 0.27 & 0.48 & 31 & \\
\hline Max. & 21.47 & 70.65 & 225 & \\
\hline
\end{tabular}

Room Breathing Zone NH3 (ppm)

\begin{tabular}{|l|c|c|c|c|c|c|c|c|c|c|}
\hline Day & 1 & 2 & 3 & 4 & 5 & 6 & 7 & 8 & 9 & 10 \\
\hline Mean & 0.03 & 0.06 & 0.10 & 0.16 & 0.26 & 0.37 & 0.54 & 0.69 & 0.86 & 1.01 \\
\hline Max. & 0.12 & 0.23 & 0.35 & 0.58 & 0.91 & 1.31 & 1.90 & 2.43 & 3.04 & 3.56 \\
\hline
\end{tabular}




\section{Histogram Distributions}

Cage occupied zone average temperature $\left({ }^{\circ} \mathrm{C}\right)$ distribution

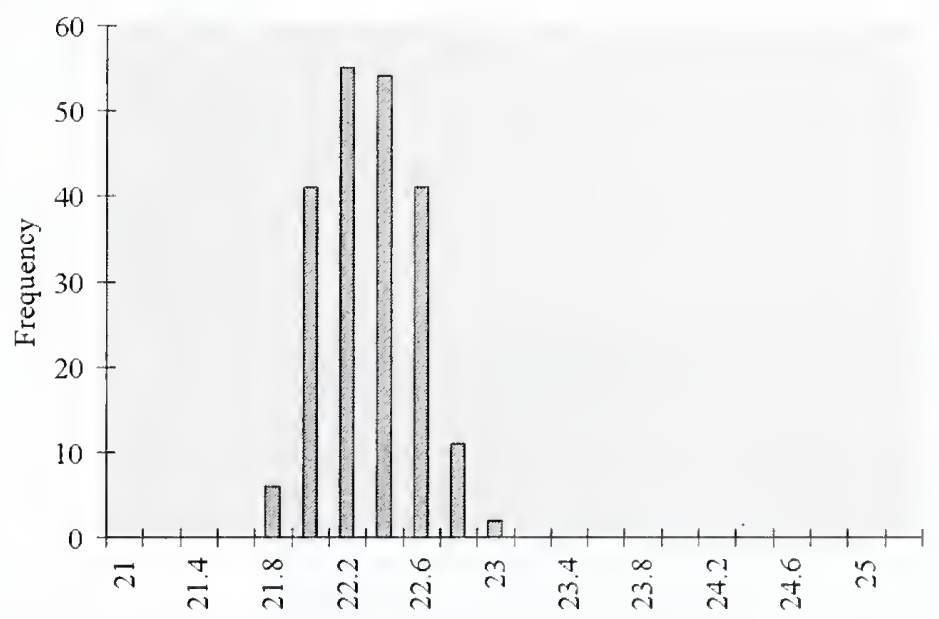

Cage occupied zone average contamination $(\mathrm{kg} / \mathrm{kg})$ distribution

Contamination conversion factors $(\mathrm{kg} / \mathrm{kg} \rightarrow \mathrm{ppm})$

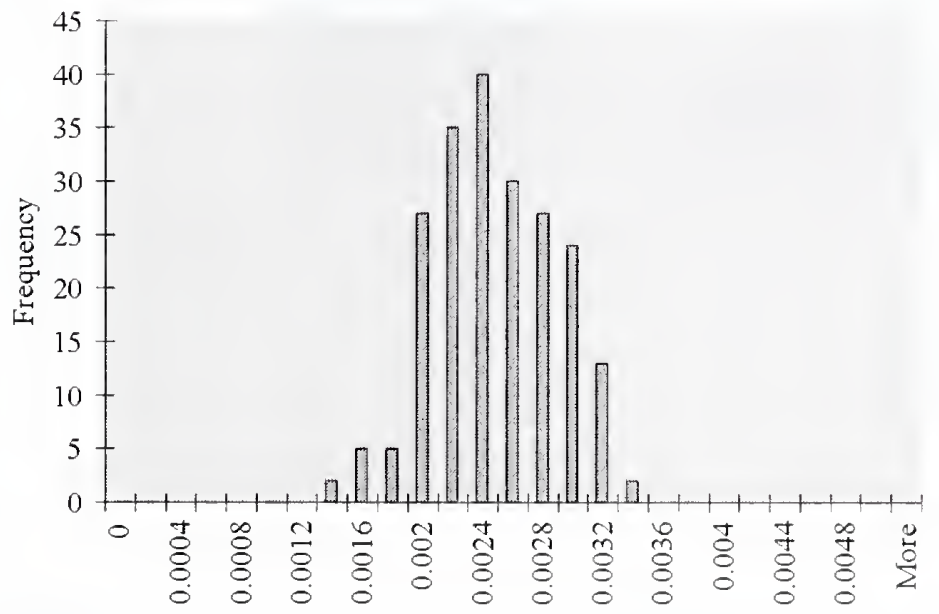

\begin{tabular}{|c|c|c|}
\hline Day & $\mathrm{CO}_{2}$ & $\mathrm{NH}_{3}$ \\
\hline $\mathbf{1}$ & 785000 & 413 \\
\hline 2 & 785000 & 795 \\
\hline 3 & 785000 & 1225 \\
\hline 4 & 785000 & 2027 \\
\hline 5 & 785000 & 3160 \\
\hline 6 & 785000 & 4565 \\
\hline 7 & 785000 & 6625 \\
\hline 8 & 785000 & 8483 \\
\hline 9 & 785000 & 10586 \\
\hline 10 & 785000 & 11384 \\
\hline
\end{tabular}

Cage occupied zone average relative humidity (\%) distribution

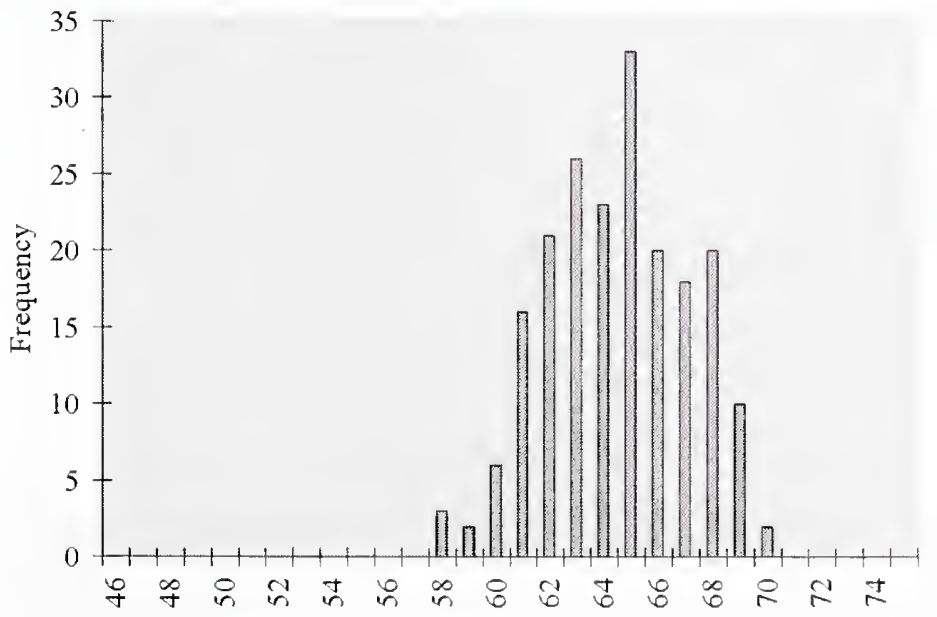


Casename

Case 09

Description

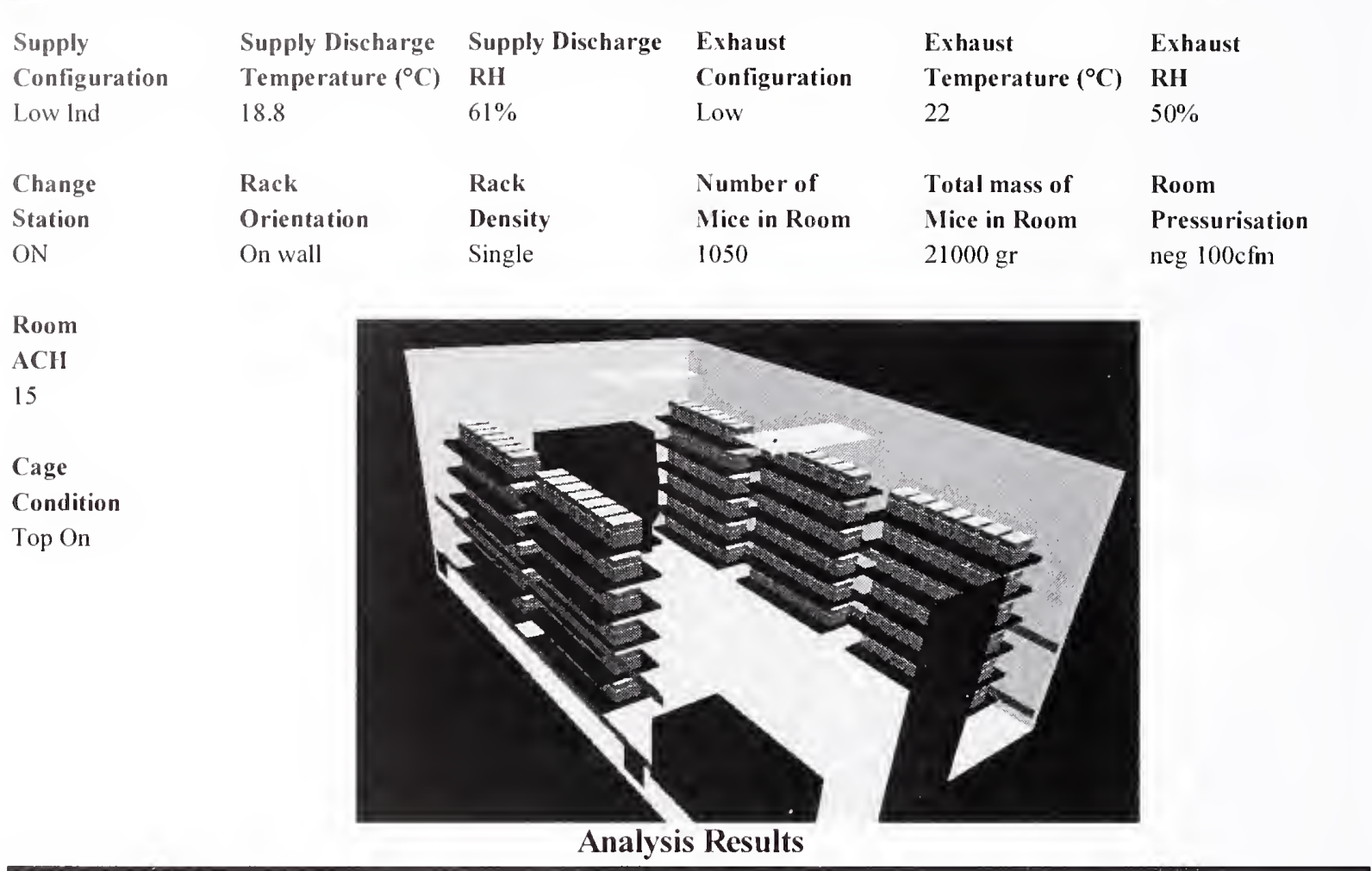

Cage Occupied Zone

\begin{tabular}{|l|cc|c|c|}
\cline { 2 - 5 } \multicolumn{1}{c|}{} & \multicolumn{2}{c|}{ Temperature } & $\mathrm{CO}_{2}$ & RH \\
\cline { 2 - 5 } \multicolumn{1}{c|}{} & ${ }^{\circ} \mathrm{C}$ & ${ }^{\circ} \mathrm{F}$ & $(\mathbf{p p m})$ & \\
\hline Mean & 23.61 & 74.49 & 1725 & $57.46 \%$ \\
\hline S.D. & 0.54 & 0.97 & 335 & $3.18 \%$ \\
\hline Max. & 24.78 & 76.61 & 2931 & $64.20 \%$ \\
\hline
\end{tabular}

Cage Occupied Zone NH3 (ppm)

\begin{tabular}{|l|c|c|c|c|c|c|c|c|c|c|}
\hline Day & $\mathbf{1}$ & $\mathbf{2}$ & $\mathbf{3}$ & $\mathbf{4}$ & $\mathbf{5}$ & $\mathbf{6}$ & $\mathbf{7}$ & $\mathbf{8}$ & $\mathbf{9}$ & $\mathbf{1 0}$ \\
\hline Mean & 0.90 & 1.75 & 2.69 & 3.88 & 5.46 & 7.57 & 10.36 & 13.97 & 18.55 & 24.25 \\
\hline Max. & 1.52 & 2.97 & 4.57 & 6.59 & 9.27 & 12.86 & 17.60 & 23.74 & 31.53 & 41.21 \\
\hline
\end{tabular}

Room Breathing Zone

\begin{tabular}{|l|cc|c|c|}
\cline { 2 - 5 } \multicolumn{1}{c|}{} & \multicolumn{2}{c|}{ Temperature } & $\mathrm{CO}_{2}$ & RH \\
\cline { 2 - 5 } \multicolumn{1}{c|}{} & ${ }^{\circ} \mathrm{C}$ & ${ }^{\circ} \mathrm{F}$ & $(\mathrm{ppm})$ & \\
\hline Mean & 22.57 & 72.62 & 88 & $48.37 \%$ \\
\hline S.D. & 0.60 & 1.08 & 31 & \\
\hline Max. & 23.76 & 74.76 & 277 & \\
\hline
\end{tabular}

Room Breathing Zone NH3 (ppm)

\begin{tabular}{|l|c|c|c|c|c|c|c|c|c|c|}
\hline Day & $\mathbf{1}$ & $\mathbf{2}$ & $\mathbf{3}$ & $\mathbf{4}$ & $\mathbf{5}$ & $\mathbf{6}$ & $\mathbf{7}$ & $\mathbf{8}$ & $\mathbf{9}$ & $\mathbf{1 0}$ \\
\hline Mean & 0.05 & 0.09 & 0.14 & 0.20 & 0.28 & 0.38 & 0.53 & 0.71 & 0.94 & 1.23 \\
\hline Max. & 0.14 & 0.28 & 0.43 & 0.62 & 0.88 & 1.21 & 1.66 & 2.24 & 2.98 & 3.89 \\
\hline
\end{tabular}




\section{Histogram Distributions}

Cage occupied zone average temperature $\left({ }^{\circ} \mathrm{C}\right)$ distribution

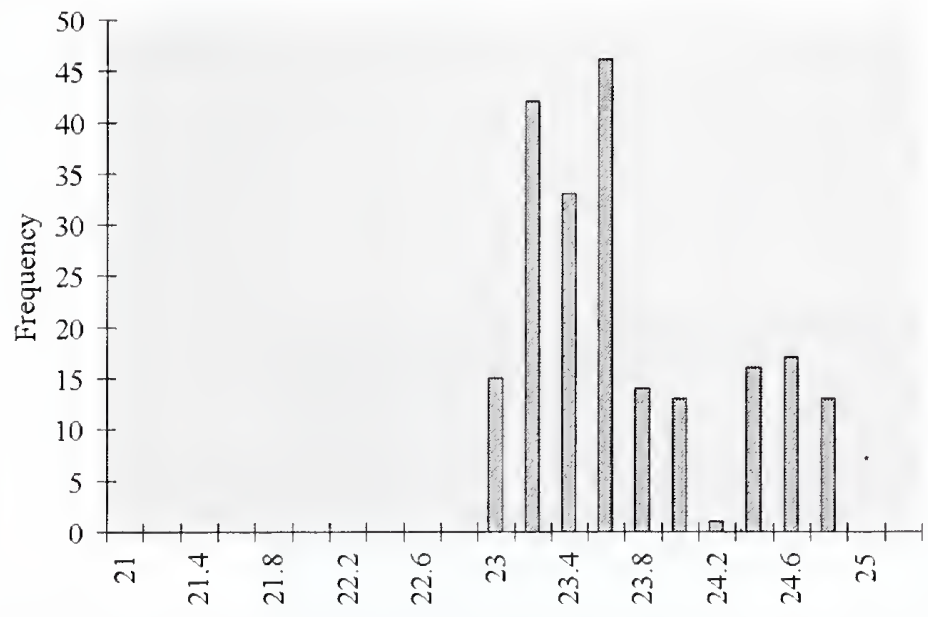

Cage occupied zone average contamination $(\mathrm{kg} / \mathrm{kg})$ distribution Contamination conversion factors $(\mathrm{kg} / \mathrm{kg} \rightarrow \mathrm{ppm})$

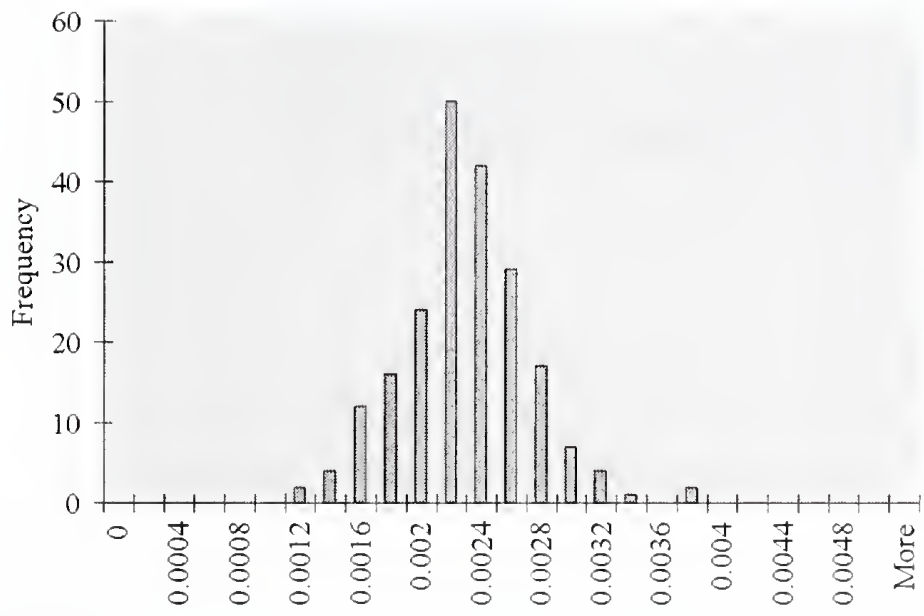

\begin{tabular}{|c|c|c|}
\hline Day & $\mathrm{CO}_{2}$ & $\mathrm{NH}_{3}$ \\
\hline $\mathbf{1}$ & 785000 & 408 \\
\hline $\mathbf{2}$ & 785000 & 795 \\
\hline $\mathbf{3}$ & 785000 & 1225 \\
\hline $\mathbf{4}$ & 785000 & 1766 \\
\hline $\mathbf{5}$ & 785000 & 2483 \\
\hline $\mathbf{6}$ & 785000 & 3443 \\
\hline $\mathbf{7}$ & 785000 & 4712 \\
\hline $\mathbf{8}$ & 785000 & 6356 \\
\hline $\mathbf{9}$ & 785000 & 8442 \\
\hline $\mathbf{1 0}$ & 785000 & 11384 \\
\hline
\end{tabular}

Cage occupied zone average relative humidity (\%) distribution

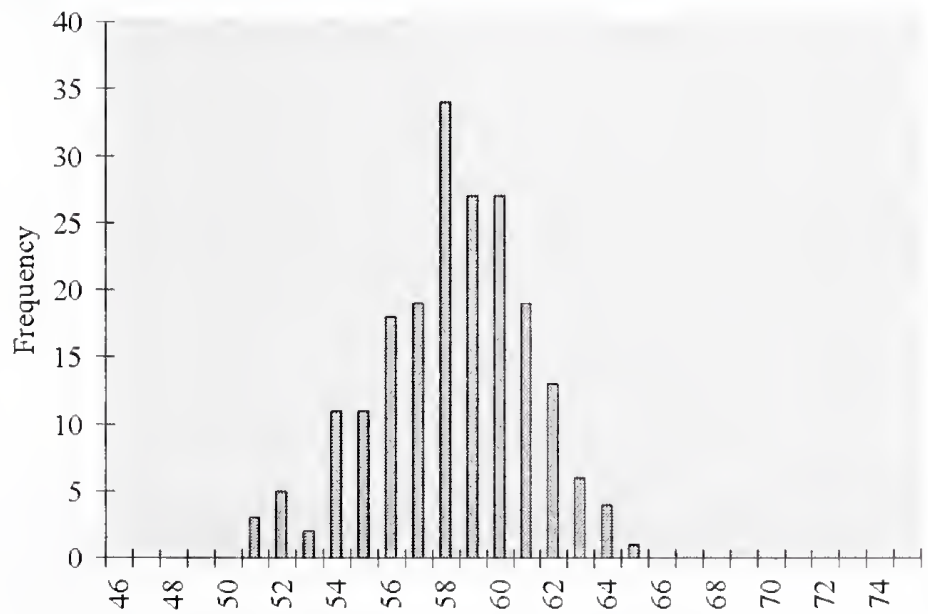


Casename

Case 10

\section{Description}

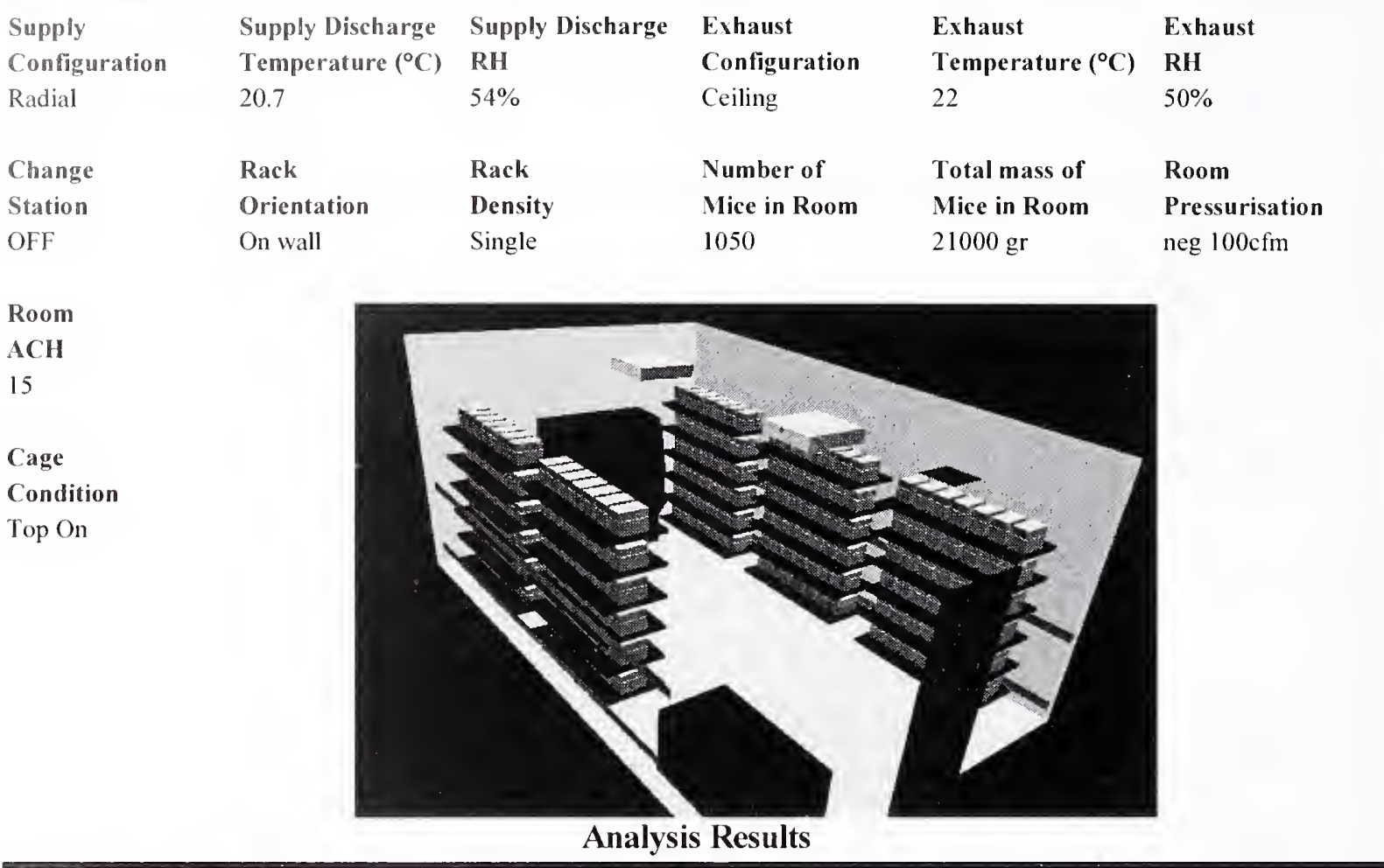

Cage Occupied Zone

\begin{tabular}{|l|cc|c|c|}
\cline { 2 - 5 } \multicolumn{1}{c|}{} & \multicolumn{2}{c|}{ Temperature } & $\mathbf{C O}_{2}$ & RH \\
\cline { 2 - 5 } \multicolumn{1}{c|}{} & ${ }^{\circ} \mathrm{C}$ & ${ }^{\circ} \mathrm{F}$ & $(\mathrm{ppm})$ & \\
\hline Mean & 23.24 & 73.84 & 2119 & $61.71 \%$ \\
\hline S.D. & 0.28 & 0.51 & 376 & $3.14 \%$ \\
\hline Max. & 23.88 & 74.98 & 3201 & $68.32 \%$ \\
\hline
\end{tabular}

Cage Occupied Zone NH3 (ppm)

\begin{tabular}{|l|c|c|c|c|c|c|c|c|c|c|}
\hline Day & $\mathbf{1}$ & $\mathbf{2}$ & $\mathbf{3}$ & $\mathbf{4}$ & $\mathbf{5}$ & $\mathbf{6}$ & $\mathbf{7}$ & $\mathbf{8}$ & $\mathbf{9}$ & $\mathbf{1 0}$ \\
\hline Mean & 1.11 & 2.14 & 3.31 & 4.94 & 7.17 & 10.06 & 14.03 & 18.61 & 24.25 & 30.72 \\
\hline Max. & 1.67 & 3.24 & 5.00 & 7.47 & 10.83 & 15.20 & 21.20 & 28.12 & 36.65 & 46.42 \\
\hline
\end{tabular}

Room Breathing Zone

\begin{tabular}{|l|cc|c|c|}
\cline { 2 - 5 } \multicolumn{1}{c|}{} & \multicolumn{2}{c|}{ Temperature } & $\mathbf{C O}_{2}$ & RH \\
\cline { 2 - 5 } \multicolumn{1}{c|}{} & ${ }^{\circ} \mathrm{C}$ & ${ }^{\circ} \mathrm{F}$ & $(\mathrm{ppm})$ & \\
\hline Mean & 20.92 & 69.66 & 29 & $53.13 \%$ \\
\hline S.D. & 0.39 & 0.70 & 44 & \\
\hline Max. & 22.21 & 71.98 & 158 & \\
\hline
\end{tabular}

Room Breathing Zone NH3 (ppm)

\begin{tabular}{|l|c|c|c|c|c|c|c|c|c|c|}
\hline Day & $\mathbf{1}$ & $\mathbf{2}$ & $\mathbf{3}$ & $\mathbf{4}$ & $\mathbf{5}$ & $\mathbf{6}$ & $\mathbf{7}$ & $\mathbf{8}$ & $\mathbf{9}$ & $\mathbf{1 0}$ \\
\hline Mean & 0.02 & 0.03 & 0.05 & 0.07 & 0.10 & 0.14 & 0.19 & 0.26 & 0.33 & 0.42 \\
\hline Max. & 0.08 & 0.16 & 0.25 & 0.37 & 0.53 & 0.75 & 1.05 & 1.39 & 1.81 & 2.29 \\
\hline
\end{tabular}




\section{Histogram Distributions}

Cage occupied zone average temperature $\left({ }^{\circ} \mathrm{C}\right)$ distribution

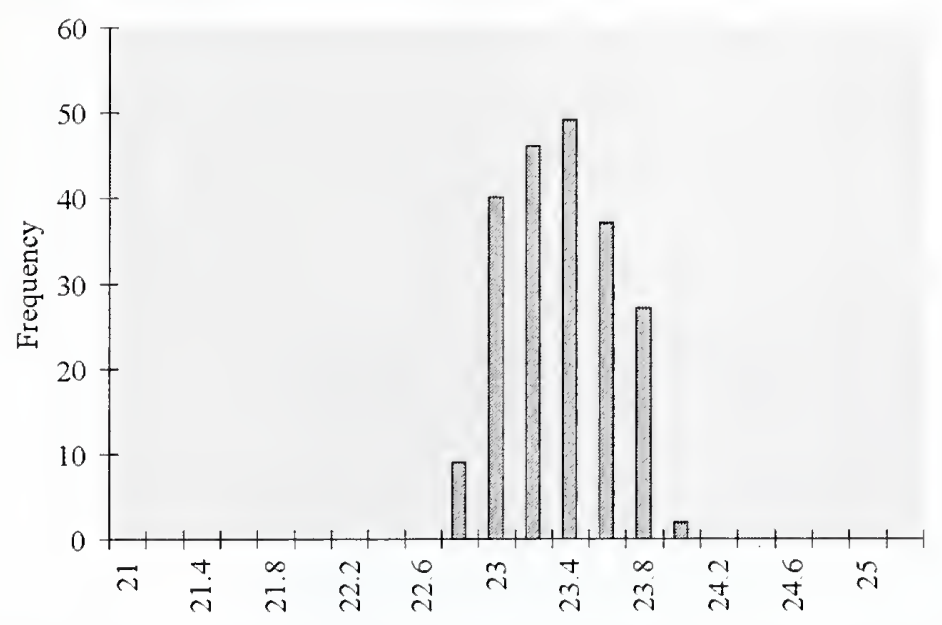

Cage occupied zone average contamination $(\mathrm{kg} / \mathrm{kg})$ distribution

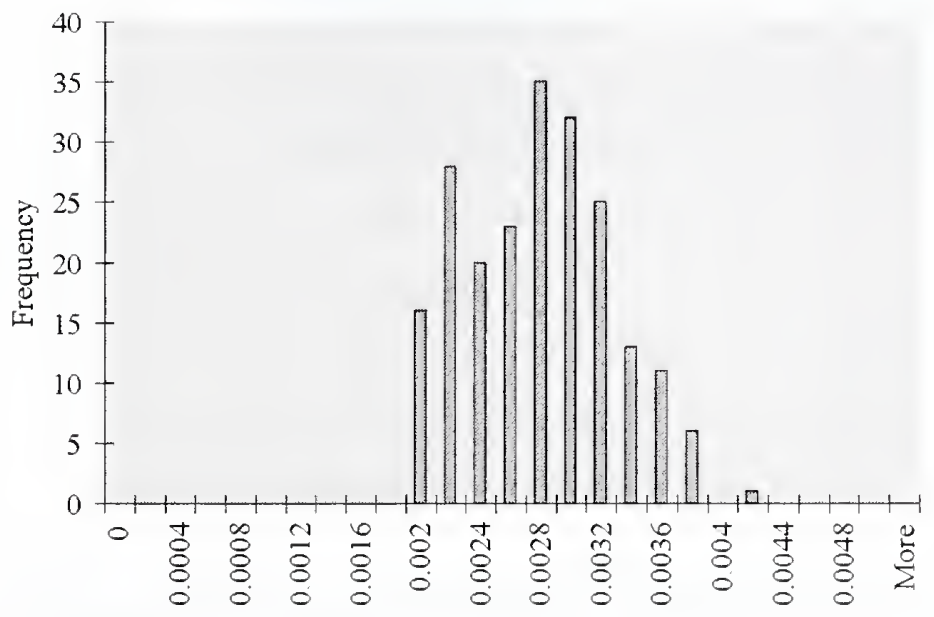

Cage occupied zone average relative humidity (\%) diștribution

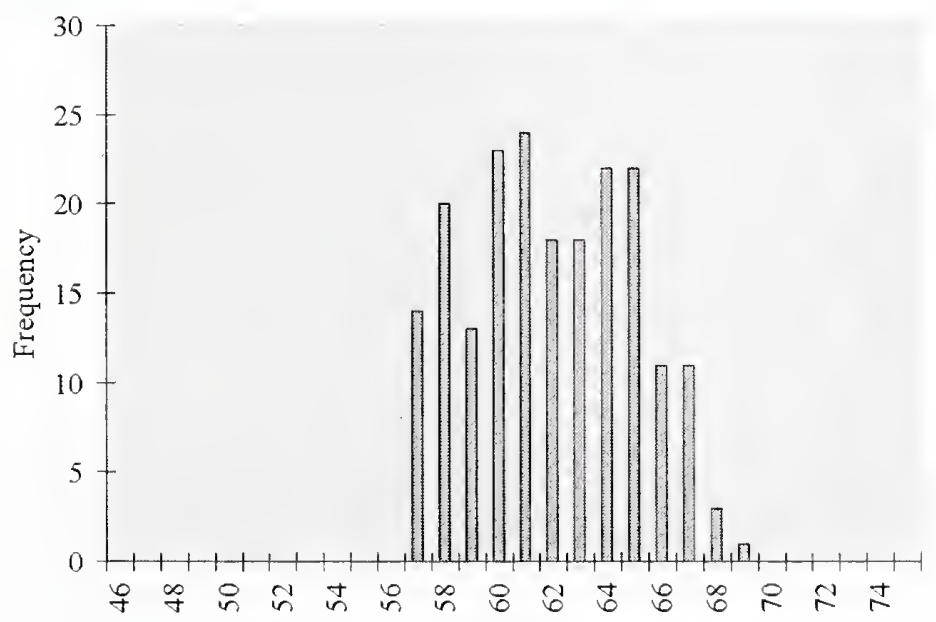

Contamination conversion factors $(\mathrm{kg} / \mathrm{kg} \rightarrow \mathrm{ppm})$

\begin{tabular}{|c|c|c|}
\hline Day & $\mathrm{CO}_{2}$ & $\mathrm{NH}_{3}$ \\
\hline $\mathbf{I}$ & 785000 & 410 \\
\hline $\mathbf{2}$ & 785000 & 795 \\
\hline $\mathbf{3}$ & 785000 & 1225 \\
\hline $\mathbf{4}$ & 785000 & 1832 \\
\hline $\mathbf{5}$ & 785000 & 2655 \\
\hline $\mathbf{6}$ & 785000 & 3728 \\
\hline 7 & 785000 & 5198 \\
\hline $\mathbf{8}$ & 785000 & 6897 \\
\hline $\mathbf{9}$ & 785000 & 8987 \\
\hline $\mathbf{1 0}$ & 785000 & 11384 \\
\hline
\end{tabular}




\section{Description}

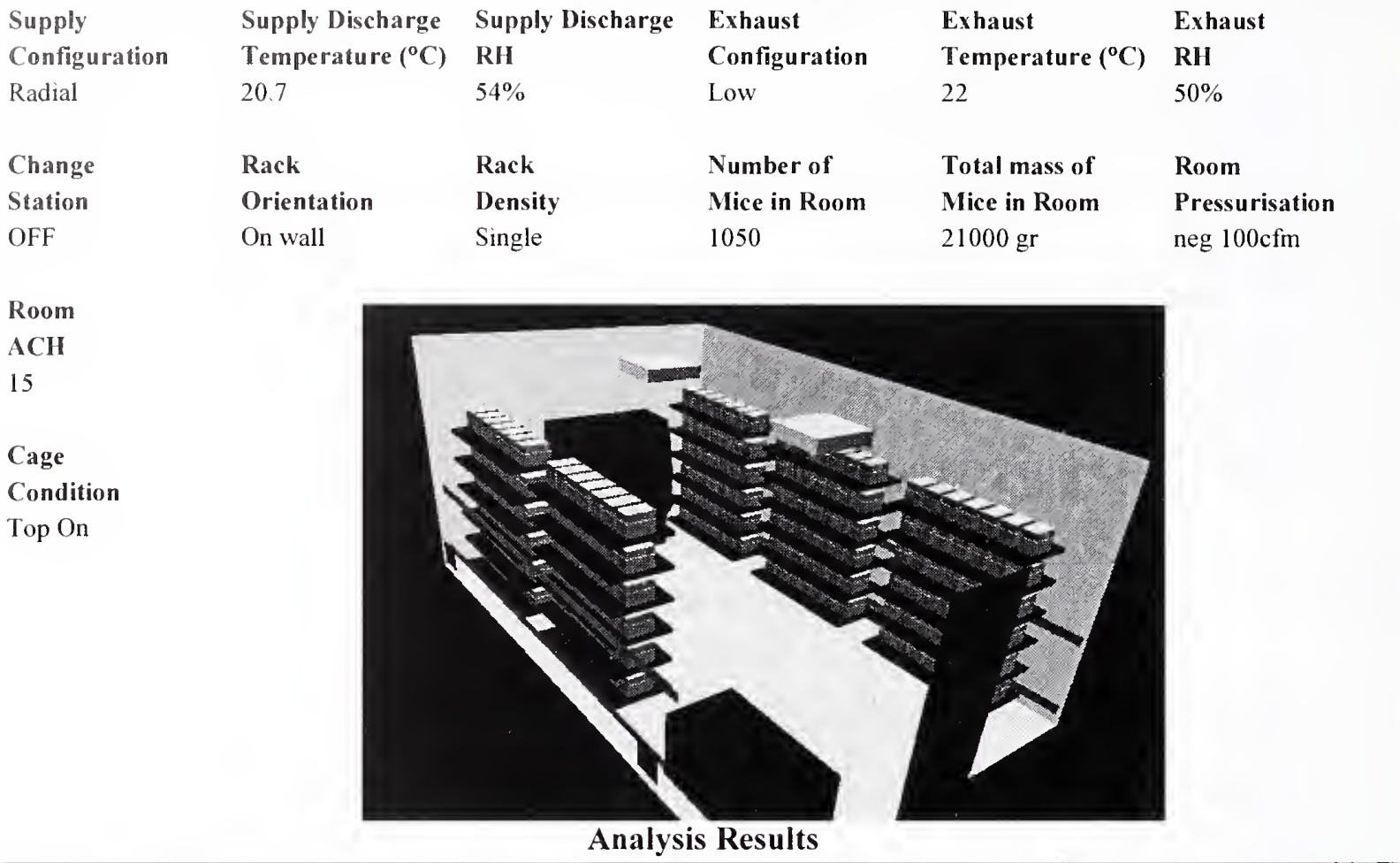

\begin{tabular}{|l|cc|c|c|}
\cline { 2 - 5 } \multicolumn{1}{c|}{} & \multicolumn{2}{c|}{ Temperature } & $\mathbf{C O}_{2}$ & RII \\
\cline { 2 - 5 } \multicolumn{1}{c|}{} & ${ }^{\circ} \mathrm{C}$ & ${ }^{\circ} \mathrm{F}$ & $(\mathbf{p p m})$ & \\
\hline Mean & 22.75 & 72.95 & 2118 & $63.61 \%$ \\
\hline S.D. & 0.27 & 0.48 & 412 & $2.69 \%$ \\
\hline Max. & 23.36 & 74.04 & 2903 & $69.23 \%$ \\
\hline
\end{tabular}

Cage Occupied Zone NH3 (ppm)

\begin{tabular}{|l|c|c|c|c|c|c|c|c|c|c|c|}
\hline Day & $\mathbf{1}$ & $\mathbf{2}$ & $\mathbf{3}$ & $\mathbf{4}$ & $\mathbf{5}$ & $\mathbf{6}$ & $\mathbf{7}$ & $\mathbf{8}$ & $\mathbf{9}$ & $\mathbf{1 0}$ \\
\hline Mean & 1.11 & 2.14 & 3.30 & 5.42 & 8.40 & $\cdot 12.10$ & 17.51 & 22.48 & 28.16 & 33.21 \\
\hline Max. & 1.53 & 2.94 & 4.53 & 7.43 & 11.51 & 16.59 & 24.01 & 30.82 & 38.60 & 45.52 \\
\hline
\end{tabular}

Room Breathing Zone

\begin{tabular}{|l|cc|c|c|}
\cline { 2 - 5 } \multicolumn{1}{c|}{} & \multicolumn{2}{c|}{ Temperature } & $\mathrm{CO}_{2}$ & RH \\
\cline { 2 - 5 } \multicolumn{1}{c|}{} & ${ }^{\circ} \mathrm{C}$ & ${ }^{\circ} \mathrm{F}$ & $(\mathbf{p p m})$ & \\
\hline Mean & 21.31 & 70.35 & 48 & $52.02 \%$ \\
\hline S.D. & 0.39 & 0.70 & 55 & \\
\hline Max. & 22.11 & 71.81 & 223 & \\
\hline
\end{tabular}

Room Breathing Zone NH3 (ppm)

\begin{tabular}{|l|c|c|c|c|c|c|c|c|c|c|}
\hline Day & $\mathbf{1}$ & $\mathbf{2}$ & $\mathbf{3}$ & $\mathbf{4}$ & $\mathbf{5}$ & $\mathbf{6}$ & $\mathbf{7}$ & $\mathbf{8}$ & $\mathbf{9}$ & $\mathbf{1 0}$ \\
\hline Mean & 0.03 & 0.05 & 0.07 & 0.12 & 0.19 & 0.27 & 0.39 & 0.51 & 0.63 & 0.75 \\
\hline Max. & 0.12 & 0.23 & 0.35 & 0.57 & 0.88 & 1.27 & 1.84 & 2.36 & 2.96 & 3.49 \\
\hline
\end{tabular}




\section{Histogram Distributions}

Cage occupied zone average temperature $\left({ }^{\circ} \mathrm{C}\right)$ distribution

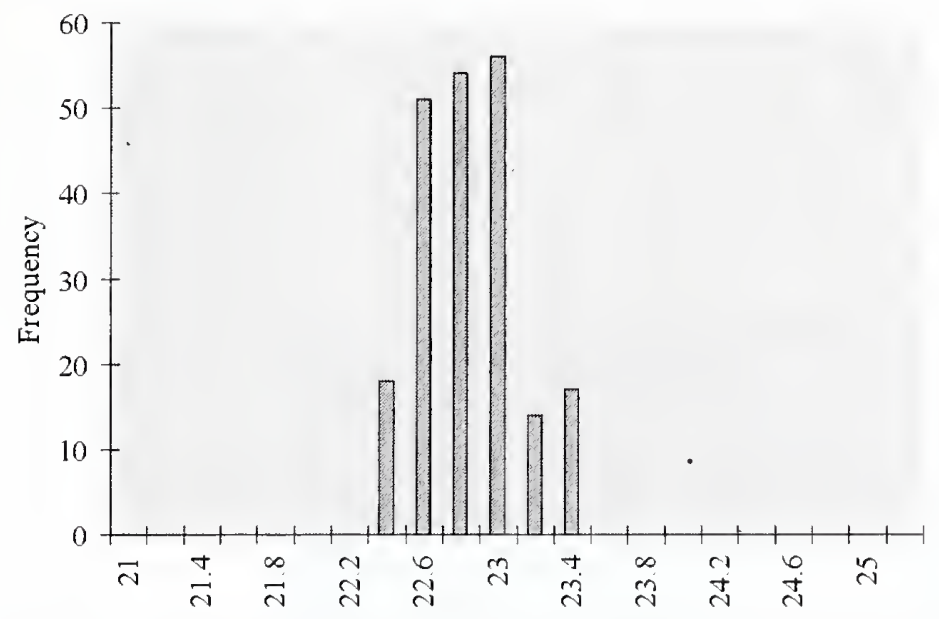

Cage occupied zone average contamination $(\mathrm{kg} / \mathrm{kg})$ distribution Contamination conversion factors $(\mathrm{kg} / \mathrm{kg} \rightarrow \mathrm{ppm})$

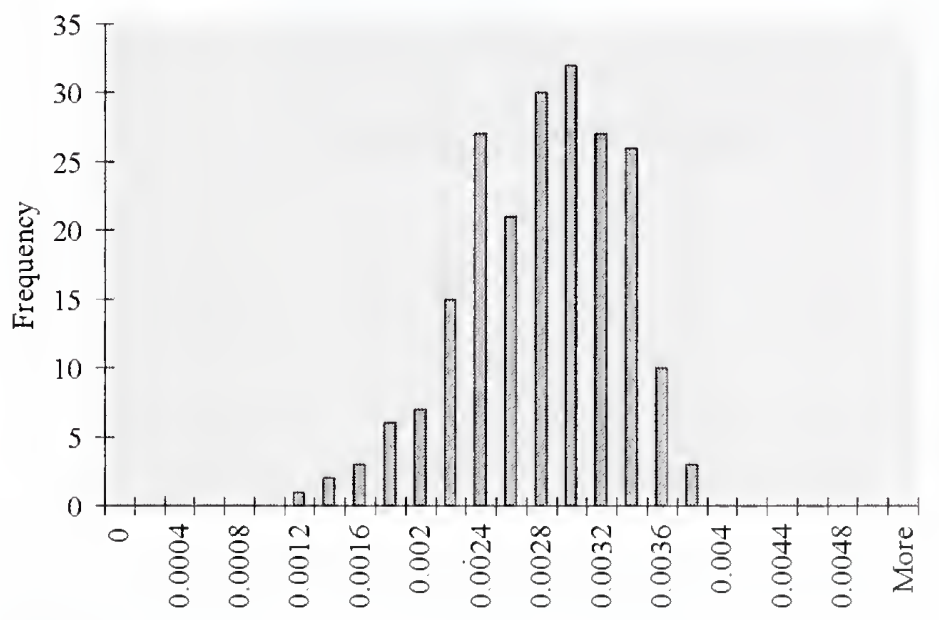

\begin{tabular}{|c|c|c|}
\hline Day & $\mathrm{CO}_{2}$ & $\mathrm{NH}_{3}$ \\
\hline 1 & 785000 & 413 \\
\hline 2 & 785000 & 795 \\
\hline 3 & 785000 & 1225 \\
\hline 4 & 785000 & 2008 \\
\hline 5 & 785000 & 3112 \\
\hline 6 & 785000 & 4486 \\
\hline 7 & 785000 & 6491 \\
\hline 8 & 785000 & 8334 \\
\hline 9 & 785000 & 10436 \\
\hline 10 & 785000 & 11384 \\
\hline
\end{tabular}

Cage occupied zone average relative humidity (\%) distribution

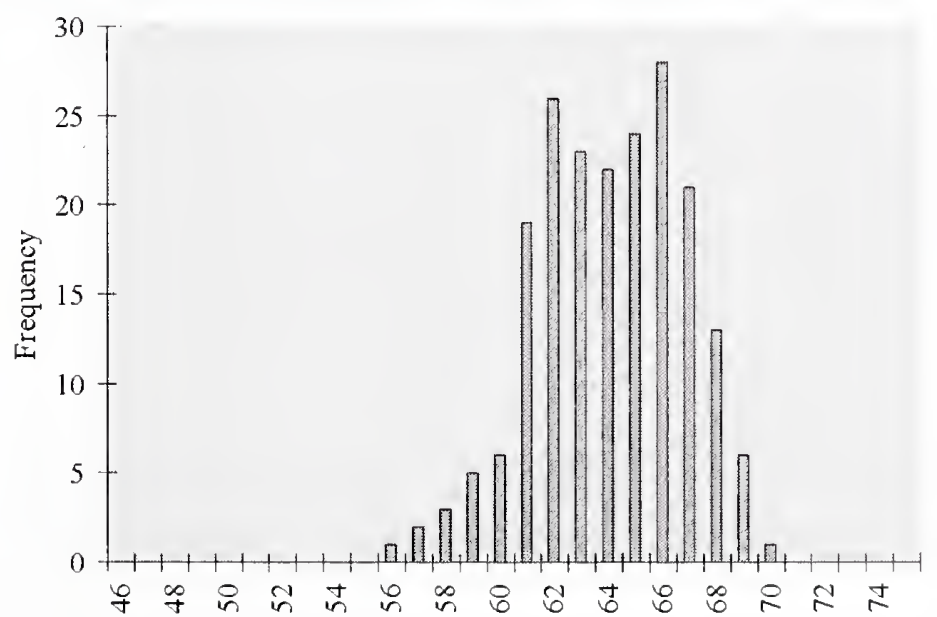


Casename

Case 12

\section{Description}

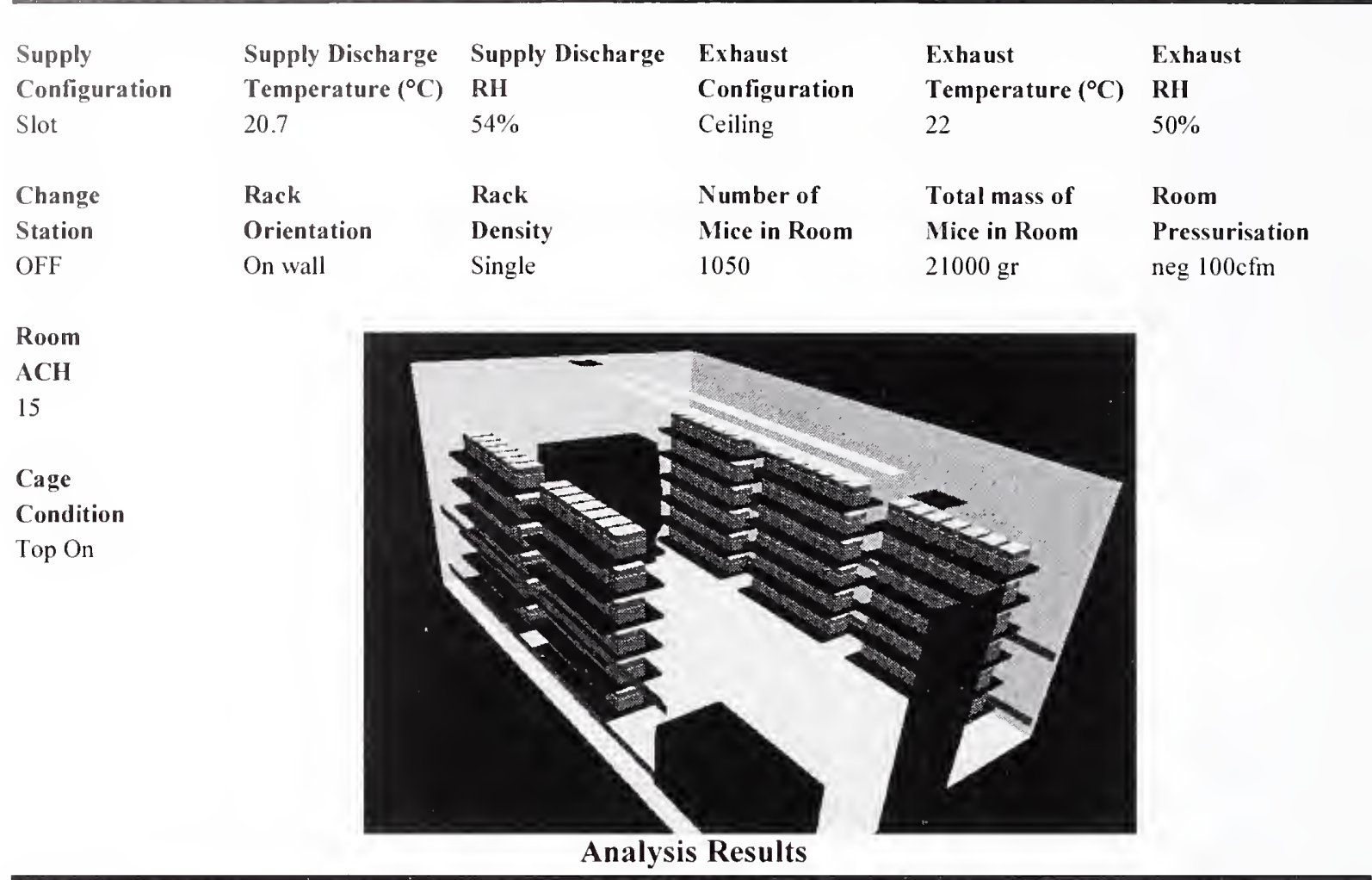

Cage Occupied Zone

\begin{tabular}{|l|cc|c|c|}
\cline { 2 - 5 } \multicolumn{1}{c|}{} & \multicolumn{2}{c|}{ Temperature } & $\mathrm{CO}_{2}$ & RH \\
\cline { 2 - 5 } \multicolumn{1}{c|}{} & ${ }^{\circ} \mathrm{C}$ & ${ }^{\circ} \mathrm{F}$ & $(\mathbf{p p m})$ & \\
\hline Mean & 22.91 & 73.24 & 2208 & $63.65 \%$ \\
\hline S.D. & 0.37 & 0.67 & 441 & $2.78 \%$ \\
\hline Max. & 23.58 & 74.45 & 3024 & $69.07 \%$ \\
\hline
\end{tabular}

Cage Occupied Zone NH3 (ppm)

\begin{tabular}{|l|c|c|c|c|c|c|c|c|c|c|}
\hline Day & $\mathbf{1}$ & $\mathbf{2}$ & $\mathbf{3}$ & $\mathbf{4}$ & $\mathbf{5}$ & $\mathbf{6}$ & $\mathbf{7}$ & $\mathbf{8}$ & $\mathbf{9}$ & $\mathbf{1 0}$ \\
\hline Mean & 1.16 & 2.23 & 3.45 & 5.66 & 8.78 & 12.66 & 18.32 & 23.52 & 29.43 & 34.67 \\
\hline Max. & 1.59 & 3.06 & 4.72 & 7.75 & 12.02 & 17.33 & 25.09 & 32.20 & 40.30 & 47.47 \\
\hline
\end{tabular}

Room Breathing Zone

\begin{tabular}{|l|cc|c|c|}
\cline { 2 - 5 } \multicolumn{1}{c|}{} & \multicolumn{2}{c|}{ Temperature } & $\mathrm{CO}_{2}$ & RI \\
\cline { 2 - 5 } \multicolumn{1}{c|}{} & ${ }^{\circ} \mathrm{C}$ & ${ }^{\circ} \mathrm{F}$ & $(\mathbf{p p m})$ & \\
\hline Mean & 21.13 & 70.03 & 17 & $52.35 \%$ \\
\hline S.D. & 0.44 & 0.80 & 42 & \\
\hline Max. & 22.14 & 71.85 & 231 & \\
\hline
\end{tabular}

Room Breathing Zone NH3 (ppm)

\begin{tabular}{|l|c|c|c|c|c|c|c|c|c|c|}
\hline Day & 1 & $\mathbf{2}$ & $\mathbf{3}$ & $\mathbf{4}$ & $\mathbf{5}$ & $\mathbf{6}$ & $\mathbf{7}$ & $\mathbf{8}$ & $\mathbf{9}$ & $\mathbf{1 0}$ \\
\hline Mean & 0.01 & 0.02 & 0.03 & 0.04 & 0.07 & 0.10 & 0.14 & 0.18 & 0.23 & 0.27 \\
\hline Max. & 0.12 & 0.23 & 0.36 & 0.59 & 0.92 & 1.32 & 1.92 & 2.46 & 3.08 & 3.63 \\
\hline
\end{tabular}




\section{Histogram Distributions}

Cage occupied zone average temperature $\left({ }^{\circ} \mathrm{C}\right)$ distribution

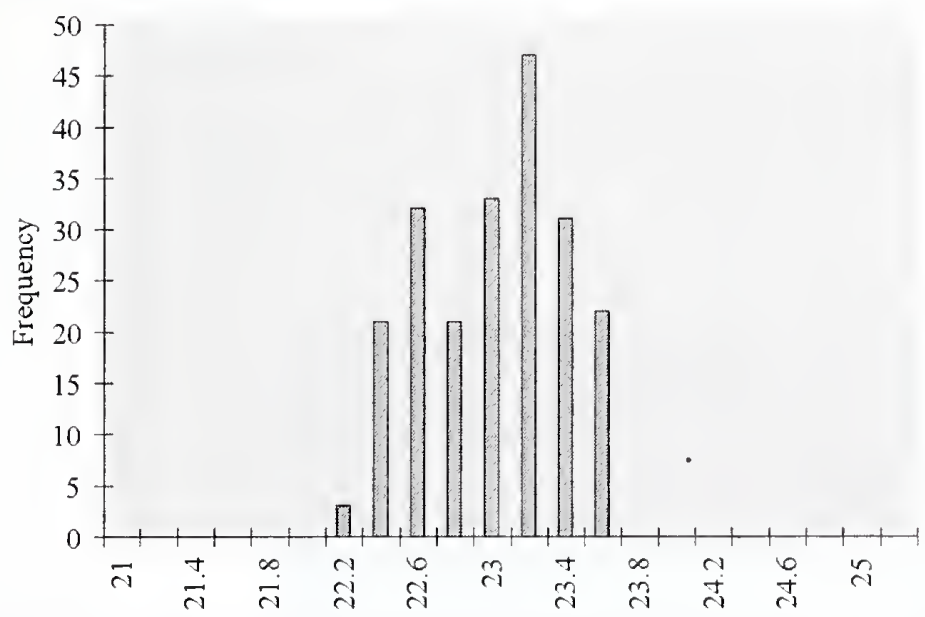

Cage occupied zone average contamination $(\mathrm{kg} / \mathrm{kg})$ distribution

Contamination conversion factors $(\mathrm{kg} / \mathrm{kg} \rightarrow \mathrm{ppm})$

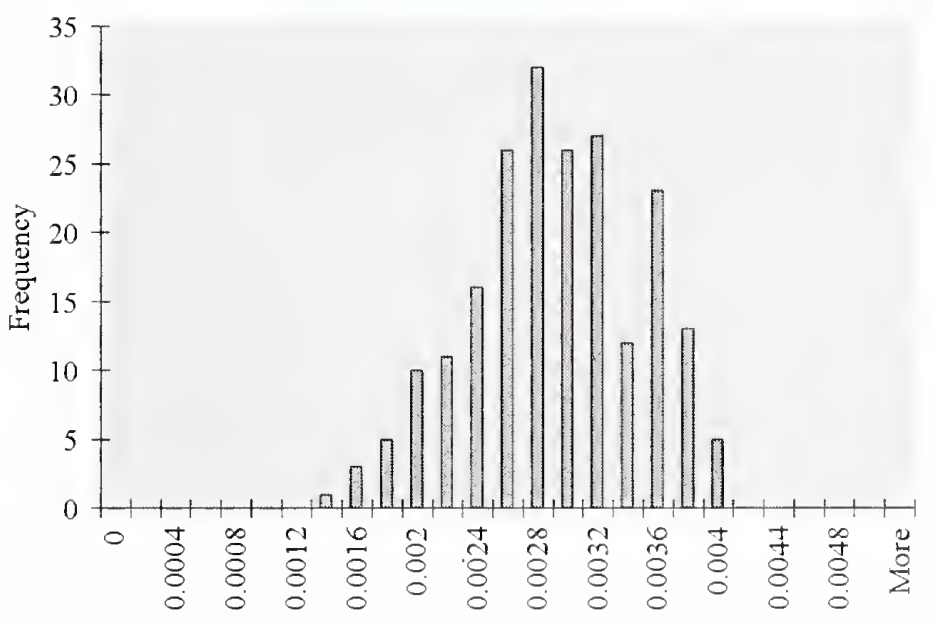

\begin{tabular}{|c|c|c|}
\hline Day & $\mathrm{CO}_{2}$ & $\mathrm{NH}_{3}$ \\
\hline $\mathbf{1}$ & 785000 & 413 \\
\hline $\mathbf{2}$ & 785000 & 795 \\
\hline $\mathbf{3}$ & 785000 & 1225 \\
\hline $\mathbf{4}$ & 785000 & 2011 \\
\hline $\mathbf{5}$ & 785000 & 3121 \\
\hline $\mathbf{6}$ & 785000 & 4500 \\
\hline 7 & 785000 & 6514 \\
\hline $\mathbf{8}$ & 785000 & 8360 \\
\hline $\mathbf{9}$ & 785000 & 10462 \\
\hline $\mathbf{1 0}$ & 785000 & 11384 \\
\hline
\end{tabular}

Cage occupied zone average relative humidity (\%) distribution

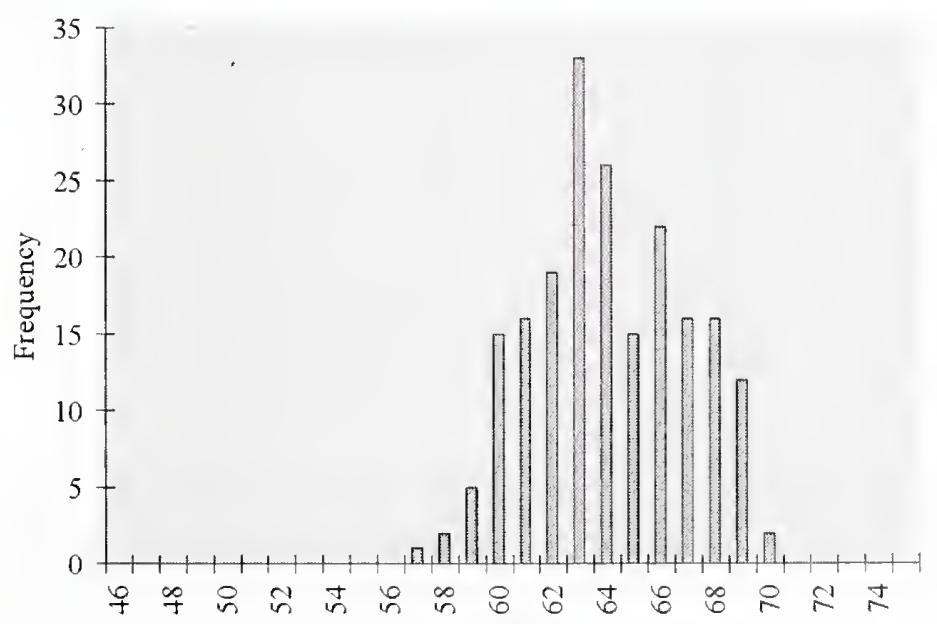


Casename

Case 13

\section{Description}

\begin{tabular}{|c|c|c|c|c|c|}
\hline Supply & Supply Discharge & Supply Discharge & Exhaust & Exhaust & Exhaust \\
\hline Configuration & Temperature $\left({ }^{\circ} \mathrm{C}\right)$ & $\mathrm{RH}$ & Configuration & Temperature $\left({ }^{\circ} \mathrm{C}\right)$ & RH \\
\hline Slot & 20.7 & $54 \%$ & Low & 22 & $50 \%$ \\
\hline $\begin{array}{l}\text { Change } \\
\text { Station } \\
\text { OFF }\end{array}$ & $\begin{array}{l}\text { Rack } \\
\text { Orientation } \\
\text { On wall }\end{array}$ & $\begin{array}{l}\text { Rack } \\
\text { Density } \\
\text { Single }\end{array}$ & $\begin{array}{l}\text { Number of } \\
\text { Mice in Room } \\
1050\end{array}$ & $\begin{array}{l}\text { Total mass of } \\
\text { Mice in Room } \\
21000 \mathrm{gr}\end{array}$ & $\begin{array}{l}\text { Room } \\
\text { Pressurisation } \\
\text { neg } 100 \mathrm{cfm}\end{array}$ \\
\hline $\begin{array}{l}\text { Room } \\
\text { ACH } \\
15\end{array}$ & & & & & \\
\hline $\begin{array}{l}\text { Cage } \\
\text { Condition } \\
\text { Top On }\end{array}$ & & & & & \\
\hline
\end{tabular}

Cage Occupied Zone

\begin{tabular}{|l|cc|c|c|}
\cline { 2 - 5 } \multicolumn{1}{c|}{} & \multicolumn{2}{c|}{ Temperature } & $\mathrm{CO}_{2}$ & RH \\
\cline { 2 - 5 } \multicolumn{1}{c|}{} & ${ }^{\circ} \mathrm{C}$ & ${ }^{\circ} \mathrm{F}$ & $(\mathrm{ppm})$ & \\
\hline Mean & 22.77 & 72.98 & 2491 & $66.46 \%$ \\
\hline S.D. & 0.21 & 0.38 & 254 & $1.81 \%$ \\
\hline Max. & 23.37 & 74.06 & 3001 & $70.61 \%$ \\
\hline
\end{tabular}

Cage Occupied Zone NH3 (ppm)

\begin{tabular}{|l|c|c|c|c|c|c|c|c|c|c|}
\hline Day & $\mathbf{1}$ & $\mathbf{2}$ & $\mathbf{3}$ & $\mathbf{4}$ & $\mathbf{5}$ & $\mathbf{6}$ & $\mathbf{7}$ & $\mathbf{8}$ & $\mathbf{9}$ & $\mathbf{1 0}$ \\
\hline Mean & 1.33 & 2.52 & 3.89 & 7.21 & 12.05 & 17.85 & 26.75 & 33.29 & 40.02 & 43.47 \\
\hline Max. & 1.60 & 3.04 & 4.68 & 8.69 & 14.52 & 21.50 & 32.23 & 40.10 & 48.21 & 52.37 \\
\hline
\end{tabular}

Room Breathing Zone

\begin{tabular}{|l|cc|c|c|}
\cline { 2 - 5 } \multicolumn{1}{c|}{} & \multicolumn{2}{c|}{ Temperature } & $\mathrm{CO}_{2}$ & RH \\
\cline { 2 - 5 } \multicolumn{1}{c|}{} & ${ }^{\circ} \mathrm{C}$ & ${ }^{\circ} \mathrm{F}$ & $(\mathrm{ppm})$ & \\
\hline Mean & 20.84 & 69.51 & 37 & $53.47 \%$ \\
\hline S.D. & 0.17 & 0.31 & 58 & \\
\hline Max. & 22.04 & 71.67 & 281 & \\
\hline
\end{tabular}

Room Breathing Zone NH3 (ppm)

\begin{tabular}{|l|c|c|c|c|c|c|c|c|c|c|}
\hline Day & $\mathbf{1}$ & $\mathbf{2}$ & $\mathbf{3}$ & $\mathbf{4}$ & $\mathbf{5}$ & $\mathbf{6}$ & $\mathbf{7}$ & $\mathbf{8}$ & $\mathbf{9}$ & $\mathbf{1 0}$ \\
\hline Mean & 0.02 & 0.04 & 0.06 & 0.11 & 0.18 & 0.26 & 0.39 & 0.49 & 0.59 & 0.64 \\
\hline Max. & 0.15 & 0.28 & 0.44 & 0.81 & 1.36 & 2.01 & 3.02 & 3.75 & 4.51 & 4.90 \\
\hline
\end{tabular}




\section{Histogram Distributions}

Cage occupied zone average temperature $\left({ }^{\circ} \mathrm{C}\right)$ distribution

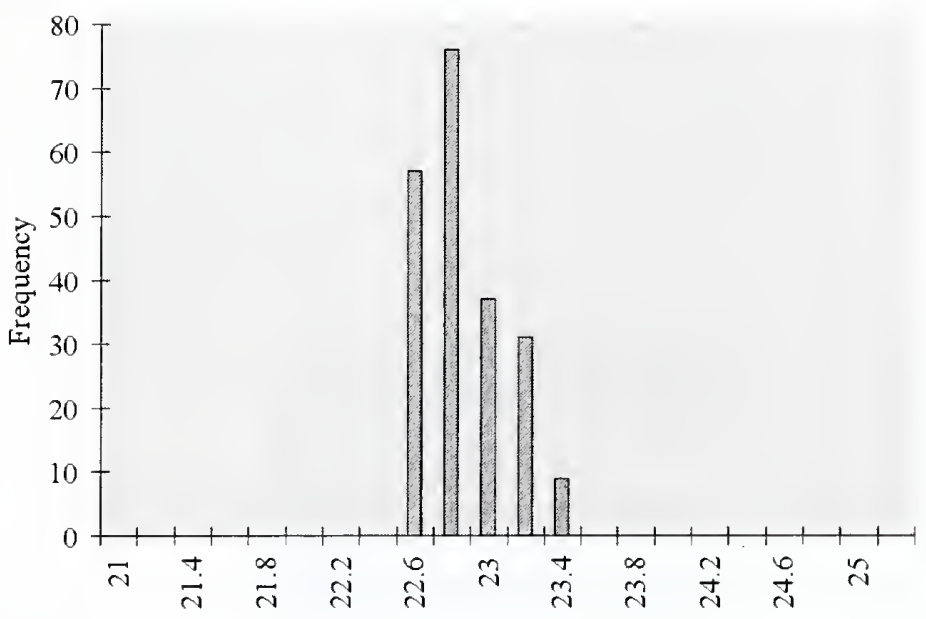

Cage occupied zone average contamination $(\mathrm{kg} / \mathrm{kg})$ distribution

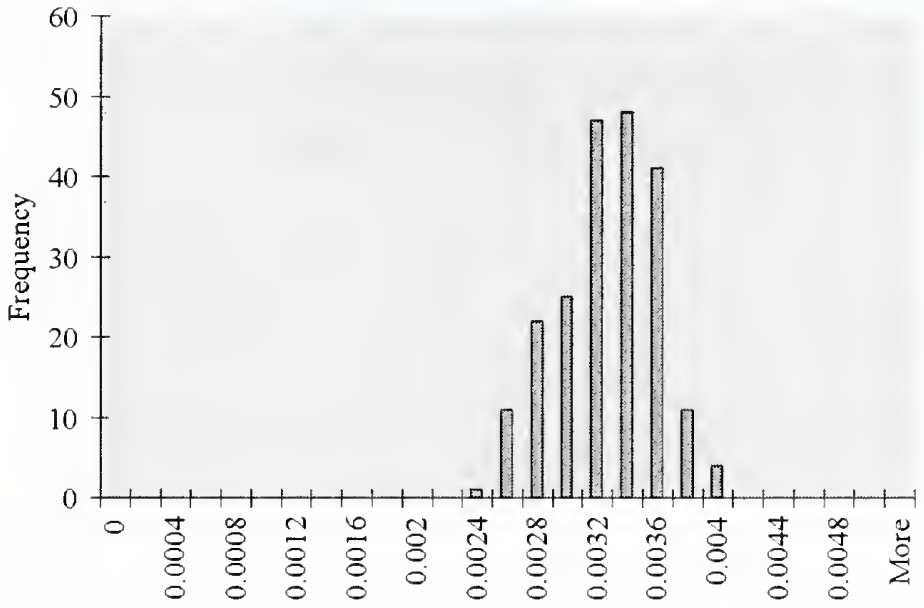

Cage occupied zone average relative humidity (\%) distribution

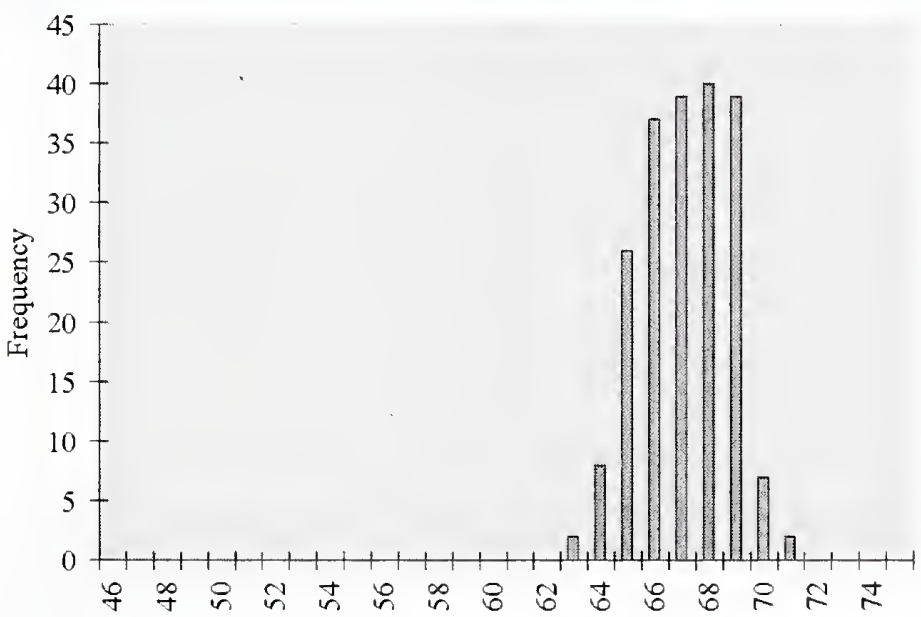

Contamination conversion factors $(\mathrm{kg} / \mathrm{kg} \rightarrow \mathrm{ppm})$

\begin{tabular}{|c|c|c|}
\hline Day & $\mathrm{CO}_{2}$ & $\mathbf{N H}_{3}$ \\
\hline 1 & 785000 & 418 \\
\hline 2 & 785000 & 795 \\
\hline 3 & 785000 & 1225 \\
\hline 4 & 785000 & 2273 \\
\hline 5 & 785000 & 3798 \\
\hline 6 & 785000 & 5624 \\
\hline 7 & 785000 & 8430 \\
\hline 8 & 785000 & 10490 \\
\hline 9 & 785000 & 12611 \\
\hline 10 & 785000 & 11384 \\
\hline
\end{tabular}


Casename

Case 14

Description

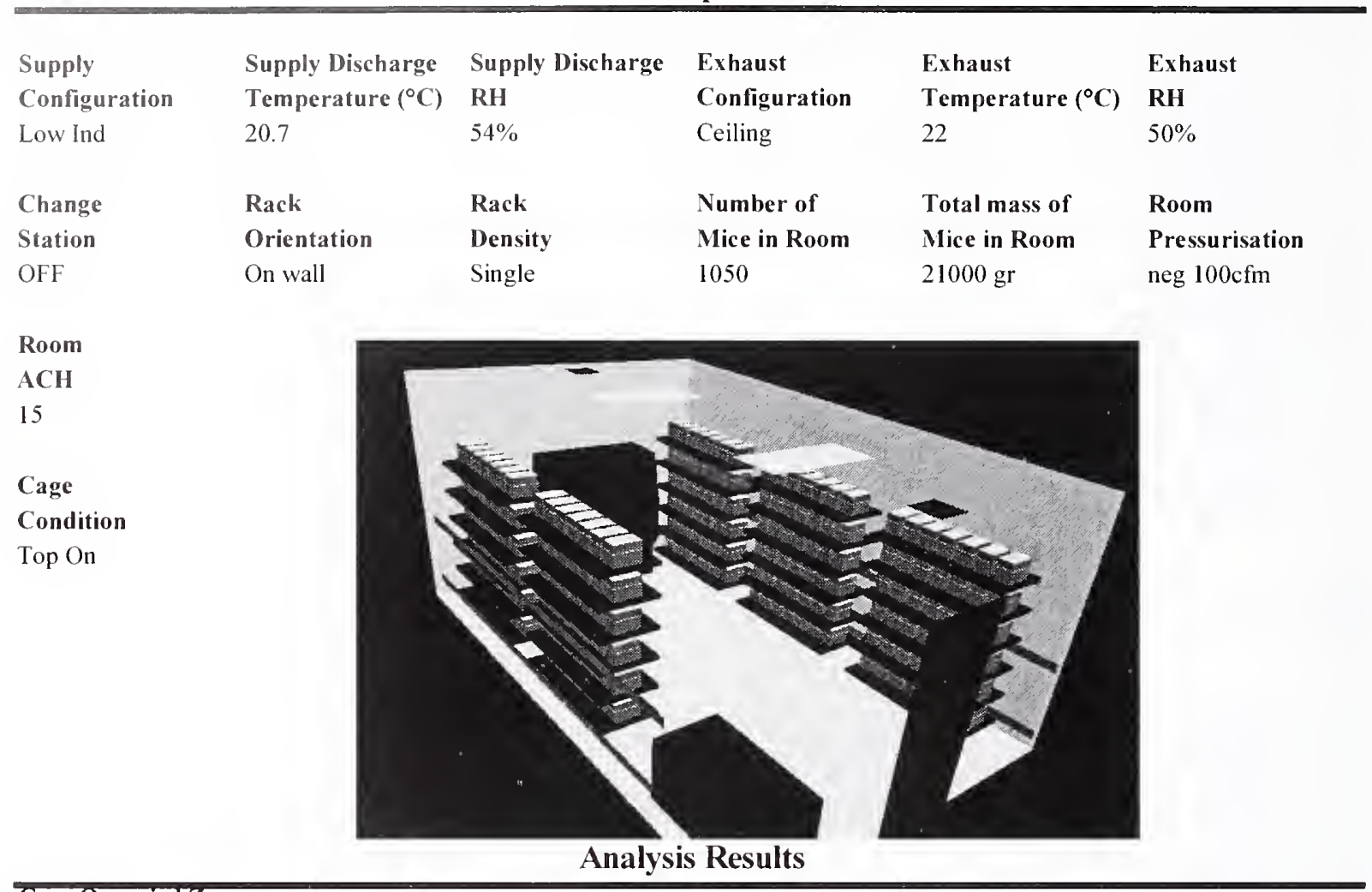

Cage Occupied Zone

\begin{tabular}{|l|cc|c|c|}
\cline { 2 - 5 } \multicolumn{1}{c|}{} & \multicolumn{2}{c|}{ Temperature } & $\mathrm{CO}_{2}$ & RH \\
\cline { 2 - 5 } \multicolumn{1}{c|}{} & ${ }^{\circ} \mathrm{C}$ & ${ }^{\circ} \mathrm{F}$ & $(\mathrm{ppm})$ & \\
\hline Mean & 22.80 & 73.04 & 2215 & $64.18 \%$ \\
\hline S.D. & 0.21 & 0.37 & 222 & $1.51 \%$ \\
\hline Max. & 23.32 & 73.97 & 2619 & $67.00 \%$ \\
\hline
\end{tabular}

Cage Occupied Zone NH3 (ppm)

\begin{tabular}{|l|c|c|c|c|c|c|c|c|c|c|}
\hline Day & $\mathbf{1}$ & $\mathbf{2}$ & $\mathbf{3}$ & $\mathbf{4}$ & $\mathbf{5}$ & $\mathbf{6}$ & $\mathbf{7}$ & $\mathbf{8}$ & $\mathbf{9}$ & $\mathbf{1 0}$ \\
\hline Mean & 1.17 & 2.24 & 3.46 & 5.82 & 9.17 & 13.30 & 19.40 & 24.73 & 30.67 & 35.51 \\
\hline Max. & 1.38 & 2.65 & 4.09 & 6.88 & 10.84 & 15.72 & 22.94 & 29.23 & 36.26 & 41.99 \\
\hline
\end{tabular}

Room Breathing Zone

\begin{tabular}{|l|cc|c|c|}
\cline { 2 - 5 } \multicolumn{1}{c|}{} & \multicolumn{2}{c|}{ Temperature } & $\mathrm{CO}_{2}$ & RH \\
\cline { 2 - 5 } \multicolumn{1}{c|}{} & ${ }^{\circ} \mathrm{C}$ & ${ }^{\circ} \mathrm{F}$ & $(\mathbf{p p m})$ & \\
\hline Mean & 21.12 & 70.01 & 52 & $52.68 \%$ \\
\hline S.D. & 0.17 & 0.31 & 23 & \\
\hline Max. & 22.07 & 71.72 & 220 & \\
\hline
\end{tabular}

Room Breathing Zone NH3 (ppm)

\begin{tabular}{|l|c|c|c|c|c|c|c|c|c|c|}
\hline Day & $\mathbf{1}$ & $\mathbf{2}$ & $\mathbf{3}$ & $\mathbf{4}$ & $\mathbf{5}$ & $\mathbf{6}$ & $\mathbf{7}$ & $\mathbf{8}$ & $\mathbf{9}$ & $\mathbf{1 0}$ \\
\hline Mean & 0.03 & 0.05 & 0.08 & 0.14 & 0.21 & 0.31 & 0.45 & 0.58 & 0.72 & 0.83 \\
\hline Max. & 0.12 & 0.22 & 0.34 & 0.58 & 0.91 & 1.32 & 1.93 & 2.45 & 3.04 & 3.52 \\
\hline
\end{tabular}




\section{Histogram Distributions}

Cage occupied zone average temperature $\left({ }^{\circ} \mathrm{C}\right)$ distribution

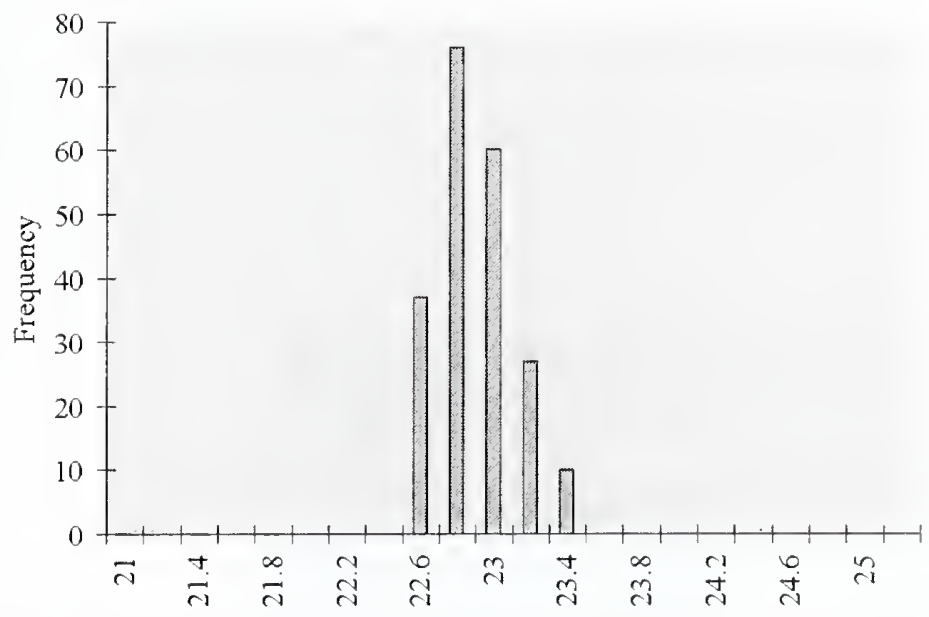

Cage occupied zone average contamination $(\mathrm{kg} / \mathrm{kg})$ distribution

Contamination conversion factors $(\mathrm{kg} / \mathrm{kg} \rightarrow \mathrm{ppm})$

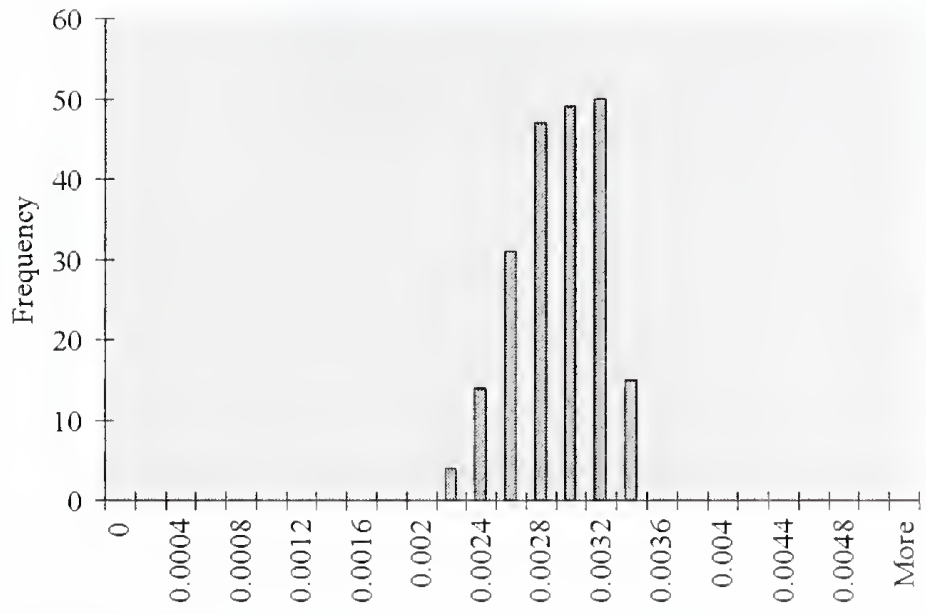

\begin{tabular}{|c|c|c|}
\hline Day & $\mathbf{C O}_{2}$ & $\mathrm{NH}_{3}$ \\
\hline $\mathbf{1}$ & 785000 & 414 \\
\hline $\mathbf{2}$ & 785000 & 795 \\
\hline $\mathbf{3}$ & 785000 & 1225 \\
\hline $\mathbf{4}$ & 785000 & 2061 \\
\hline $\mathbf{5}$ & 785000 & 3249 \\
\hline $\mathbf{6}$ & 785000 & 4713 \\
\hline $\mathbf{7}$ & 785000 & 6877 \\
\hline $\mathbf{8}$ & 785000 & 8763 \\
\hline $\mathbf{9}$ & 785000 & 10869 \\
\hline $\mathbf{1 0}$ & 785000 & 11384 \\
\hline
\end{tabular}

Cage occupied zone average relative humidity (\%) distribution

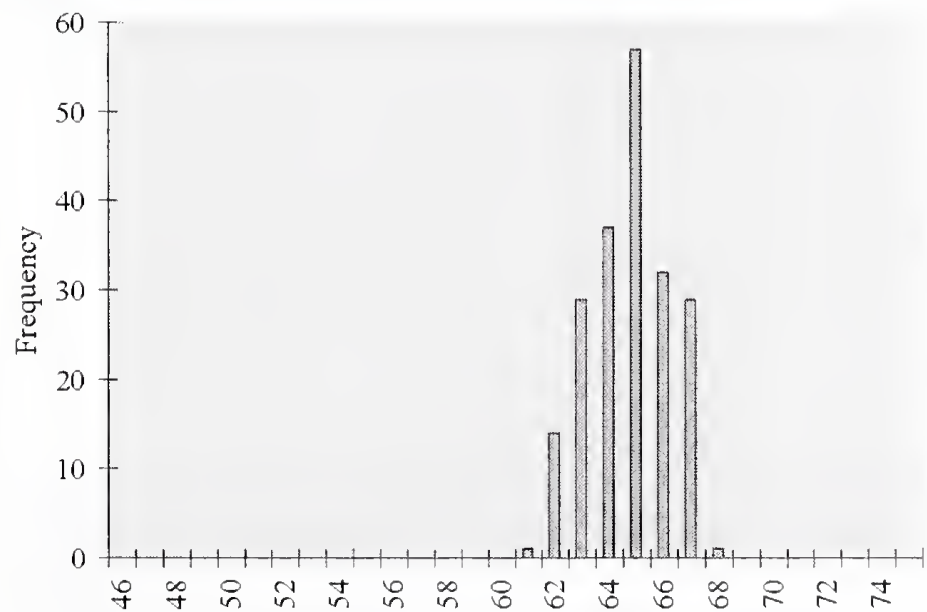


Casename

Case 15

\section{Description}

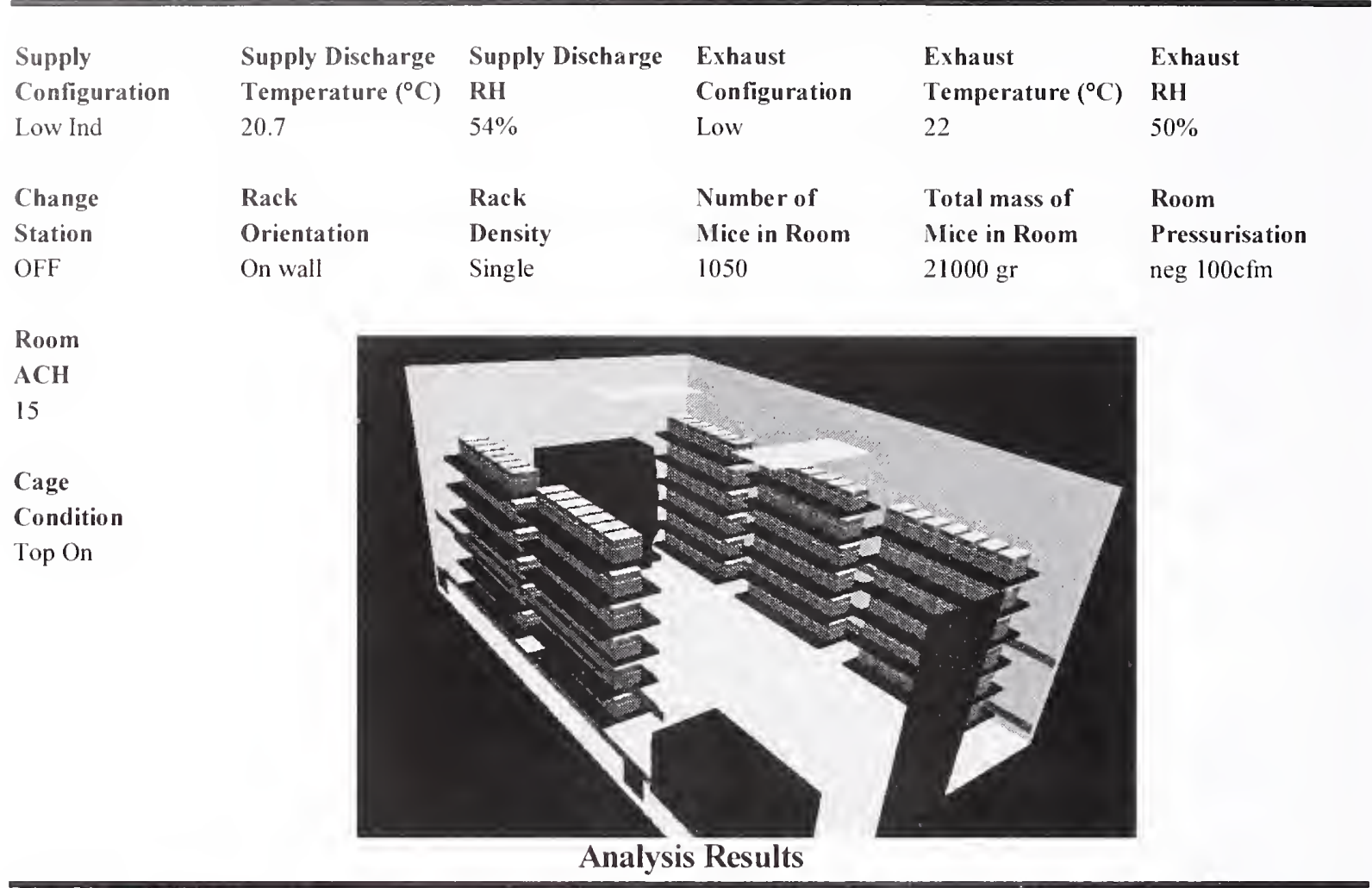

Cage Occupied Zone

\begin{tabular}{|l|cc|c|c|}
\cline { 2 - 5 } \multicolumn{1}{c|}{} & \multicolumn{2}{c|}{ Temperature } & $\mathrm{CO}_{2}$ & \multicolumn{1}{c|}{$\mathrm{RH}$} \\
\cline { 2 - 5 } \multicolumn{1}{c|}{} & ${ }^{\circ} \mathrm{C}$ & ${ }^{\circ} \mathrm{F}$ & $(\mathrm{ppm})$ & \\
\hline Mean & 23.24 & 73.84 & 2119 & $61.71 \%$ \\
\hline S.D. & 0.28 & 0.51 & 376 & $3.14 \%$ \\
\hline Max. & 23.88 & 74.98 & 3201 & $68.32 \%$ \\
\hline
\end{tabular}

Cage Occupied Zone NH3 (ppm)

\begin{tabular}{|l|c|c|c|c|c|c|c|c|c|c|}
\hline Day & $\mathbf{1}$ & $\mathbf{2}$ & $\mathbf{3}$ & $\mathbf{4}$ & $\mathbf{5}$ & $\mathbf{6}$ & $\mathbf{7}$ & $\mathbf{8}$ & $\mathbf{9}$ & $\mathbf{1 0}$ \\
\hline Mean & 1.11 & 2.14 & 3.31 & 4.94 & 7.17 & 10.06 & 14.03 & 18.61 & 24.25 & 30.72 \\
\hline Max. & 1.67 & 3.24 & 5.00 & 7.47 & 10.83 & 15.20 & 21.20 & 28.12 & 36.65 & 46.42 \\
\hline
\end{tabular}

Room Breathing Zone

\begin{tabular}{|l|cc|c|c|}
\cline { 2 - 5 } \multicolumn{1}{c|}{} & \multicolumn{2}{|c|}{ Temperature } & $\mathrm{CO}_{2}$ & RH \\
\cline { 2 - 5 } \multicolumn{1}{c|}{} & ${ }^{\circ} \mathrm{C}$ & ${ }^{\circ} \mathrm{F}$ & $(\mathrm{ppm})$ & \\
\hline Mean & 21.21 & 70.19 & 48 & $52.32 \%$ \\
\hline S.D. & 0.16 & 0.29 & 44 & \\
\hline Max. & 21.94 & 71.50 & 306 & \\
\hline
\end{tabular}

Room Breathing Zone NH3 (ppm)

\begin{tabular}{|l|c|c|c|c|c|c|c|c|c|c|}
\hline Day & $\mathbf{1}$ & $\mathbf{2}$ & $\mathbf{3}$ & $\mathbf{4}$ & $\mathbf{5}$ & $\mathbf{6}$ & $\mathbf{7}$ & $\mathbf{8}$ & $\mathbf{9}$ & $\mathbf{1 0}$ \\
\hline Mean & 0.03 & 0.05 & 0.07 & 0.11 & 0.16 & 0.23 & 0.32 & 0.42 & 0.55 & 0.70 \\
\hline Max. & 0.16 & 0.31 & 0.48 & 0.71 & 1.03 & 1.45 & 2.02 & 2.69 & 3.50 & 4.43 \\
\hline
\end{tabular}




\section{Histogram Distributions}

Cage occupied zone average temperature $\left({ }^{\circ} \mathrm{C}\right)$ distribution

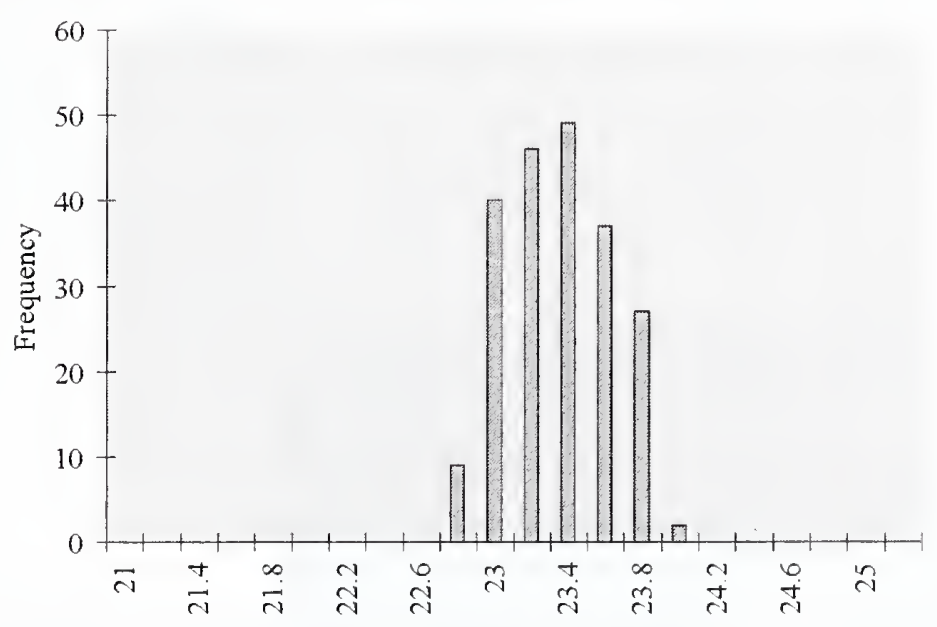

Cage occupied zone average contamination $(\mathrm{kg} / \mathrm{kg})$ distribution

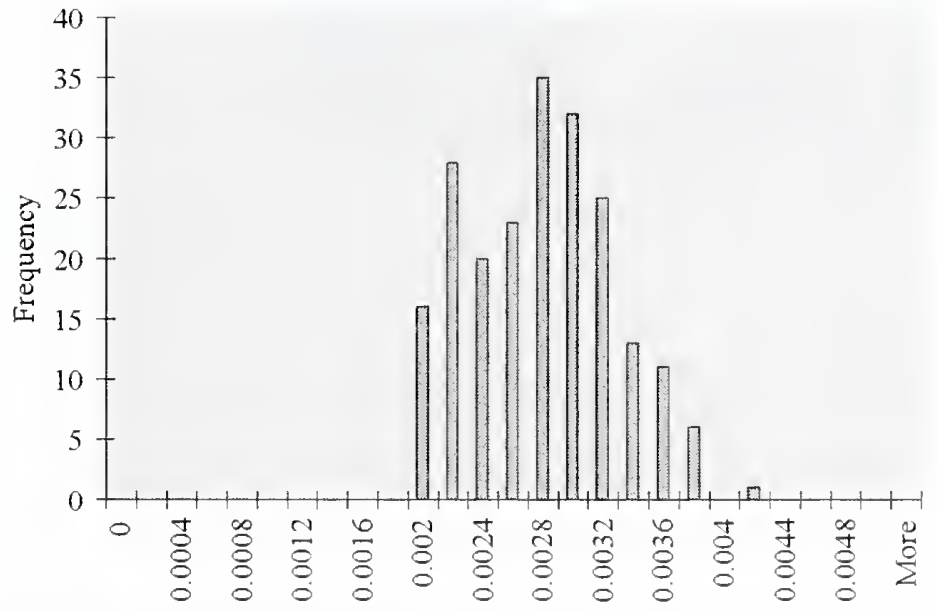

Cage occupied zone average relative humidity $(\%)$ distribution

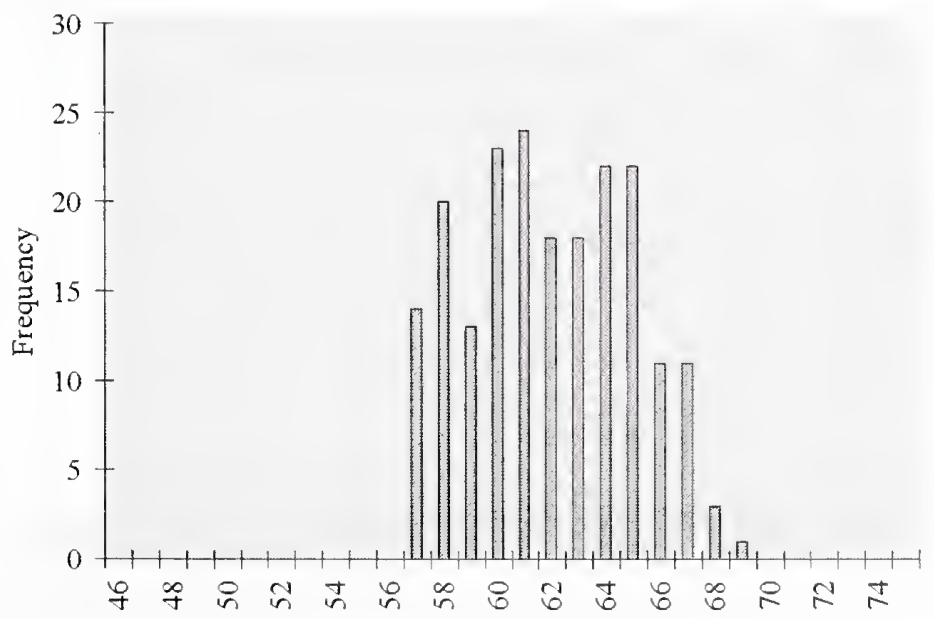

Contamination conversion factors $(\mathrm{kg} / \mathrm{kg} \rightarrow \mathrm{ppm})$

\begin{tabular}{|c|c|c|}
\hline Day & $\mathrm{CO}_{2}$ & $\mathrm{NH}_{3}$ \\
\hline $\mathbf{1}$ & 785000 & 410 \\
\hline $\mathbf{2}$ & 785000 & 795 \\
\hline 3 & 785000 & 1225 \\
\hline 4 & 785000 & 1832 \\
\hline 5 & 785000 & 2655 \\
\hline $\mathbf{6}$ & 785000 & 3728 \\
\hline 7 & 785000 & 5198 \\
\hline $\mathbf{8}$ & 785000 & 6897 \\
\hline $\mathbf{9}$ & 785000 & 8987 \\
\hline $\mathbf{1 0}$ & 785000 & 11384 \\
\hline
\end{tabular}


Casename

Case 16

Description

\begin{tabular}{|c|c|c|c|c|c|}
\hline Supply & Supply Discharge & Supply Discharge & Exhaust & Exhaust & Exhaust \\
\hline Configuration & Temperature $\left({ }^{\circ} \mathrm{C}\right)$ & RH & Configuration & Temperature $\left({ }^{\circ} \mathrm{C}\right)$ & RH \\
\hline Radial & 18.8 & $61 \%$ & Ceiling & 22 & $50 \%$ \\
\hline Change & Rack & Rack & Number of & Total mass of & Room \\
\hline Station & Orientation & Density & Mice in Room & Mice in Room & Pressurisation \\
\hline ON & On wall & Single & 1050 & $21000 \mathrm{gr}$ & pos $100 \mathrm{cfm}$ \\
\hline
\end{tabular}

Room

$\mathrm{ACH}$

15

Cage

Condition

Top On

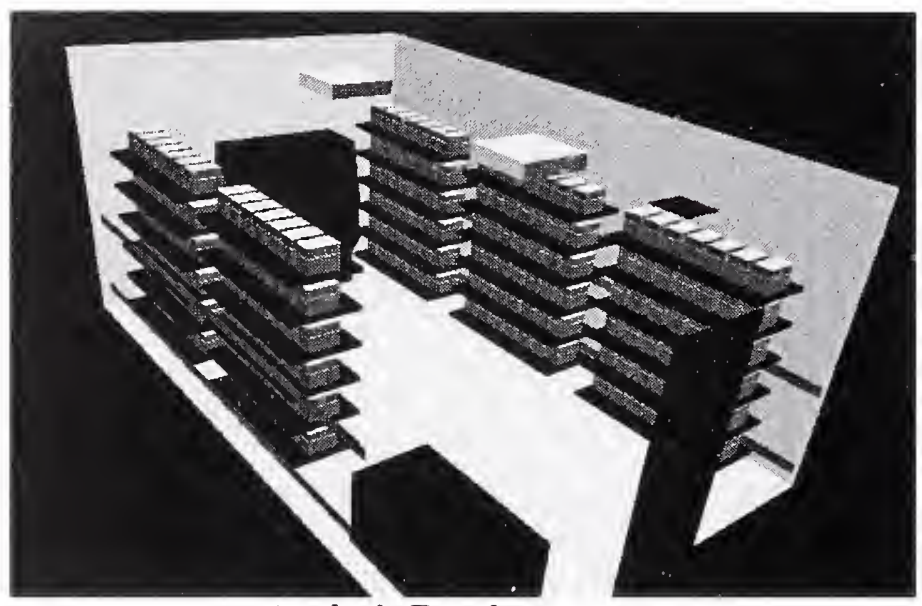

Analysis Results

Cage Occupied Zone

\begin{tabular}{|l|cc|c|c|}
\cline { 2 - 5 } \multicolumn{1}{c|}{} & \multicolumn{2}{c|}{ Temperature } & $\mathrm{CO}_{2}$ & RH \\
\cline { 2 - 5 } \multicolumn{1}{c|}{} & ${ }^{\circ} \mathrm{C}$ & ${ }^{\circ} \mathrm{F}$ & $(\mathbf{p p m})$ & \\
\hline Mean & 22.85 & 73.13 & 2213 & $63.97 \%$ \\
\hline S.D. & 0.81 & 1.45 & 450 & $3.43 \%$ \\
\hline Max. & 24.17 & 75.50 & 3126 & $74.23 \%$ \\
\hline
\end{tabular}

Cage Occupied Zone NH3 (ppm)

\begin{tabular}{|l|c|c|c|c|c|c|c|c|c|c|}
\hline Day & $\mathbf{1}$ & $\mathbf{2}$ & $\mathbf{3}$ & $\mathbf{4}$ & $\mathbf{5}$ & $\mathbf{6}$ & $\mathbf{7}$ & $\mathbf{8}$ & $\mathbf{9}$ & $\mathbf{1 0}$ \\
\hline Mean & 1.17 & 2.24 & 3.45 & 5.76 & 9.02 & 13.05 & 18.99 & 24.26 & 30.19 & 35.20 \\
\hline Max. & 1.65 & 3.16 & 4.88 & 8.13 & 12.74 & 18.43 & 26.82 & 34.26 & 42.65 & 49.71 \\
\hline
\end{tabular}

Room Breathing Zone

\begin{tabular}{|l|cc|c|c|}
\cline { 2 - 5 } \multicolumn{1}{c|}{} & \multicolumn{2}{c|}{ Temperature } & $\mathrm{CO}_{2}$ & RH \\
\cline { 2 - 5 } \multicolumn{1}{c|}{} & ${ }^{\circ} \mathrm{C}$ & ${ }^{\circ} \mathrm{F}$ & $(\mathrm{ppm})$ & \\
\hline Mean & 20.07 & 68.12 & 37 & $56.18 \%$ \\
\hline S.D. & 1.29 & 2.33 & 47 & \\
\hline Max. & 23.06 & 73.52 & 251 & \\
\hline
\end{tabular}

Room Breathing Zone NH3 (ppm)

\begin{tabular}{|l|c|c|c|c|c|c|c|c|c|c|}
\hline Day & $\mathbf{1}$ & $\mathbf{2}$ & $\mathbf{3}$ & $\mathbf{4}$ & $\mathbf{5}$ & $\mathbf{6}$ & $\mathbf{7}$ & $\mathbf{8}$ & $\mathbf{9}$ & $\mathbf{1 0}$ \\
\hline Mean & 0.02 & 0.04 & 0.06 & 0.10 & 0.15 & 0.22 & 0.31 & 0.40 & 0.50 & 0.58 \\
\hline Max. & 0.13 & 0.25 & 0.39 & 0.65 & 1.02 & 1.48 & 2.16 & 2.76 & 3.43 & 4.00 \\
\hline
\end{tabular}




\section{Histogram Distributions}

Cage occupied zone average temperature $\left({ }^{\circ} \mathrm{C}\right)$ distribution

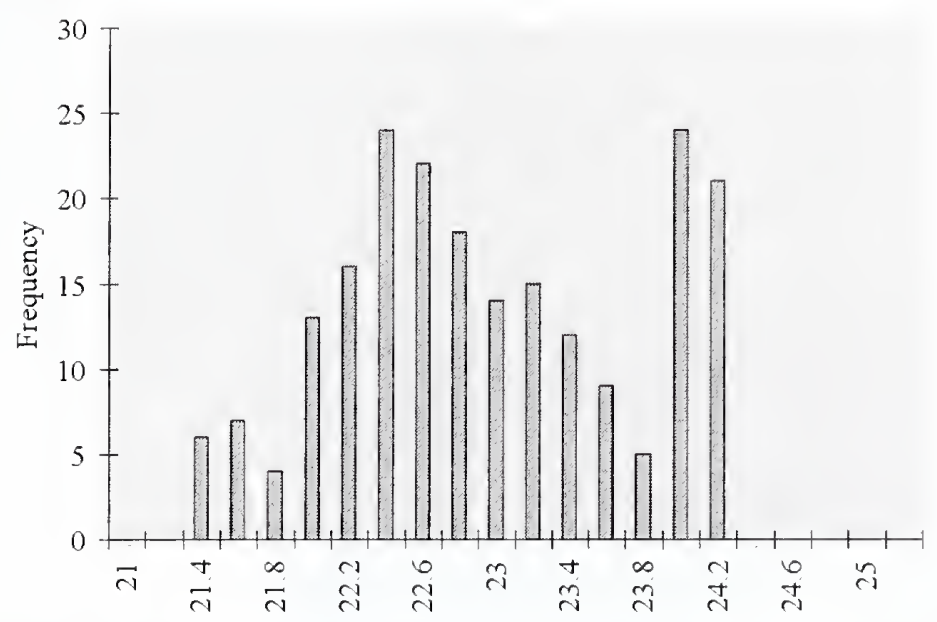

Cage occupied zone average contamination $(\mathrm{kg} / \mathrm{kg})$ distribution

Contamination conversion factors $(\mathrm{kg} / \mathrm{kg} \rightarrow \mathrm{ppm})$

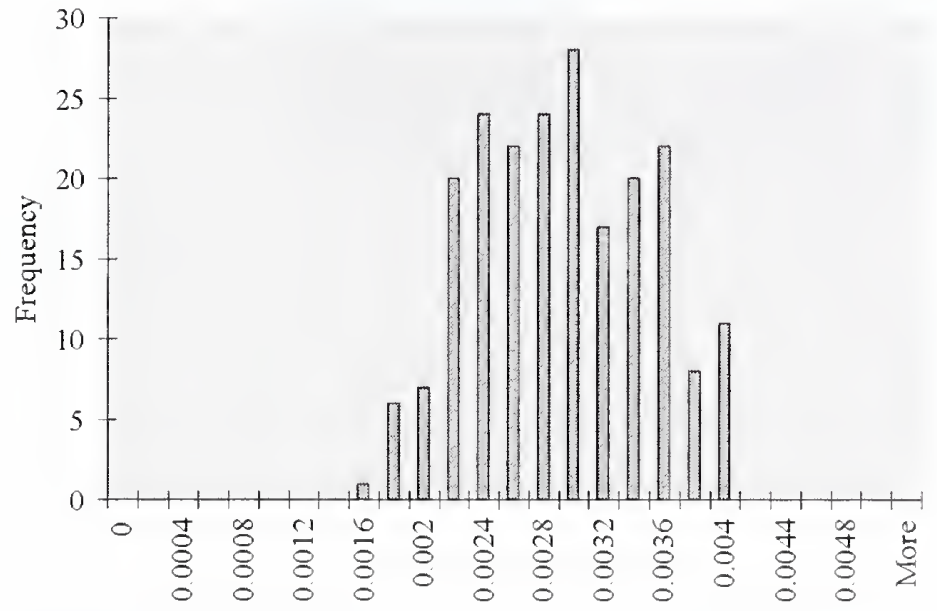

Cage occupied zone average relative humidity (\%) distribution

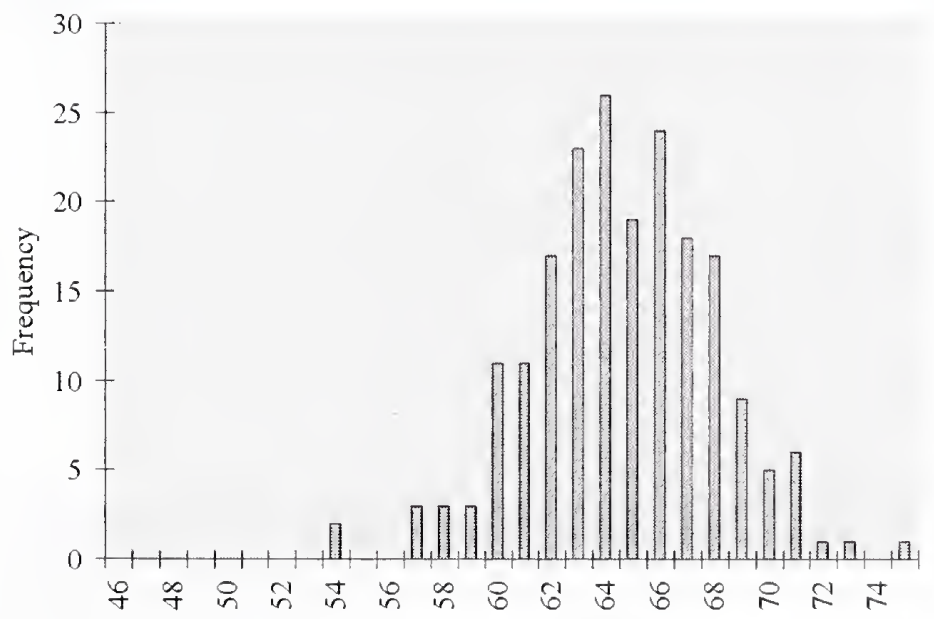


Casename

Case 17

Description

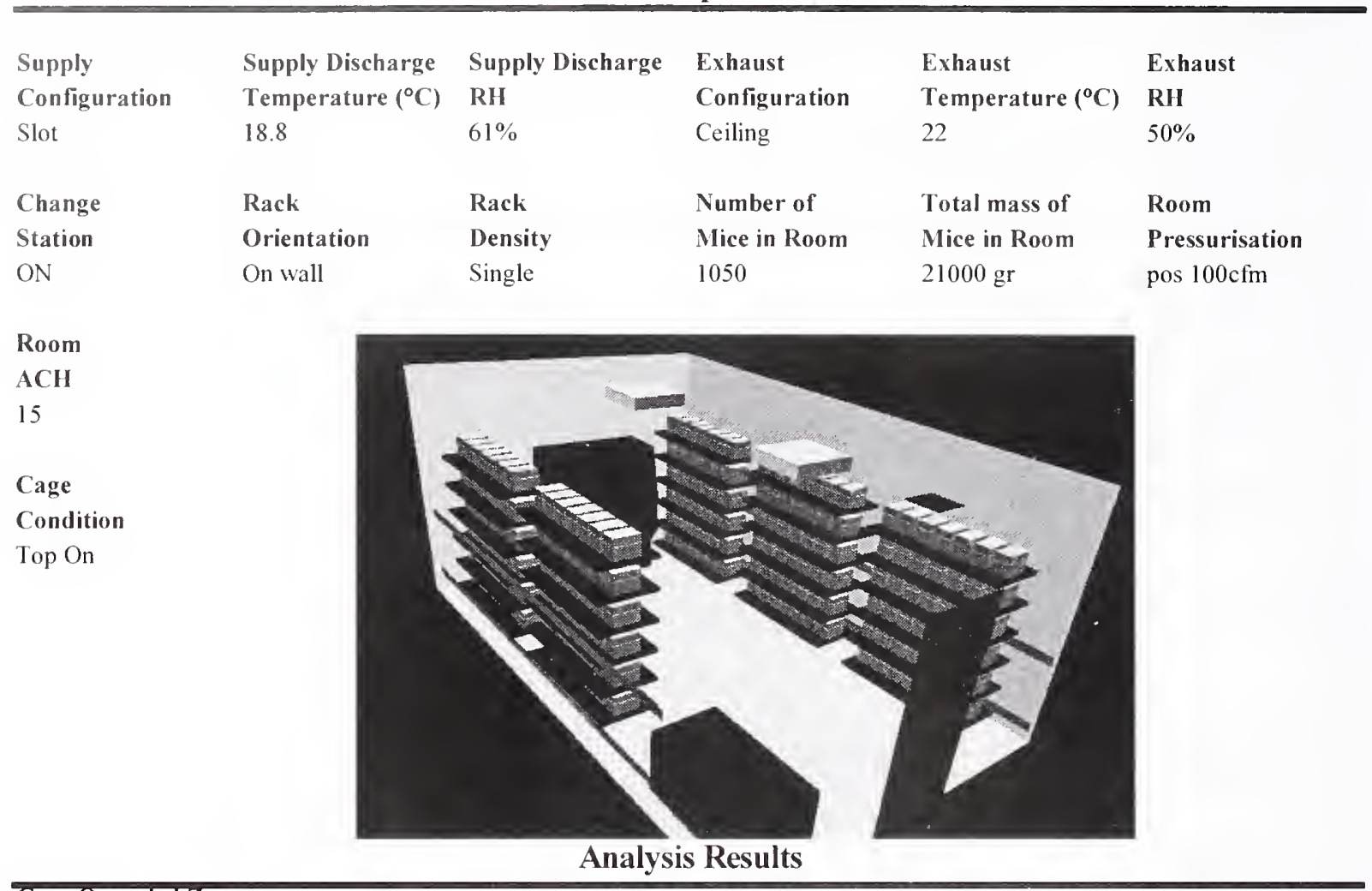

Cage Occupied Zone

\begin{tabular}{|l|cc|c|c|}
\cline { 2 - 5 } \multicolumn{1}{c|}{} & \multicolumn{2}{c|}{ Temperature } & $\mathrm{CO}_{2}$ & RH \\
\cline { 2 - 5 } \multicolumn{1}{c|}{} & ${ }^{\circ} \mathrm{C}$ & ${ }^{\circ} \mathrm{F}$ & $(\mathrm{ppm})$ & \\
\hline Mean & 22.07 & 71.73 & 1815 & $63.94 \%$ \\
\hline S.D. & 0.29 & 0.52 & 292 & $2.38 \%$ \\
\hline Max. & 23.08 & 73.55 & 2464 & $68.92 \%$ \\
\hline
\end{tabular}

Cage Occupied Zone NH3 (ppm)

\begin{tabular}{|l|c|c|c|c|c|c|c|c|c|c|}
\hline Day & $\mathbf{1}$ & $\mathbf{2}$ & $\mathbf{3}$ & $\mathbf{4}$ & $\mathbf{5}$ & $\mathbf{6}$ & $\mathbf{7}$ & $\mathbf{8}$ & $\mathbf{9}$ & $\mathbf{1 0}$ \\
\hline Mean & 0.96 & 1.84 & 2.83 & 4.71 & 7.38 & 10.67 & 15.52 & 19.84 & 24.70 & 28.82 \\
\hline Max. & 1.30 & 2.49 & 3.84 & 6.40 & 10.01 & 14.49 & 21.07 & 26.93 & 33.54 & 39.13 \\
\hline
\end{tabular}

Room Breathing Zone

\begin{tabular}{|l|cc|c|c|}
\cline { 2 - 5 } \multicolumn{1}{c|}{} & \multicolumn{2}{c|}{ Temperature } & $\mathrm{CO}_{2}$ & \multicolumn{1}{c|}{$\mathrm{RH}$} \\
\cline { 2 - 5 } \multicolumn{1}{c|}{} & ${ }^{\circ} \mathrm{C}$ & ${ }^{\circ} \mathrm{F}$ & $(\mathrm{ppm})$ & \\
\hline Mean & 20.23 & 68.41 & 78 & $55.99 \%$ \\
\hline S.D. & 0.13 & 0.23 & 28 & \\
\hline Max. & 21.35 & 70.43 & 181 & \\
\hline
\end{tabular}

Room Breathing Zone NHI3 (ppm)

\begin{tabular}{|l|c|c|c|c|c|c|c|c|c|c|}
\hline Day & $\mathbf{1}$ & $\mathbf{2}$ & $\mathbf{3}$ & $\mathbf{4}$ & $\mathbf{5}$ & $\mathbf{6}$ & $\mathbf{7}$ & $\mathbf{8}$ & $\mathbf{9}$ & $\mathbf{1 0}$ \\
\hline Mean & 0.04 & 0.08 & 0.12 & 0.20 & 0.32 & 0.46 & 0.66 & 0.85 & 1.06 & 1.23 \\
\hline Max. & 0.10 & 0.18 & 0.28 & 0.47 & 0.73 & 1.06 & 1.54 & 1.97 & 2.46 & 2.87 \\
\hline
\end{tabular}




\section{Histogram Distributions}

Cage occupied zone average temperature $\left({ }^{\circ} \mathrm{C}\right)$ distribution

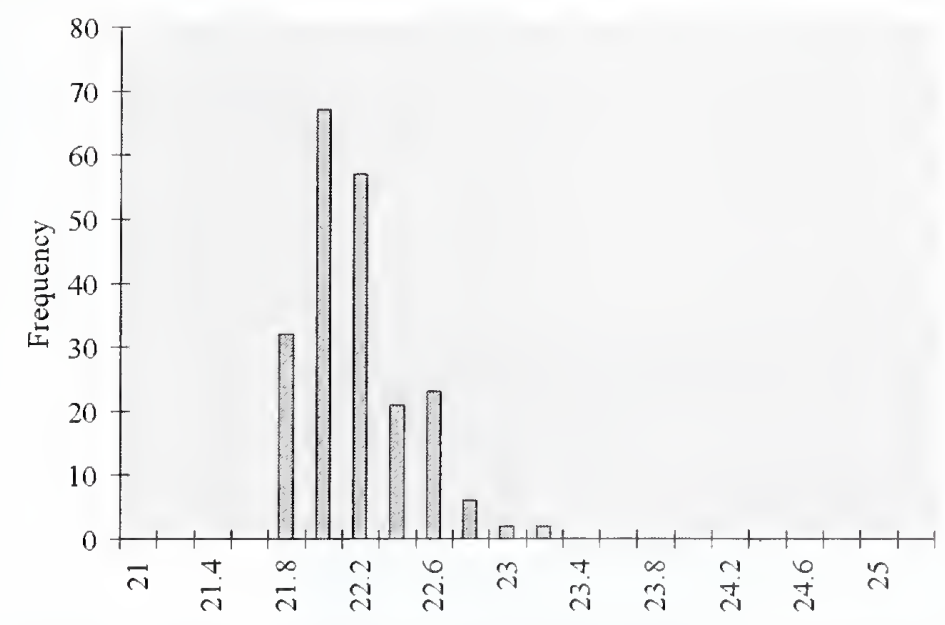

Cage occupied zone average contamination $(\mathrm{kg} / \mathrm{kg})$ distribution

Contamination conversion factors $(\mathrm{kg} / \mathrm{kg} \rightarrow \mathrm{ppm})$

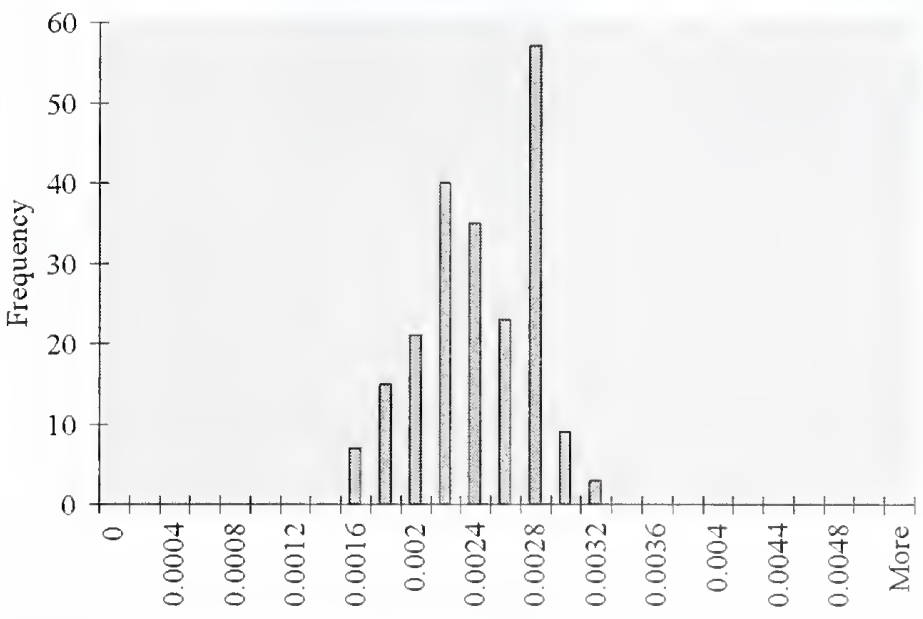

Cage occupied zone average relative humidity (\%) distribution

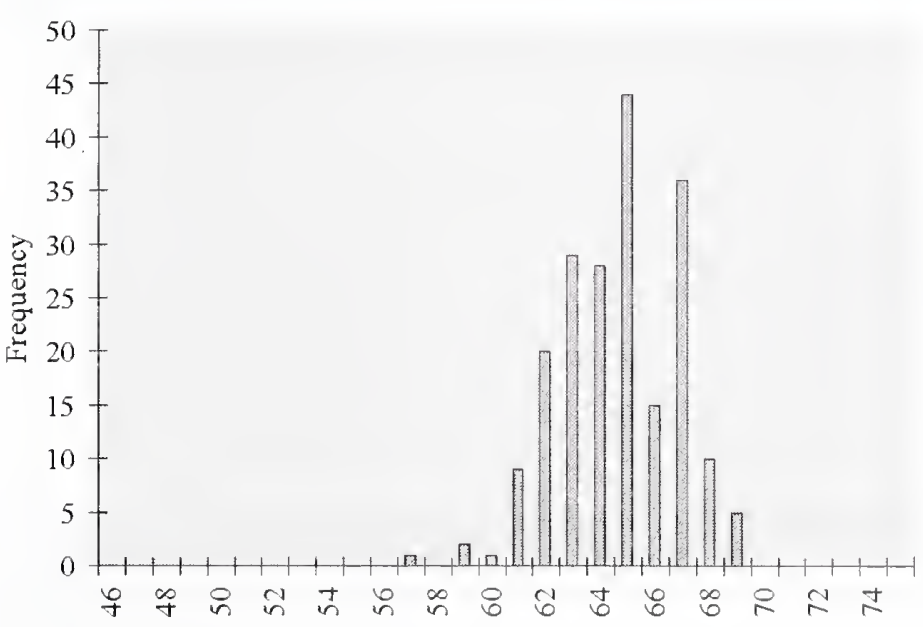


Casename

\section{Case 18}

\section{Description}

\begin{tabular}{|c|c|c|c|c|c|}
\hline Supply & Supply Discharge & Supply Discharge & Exhaust & Exhaust & Exhaust \\
\hline Configuration & Temperature $\left({ }^{\circ} \mathrm{C}\right)$ & RH & Configuration & Temperature $\left({ }^{\circ} \mathrm{C}\right)$ & RH \\
\hline Low Ind & 18.8 & $61 \%$ & Ceiling & 22 & $50 \%$ \\
\hline Change & Rack & Rack & Number of & Total mass of & Room \\
\hline Station & Orientation & Density & Mice in Room & Mice in Room & Pressurisation \\
\hline ON & On wall & Single & 1050 & $21000 \mathrm{gr}$ & pos $100 \mathrm{cfm}$ \\
\hline
\end{tabular}

Roons

ACHI

15

Cage

Condition

Top On

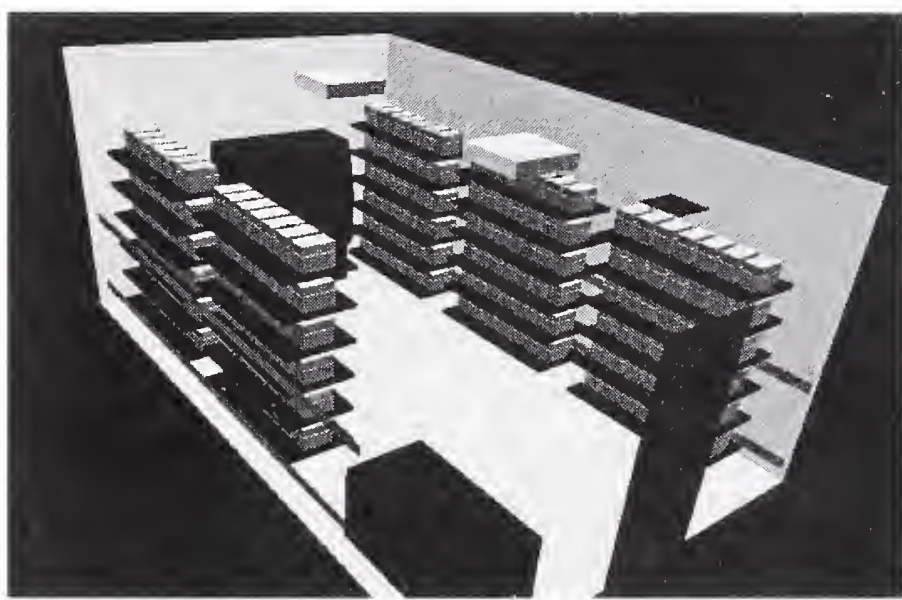

Analysis Results

Cage Occupied Zone

\begin{tabular}{|l|cc|c|c|}
\cline { 2 - 5 } \multicolumn{1}{c|}{} & \multicolumn{2}{c|}{ Temperature } & $\mathrm{CO}_{2}$ & RH \\
\cline { 2 - 5 } \multicolumn{1}{c|}{} & ${ }^{\circ} \mathrm{C}$ & ${ }^{\circ} \mathrm{F}$ & $(\mathrm{ppm})$ & \\
\hline Mean & 22.06 & 71.71 & 1791 & $63.78 \%$ \\
\hline S.D. & 0.29 & 0.52 & 287 & $2.35 \%$ \\
\hline Max. & 23.08 & 73.55 & 2435 & $68.75 \%$ \\
\hline
\end{tabular}

Cage Occupied Zone NH3 (ppm)

\begin{tabular}{|l|c|c|c|c|c|c|c|c|c|c|}
\hline Day & $\mathbf{1}$ & $\mathbf{2}$ & $\mathbf{3}$ & $\mathbf{4}$ & $\mathbf{5}$ & $\mathbf{6}$ & $\mathbf{7}$ & $\mathbf{8}$ & $\mathbf{9}$ & $\mathbf{1 0}$ \\
\hline Mean & 0.94 & 1.81 & 2.79 & 4.62 & 7.19 & 10.39 & 15.07 & 19.30 & 24.10 & 28.27 \\
\hline Max. & 1.28 & 2.47 & 3.80 & 6.28 & 9.78 & 14.12 & 20.48 & 26.24 & 32.77 & 38.44 \\
\hline
\end{tabular}

Room Breathing Zone

\begin{tabular}{|l|cc|c|c|}
\cline { 2 - 5 } \multicolumn{1}{c|}{} & \multicolumn{2}{c|}{ Temperature } & $\mathrm{CO}_{z}$ & RH \\
\cline { 2 - 5 } \multicolumn{1}{c|}{} & ${ }^{\circ} \mathrm{C}$ & ${ }^{\circ} \mathrm{F}$ & $(\mathrm{ppm})$ & \\
\hline Mean & 20.22 & 68.40 & 79 & $56.00 \%$ \\
\hline S.D. & 0.13 & 0.24 & 28 & \\
\hline Max. & 21.35 & 70.43 & 181 & \\
\hline
\end{tabular}

Room Breathing Zone NH3 (ppm)

\begin{tabular}{|l|c|c|c|c|c|c|c|c|c|c|}
\hline Day & $\mathbf{1}$ & $\mathbf{2}$ & $\mathbf{3}$ & $\mathbf{4}$ & $\mathbf{5}$ & $\mathbf{6}$ & $\mathbf{7}$ & $\mathbf{8}$ & $\mathbf{9}$ & $\mathbf{1 0}$ \\
\hline Mean & 0.04 & 0.08 & 0.12 & 0.20 & 0.32 & 0.46 & 0.67 & 0.85 & 1.06 & 1.25 \\
\hline Max. & 0.10 & 0.18 & 0.28 & 0.47 & 0.73 & 1.05 & 1.53 & 1.96 & 2.44 & 2.86 \\
\hline
\end{tabular}




\section{Histogram Distributions}

Cage occupied zone average temperature $\left({ }^{\circ} \mathrm{C}\right)$ distribution

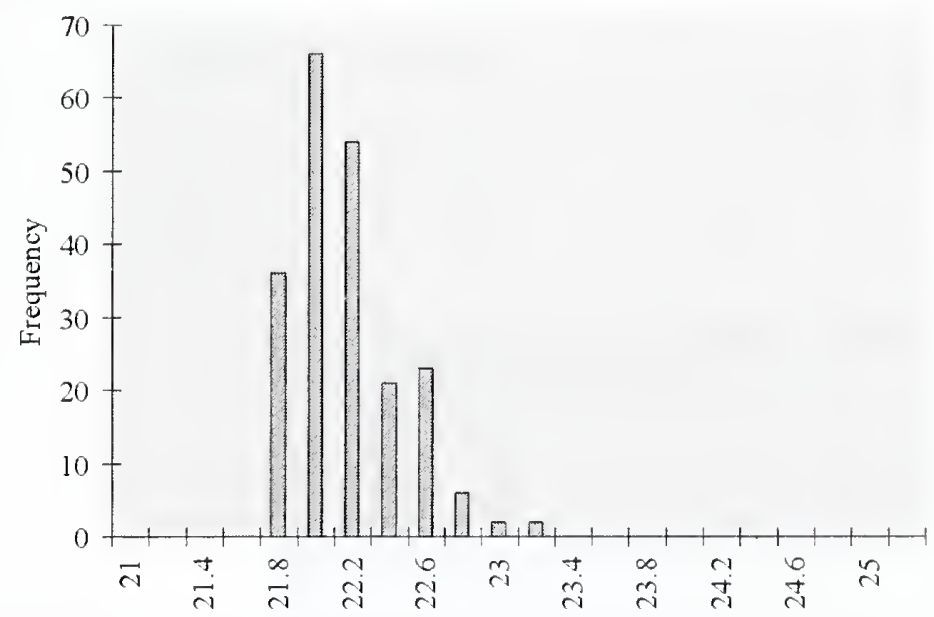

Cage occupied zone average contamination $(\mathrm{kg} / \mathrm{kg})$ distribution

Contamination conversion factors $(\mathrm{kg} / \mathrm{kg} \rightarrow \mathrm{ppm})$

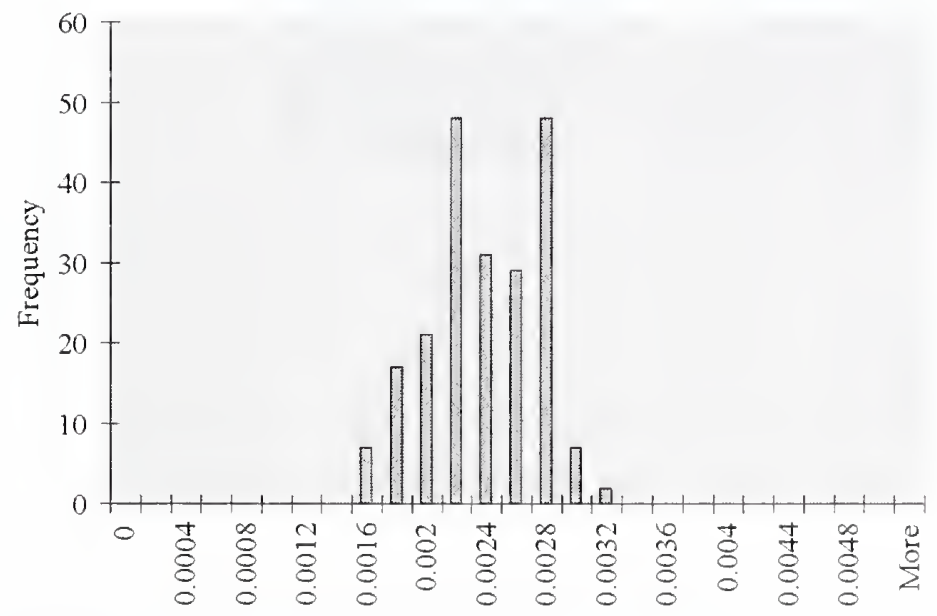

\begin{tabular}{|c|c|c|}
\hline Day & $\mathrm{CO}_{2}$ & $\mathrm{NH}_{3}$ \\
\hline $\mathbf{1}$ & 785000 & 413 \\
\hline $\mathbf{2}$ & 785000 & 795 \\
\hline $\mathbf{3}$ & 785000 & 1225 \\
\hline $\mathbf{4}$ & 785000 & 2024 \\
\hline $\mathbf{5}$ & 785000 & 3152 \\
\hline $\mathbf{6}$ & 785000 & 4552 \\
\hline 7 & 785000 & 6603 \\
\hline $\mathbf{8}$ & 785000 & 8458 \\
\hline $\mathbf{9}$ & 785000 & 10562 \\
\hline $\mathbf{1 0}$ & 785000 & 11384 \\
\hline
\end{tabular}

Cage occupied zone average relative humidity (\%) distribution

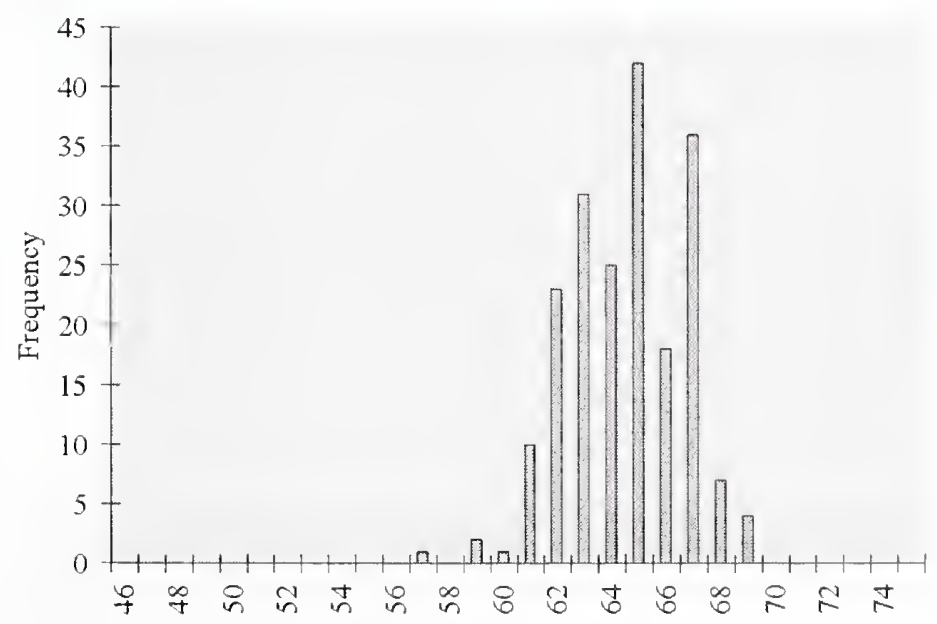


Casename

Case 19

Description

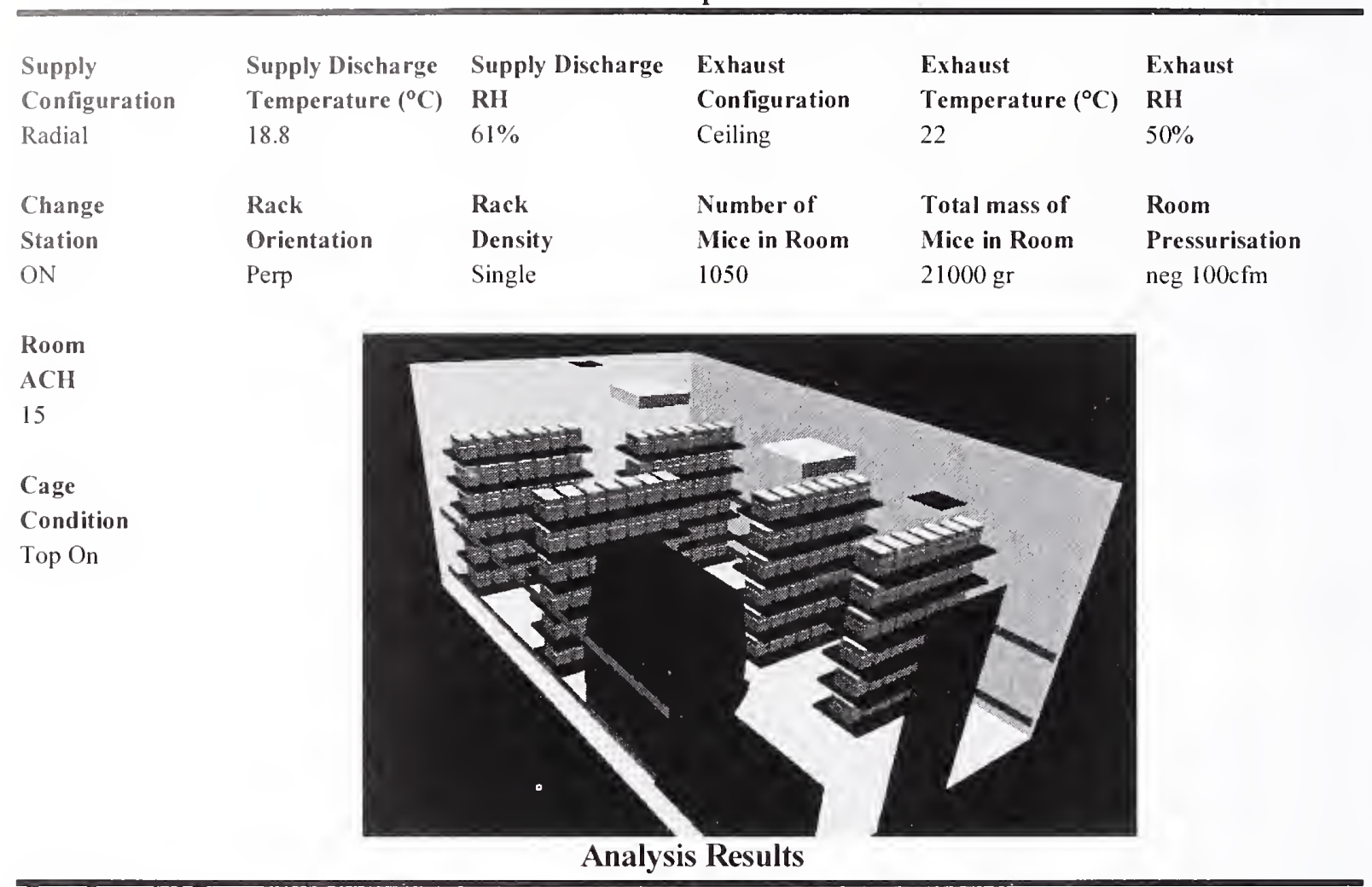

Cage Occupied Zone

\begin{tabular}{|l|cc|c|c|}
\cline { 2 - 5 } \multicolumn{1}{c|}{} & \multicolumn{2}{c|}{ Temperature } & $\mathrm{CO}_{2}$ & RH \\
\cline { 2 - 5 } \multicolumn{1}{c|}{} & ${ }^{\circ} \mathrm{C}$ & ${ }^{\circ} \mathrm{F}$ & $(\mathrm{ppm})$ & \\
\hline Mean & 21.73 & 71.11 & 1725 & $64.59 \%$ \\
\hline S.D. & 0.20 & 0.37 & 346 & $2.80 \%$ \\
\hline Max. & 22.37 & 72.27 & 2353 & $69.85 \%$ \\
\hline
\end{tabular}

Cage Occupied Zone NH3 (ppm)

\begin{tabular}{|l|c|c|c|c|c|c|c|c|c|c|}
\hline Day & $\mathbf{1}$ & $\mathbf{2}$ & $\mathbf{3}$ & $\mathbf{4}$ & $\mathbf{5}$ & $\mathbf{6}$ & $\mathbf{7}$ & $\mathbf{8}$ & $\mathbf{9}$ & $\mathbf{1 0}$ \\
\hline Mean & 0.91 & 1.75 & 2.69 & 4.61 & 7.36 & 10.72 & 15.74 & 19.95 & 24.59 & 28.11 \\
\hline Max. & 1.24 & 2.38 & 3.67 & 6.29 & 10.04 & 14.63 & 21.46 & 27.21 & 33.53 & 38.34 \\
\hline
\end{tabular}

Room Breathing Zone

\begin{tabular}{|l|cc|c|c|}
\cline { 2 - 5 } \multicolumn{1}{c|}{} & \multicolumn{2}{c|}{ Temperature } & $\mathrm{CO}_{2}$ & RH \\
\cline { 2 - 5 } \multicolumn{1}{c|}{} & ${ }^{\circ} \mathrm{C}$ & ${ }^{\circ} \mathrm{F}$ & $(\mathrm{ppm})$ & \\
\hline Mean & 20.20 & 68.36 & 59 & $55.90 \%$ \\
\hline S.D. & 0.57 & 1.03 & 20 & \\
\hline Max. & 26.19 & 79.14 & 222 & \\
\hline
\end{tabular}

Room Breathing Zone NH3 (ppm)

\begin{tabular}{|l|c|c|c|c|c|c|c|c|c|c|}
\hline Day & $\mathbf{1}$ & $\mathbf{2}$ & $\mathbf{3}$ & $\mathbf{4}$ & $\mathbf{5}$ & $\mathbf{6}$ & $\mathbf{7}$ & $\mathbf{8}$ & $\mathbf{9}$ & $\mathbf{1 0}$ \\
\hline Mean & 0.03 & 0.06 & 0.09 & 0.16 & 0.25 & 0.36 & 0.54 & 0.68 & 0.84 & 0.96 \\
\hline Max. & 0.12 & 0.22 & 0.35 & 0.59 & 0.95 & 1.38 & 2.03 & 2.57 & 3.16 & 3.62 \\
\hline
\end{tabular}




\section{Histogram Distributions}

Cage occupied zone average temperature $\left({ }^{\circ} \mathrm{C}\right)$ distribution

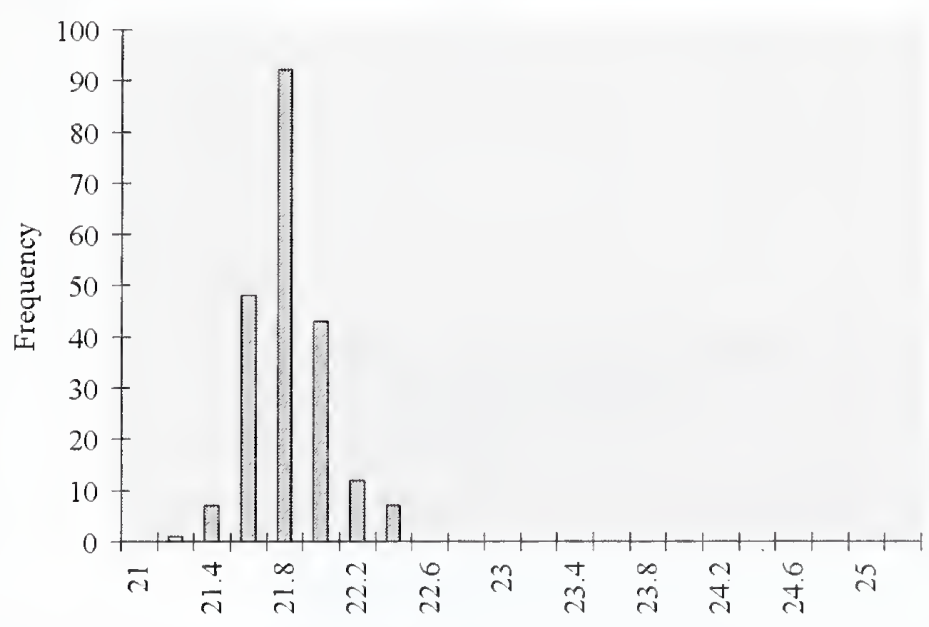

Cage occupied zone average contamination $(\mathrm{kg} / \mathrm{kg})$ distribution

Contamination conversion factors $(\mathrm{kg} / \mathrm{kg} \rightarrow \mathrm{ppm})$

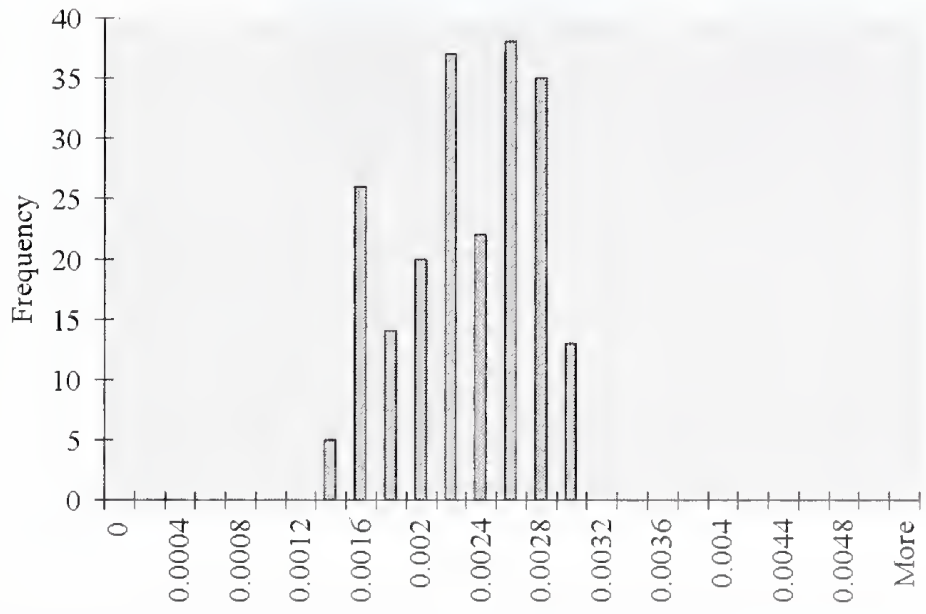

\begin{tabular}{|c|c|c|}
\hline Day & $\mathrm{CO}_{2}$ & $\mathrm{NH}_{3}$ \\
\hline 1 & 785000 & 415 \\
\hline 2 & 785000 & 795 \\
\hline 3 & 785000 & 1225 \\
\hline 4 & 785000 & 2100 \\
\hline 5 & 785000 & 3349 \\
\hline 6 & 785000 & 4879 \\
\hline 7 & 785000 & 7160 \\
\hline 8 & 785000 & 9078 \\
\hline 9 & 785000 & 11186 \\
\hline 10 & 785000 & 11384 \\
\hline
\end{tabular}

Cage occupied zone average relative humidity (\%) distribution

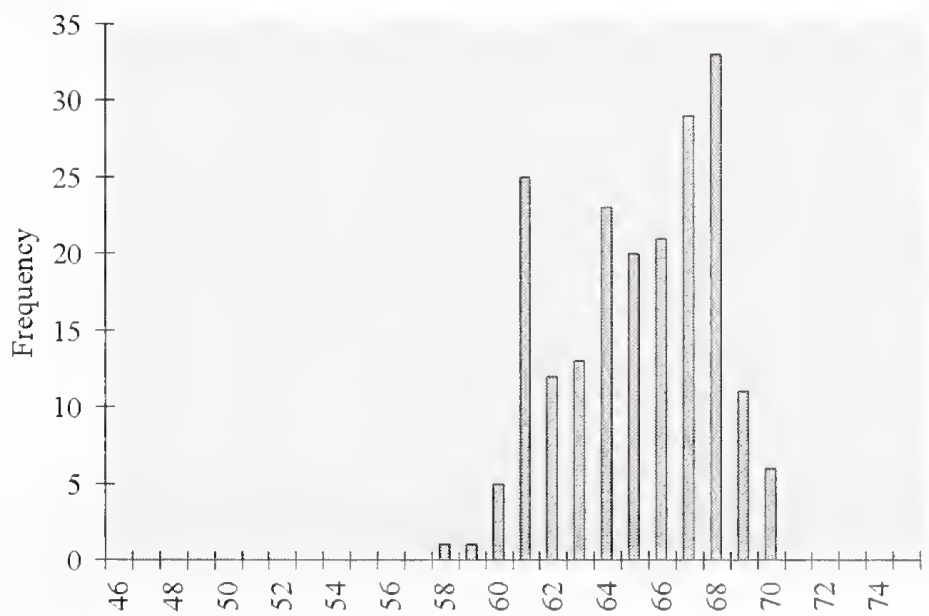


Casename

Case 20

Description

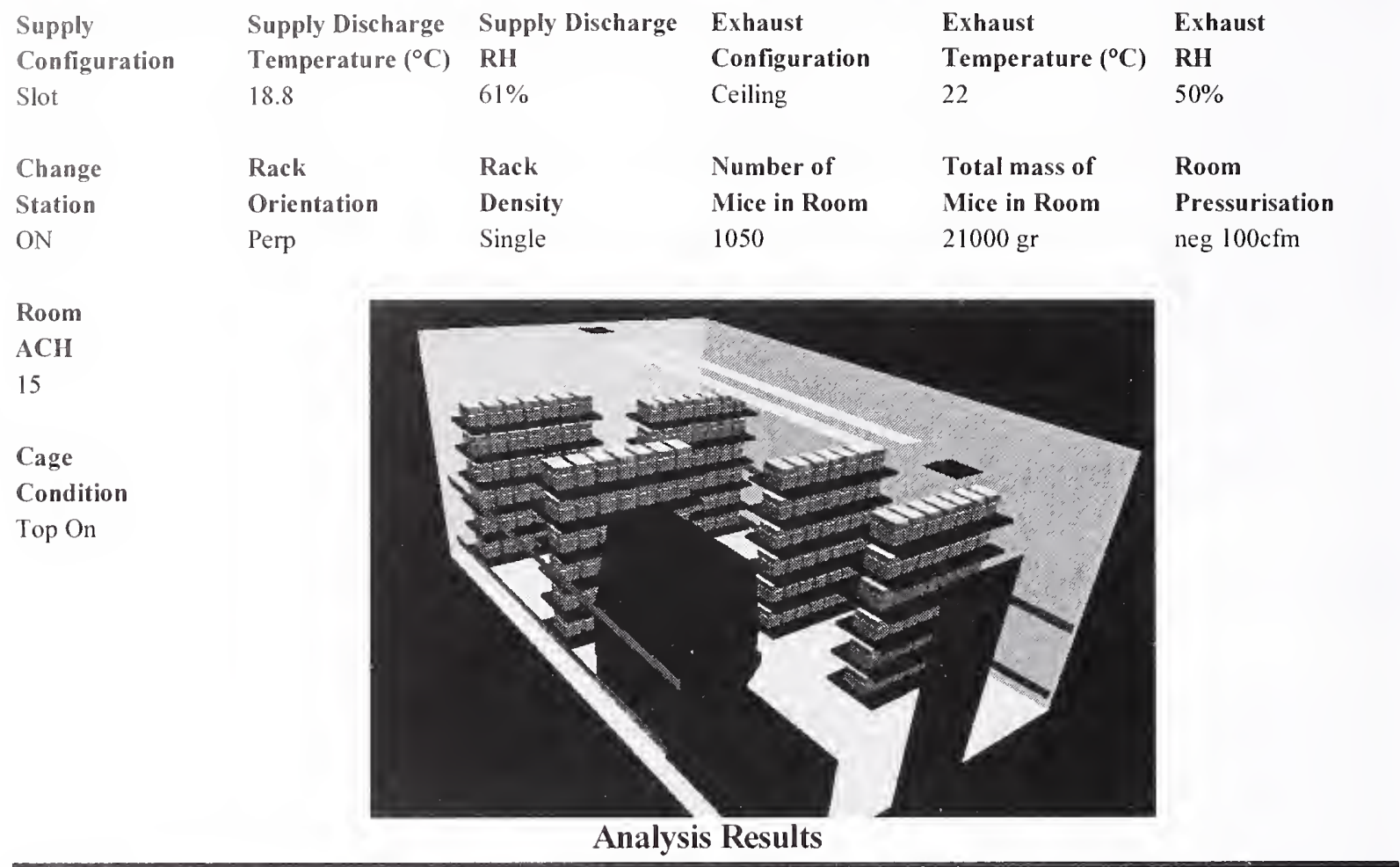

Cage Occupied Zone

\begin{tabular}{|l|cc|c|c|}
\cline { 2 - 5 } \multicolumn{1}{c|}{} & \multicolumn{2}{c|}{ Temperature } & $\mathrm{CO}_{2}$ & RH \\
\cline { 2 - 5 } \multicolumn{1}{c|}{} & ${ }^{\circ} \mathrm{C}$ & ${ }^{\circ} \mathrm{F}$ & $(\mathrm{ppm})$ & \\
\hline Mean & 22.08 & 71.74 & 1656 & $62.61 \%$ \\
\hline S.D. & 0.29 & 0.52 & 296 & $2.07 \%$ \\
\hline Max. & 22.72 & 72.89 & 2352 & $67.60 \%$ \\
\hline
\end{tabular}

Cage Occupied Zone NH3 (ppm)

\begin{tabular}{|l|c|c|c|c|c|c|c|c|c|c|}
\hline Day & $\mathbf{1}$ & $\mathbf{2}$ & $\mathbf{3}$ & $\mathbf{4}$ & $\mathbf{5}$ & $\mathbf{6}$ & $\mathbf{7}$ & $\mathbf{8}$ & $\mathbf{9}$ & $\mathbf{1 0}$ \\
\hline Mean & 0.87 & 1.68 & 2.58 & 4.04 & 6.06 & 8.62 & 12.26 & 15.98 & 20.40 & 24.94 \\
\hline Max. & 1.23 & 2.38 & 3.67 & 5.74 & 8.60 & 12.25 & 17.41 & 22.70 & 28.98 & 35.42 \\
\hline
\end{tabular}

Room Breathing Zone

\begin{tabular}{|l|cc|c|c|}
\cline { 2 - 5 } \multicolumn{1}{c|}{} & \multicolumn{2}{c|}{ Temperature } & $\mathrm{CO}_{2}$ & RI \\
\cline { 2 - 5 } \multicolumn{1}{c|}{} & ${ }^{\circ} \mathrm{C}$ & ${ }^{\circ} \mathrm{F}$ & $(\mathrm{ppm})$ & \\
\hline Mean & 20.56 & 69.00 & 75 & $54.79 \%$ \\
\hline S.D. & 0.58 & 1.05 & 28 & \\
\hline Max. & 27.19 & 80.94 & 174 & \\
\hline
\end{tabular}

Room Breathing Zone NH3 (ppm)

\begin{tabular}{|l|c|c|c|c|c|c|c|c|c|c|}
\hline Day & $\mathbf{1}$ & $\mathbf{2}$ & $\mathbf{3}$ & $\mathbf{4}$ & $\mathbf{5}$ & $\mathbf{6}$ & $\mathbf{7}$ & $\mathbf{8}$ & $\mathbf{9}$ & $\mathbf{1 0}$ \\
\hline Mean & 0.04 & 0.08 & 0.12 & 0.18 & 0.27 & 0.39 & 0.55 & 0.72 & 0.92 & 1.13 \\
\hline Max. & 0.09 & 0.18 & 0.27 & 0.43 & 0.64 & 0.91 & 1.29 & 1.68 & 2.15 & 2.62 \\
\hline
\end{tabular}




\section{Histogram Distributions}

Cage occupied zone average temperature $\left({ }^{\circ} \mathrm{C}\right)$ distribution

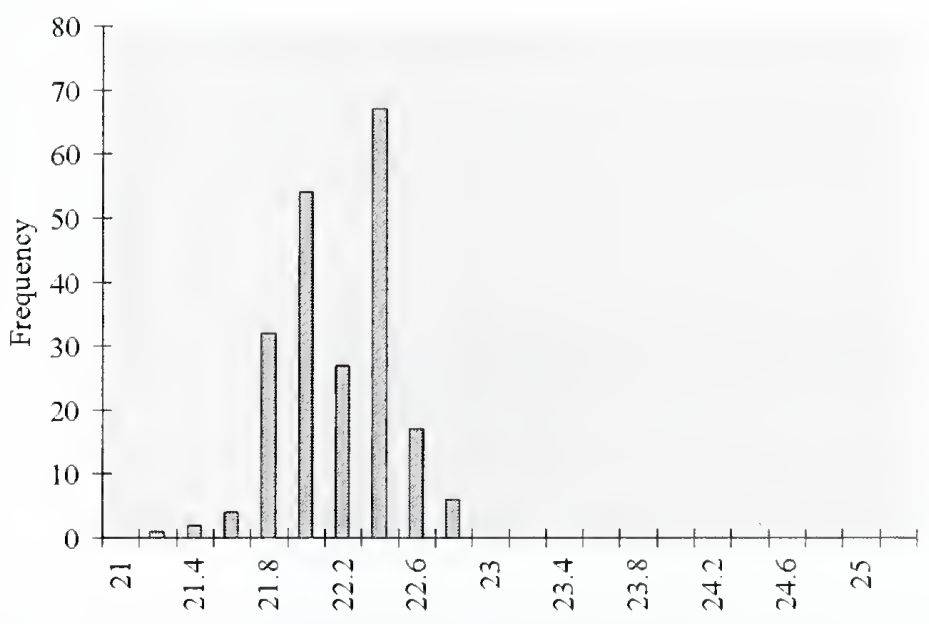

Cage occupied zone average contamination $(\mathrm{kg} / \mathrm{kg})$ distribution

Contamination conversion factors $(\mathrm{kg} / \mathrm{kg} \rightarrow \mathrm{ppm})$

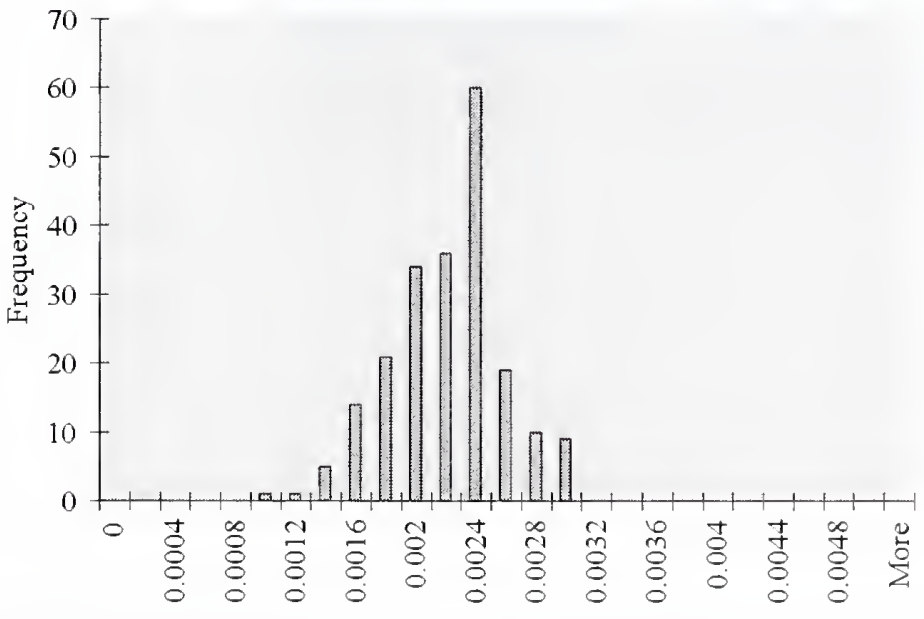

\begin{tabular}{|c|c|c|}
\hline Day & $\mathbf{C O}_{2}$ & $\mathbf{N H}_{3}$ \\
\hline $\mathbf{1}$ & 785000 & 411 \\
\hline $\mathbf{2}$ & 785000 & 795 \\
\hline $\mathbf{3}$ & 785000 & 1225 \\
\hline 4 & 785000 & 1915 \\
\hline $\mathbf{5}$ & 785000 & 2871 \\
\hline $\mathbf{6}$ & 785000 & 4087 \\
\hline $\mathbf{7}$ & 785000 & 5810 \\
\hline $\mathbf{8}$ & 785000 & 7576 \\
\hline $\mathbf{9}$ & 785000 & 9672 \\
\hline $\mathbf{1 0}$ & 785000 & 11384 \\
\hline
\end{tabular}

Cage occupied zone average relative humidity (\%) distribution

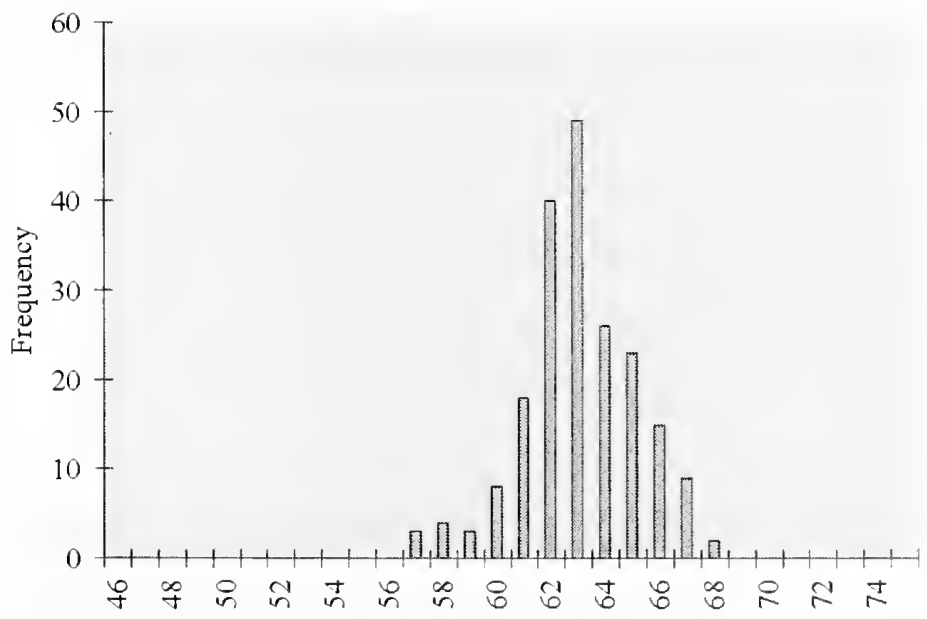


Casename

Case 21

Description

\begin{tabular}{|c|c|c|c|c|c|}
\hline Supply & Supply Discharge & Supply Discharge & Exhaust & Exhaust & Exhaust \\
\hline Configuration & Temperature $\left({ }^{\circ} \mathrm{C}\right)$ & RH & Configuration & Temperature $\left({ }^{\circ} \mathrm{C}\right)$ & $\mathbf{R H}$ \\
\hline Low lnd & 18.8 & $61 \%$ & Ceiling & 22 & $50 \%$ \\
\hline Change & Rack & Rack & Number of & Total mass of & Room \\
\hline Station & Orientation & Density & Mice in Room & Mice in Room & Pressurisatior \\
\hline ON & Perp & Single & 1050 & $21000 \mathrm{gr}$ & neg $100 \mathrm{cfm}$ \\
\hline
\end{tabular}

\section{Room}

$\mathrm{ACH}$

15

Cage

Condition

Top On

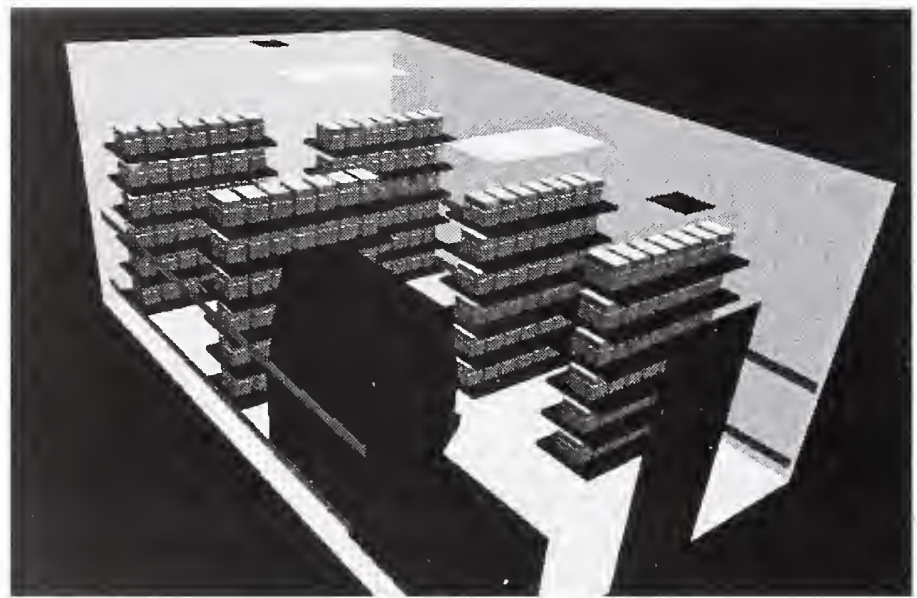

Analysis Results

Cage Occupied Zone

\begin{tabular}{|l|cc|c|c|}
\cline { 2 - 5 } \multicolumn{1}{c|}{} & \multicolumn{2}{c|}{ Temperature } & $\mathrm{CO}_{2}$ & RH \\
\cline { 2 - 5 } \multicolumn{1}{c|}{} & ${ }^{\circ} \mathrm{C}$ & ${ }^{\circ} \mathrm{F}$ & $(\mathbf{p p m})$ & \\
\hline Mean & 22.01 & 71.62 & 1808 & $64.10 \%$ \\
\hline S.D. & 0.23 & 0.42 & 309 & $2.32 \%$ \\
\hline Max. & 22.60 & 72.68 & 2555 & $69.39 \%$ \\
\hline
\end{tabular}

Cage Occupied Zone NH3 (ppm)

\begin{tabular}{|l|c|c|c|c|c|c|c|c|c|c|}
\hline Day & $\mathbf{1}$ & $\mathbf{2}$ & $\mathbf{3}$ & $\mathbf{4}$ & $\mathbf{5}$ & $\mathbf{6}$ & $\mathbf{7}$ & $\mathbf{8}$ & $\mathbf{9}$ & $\mathbf{1 0}$ \\
\hline Mean & 0.95 & $\mathbf{1 . 8 3}$ & 2.82 & 4.73 & 7.44 & 10.79 & 15.72 & 20.05 & 24.90 & 28.90 \\
\hline Max. & 1.35 & 2.59 & 3.99 & 6.69 & 10.52 & 15.24 & 22.22 & 28.34 & 35.20 & 40.85 \\
\hline
\end{tabular}

Room Breathing Zone

\begin{tabular}{|l|cc|c|c|}
\cline { 2 - 5 } \multicolumn{1}{c|}{} & \multicolumn{2}{c|}{ Temperature } & $\mathrm{CO}_{2}$ & RH \\
\cline { 2 - 5 } \multicolumn{1}{c|}{} & ${ }^{\circ} \mathrm{C}$ & ${ }^{\circ} \mathrm{F}$ & $(\mathrm{ppm})$ & \\
\hline Mean & 20.40 & 68.71 & 62 & $55.24 \%$ \\
\hline S.D. & 0.60 & 1.08 & 24 & \\
\hline Max. & 27.06 & 80.71 & 186 & \\
\hline
\end{tabular}

Room Breathing Zone NH3 (ppm)

\begin{tabular}{|l|c|c|c|c|c|c|c|c|c|c|}
\hline Day & 1 & 2 & 3 & 4 & 5 & 6 & 7 & 8 & 9 & 10 \\
\hline Mean & 0.03 & 0.06 & 0.10 & 0.16 & 0.26 & 0.37 & 0.54 & 0.69 & 0.85 & 0.99 \\
\hline Max. & 0.10 & 0.19 & 0.29 & 0.49 & 0.77 & 1.11 & 1.62 & 2.06 & 2.56 & 2.98 \\
\hline
\end{tabular}




\section{Histogram Distributions}

Cage occupied zone average temperature $\left({ }^{\circ} \mathrm{C}\right)$ distribution

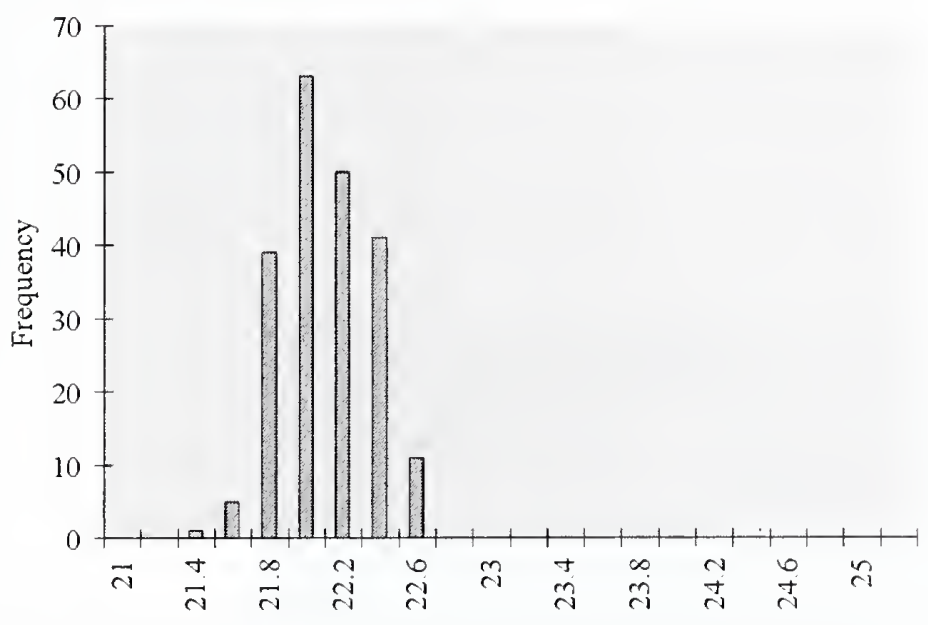

Cage occupied zone average contamination $(\mathrm{kg} / \mathrm{kg})$ distribution

Contamination conversion factors $(\mathrm{kg} / \mathrm{kg} \rightarrow \mathrm{ppm})$

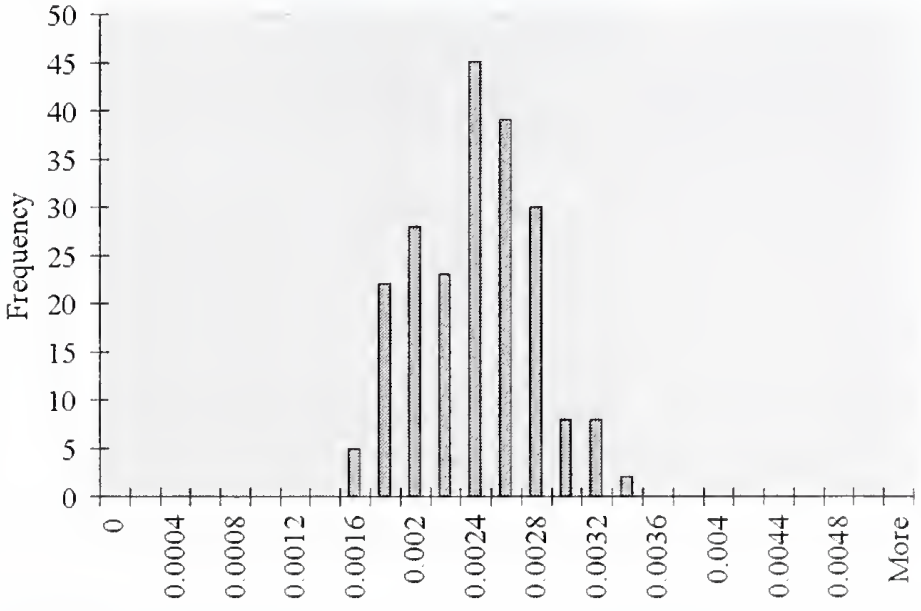

\begin{tabular}{|c|c|c|}
\hline Day & $\mathbf{C O}_{2}$ & $\mathbf{N H}_{3}$ \\
\hline $\mathbf{1}$ & 785000 & 414 \\
\hline $\mathbf{2}$ & 785000 & 795 \\
\hline $\mathbf{3}$ & 785000 & 1225 \\
\hline $\mathbf{4}$ & 785000 & 2054 \\
\hline $\mathbf{5}$ & 785000 & 3231 \\
\hline $\mathbf{6}$ & 785000 & 4683 \\
\hline $\mathbf{7}$ & 785000 & 6826 \\
\hline $\mathbf{8}$ & 785000 & 8706 \\
\hline $\mathbf{9}$ & 785000 & 10811 \\
\hline $\mathbf{1 0}$ & 785000 & 11384 \\
\hline
\end{tabular}

Cage occupied zone average relative humidity (\%) distribution

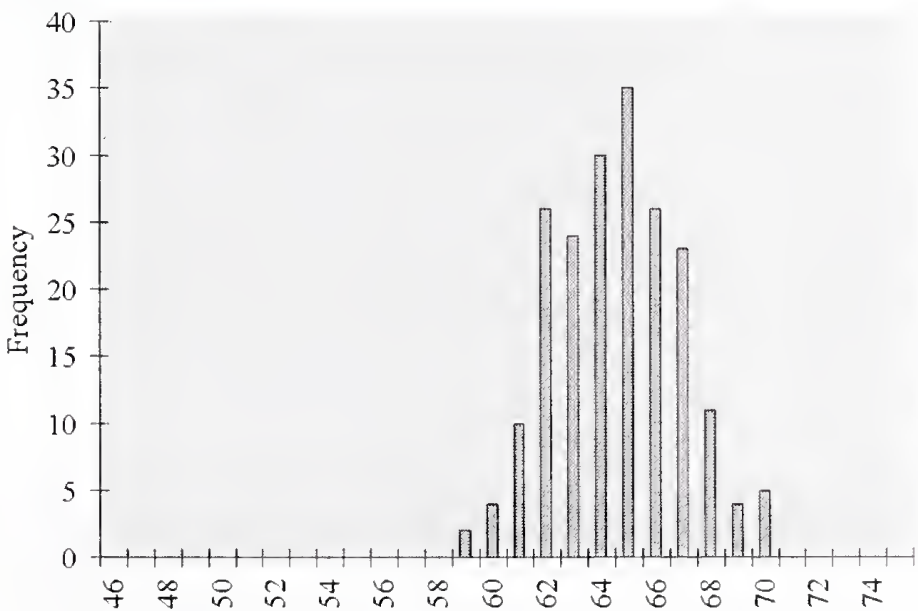


Casename

Case 22

\section{Description}

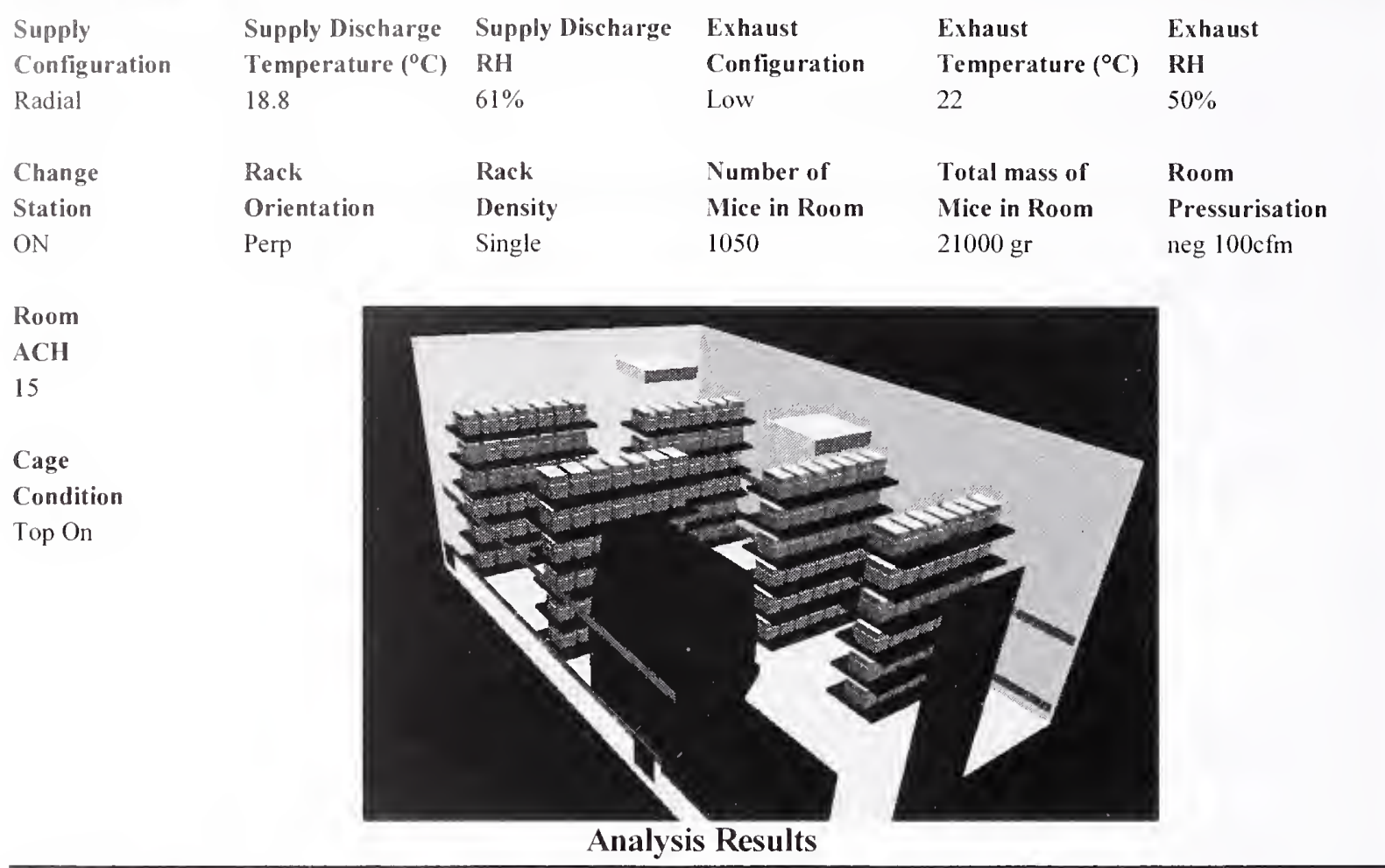

Cage Occupied Zone

\begin{tabular}{|l|cc|c|c|}
\cline { 2 - 5 } \multicolumn{1}{c|}{} & \multicolumn{2}{c|}{ Temperature } & $\mathrm{CO}_{2}$ & RIH \\
\cline { 2 - 5 } \multicolumn{1}{c|}{} & ${ }^{\circ} \mathrm{C}$ & ${ }^{\circ} \mathrm{F}$ & $(\mathbf{p p m})$ & \\
\hline Mean & 22.47 & 72.45 & 1665 & $61.14 \%$ \\
\hline S.D. & 0.34 & 0.62 & 446 & $3.32 \%$ \\
\hline Max. & 23.41 & 74.13 & 2623 & $67.76 \%$ \\
\hline
\end{tabular}

Cage Occupied Zone NH3 (ppm)

\begin{tabular}{|l|c|c|c|c|c|c|c|c|c|c|}
\hline Day & $\mathbf{1}$ & $\mathbf{2}$ & $\mathbf{3}$ & $\mathbf{4}$ & $\mathbf{5}$ & $\mathbf{6}$ & $\mathbf{7}$ & $\mathbf{8}$ & $\mathbf{9}$ & $\mathbf{1 0}$ \\
\hline Mean & 0.87 & 1.69 & 2.60 & 3.77 & 5.34 & 7.42 & 10.20 & 13.71 & 18.13 & 23.55 \\
\hline Max. & 1.37 & 2.66 & 4.09 & 5.94 & 8.41 & 11.69 & 16.06 & 21.59 & 28.57 & 37.11 \\
\hline
\end{tabular}

Room Breathing Zone

\begin{tabular}{|l|cc|c|c|}
\cline { 2 - 5 } \multicolumn{1}{c|}{} & \multicolumn{2}{c|}{ Temperature } & $\mathrm{CO}_{2}$ & RH \\
\cline { 2 - 5 } \multicolumn{1}{c|}{} & ${ }^{\circ} \mathrm{C}$ & ${ }^{\circ} \mathrm{F}$ & $(\mathrm{ppm})$ & \\
\hline Mean & 21.24 & 70.24 & 61 & $52.34 \%$ \\
\hline S.D. & 0.71 & 1.27 & 28 & \\
\hline Max. & 26.82 & 80.28 & 217 & \\
\hline
\end{tabular}

Room Breathing Zone NH3 (ppm)

\begin{tabular}{|l|c|c|c|c|c|c|c|c|c|c|}
\hline Day & $\mathbf{1}$ & $\mathbf{2}$ & $\mathbf{3}$ & $\mathbf{4}$ & $\mathbf{5}$ & $\mathbf{6}$ & $\mathbf{7}$ & $\mathbf{8}$ & $\mathbf{9}$ & $\mathbf{1 0}$ \\
\hline Mean & 0.03 & 0.06 & 0.10 & 0.14 & 0.20 & 0.27 & 0.37 & 0.50 & 0.66 & 0.86 \\
\hline Max. & 0.11 & 0.22 & 0.34 & 0.49 & 0.69 & 0.97 & 1.33 & 1.78 & 2.36 & 3.06 \\
\hline
\end{tabular}




\section{Histogram Distributions}

Cage occupied zone average temperature $\left({ }^{\circ} \mathrm{C}\right)$ distribution

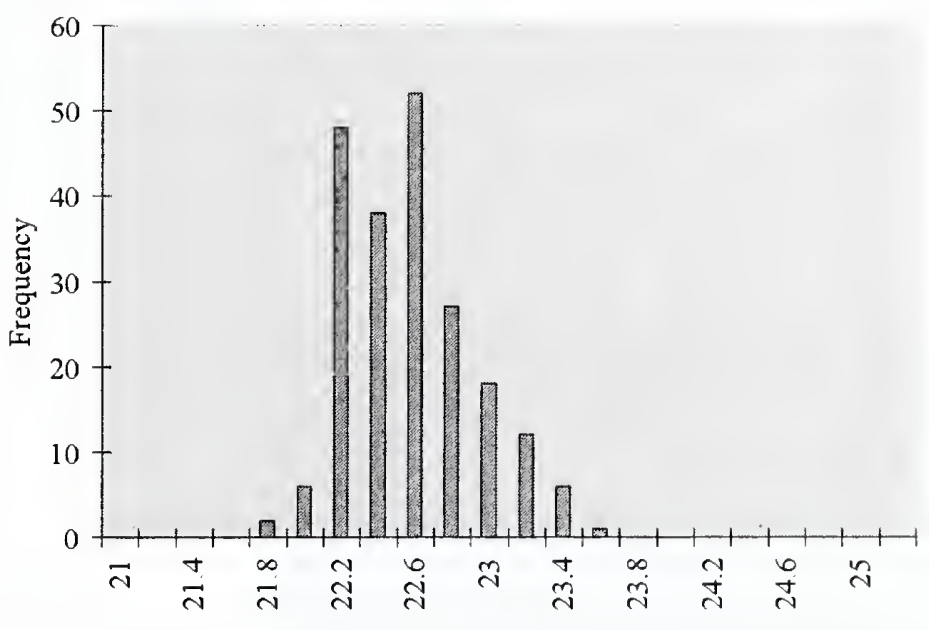

Cage occupied zone average contamination $(\mathrm{kg} / \mathrm{kg})$ distribution

Contamination conversion factors $(\mathrm{kg} / \mathrm{kg} \rightarrow \mathrm{ppm})$

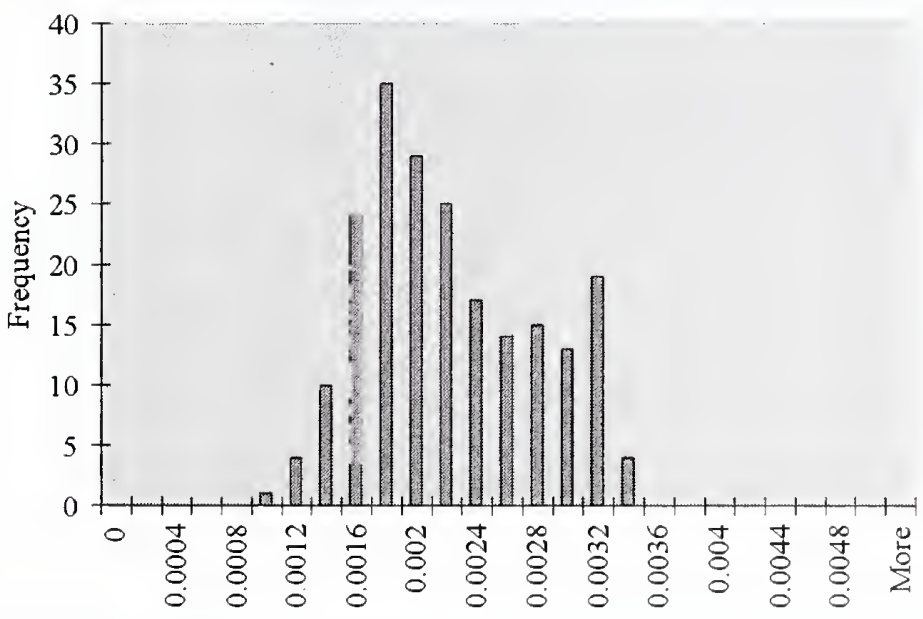

\begin{tabular}{|c|c|c|}
\hline Day & $\mathrm{CO}_{2}$ & $\mathrm{NH}_{3}$ \\
\hline $\mathbf{1}$ & 785000 & 409 \\
\hline 2 & 785000 & 795 \\
\hline 3 & 785000 & 1225 \\
\hline 4 & 785000 & 1779 \\
\hline 5 & 785000 & 2516 \\
\hline 6 & 785000 & 3498 \\
\hline 7 & 785000 & 4807 \\
\hline 8 & 785000 & 6461 \\
\hline 9 & 785000 & 8548 \\
\hline 10 & 785000 & 11384 \\
\hline
\end{tabular}

Cage occupied zone average relative humidity (\%) distribution

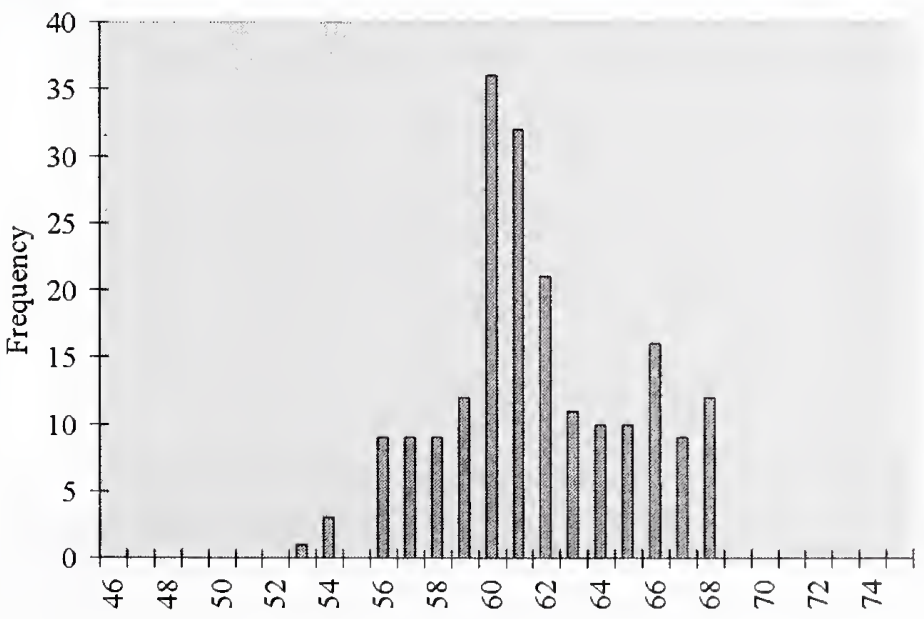


Casename

Case 23

Description

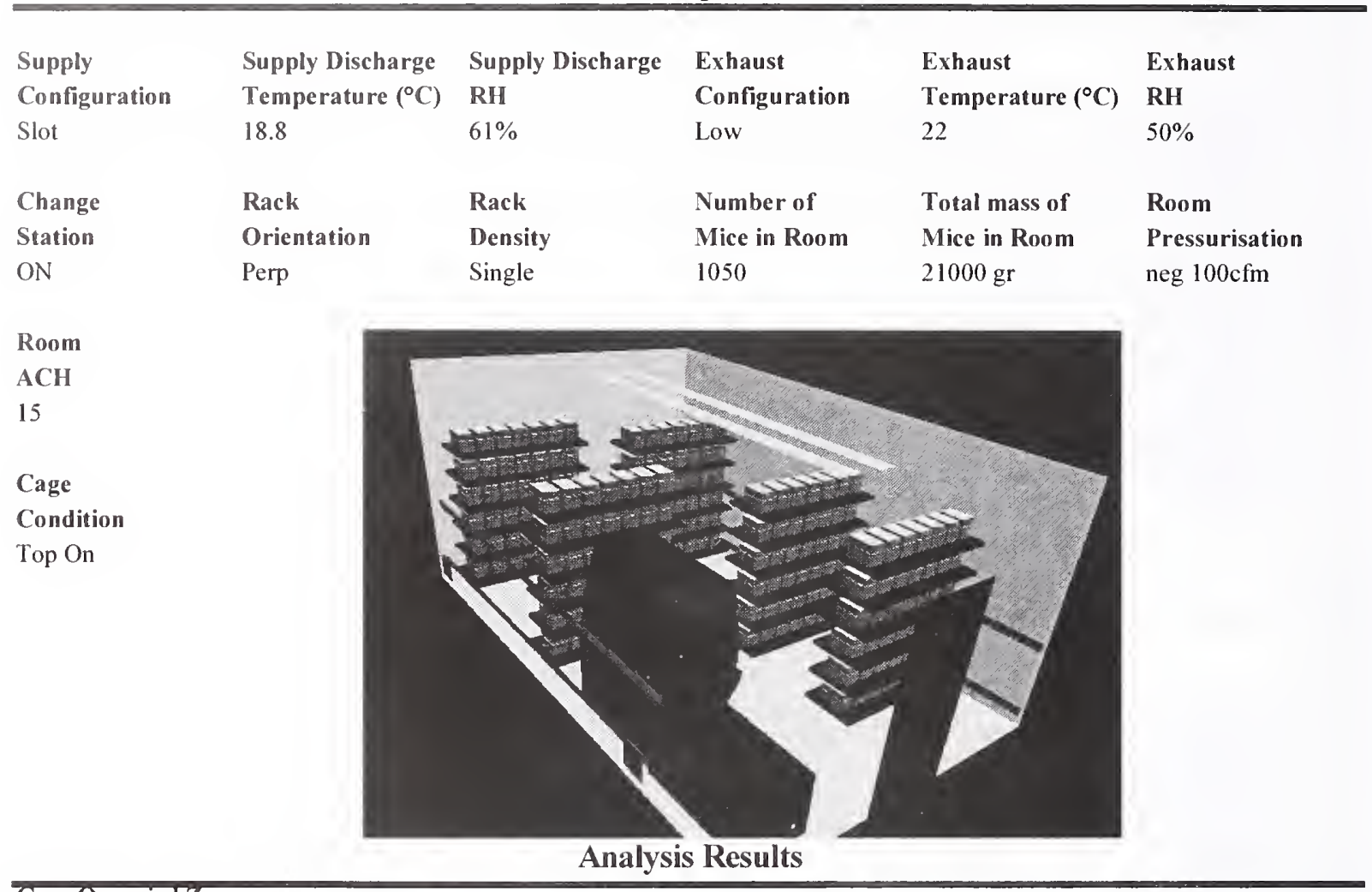

Cage Occupied Zone

\begin{tabular}{|l|cc|c|c|}
\cline { 2 - 5 } \multicolumn{1}{c|}{} & \multicolumn{2}{c|}{ Temperature } & $\mathrm{CO}_{2}$ & RH \\
\cline { 2 - 5 } \multicolumn{1}{c|}{} & ${ }^{\circ} \mathrm{C}$ & ${ }^{\circ} \mathrm{F}$ & $(\mathrm{ppm})$ & \\
\hline Mean & 22.70 & 72.86 & 1652 & $60.16 \%$ \\
\hline S.D. & 0.47 & 0.85 & 375 & $2.57 \%$ \\
\hline Max. & 23.54 & 74.38 & 2552 & $66.04 \%$ \\
\hline
\end{tabular}

Cage Occupied Zone NH3 (ppm)

\begin{tabular}{|l|c|c|c|c|c|c|c|c|c|c|}
\hline Day & $\mathbf{1}$ & $\mathbf{2}$ & $\mathbf{3}$ & $\mathbf{4}$ & $\mathbf{5}$ & $\mathbf{6}$ & $\mathbf{7}$ & $\mathbf{8}$ & $\mathbf{9}$ & $\mathbf{1 0}$ \\
\hline Mean & 0.86 & 1.67 & 2.58 & 3.71 & 5.22 & 7.24 & 9.91 & 13.37 & 17.76 & 23.22 \\
\hline Max. & 1.33 & 2.58 & 3.98 & 5.74 & 8.07 & 11.19 & 15.32 & 20.67 & 27.45 & 35.88 \\
\hline
\end{tabular}

Room Breathing Zone

\begin{tabular}{|l|cc|c|c|}
\cline { 2 - 5 } \multicolumn{1}{c|}{} & \multicolumn{2}{c|}{ Temperature } & $\mathrm{CO}_{2}$ & RH \\
\cline { 2 - 5 } \multicolumn{1}{c|}{} & ${ }^{\circ} \mathrm{C}$ & ${ }^{\circ} \mathrm{F}$ & $(\mathrm{ppm})$ & \\
\hline Mean & 21.76 & 71.17 & 63 & $50.67 \%$ \\
\hline S.D. & 0.64 & 1.15 & 26 & \\
\hline Max. & 27.45 & 81.41 & 181 & \\
\hline
\end{tabular}

Room Breathing Zone NH33 (ppm)

\begin{tabular}{|l|c|c|c|c|c|c|c|c|c|c|}
\hline Day & $\mathbf{1}$ & $\mathbf{2}$ & $\mathbf{3}$ & $\mathbf{4}$ & $\mathbf{5}$ & $\mathbf{6}$ & $\mathbf{7}$ & $\mathbf{8}$ & $\mathbf{9}$ & $\mathbf{1 0}$ \\
\hline Mean & 0.03 & 0.06 & 0.10 & 0.14 & 0.20 & 0.28 & 0.38 & 0.51 & 0.68 & 0.89 \\
\hline Max. & 0.09 & 0.18 & 0.28 & 0.41 & 0.57 & 0.79 & 1.08 & 1.46 & 1.94 & 2.54 \\
\hline
\end{tabular}




\section{Histogram Distributions}

Cage occupied zone average temperature $\left({ }^{\circ} \mathrm{C}\right)$ distribution

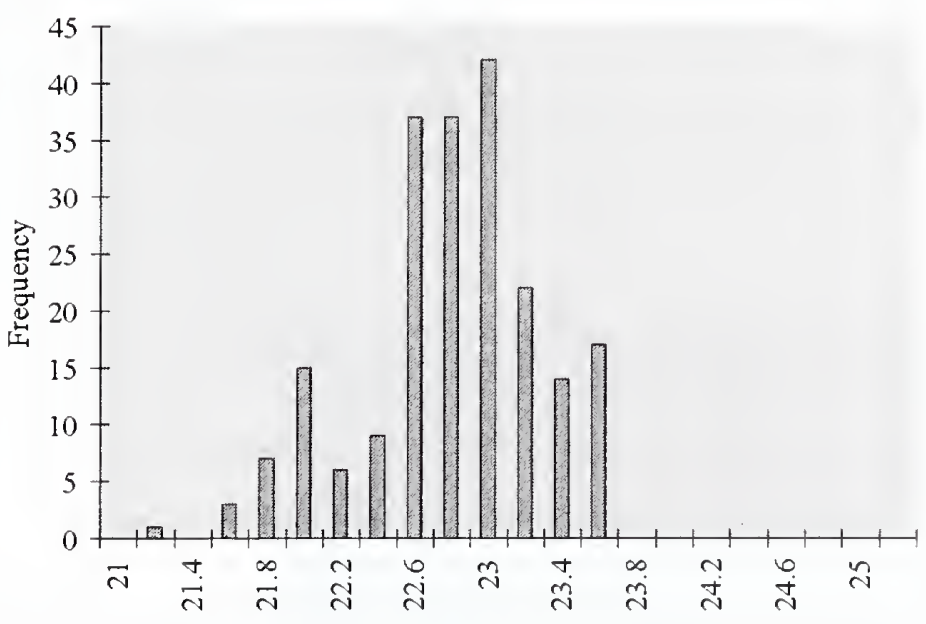

Cage occupied zone average contamination $(\mathrm{kg} / \mathrm{kg})$ distribution

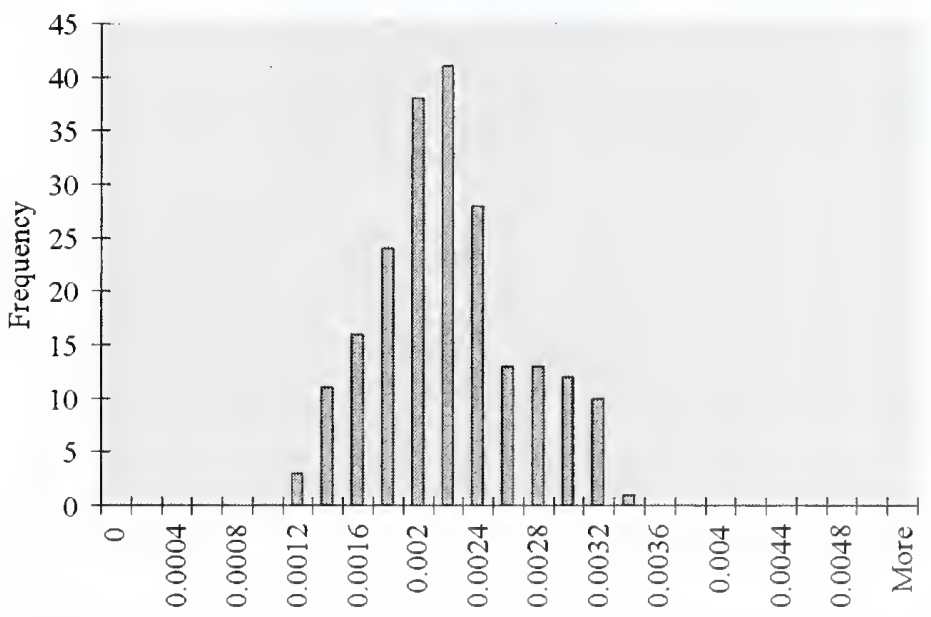

Cage occupied zone average relative humidity (\%) distribution

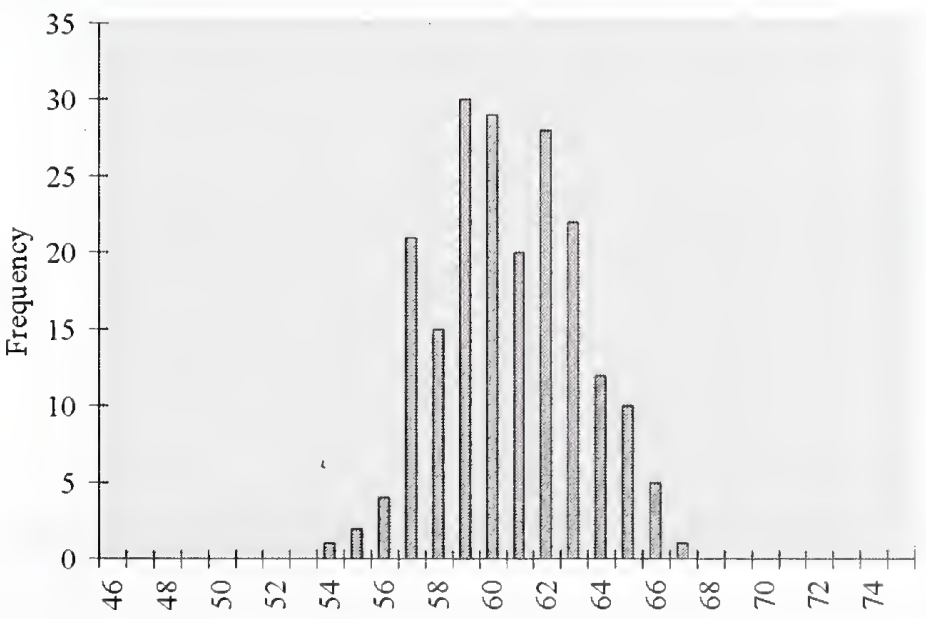

Contamination conversion factors $(\mathrm{kg} / \mathrm{kg} \rightarrow \mathrm{ppm})$

\begin{tabular}{|c|c|c|}
\hline Day & $\mathrm{CO}_{2}$ & $\mathrm{NH}_{3}$ \\
\hline 1 & 785000 & 408 \\
\hline 2 & 785000 & 795 \\
\hline 3 & 785000 & 1225 \\
\hline 4 & 785000 & 1766 \\
\hline 5 & 785000 & 2483 \\
\hline 6 & 785000 & 3443 \\
\hline 7 & 785000 & 4712 \\
\hline 8 & 785000 & 6356 \\
\hline 9 & 785000 & 8442 \\
\hline 10 & 785000 & 11384 \\
\hline
\end{tabular}


Casename Case 24

Description

\begin{tabular}{|c|c|c|c|c|c|}
\hline Supply & Supply Discharge & Supply Discharge & Exhaust & Exhaust & Exhaust \\
\hline Configuration & Temperature $\left({ }^{\circ} \mathrm{C}\right.$ & RH & Configuration & Temperature $\left({ }^{\circ} \mathrm{C}\right)$ & RH \\
\hline Low Ind & 188 & $61 \%$ & Low & 22 & $50 \%$ \\
\hline Change & Rack & Rack & Number of & Total mass of & Room \\
\hline Station & Orientation & Density & Mice in Room & Mice in Room & Pressurisation \\
\hline ON & Perp & Single & 1050 & $21000 \mathrm{gr}$ & neg $100 \mathrm{cfm}$ \\
\hline \multicolumn{6}{|l|}{ Room } \\
\hline \multicolumn{6}{|l|}{ ACH } \\
\hline \multicolumn{6}{|l|}{15} \\
\hline \multicolumn{6}{|l|}{ Cage } \\
\hline \multicolumn{6}{|l|}{ Condition } \\
\hline \multicolumn{5}{|l|}{ Top On } & \\
\hline
\end{tabular}

Cage Occupied Zone

\begin{tabular}{|l|cc|c|c|}
\cline { 2 - 5 } \multicolumn{1}{c|}{} & \multicolumn{2}{c|}{ Temperature } & $\mathrm{CO}_{2}$ & RH \\
\cline { 2 - 5 } \multicolumn{1}{c|}{} & ${ }^{\circ} \mathrm{C}$ & ${ }^{\circ} \mathrm{F}$ & $(\mathrm{ppm})$ & \\
\hline Mean & 23.16 & 73.68 & 1883 & $60.24 \%$ \\
\hline S.D. & 0.46 & 0.83 & 495 & $3.53 \%$ \\
\hline Max. & 24.13 & 75.44 & 3022 & $67.04 \%$ \\
\hline
\end{tabular}

Cage Occupied Zone NH3 (ppm)

\begin{tabular}{|l|c|c|c|c|c|c|c|c|c|c|}
\hline Day & $\mathbf{1}$ & $\mathbf{2}$ & $\mathbf{3}$ & $\mathbf{4}$ & $\mathbf{5}$ & $\mathbf{6}$ & $\mathbf{7}$ & $\mathbf{8}$ & $\mathbf{9}$ & $\mathbf{1 0}$ \\
\hline Mean & 0.98 & 1.91 & 2.94 & 4.24 & 5.96 & 8.26 & 11.30 & 15.25 & 20.25 & 2647 \\
\hline Max. & 1.57 & 3.06 & 4.72 & 6.80 & 9.56 & 13.26 & 18.14 & 24.47 & 32.50 & 42.49 \\
\hline
\end{tabular}

Room Breathing Zone

\begin{tabular}{|l|cc|c|c|}
\cline { 2 - 5 } \multicolumn{1}{c|}{} & \multicolumn{2}{c|}{ Temperature } & $\mathrm{CO}_{2}$ & RH \\
\cline { 2 - 5 } \multicolumn{1}{c|}{} & ${ }^{\circ} \mathrm{C}$ & ${ }^{\circ} \mathrm{F}$ & $(\mathrm{ppm})$ & \\
\hline Mean & 22.10 & 71.78 & 81 & $49.75 \%$ \\
\hline S.D. & 0.78 & 1.41 & 47 & \\
\hline Max. & 27.78 & 82.00 & 427 & \\
\hline
\end{tabular}

Room Breathing Zone NH3 (ppm)

\begin{tabular}{|l|c|c|c|c|c|c|c|c|c|c|}
\hline Day & $\mathbf{1}$ & $\mathbf{2}$ & $\mathbf{3}$ & $\mathbf{4}$ & $\mathbf{5}$ & $\mathbf{6}$ & $\mathbf{7}$ & $\mathbf{8}$ & $\mathbf{9}$ & $\mathbf{1 0}$ \\
\hline Mean & 0.04 & 0.08 & 0.13 & 0.18 & 0.26 & 0.36 & 0.49 & 0.66 & 0.88 & 1.15 \\
\hline Max. & 0.22 & 0.43 & 0.67 & 0.96 & 1.35 & 187 & 2.56 & 3.46 & 4.59 & 6.00 \\
\hline
\end{tabular}




\section{Histogram Distributions}

Cage occupied zone average temperature $\left({ }^{\circ} \mathrm{C}\right)$ distribution

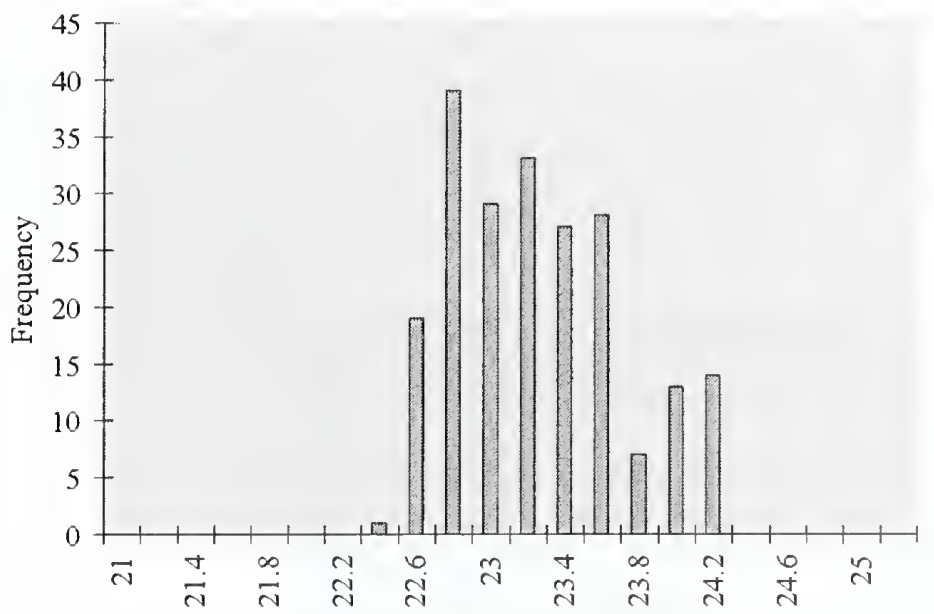

Cage occupied zone average contamination $(\mathrm{kg} / \mathrm{kg})$ distribution

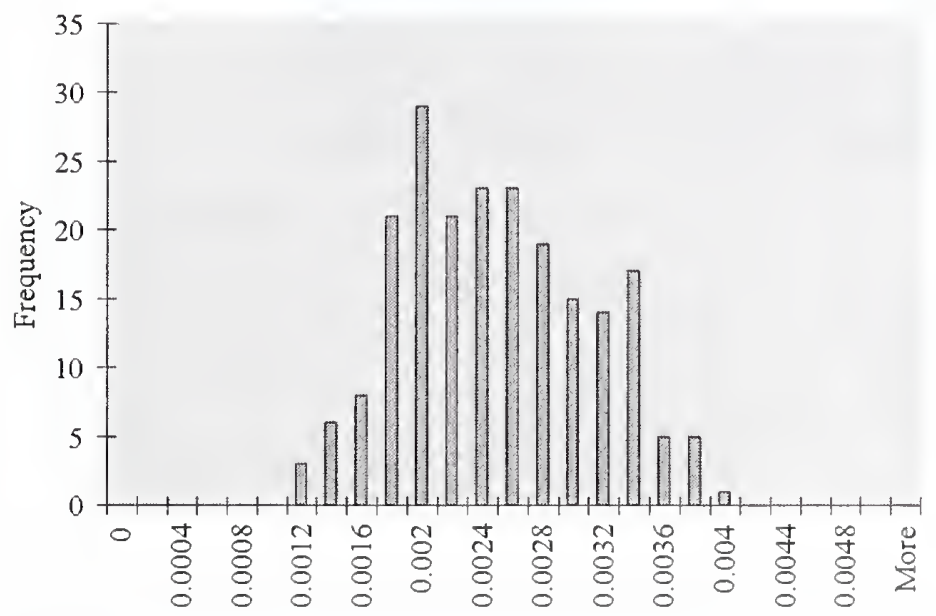

Cage occupied zone average relative humidity (\%) distribution

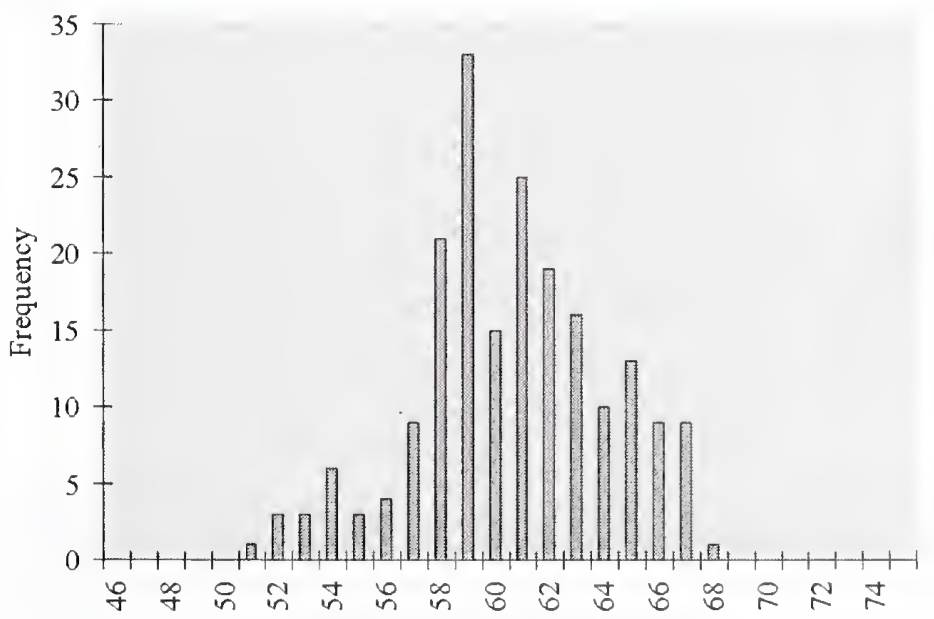

Contamination conversion factors $(\mathrm{kg} / \mathrm{kg} \rightarrow \mathrm{ppm})$

\begin{tabular}{|c|c|c|}
\hline Day & $\mathbf{C O}_{2}$ & $\mathrm{NH}_{3}$ \\
\hline $\mathbf{1}$ & 785000 & 408 \\
\hline $\mathbf{2}$ & 785000 & 795 \\
\hline $\mathbf{3}$ & 785000 & 1225 \\
\hline 4 & 785000 & 1766 \\
\hline 5 & 785000 & 2483 \\
\hline $\mathbf{6}$ & 785000 & 3443 \\
\hline 7 & 785000 & 4712 \\
\hline 8 & 785000 & 6356 \\
\hline 9 & 785000 & 8442 \\
\hline 10 & 785000 & 11384 \\
\hline
\end{tabular}


Casename $\quad$ Case 25

\section{Description}

Supply

Configuration

Radial

Change

Station

ON

Room
ACH
15
Cage
Condition
Sealed

\section{Supply Discharge Supply Discharge}

Temperature $\left({ }^{\circ} \mathrm{C}\right)$

18.8

RH

$61 \%$

Rack

Orientation

On wall
Rack

Density

Single
Exhaust

Configuration

Ceiling

Number of

Mice in Room

1050

\section{Exha ust}

Temperature $\left({ }^{\circ} \mathrm{C}\right)$

22

Total mass of
Mice in Room
$21000 \mathrm{gr}$
Exhaust

RH

$50 \%$

Room

Pressurisation

neg $100 \mathrm{cfm}$

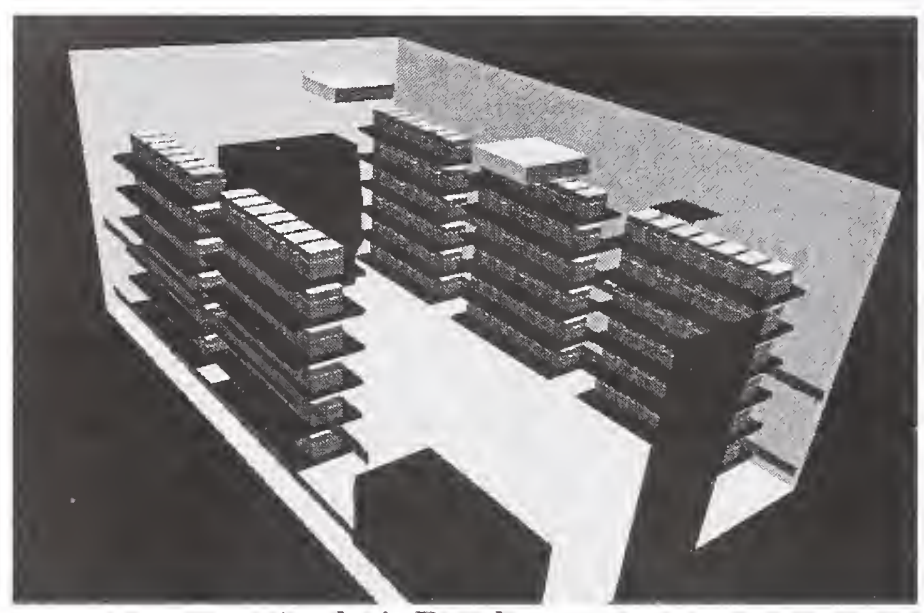

Analysis Results

\begin{tabular}{|l|cc|c|c|}
\cline { 2 - 5 } \multicolumn{1}{c|}{} & \multicolumn{2}{c|}{ Temperature } & $\mathrm{CO}_{2}$ & $\mathrm{RH}$ \\
\cline { 2 - 5 } \multicolumn{1}{c|}{} & ${ }^{\circ} \mathrm{C}$ & ${ }^{\circ} \mathrm{F}$ & $(\mathrm{ppm})$ & \\
\hline Mean & 22.20 & 71.96 & 3008 & $73.04 \%$ \\
\hline S.D. & 0.25 & 0.45 & 492 & $4.17 \%$ \\
\hline Max. & 23.07 & 73.52 & 3939 & $80.80 \%$ \\
\hline
\end{tabular}

Cage Occupied Zone NH3 (ppm)

\begin{tabular}{|l|c|c|c|c|c|c|c|c|c|c|}
\hline Day & $\mathbf{1}$ & $\mathbf{2}$ & $\mathbf{3}$ & $\mathbf{4}$ & $\mathbf{5}$ & $\mathbf{6}$ & $\mathbf{7}$ & $\mathbf{8}$ & $\mathbf{9}$ & $\mathbf{1 0}$ \\
\hline Mean & 1.64 & 3.04 & 4.69 & 11.05 & 20.64 & 31.63 & 49.49 & 59.31 & 67.59 & 64.80 \\
\hline Max. & 2.15 & 3.99 & 6.15 & 14.47 & 27.02 & 41.42 & 64.81 & 77.66 & 88.51 & 84.85 \\
\hline
\end{tabular}

Room Breathing Zone

\begin{tabular}{|l|cc|c|c|}
\cline { 2 - 5 } \multicolumn{1}{c|}{} & \multicolumn{2}{c|}{ Temperature } & $\mathrm{CO}_{2}$ & $\mathrm{RH}$ \\
\cline { 2 - 5 } \multicolumn{1}{c|}{} & ${ }^{\circ} \mathrm{C}$ & ${ }^{\circ} \mathrm{F}$ & $(\mathrm{ppm})$ & \\
\hline Mean & 20.30 & 68.53 & 60 & $55.58 \%$ \\
\hline S.D. & 0.22 & 0.40 & 24 & \\
\hline Max. & 21.27 & 70.28 & 233 & \\
\hline
\end{tabular}

Room Breathing Zone NH3 (ppm)

\begin{tabular}{|l|c|c|c|c|c|c|c|c|c|c|}
\hline Day & $\mathbf{1}$ & $\mathbf{2}$ & $\mathbf{3}$ & $\mathbf{4}$ & $\mathbf{5}$ & $\mathbf{6}$ & $\mathbf{7}$ & $\mathbf{8}$ & $\mathbf{9}$ & $\mathbf{1 0}$ \\
\hline Mean & 0.03 & 0.06 & 0.09 & 0.22 & 0.41 & 0.63 & 0.99 & 1.19 & 1.36 & 1.30 \\
\hline Max. & 0.13 & 0.24 & 0.36 & 0.86 & 1.60 & 2.45 & 3.83 & 4.59 & 5.23 & 5.01 \\
\hline
\end{tabular}




\section{Histogram Distributions}

Cage occupied zone average temperature $\left({ }^{\circ} \mathrm{C}\right)$ distribution

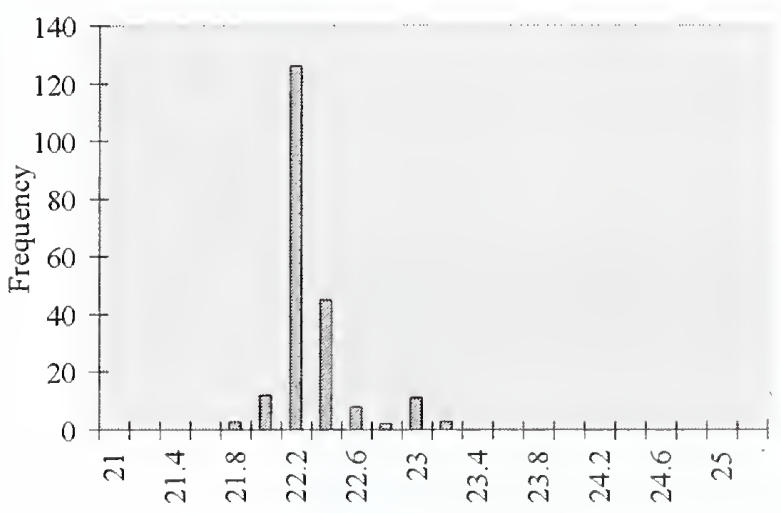

Cage occupied zone average contamination $(\mathrm{kg} / \mathrm{kg})$ distribution

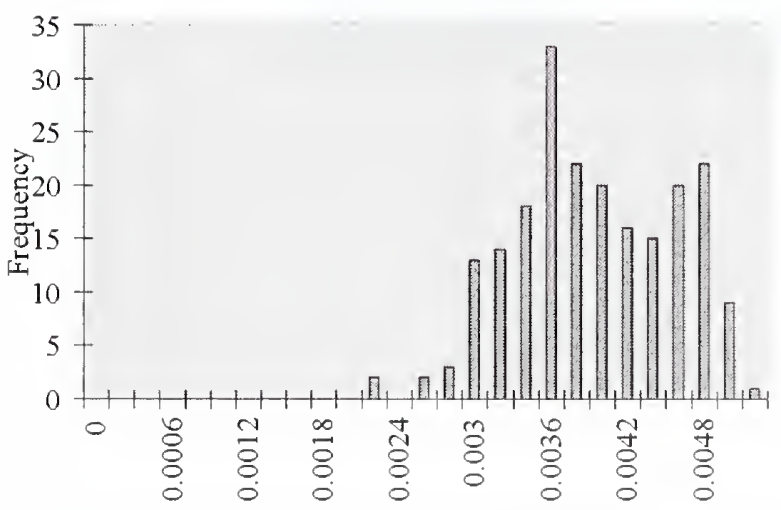

Cage occupied zone average relative humidity (\%) distribution

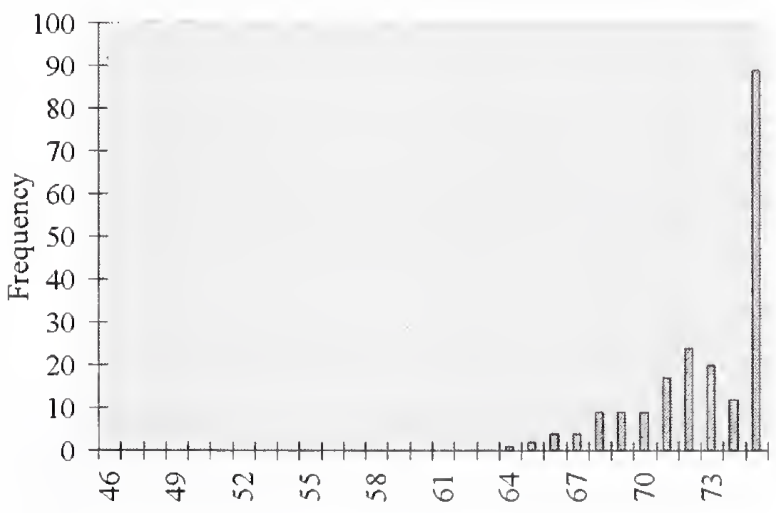

Contamination conversion factors $(\mathrm{kg} / \mathrm{kg} \rightarrow \mathrm{ppm})$

\begin{tabular}{|c|c|c|}
\hline Day & $\mathrm{CO}_{2}$ & $\mathrm{NH}_{3}$ \\
\hline $\mathbf{1}$ & 785000 & 429 \\
\hline $\mathbf{2}$ & 785000 & 795 \\
\hline $\mathbf{3}$ & 785000 & 1225 \\
\hline $\mathbf{4}$ & 785000 & 2885 \\
\hline $\mathbf{5}$ & 785000 & 5386 \\
\hline $\mathbf{6}$ & 785000 & 8256 \\
\hline 7 & 785000 & 12918 \\
\hline $\mathbf{8}$ & 785000 & 15479 \\
\hline $\mathbf{9}$ & 785000 & 17641 \\
\hline 10 & 785000 & 11384 \\
\hline
\end{tabular}


Casename

Case 26

Description

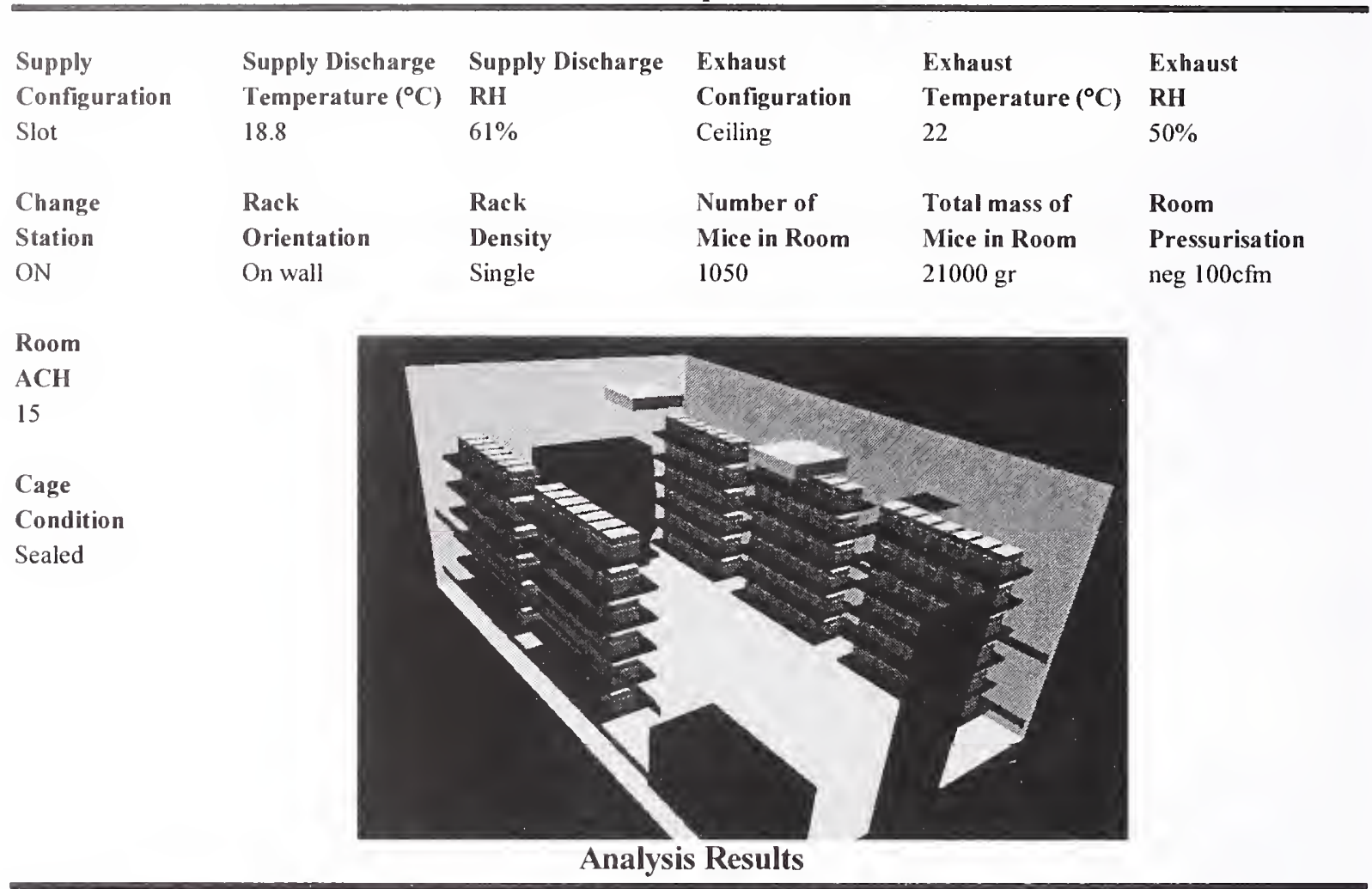

Cage Occupied Zone

\begin{tabular}{|l|cc|c|c|}
\cline { 2 - 5 } \multicolumn{1}{c|}{} & \multicolumn{2}{c|}{ Temperature } & $\mathrm{CO}_{2}$ & RH \\
\cline { 2 - 5 } \multicolumn{1}{c|}{} & ${ }^{\circ} \mathrm{C}$ & ${ }^{\circ} \mathrm{F}$ & $(\mathrm{ppm})$ & \\
\hline Mean & 22.14 & 71.85 & 3975 & $81.15 \%$ \\
\hline S.D. & 0.38 & 0.68 & 422 & $3.46 \%$ \\
\hline Max. & 23.03 & 73.45 & 4836 & $88.54 \%$ \\
\hline
\end{tabular}

Cage Occupied Zone NH3 (ppm)

\begin{tabular}{|l|c|c|c|c|c|c|c|c|c|c|}
\hline Day & 1 & 2 & 3 & 4 & 5 & 6 & 7 & 8 & 9 & 10 \\
\hline Mean & 2.25 & 4.02 & 6.20 & 18.42 & 37.17 & 58.21 & 93.38 & 109.47 & 120.68 & 105.66 \\
\hline Max. & 2.73 & 4.90 & 7.55 & 22.42 & 45.23 & 70.83 & 113.63 & 133.21 & 146.85 & 128.57 \\
\hline
\end{tabular}

Room Breathing Zone

\begin{tabular}{|l|cc|c|c|}
\cline { 2 - 5 } \multicolumn{1}{c|}{} & \multicolumn{2}{c|}{ Temperature } & $\mathrm{CO}_{2}$ & RH \\
\cline { 2 - 5 } \multicolumn{1}{c|}{} & ${ }^{\circ} \mathrm{C}$ & ${ }^{\circ} \mathrm{F}$ & $(\mathrm{ppm})$ & \\
\hline Mean & 20.40 & 68.72 & 35 & $54.98 \%$ \\
\hline S.I. & 0.55 & 0.99 & 28 & \\
\hline Max. & 21.81 & 71.27 & 149 & \\
\hline
\end{tabular}

Room Breathing Zone NH3 (ppm)

\begin{tabular}{|l|c|c|c|c|c|c|c|c|c|c|}
\hline Day & 1 & 2 & 3 & 4 & 5 & 6 & 7 & 8 & 9 & 10 \\
\hline Mean & 0.02 & 0.04 & 0.05 & 0.16 & 0.32 & 0.51 & 0.81 & 0.95 & 1.05 & 0.92 \\
\hline Max. & 0.08 & 0.15 & 0.23 & 0.69 & 1.39 & 2.18 & 3.50 & 4.10 & 4.52 & 3.96 \\
\hline
\end{tabular}




\section{Histogram Distributions}

Cage occupied zone average temperature $\left({ }^{\circ} \mathrm{C}\right)$ distribution

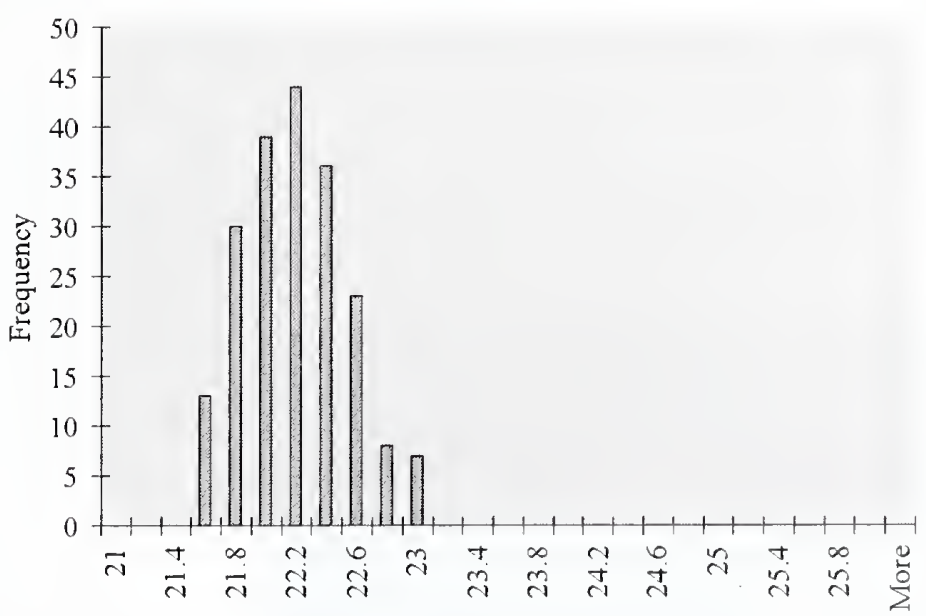

Cage occupied zone average contamination $(\mathrm{kg} / \mathrm{kg})$ distribution

Contamination conversion factors $(\mathrm{kg} / \mathrm{kg} \rightarrow \mathrm{ppm})$

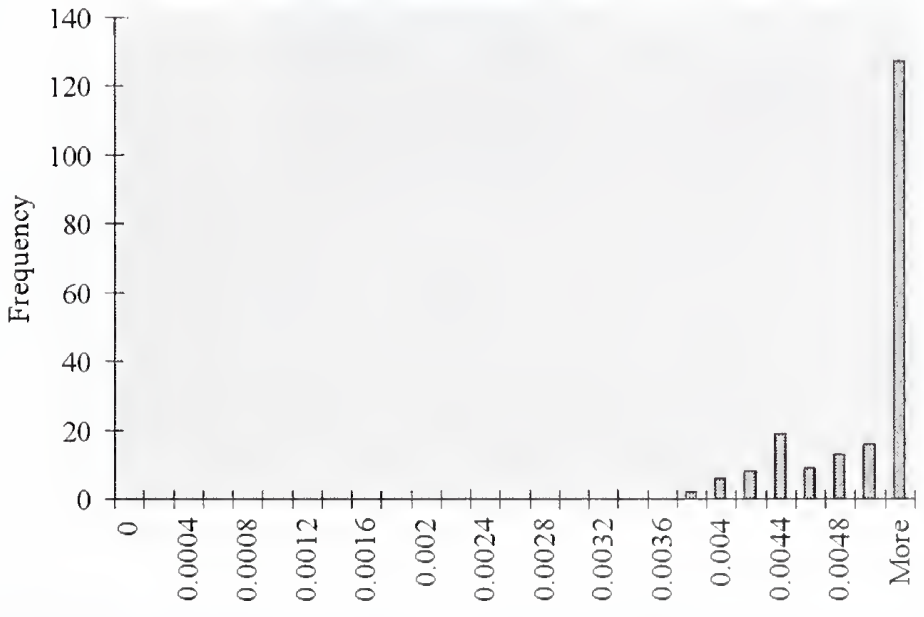

\begin{tabular}{|c|c|c|}
\hline Day & $\mathrm{CO}_{2}$ & $\mathrm{NH}_{3}$ \\
\hline $\mathbf{1}$ & 785000 & 443 \\
\hline $\mathbf{2}$ & 785000 & 795 \\
\hline $\mathbf{3}$ & 785000 & 1225 \\
\hline $\mathbf{4}$ & 785000 & 3639 \\
\hline $\mathbf{5}$ & 785000 & 7341 \\
\hline $\mathbf{6}$ & 785000 & 11497 \\
\hline $\mathbf{7}$ & 785000 & 18443 \\
\hline $\mathbf{8}$ & 785000 & 21621 \\
\hline $\mathbf{9}$ & 785000 & 23835 \\
\hline $\mathbf{1 0}$ & 785000 & 11384 \\
\hline
\end{tabular}

Cage occupied zone average relative humidity (\%) distribution

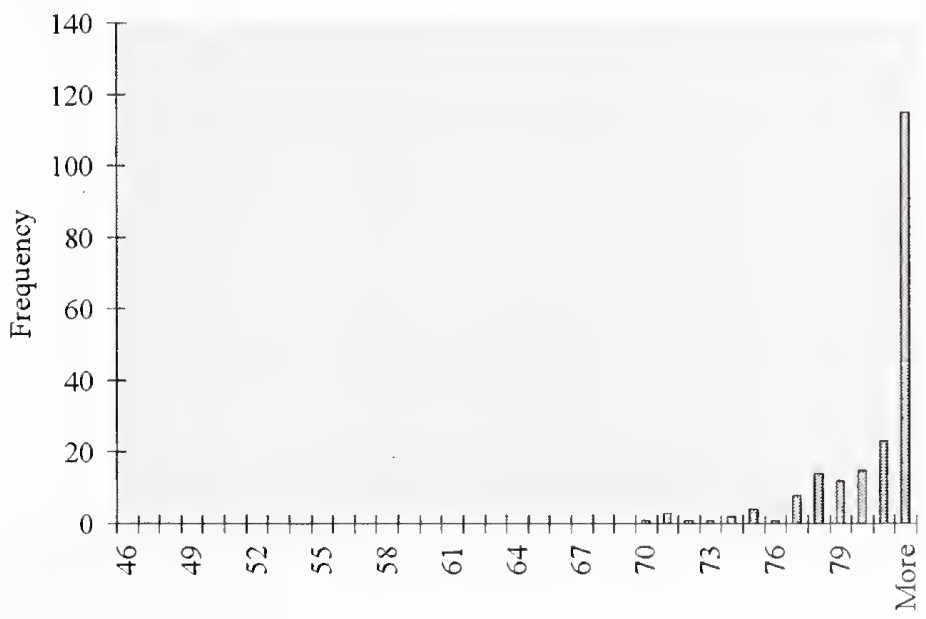




\section{Description}

\begin{tabular}{|c|c|c|c|c|c|}
\hline Supply & Supply Discharge & Supply Discharge & Exhaust & Exhaust & Exhaust \\
\hline Configuration & Temperature $\left({ }^{\circ} \mathrm{C}\right)$ & $\mathrm{RH}$ & Configuration & Temperature $\left({ }^{\circ} \mathrm{C}\right)$ & RH \\
\hline Low Ind & 18.8 & $61 \%$ & Ceiling & 22 & $50 \%$ \\
\hline Change & Rack & Rack & Number of & Total mass of & Room \\
\hline Station & Orientation & Density & Mice in Room & Mice in Room & Pressurisation \\
\hline $\mathrm{ON}$ & On wall & Single & 1050 & $21000 \mathrm{gr}$ & neg $100 \mathrm{cfm}$ \\
\hline
\end{tabular}

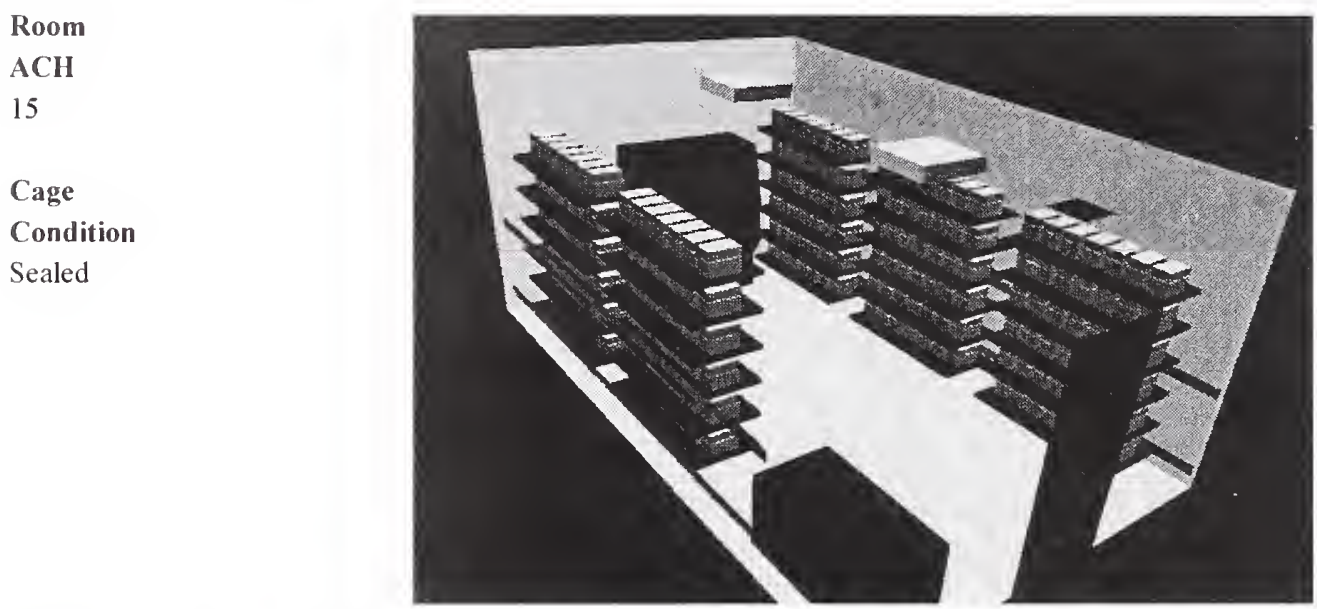

Analysis Results

Cage Occupied Zone

\begin{tabular}{|l|cc|c|c|}
\cline { 2 - 5 } \multicolumn{1}{c|}{} & \multicolumn{2}{c|}{ Temperature } & $\mathrm{CO}_{2}$ & RH \\
\cline { 2 - 5 } \multicolumn{1}{c|}{} & ${ }^{\circ} \mathrm{C}$ & ${ }^{\circ} \mathrm{F}$ & $(\mathrm{ppm})$ & \\
\hline Mean & 22.10 & 71.79 & 3171 & $74.81 \%$ \\
\hline S.D. & 0.24 & 0.43 & 569 & $4.79 \%$ \\
\hline Max. & 23.12 & 73.62 & 4558 & $86.18 \%$ \\
\hline
\end{tabular}

Cage Occupied Zone NH3 (ppm)

\begin{tabular}{|l|c|c|c|c|c|c|c|c|c|c|}
\hline Day & $\mathbf{1}$ & $\mathbf{2}$ & $\mathbf{3}$ & $\mathbf{4}$ & $\mathbf{5}$ & $\mathbf{6}$ & $\mathbf{7}$ & $\mathbf{8}$ & $\mathbf{9}$ & $\mathbf{1 0}$ \\
\hline Mean & 1.75 & 3.21 & 4.95 & 12.31 & 23.47 & 36.19 & 57.03 & 67.91 & 76.69 & 71.78 \\
\hline Max. & 2.51 & 4.61 & 7.11 & 17.70 & 33.74 & 52.03 & 81.98 & 97.63 & 110.26 & 103.19 \\
\hline
\end{tabular}

Room Breathing Zone

\begin{tabular}{|l|cc|c|c|}
\cline { 2 - 5 } \multicolumn{1}{c|}{} & \multicolumn{2}{|c|}{ Temperature } & $\mathrm{CO}_{2}$ & RH \\
\cline { 2 - 5 } \multicolumn{1}{c|}{} & ${ }^{\circ} \mathrm{C}$ & ${ }^{\circ} \mathrm{F}$ & $(\mathrm{ppm})$ & \\
\hline Mean & 20.31 & 68.56 & 61 & $55.54 \%$ \\
\hline S.D. & 0.25 & 0.46 & 26 & \\
\hline Max. & 21.95 & 71.51 & 239 & \\
\hline
\end{tabular}

Room Breathing Zone NH3 (ppm)

\begin{tabular}{|l|c|c|c|c|c|c|c|c|c|c|}
\hline Day & 1 & 2 & 3 & 4 & 5 & 6 & 7 & 8 & 9 & 10 \\
\hline Mean & 0.03 & 0.06 & 0.10 & 0.24 & 0.45 & 0.70 & 1.10 & 1.31 & 1.48 & 1.39 \\
\hline Max. & 0.13 & 0.24 & 0.37 & 0.93 & 1.77 & 2.72 & 4.29 & 5.11 & 5.77 & 5.40 \\
\hline
\end{tabular}




\section{Histogram Distributions}

Cage occupied zone average temperature $\left({ }^{\circ} \mathrm{C}\right)$ distribution

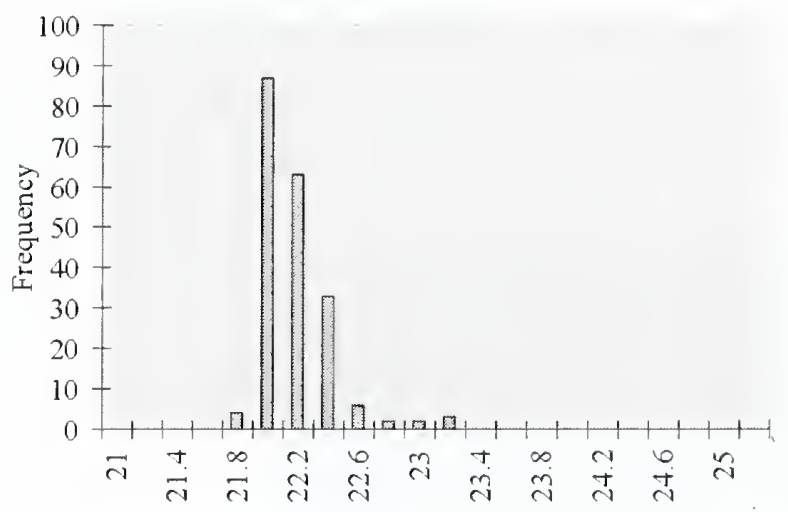

Cage occupied zone average contamination $(\mathrm{kg} / \mathrm{kg})$ distribution

Contamination conversion factors $(\mathrm{kg} / \mathrm{kg} \rightarrow \mathrm{ppm})$

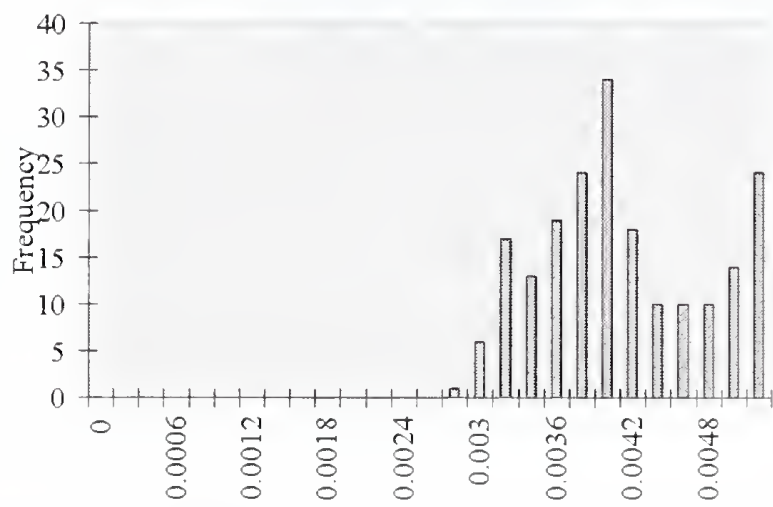

\begin{tabular}{|c|c|c|}
\hline Day & $\mathbf{C O}_{2}$ & $\mathbf{N H}_{3}$ \\
\hline $\mathbf{1}$ & 785000 & 432 \\
\hline $\mathbf{2}$ & 785000 & 795 \\
\hline $\mathbf{3}$ & 785000 & 1225 \\
\hline $\mathbf{4}$ & 785000 & 3049 \\
\hline $\mathbf{5}$ & 785000 & 5811 \\
\hline $\mathbf{6}$ & 785000 & 8960 \\
\hline $\mathbf{7}$ & 785000 & 14119 \\
\hline $\mathbf{8}$ & 785000 & 16814 \\
\hline $\mathbf{9}$ & 785000 & 18988 \\
\hline $\mathbf{1 0}$ & 785000 & 11384 \\
\hline
\end{tabular}

Cage occupied zone average relative humidity $(\%)$ distribution

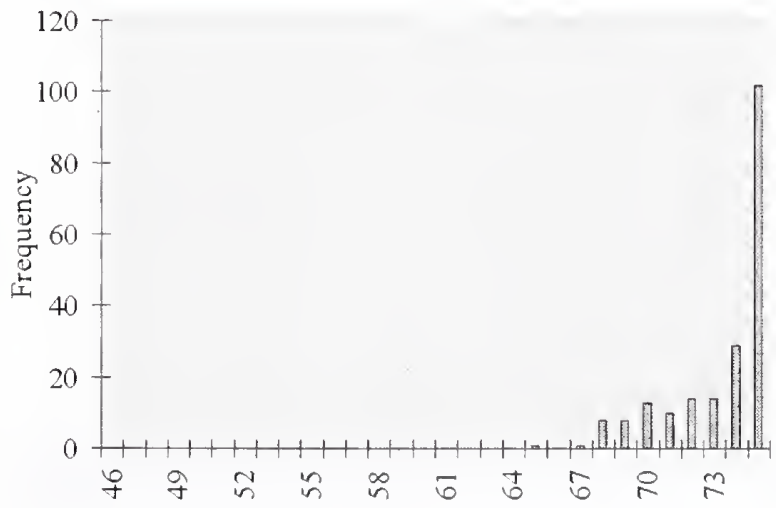


Volume II - 112 Ventilation Design Handbook on Animal Research Facilities Using Static Microisolators

Casename $\quad$ Case 28

Description

\begin{tabular}{|c|c|c|c|c|c|}
\hline Supply & Supply Discharge & Supply Discharge & Exhaust & Exhaust & Exhaust \\
\hline Configuration & Temperature $\left({ }^{\circ} \mathrm{C}\right)$ & $\mathbf{R H}$ & Configuration & Temperature $\left({ }^{\circ} \mathrm{C}\right)$ & $\mathbf{R H}$ \\
\hline Low Ind & 16.8 & $69 \%$ & Low & 22 & $50 \%$ \\
\hline Change & Rack & Rack & Number of & Total mass of & Room \\
\hline Station & Orientation & Density & Mice in Room & Mice in Room & Pressurisation \\
\hline ON & On wall & Single & 1050 & $21000 \mathrm{gr}$ & neg $100 \mathrm{cfm}$ \\
\hline
\end{tabular}

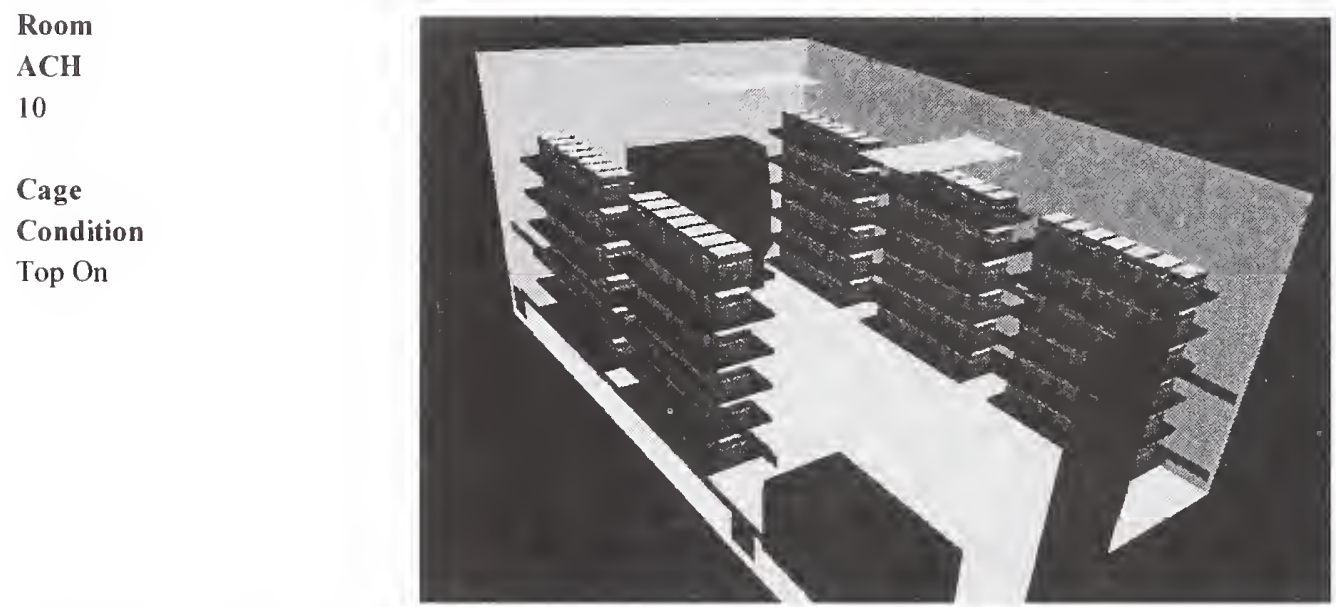

Analysis Results

Cage Occupied Zone

\begin{tabular}{|l|cc|c|c|}
\cline { 2 - 5 } \multicolumn{1}{c|}{} & \multicolumn{2}{c|}{ Temperature } & $\mathrm{CO}_{2}$ & RH \\
\cline { 2 - 5 } \multicolumn{1}{c|}{} & ${ }^{\circ} \mathrm{C}$ & ${ }^{\circ} \mathrm{F}$ & $(\mathrm{ppm})$ & \\
\hline Mean & 23.32 & 73.97 & 1749 & $58.69 \%$ \\
\hline S.1. & 0.60 & 1.08 & 333 & $3.37 \%$ \\
\hline Max. & 24.56 & 76.21 & 2705 & $65.58 \%$ \\
\hline
\end{tabular}

Cage Occupied Zone NH3 (ppm)

\begin{tabular}{|l|c|c|c|c|c|c|c|c|c|c|}
\hline Day & $\mathbf{1}$ & $\mathbf{2}$ & $\mathbf{3}$ & $\mathbf{4}$ & $\mathbf{5}$ & $\mathbf{6}$ & $\mathbf{7}$ & $\mathbf{8}$ & $\mathbf{9}$ & $\mathbf{1 0}$ \\
\hline Mean & 0.91 & 1.77 & 2.73 & 3.93 & 5.53 & 7.67 & 10.50 & 14.16 & 18.81 & 24.59 \\
\hline Max. & 1.41 & 2.74 & 4.22 & 6.08 & 8.56 & 11.86 & 16.24 & 21.90 & 29.09 & 38.02 \\
\hline
\end{tabular}

Room Breathing Zone

\begin{tabular}{|l|cc|c|c|}
\cline { 2 - 5 } \multicolumn{1}{c|}{} & \multicolumn{2}{c|}{ Temperature } & $\mathrm{CO}_{2}$ & RH \\
\cline { 2 - 5 } \multicolumn{1}{c|}{} & ${ }^{\circ} \mathrm{C}$ & ${ }^{\circ} \mathrm{F}$ & $(\mathrm{ppm})$ & \\
\hline Mean & 22.28 & 72.10 & 113 & $49.45 \%$ \\
\hline S.D. & 0.61 & 1.10 & 29 & \\
\hline Max. & 23.38 & 74.09 & 307 & \\
\hline
\end{tabular}

Room Breathing Zone NH3 (ppm)

\begin{tabular}{|l|c|c|c|c|c|c|c|c|c|c|}
\hline Day & $\mathbf{1}$ & $\mathbf{2}$ & $\mathbf{3}$ & $\mathbf{4}$ & $\mathbf{5}$ & $\mathbf{6}$ & $\mathbf{7}$ & $\mathbf{8}$ & $\mathbf{9}$ & $\mathbf{1 0}$ \\
\hline Mean & 0.06 & 0.11 & 0.18 & 0.25 & 0.36 & 0.50 & 0.68 & 0.92 & 1.22 & 1.59 \\
\hline Max. & 0.16 & 0.31 & 0.48 & 0.69 & 0.97 & 1.35 & 1.84 & 2.48 & 3.30 & 4.31 \\
\hline
\end{tabular}




\section{Histogram Distributions}

Cage occupied zone average temperature $\left({ }^{\circ} \mathrm{C}\right)$ distribution

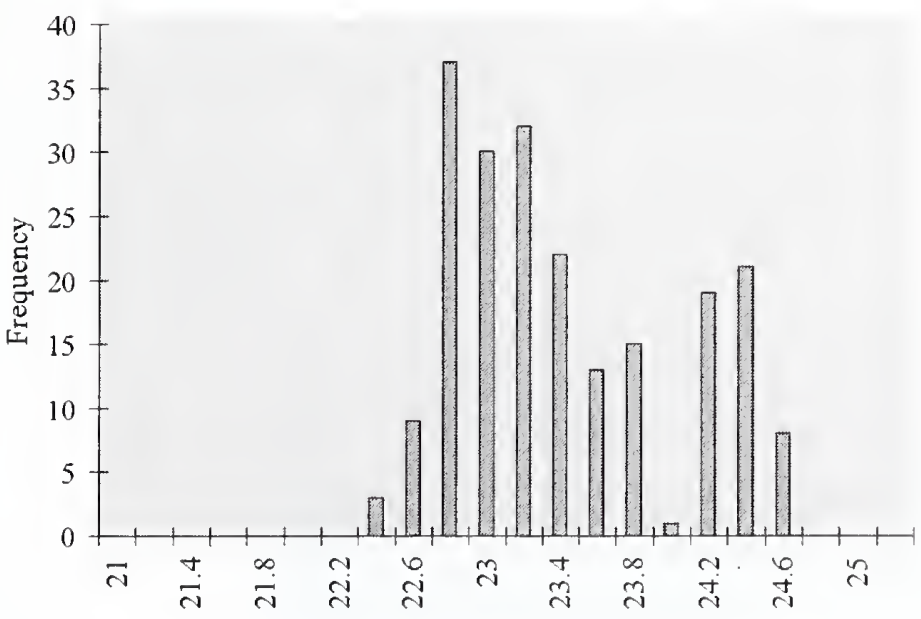

Cage occupied zone average contamination $(\mathrm{kg} / \mathrm{kg})$ distribution

Contamination conversion factors $(\mathrm{kg} / \mathrm{kg} \rightarrow \mathrm{ppm})$

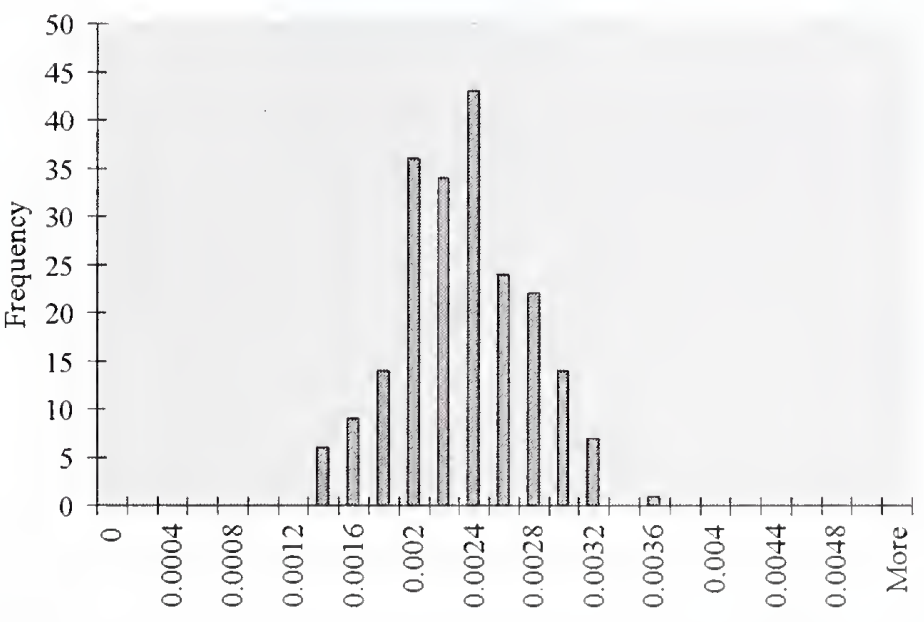

\begin{tabular}{|c|c|c|}
\hline Day & $\mathbf{C O}_{2}$ & $\mathbf{N H}_{3}$ \\
\hline $\mathbf{1}$ & 785000 & 408 \\
\hline $\mathbf{2}$ & 785000 & 795 \\
\hline $\mathbf{3}$ & 785000 & 1225 \\
\hline $\mathbf{4}$ & 785000 & 1766 \\
\hline $\mathbf{5}$ & 785000 & 2483 \\
\hline $\mathbf{6}$ & 785000 & 3443 \\
\hline $\mathbf{7}$ & 785000 & 4712 \\
\hline $\mathbf{8}$ & 785000 & 6356 \\
\hline $\mathbf{9}$ & 785000 & 8442 \\
\hline $\mathbf{1 0}$ & 785000 & 11384 \\
\hline
\end{tabular}

Cage occupied zone average relative humidity (\%) distribŭtion

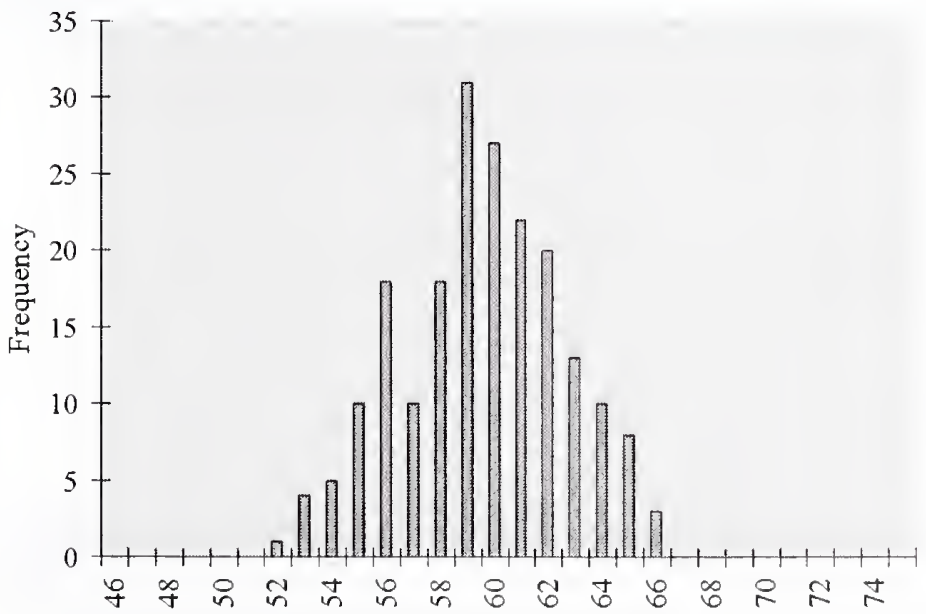


Volume III - $114 \quad$ Ventilation Design Handbook on Animal Research Facilities Using Static Microisolators

Casename

Case 29

\section{Description}

\begin{tabular}{|c|c|c|c|c|c|}
\hline Supply & Supply Discharge & Supply Discharge & Exhaust & Exhaust & Exhaust \\
\hline Configuration & Temperature $\left({ }^{\circ} \mathrm{C}\right)$ & RH & Configuration & Temperature $\left({ }^{\circ} \mathrm{C}\right)$ & RH \\
\hline Low Ind & 11 & $65 \%$ & Low & 22 & $50 \%$ \\
\hline Change & Rack & Rack & Number of & Total mass of & Room \\
\hline Station & Orientation & Density & Mice in Room & Mice in Room & Pressurisation \\
\hline ON & On wall & Single & 1050 & $21000 \mathrm{gr}$ & neg $100 \mathrm{cfm}$ \\
\hline
\end{tabular}

Room

$\mathrm{ACH}$

5

Cage

Condition

Top On

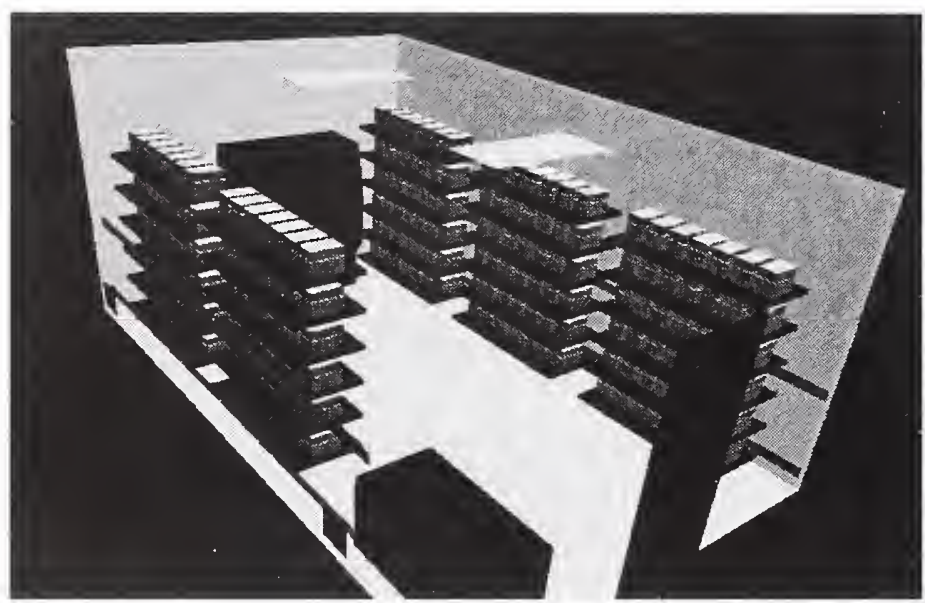

Analysis Results

Cage Occupied Zone

\begin{tabular}{|l|cc|c|c|}
\cline { 2 - 5 } \multicolumn{1}{c|}{} & \multicolumn{2}{c|}{ Temperature } & $\mathrm{CO}_{2}$ & RH \\
\cline { 2 - 5 } \multicolumn{1}{c|}{} & ${ }^{\circ} \mathrm{C}$ & ${ }^{\circ} \mathrm{F}$ & $(\mathrm{ppm})$ & \\
\hline Mean & 22.64 & 72.75 & 1764 & $44.37 \%$ \\
\hline S.D. & 0.54 & 0.97 & 314 & $289 \%$ \\
\hline Max. & 23.83 & 74.89 & 2556 & $50.77 \%$ \\
\hline
\end{tabular}

Cage Occupied Zone NH3 (ppm)

\begin{tabular}{|l|c|c|c|c|c|c|c|c|c|c|}
\hline Day & $\mathbf{1}$ & $\mathbf{2}$ & $\mathbf{3}$ & $\mathbf{4}$ & $\mathbf{5}$ & $\mathbf{6}$ & $\mathbf{7}$ & $\mathbf{8}$ & $\mathbf{9}$ & $\mathbf{1 0}$ \\
\hline Mean & 0.92 & 1.79 & 2.75 & 3.97 & 5.58 & 7.74 & 10.59 & 14.28 & 18.97 & 24.80 \\
\hline Max. & 1.33 & 2.59 & 3.99 & 5.75 & 8.08 & 11.21 & 15.34 & 20.70 & 27.49 & 35.93 \\
\hline
\end{tabular}

Room Breathing Zone

\begin{tabular}{|l|cc|c|c|}
\cline { 2 - 5 } \multicolumn{1}{c|}{} & \multicolumn{2}{c|}{ Temperature } & $\mathrm{CO}_{2}$ & RH \\
\cline { 2 - 5 } \multicolumn{1}{c|}{} & ${ }^{\circ} \mathrm{C}$ & ${ }^{\circ} \mathrm{F}$ & $(\mathrm{ppm})$ & \\
\hline Mean & 21.19 & 70.14 & 140 & $53.19 \%$ \\
\hline S.D. & 0.53 & 0.96 & 33 & \\
\hline Max. & 22.18 & 71.93 & 267 & \\
\hline
\end{tabular}

Room Breathing Zone NH3 (ppm)

\begin{tabular}{|l|c|c|c|c|c|c|c|c|c|c|}
\hline Day & $\mathbf{1}$ & $\mathbf{2}$ & $\mathbf{3}$ & $\mathbf{4}$ & $\mathbf{5}$ & $\mathbf{6}$ & $\mathbf{7}$ & $\mathbf{8}$ & $\mathbf{9}$ & $\mathbf{1 0}$ \\
\hline Mean & 0.07 & 0.14 & 0.22 & 0.32 & 0.44 & 0.62 & 0.84 & 1.14 & 1.51 & 197 \\
\hline Max. & 0.14 & 0.27 & 0.42 & 0.60 & 0.85 & 1.17 & 1.60 & 2.16 & 2.88 & 3.76 \\
\hline
\end{tabular}




\section{Histogram Distributions}

Cage occupied zone average temperature $\left({ }^{\circ} \mathrm{C}\right)$ distribution

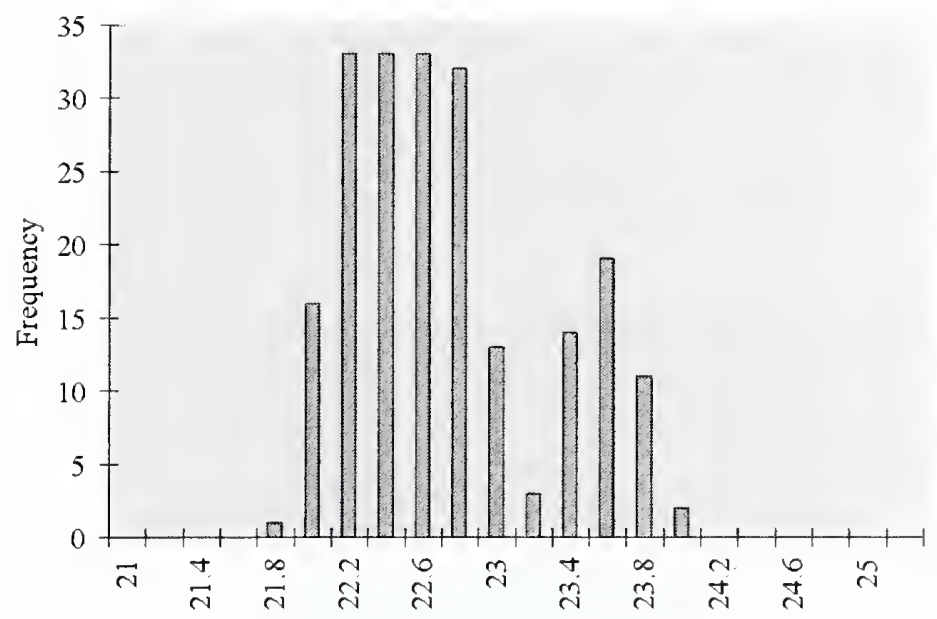

Cage occupied zone average contamination $(\mathrm{kg} / \mathrm{kg})$ distribution

Contamination conversion factors $(\mathrm{kg} / \mathrm{kg} \rightarrow \mathrm{ppm})$

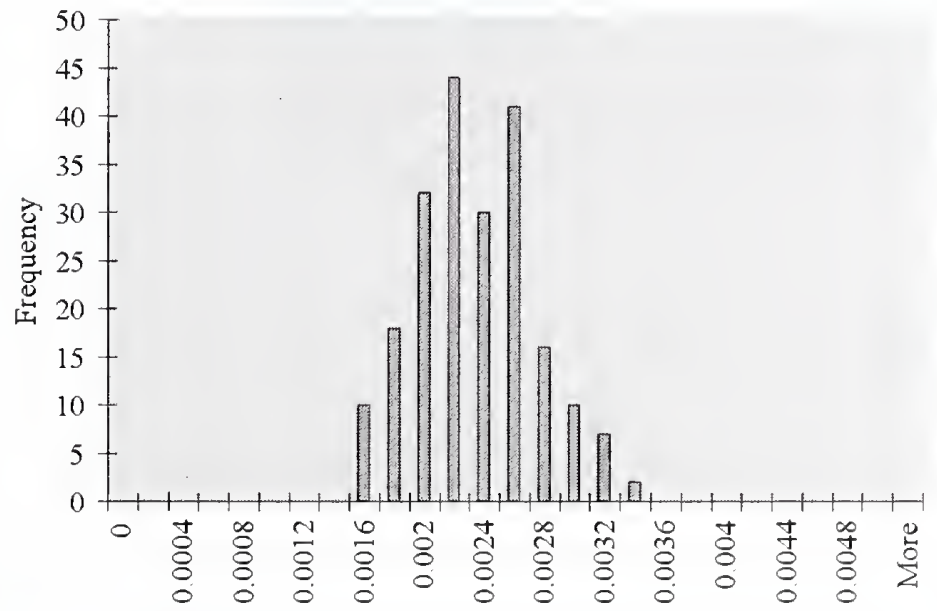

\begin{tabular}{|c|c|c|}
\hline Day & $\mathrm{CO}_{2}$ & $\mathrm{NH}_{3}$ \\
\hline $\mathbf{1}$ & 785000 & 408 \\
\hline $\mathbf{2}$ & 785000 & 795 \\
\hline $\mathbf{3}$ & 785000 & 1225 \\
\hline $\mathbf{4}$ & 785000 & 1766 \\
\hline $\mathbf{5}$ & 785000 & 2483 \\
\hline $\mathbf{6}$ & 785000 & 3443 \\
\hline 7 & 785000 & 4712 \\
\hline $\mathbf{8}$ & 785000 & 6356 \\
\hline $\mathbf{9}$ & 785000 & 8442 \\
\hline $\mathbf{1 0}$ & 785000 & 11384 \\
\hline
\end{tabular}

Cage occupied zone average relative humidity (\%) distribution

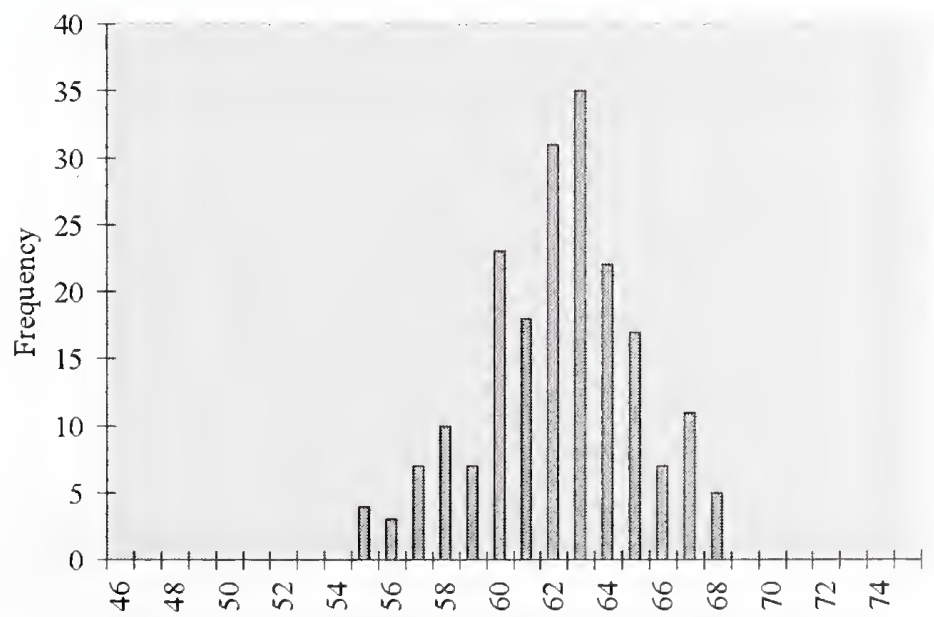


Description

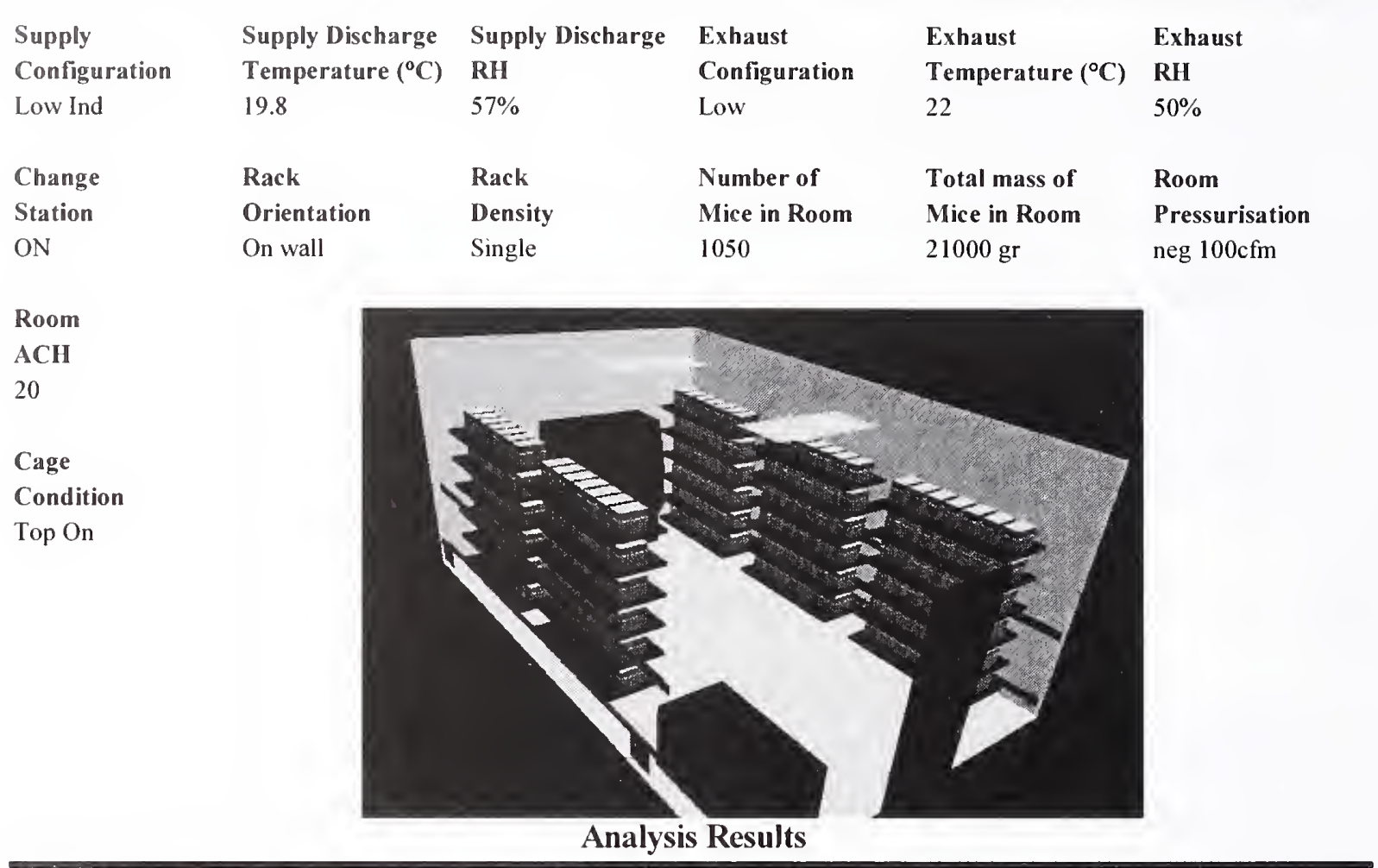

Cage Occupied Zone

\begin{tabular}{|l|cc|c|c|}
\cline { 2 - 5 } \multicolumn{1}{c|}{} & \multicolumn{2}{c|}{ Temperature } & $\mathrm{CO}_{2}$ & RH \\
\cline { 2 - 5 } \multicolumn{1}{c|}{} & ${ }^{\circ} \mathrm{C}$ & ${ }^{\circ}$ & $(\mathbf{p p m})$ & \\
\hline Mean & 23.64 & 74.56 & 1667 & $56.87 \%$ \\
\hline S.D. & 0.47 & 0.84 & 386 & $3.17 \%$ \\
\hline Max. & 24.70 & 76.47 & 2576 & $63.64 \%$ \\
\hline
\end{tabular}

Cage Occupied Zone NH3 (ppm)

\begin{tabular}{|l|c|c|c|c|c|c|c|c|c|c|}
\hline Day & $\mathbf{1}$ & $\mathbf{2}$ & $\mathbf{3}$ & $\mathbf{4}$ & $\mathbf{5}$ & $\mathbf{6}$ & $\mathbf{7}$ & $\mathbf{8}$ & $\mathbf{9}$ & $\mathbf{1 0}$ \\
\hline Mean & 0.87 & 1.69 & 2.60 & 3.75 & 5.27 & 7.31 & 10.01 & 13.50 & 17.93 & 23.44 \\
\hline Max. & 1.34 & 2.61 & 4.02 & 5.79 & 8.15 & 11.30 & 15.47 & 20.86 & 27.71 & 36.22 \\
\hline
\end{tabular}

Room Breathing Zone

\begin{tabular}{|l|cc|c|c|}
\cline { 2 - 5 } \multicolumn{1}{c|}{} & \multicolumn{2}{c|}{ Temperature } & $\mathrm{CO}_{2}$ & RH \\
\cline { 2 - 5 } \multicolumn{1}{c|}{} & ${ }^{\circ} \mathrm{C}$ & ${ }^{\circ} \mathrm{F}$ & $(\mathrm{ppm})$ & \\
\hline Mean & 22.57 & 72.63 & 64 & $48.16 \%$ \\
\hline S.D. & 0.49 & 0.89 & 26 & \\
\hline Max. & 24.17 & 75.50 & 252 & \\
\hline
\end{tabular}

Room Breathing Zone NH3 (ppm)

\begin{tabular}{|l|c|c|c|c|c|c|c|c|c|c|}
\hline Day & 1 & 2 & 3 & 4 & 5 & 6 & 7 & 8 & 9 & I0 \\
\hline Mean & 0.03 & 0.06 & 0.10 & 0.14 & 0.20 & 0.28 & 0.38 & 0.52 & 0.68 & 0.89 \\
\hline Max. & 0.13 & 0.25 & 0.39 & 0.57 & 0.80 & 1.10 & 1.51 & 2.04 & 2.71 & 3.54 \\
\hline
\end{tabular}




\section{Histogram Distributions}

Cage occupied zone average temperature $\left({ }^{\circ} \mathrm{C}\right)$ distribution

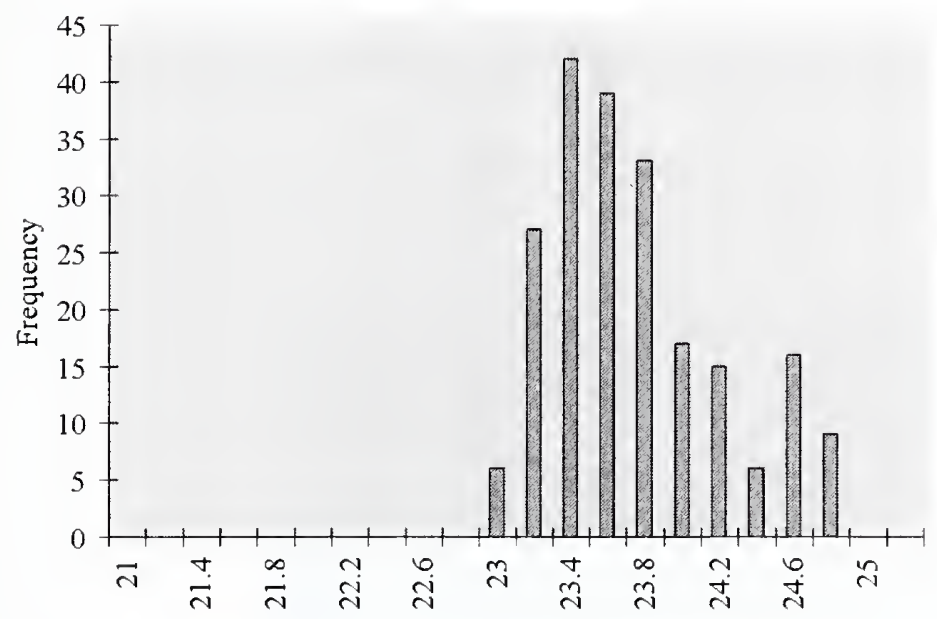

Cage occupied zone average contamination $(\mathrm{kg} / \mathrm{kg})$ distribution

Contamination conversion factors $(\mathrm{kg} / \mathrm{kg} \rightarrow \mathrm{ppm})$

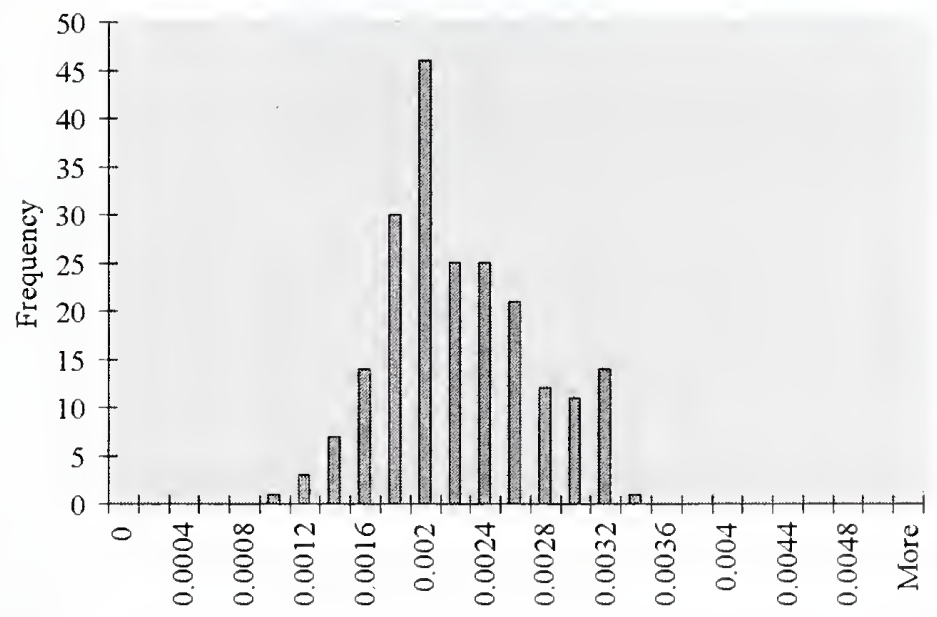

\begin{tabular}{|c|c|c|}
\hline Day & $\mathrm{CO}_{2}$ & $\mathrm{NH}_{3}$ \\
\hline $\mathbf{1}$ & 785000 & 408 \\
\hline $\mathbf{2}$ & 785000 & 795 \\
\hline $\mathbf{3}$ & 785000 & 1225 \\
\hline $\mathbf{4}$ & 785000 & 1766 \\
\hline $\mathbf{5}$ & 785000 & 2483 \\
\hline $\mathbf{6}$ & 785000 & 3443 \\
\hline 7 & 785000 & 4712 \\
\hline $\mathbf{8}$ & 785000 & 6356 \\
\hline $\mathbf{9}$ & 785000 & 8442 \\
\hline $\mathbf{1 0}$ & 785000 & 11384 \\
\hline
\end{tabular}

Cage occupied zone average relative humidity (\%) distribution

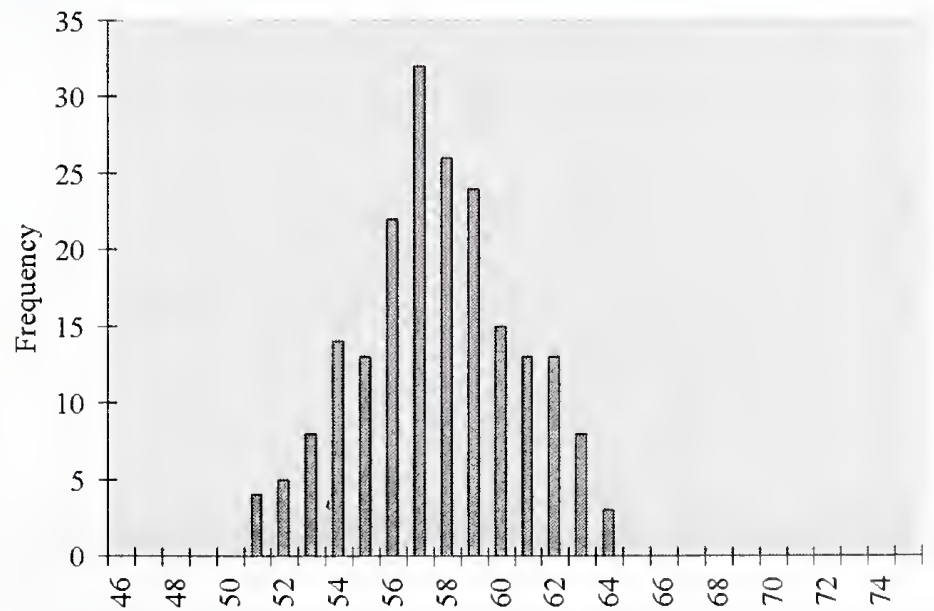


Description

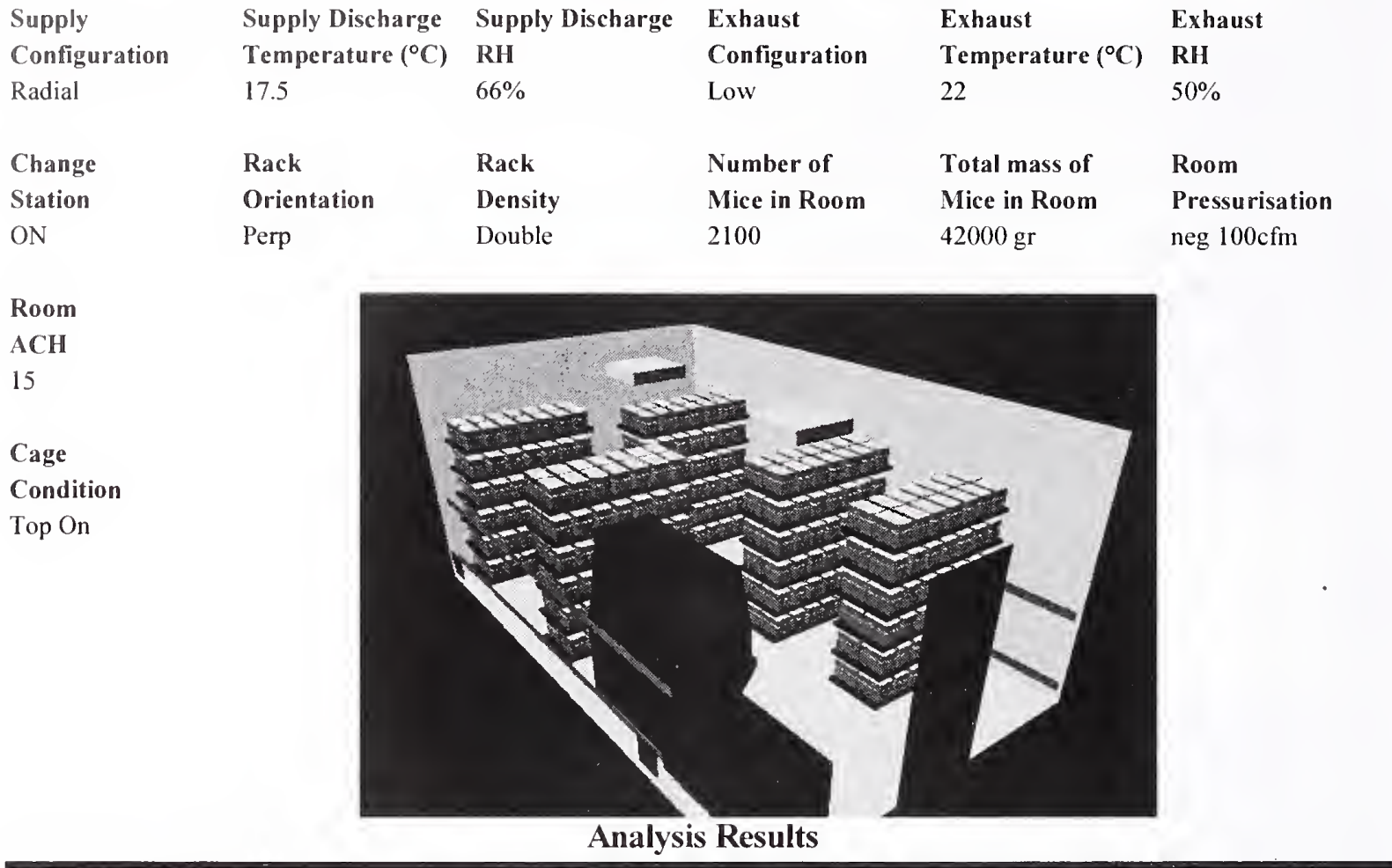

Cage Occupied Zone

\begin{tabular}{|l|cc|c|c|}
\cline { 2 - 5 } \multicolumn{1}{c|}{} & \multicolumn{2}{c|}{ Temperature } & $\mathrm{CO}_{2}$ & RH \\
\cline { 2 - 5 } \multicolumn{1}{c|}{} & ${ }^{\circ} \mathrm{C}$ & ${ }^{\circ} \mathrm{F}$ & $(\mathrm{ppm})$ & \\
\hline Mean & 22.67 & 72.80 & 1938 & $61.40 \%$ \\
\hline S.D. & 0.28 & 0.50 & 465 & $3.41 \%$ \\
\hline Max. & 23.39 & 74.09 & 2839 & $67.52 \%$ \\
\hline
\end{tabular}

Cage Occupied Zone NH3 (ppm)

\begin{tabular}{|l|c|c|c|c|c|c|c|c|c|c|}
\hline Day & $\mathbf{1}$ & $\mathbf{2}$ & $\mathbf{3}$ & $\mathbf{4}$ & $\mathbf{5}$ & $\mathbf{6}$ & $\mathbf{7}$ & $\mathbf{8}$ & $\mathbf{9}$ & $\mathbf{1 0}$ \\
\hline Mean & 1.01 & 1.96 & 3.02 & 4.45 & 6.37 & 8.89 & 12.30 & 16.44 & 21.59 & 27.73 \\
\hline Max. & 1.48 & 2.87 & 4.43 & 6.52 & 9.33 & 13.03 & 18.02 & 24.08 & 31.63 & 40.61 \\
\hline
\end{tabular}

Room Breathing Zone

\begin{tabular}{|l|cc|c|c|}
\cline { 2 - 5 } \multicolumn{1}{c|}{} & \multicolumn{2}{c|}{ Temperature } & $\mathrm{CO}_{2}$ & RH \\
\cline { 2 - 5 } \multicolumn{1}{c|}{} & ${ }^{\circ} \mathrm{C}$ & ${ }^{\circ} \mathrm{F}$ & $(\mathrm{ppm})$ & \\
\hline Mean & 21.37 & 70.46 & 107 & $52.32 \%$ \\
\hline S.D. & 0.73 & 1.31 & 43 & \\
\hline Max. & 28.10 & 82.59 & 315 & \\
\hline
\end{tabular}

Room Breathing Zone NH3 (ppm)

\begin{tabular}{|l|c|c|c|c|c|c|c|c|c|c|}
\hline Day & $\mathbf{1}$ & $\mathbf{2}$ & $\mathbf{3}$ & $\mathbf{4}$ & $\mathbf{5}$ & $\mathbf{6}$ & $\mathbf{7}$ & $\mathbf{8}$ & $\mathbf{9}$ & $\mathbf{1 0}$ \\
\hline Mean & 0.06 & 0.11 & 0.17 & 0.24 & 0.35 & 0.49 & 0.68 & 0.90 & 1.19 & 1.52 \\
\hline Max. & 0.16 & 0.32 & 0.49 & 0.72 & 1.04 & 1.45 & 2.00 & 2.68 & 3.51 & 4.51 \\
\hline
\end{tabular}




\section{Histogram Distributions}

Cage occupied zone average temperature $\left({ }^{\circ} \mathrm{C}\right)$ distribution

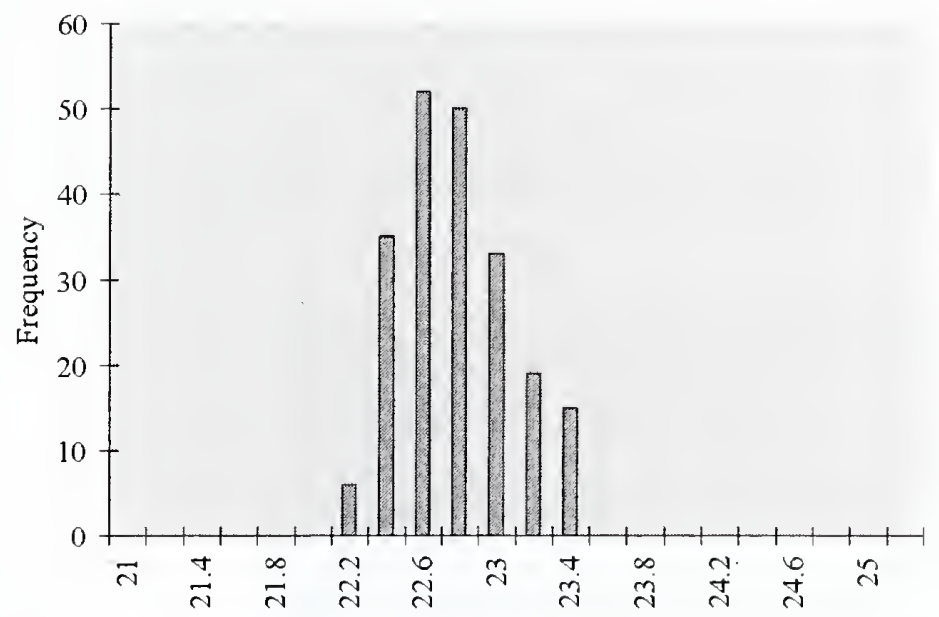

Cage occupied zone average contamination $(\mathrm{kg} / \mathrm{kg})$ distribution

Contamination conversion factors $(\mathrm{kg} / \mathrm{kg} \rightarrow \mathrm{ppm})$

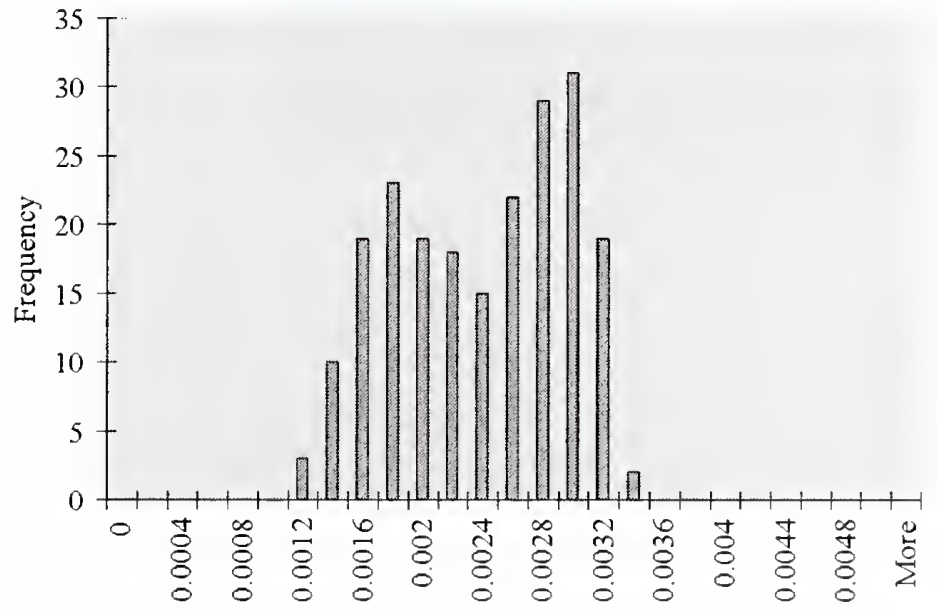

\begin{tabular}{|c|c|c|}
\hline Day & $\mathbf{C O}_{\mathbf{2}}$ & $\mathbf{N H}_{\mathbf{3}}$ \\
\hline $\mathbf{1}$ & 785000 & 409 \\
\hline $\mathbf{2}$ & 785000 & 795 \\
\hline $\mathbf{3}$ & 785000 & 1225 \\
\hline $\mathbf{4}$ & 785000 & 1803 \\
\hline $\mathbf{5}$ & 785000 & 2579 \\
\hline $\mathbf{6}$ & 785000 & 3602 \\
\hline $\mathbf{7}$ & 785000 & 4984 \\
\hline $\mathbf{8}$ & 785000 & 6658 \\
\hline $\mathbf{9}$ & 785000 & 8747 \\
\hline $\mathbf{1 0}$ & 785000 & 11384 \\
\hline
\end{tabular}

Cage occupied zone average relative humidity (\%) distribution

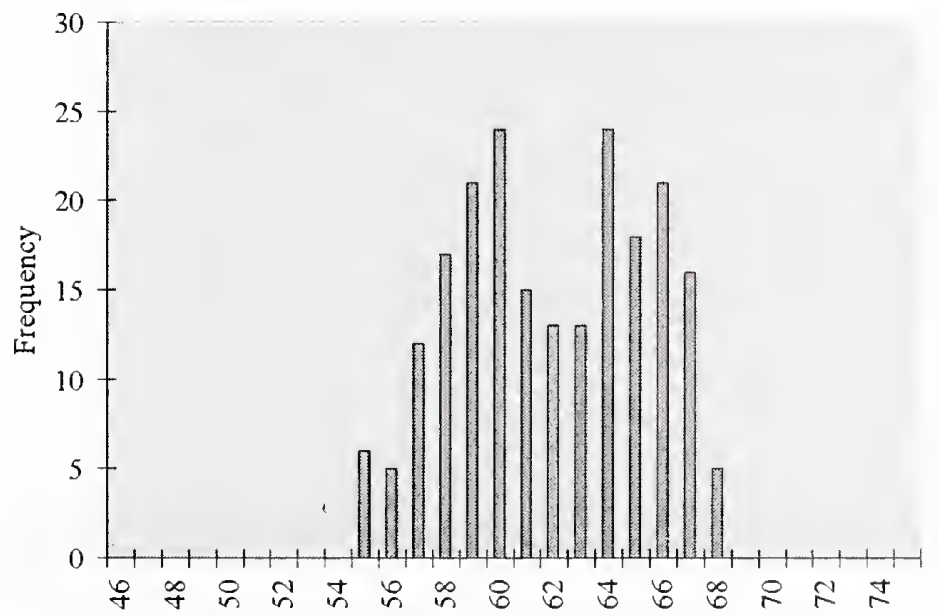


Volume III - $120 \quad$ Ventilation Design Handbook on Animal Research Facilities Using Static Microisolators

Casename Case 32

Description

\begin{tabular}{|c|c|c|c|c|c|}
\hline Supply & Supply Discharge & Supply Discharge & Exhaust & Exhaust & Exhaust \\
\hline Configuration & Temperature $\left({ }^{\circ} \mathrm{C}\right)$ & RH & Configuration & Temperature $\left({ }^{\circ} \mathrm{C}\right)$ & RH \\
\hline Slot & 17.5 & $66 \%$ & Low & 22 & $50 \%$ \\
\hline Change & Rack & Rack & Number of & Total mass of & Room \\
\hline Station & Orientation & Density & Mice in Room & Mice in Room & Pressurisation \\
\hline ON & Perp & Double & 2100 & $42000 \mathrm{gr}$ & neg $100 \mathrm{cfm}$ \\
\hline
\end{tabular}

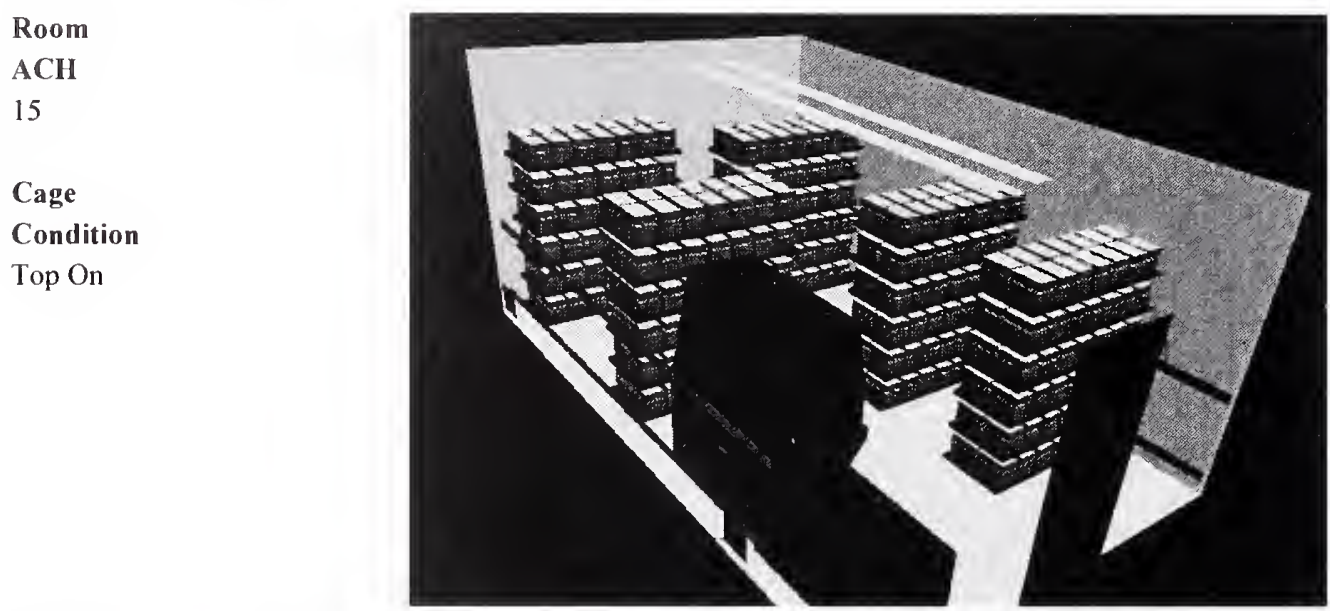

Analysis Results

Cage Occupied Zone

\begin{tabular}{|l|cc|c|c|}
\cline { 2 - 5 } \multicolumn{1}{c|}{} & \multicolumn{2}{c|}{ Temperature } & $\mathrm{CO}_{2}$ & RH \\
\cline { 2 - 5 } \multicolumn{1}{c|}{} & ${ }^{\circ} \mathrm{C}$ & ${ }^{\circ} \mathrm{F}$ & $(\mathbf{p p m})$ & \\
\hline Mean & 22.90 & 73.22 & 1747 & $59.10 \%$ \\
\hline S.D. & 0.52 & 0.93 & 426 & $2.60 \%$ \\
\hline Max. & 23.96 & 75.12 & 2789 & $65.01 \%$ \\
\hline
\end{tabular}

Cage Occupied Zone NH3 (ppm)

\begin{tabular}{|l|c|c|c|c|c|c|c|c|c|c|}
\hline Day & $\mathbf{1}$ & $\mathbf{2}$ & $\mathbf{3}$ & $\mathbf{4}$ & $\mathbf{5}$ & $\mathbf{6}$ & $\mathbf{7}$ & $\mathbf{8}$ & $\mathbf{9}$ & $\mathbf{1 0}$ \\
\hline Mean & 0.91 & 1.77 & 2.73 & 3.93 & 5.53 & 7.66 & 10.49 & 14.15 & 18.79 & 24.57 \\
\hline Max. & 1.45 & 2.82 & 4.35 & 6.27 & 8.82 & 12.23 & 16.74 & 22.59 & 30.00 & 39.21 \\
\hline
\end{tabular}

Room Breathing Zone

\begin{tabular}{|l|cc|c|c|}
\cline { 2 - 5 } \multicolumn{1}{c|}{} & \multicolumn{2}{c|}{ Temperature } & $\mathrm{CO}_{2}$ & RH \\
\cline { 2 - 5 } \multicolumn{1}{c|}{} & ${ }^{\circ} \mathrm{C}$ & ${ }^{\circ} \mathrm{F}$ & $(\mathbf{p p m})$ & \\
\hline Mean & 21.96 & 71.54 & 125 & $50.53 \%$ \\
\hline S.D. & 0.66 & 1.19 & 51 & \\
\hline Max. & 27.92 & 82.26 & 337 & \\
\hline
\end{tabular}

Room Breathing Zone NH3 (ppm)

\begin{tabular}{|l|c|c|c|c|c|c|c|c|c|c|}
\hline Day & $\mathbf{1}$ & $\mathbf{2}$ & $\mathbf{3}$ & $\mathbf{4}$ & $\mathbf{5}$ & $\mathbf{6}$ & $\mathbf{7}$ & $\mathbf{8}$ & $\mathbf{9}$ & $\mathbf{1 0}$ \\
\hline Mean & 0.06 & 0.13 & 0.19 & 0.28 & 0.39 & 0.55 & 0.75 & 1.01 & 1.34 & 1.75 \\
\hline Max. & 0.18 & 0.34 & 0.53 & 0.76 & 1.07 & 1.48 & 2.02 & 2.73 & 3.63 & 4.74 \\
\hline
\end{tabular}




\section{Histogram Distributions}

Cage occupied zone average temperature $\left({ }^{\circ} \mathrm{C}\right)$ distribution

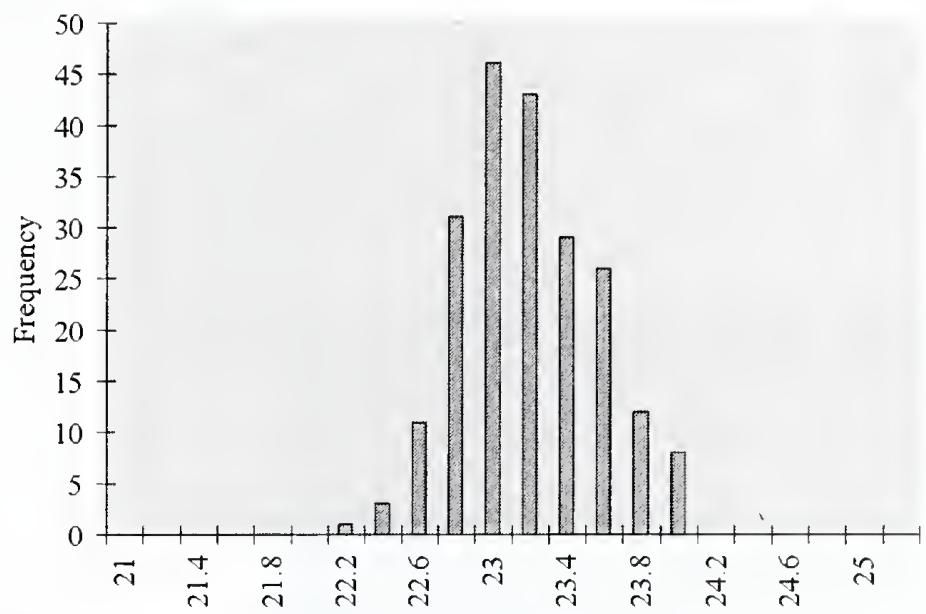

Cage occupied zone average contamination $(\mathrm{kg} / \mathrm{kg})$ distribution Contamination conversion factors $(\mathrm{kg} / \mathrm{kg} \rightarrow \mathrm{ppm})$

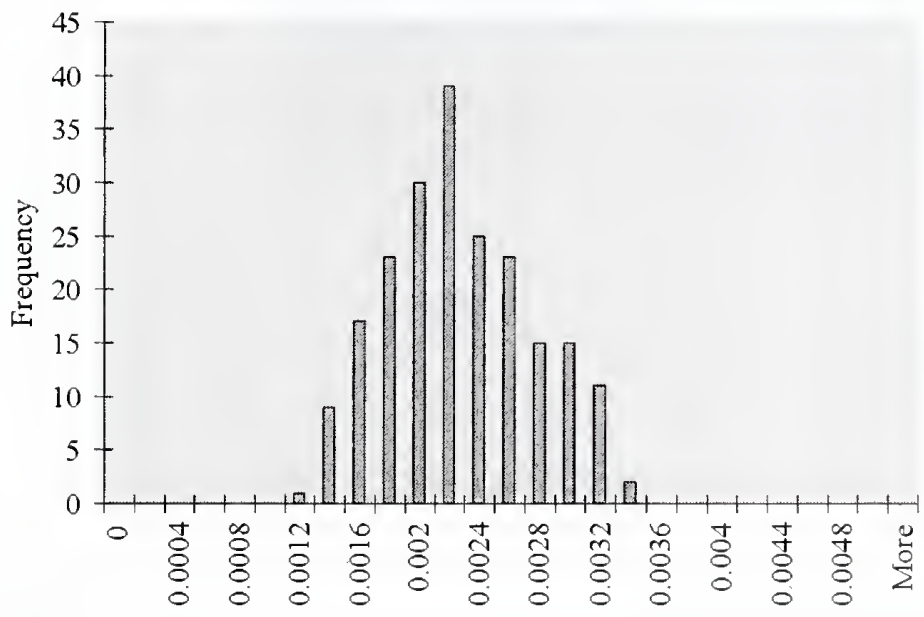

\begin{tabular}{|c|c|c|}
\hline Day & $\mathrm{CO}_{2}$ & $\mathrm{NH}_{3}$ \\
\hline $\mathbf{I}$ & 785000 & 408 \\
\hline $\mathbf{2}$ & 785000 & 795 \\
\hline $\mathbf{3}$ & 785000 & 1225 \\
\hline $\mathbf{4}$ & 785000 & 1766 \\
\hline $\mathbf{5}$ & 785000 & 2483 \\
\hline $\mathbf{6}$ & 785000 & 3443 \\
\hline 7 & 785000 & 4712 \\
\hline $\mathbf{8}$ & 785000 & 6356 \\
\hline 9 & 785000 & 8442 \\
\hline 10 & 785000 & 11384 \\
\hline
\end{tabular}

Cage occupied zone average relative humidity (\%) distribution

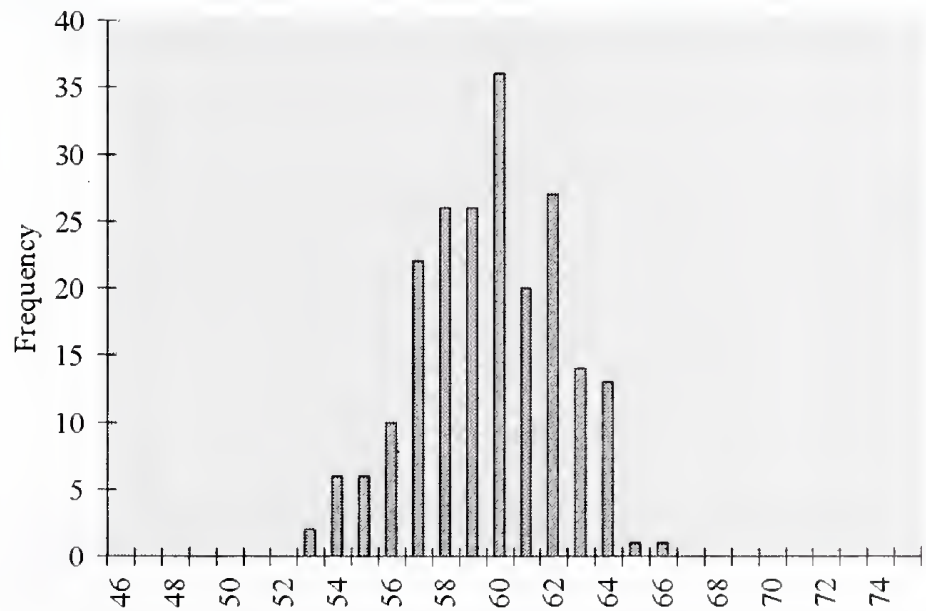




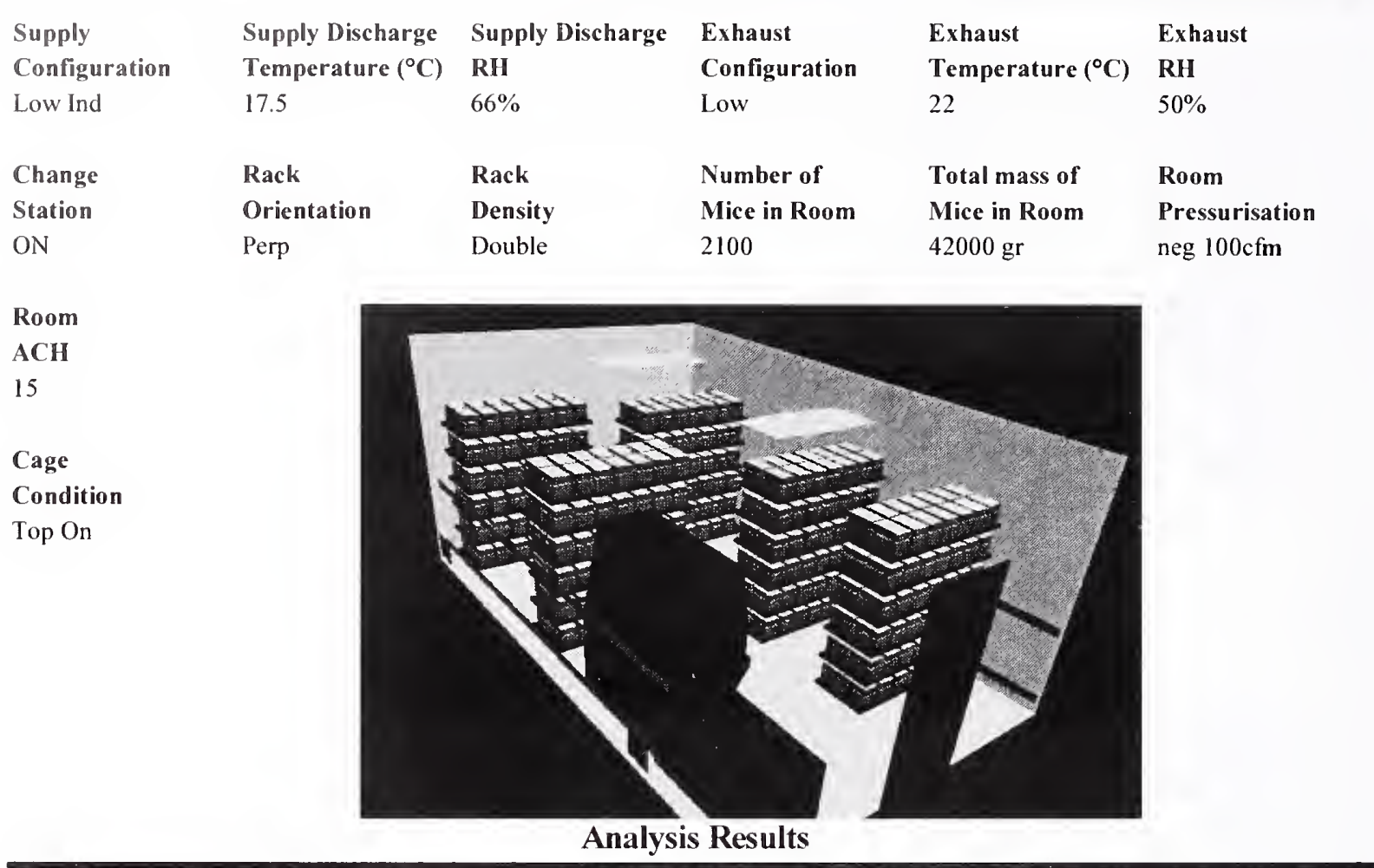

Cage Occupied Zone

\begin{tabular}{|l|cc|c|c|}
\cline { 2 - 5 } \multicolumn{1}{c|}{} & \multicolumn{2}{c|}{ Temperature } & $\mathrm{CO}_{2}$ & RH \\
\cline { 2 - 5 } \multicolumn{1}{c|}{} & ${ }^{\circ} \mathrm{C}$ & ${ }^{\circ} \mathrm{F}$ & $(\mathbf{p p m})$ & \\
\hline Mean & 23.16 & 73.68 & 1974 & $59.06 \%$ \\
\hline S.D. & 0.36 & 0.64 & 485 & $2.81 \%$ \\
\hline Max. & 24.12 & 75.41 & 3133 & $65.78 \%$ \\
\hline
\end{tabular}

Cage Occupied Zone NH3 (ppm)

\begin{tabular}{|l|c|c|c|c|c|c|c|c|c|c|}
\hline Day & $\mathbf{1}$ & $\mathbf{2}$ & $\mathbf{3}$ & $\mathbf{4}$ & $\mathbf{5}$ & $\mathbf{6}$ & $\mathbf{7}$ & $\mathbf{8}$ & $\mathbf{9}$ & $\mathbf{1 0}$ \\
\hline Mean & 1.03 & 2.00 & 3.08 & 4.44 & 6.24 & 8.66 & 11.85 & 15.98 & 21.23 & 27.75 \\
\hline Max. & 1.63 & 3.17 & 4.89 & 7.05 & 9.91 & 13.74 & 18.81 & 25.37 & 33.69 & 44.04 \\
\hline
\end{tabular}

Room Breathing Zone

\begin{tabular}{|l|cc|c|c|}
\cline { 2 - 5 } \multicolumn{1}{c|}{} & \multicolumn{2}{c|}{ Temperature } & \multicolumn{1}{c|}{$\mathrm{CO}_{2}$} & \multicolumn{1}{c|}{ RH } \\
\cline { 2 - 5 } \multicolumn{1}{c|}{} & ${ }^{\circ} \mathrm{C}$ & ${ }^{\circ} \mathrm{F}$ & $(\mathrm{ppm})$ & \\
\hline Mean & 21.97 & 71.54 & 143 & $50.67 \%$ \\
\hline S.D. & 0.74 & 1.33 & 67 & \\
\hline Max. & 27.68 & 81.82 & 433 & \\
\hline
\end{tabular}

Room Breathing Zone NH3 (ppm)

\begin{tabular}{|l|c|c|c|c|c|c|c|c|c|c|}
\hline Day & $\mathbf{1}$ & $\mathbf{2}$ & $\mathbf{3}$ & $\mathbf{4}$ & $\mathbf{5}$ & $\mathbf{6}$ & $\mathbf{7}$ & $\mathbf{8}$ & $\mathbf{9}$ & $\mathbf{1 0}$ \\
\hline Mean & 0.07 & 0.14 & 0.22 & 0.32 & 0.45 & 0.63 & 0.86 & 1.16 & 1.54 & 2.01 \\
\hline Max. & 0.22 & 0.44 & 0.67 & 0.97 & 1.37 & 1.90 & 2.60 & 3.50 & 4.65 & 6.08 \\
\hline
\end{tabular}




\section{Histogram Distributions}

Cage occupied zone average temperature $\left({ }^{\circ} \mathrm{C}\right)$ distribution

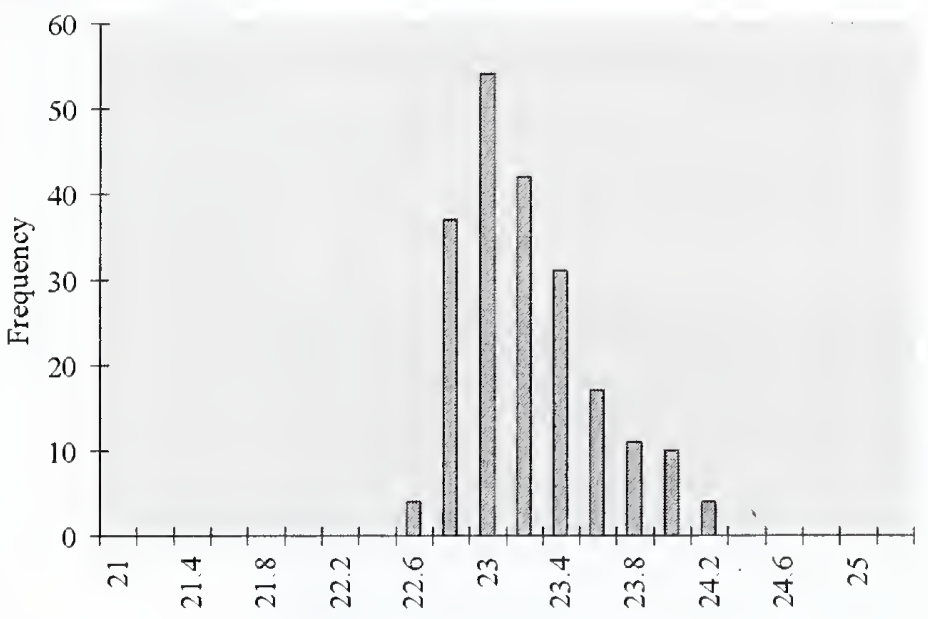

Cage occupied zone average contamination $(\mathrm{kg} / \mathrm{kg})$ distribution

Contamination conversion factors $(\mathrm{kg} / \mathrm{kg} \rightarrow \mathrm{ppm})$

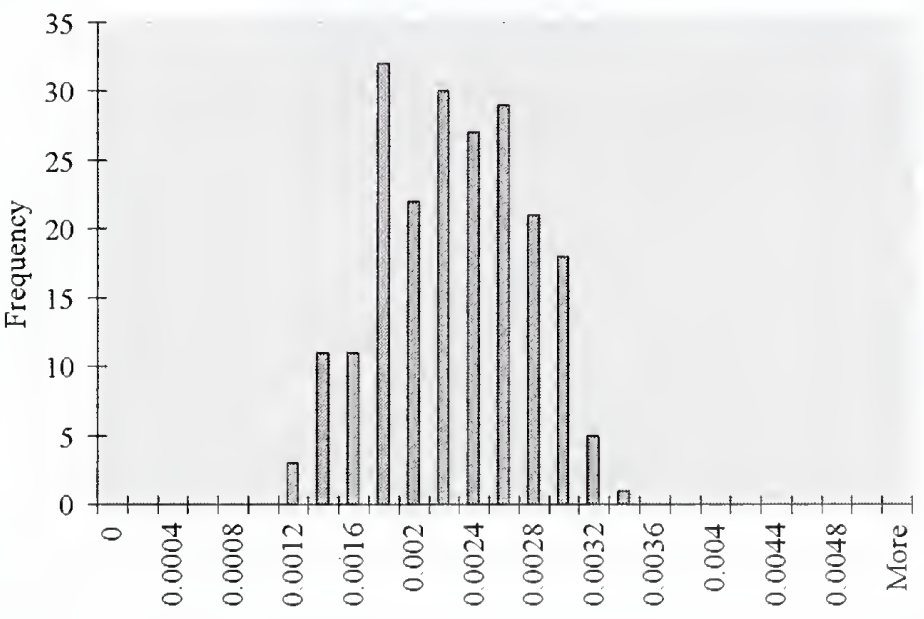

\begin{tabular}{|c|c|c|}
\hline Day & $\mathbf{C O}_{2}$ & $\mathrm{NH}_{3}$ \\
\hline $\mathbf{1}$ & 785000 & 408 \\
\hline $\mathbf{2}$ & 785000 & 795 \\
\hline $\mathbf{3}$ & 785000 & 1225 \\
\hline $\mathbf{4}$ & 785000 & 1766 \\
\hline $\mathbf{5}$ & 785000 & 2483 \\
\hline $\mathbf{6}$ & 785000 & 3443 \\
\hline 7 & 785000 & 4712 \\
\hline $\mathbf{8}$ & 785000 & 6356 \\
\hline $\mathbf{9}$ & 785000 & 8442 \\
\hline $\mathbf{1 0}$ & 785000 & 11384 \\
\hline
\end{tabular}

Cage occupied zone average relative humidity (\%) distribution

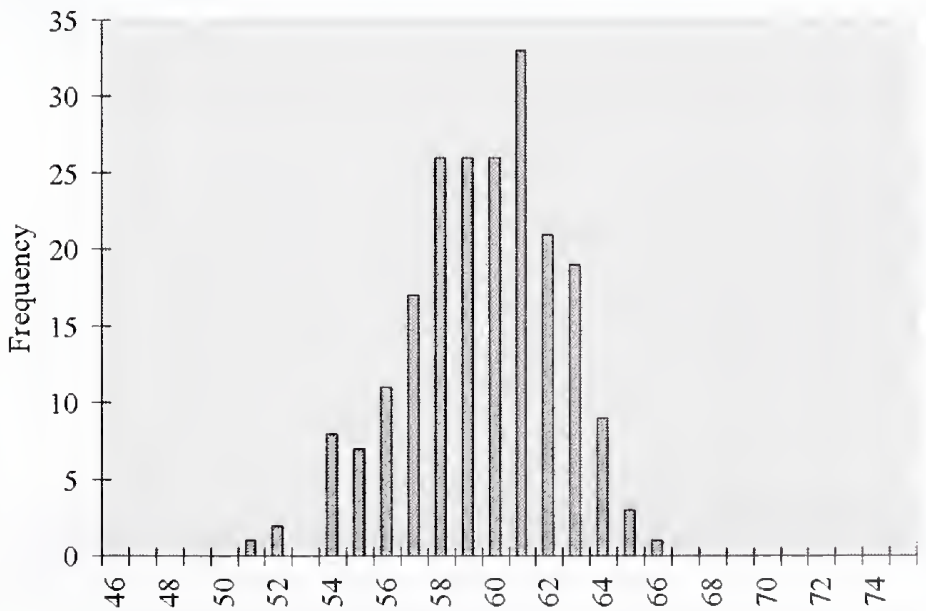


Casename

Case 34

Description

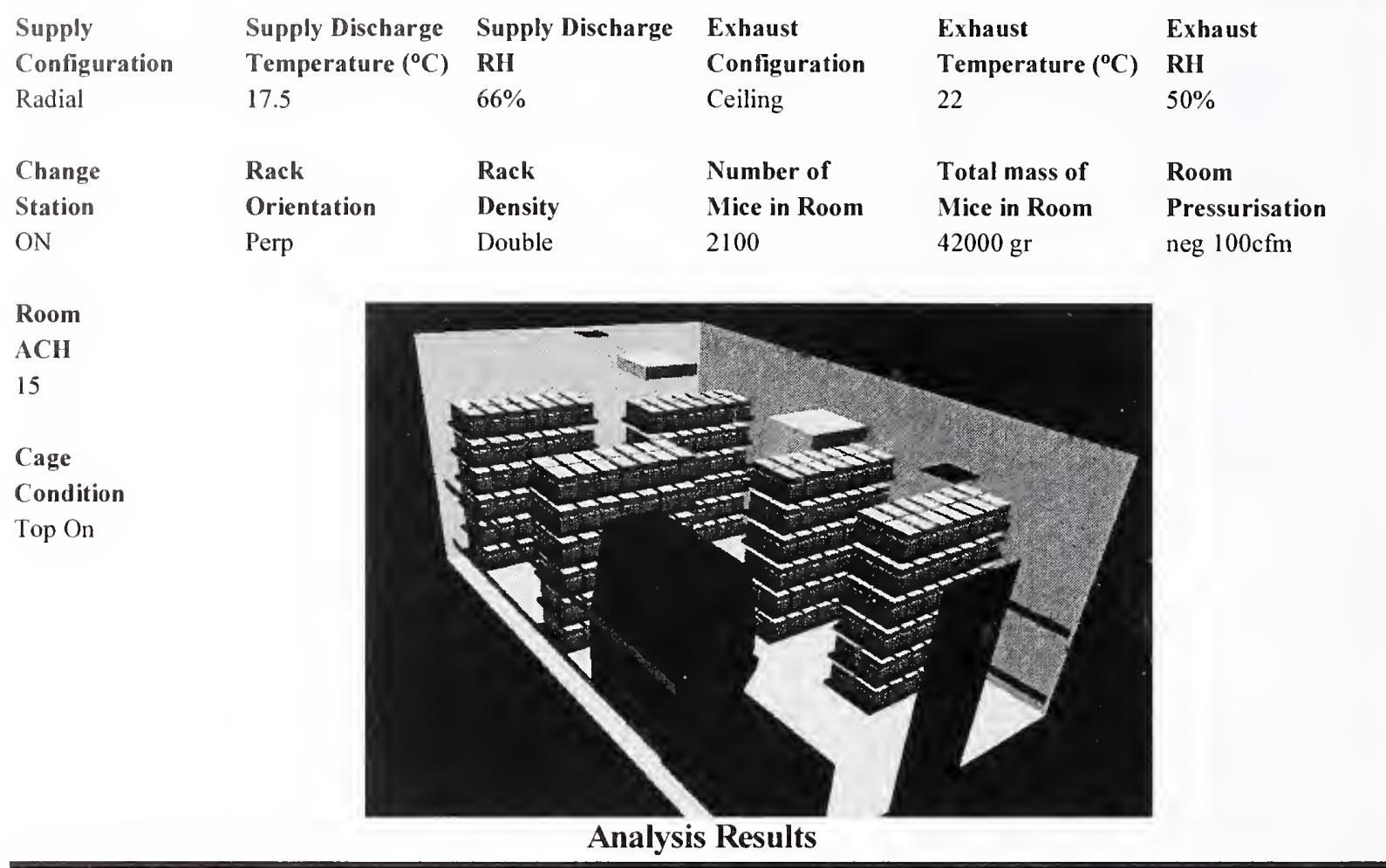

Cage Occupied Zone

\begin{tabular}{|l|cc|c|c|}
\cline { 2 - 5 } \multicolumn{1}{c|}{} & \multicolumn{2}{c|}{ Temperature } & $\mathrm{CO}_{2}$ & RH \\
\cline { 2 - 5 } \multicolumn{1}{c|}{} & ${ }^{\circ} \mathrm{C}$ & ${ }^{\circ} \mathrm{F}$ & $(\mathbf{p p m})$ & \\
\hline Mean & 21.36 & 70.45 & 1896 & $67.24 \%$ \\
\hline S.D. & 0.27 & 0.49 & 374 & $2.62 \%$ \\
\hline Max. & 22.02 & 71.63 & 2689 & $72.42 \%$ \\
\hline
\end{tabular}

Cage Occupied Zone NH3 (ppm)

\begin{tabular}{|l|c|c|c|c|c|c|c|c|c|c|}
\hline Day & $\mathbf{1}$ & $\mathbf{2}$ & $\mathbf{3}$ & $\mathbf{4}$ & $\mathbf{5}$ & $\mathbf{6}$ & $\mathbf{7}$ & $\mathbf{8}$ & $\mathbf{9}$ & $\mathbf{1 0}$ \\
\hline Mean & 1.01 & 1.92 & 2.96 & 5.66 & 9.63 & 14.34 & 21.65 & 26.77 & 31.90 & 34.00 \\
\hline Max. & 1.44 & 2.72 & 4.20 & 8.03 & 13.66 & 20.34 & 30.71 & 37.96 & 45.24 & 48.23 \\
\hline
\end{tabular}

Room Breathing Zone

\begin{tabular}{|l|cc|c|c|}
\cline { 2 - 5 } \multicolumn{1}{c|}{} & \multicolumn{2}{c|}{ Temperature } & $\mathrm{CO}_{2}$ & RH \\
\cline { 2 - 5 } \multicolumn{1}{c|}{} & ${ }^{\circ} \mathrm{C}$ & ${ }^{\circ}$ & $(\mathrm{ppm})$ & \\
\hline Mean & 19.96 & 67.93 & 136 & $57.48 \%$ \\
\hline S.D. & 0.93 & 1.67 & 56 & \\
\hline Max. & 26.86 & 80.35 & 318 & \\
\hline
\end{tabular}

Room Breathing Zone NH3 (ppm)

\begin{tabular}{|l|c|c|c|c|c|c|c|c|c|c|}
\hline Day & $\mathbf{1}$ & $\mathbf{2}$ & $\mathbf{3}$ & $\mathbf{4}$ & $\mathbf{5}$ & $\mathbf{6}$ & $\mathbf{7}$ & $\mathbf{8}$ & $\mathbf{9}$ & $\mathbf{1 0}$ \\
\hline Mean & 0.07 & 0.14 & 0.21 & 0.41 & 0.69 & 1.03 & 1.55 & 1.92 & 2.29 & 2.44 \\
\hline Max. & 0.17 & 0.32 & 0.50 & 0.95 & 1.61 & 2.40 & 3.63 & 4.49 & 5.35 & 5.70 \\
\hline
\end{tabular}




\section{Histogram Distributions}

Cage occupied zone average temperature $\left({ }^{\circ} \mathrm{C}\right)$ distribution

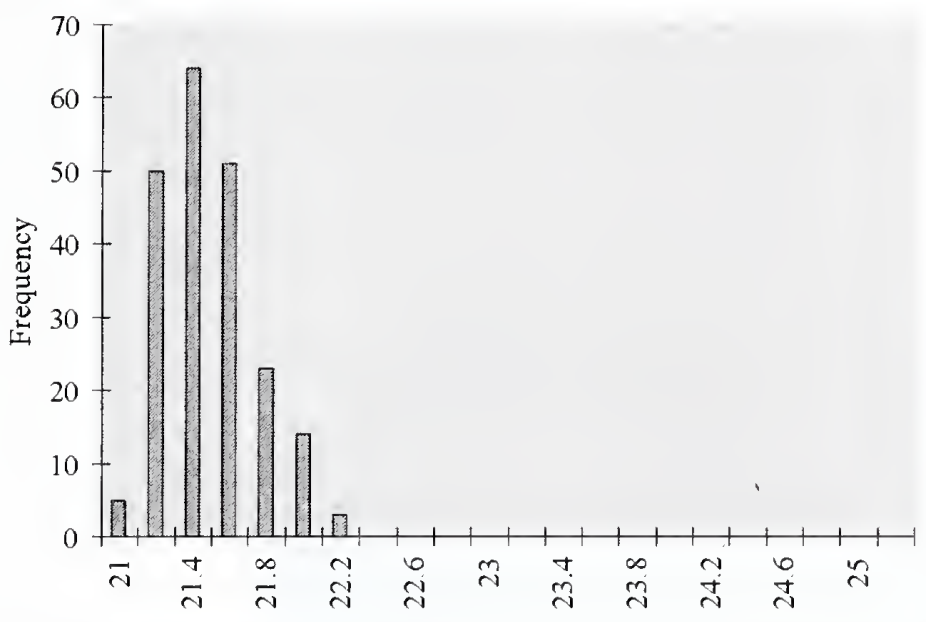

Cage occupied zone average contamination $(\mathrm{kg} / \mathrm{kg})$ distribution

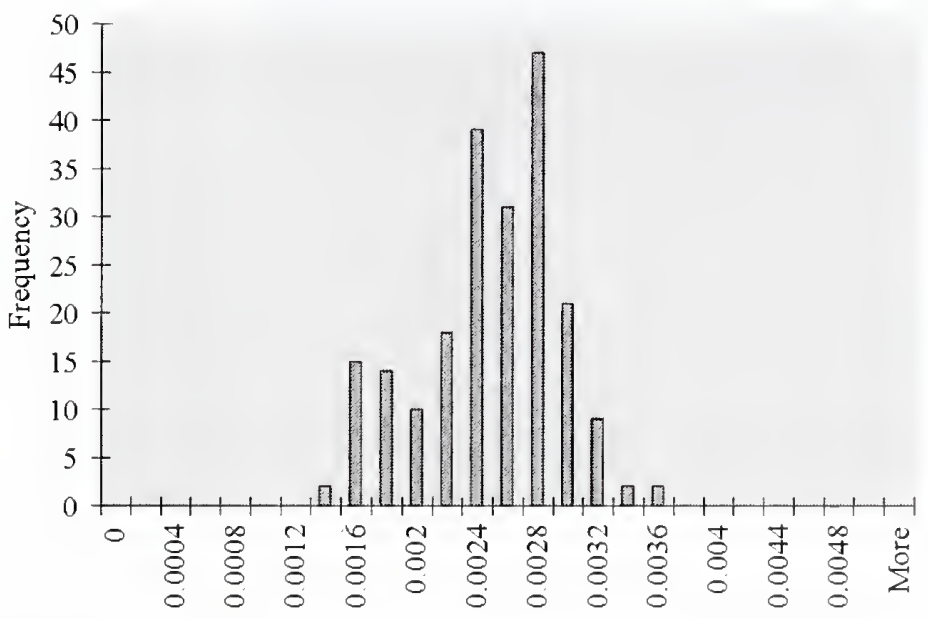

Cage occupied zone average relative humidity (\%) distribution

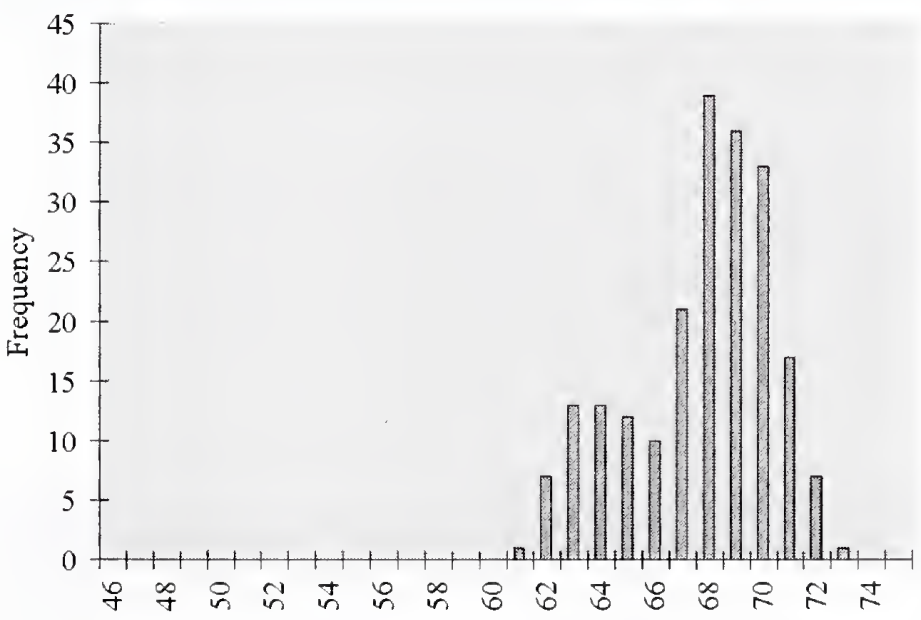

Contamination conversion factors $(\mathrm{kg} / \mathrm{kg} \rightarrow \mathrm{ppm})$

\begin{tabular}{|c|c|c|}
\hline Day & $\mathrm{CO}_{2}$ & $\mathrm{NH}_{3}$ \\
\hline $\mathbf{1}$ & 785000 & 419 \\
\hline 2 & 785000 & 795 \\
\hline $\mathbf{3}$ & 785000 & 1225 \\
\hline $\mathbf{4}$ & 785000 & 2346 \\
\hline $\mathbf{5}$ & 785000 & 3988 \\
\hline $\mathbf{6}$ & 785000 & 5938 \\
\hline 7 & 785000 & 8966 \\
\hline $\mathbf{8}$ & 785000 & 11085 \\
\hline 9 & 785000 & 13210 \\
\hline 10 & 785000 & 11384 \\
\hline
\end{tabular}


Casename

Case 35

Description

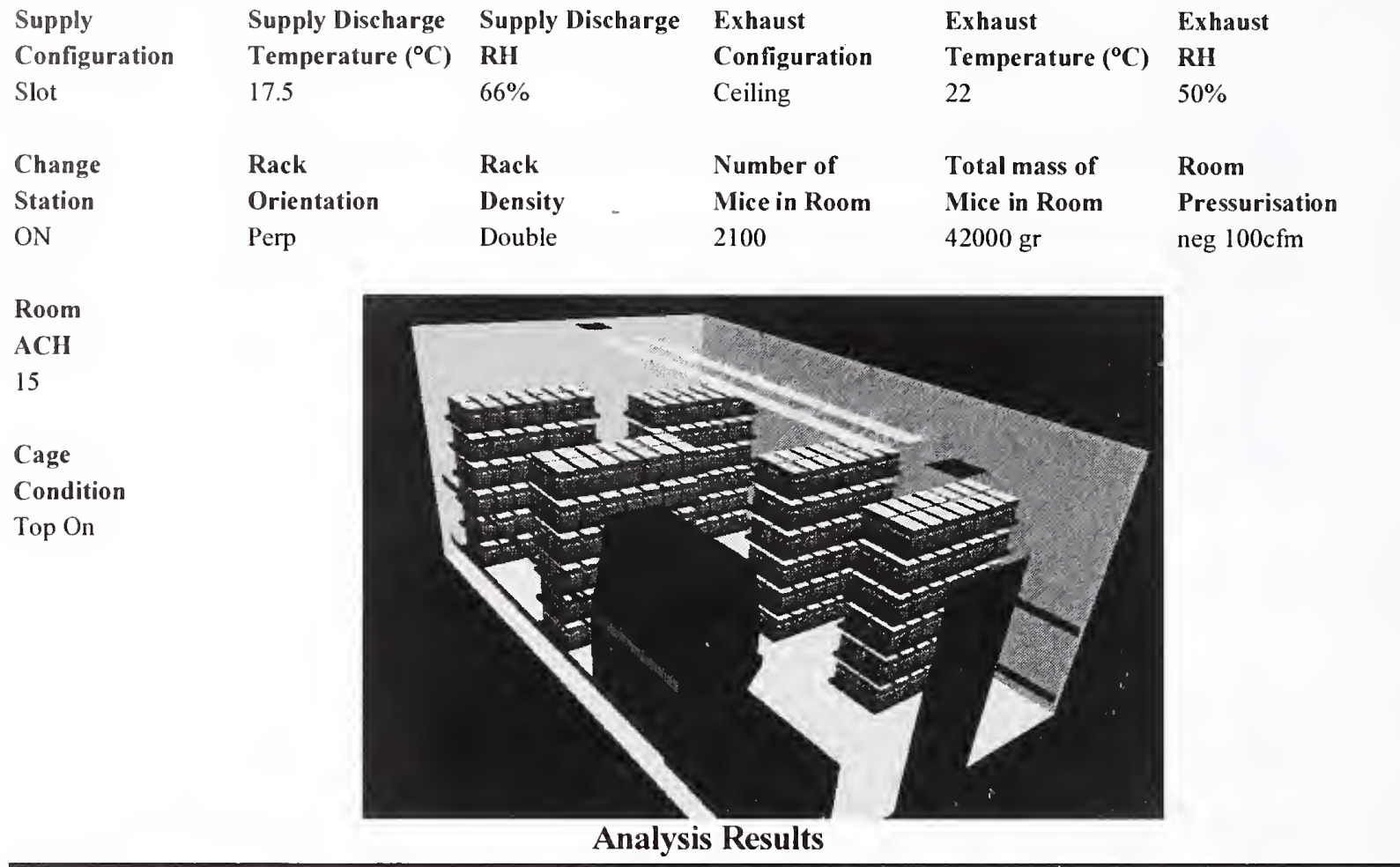

Cage Occupied Zone

\begin{tabular}{|l|cc|c|c|}
\cline { 2 - 5 } \multicolumn{1}{c|}{} & \multicolumn{2}{c|}{ Temperature } & $\mathrm{CO}_{2}$ & \multicolumn{1}{c|}{ RH } \\
\cline { 2 - 5 } \multicolumn{1}{c|}{} & ${ }^{\circ} \mathrm{C}$ & ${ }^{\circ} \mathrm{F}$ & $(\mathrm{ppm})$ & \\
\hline Mean & 21.33 & 70.40 & 1761 & $65.51 \%$ \\
\hline S.D. & 0.47 & 0.84 & 403 & $2.79 \%$ \\
\hline Max. & 22.43 & 72.37 & 2691 & $71.14 \%$ \\
\hline
\end{tabular}

Cage Occupied Zone NH3 (ppm)

\begin{tabular}{|l|c|c|c|c|c|c|c|c|c|c|}
\hline Day & $\mathbf{1}$ & $\mathbf{2}$ & $\mathbf{3}$ & $\mathbf{4}$ & $\mathbf{5}$ & $\mathbf{6}$ & $\mathbf{7}$ & $\mathbf{8}$ & $\mathbf{9}$ & $\mathbf{1 0}$ \\
\hline Mean & 0.93 & 1.78 & 2.75 & 4.90 & 8.01 & 11.77 & 17.46 & 21.92 & 26.67 & 29.69 \\
\hline Max. & 1.43 & 2.72 & 4.20 & 7.49 & 12.24 & 17.99 & 26.70 & 33.51 & 40.76 & 45.38 \\
\hline
\end{tabular}

Room Breathing Zone

\begin{tabular}{|l|cc|c|c|}
\cline { 2 - 5 } \multicolumn{1}{c|}{} & \multicolumn{2}{c|}{ Temperature } & $\mathrm{CO}_{2}$ & RH \\
\cline { 2 - 5 } \multicolumn{1}{c|}{} & ${ }^{\circ} \mathrm{C}$ & ${ }^{\circ} \mathrm{F}$ & $(\mathrm{ppm})$ & \\
\hline Mean & 19.96 & 67.92 & 122 & $57.37 \%$ \\
\hline S.D. & 1.01 & 182 & 59 & \\
\hline Max. & 25.93 & 78.67 & 291 & \\
\hline
\end{tabular}

Room Breathing Zone NH3 (ppm)

\begin{tabular}{|l|c|c|c|c|c|c|c|c|c|c|}
\hline Day & $\mathbf{1}$ & $\mathbf{2}$ & $\mathbf{3}$ & $\mathbf{4}$ & $\mathbf{5}$ & $\mathbf{6}$ & $\mathbf{7}$ & $\mathbf{8}$ & 9 & 10 \\
\hline Mean & 0.06 & 0.12 & 0.19 & 0.34 & 0.56 & 0.82 & 1.21 & 1.52 & 1.85 & 2.06 \\
\hline Max. & 0.15 & 0.29 & 0.45 & 0.81 & 1.32 & 1.95 & 2.89 & 3.63 & 4.41 & 4.91 \\
\hline
\end{tabular}




\section{Histogram Distributions}

Cage occupied zone average temperature $\left({ }^{\circ} \mathrm{C}\right)$ distribution

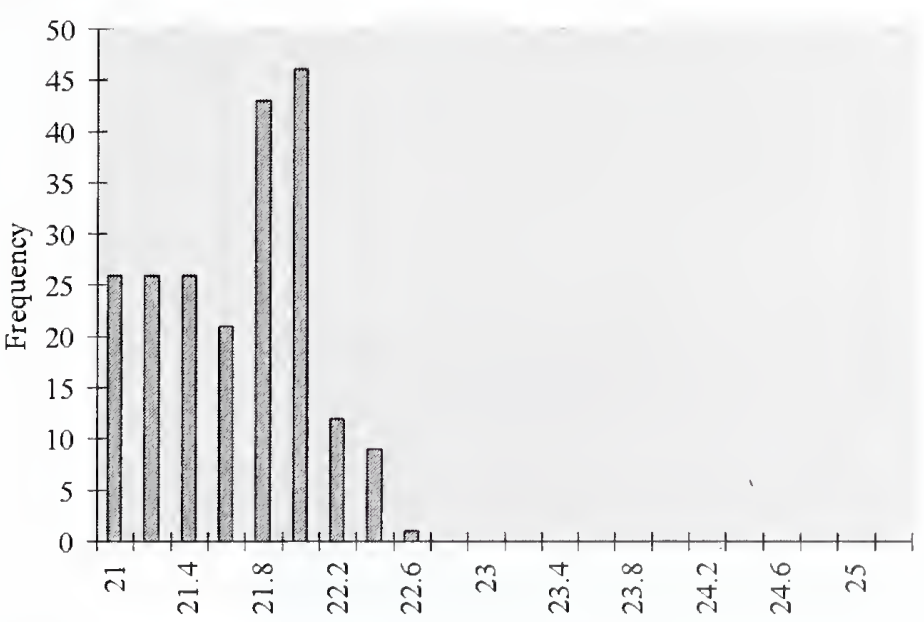

Cage occupied zone average contamination $(\mathrm{kg} / \mathrm{kg})$ distribution

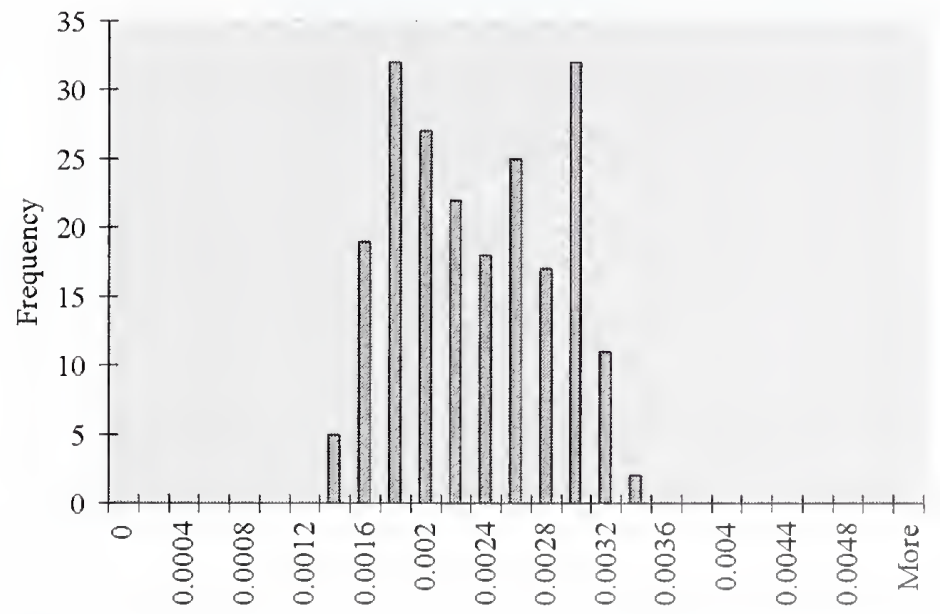

Cage occupied zone average relative humidity (\%) distribution

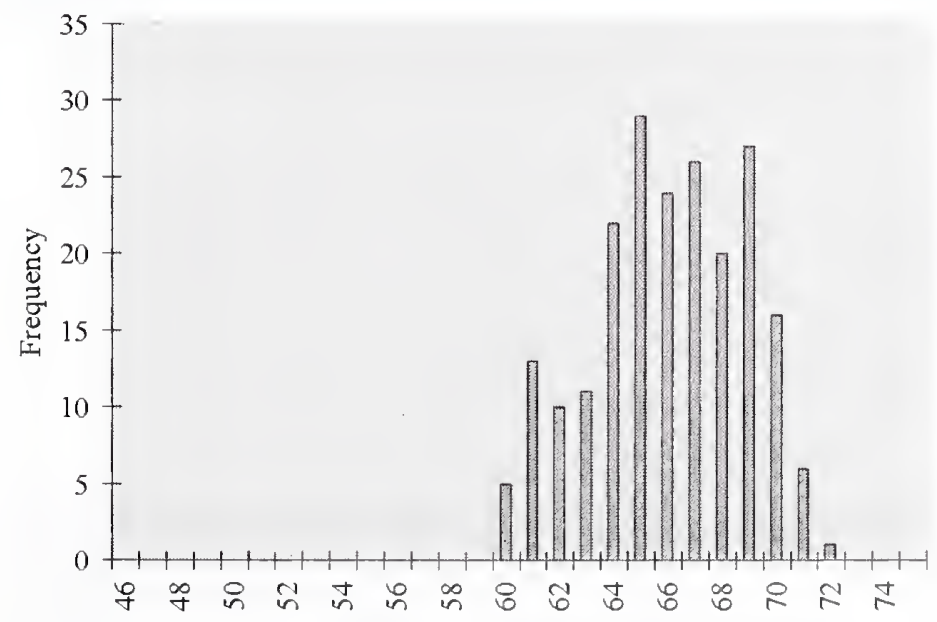

Contamination conversion factors $(\mathrm{kg} / \mathrm{kg} \rightarrow \mathrm{ppm})$

\begin{tabular}{|c|c|c|}
\hline Day & $\mathrm{CO}_{2}$ & $\mathrm{NH}_{3}$ \\
\hline 1 & 785000 & 416 \\
\hline 2 & 785000 & 795 \\
\hline 3 & 785000 & 1225 \\
\hline 4 & 785000 & 2185 \\
\hline 5 & 785000 & 3571 \\
\hline 6 & 785000 & 5246 \\
\hline 7 & 785000 & 7787 \\
\hline 8 & 785000 & 9774 \\
\hline 9 & 785000 & 11889 \\
\hline 10 & 785000 & 11384 \\
\hline
\end{tabular}


Casename

Case 36

Description

\begin{tabular}{|c|c|c|c|c|c|}
\hline Supply & Supply Discharge & Supply Discharge & Exhaust & Exhaust & Exhaust \\
\hline Configuration & Temperature $\left({ }^{\circ} \mathrm{C}\right)$ & RH & Configuration & Temperature $\left({ }^{\circ} \mathrm{C}\right)$ & RH \\
\hline Low Ind & 17.5 & $66 \%$ & Ceiling & 22 & $50 \%$ \\
\hline Change & Rack & Rack & Number of & Total mass of & Room \\
\hline Station & Orientation & Density & Mice in Room & Mice in Room & Pressurisation \\
\hline ON & Perp & Double & 2100 & $42000 \mathrm{gr}$ & neg $100 \mathrm{cfm}$ \\
\hline \multicolumn{6}{|l|}{ Room } \\
\hline \multicolumn{6}{|l|}{$\mathbf{A C H}$} \\
\hline \multicolumn{6}{|l|}{15} \\
\hline \multicolumn{6}{|l|}{ Cage } \\
\hline \multicolumn{6}{|l|}{ Condition } \\
\hline \multicolumn{6}{|l|}{ Top On } \\
\hline
\end{tabular}

Cage Occupied Zone

\begin{tabular}{|l|cc|c|c|}
\cline { 2 - 5 } \multicolumn{1}{c|}{} & \multicolumn{2}{c|}{ Temperature } & $\mathrm{CO}_{2}$ & RH \\
\cline { 2 - 5 } \multicolumn{1}{c|}{} & ${ }^{\circ} \mathrm{C}$ & \multicolumn{1}{c|}{} & $(\mathrm{ppm})$ & \\
\hline Mean & 21.47 & 70.64 & 1878 & $66.47 \%$ \\
\hline S.D. & 0.27 & 0.49 & 351 & $2.61 \%$ \\
\hline Max. & 22.20 & 71.97 & 2594 & $71.66 \%$ \\
\hline
\end{tabular}

Cage Occupied Zone NH3 (ppm)

\begin{tabular}{|l|c|c|c|c|c|c|c|c|c|c|}
\hline Day & $\mathbf{1}$ & $\mathbf{2}$ & $\mathbf{3}$ & $\mathbf{4}$ & $\mathbf{5}$ & $\mathbf{6}$ & $\mathbf{7}$ & $\mathbf{8}$ & $\mathbf{9}$ & $\mathbf{1 0}$ \\
\hline Mean & 100 & 1.90 & 2.93 & 5.44 & 9.09 & 13.47 & 20.19 & 25.12 & 30.19 & 32.78 \\
\hline Max. & 1.38 & 2.63 & 4.05 & 7.51 & 12.56 & 1860 & 2788 & 34.69 & 41.70 & 45.28 \\
\hline
\end{tabular}

Room Breathing Zone

\begin{tabular}{|l|cc|c|c|}
\cline { 2 - 5 } \multicolumn{1}{c|}{} & \multicolumn{2}{c|}{ Temperature } & $\mathbf{C O}_{2}$ & RH \\
\cline { 2 - 5 } \multicolumn{1}{c|}{} & ${ }^{\circ} \mathrm{C}$ & ${ }^{\circ} \mathrm{F}$ & $(\mathbf{p p m})$ & \\
\hline Mean & 19.90 & 67.81 & 134 & $57.69 \%$ \\
\hline S.D. & 0.97 & 1.74 & 59 & \\
\hline Max. & 25.63 & 78.13 & 303 & \\
\hline
\end{tabular}

Room Breathing Zone NH3 (ppm)

\begin{tabular}{|l|c|c|c|c|c|c|c|c|c|c|}
\hline Day & $\mathbf{1}$ & $\mathbf{2}$ & $\mathbf{3}$ & $\mathbf{4}$ & $\mathbf{5}$ & $\mathbf{6}$ & $\mathbf{7}$ & $\mathbf{8}$ & $\mathbf{9}$ & $\mathbf{1 0}$ \\
\hline Mean & 0.07 & 0.14 & 0.21 & 0.39 & 0.65 & 0.96 & 1.44 & 1.79 & 2.15 & 2.34 \\
\hline Max. & 0.16 & 0.31 & 0.47 & 0.88 & 1.47 & 2.18 & 3.26 & 4.06 & 4.88 & 530 \\
\hline
\end{tabular}




\section{Histogram Distributions}

Cage occupied zone average temperature $\left({ }^{\circ} \mathrm{C}\right)$ distribution

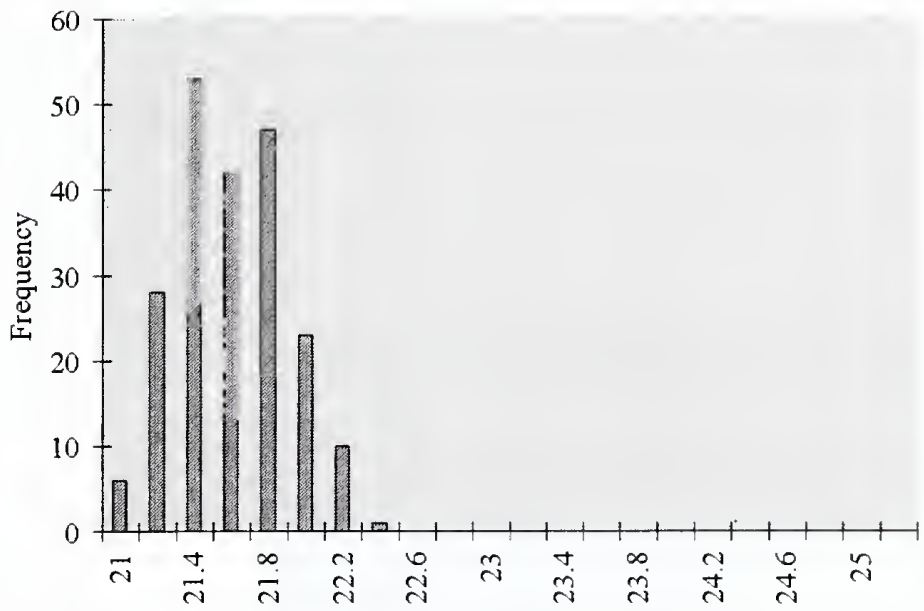

Cage occupied zone average contamination $(\mathrm{kg} / \mathrm{kg})$ distribution

Contamination conversion factors $(\mathrm{kg} / \mathrm{kg} \rightarrow \mathrm{ppm})$

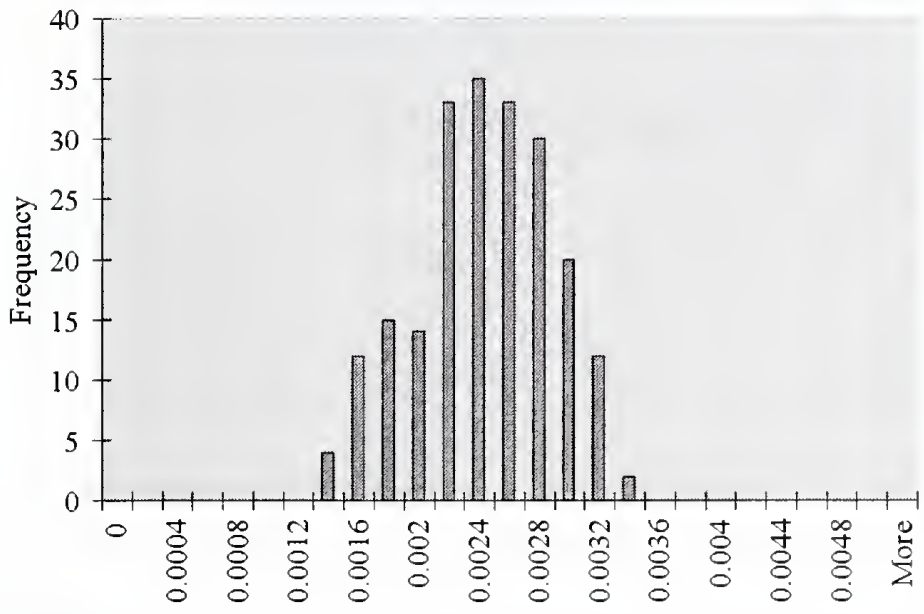

\begin{tabular}{|c|c|c|}
\hline Day & $\mathbf{C O}_{2}$ & $\mathbf{N H}_{3}$ \\
\hline $\mathbf{I}$ & 785000 & 418 \\
\hline $\mathbf{2}$ & 785000 & 795 \\
\hline $\mathbf{3}$ & 785000 & 1225 \\
\hline $\mathbf{4}$ & 785000 & 2274 \\
\hline $\mathbf{5}$ & 785000 & 3802 \\
\hline $\mathbf{6}$ & 785000 & 5629 \\
\hline 7 & 785000 & 8439 \\
\hline $\mathbf{8}$ & 785000 & 10500 \\
\hline $\mathbf{9}$ & 785000 & 12620 \\
\hline $\mathbf{1 0}$ & 785000 & 11384 \\
\hline
\end{tabular}

Cage occupied zone average relative humidity (\%) distribution

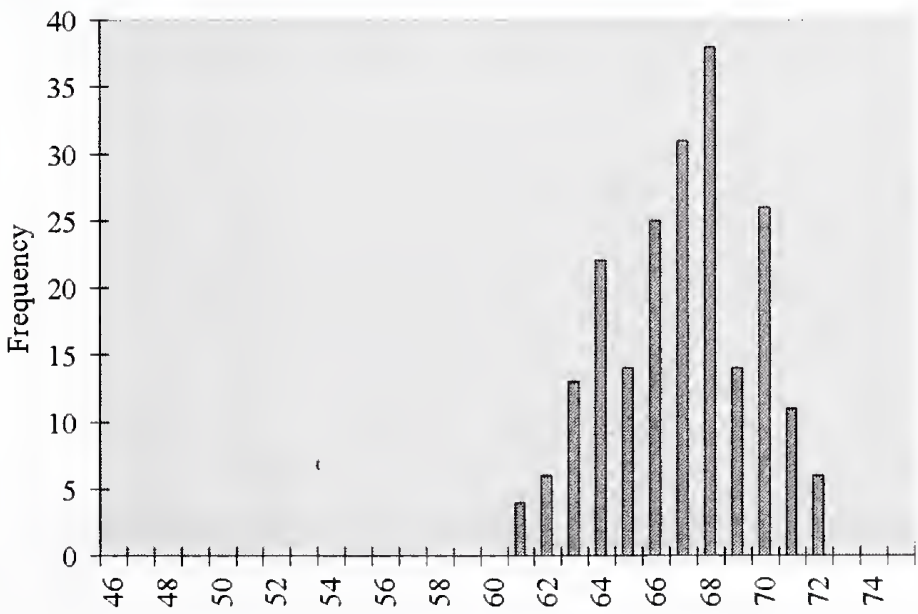


Casename

Case 37

\section{Description}

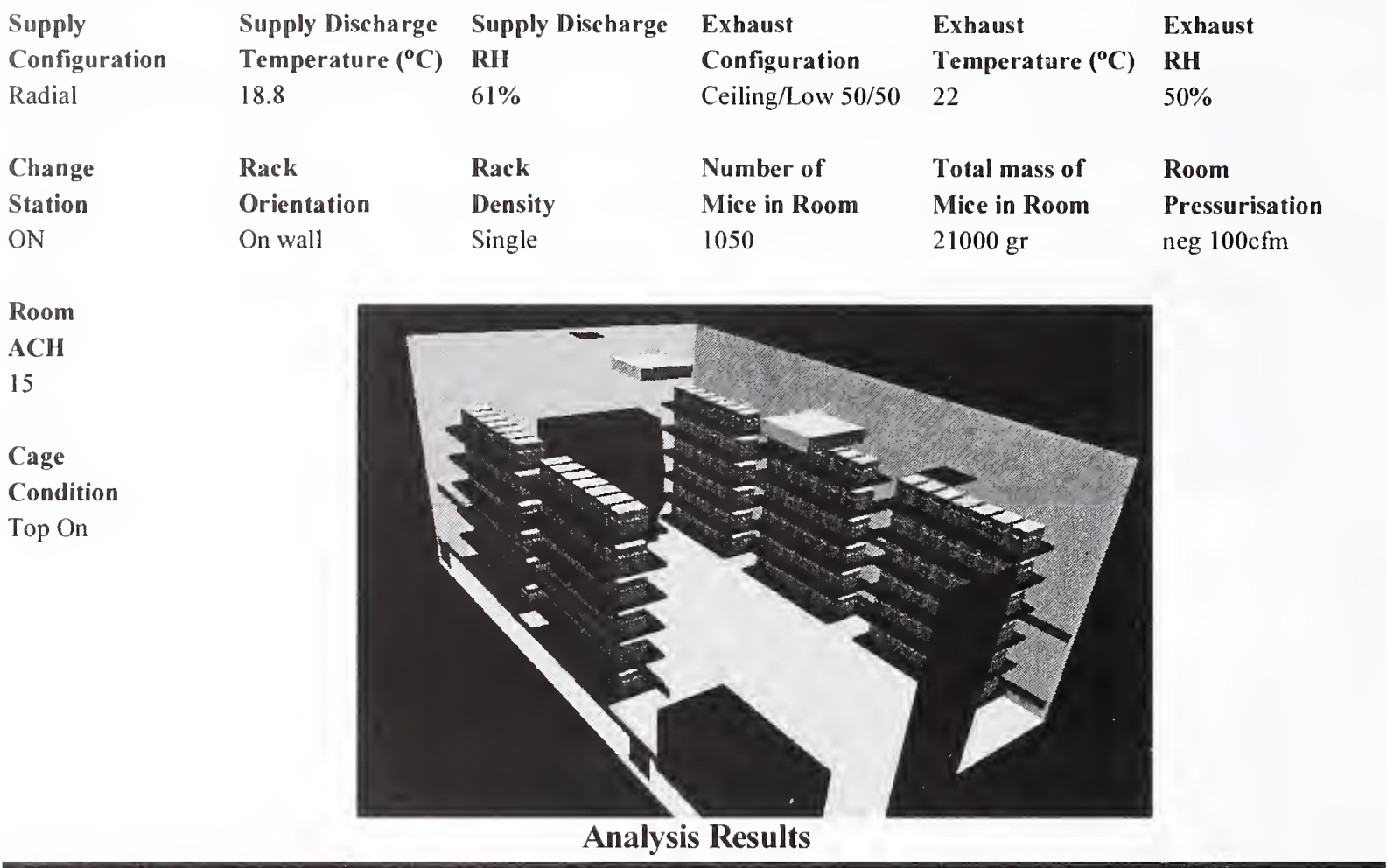

Cage Occupied Zone

\begin{tabular}{|l|cc|c|c|}
\cline { 2 - 5 } \multicolumn{1}{c|}{} & \multicolumn{2}{c|}{ Temperature } & $\mathrm{CO}_{2}$ & RH \\
\cline { 2 - 5 } \multicolumn{1}{c|}{} & ${ }^{\circ} \mathrm{C}$ & ${ }^{\circ}$ & $(\mathbf{p p m})$ & \\
\hline Mean & 22.27 & 72.08 & 1715 & $62.35 \%$ \\
\hline S.D. & 0.24 & 0.44 & 302 & $2.42 \%$ \\
\hline Max. & 23.04 & 73.48 & 2244 & $66.63 \%$ \\
\hline
\end{tabular}

Cage Occupied Zone NH3 (ppm)

\begin{tabular}{|l|c|c|c|c|c|c|c|c|c|c|}
\hline Day & $\mathbf{1}$ & $\mathbf{2}$ & $\mathbf{3}$ & $\mathbf{4}$ & $\mathbf{5}$ & $\mathbf{6}$ & $\mathbf{7}$ & $\mathbf{8}$ & $\mathbf{9}$ & $\mathbf{1 0}$ \\
\hline Mean & 0.90 & 1.74 & 2.68 & 4.13 & 6.14 & 8.70 & 12.31 & 16.12 & 20.70 & 25.55 \\
\hline Max. & 1.17 & 2.27 & 3.50 & 5.41 & 8.03 & 11.38 & 16.10 & 21.09 & 27.08 & 33.42 \\
\hline
\end{tabular}

Room Breathing Zone

\begin{tabular}{|l|cc|c|c|}
\cline { 2 - 5 } \multicolumn{1}{c|}{} & \multicolumn{2}{c|}{ Temperature } & $\mathbf{C O}_{2}$ & RH \\
\cline { 2 - 5 } \multicolumn{1}{c|}{} & ${ }^{\circ} \mathrm{C}$ & ${ }^{\circ}$ & $(\mathbf{p p m})$ & \\
\hline Mean & 20.70 & 69.25 & 59 & $54.16 \%$ \\
\hline S.D. & 0.22 & 0.40 & 26 & \\
\hline Max. & 21.76 & 71.16 & 178 & \\
\hline
\end{tabular}

Room Breathing Zone NH3 (ppm)

\begin{tabular}{|l|c|c|c|c|c|c|c|c|c|c|}
\hline Day & 1 & 2 & 3 & 4 & 5 & 6 & 7 & 8 & 9 & 10 \\
\hline Mean & 0.03 & 0.06 & 0.09 & 0.14 & 0.21 & 0.30 & 0.42 & 0.55 & 0.71 & 0.87 \\
\hline Max. & 0.09 & 0.18 & 0.28 & 0.43 & 0.64 & 0.90 & 1.28 & 1.68 & 2.15 & 2.66 \\
\hline
\end{tabular}




\section{Histogram Distributions}

Cage occupied zone average temperature $\left({ }^{\circ} \mathrm{C}\right)$ distribution

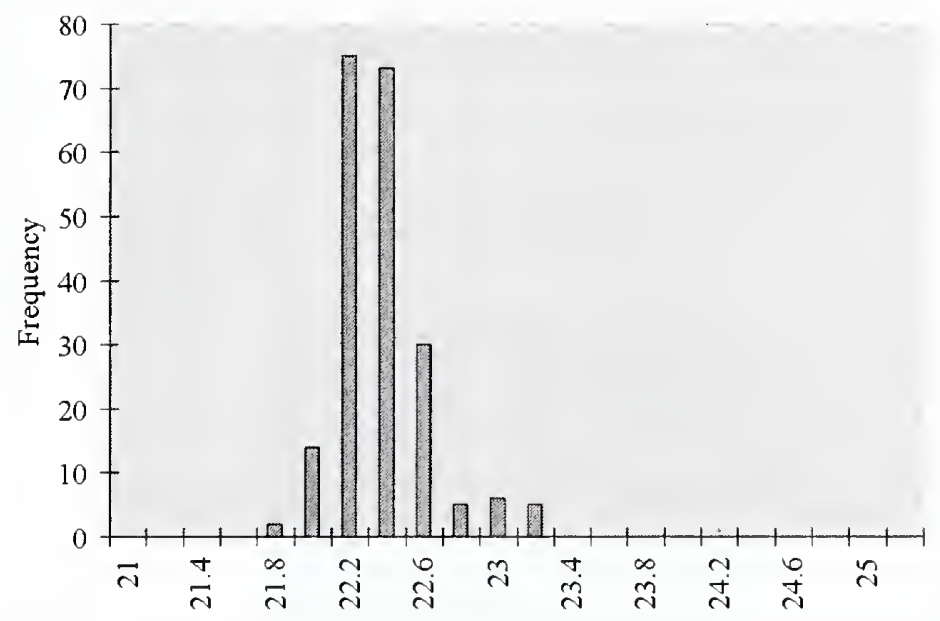

Cage occupied zone average contamination $(\mathrm{kg} / \mathrm{kg})$ distribution Contamination conversion factors $(\mathrm{kg} / \mathrm{kg} \rightarrow \mathrm{ppm})$

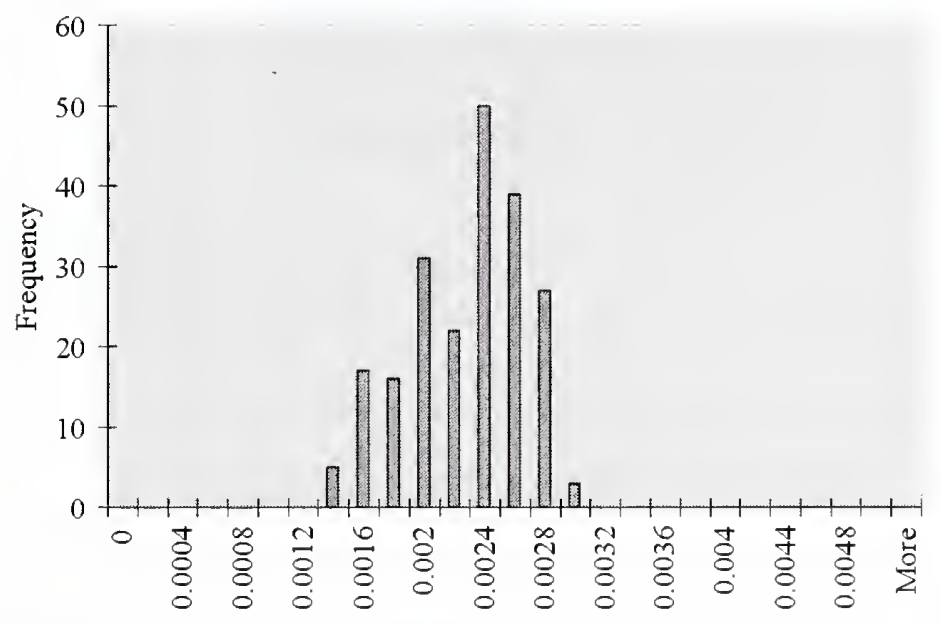

\begin{tabular}{|c|c|c|}
\hline Day & $\mathrm{CO}_{2}$ & $\mathrm{NH}_{3}$ \\
\hline $\mathbf{1}$ & 785000 & 411 \\
\hline $\mathbf{2}$ & 785000 & 795 \\
\hline $\mathbf{3}$ & 785000 & 1225 \\
\hline $\mathbf{4}$ & 785000 & 1891 \\
\hline $\mathbf{5}$ & 785000 & 2808 \\
\hline $\mathbf{6}$ & 785000 & 3983 \\
\hline 7 & 785000 & 5632 \\
\hline $\mathbf{8}$ & 785000 & 7379 \\
\hline 9 & 785000 & 9473 \\
\hline 10 & 785000 & 11384 \\
\hline
\end{tabular}

Cage occupied zone average relative humidity (\%) distribution

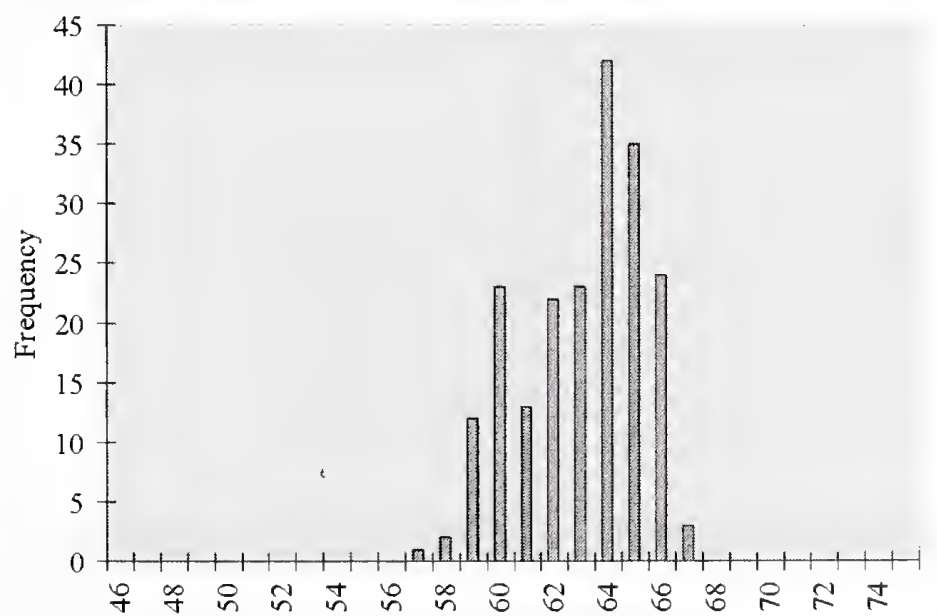


Casename $\quad$ Case 38

\section{Description}

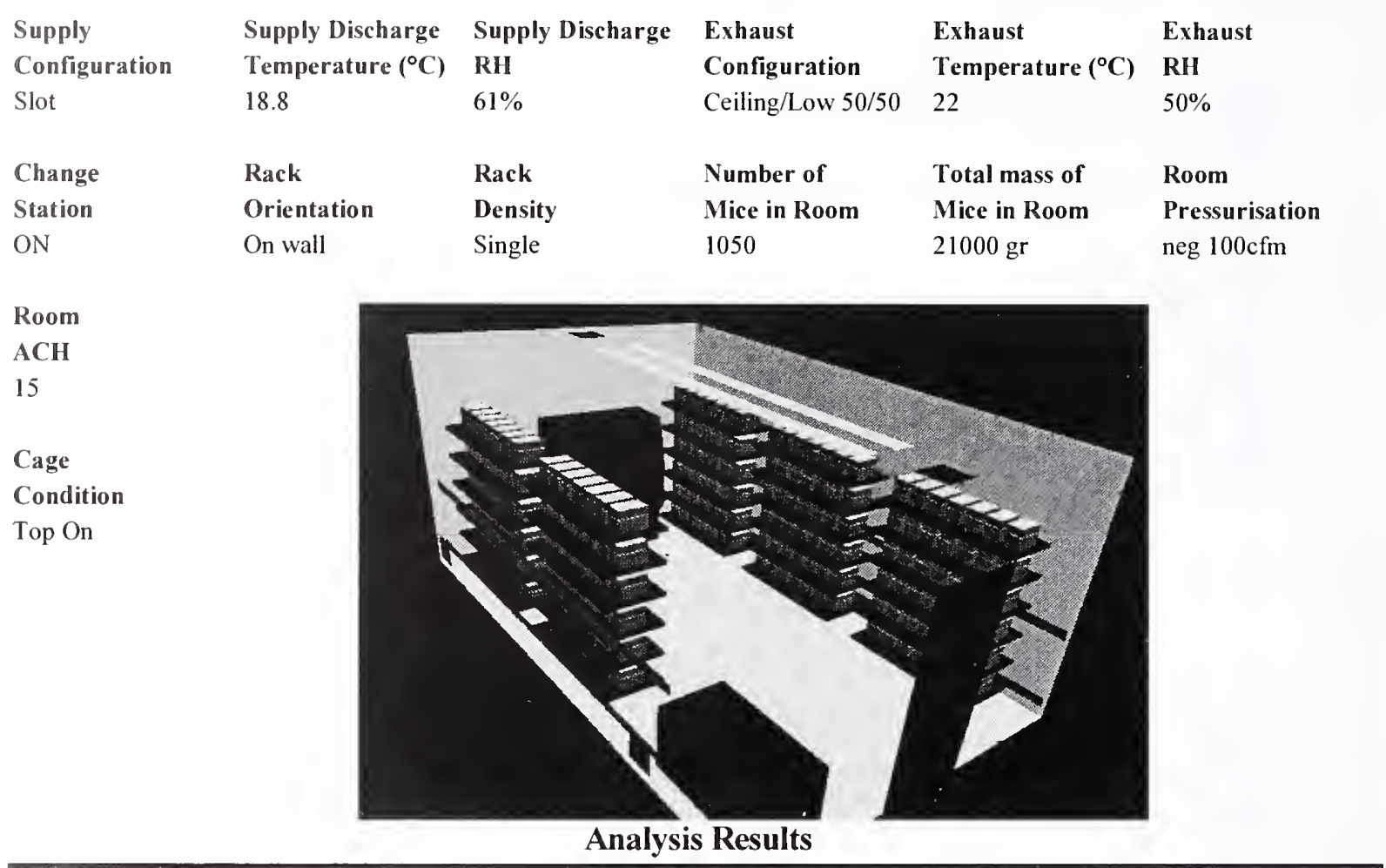

Cage Occupied Zone

\begin{tabular}{|l|cc|c|c|}
\cline { 2 - 5 } \multicolumn{1}{c|}{} & \multicolumn{2}{c|}{ Temperature } & $\mathrm{CO}_{2}$ & $\mathrm{RH}$ \\
\cline { 2 - 5 } \multicolumn{1}{c|}{} & ${ }^{\circ} \mathrm{C}$ & ${ }^{\circ} \mathrm{F}$ & $(\mathrm{ppm})$ & \\
\hline Mean & 22.39 & 72.29 & 1776 & $62.36 \%$ \\
\hline S.D. & 0.29 & 0.53 & 342 & $2.50 \%$ \\
\hline Max. & 23.31 & 73.96 & 2666 & $68.62 \%$ \\
\hline
\end{tabular}

Cage Occupied Zone NH3 (ppm)

\begin{tabular}{|l|c|c|c|c|c|c|c|c|c|c|}
\hline Day & $\mathbf{1}$ & $\mathbf{2}$ & $\mathbf{3}$ & $\mathbf{4}$ & $\mathbf{5}$ & $\mathbf{6}$ & $\mathbf{7}$ & $\mathbf{8}$ & $\mathbf{9}$ & $\mathbf{1 0}$ \\
\hline Mean & 0.93 & 1.80 & 2.77 & 4.28 & 6.36 & 9.02 & 12.75 & 16.71 & 21.45 & 26.46 \\
\hline Max. & 1.39 & 2.70 & 4.16 & 6.42 & 9.54 & 13.54 & 19.15 & 25.08 & 32.19 & 39.73 \\
\hline
\end{tabular}

Room Breathing Zone

\begin{tabular}{|l|cc|c|c|}
\cline { 2 - 5 } \multicolumn{1}{c|}{} & \multicolumn{2}{c|}{ Temperature } & $\mathrm{CO}_{2}$ & RH \\
\cline { 2 - 5 } \multicolumn{1}{c|}{} & ${ }^{\circ} \mathrm{C}$ & ${ }^{\circ} \mathrm{F}$ & $(\mathrm{ppm})$ & \\
\hline Mean & 20.77 & 69.38 & 62 & $53.95 \%$ \\
\hline S.D. & 0.26 & 0.47 & 21 & \\
\hline Max. & 22.03 & 71.66 & 197 & \\
\hline
\end{tabular}

Room Breathing Zone NH3 (ppm)

\begin{tabular}{|l|c|c|c|c|c|c|c|c|c|c|}
\hline Day & $\mathbf{1}$ & $\mathbf{2}$ & $\mathbf{3}$ & $\mathbf{4}$ & $\mathbf{5}$ & $\mathbf{6}$ & $\mathbf{7}$ & $\mathbf{8}$ & $\mathbf{9}$ & $\mathbf{1 0}$ \\
\hline Mean & 0.03 & 0.06 & 0.10 & 0.15 & 0.22 & 0.32 & 0.45 & 0.59 & 0.75 & 0.93 \\
\hline Max. & 0.10 & 0.20 & 0.31 & 0.47 & 0.70 & 1.00 & 1.41 & 1.85 & 2.38 & 2.93 \\
\hline
\end{tabular}




\section{Histogram Distributions}

Cage occupied zone average temperature $\left({ }^{\circ} \mathrm{C}\right)$ distribution

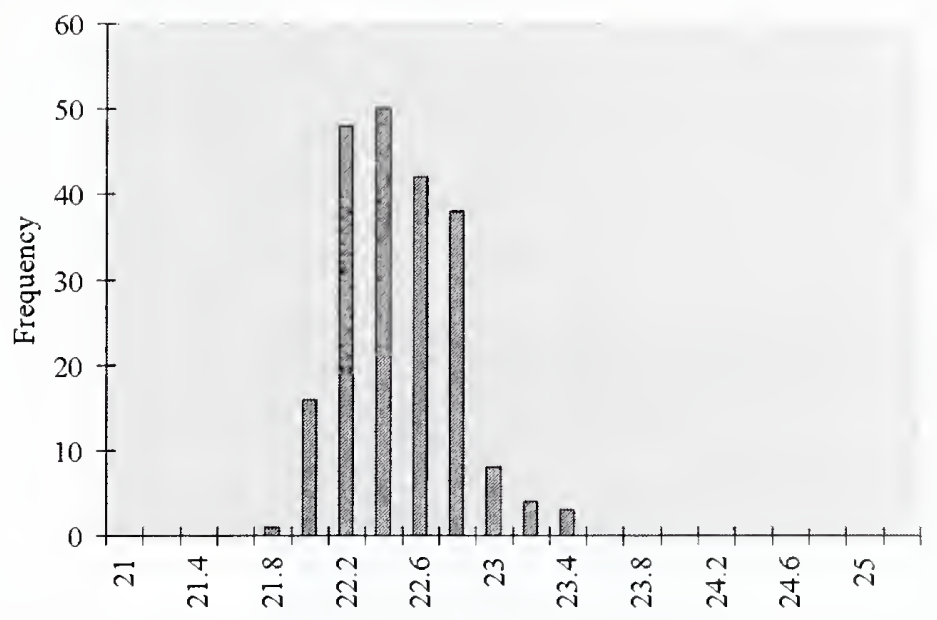

Cage occupied zone average contamination $(\mathrm{kg} / \mathrm{kg})$ distribution Contamination conversion factors $(\mathrm{kg} / \mathrm{kg} \rightarrow \mathrm{ppm})$

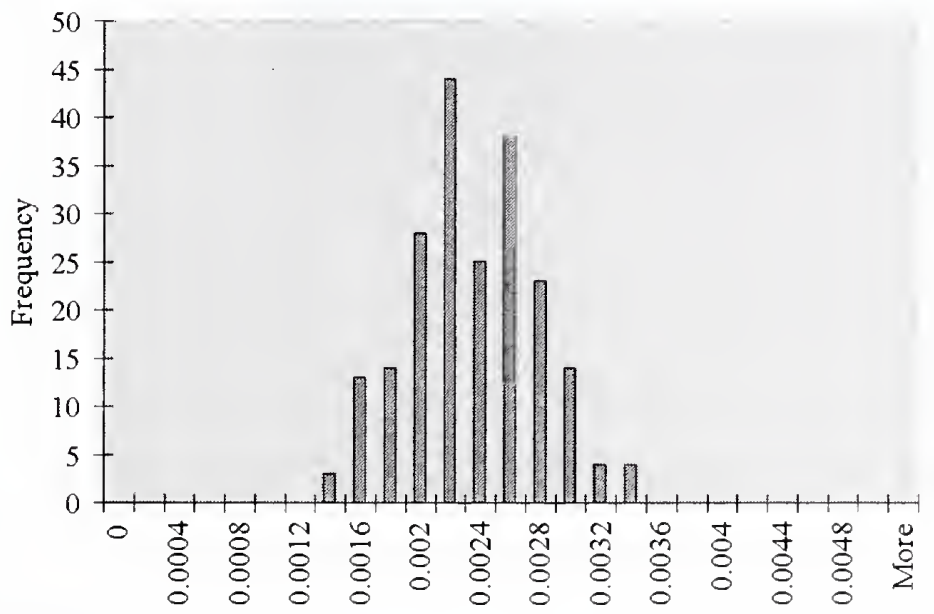

\begin{tabular}{|c|c|c|}
\hline Day & $\mathbf{C O}_{2}$ & $\mathrm{NH}_{3}$ \\
\hline $\mathbf{1}$ & 785000 & 411 \\
\hline $\mathbf{2}$ & 785000 & 795 \\
\hline $\mathbf{3}$ & 785000 & 1225 \\
\hline $\mathbf{4}$ & 785000 & 1892 \\
\hline $\mathbf{5}$ & 785000 & 2811 \\
\hline $\mathbf{6}$ & 785000 & 3986 \\
\hline 7 & 785000 & 5639 \\
\hline $\mathbf{8}$ & 785000 & 7386 \\
\hline $\mathbf{9}$ & 785000 & 9481 \\
\hline 10 & 785000 & 11384 \\
\hline
\end{tabular}

Cage occupied zone average relative humidity (\%) distribution

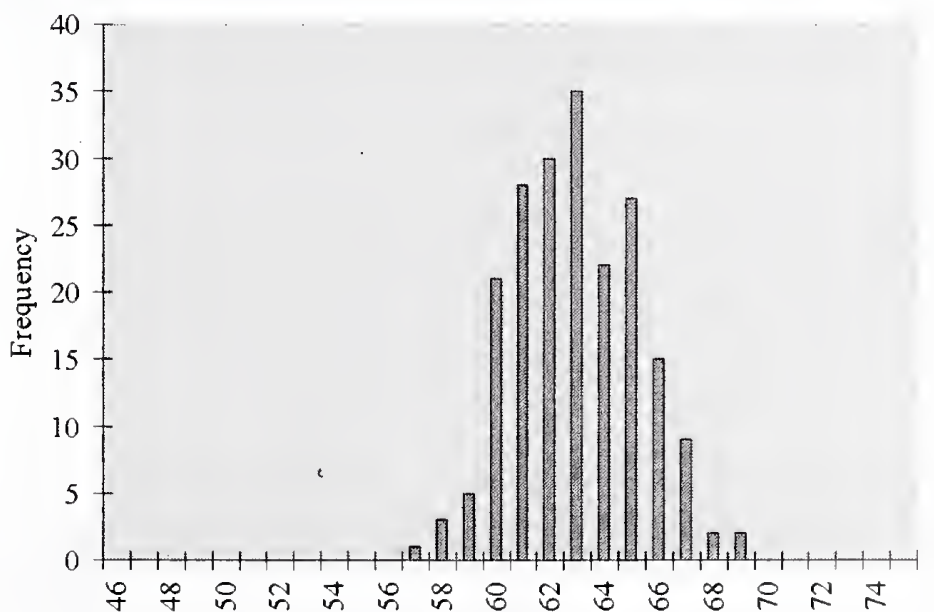


Casename

Case 39

Description

\begin{tabular}{|c|c|c|c|c|c|}
\hline Supply & Supply Discharge & Supply Discharge & Exhaust & Exhaust & Exhaust \\
\hline Configuration & Temperature $\left({ }^{\circ} \mathrm{C}\right)$ & $\mathbf{R H}$ & Configuration & Temperature $\left({ }^{\circ} \mathrm{C}\right)$ & RH \\
\hline Low Ind & 18.8 & $61 \%$ & Ceiling/Low $50 / 50$ & 22 & $50 \%$ \\
\hline Change & Rack & Rack & Number of & Total mass of & Room \\
\hline Station & Orientation & Density & Mice in Room & Mice in Room & Pressurisation \\
\hline $\mathrm{ON}$ & On wall & Single & 1050 & $21000 \mathrm{gr}$ & neg $100 \mathrm{cfm}$ \\
\hline
\end{tabular}

Room

ACH

15

Cage

Condition

Top On

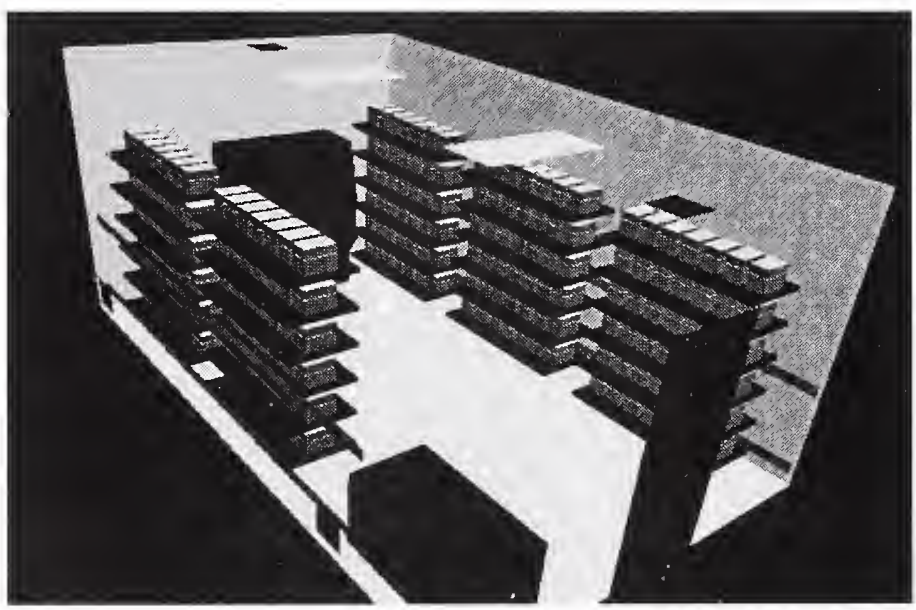

Analysis Results

\section{Cage Occupied Zone}

\begin{tabular}{|l|cc|c|c|}
\cline { 2 - 5 } \multicolumn{1}{c|}{} & \multicolumn{2}{c|}{ Temperature } & $\mathrm{CO}_{2}$ & RH \\
\cline { 2 - 5 } \multicolumn{1}{c|}{} & ${ }^{\circ} \mathrm{C}$ & ${ }^{\circ} \mathrm{F}$ & $(\mathrm{ppm})$ & \\
\hline Mean & 22.39 & 72.30 & 1848 & $62.92 \%$ \\
\hline S.D. & 0.26 & 0.47 & 331 & $2.51 \%$ \\
\hline Max. & 23.14 & 73.65 & 2684 & $68.59 \%$ \\
\hline
\end{tabular}

Cage Occupied Zone NH3 (ppm)

\begin{tabular}{|l|c|c|c|c|c|c|c|c|c|c|}
\hline Day & $\mathbf{1}$ & $\mathbf{2}$ & $\mathbf{3}$ & $\mathbf{4}$ & $\mathbf{5}$ & $\mathbf{6}$ & $\mathbf{7}$ & $\mathbf{8}$ & $\mathbf{9}$ & $\mathbf{1 0}$ \\
\hline Mean & 0.97 & 1.87 & 2.88 & 4.58 & 6.94 & 9.92 & 14.18 & 18.39 & 23.33 & 28.19 \\
\hline Max. & 1.41 & 2.72 & 4.19 & 6.65 & 10.08 & 14.40 & 20.59 & 26.72 & 33.89 & 40.94 \\
\hline
\end{tabular}

Room Breathing Zone

\begin{tabular}{|l|cc|c|c|}
\cline { 2 - 5 } \multicolumn{1}{c|}{} & \multicolumn{2}{c|}{ Temperature } & $\mathrm{CO}_{2}$ & RH \\
\cline { 2 - 5 } \multicolumn{1}{c|}{} & ${ }^{\circ} \mathrm{C}$ & ${ }^{\circ} \mathrm{F}$ & $(\mathrm{ppm})$ & \\
\hline Mean & 20.77 & 69.38 & 71 & $54.02 \%$ \\
\hline S.D. & 0.33 & 0.60 & 31 & \\
\hline Max. & 22.24 & 72.03 & 249 & \\
\hline
\end{tabular}

Room Breathing Zone NH3 (ppm)

\begin{tabular}{|l|c|c|c|c|c|c|c|c|c|c|}
\hline Day & $\mathbf{1}$ & $\mathbf{2}$ & $\mathbf{3}$ & $\mathbf{4}$ & $\mathbf{5}$ & $\mathbf{6}$ & $\mathbf{7}$ & $\mathbf{8}$ & $\mathbf{9}$ & $\mathbf{1 0}$ \\
\hline Mean & 0.04 & 0.07 & 0.11 & 0.18 & 0.27 & 0.38 & 0.55 & 0.71 & 0.90 & 1.08 \\
\hline Max. & 0.13 & 0.25 & 0.39 & 0.62 & 0.94 & 1.34 & 1.91 & 2.48 & 3.15 & 3.80 \\
\hline
\end{tabular}




\section{Histogram Distributions}

Cage occupied zone average temperature $\left({ }^{\circ} \mathrm{C}\right)$ distribution

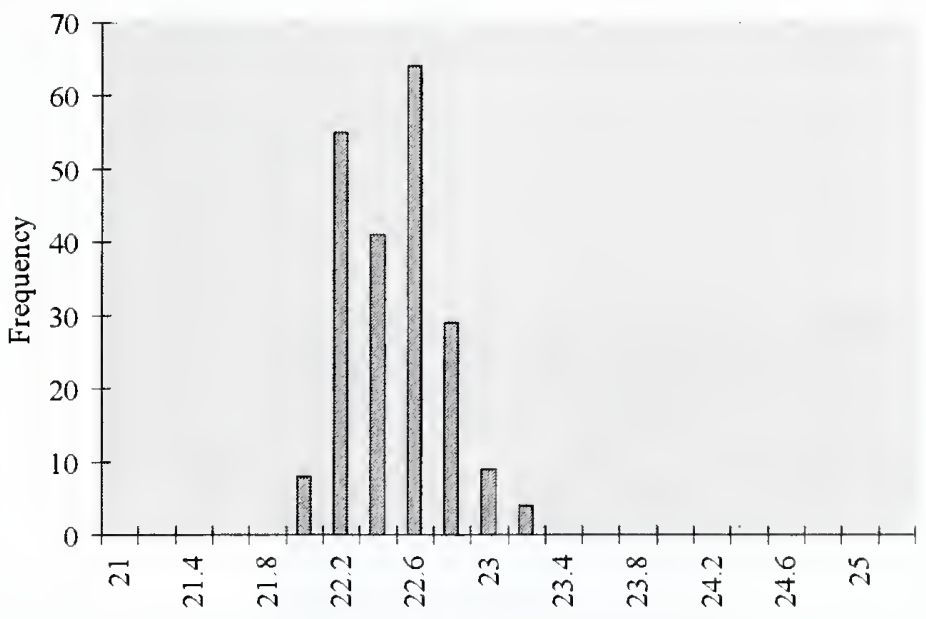

Cage occupied zone average contamination $(\mathrm{kg} / \mathrm{kg})$ distribution

Contamination conversion factors $(\mathrm{kg} / \mathrm{kg} \rightarrow \mathrm{ppm})$

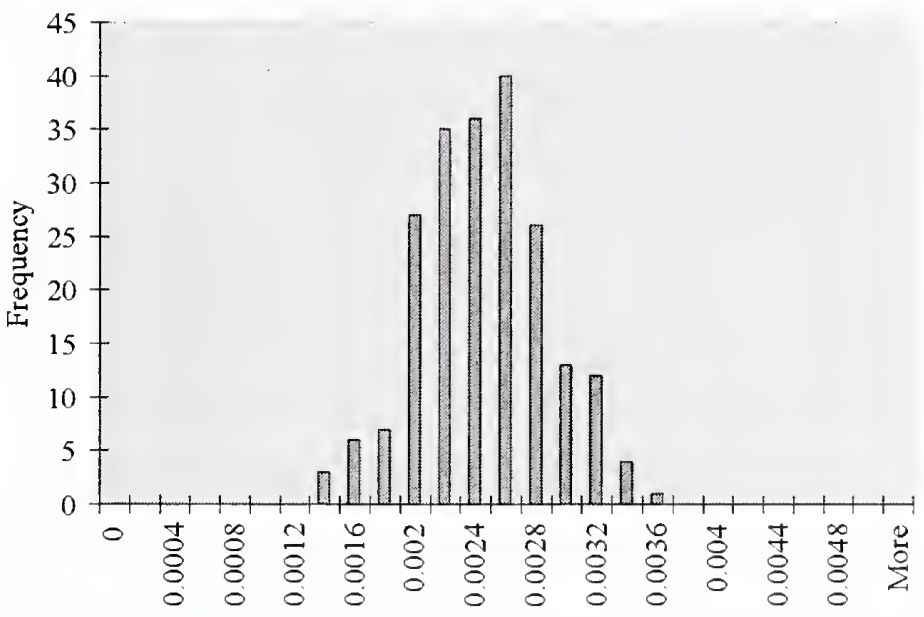

\begin{tabular}{|c|c|c|}
\hline Day & $\mathrm{CO}_{2}$ & $\mathrm{NH}_{3}$ \\
\hline $\mathbf{1}$ & 785000 & 412 \\
\hline $\mathbf{2}$ & 785000 & 795 \\
\hline $\mathbf{3}$ & 785000 & 1225 \\
\hline $\mathbf{4}$ & 785000 & 1944 \\
\hline $\mathbf{5}$ & 785000 & 2947 \\
\hline $\mathbf{6}$ & 785000 & 4212 \\
\hline 7 & 785000 & 6023 \\
\hline $\mathbf{8}$ & 785000 & 7813 \\
\hline $\mathbf{9}$ & 785000 & 9911 \\
\hline 10 & 785000 & 11384 \\
\hline
\end{tabular}

Cage occupied zone average relative humidity (\%) distribution

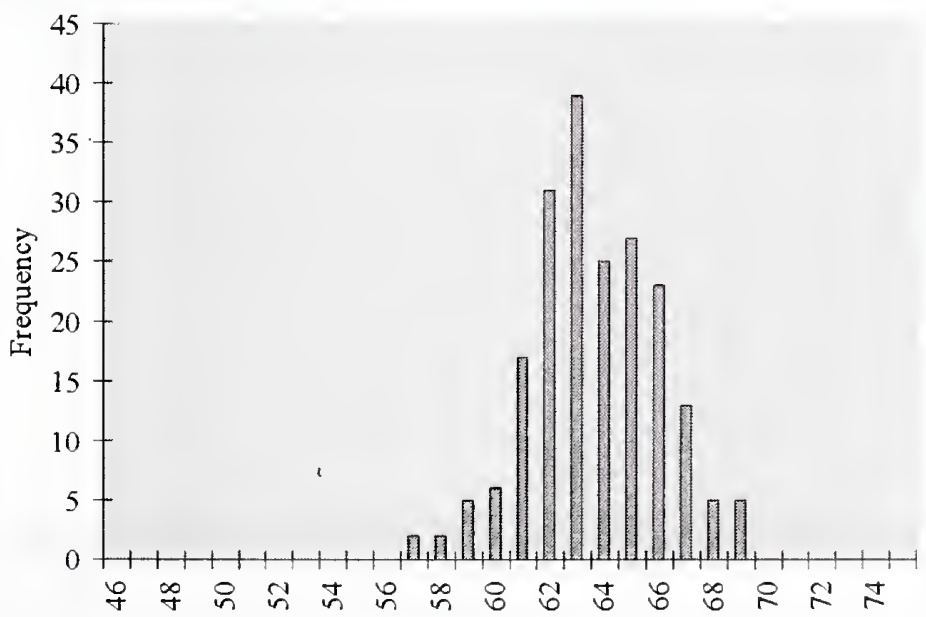




\section{Description}

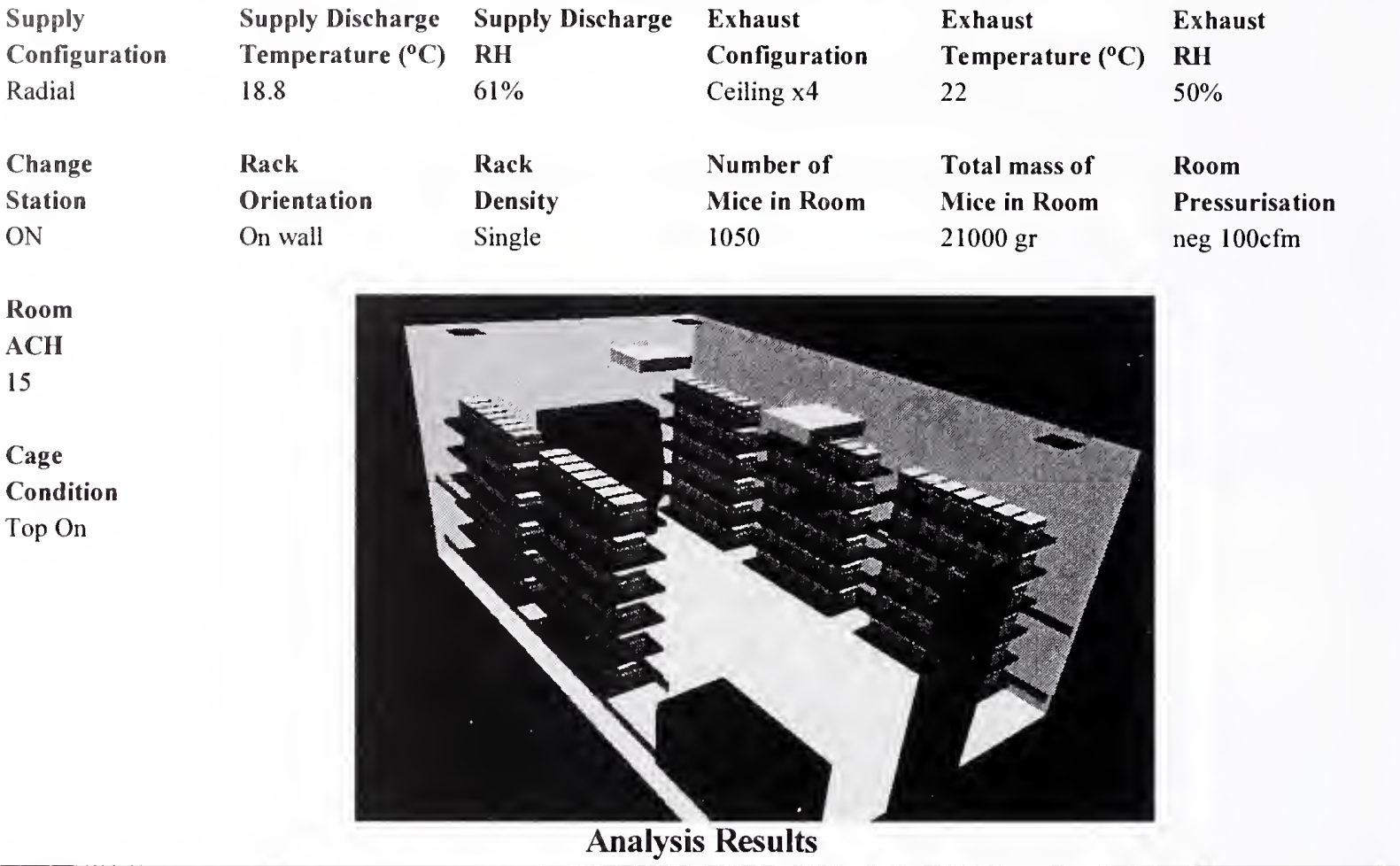

Cage Occupied Zone

\begin{tabular}{|l|cc|c|c|}
\cline { 2 - 5 } \multicolumn{1}{c|}{} & \multicolumn{2}{c|}{ Temperature } & $\mathbf{C O}_{2}$ & RH \\
\cline { 2 - 5 } \multicolumn{1}{c|}{} & ${ }^{\circ} \mathrm{C}$ & ${ }^{\circ}$ & $(\mathbf{p p m})$ & \\
\hline Mean & 22.18 & 71.92 & 1975 & $64.79 \%$ \\
\hline S.D. & 0.30 & 0.54 & 326 & $2.55 \%$ \\
\hline Max. & 23.11 & 73.60 & 2778 & $70.34 \%$ \\
\hline
\end{tabular}

Cage Occupied Zone NH3 (ppm)

\begin{tabular}{|l|c|c|c|c|c|c|c|c|c|c|}
\hline Day & $\mathbf{1}$ & $\mathbf{2}$ & $\mathbf{3}$ & $\mathbf{4}$ & $\mathbf{5}$ & $\mathbf{6}$ & $\mathbf{7}$ & $\mathbf{8}$ & $\mathbf{9}$ & $\mathbf{1 0}$ \\
\hline Mean & 1.04 & 2.00 & 3.08 & 5.33 & 8.55 & 12.48 & 18.36 & 23.22 & 28.53 & 32.42 \\
\hline Max. & 1.47 & 2.81 & 4.34 & 7.50 & 12.02 & 17.55 & 25.82 & 32.66 & 40.12 & 45.60 \\
\hline
\end{tabular}

Room Breathing Zone

\begin{tabular}{|l|cc|c|c|}
\cline { 2 - 5 } \multicolumn{1}{c|}{} & \multicolumn{2}{c|}{ Temperature } & $\mathrm{CO}_{2}$ & RH \\
\cline { 2 - 5 } \multicolumn{1}{c|}{} & ${ }^{\circ} \mathrm{C}$ & ${ }^{\circ} \mathrm{F}$ & $(\mathbf{p p m})$ & \\
\hline Mean & 20.54 & 68.97 & 21 & $54.36 \%$ \\
\hline S.D. & 0.25 & 0.45 & 9 & \\
\hline Max. & 21.53 & 70.75 & 85 & \\
\hline
\end{tabular}

Room Breathing Zone NH3 (ppm)

\begin{tabular}{|l|c|c|c|c|c|c|c|c|c|c|}
\hline Day & $\mathbf{1}$ & $\mathbf{2}$ & $\mathbf{3}$ & $\mathbf{4}$ & $\mathbf{5}$ & $\mathbf{6}$ & $\mathbf{7}$ & $\mathbf{8}$ & $\mathbf{9}$ & $\mathbf{1 0}$ \\
\hline Mean & 0.01 & 0.02 & 0.03 & 0.06 & 0.09 & 0.13 & 0.20 & 0.25 & 0.31 & 0.35 \\
\hline Max. & 0.05 & 0.09 & 0.13 & 0.23 & 0.37 & 0.54 & 0.79 & 1.00 & 1.23 & 1.40 \\
\hline
\end{tabular}




\section{Histogram Distributions}

Cage occupied zone average temperature $\left({ }^{\circ} \mathrm{C}\right)$ distribution

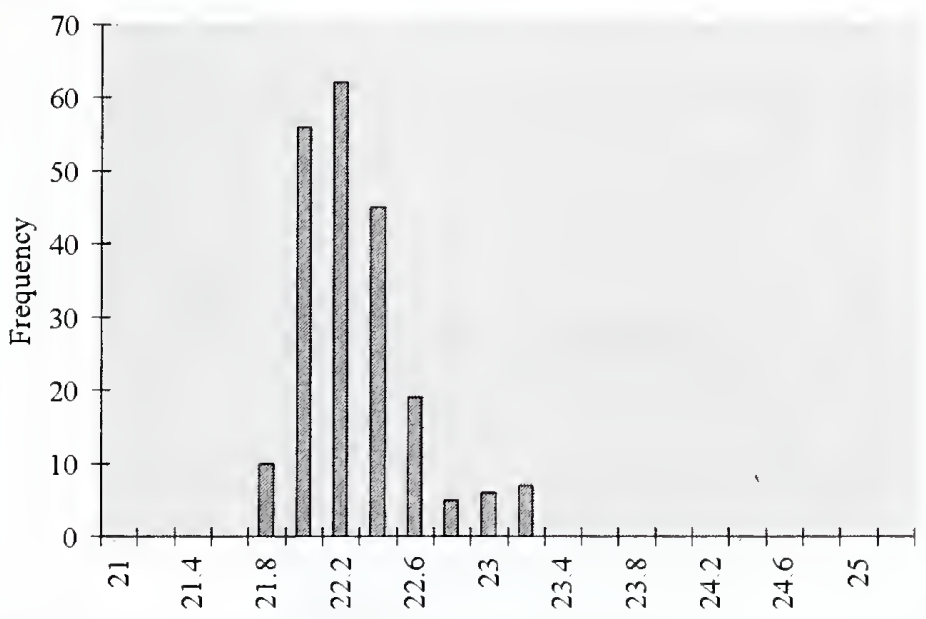

Cage occupied zone average contamination $(\mathrm{kg} / \mathrm{kg})$ distribution

Contamination conversion factors $(\mathrm{kg} / \mathrm{kg} \rightarrow \mathrm{ppm})$

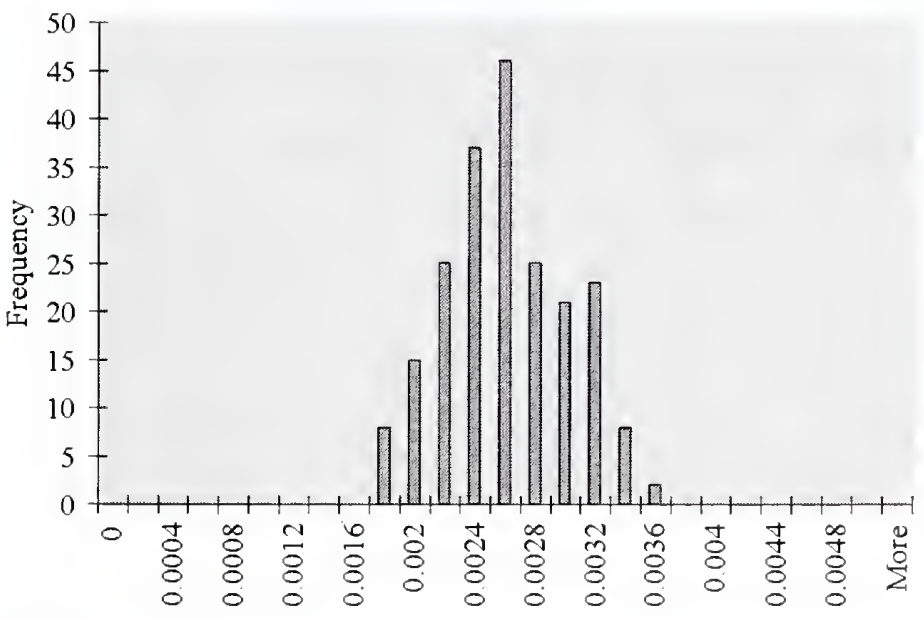

\begin{tabular}{|c|c|c|}
\hline Day & $\mathrm{CO}_{2}$ & $\mathrm{NH}_{3}$ \\
\hline $\mathbf{1}$ & 785000 & 415 \\
\hline $\mathbf{2}$ & 785000 & 795 \\
\hline $\mathbf{3}$ & 785000 & 1225 \\
\hline $\mathbf{4}$ & 785000 & 2118 \\
\hline $\mathbf{5}$ & 785000 & 3396 \\
\hline $\mathbf{6}$ & 785000 & 4957 \\
\hline 7 & 785000 & 7294 \\
\hline $\mathbf{8}$ & 785000 & 9227 \\
\hline $\mathbf{9}$ & 785000 & 11337 \\
\hline 10 & 785000 & 11384 \\
\hline
\end{tabular}

Cage occupied zone average relative humidity (\%) distribution

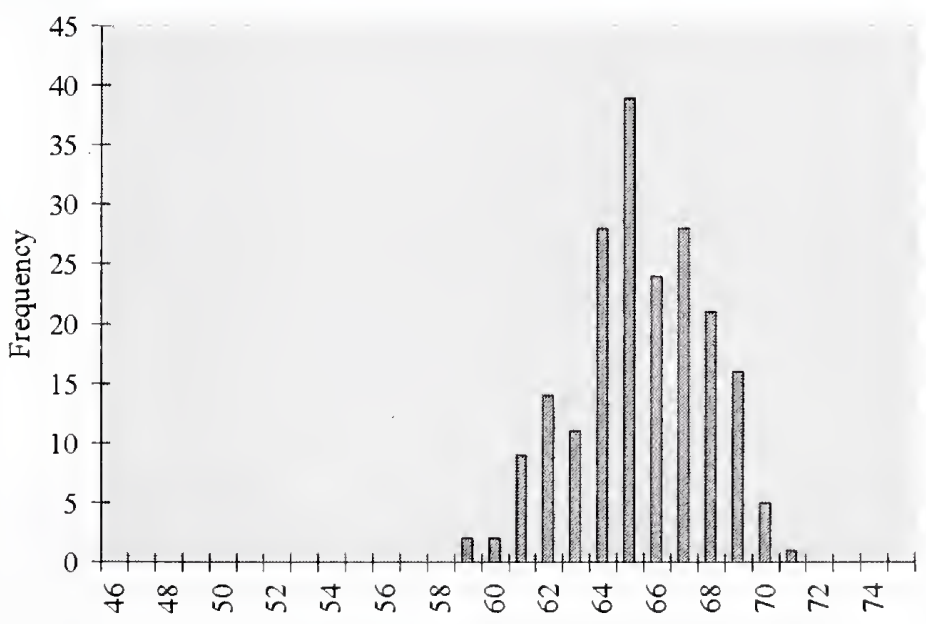


Casename

Case 41

Description

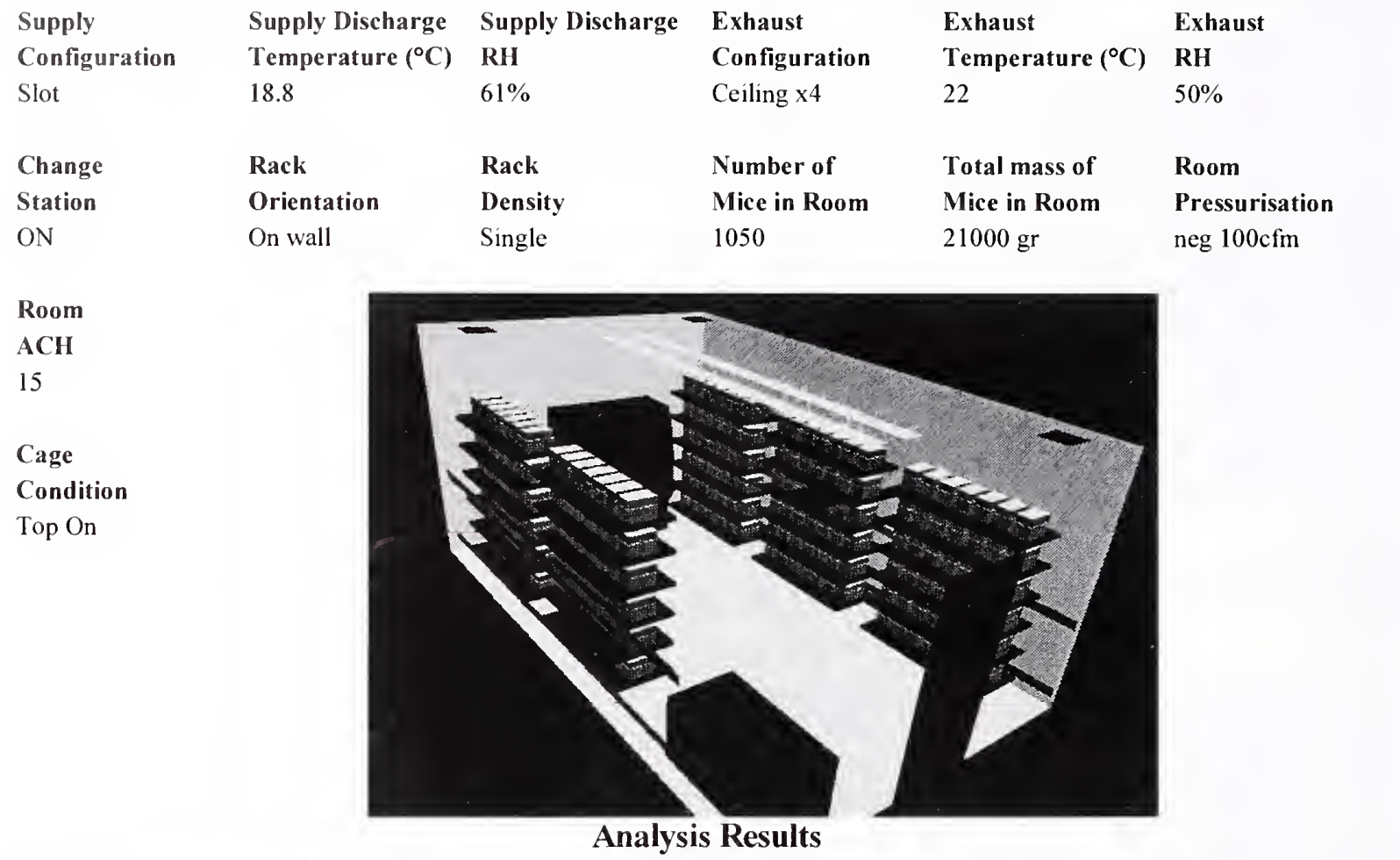

Cage Occupied Zone

\begin{tabular}{|l|cc|c|c|}
\cline { 2 - 5 } \multicolumn{1}{c|}{} & \multicolumn{2}{c|}{ Temperature } & $\mathbf{C O}_{2}$ & RH \\
\cline { 2 - 5 } \multicolumn{1}{c|}{} & ${ }^{\circ} \mathrm{C}$ & ${ }^{\circ} \mathrm{F}$ & $(\mathrm{ppm})$ & \\
\hline Mean & 22.23 & 72.02 & 1690 & $62.28 \%$ \\
\hline S.D. & 0.26 & 0.47 & 298 & $2.30 \%$ \\
\hline Max. & 22.88 & 73.19 & 2381 & $67.44 \%$ \\
\hline
\end{tabular}

Cage Occupied Zone NH3 (ppm)

\begin{tabular}{|l|c|c|c|c|c|c|c|c|c|c|}
\hline Day & $\mathbf{1}$ & $\mathbf{2}$ & $\mathbf{3}$ & $\mathbf{4}$ & $\mathbf{5}$ & $\mathbf{6}$ & $\mathbf{7}$ & $\mathbf{8}$ & $\mathbf{9}$ & $\mathbf{1 0}$ \\
\hline Mean & 0.88 & 1.71 & 2.64 & 4.06 & 6.01 & 8.51 & 12.02 & 15.77 & 20.28 & 25.10 \\
\hline Max. & 1.25 & 2.41 & 3.71 & 5.72 & 8.47 & 11.99 & 16.93 & 22.21 & 28.56 & 35.36 \\
\hline
\end{tabular}

Room Breathing Zone

\begin{tabular}{|l|cc|c|c|}
\cline { 2 - 5 } \multicolumn{1}{c|}{} & \multicolumn{2}{c|}{ Temperature } & $\mathbf{C O}_{2}$ & RH \\
\cline { 2 - 5 } \multicolumn{1}{c|}{} & ${ }^{\circ} \mathrm{C}$ & ${ }^{\circ}$ & $(\mathbf{p p m})$ & \\
\hline Mean & 20.54 & 68.96 & 51 & $54.65 \%$ \\
\hline S.D. & 0.16 & 0.29 & 23 & \\
\hline Max. & 21.58 & 70.84 & 140 & \\
\hline
\end{tabular}

Room Breathing Zone NH3 (ppm)

\begin{tabular}{|l|c|c|c|c|c|c|c|c|c|c|}
\hline Day & $\mathbf{1}$ & $\mathbf{2}$ & $\mathbf{3}$ & $\mathbf{4}$ & $\mathbf{5}$ & $\mathbf{6}$ & $\mathbf{7}$ & $\mathbf{8}$ & $\mathbf{9}$ & 10 \\
\hline Mean & 0.03 & 0.05 & 0.08 & 0.12 & 0.18 & 0.26 & 0.36 & 0.47 & 0.61 & 0.75 \\
\hline Max. & 0.07 & 0.14 & 0.22 & 0.34 & 0.50 & 0.70 & 0.99 & 1.30 & 1.68 & 2.08 \\
\hline
\end{tabular}




\section{Histogram Distributions}

Cage occupied zone average temperature $\left({ }^{\circ} \mathrm{C}\right)$ distribution

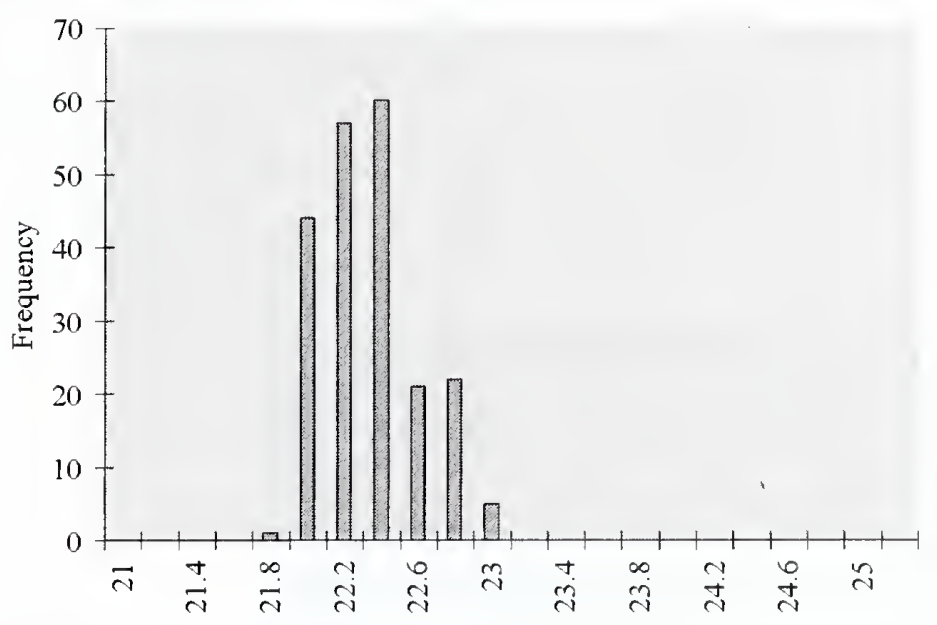

Cage occupied zone average contamination $(\mathrm{kg} / \mathrm{kg})$ distribution

Contamination conversion factors $(\mathrm{kg} / \mathrm{kg} \rightarrow \mathrm{ppm})$

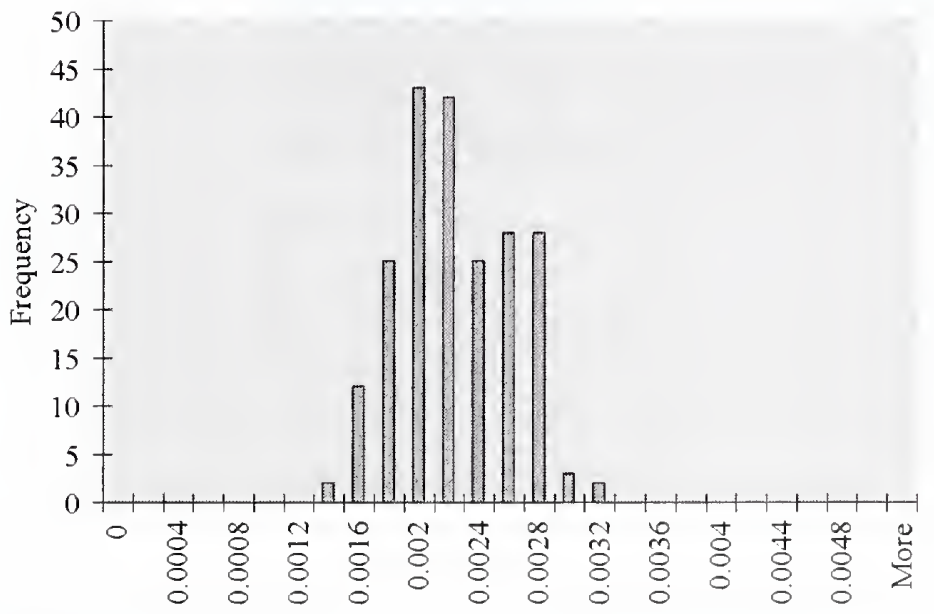

\begin{tabular}{|c|c|c|}
\hline Day & $\mathrm{CO}_{2}$ & $\mathrm{NH}_{3}$ \\
\hline 1 & 785000 & 411 \\
\hline 2 & 785000 & 795 \\
\hline 3 & 785000 & 1225 \\
\hline 4 & 785000 & 1885 \\
\hline 5 & 785000 & 2791 \\
\hline 6 & 785000 & 3954 \\
\hline 7 & 785000 & 5584 \\
\hline $\mathbf{8}$ & 785000 & 7325 \\
\hline 9 & 785000 & 9419 \\
\hline 10 & 785000 & 11384 \\
\hline
\end{tabular}

Cage occupied zone average relative humidity (\%) distribution

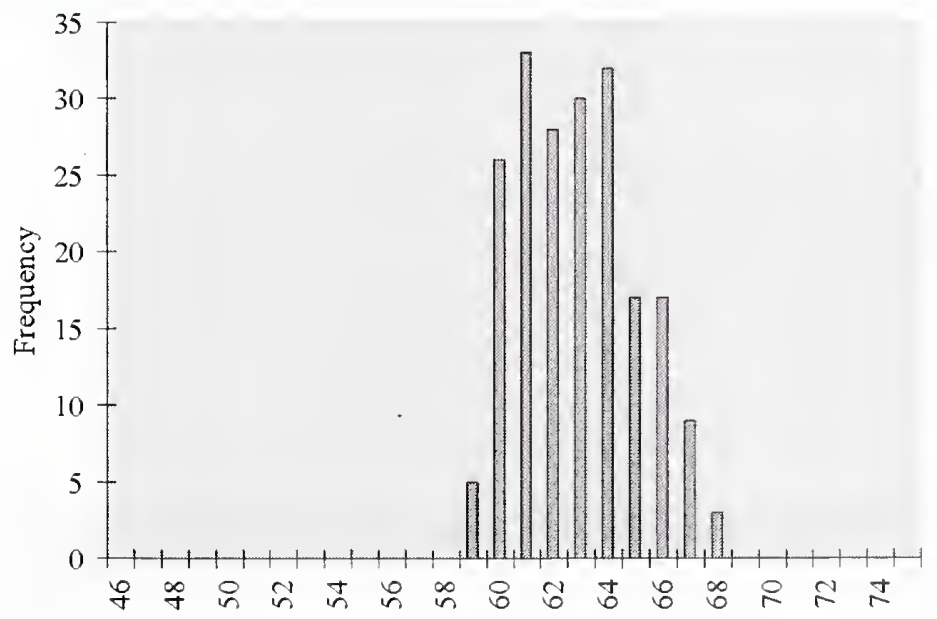


Casename

Case 42

\section{Description}

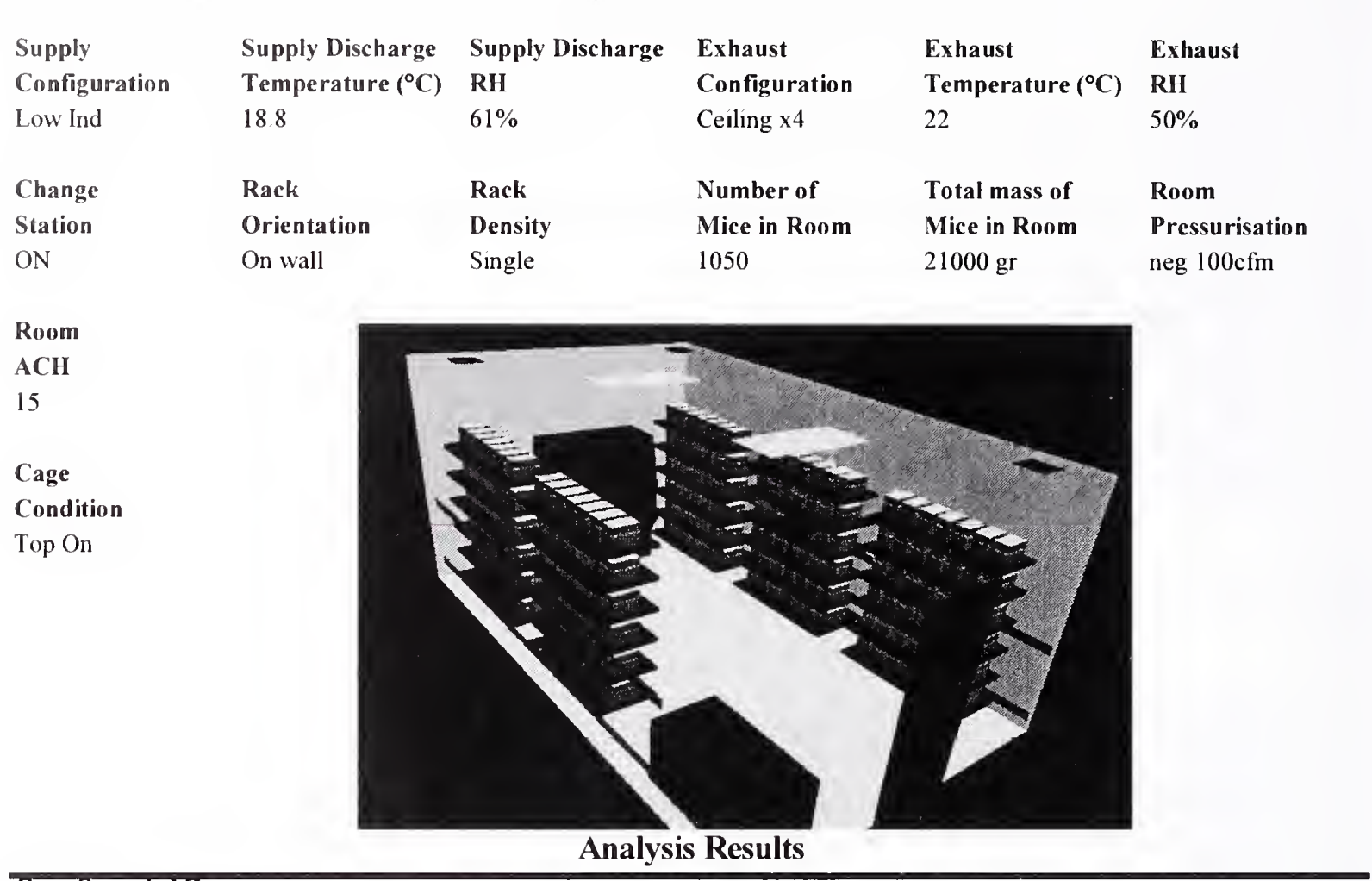

Cage Occupied Zone

\begin{tabular}{|l|cc|c|c|}
\cline { 2 - 5 } \multicolumn{1}{c|}{} & \multicolumn{2}{c|}{ Temperature } & $\mathbf{C O}_{2}$ & RH \\
\cline { 2 - 5 } \multicolumn{1}{c|}{} & ${ }^{\circ} \mathrm{C}$ & ${ }^{2} \mathrm{~F}$ & $(\mathbf{p p m})$ & \\
\hline Mean & 22.19 & 71.93 & 1728 & $62.77 \%$ \\
\hline S.D. & 0.24 & 0.44 & 326 & $2.64 \%$ \\
\hline Max. & 22.86 & 73.15 & 2535 & $69.49 \%$ \\
\hline
\end{tabular}

Cage Occupied Zone NH3 (ppm)

\begin{tabular}{|l|c|c|c|c|c|c|c|c|c|c|}
\hline Day & $\mathbf{1}$ & $\mathbf{2}$ & $\mathbf{3}$ & $\mathbf{4}$ & $\mathbf{5}$ & $\mathbf{6}$ & $\mathbf{7}$ & $\mathbf{8}$ & $\mathbf{9}$ & $\mathbf{1 0}$ \\
\hline Mean & 0.91 & 1.75 & 2.70 & 4.25 & 6.41 & 9.14 & 13.03 & 16.94 & 21.56 & 26.19 \\
\hline Max. & 1.33 & 2.57 & 3.96 & 623 & 9.40 & 13.40 & 19.11 & 24.86 & 31.63 & 3842 \\
\hline
\end{tabular}

Room Breathing Zone

\begin{tabular}{|l|cc|c|c|}
\cline { 2 - 5 } \multicolumn{1}{c|}{} & \multicolumn{2}{c|}{ Temperature } & $\mathbf{C O}_{2}$ & RH \\
\cline { 2 - 5 } \multicolumn{1}{c|}{} & ${ }^{\circ} \mathrm{C}$ & ${ }^{\circ} \mathrm{F}$ & $(\mathbf{p p m})$ & \\
\hline Mean & 20.53 & 6895 & 63 & $54.79 \%$ \\
\hline S.D. & 0.24 & 0.44 & 26 & \\
\hline Max. & 2153 & 70.75 & 189 & \\
\hline
\end{tabular}

Room Breathing Zone NH3 (ppm)

\begin{tabular}{|l|c|c|c|c|c|c|c|c|c|c|}
\hline Day & $\mathbf{1}$ & $\mathbf{2}$ & $\mathbf{3}$ & $\mathbf{4}$ & $\mathbf{5}$ & $\mathbf{6}$ & $\mathbf{7}$ & $\mathbf{8}$ & $\mathbf{9}$ & $\mathbf{1 0}$ \\
\hline Mean & 0.03 & 0.06 & 0.10 & 0.16 & 0.23 & 0.33 & 0.48 & 0.62 & 0.79 & 0.96 \\
\hline Max. & 0.10 & 0.19 & 0.30 & 0.47 & 0.70 & 1.00 & 1.43 & 1.85 & 2.36 & 287 \\
\hline
\end{tabular}




\section{Histogram Distributions}

Cage occupied zone average temperature $\left({ }^{\circ} \mathrm{C}\right)$ distribution

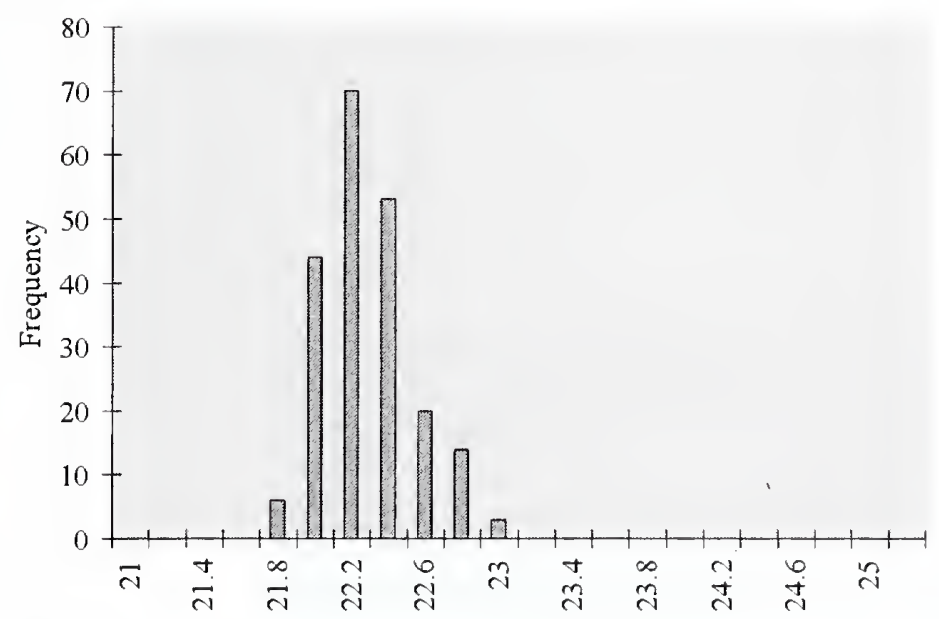

Cage occupied zone average contamination $(\mathrm{kg} / \mathrm{kg})$ distribution

Contamination conversion factors $(\mathrm{kg} / \mathrm{kg} \rightarrow \mathrm{ppm})$

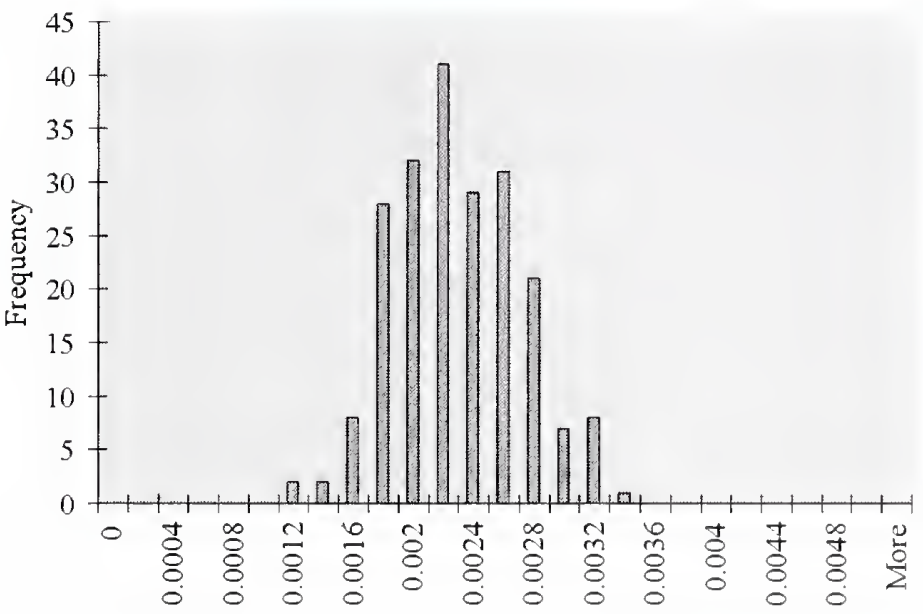

\begin{tabular}{|c|c|c|}
\hline Day & $\mathbf{C O}_{2}$ & $\mathbf{N H}_{3}$ \\
\hline 1 & 785000 & 411 \\
\hline $\mathbf{2}$ & 785000 & 795 \\
\hline $\mathbf{3}$ & 785000 & 1225 \\
\hline 4 & 785000 & 1930 \\
\hline 5 & 785000 & 2910 \\
\hline $\mathbf{6}$ & 785000 & 4151 \\
\hline 7 & 785000 & 5919 \\
\hline $\mathbf{8}$ & 785000 & 7698 \\
\hline $\mathbf{9}$ & 785000 & 9795 \\
\hline 10 & 785000 & 11384 \\
\hline
\end{tabular}

Cage occupied zone average relative humidity (\%) distribution

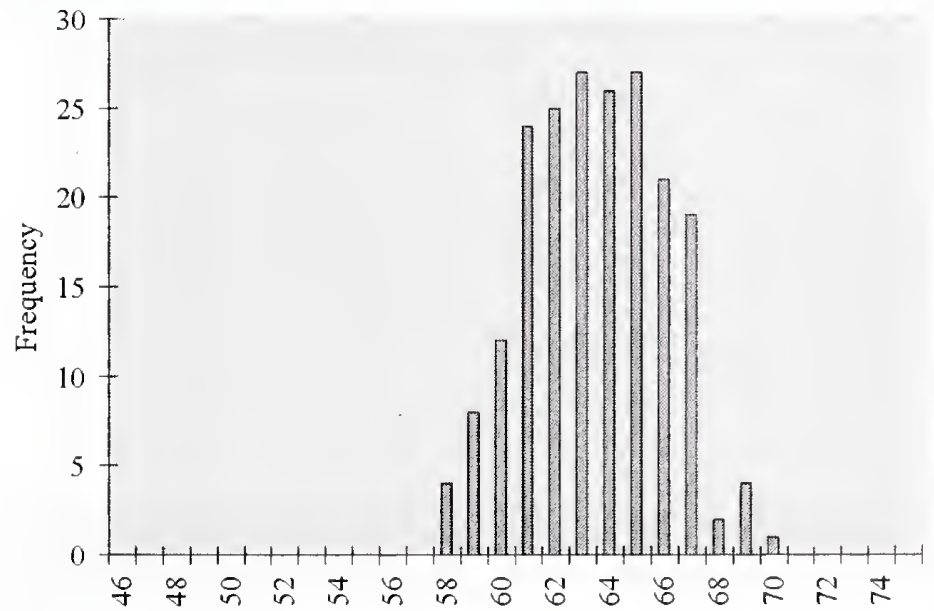


Casename

Case 43

Description

\begin{tabular}{|c|c|c|c|c|c|}
\hline Supply & Supply Discharge & Supply Discharge & Exhaust & Exhaust & Exhaust \\
\hline Configuration & Temperature $\left({ }^{\circ} \mathrm{C}\right)$ & RH & Configuration & Temperature $\left({ }^{\circ} \mathrm{C}\right)$ & RH \\
\hline Low Ind $\left(\operatorname{rot} 90^{\circ}\right)$ & 18.8 & $61 \%$ & Ceiling & 22 & $50 \%$ \\
\hline $\begin{array}{l}\text { Change } \\
\text { Station } \\
\text { ON }\end{array}$ & $\begin{array}{l}\text { Rack } \\
\text { Orientation } \\
\text { On wall }\end{array}$ & $\begin{array}{l}\text { Rack } \\
\text { Density } \\
\text { Single }\end{array}$ & $\begin{array}{l}\text { Number of } \\
\text { Mice in Room } \\
1050\end{array}$ & $\begin{array}{l}\text { Total mass of } \\
\text { Mice in Room } \\
21000 \mathrm{gr}\end{array}$ & $\begin{array}{l}\text { Room } \\
\text { Pressurisation } \\
\text { neg } 100 \mathrm{cfm}\end{array}$ \\
\hline $\begin{array}{l}\text { Room } \\
\mathrm{ACH} \\
15\end{array}$ & & & & & \\
\hline $\begin{array}{l}\text { Cage } \\
\text { Condition } \\
\text { Top On }\end{array}$ & & & & & \\
\hline
\end{tabular}

Cage Occupied Zone

\begin{tabular}{|l|cc|c|c|}
\cline { 2 - 5 } \multicolumn{1}{c|}{} & \multicolumn{2}{c|}{ Temperature } & $\mathrm{CO}_{2}$ & RH \\
\cline { 2 - 5 } \multicolumn{1}{c|}{} & ${ }^{\circ} \mathrm{C}$ & ${ }^{\circ}$ & $(\mathrm{ppm})$ & \\
\hline Mean & 22.00 & 71.59 & 1855 & $64.56 \%$ \\
\hline S.D. & 0.23 & 0.41 & 271 & $2.03 \%$ \\
\hline Max. & 22.56 & 72.61 & 2429 & $69.24 \%$ \\
\hline
\end{tabular}

Cage Occupied Zone NH3 (ppm)

\begin{tabular}{|l|c|c|c|c|c|c|c|c|c|c|}
\hline Day & $\mathbf{1}$ & $\mathbf{2}$ & $\mathbf{3}$ & $\mathbf{4}$ & $\mathbf{5}$ & $\mathbf{6}$ & $\mathbf{7}$ & $\mathbf{8}$ & $\mathbf{9}$ & $\mathbf{1 0}$ \\
\hline Mean & 0.98 & 1.88 & 2.90 & 4.96 & 7.90 & 11.50 & 16.87 & 21.40 & 26.38 & 30.19 \\
\hline Max. & 1.28 & 2.46 & 3.79 & 6.49 & 10.34 & 15.06 & 22.09 & 28.02 & 34.54 & 39.52 \\
\hline
\end{tabular}

Room Breathing Zone

\begin{tabular}{|l|cc|c|c|}
\cline { 2 - 5 } \multicolumn{1}{c|}{} & \multicolumn{2}{|c|}{ Temperature } & $\mathrm{CO}_{2}$ & RH \\
\cline { 2 - 5 } \multicolumn{1}{c|}{} & ${ }^{\circ} \mathrm{C}$ & ${ }^{\circ} \mathrm{F}$ & $(\mathrm{ppm})$ & \\
\hline Mean & 20.24 & 68.44 & 65 & $55.81 \%$ \\
\hline S.D. & 0.22 & 0.39 & 23 & \\
\hline Max. & 21.94 & 71.49 & 164 & \\
\hline
\end{tabular}

Room Breathing Zone NH3 (ppm)

\begin{tabular}{|l|c|c|c|c|c|c|c|c|c|c|}
\hline Day & $\mathbf{1}$ & $\mathbf{2}$ & $\mathbf{3}$ & $\mathbf{4}$ & $\mathbf{5}$ & $\mathbf{6}$ & $\mathbf{7}$ & $\mathbf{8}$ & $\mathbf{9}$ & $\mathbf{1 0}$ \\
\hline Mean & 0.03 & 0.07 & 0.10 & 0.17 & 0.28 & 0.40 & 0.59 & 0.75 & 0.93 & 1.06 \\
\hline Max. & 0.09 & 0.17 & 0.26 & 0.44 & 0.70 & 1.01 & 1.49 & 1.89 & 2.33 & 2.66 \\
\hline
\end{tabular}




\section{Histogram Distributions}

Cage occupied zone average temperature $\left({ }^{\circ} \mathrm{C}\right)$ distribution

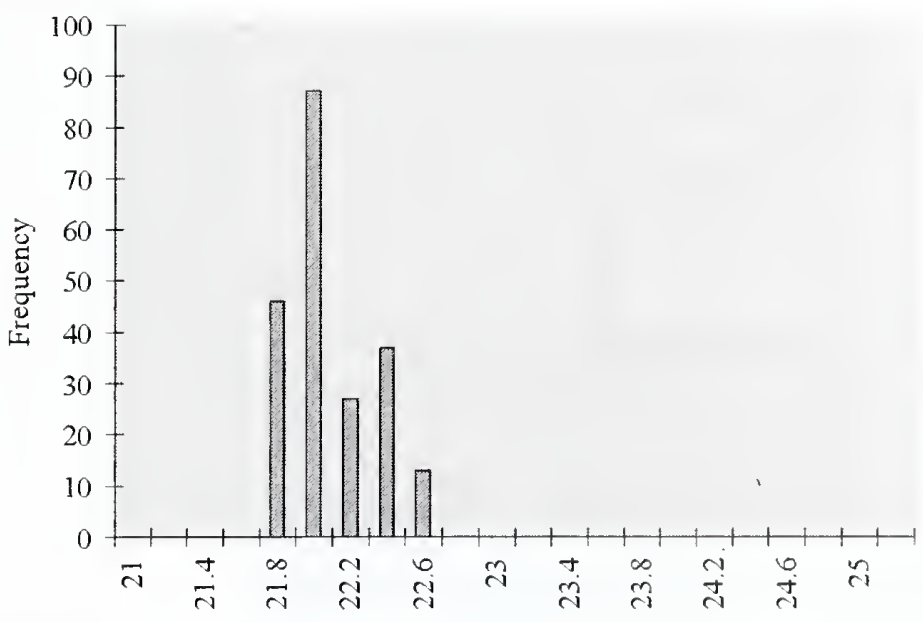

Cage occupied zone average contamination $(\mathrm{kg} / \mathrm{kg})$ distribution

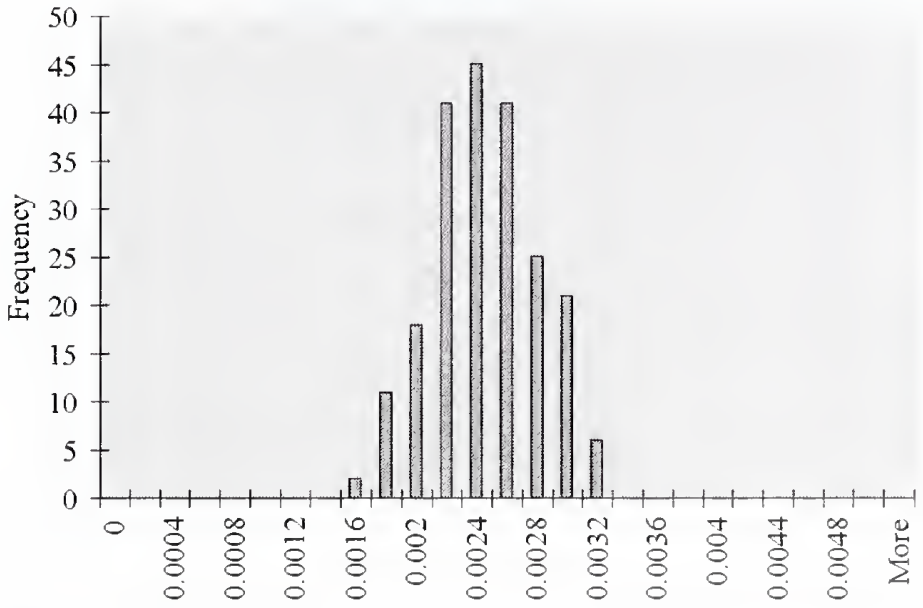

Cage occupied zone average relative humidity (\%) distribution

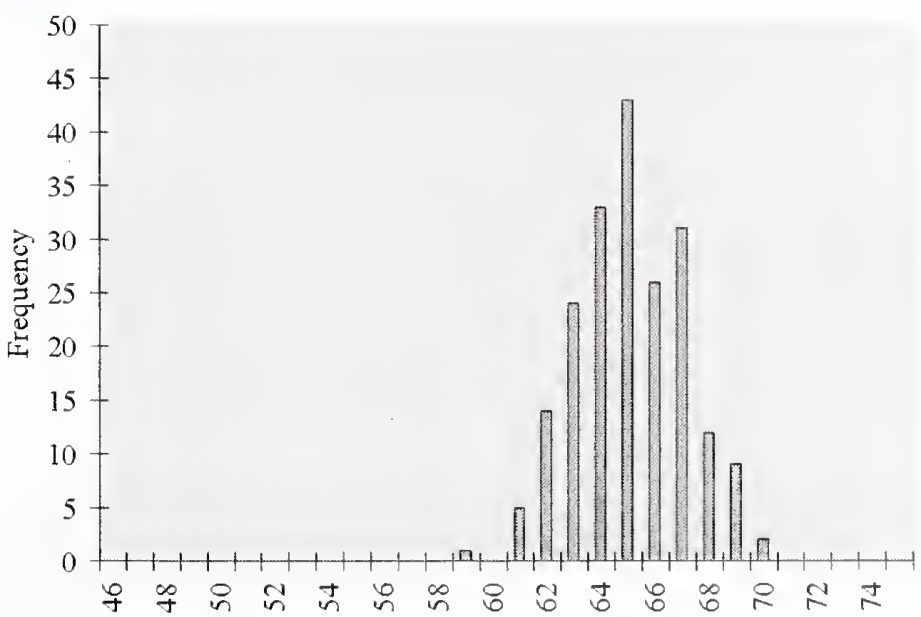

Contamination conversion factors $(\mathrm{kg} / \mathrm{kg} \rightarrow \mathrm{ppm})$

\begin{tabular}{|c|c|c|}
\hline Day & $\mathrm{CO}_{2}$ & $\mathrm{NH}_{3}$ \\
\hline 1 & 785000 & 415 \\
\hline $\mathbf{2}$ & 785000 & 795 \\
\hline $\mathbf{3}$ & 785000 & 1225 \\
\hline $\mathbf{4}$ & 785000 & 2097 \\
\hline 5 & 785000 & 3342 \\
\hline $\mathbf{6}$ & 785000 & 4867 \\
\hline 7 & 785000 & 7139 \\
\hline $\mathbf{8}$ & 785000 & 9054 \\
\hline $\mathbf{9}$ & 785000 & 1163 \\
\hline 10 & 785000 & 11384 \\
\hline
\end{tabular}




\section{Description}

\begin{tabular}{|c|c|c|c|c|c|}
\hline Supply & Supply Discharge & Supply Discharge & Exhaust & Exhaust & Exhaust \\
\hline Configuration & Temperature $\left({ }^{\circ} \mathrm{C}\right)$ & RH & Configuration & Temperature $\left({ }^{\circ} \mathrm{C}\right)$ & RH \\
\hline Radial $\left(\operatorname{rot} 90^{\circ}\right)$ & 18.8 & $61 \%$ & Ceiling & 22 & $50 \%$ \\
\hline Change & Rack & Rack & Number of & Total mass of & Room \\
\hline Station & Orientation & Density & Mice in Room & Mice in Room & Pressurisation \\
\hline $\mathrm{ON}$ & On wall & Single & 1050 & $21000 \mathrm{gr}$ & neg $100 \mathrm{cfm}$ \\
\hline
\end{tabular}

\section{Room}

$\mathrm{ACH}$

15

Cage

Condition

Top On

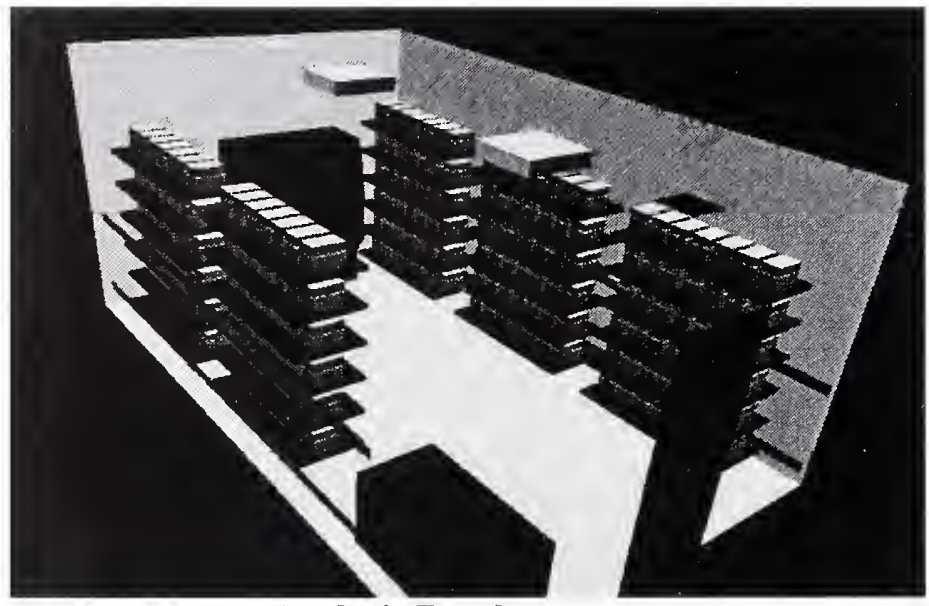

Analysis Results

Cage Occupied Zone

\begin{tabular}{|l|cc|c|c|}
\cline { 2 - 5 } \multicolumn{1}{c|}{} & \multicolumn{2}{c|}{ Temperature } & $\mathrm{CO}_{2}$ & RH \\
\cline { 2 - 5 } \multicolumn{1}{c|}{} & ${ }^{\circ} \mathrm{C}$ & ${ }^{\circ} \mathrm{F}$ & $(\mathbf{p p m})$ & \\
\hline Mean & 22.16 & 71.89 & 2118 & $66.03 \%$ \\
\hline S.D. & 0.29 & 0.52 & 286 & $2.26 \%$ \\
\hline Max. & 22.97 & 73.35 & 2732 & $71.46 \%$ \\
\hline
\end{tabular}

Cage Occupied Zone NH3 (ppm)

\begin{tabular}{|l|c|c|c|c|c|c|c|c|c|c|}
\hline Day & 1 & 2 & 3 & 4 & 5 & 6 & 7 & 8 & 9 & 10 \\
\hline Mean & 1.13 & 2.14 & 3.31 & 6.03 & 9.97 & 14.71 & 21.96 & 27.43 & 33.15 & 36.40 \\
\hline Max. & 1.45 & 2.77 & 4.26 & 7.77 & 12.86 & 18.98 & 28.33 & 35.38 & 42.75 & 46.95 \\
\hline
\end{tabular}

Room Breathing Zone

\begin{tabular}{|l|cc|c|c|}
\cline { 2 - 5 } \multicolumn{1}{c|}{} & \multicolumn{2}{c|}{ Temperature } & $\mathrm{CO}_{\mathbf{z}}$ & RH \\
\cline { 2 - 5 } \multicolumn{1}{c|}{} & ${ }^{\circ} \mathrm{C}$ & ${ }^{\circ} \mathrm{F}$ & $(\mathbf{p p m})$ & \\
\hline Mean & 20.45 & 68.80 & 74 & $55.17 \%$ \\
\hline S.D. & 0.21 & 0.38 & 35 & \\
\hline Max. & 21.87 & 71.36 & 237 & \\
\hline
\end{tabular}

Room Breathing Zone NH3 (ppm)

\begin{tabular}{|l|c|c|c|c|c|c|c|c|c|c|}
\hline Day & 1 & 2 & 3 & 4 & 5 & 6 & 7 & 8 & 9 & 10 \\
\hline Mean & 0.04 & 0.07 & 0.11 & 0.21 & 0.35 & 0.51 & 0.76 & 0.95 & 1.15 & 1.27 \\
\hline Max. & 0.13 & 0.24 & 0.37 & 0.67 & 1.11 & 1.65 & 2.46 & 3.07 & 3.71 & 4.07 \\
\hline
\end{tabular}




\section{Histogram Distributions}

Cage occupied zone average temperature $\left({ }^{\circ} \mathrm{C}\right)$ distribution

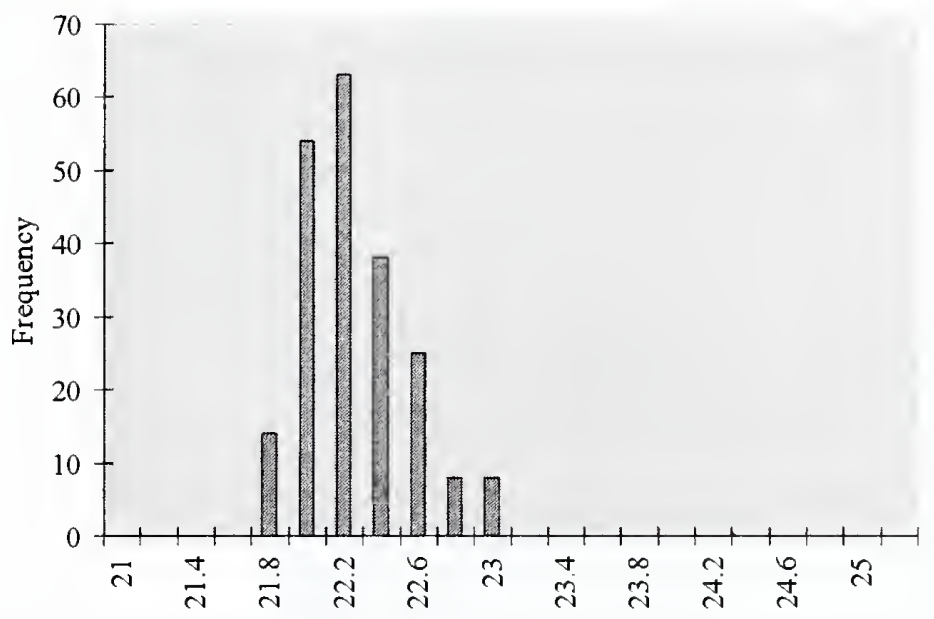

Cage occupied zone average contamination $(\mathrm{kg} / \mathrm{kg})$ distribution Contamination conversion factors $(\mathrm{kg} / \mathrm{kg} \rightarrow \mathrm{ppm})$

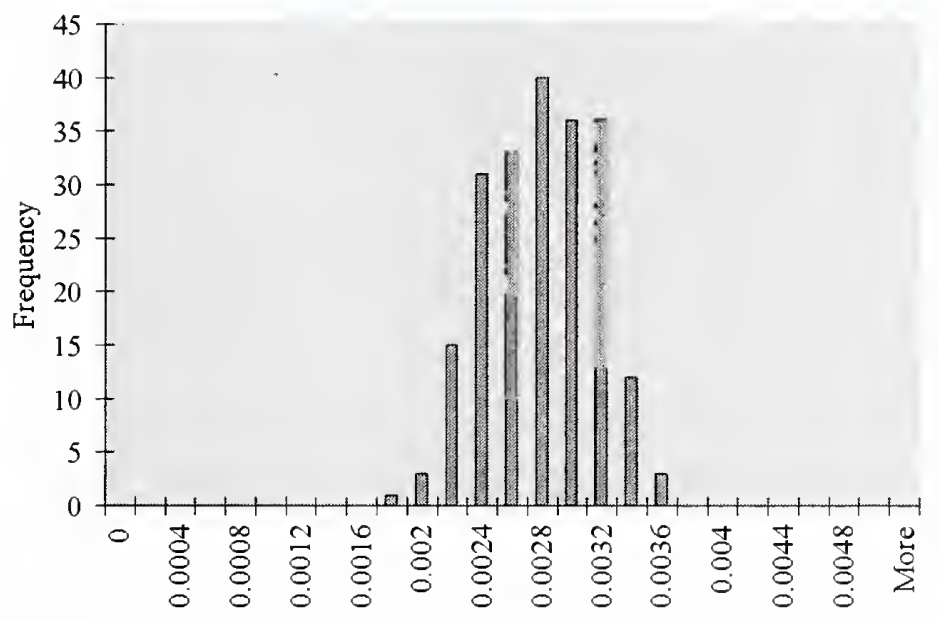

\begin{tabular}{|c|c|c|}
\hline Day & $\mathbf{C O}_{2}$ & $\mathbf{N H}_{3}$ \\
\hline $\mathbf{1}$ & 785000 & 417 \\
\hline $\mathbf{2}$ & 785000 & 795 \\
\hline $\mathbf{3}$ & 785000 & 1225 \\
\hline $\mathbf{4}$ & 785000 & 2233 \\
\hline $\mathbf{5}$ & 785000 & 3696 \\
\hline $\mathbf{6}$ & 785000 & 5454 \\
\hline $\mathbf{7}$ & 785000 & 8140 \\
\hline $\mathbf{8}$ & 785000 & 10167 \\
\hline $\mathbf{9}$ & 785000 & 12285 \\
\hline $\mathbf{1 0}$ & 785000 & 11384 \\
\hline
\end{tabular}

Cage occupied zone average relative humidity (\%) distribution

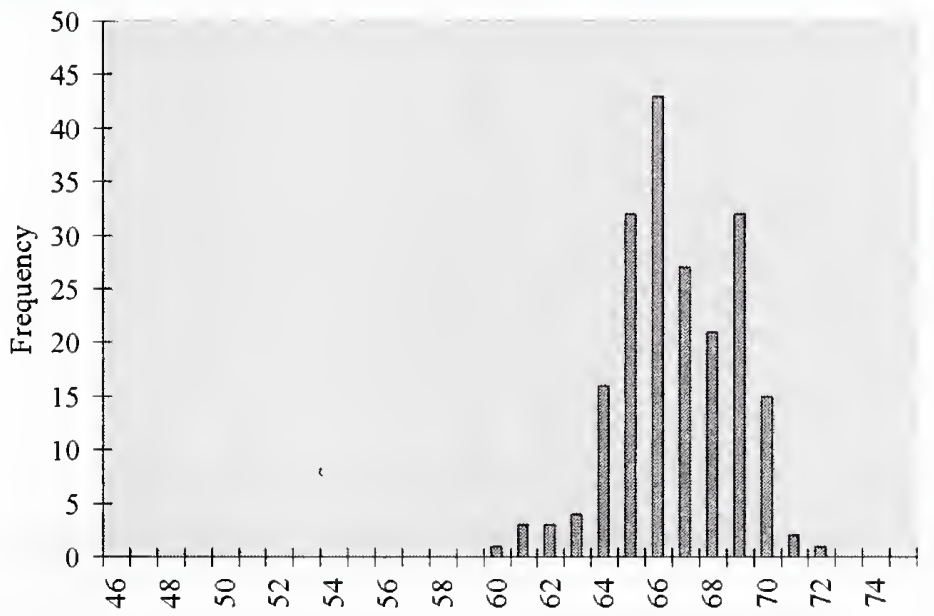


Casename Case 45

\section{Description}

\begin{tabular}{|c|c|c|c|c|c|}
\hline Supply & Supply Discharge & Supply Discharge & Exhaust & Exhaust & Exhaust \\
\hline Configuration & Temperature $\left({ }^{\circ} \mathrm{C}\right)$ & RH & Configuration & Temperature $\left({ }^{\circ} \mathrm{C}\right)$ & RH \\
\hline Radial & 18.8 & $61 \%$ & Ceiling & 22 & $50 \%$ \\
\hline Change & Rack & Rack & Number of & Total mass of & Room \\
\hline Station & Orientation & Density & Mice in Room & Mice in Room & Pressurisation \\
\hline $\mathrm{ON}$ & On wall & Single & 1050 & $21000 \mathrm{gr}$ & neg $50 \mathrm{cfm}$ \\
\hline
\end{tabular}

Room

$\mathrm{ACH}$

15

Cage

Condition

Top On

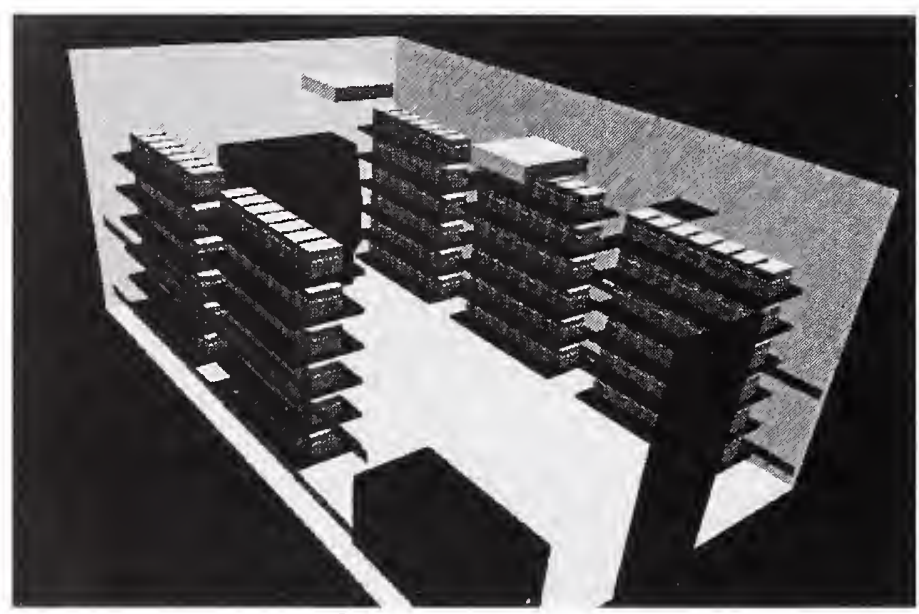

Analysis Results

Cage Occupied Zone

\begin{tabular}{|l|cc|c|c|}
\cline { 2 - 5 } \multicolumn{1}{c|}{} & \multicolumn{2}{c|}{ Temperature } & $\mathrm{CO}_{2}$ & RH \\
\cline { 2 - 5 } \multicolumn{1}{c|}{} & ${ }^{\circ} \mathrm{C}$ & ${ }^{\circ} \mathrm{F}$ & $(\mathrm{ppm})$ & \\
\hline Mean & 22.06 & 71.72 & 1929 & $64.88 \%$ \\
\hline S.D. & 0.27 & 0.48 & 325 & $2.55 \%$ \\
\hline Max. & 22.92 & 73.26 & 2681 & $69.79 \%$ \\
\hline
\end{tabular}

Cage Occupied Zone NH3 (ppm)

\begin{tabular}{|l|c|c|c|c|c|c|c|c|c|c|}
\hline Day & $\mathbf{1}$ & $\mathbf{2}$ & $\mathbf{3}$ & $\mathbf{4}$ & $\mathbf{5}$ & $\mathbf{6}$ & $\mathbf{7}$ & $\mathbf{8}$ & $\mathbf{9}$ & $\mathbf{1 0}$ \\
\hline Mean & 1.02 & 1.95 & 3.01 & 5.22 & 8.40 & 12.27 & 18.08 & 22.84 & 28.03 & 31.77 \\
\hline Max. & 1.42 & 2.71 & 4.18 & 7.26 & 11.68 & 17.06 & 25.13 & 31.76 & 38.96 & 44.16 \\
\hline
\end{tabular}

Room Breathing Zone

\begin{tabular}{|l|cc|c|c|}
\cline { 2 - 5 } \multicolumn{1}{c|}{} & \multicolumn{2}{c|}{ Temperature } & $\mathrm{CO}_{2}$ & RH \\
\cline { 2 - 5 } \multicolumn{1}{c|}{} & ${ }^{\circ} \mathrm{C}$ & ${ }^{\circ} \mathrm{F}$ & $(\mathbf{p p m})$ & \\
\hline Mean & 20.27 & 68.48 & 52 & $55.61 \%$ \\
\hline S.D. & 0.20 & 0.37 & 23 & \\
\hline Max. & 21.74 & 71.14 & 246 & \\
\hline
\end{tabular}

Room Breathing Zone NH3 (ppm)

\begin{tabular}{|l|c|c|c|c|c|c|c|c|c|c|}
\hline Day & $\mathbf{1}$ & $\mathbf{2}$ & $\mathbf{3}$ & $\mathbf{4}$ & $\mathbf{5}$ & $\mathbf{6}$ & $\mathbf{7}$ & $\mathbf{8}$ & $\mathbf{9}$ & $\mathbf{1 0}$ \\
\hline Mean & 0.03 & 0.05 & 0.08 & 0.14 & 0.23 & 0.33 & 0.49 & 0.62 & 0.76 & 0.86 \\
\hline Max. & 0.13 & 0.25 & 0.38 & 0.67 & 1.07 & 1.56 & 2.30 & 2.91 & 3.57 & 4.05 \\
\hline
\end{tabular}




\section{Histogram Distributions}

Cage occupied zone average temperature $\left({ }^{\circ} \mathrm{C}\right)$ distribution

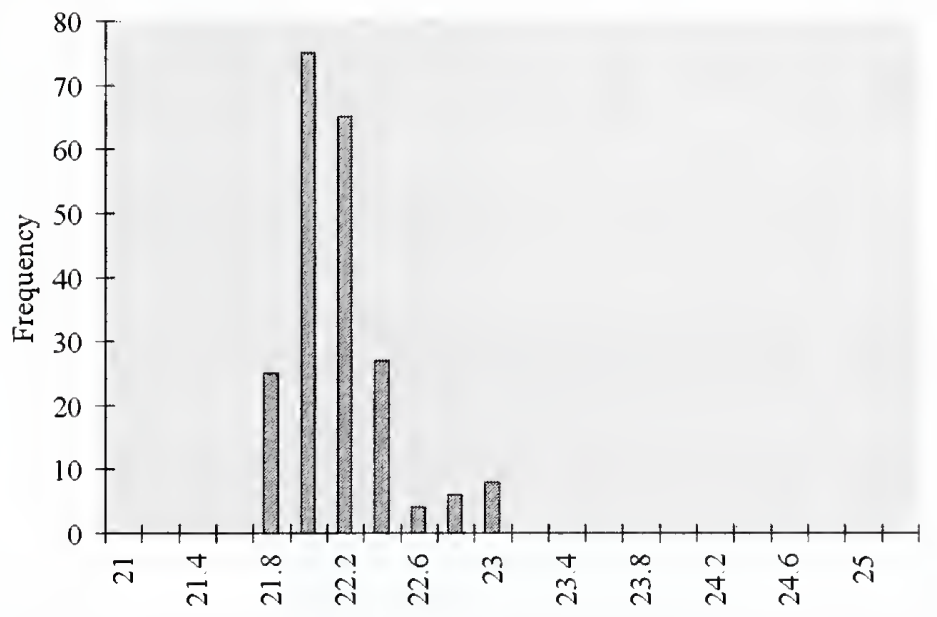

Cage occupied zone average contamination $(\mathrm{kg} / \mathrm{kg})$ distribution

Contamination conversion factors $(\mathrm{kg} / \mathrm{kg} \rightarrow \mathrm{ppm})$

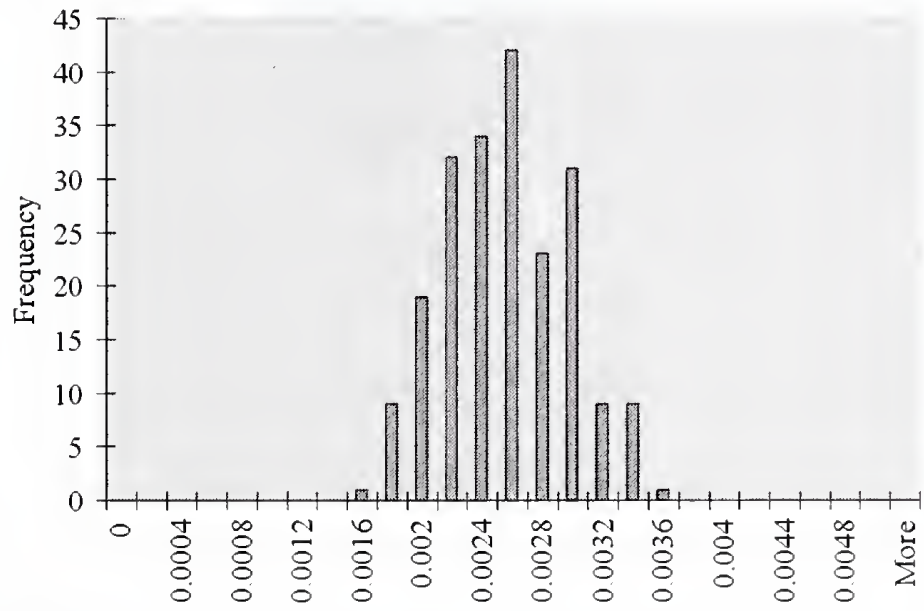

\begin{tabular}{|c|c|c|}
\hline Day & $\mathbf{C O}_{2}$ & $\mathbf{N H}_{\mathbf{3}}$ \\
\hline $\mathbf{1}$ & 785000 & 415 \\
\hline $\mathbf{2}$ & 785000 & 795 \\
\hline $\mathbf{3}$ & 785000 & 1225 \\
\hline $\mathbf{4}$ & 785000 & 2127 \\
\hline $\mathbf{5}$ & 785000 & 3419 \\
\hline $\mathbf{6}$ & 785000 & 4995 \\
\hline $\mathbf{7}$ & 785000 & 7358 \\
\hline $\mathbf{8}$ & 785000 & 9298 \\
\hline $\mathbf{9}$ & 785000 & 11408 \\
\hline $\mathbf{1 0}$ & 785000 & 11384 \\
\hline
\end{tabular}

Cage occupied zone average relative humidity (\%) distribution

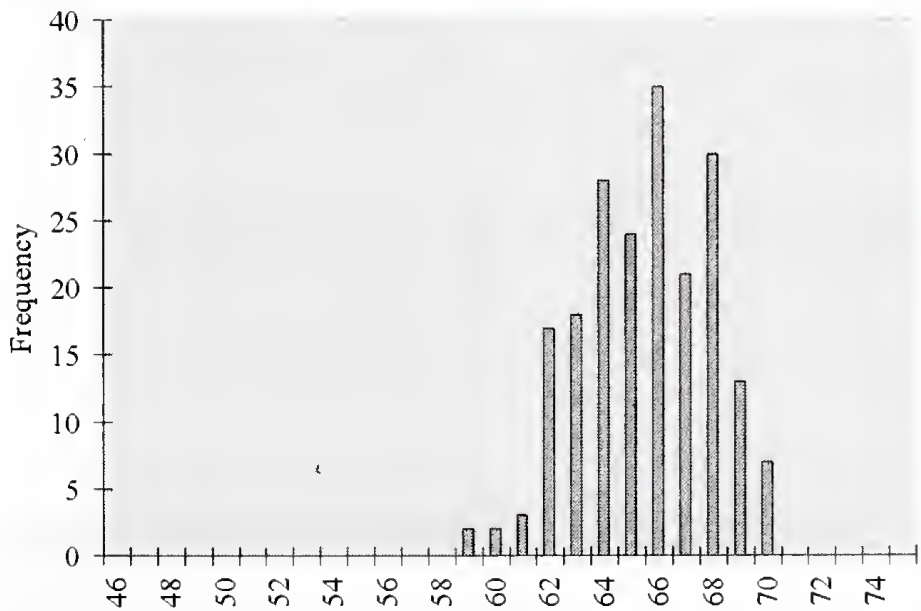




\section{Casename Case 46}

\section{Description}

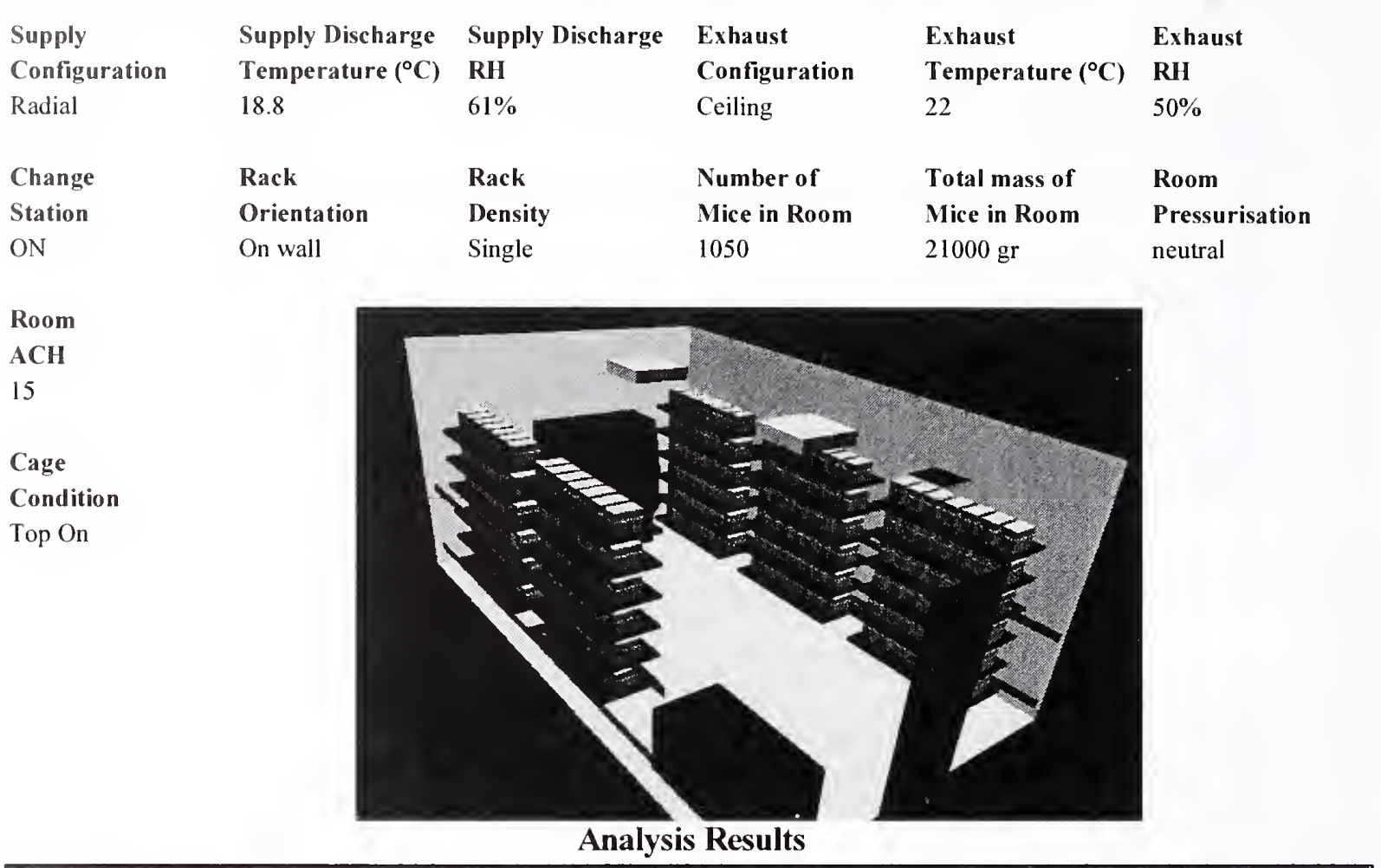

Cage Occupied Zone

\begin{tabular}{|l|cc|c|c|}
\cline { 2 - 5 } \multicolumn{1}{c|}{} & \multicolumn{2}{c|}{ Temperature } & $\mathrm{CO}_{2}$ & RH \\
\cline { 2 - 5 } \multicolumn{1}{c|}{} & ${ }^{\circ} \mathrm{C}$ & ${ }^{\circ} \mathrm{F}$ & $(\mathbf{p p m})$ & \\
\hline Mean & 22.00 & 71.61 & 1943 & $65.24 \%$ \\
\hline S.D. & 0.29 & 0.52 & 346 & $2.71 \%$ \\
\hline Max. & 22.91 & 73.24 & 2746 & $71.02 \%$ \\
\hline
\end{tabular}

Cage Occupied Zone NH3 (ppm)

\begin{tabular}{|l|c|c|c|c|c|c|c|c|c|c|}
\hline Day & $\mathbf{1}$ & $\mathbf{2}$ & $\mathbf{3}$ & $\mathbf{4}$ & $\mathbf{5}$ & $\mathbf{6}$ & $\mathbf{7}$ & $\mathbf{8}$ & $\mathbf{9}$ & $\mathbf{1 0}$ \\
\hline Mean & 1.03 & 1.97 & 3.03 & 5.35 & 8.68 & 12.72 & 18.82 & 23.69 & 28.92 & 32.44 \\
\hline Max. & 1.45 & 2.78 & 4.28 & 7.56 & 12.26 & 17.97 & 26.60 & 33.48 & 40.87 & 45.84 \\
\hline
\end{tabular}

Room Breathing Zone

\begin{tabular}{|l|cc|c|c|}
\cline { 2 - 5 } \multicolumn{1}{c|}{} & \multicolumn{2}{c|}{ Temperature } & $\mathrm{CO}_{2}$ & RH \\
\cline { 2 - 5 } \multicolumn{1}{c|}{} & ${ }^{\circ} \mathrm{C}$ & ${ }^{\circ} \mathrm{F}$ & $(\mathbf{p p m})$ & \\
\hline Mean & 20.16 & 68.29 & 43 & $55.89 \%$ \\
\hline S.D. & 0.23 & 0.41 & 18 & \\
\hline Max. & 20.82 & 69.48 & 200 & \\
\hline
\end{tabular}

Room Breathing Zone NH3 (ppm)

\begin{tabular}{|l|c|c|c|c|c|c|c|c|c|c|}
\hline Day & $\mathbf{1}$ & $\mathbf{2}$ & $\mathbf{3}$ & $\mathbf{4}$ & $\mathbf{5}$ & $\mathbf{6}$ & $\mathbf{7}$ & $\mathbf{8}$ & $\mathbf{9}$ & $\mathbf{1 0}$ \\
\hline Mean & 0.02 & 0.04 & 0.07 & 0.12 & 0.19 & 0.28 & 0.42 & 0.53 & 0.64 & 0.72 \\
\hline Max. & 0.11 & 0.20 & 0.31 & 0.55 & 0.89 & 1.31 & 1.94 & 2.44 & 2.98 & 3.34 \\
\hline
\end{tabular}




\section{Histogram Distributions}

Cage occupied zone average temperature $\left({ }^{\circ} \mathrm{C}\right)$ distribution

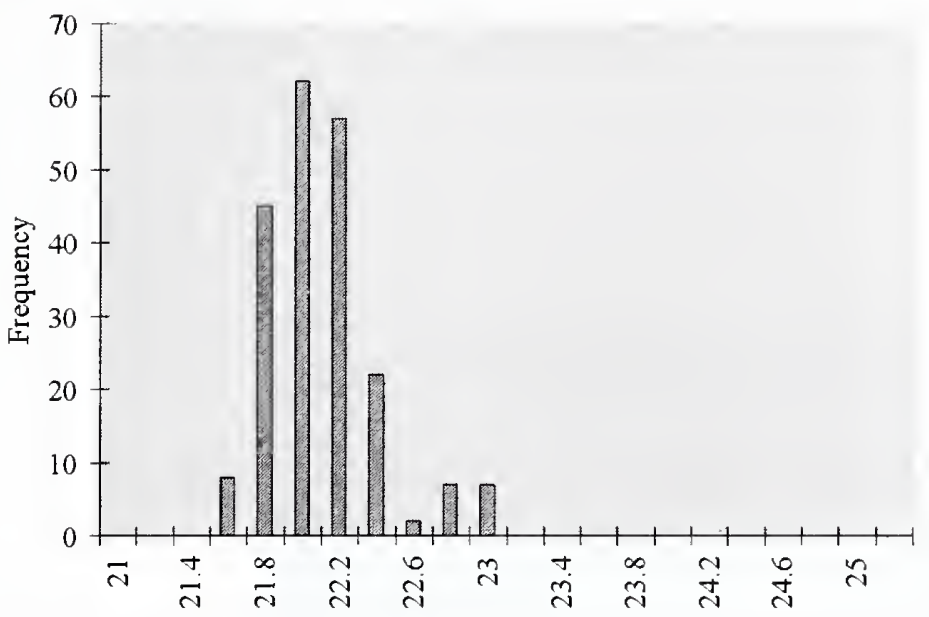

Cage occupied zone average contamination $(\mathrm{kg} / \mathrm{kg})$ distribution

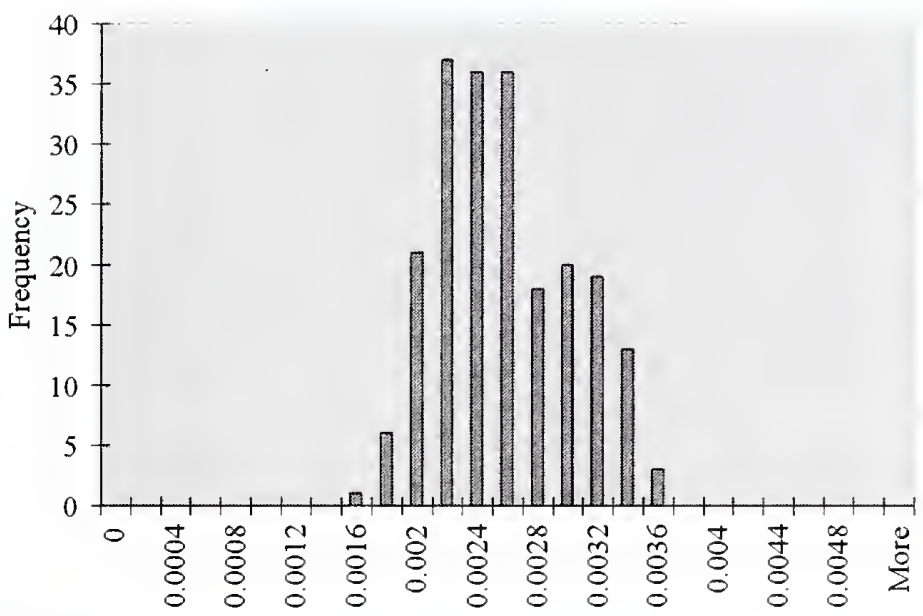

Cage occupied zone average relative humidity (\%) distribution

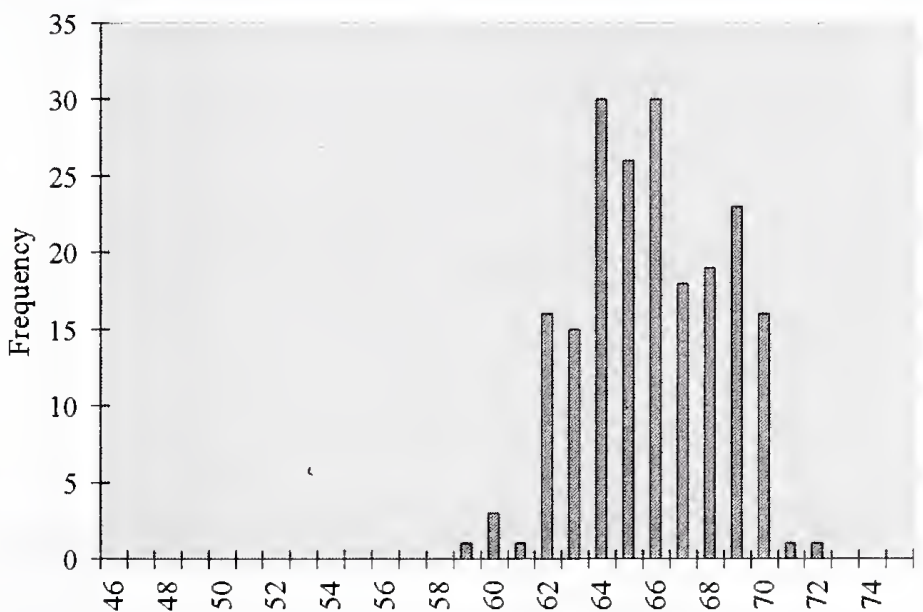

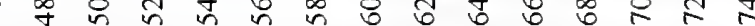

Contamination conversion factors $(\mathrm{kg} / \mathrm{kg} \rightarrow \mathrm{ppm})$

\begin{tabular}{|c|c|c|}
\hline Day & $\mathbf{C O}_{2}$ & $\mathbf{N H}_{\mathbf{3}}$ \\
\hline $\mathbf{1}$ & 785000 & 416 \\
\hline $\mathbf{2}$ & 785000 & 795 \\
\hline $\mathbf{3}$ & 785000 & 1225 \\
\hline $\mathbf{4}$ & 785000 & 2160 \\
\hline $\mathbf{5}$ & 785000 & 3506 \\
\hline $\mathbf{6}$ & 785000 & 5139 \\
\hline $\mathbf{7}$ & 785000 & 7604 \\
\hline $\mathbf{8}$ & 785000 & 9571 \\
\hline $\mathbf{9}$ & 785000 & 11684 \\
\hline $\mathbf{1 0}$ & 785000 & 11384 \\
\hline
\end{tabular}




\section{Description}

\begin{tabular}{|c|c|c|c|c|c|}
\hline Supply & Supply Discharge & Supply Discharge & Exhaust & Exhaust & Exhaust \\
\hline Configuration & Temperature $\left({ }^{\circ} \mathrm{C}\right)$ & RH & Configuration & Temperature $\left({ }^{\circ} \mathrm{C}\right)$ & RH \\
\hline Radial & 18.8 & $61 \%$ & Ceiling & 22 & $50 \%$ \\
\hline Change & Rack & Rack & Number of & Total mass of & Room \\
\hline Station & Orientation & Density & Mice in Room & Mice in Room & Pressurisation \\
\hline $\mathrm{ON}$ & On wall & Single & 1050 & $21000 \mathrm{gr}$ & pos $50 \mathrm{cfm}$ \\
\hline
\end{tabular}

Room

$\mathrm{ACH}$

15

Cage

Condition

Top On

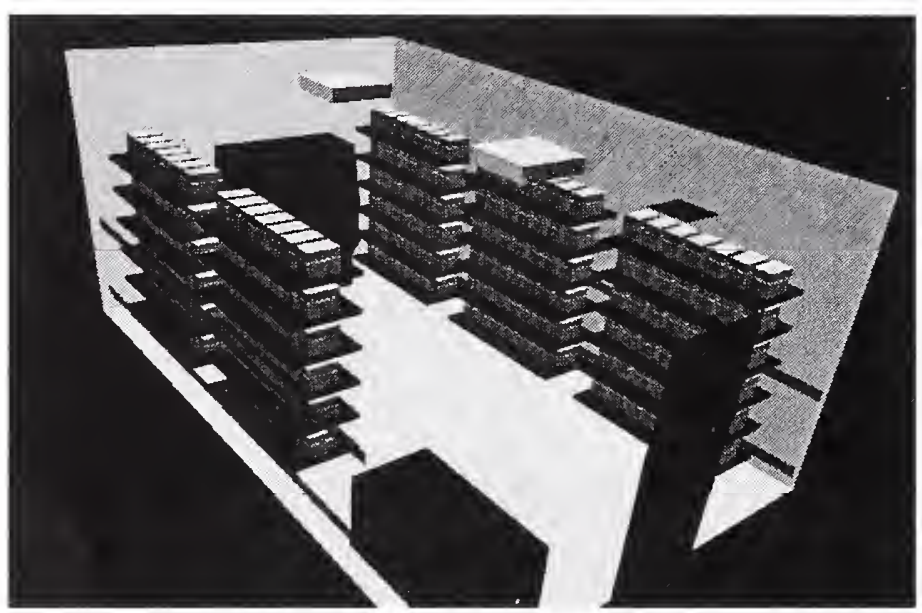

Analysis Results

Cage Occupied Zone

\begin{tabular}{|l|cc|c|c|}
\cline { 2 - 5 } \multicolumn{1}{c|}{} & \multicolumn{2}{c|}{ Temperature } & $\mathrm{CO}_{2}$ & RH \\
\cline { 2 - 5 } \multicolumn{1}{c|}{} & ${ }^{\circ} \mathrm{C}$ & ${ }^{\circ} \mathrm{F}$ & $(\mathrm{ppm})$ & \\
\hline Mean & 22.08 & 71.74 & 1961 & $65.09 \%$ \\
\hline S.D. & 0.31 & 0.55 & 363 & $2.81 \%$ \\
\hline Max. & 23.03 & 73.46 & 2805 & $70.99 \%$ \\
\hline
\end{tabular}

Cage Occupied Zone NH3 (ppm)

\begin{tabular}{|l|c|c|c|c|c|c|c|c|c|c|}
\hline Day & $\mathbf{1}$ & $\mathbf{2}$ & $\mathbf{3}$ & $\mathbf{4}$ & $\mathbf{5}$ & $\mathbf{6}$ & $\mathbf{7}$ & $\mathbf{8}$ & $\mathbf{9}$ & $\mathbf{1 0}$ \\
\hline Mean & 1.04 & 1.98 & 3.06 & 5.36 & 8.67 & 12.68 & 18.73 & 23.62 & 28.89 & 32.55 \\
\hline Max. & 1.48 & 2.84 & 4.38 & 7.67 & 12.40 & 18.15 & 26.80 & 33.79 & 41.34 & 46.57 \\
\hline
\end{tabular}

Room Breathing Zone

\begin{tabular}{|l|cc|c|c|}
\cline { 2 - 5 } \multicolumn{1}{c|}{} & \multicolumn{2}{c|}{ Temperature } & $\mathrm{CO}_{2}$ & RH \\
\cline { 2 - 5 } \multicolumn{1}{c|}{} & ${ }^{\circ} \mathrm{C}$ & ${ }^{\circ} \mathrm{F}$ & $(\mathrm{ppm})$ & \\
\hline Mean & 20.26 & 68.46 & 40 & $55.53 \%$ \\
\hline S.D. & 0.23 & 0.41 & 16 & \\
\hline Max. & 20.93 & 69.68 & 176 & \\
\hline
\end{tabular}

Room Breathing Zone NH3 (ppm)

\begin{tabular}{|l|c|c|c|c|c|c|c|c|c|c|}
\hline Day & $\mathbf{1}$ & $\mathbf{2}$ & $\mathbf{3}$ & $\mathbf{4}$ & $\mathbf{5}$ & $\mathbf{6}$ & $\mathbf{7}$ & $\mathbf{8}$ & $\mathbf{9}$ & $\mathbf{1 0}$ \\
\hline Mean & 0.02 & 0.04 & 0.06 & 0.11 & 0.18 & 0.26 & 0.38 & 0.48 & 0.58 & 0.66 \\
\hline Max. & 0.09 & 0.18 & 0.27 & 0.48 & 0.78 & 1.14 & 1.68 & 2.12 & 2.59 & 2.92 \\
\hline
\end{tabular}




\section{Histogram Distributions}

Cage occupied zone average temperature $\left({ }^{\circ} \mathrm{C}\right)$ distribution

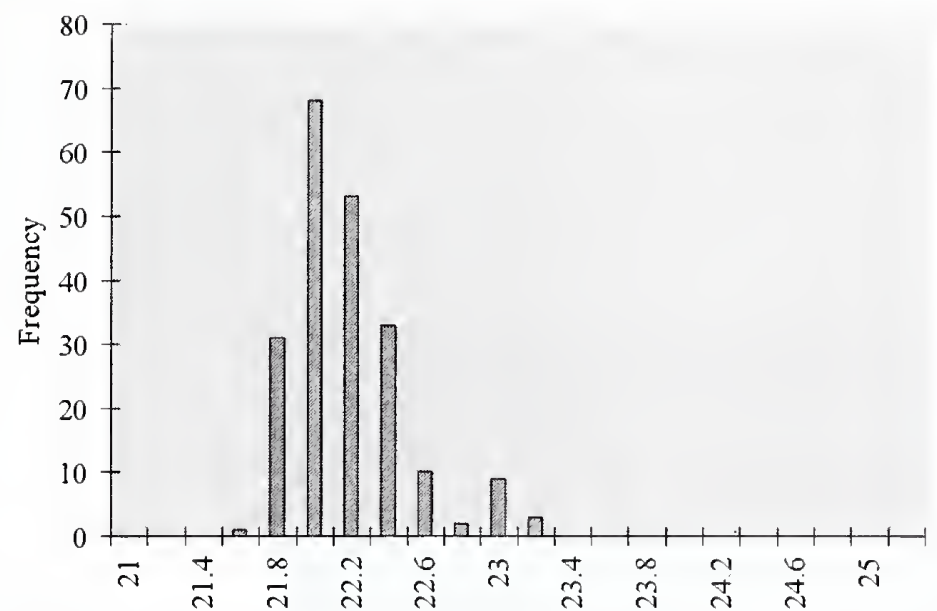

Cage occupied zone average contamination $(\mathrm{kg} / \mathrm{kg})$ distribution

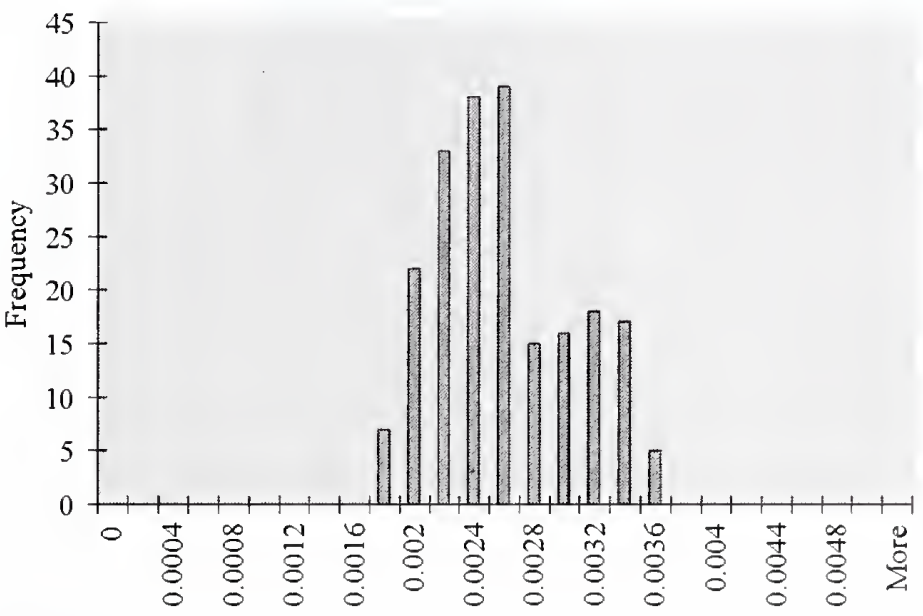

Cage occupied zone average relative humidity (\%) distribution

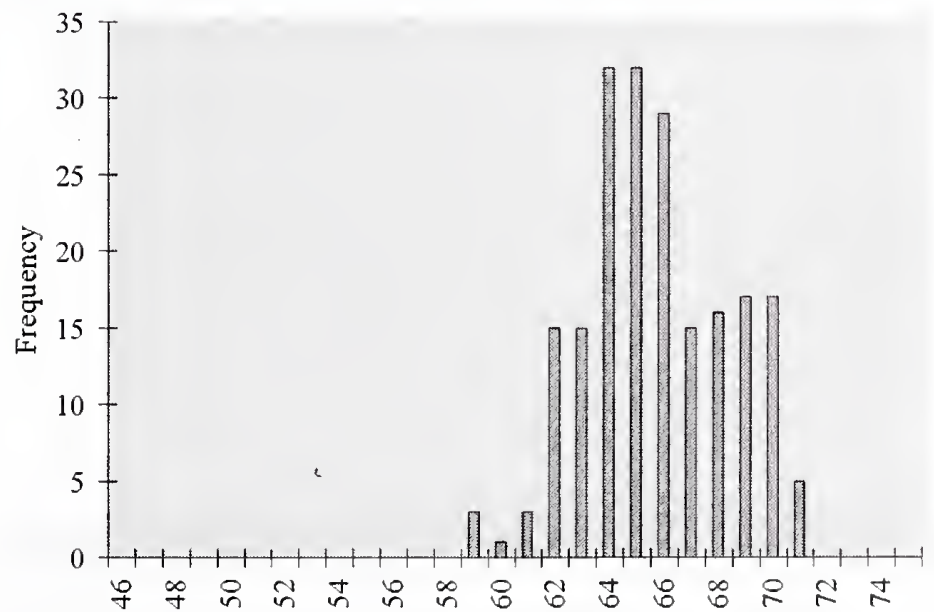

Contamination conversion factors $(\mathrm{kg} / \mathrm{kg} \rightarrow \mathrm{ppm})$

\begin{tabular}{|c|c|c|}
\hline Day & $\mathbf{C O}_{2}$ & $\mathbf{N H}_{3}$ \\
\hline $\mathbf{1}$ & 785000 & 415 \\
\hline $\mathbf{2}$ & 785000 & 795 \\
\hline $\mathbf{3}$ & 785000 & 1225 \\
\hline $\mathbf{4}$ & 785000 & 2146 \\
\hline $\mathbf{5}$ & 785000 & 3470 \\
\hline $\mathbf{6}$ & 785000 & 5079 \\
\hline $\mathbf{7}$ & 785000 & 7501 \\
\hline $\mathbf{8}$ & 785000 & 9456 \\
\hline $\mathbf{9}$ & 785000 & 11568 \\
\hline $\mathbf{1 0}$ & 785000 & 11384 \\
\hline
\end{tabular}


Casename $\quad$ Case 48

\section{Description}

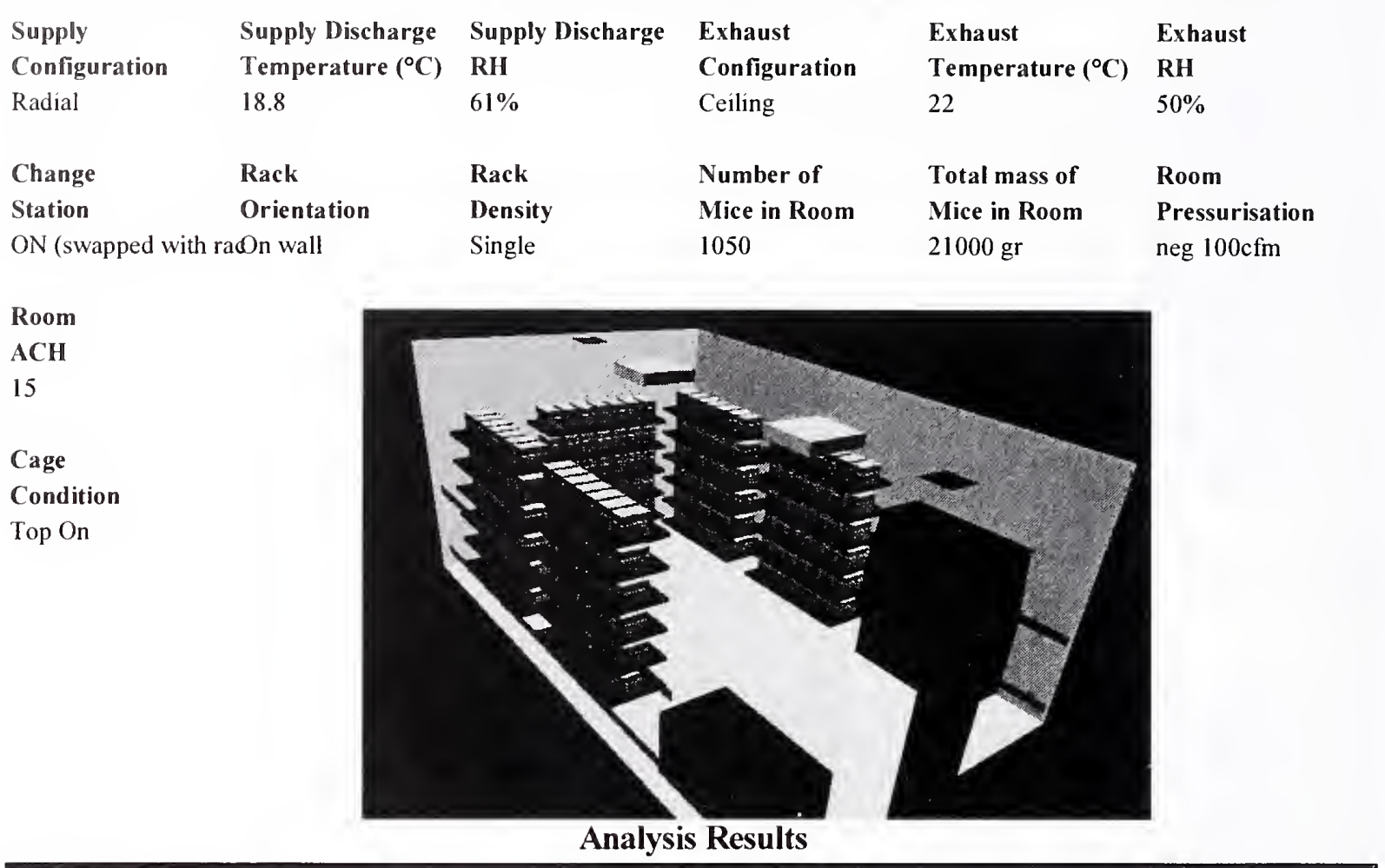

Cage Occupied Zone

\begin{tabular}{|l|cc|c|c|}
\cline { 2 - 5 } \multicolumn{1}{c|}{} & \multicolumn{2}{c|}{ Temperature } & $\mathbf{C O}_{2}$ & RH \\
\cline { 2 - 5 } \multicolumn{1}{c|}{} & ${ }^{\circ} \mathrm{C}$ & ${ }^{\circ} \mathrm{F}$ & $(\mathrm{ppm})$ & \\
\hline Mean & 22.11 & 71.80 & 2099 & $66.07 \%$ \\
\hline S.D. & 0.21 & 0.38 & 368 & $2.74 \%$ \\
\hline Max. & 22.62 & 72.72 & 2715 & $70.68 \%$ \\
\hline
\end{tabular}

\section{Cage Occupied Zone NH3 (ppm)}

\begin{tabular}{|l|c|c|c|c|c|c|c|c|c|c|}
\hline Day & $\mathbf{1}$ & $\mathbf{2}$ & $\mathbf{3}$ & $\mathbf{4}$ & $\mathbf{5}$ & $\mathbf{6}$ & $\mathbf{7}$ & $\mathbf{8}$ & $\mathbf{9}$ & $\mathbf{1 0}$ \\
\hline Mean & 1.12 & 2.12 & 3.28 & 5.98 & 9.91 & 14.62 & 21.83 & 27.26 & 32.92 & 36.12 \\
\hline Max. & 1.44 & 2.75 & 4.24 & 7.73 & 12.81 & 18.91 & 28.23 & 35.25 & 42.58 & 46.71 \\
\hline
\end{tabular}

Room Breathing Zone

\begin{tabular}{|l|cc|c|c|}
\cline { 2 - 5 } \multicolumn{1}{c|}{} & \multicolumn{2}{c|}{ Temperature } & $\mathrm{CO}_{2}$ & RH \\
\cline { 2 - 5 } \multicolumn{1}{c|}{} & ${ }^{\circ} \mathrm{C}$ & ${ }^{\circ} \mathrm{F}$ & $(\mathbf{p p m})$ & \\
\hline Mean & 20.61 & 69.09 & 65 & $54.53 \%$ \\
\hline S.D. & 0.52 & 0.93 & 44 & \\
\hline Max. & 25.89 & 78.59 & 302 & \\
\hline
\end{tabular}

Room Breathing Zone NH3 (ppm)

\begin{tabular}{|l|c|c|c|c|c|c|c|c|c|c|}
\hline Day & $\mathbf{1}$ & $\mathbf{2}$ & $\mathbf{3}$ & $\mathbf{4}$ & $\mathbf{5}$ & $\mathbf{6}$ & $\mathbf{7}$ & $\mathbf{8}$ & $\mathbf{9}$ & $\mathbf{1 0}$ \\
\hline Mean & 0.03 & 0.07 & 0.10 & 0.18 & 0.30 & 0.45 & 0.67 & 0.84 & 1.01 & 1.11 \\
\hline Max. & 0.16 & 0.31 & 0.47 & 0.86 & 1.42 & 2.10 & 3.14 & 3.92 & 4.73 & 5.19 \\
\hline
\end{tabular}




\section{Histogram Distributions}

Cage occupied zone average temperature $\left({ }^{\circ} \mathrm{C}\right)$ distribution

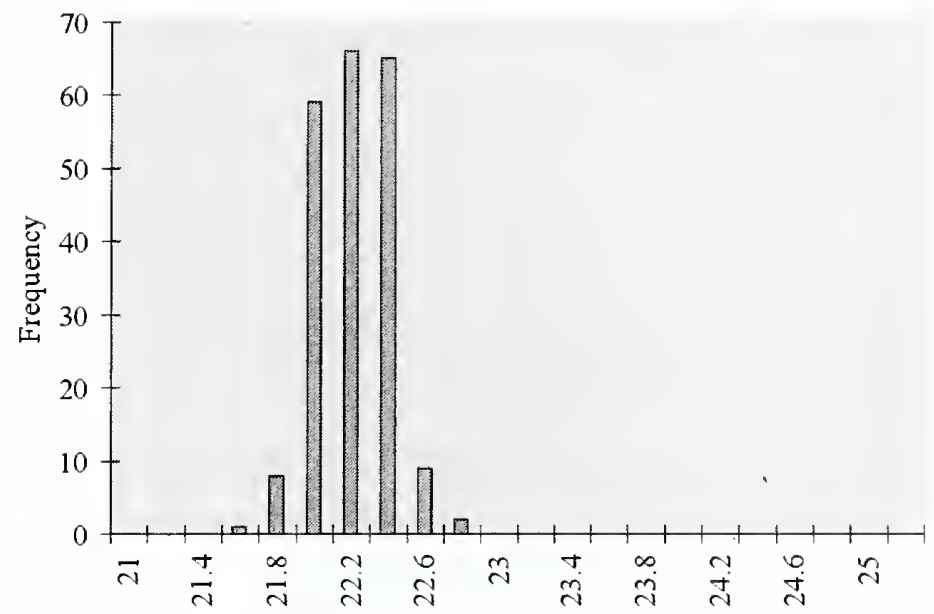

Cage occupied zone average contamination $(\mathrm{kg} / \mathrm{kg})$ distribution Contamination conversion factors $(\mathrm{kg} / \mathrm{kg} \rightarrow \mathrm{ppm})$

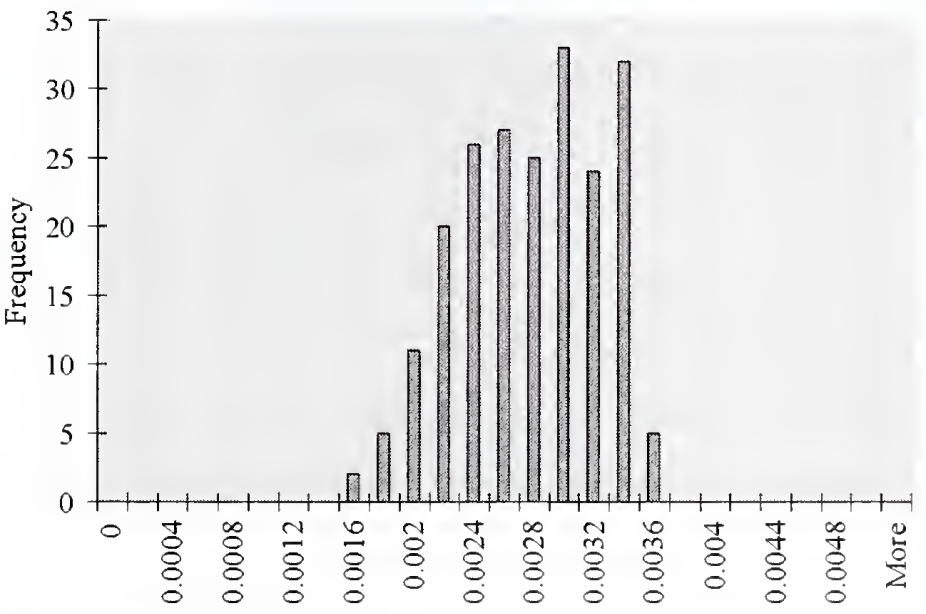

\begin{tabular}{|c|c|c|}
\hline Day & $\mathrm{CO}_{2}$ & $\mathbf{N H}_{3}$ \\
\hline $\mathbf{1}$ & 785000 & 417 \\
\hline $\mathbf{2}$ & 785000 & 795 \\
\hline $\mathbf{3}$ & 785000 & 1225 \\
\hline $\mathbf{4}$ & 785000 & 2237 \\
\hline $\mathbf{5}$ & 785000 & 3704 \\
\hline $\mathbf{6}$ & 785000 & 5468 \\
\hline $\mathbf{7}$ & 785000 & 8165 \\
\hline $\mathbf{8}$ & 785000 & 10195 \\
\hline $\mathbf{9}$ & 785000 & 12313 \\
\hline $\mathbf{1 0}$ & 785000 & 11384 \\
\hline
\end{tabular}

Cage occupied zone average relative humidity (\%) distribution

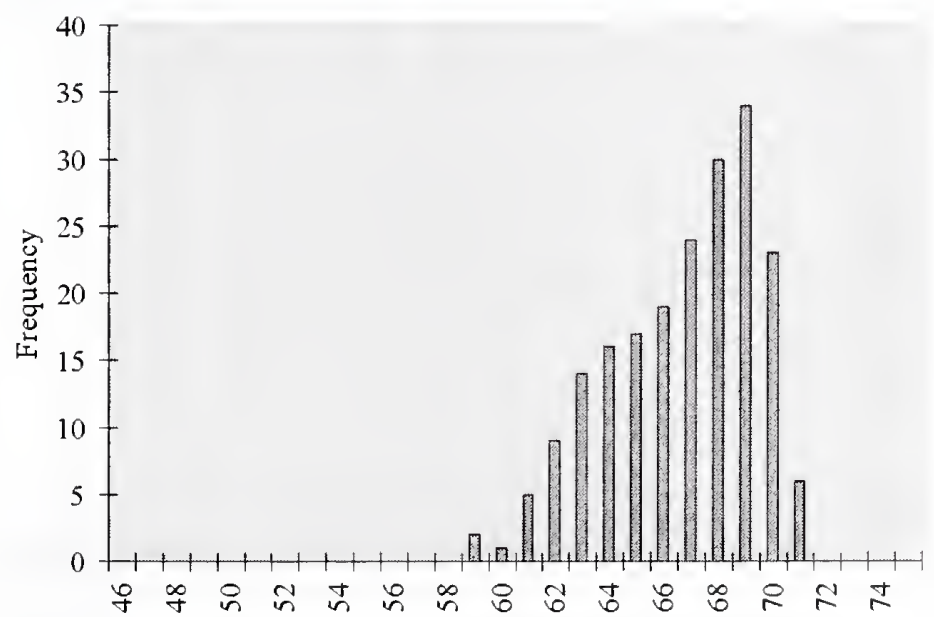


Casename $\quad$ Case 49

\section{Description}

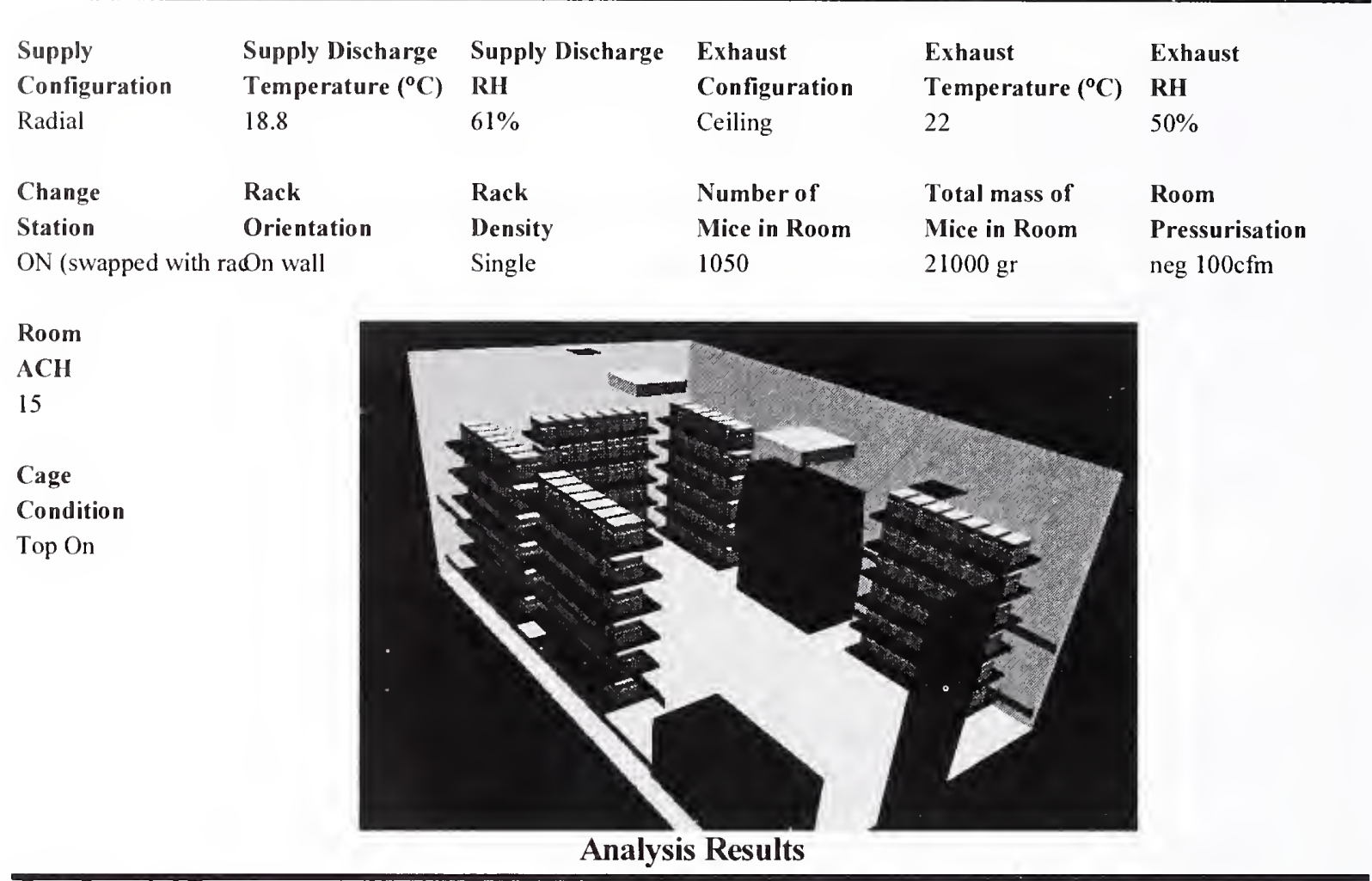

Cage Occupied Zone

\begin{tabular}{|l|cc|c|c|}
\cline { 2 - 5 } \multicolumn{1}{c|}{} & \multicolumn{2}{c|}{ Temperature } & $\mathrm{CO}_{2}$ & RH \\
\cline { 2 - 5 } \multicolumn{1}{c|}{} & ${ }^{\circ} \mathrm{C}$ & ${ }^{\circ} \mathrm{F}$ & $(\mathrm{ppm})$ & \\
\hline Mean & 22.18 & 71.92 & 2025 & $65.20 \%$ \\
\hline S.D. & 0.22 & 0.39 & 351 & $2.99 \%$ \\
\hline Max. & 23.20 & 73.77 & 2683 & $69.92 \%$ \\
\hline
\end{tabular}

Cage Occupied Zone NH3 (ppm)

\begin{tabular}{|l|c|c|c|c|c|c|c|c|c|c|}
\hline Day & $\mathbf{1}$ & $\mathbf{2}$ & $\mathbf{3}$ & $\mathbf{4}$ & $\mathbf{5}$ & $\mathbf{6}$ & $\mathbf{7}$ & $\mathbf{8}$ & $\mathbf{9}$ & $\mathbf{1 0}$ \\
\hline Mean & 1.07 & 2.05 & 3.16 & 5.56 & 9.02 & 13.21 & 19.54 & 24.61 & 30.06 & 33.76 \\
\hline Max. & 1.42 & 2.72 & 4.19 & 7.37 & 11.95 & 17.51 & 25.89 & 32.60 & 39.82 & 44.72 \\
\hline
\end{tabular}

Room Breathing Zone

\begin{tabular}{|l|cc|c|c|}
\cline { 2 - 5 } \multicolumn{1}{c|}{} & \multicolumn{2}{c|}{ Temperature } & $\mathrm{CO}_{2}$ & RH \\
\cline { 2 - 5 } \multicolumn{1}{c|}{} & ${ }^{\circ} \mathrm{C}$ & ${ }^{\circ} \mathrm{F}$ & $(\mathrm{ppm})$ & \\
\hline Mean & 20.55 & 68.98 & 52 & $54.62 \%$ \\
\hline S.D. & 0.56 & 1.02 & 39 & \\
\hline Max. & 26.07 & 78.93 & 246 & \\
\hline
\end{tabular}

Room Breathing Zone NH3 (ppm)

\begin{tabular}{|l|c|c|c|c|c|c|c|c|c|c|}
\hline Day & $\mathbf{1}$ & $\mathbf{2}$ & $\mathbf{3}$ & $\mathbf{4}$ & $\mathbf{5}$ & $\mathbf{6}$ & $\mathbf{7}$ & $\mathbf{8}$ & $\mathbf{9}$ & $\mathbf{1 0}$ \\
\hline Mean & 0.03 & 0.05 & 0.08 & 0.14 & 0.23 & 0.34 & 0.50 & 0.63 & 0.77 & 0.86 \\
\hline Max. & 0.13 & 0.25 & 0.38 & 0.68 & 1.10 & 1.61 & 2.38 & 3.00 & 3.66 & 4.11 \\
\hline
\end{tabular}




\section{Histogram Distributions}

Cage occupied zone average temperature $\left({ }^{\circ} \mathrm{C}\right)$ distribution

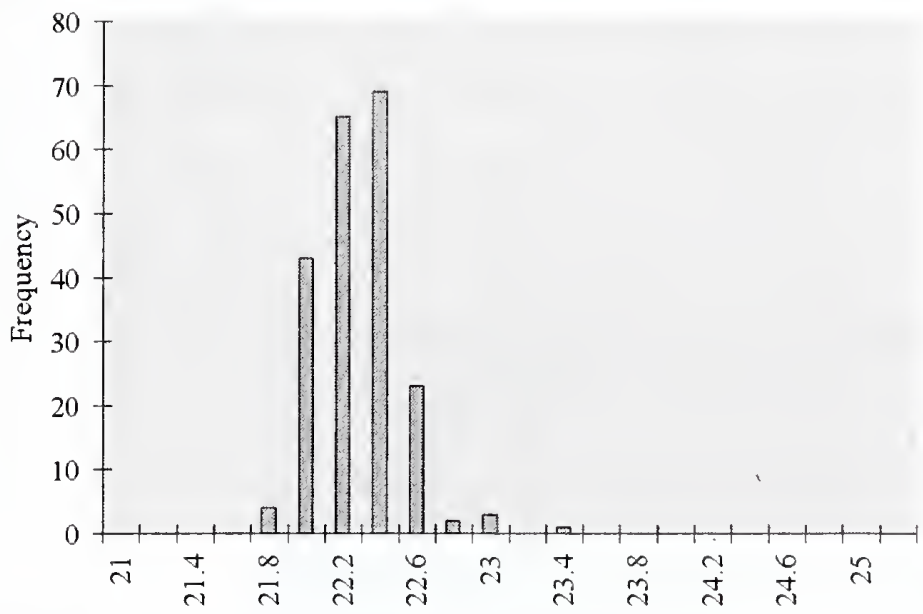

Cage occupied zone average contamination $(\mathrm{kg} / \mathrm{kg})$ distribution

Contamination conversion factors $(\mathrm{kg} / \mathrm{kg} \rightarrow \mathrm{ppm})$

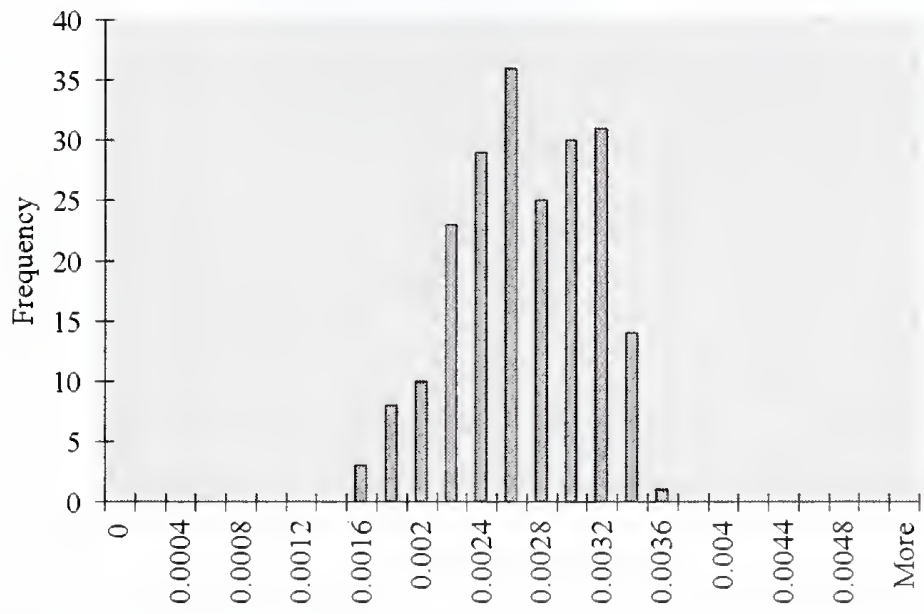

\begin{tabular}{|c|c|c|}
\hline Day & $\mathbf{C O}_{2}$ & $\mathbf{N H}_{3}$ \\
\hline $\mathbf{1}$ & 785000 & 416 \\
\hline $\mathbf{2}$ & 785000 & 795 \\
\hline $\mathbf{3}$ & 785000 & 1225 \\
\hline $\mathbf{4}$ & 785000 & 2156 \\
\hline $\mathbf{5}$ & 785000 & 3496 \\
\hline $\mathbf{6}$ & 785000 & 5123 \\
\hline $\mathbf{7}$ & 785000 & 7576 \\
\hline $\mathbf{8}$ & 785000 & 9540 \\
\hline $\mathbf{9}$ & 785000 & 11652 \\
\hline $\mathbf{1 0}$ & 785000 & 11384 \\
\hline
\end{tabular}

Cage occupied zone average relative humidity (\%) distribution

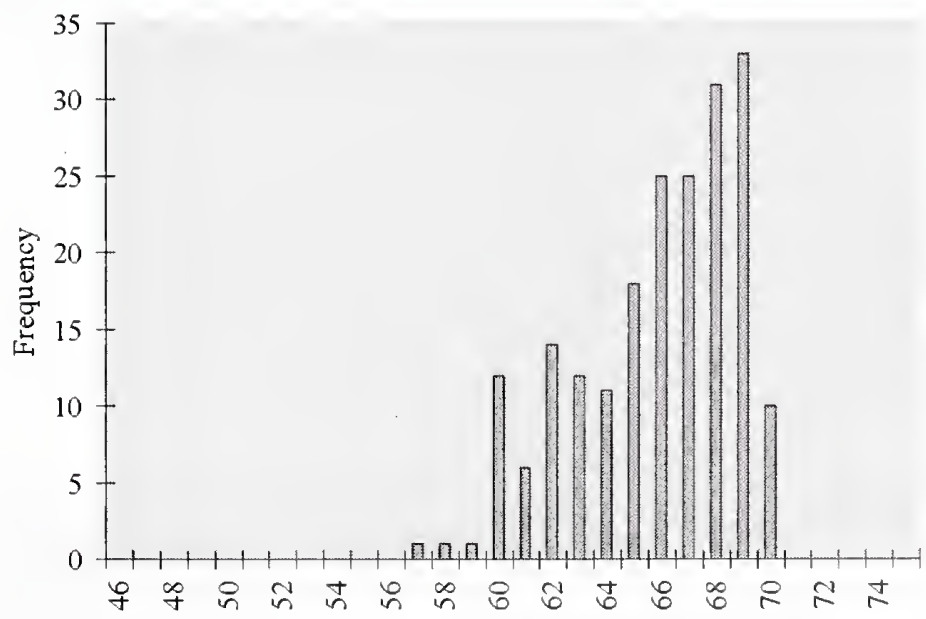




\section{Casename $\quad$ Case $\mathbf{5 0}$}

\section{Description}

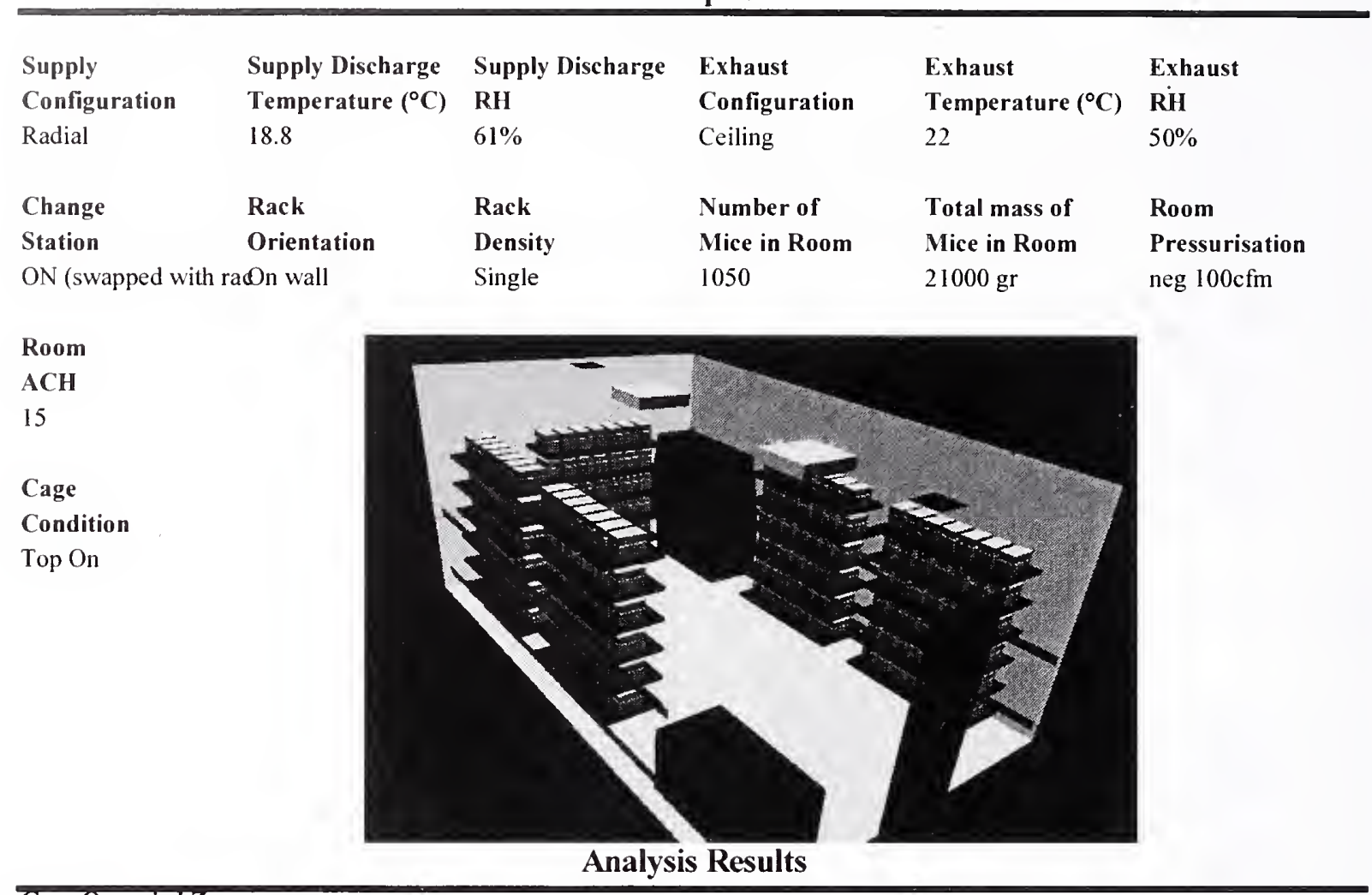

Cage Occupied Zone

\begin{tabular}{|l|cc|c|c|}
\cline { 2 - 5 } \multicolumn{1}{c|}{} & \multicolumn{2}{c|}{ Temperature } & $\mathrm{CO}_{2}$ & RH \\
\cline { 2 - 5 } \multicolumn{1}{c|}{} & ${ }^{\circ} \mathrm{C}$ & ${ }^{\circ} \mathrm{F}$ & $(\mathbf{p p m})$ & \\
\hline Mean & 22.17 & 71.91 & 1840 & $63.72 \%$ \\
\hline S.D. & 0.17 & 0.31 & 402 & $3.09 \%$ \\
\hline Max. & 22.58 & 72.64 & 2594 & $69.58 \%$ \\
\hline
\end{tabular}

Cage Occupied Zone NH3 (ppm)

\begin{tabular}{|l|c|c|c|c|c|c|c|c|c|c|}
\hline Day & $\mathbf{1}$ & $\mathbf{2}$ & $\mathbf{3}$ & $\mathbf{4}$ & $\mathbf{5}$ & $\mathbf{6}$ & $\mathbf{7}$ & $\mathbf{8}$ & $\mathbf{9}$ & $\mathbf{1 0}$ \\
\hline Mean & 0.97 & 1.86 & 2.87 & 4.73 & 7.36 & 10.62 & 15.40 & 19.74 & 24.67 & 28.99 \\
\hline Max. & 1.36 & 2.63 & 4.05 & 6.67 & 10.37 & 14.97 & 21.70 & 27.81 & 34.76 & 40.85 \\
\hline
\end{tabular}

Room Breathing Zone

\begin{tabular}{|l|cc|c|c|}
\cline { 2 - 5 } \multicolumn{1}{c|}{} & \multicolumn{2}{c|}{ Temperature } & $\mathrm{CO}_{2}$ & RH \\
\cline { 2 - 5 } \multicolumn{1}{c|}{} & ${ }^{\circ} \mathrm{C}$ & ${ }^{\circ} \mathrm{F}$ & $(\mathbf{p p m})$ & \\
\hline Mean & 20.46 & 68.83 & 55 & $54.94 \%$ \\
\hline S.D. & 0.29 & 0.52 & 26 & \\
\hline Max. & 25.99 & 78.78 & 198 & \\
\hline
\end{tabular}

Room Breathing Zone NH3 (ppm)

\begin{tabular}{|l|c|c|c|c|c|c|c|c|c|c|}
\hline Day & $\mathbf{1}$ & $\mathbf{2}$ & $\mathbf{3}$ & $\mathbf{4}$ & $\mathbf{5}$ & $\mathbf{6}$ & $\mathbf{7}$ & $\mathbf{8}$ & 9 & $\mathbf{1 0}$ \\
\hline Mean & 0.03 & 0.06 & 0.09 & 0.14 & 0.22 & 0.32 & 0.46 & 0.59 & 0.74 & 0.86 \\
\hline Max. & 0.10 & 0.20 & 0.31 & 0.51 & 0.79 & 1.14 & 1.65 & 2.12 & 2.65 & 3.11 \\
\hline
\end{tabular}




\section{Histogram Distributions}

Cage occupied zone average temperature $\left({ }^{\circ} \mathrm{C}\right)$ distribution

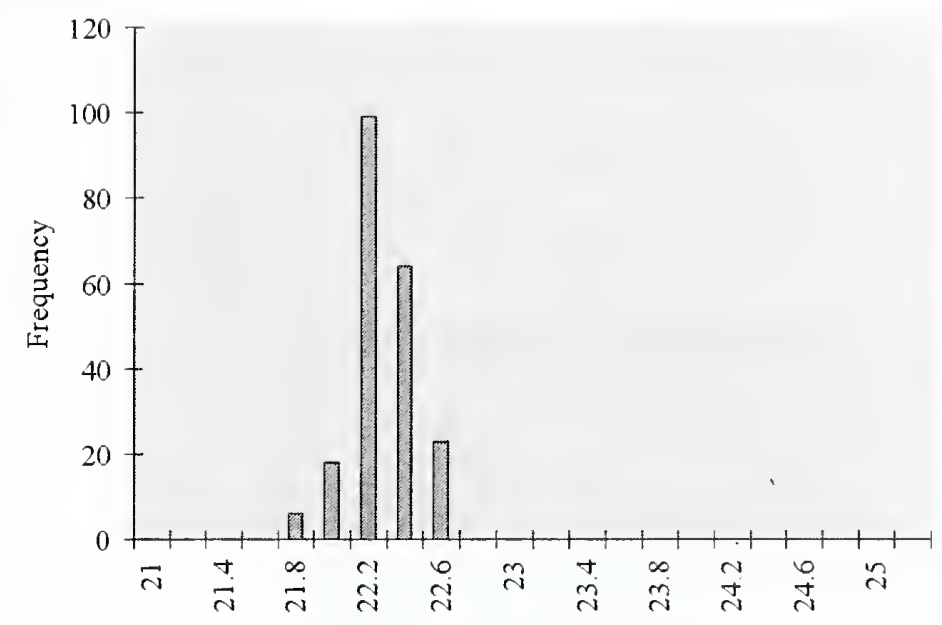

Cage occupied zone average contamination $(\mathrm{kg} / \mathrm{kg})$ distribution

Contamination conversion factors $(\mathrm{kg} / \mathrm{kg} \rightarrow \mathrm{ppm})$

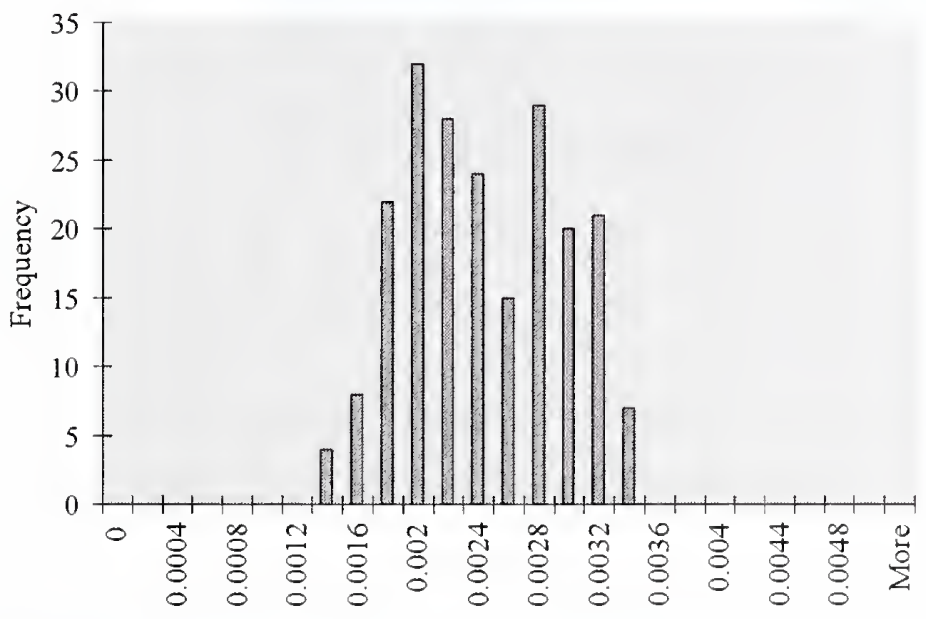

\begin{tabular}{|c|c|c|}
\hline Day & $\mathbf{C O}_{\mathbf{2}}$ & $\mathbf{N H}_{\mathbf{3}}$ \\
\hline $\mathbf{1}$ & 785000 & 413 \\
\hline $\mathbf{2}$ & 785000 & 795 \\
\hline $\mathbf{3}$ & 785000 & 1225 \\
\hline $\mathbf{4}$ & 785000 & 2019 \\
\hline $\mathbf{5}$ & 785000 & 3139 \\
\hline $\mathbf{6}$ & 785000 & 4531 \\
\hline $\mathbf{7}$ & 785000 & 6567 \\
\hline $\mathbf{8}$ & 785000 & 8418 \\
\hline $\mathbf{9}$ & 785000 & 10521 \\
\hline $\mathbf{1 0}$ & 785000 & 11384 \\
\hline
\end{tabular}

Cage occupied zone average relative humidity (\%) distribution

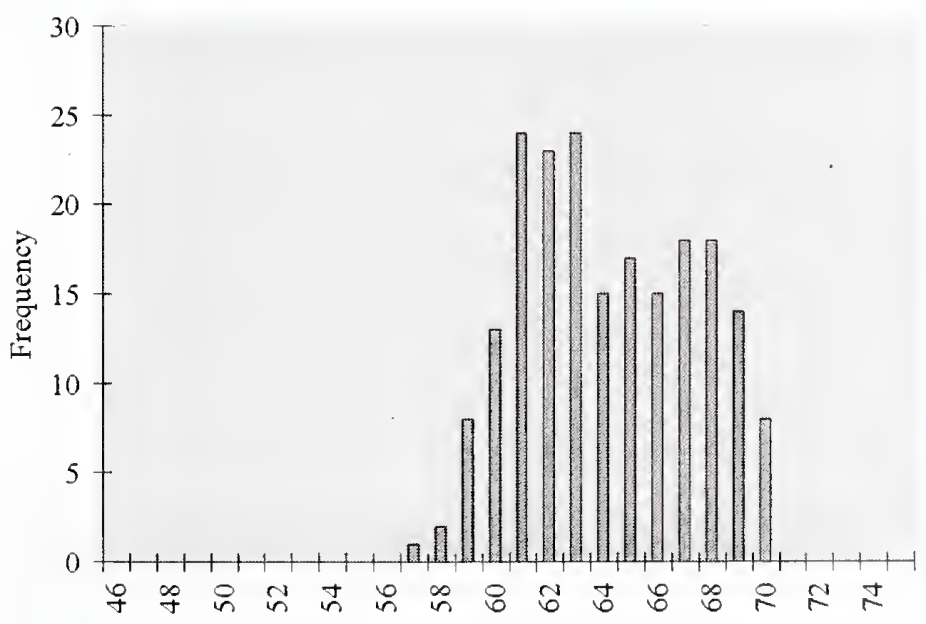


Casename

Case 51

Description

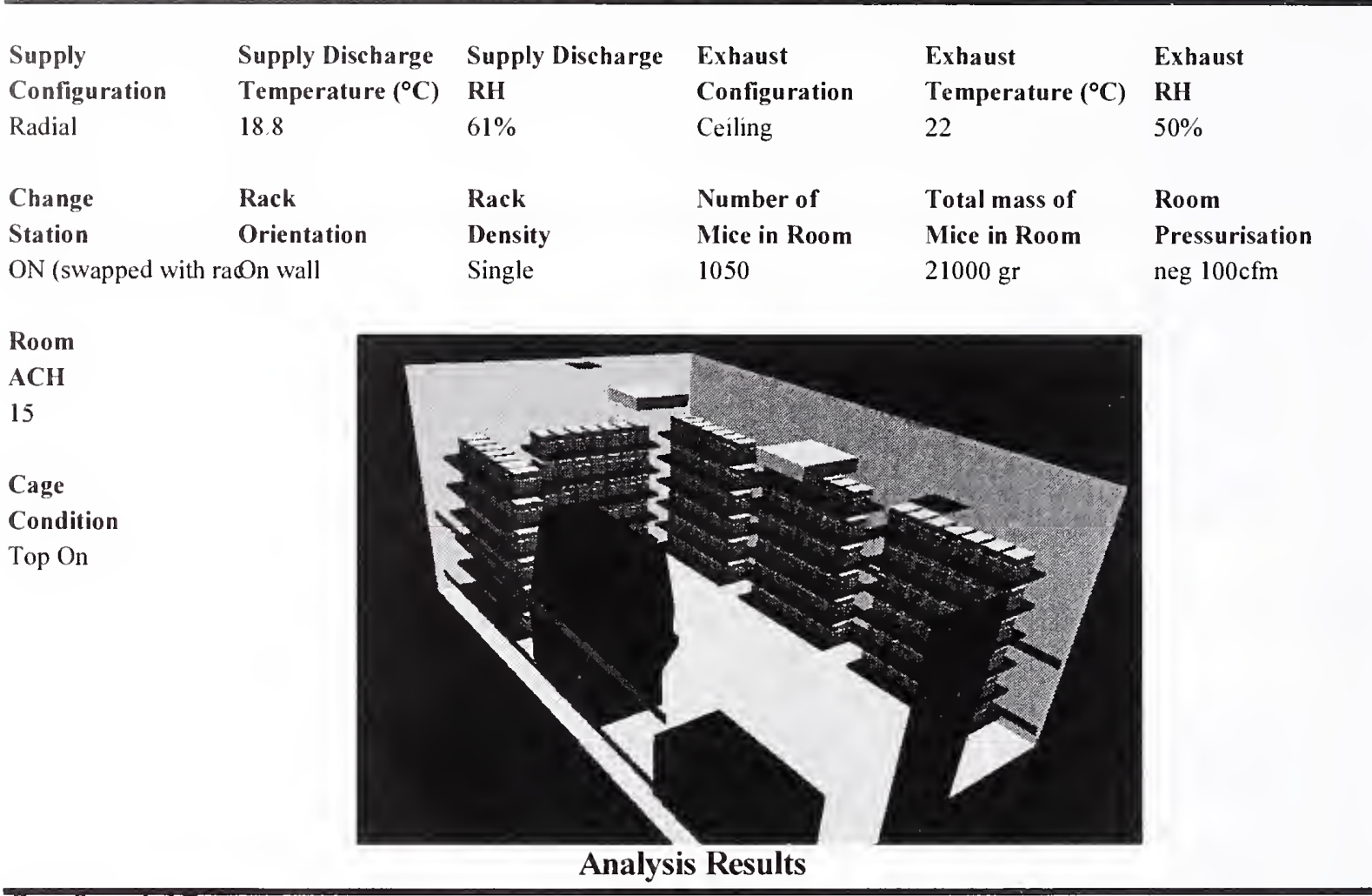

Cage Occupied Zone

\begin{tabular}{|l|cc|c|c|}
\cline { 2 - 5 } \multicolumn{1}{c|}{} & \multicolumn{2}{c|}{ Temperature } & $\mathbf{C O}_{2}$ & RH \\
\cline { 2 - 5 } \multicolumn{1}{c|}{} & ${ }^{\circ} \mathrm{C}$ & ${ }^{\circ}$ & $(\mathbf{p p m})$ & \\
\hline Mean & 22.19 & 71.95 & 2045 & $65.30 \%$ \\
\hline S.D. & 0.23 & 0.41 & 341 & $2.93 \%$ \\
\hline Max. & 23.17 & 73.71 & 2710 & $70.21 \%$ \\
\hline
\end{tabular}

Cage Occupied Zone NH3 (ppm)

\begin{tabular}{|l|c|c|c|c|c|c|c|c|c|c|}
\hline Day & $\mathbf{1}$ & $\mathbf{2}$ & $\mathbf{3}$ & $\mathbf{4}$ & $\mathbf{5}$ & $\mathbf{6}$ & $\mathbf{7}$ & $\mathbf{8}$ & $\mathbf{9}$ & $\mathbf{1 0}$ \\
\hline Mean & 1.08 & 2.07 & 3.19 & 5.64 & 9.17 & 13.45 & 19.91 & 25.05 & 30.56 & 34.22 \\
\hline Max. & 1.44 & 2.74 & 4.23 & 7.48 & 12.15 & 17.82 & 26.39 & 33.19 & 40.49 & 45.34 \\
\hline
\end{tabular}

Room Breathing Zone

\begin{tabular}{|l|cc|c|c|}
\cline { 2 - 5 } \multicolumn{1}{c|}{} & \multicolumn{2}{c|}{ Temperature } & $\mathrm{CO}_{2}$ & RH \\
\cline { 2 - 5 } \multicolumn{1}{c|}{} & ${ }^{\circ} \mathrm{C}$ & ${ }^{\circ} \mathrm{F}$ & $(\mathbf{p p m})$ & \\
\hline Mean & 20.54 & 68.96 & 67 & $54.79 \%$ \\
\hline S.D. & 0.41 & 0.74 & 37 & \\
\hline Max. & 25.57 & 78.03 & 229 & \\
\hline
\end{tabular}

Room Breathing Zone NH3 (ppm)

\begin{tabular}{|l|c|c|c|c|c|c|c|c|c|c|}
\hline Day & $\mathbf{l}$ & $\mathbf{2}$ & $\mathbf{3}$ & $\mathbf{4}$ & $\mathbf{5}$ & $\mathbf{6}$ & $\mathbf{7}$ & $\mathbf{8}$ & 9 & $\mathbf{1 0}$ \\
\hline Mean & 0.04 & 0.07 & 0.10 & 0.18 & 0.30 & 0.44 & 0.65 & 0.82 & 1.00 & 1.12 \\
\hline Max. & 0.12 & 0.23 & 0.36 & 0.63 & 1.03 & 1.51 & 2.23 & 2.81 & 3.42 & 3.83 \\
\hline
\end{tabular}




\section{Histogram Distributions}

Cage occupied zone average temperature $\left({ }^{\circ} \mathrm{C}\right)$ distribution

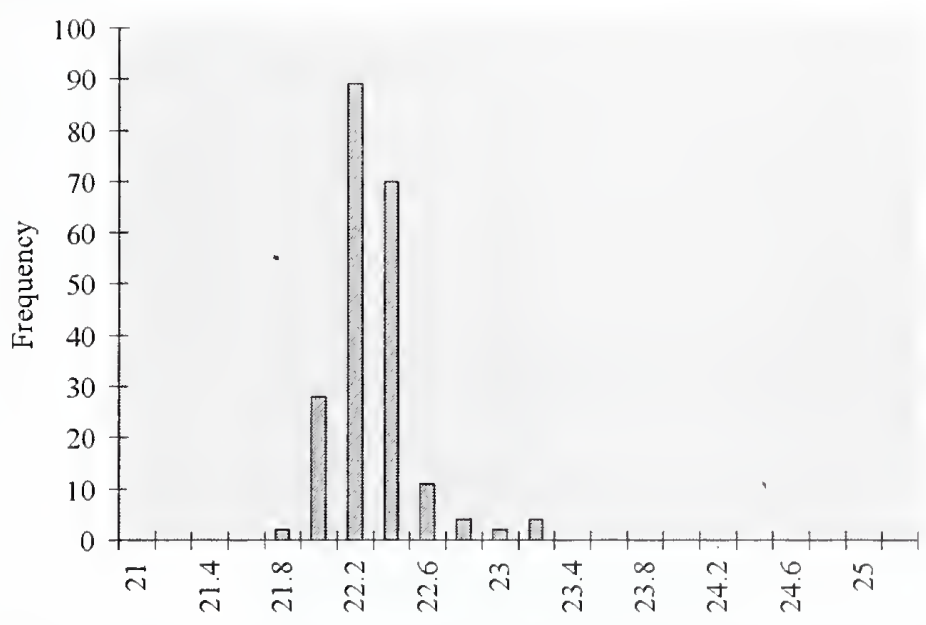

Cage occupied zone average contamination $(\mathrm{kg} / \mathrm{kg})$ distribution

Contamination conversion factors $(\mathrm{kg} / \mathrm{kg} \rightarrow \mathrm{ppm})$

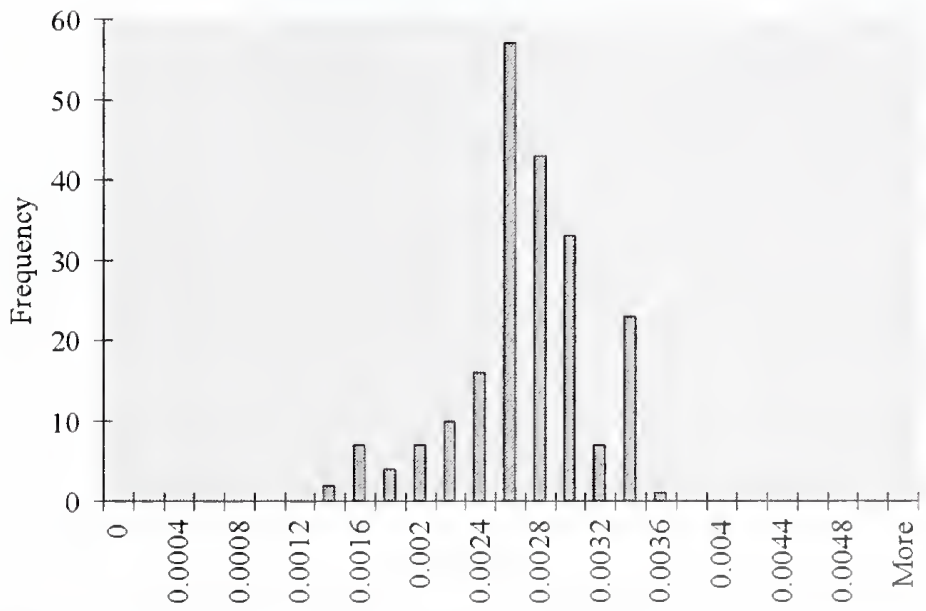

\begin{tabular}{|c|c|c|}
\hline Day & $\mathrm{CO}_{2}$ & $\mathrm{NH}_{3}$ \\
\hline $\mathbf{1}$ & 785000 & 416 \\
\hline $\mathbf{2}$ & 785000 & 795 \\
\hline $\mathbf{3}$ & 785000 & 1225 \\
\hline $\mathbf{4}$ & 785000 & 2166 \\
\hline $\mathbf{5}$ & 785000 & 3521 \\
\hline $\mathbf{6}$ & 785000 & 5163 \\
\hline $\mathbf{7}$ & 785000 & 7645 \\
\hline $\mathbf{8}$ & 785000 & 9617 \\
\hline $\mathbf{9}$ & 785000 & 11730 \\
\hline $\mathbf{1 0}$ & 785000 & 11384 \\
\hline
\end{tabular}

Cage occupied zone average relative humidity (\%) distribution

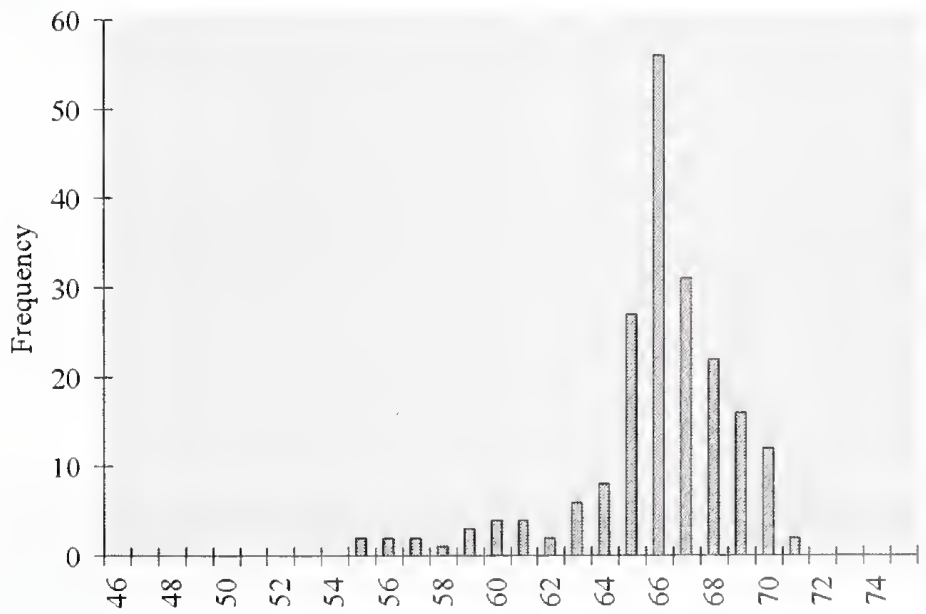


Casename

\section{Case 52}

\section{Description}

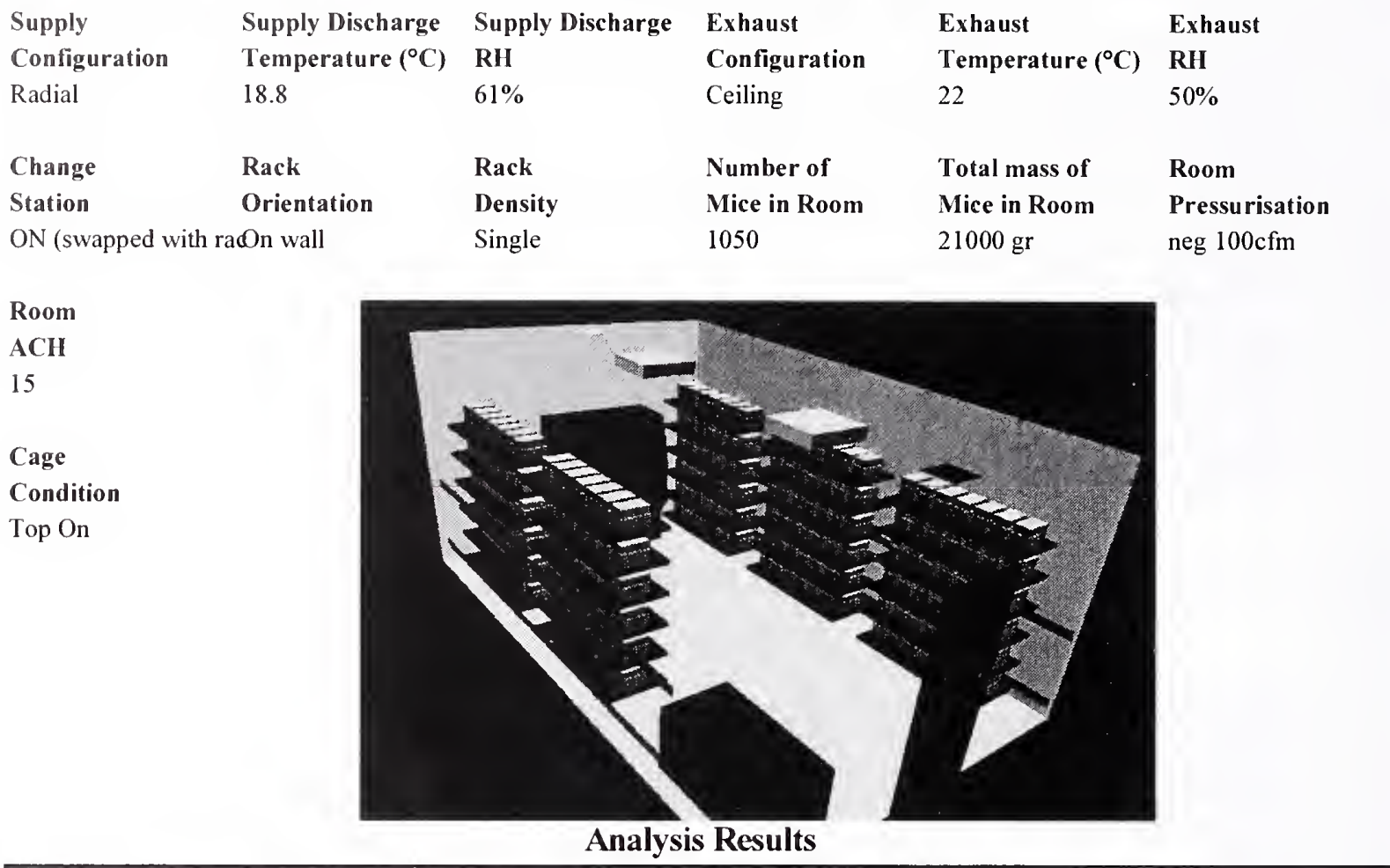

Cage Occupied Zone

\begin{tabular}{|l|cc|c|c|}
\cline { 2 - 5 } \multicolumn{1}{c|}{} & \multicolumn{2}{c|}{ Temperature } & $\mathbf{C O}_{2}$ & RH \\
\cline { 2 - 5 } \multicolumn{1}{c|}{} & ${ }^{\circ} \mathrm{C}$ & ${ }^{\circ} \mathrm{F}$ & $(\mathrm{ppm})$ & \\
\hline Mean & 22.47 & 72.45 & 1665 & $64.79 \%$ \\
\hline S.D. & 0.34 & 0.62 & 446 & $3.28 \%$ \\
\hline Max. & 23.41 & 74.13 & 2623 & $71.21 \%$ \\
\hline
\end{tabular}

Cage Occupied Zone NH3 (ppm)

\begin{tabular}{|l|c|c|c|c|c|c|c|c|c|c|}
\hline Day & $\mathbf{1}$ & $\mathbf{2}$ & $\mathbf{3}$ & $\mathbf{4}$ & $\mathbf{5}$ & $\mathbf{6}$ & $\mathbf{7}$ & $\mathbf{8}$ & $\mathbf{9}$ & $\mathbf{1 0}$ \\
\hline Mean & 0.88 & 1.69 & 2.60 & 4.49 & 7.20 & 10.51 & 15.47 & 19.57 & 24.04 & 27.33 \\
\hline Max. & 1.39 & 2.66 & 4.09 & 7.08 & 11.35 & 16.56 & 24.37 & 30.83 & 37.88 & 43.05 \\
\hline
\end{tabular}

Room Breathing Zone

\begin{tabular}{|l|cc|c|c|}
\cline { 2 - 5 } \multicolumn{1}{c|}{} & \multicolumn{2}{c|}{ Temperature } & $\mathrm{CO}_{2}$ & RH \\
\cline { 2 - 5 } \multicolumn{1}{c|}{} & ${ }^{\circ} \mathrm{C}$ & ${ }^{\circ}$ & $(\mathbf{p p m})$ & \\
\hline Mean & 20.46 & 68.83 & 65 & $55.03 \%$ \\
\hline S.D. & 0.23 & 0.41 & 46 & \\
\hline Max. & 25.02 & 77.03 & 363 & \\
\hline
\end{tabular}

Room Breathing Zone NH3 (ppm)

\begin{tabular}{|l|c|c|c|c|c|c|c|c|c|c|}
\hline Day & $\mathbf{1}$ & $\mathbf{2}$ & $\mathbf{3}$ & $\mathbf{4}$ & $\mathbf{5}$ & $\mathbf{6}$ & $\mathbf{7}$ & $\mathbf{8}$ & $\mathbf{9}$ & $\mathbf{1 0}$ \\
\hline Mean & 0.03 & 0.07 & 0.10 & 0.18 & 0.28 & 0.41 & 0.60 & 0.76 & 0.94 & 1.07 \\
\hline Max. & 0.19 & 0.37 & 0.57 & 0.98 & 1.57 & 2.29 & 3.37 & 4.26 & 5.24 & 5.95 \\
\hline
\end{tabular}




\section{Histogram Distributions}

Cage occupied zone average temperature $\left({ }^{\circ} \mathrm{C}\right)$ distribution

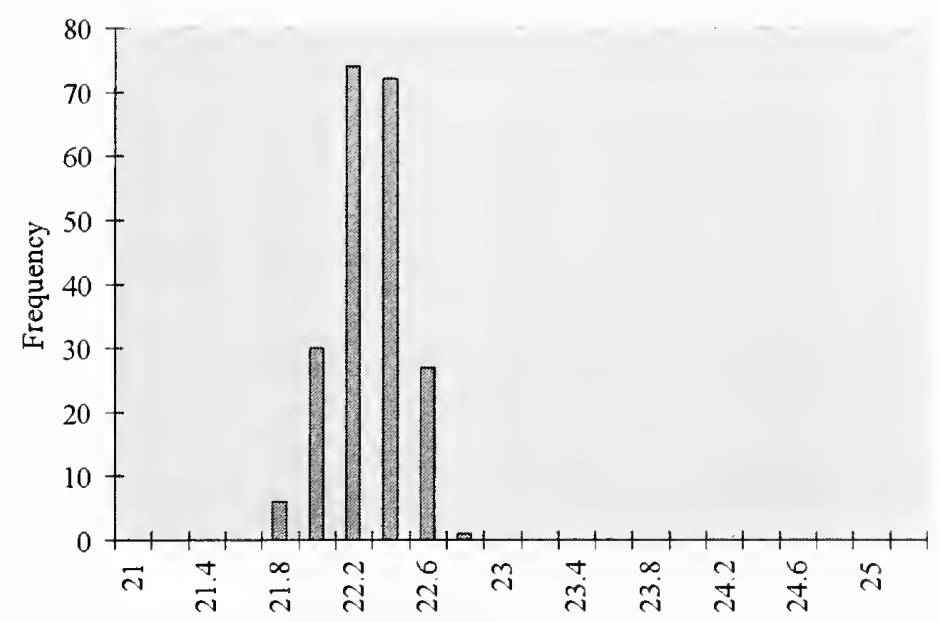

Cage occupied zone average contamination $(\mathrm{kg} / \mathrm{kg})$ distribution

Contamination conversion factors $(\mathrm{kg} / \mathrm{kg} \rightarrow \mathrm{ppm})$

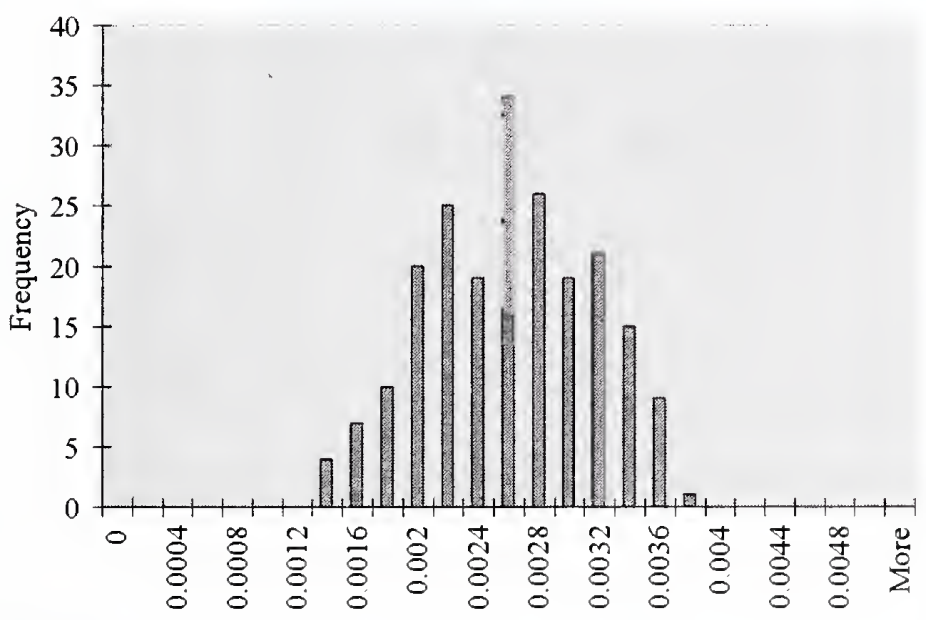

\begin{tabular}{|c|c|c|}
\hline Day & $\mathrm{CO}_{2}$ & $\mathrm{NH}_{3}$ \\
\hline $\mathbf{1}$ & 785000 & 415 \\
\hline $\mathbf{2}$ & 785000 & 795 \\
\hline $\mathbf{3}$ & 785000 & 1225 \\
\hline $\mathbf{4}$ & 785000 & 2118 \\
\hline $\mathbf{5}$ & 785000 & 3396 \\
\hline $\mathbf{6}$ & 785000 & 4956 \\
\hline $\mathbf{7}$ & 785000 & 7292 \\
\hline $\mathbf{8}$ & 785000 & 9224 \\
\hline $\mathbf{9}$ & 785000 & 11334 \\
\hline $\mathbf{1 0}$ & 785000 & 11384 \\
\hline
\end{tabular}

Cage occupied zone average relative humidity (\%) distribution

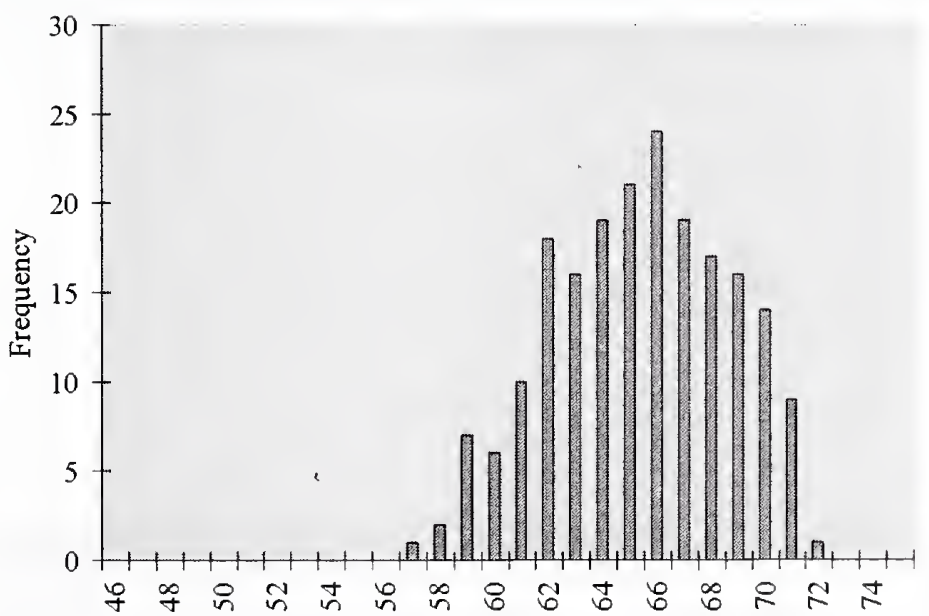


Casename Case 53

Description

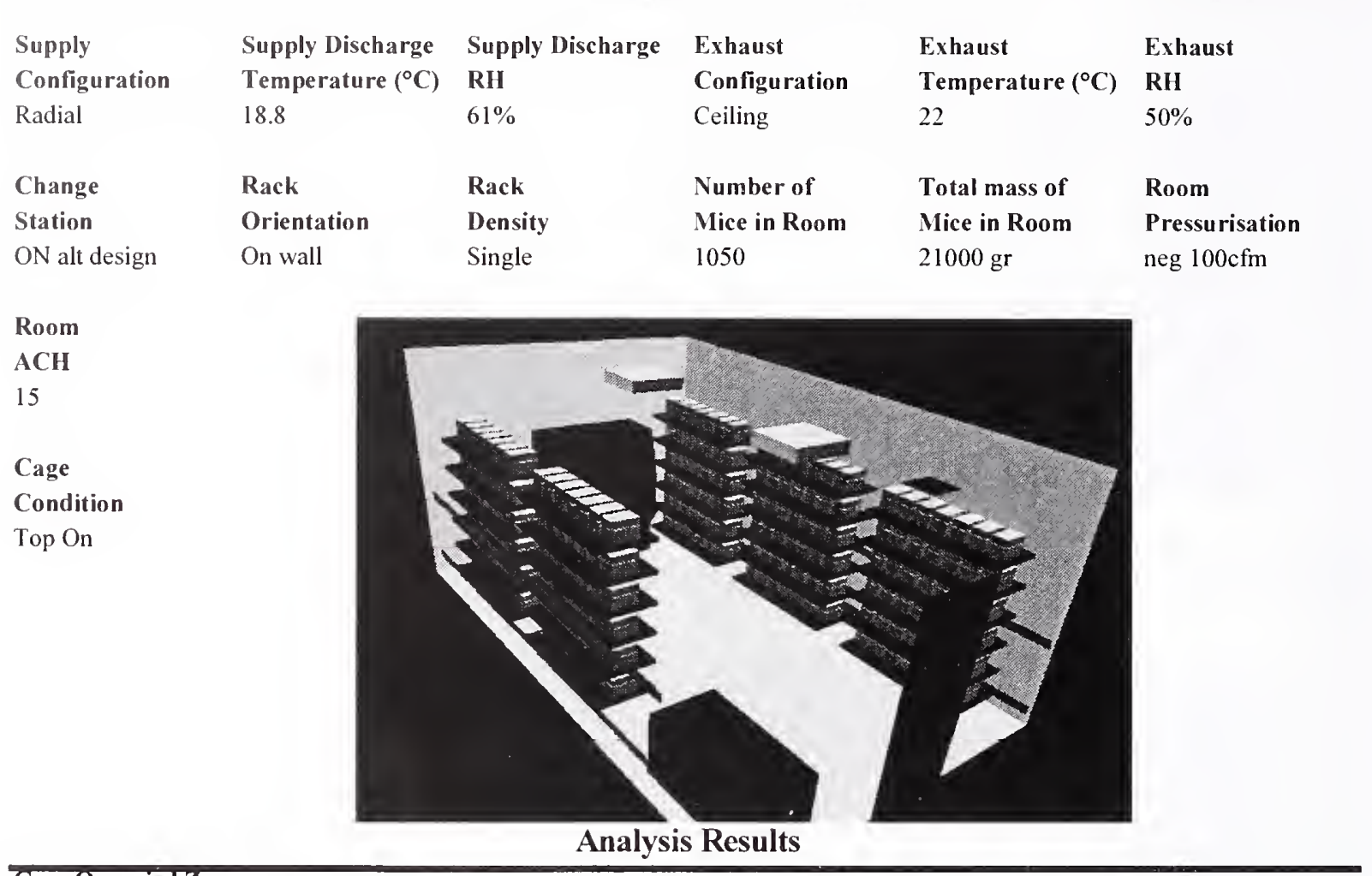

Cage Occupied Zone

\begin{tabular}{|l|cc|c|c|}
\cline { 2 - 5 } \multicolumn{1}{c|}{} & \multicolumn{2}{c|}{ Temperature } & $\mathrm{CO}_{2}$ & RH \\
\cline { 2 - 5 } \multicolumn{1}{c|}{} & ${ }^{\circ} \mathrm{C}$ & ${ }^{\circ} \mathrm{F}$ & $(\mathrm{ppm})$ & \\
\hline Mean & 21.69 & 71.03 & 2212 & $68.80 \%$ \\
\hline S.D. & 0.15 & 0.26 & 353 & $2.63 \%$ \\
\hline Max. & 21.98 & 71.56 & 2826 & $73.06 \%$ \\
\hline
\end{tabular}

Cage Occupied Zone NH3 (ppm)

\begin{tabular}{|l|c|c|c|c|c|c|c|c|c|c|}
\hline Day & $\mathbf{1}$ & $\mathbf{2}$ & $\mathbf{3}$ & $\mathbf{4}$ & $\mathbf{5}$ & $\mathbf{6}$ & $\mathbf{7}$ & $\mathbf{8}$ & $\mathbf{9}$ & $\mathbf{1 0}$ \\
\hline Mean & 1.19 & 2.24 & 3.45 & 7.02 & 12.29 & 18.48 & 28.24 & 34.54 & 40.56 & 41.81 \\
\hline Max. & 1.52 & 2.86 & 4.41 & 8.96 & 15.70 & 23.61 & 36.08 & 44.13 & 51.82 & 53.41 \\
\hline
\end{tabular}

Room Breathing Zone

\begin{tabular}{|l|cc|c|c|}
\cline { 2 - 5 } \multicolumn{1}{c|}{} & \multicolumn{2}{c|}{ Temperature } & $\mathrm{CO}_{2}$ & RH \\
\cline { 2 - 5 } \multicolumn{1}{c|}{} & ${ }^{\circ} \mathrm{C}$ & ${ }^{\circ} \mathrm{F}$ & $(\mathrm{ppm})$ & \\
\hline Mean & 19.77 & 67.59 & 58 & $57.45 \%$ \\
\hline S.D. & 0.16 & 0.28 & 24 & \\
\hline Max. & 21.55 & 70.80 & 168 & \\
\hline
\end{tabular}

Room Breathing Zone NH3 (ppm)

\begin{tabular}{|l|c|c|c|c|c|c|c|c|c|c|}
\hline Day & $\mathbf{1}$ & $\mathbf{2}$ & $\mathbf{3}$ & $\mathbf{4}$ & $\mathbf{5}$ & $\mathbf{6}$ & $\mathbf{7}$ & $\mathbf{8}$ & $\mathbf{9}$ & $\mathbf{1 0}$ \\
\hline Mean & 0.03 & 0.06 & 0.09 & 0.18 & 0.32 & 0.49 & 0.74 & 0.91 & 1.07 & 1.10 \\
\hline Max. & 0.09 & 0.17 & 0.26 & 0.53 & 0.93 & 1.40 & 2.14 & 2.62 & 3.08 & 3.18 \\
\hline
\end{tabular}




\section{Histogram Distributions}

Cage occupied zone average temperature $\left({ }^{\circ} \mathrm{C}\right)$ distribution

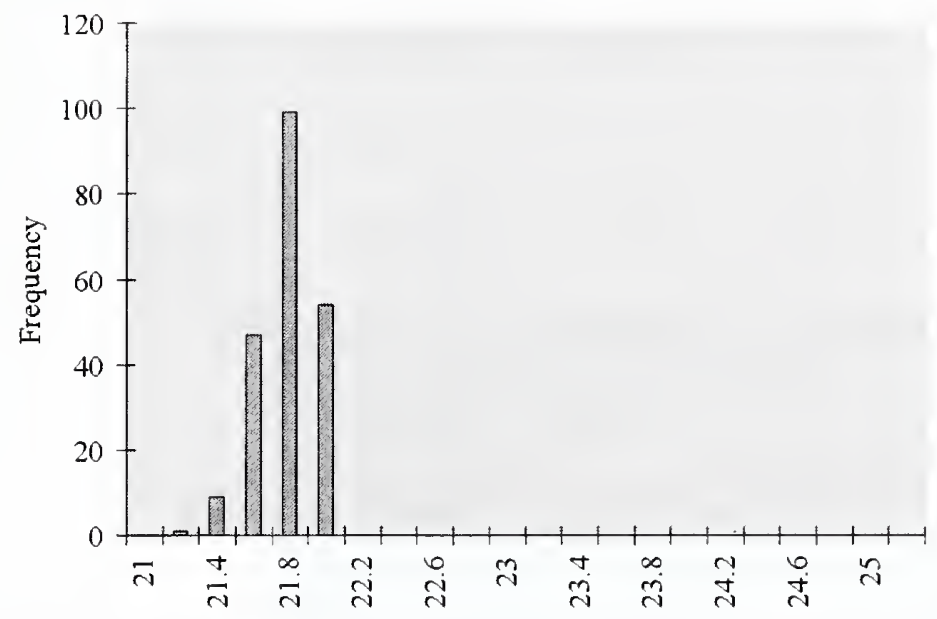

Cage occupied zone average contamination $(\mathrm{kg} / \mathrm{kg})$ distribution

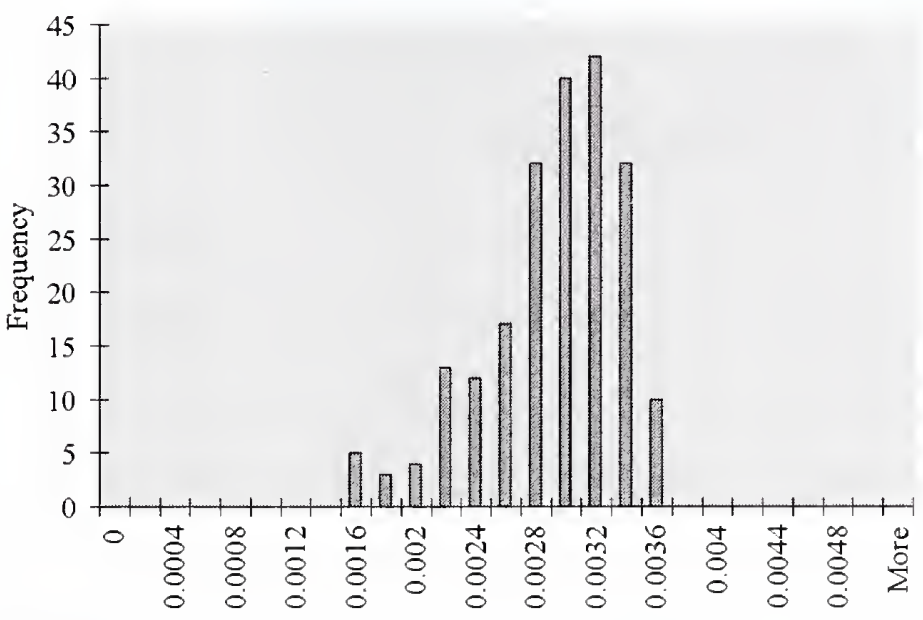

Cage occupied zone average relative humidity (\%) distribution

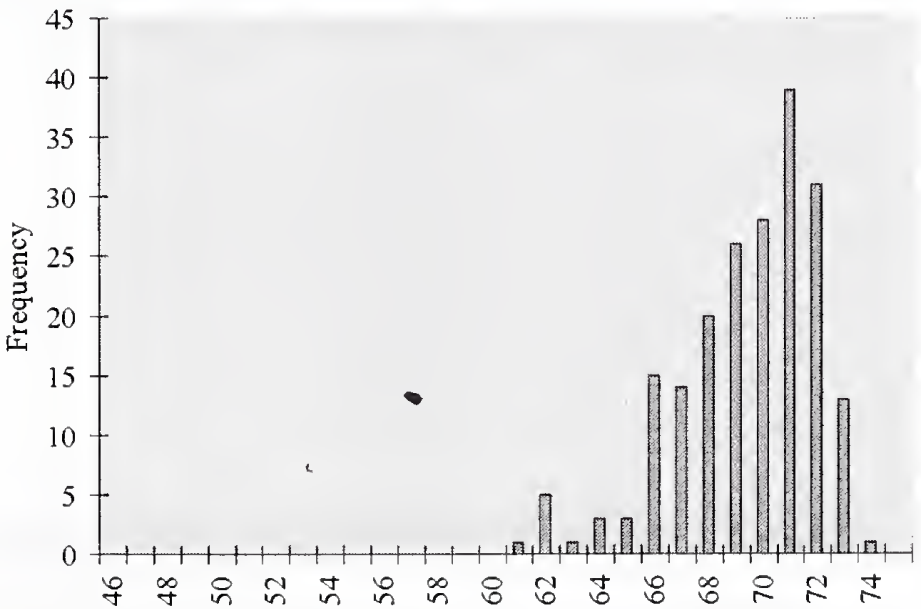

Contamination conversion factors $(\mathrm{kg} / \mathrm{kg} \rightarrow \mathrm{ppm})$

\begin{tabular}{|c|c|c|}
\hline Day & $\mathrm{CO}_{2}$ & $\mathrm{NH}_{3}$ \\
\hline $\mathbf{1}$ & 785000 & 422 \\
\hline $\mathbf{2}$ & 785000 & 795 \\
\hline $\mathbf{3}$ & 785000 & 1225 \\
\hline $\mathbf{4}$ & 785000 & 2490 \\
\hline $\mathbf{5}$ & 785000 & 4362 \\
\hline $\mathbf{6}$ & 785000 & 6558 \\
\hline $\mathbf{7}$ & 785000 & 10023 \\
\hline $\mathbf{8}$ & 785000 & 12260 \\
\hline $\mathbf{9}$ & 785000 & 14396 \\
\hline $\mathbf{1 0}$ & 785000 & 11384 \\
\hline
\end{tabular}




\section{Description}

$\begin{array}{llllll}\begin{array}{l}\text { Supply } \\ \text { Configuration } \\ \text { Slot }\end{array} & \begin{array}{l}\text { Supply Discharge } \\ \text { Temperature }\left({ }^{\circ} \mathrm{C}\right)\end{array} & \begin{array}{l}\text { Supply Discharge } \\ \text { RH }\end{array} & \begin{array}{l}\text { Exhaust } \\ \text { Configuration } \\ \text { Ceiling }\end{array} & \begin{array}{l}\text { Exhaust } \\ \text { Temperature }\left({ }^{\circ} \mathrm{C}\right)\end{array} & \begin{array}{l}\text { Exhaust } \\ \text { RH }\end{array} \\ & 18.8 & 61 \% & & & 50 \% \\ \text { Change } & \text { Rack } & \text { Rack } & \text { Number of } & \text { Total mass of } & \text { Room } \\ \begin{array}{l}\text { Station } \\ \text { ON alt design }\end{array} & \text { Orientation } & \text { Density } & \text { Mice in Room } & \text { Mice in Room } & \text { Pressurisation } \\ & \text { On wall } & \text { Single } & 1050 & 21000 \text { gr } & \text { neg } 100 \mathrm{cfm}\end{array}$

Room
ACH
15
Cage
Condition
Top On

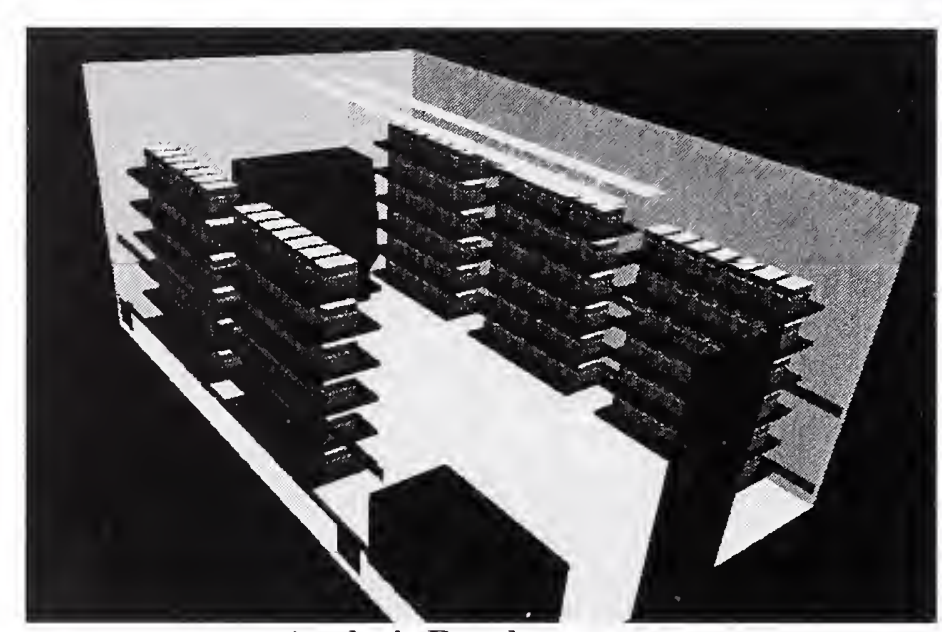

Analysis Results

Cage Occupied Zone

\begin{tabular}{|c|c|c|c|c|}
\hline & \multicolumn{2}{|c|}{ Temperature } & $\mathrm{CO}_{2}$ & RH \\
\hline & ${ }^{\circ} \mathrm{C}$ & ${ }^{\circ} \mathrm{F}$ & (ppm) & \\
\hline Mean & 21.67 & 71.00 & 1869 & $66.03 \%$ \\
\hline S.D. & 0.15 & 0.27 & 301 & $2.26 \%$ \\
\hline Max. & 22.00 & 71.60 & 2677 & $72.37 \%$ \\
\hline
\end{tabular}

Cage Occupied Zone NH3 (ppm)

\begin{tabular}{|l|c|c|c|c|c|c|c|c|c|c|}
\hline Day & $\mathbf{1}$ & $\mathbf{2}$ & $\mathbf{3}$ & $\mathbf{4}$ & $\mathbf{5}$ & $\mathbf{6}$ & $\mathbf{7}$ & $\mathbf{8}$ & $\mathbf{9}$ & $\mathbf{1 0}$ \\
\hline Mean & 0.99 & 1.89 & 2.92 & 5.32 & 8.80 & 12.98 & 19.37 & 24.20 & 29.24 & 32.12 \\
\hline Max. & 1.42 & 2.71 & 4.18 & 7.61 & 12.60 & 18.59 & 27.75 & 34.66 & 41.88 & 46.00 \\
\hline
\end{tabular}

Room Breathing Zone

\begin{tabular}{|l|cc|c|c|}
\cline { 2 - 5 } \multicolumn{1}{c|}{} & \multicolumn{2}{c|}{ Temperature } & $\mathbf{C O}_{2}$ & RH \\
\cline { 2 - 5 } \multicolumn{1}{c|}{} & ${ }^{\circ} \mathrm{C}$ & ${ }^{\circ}$ & $(\mathbf{p p m})$ & \\
\hline Mean & 19.79 & 67.61 & 60 & $57.41 \%$ \\
\hline S.D. & 0.16 & 0.29 & 33 & \\
\hline Max. & 20.81 & 69.47 & 283 & \\
\hline
\end{tabular}

Room Breathing Zone NH3 (ppm)

\begin{tabular}{|l|c|c|c|c|c|c|c|c|c|c|}
\hline Day & $\mathbf{1}$ & $\mathbf{2}$ & $\mathbf{3}$ & $\mathbf{4}$ & $\mathbf{5}$ & $\mathbf{6}$ & $\mathbf{7}$ & $\mathbf{8}$ & $\mathbf{9}$ & $\mathbf{1 0}$ \\
\hline Mean & 0.03 & 0.06 & 0.09 & 0.17 & 0.28 & 0.42 & 0.62 & 0.78 & 0.94 & 1.03 \\
\hline Max. & 0.15 & 0.29 & 0.44 & 0.81 & 1.33 & 1.97 & 2.94 & 3.67 & 4.43 & 4.87 \\
\hline
\end{tabular}




\section{Histogram Distributions}

Cage occupied zone average temperature $\left({ }^{\circ} \mathrm{C}\right)$ distribution

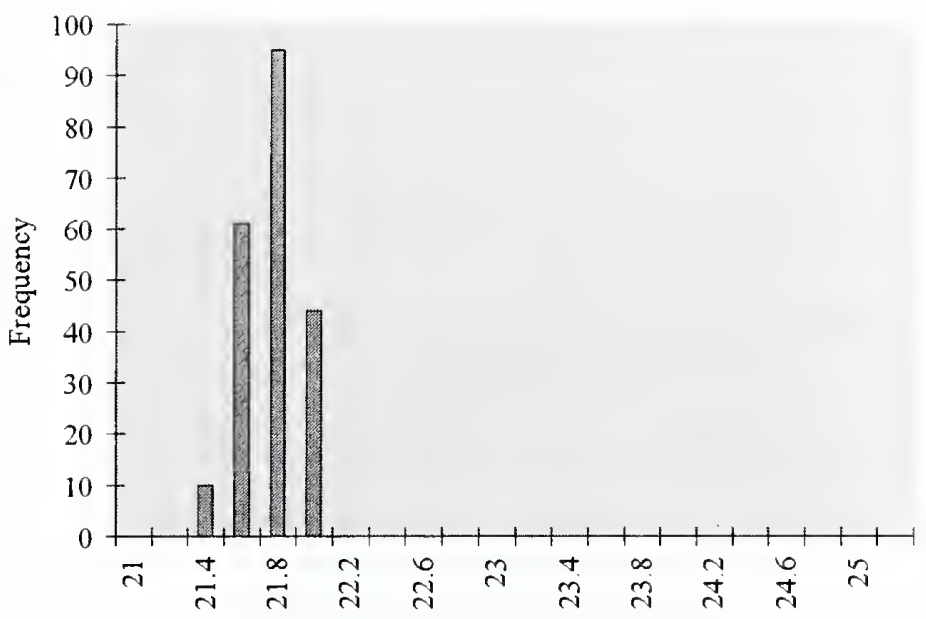

Cage occupied zone average contamination $(\mathrm{kg} / \mathrm{kg})$ distribution

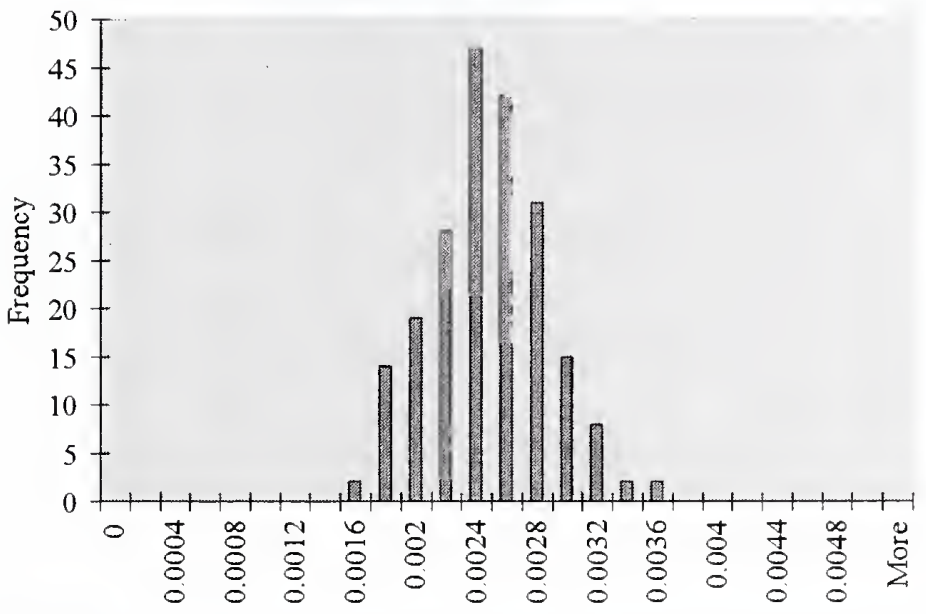

Cage occupied zone average relative humidity (\%) distribution

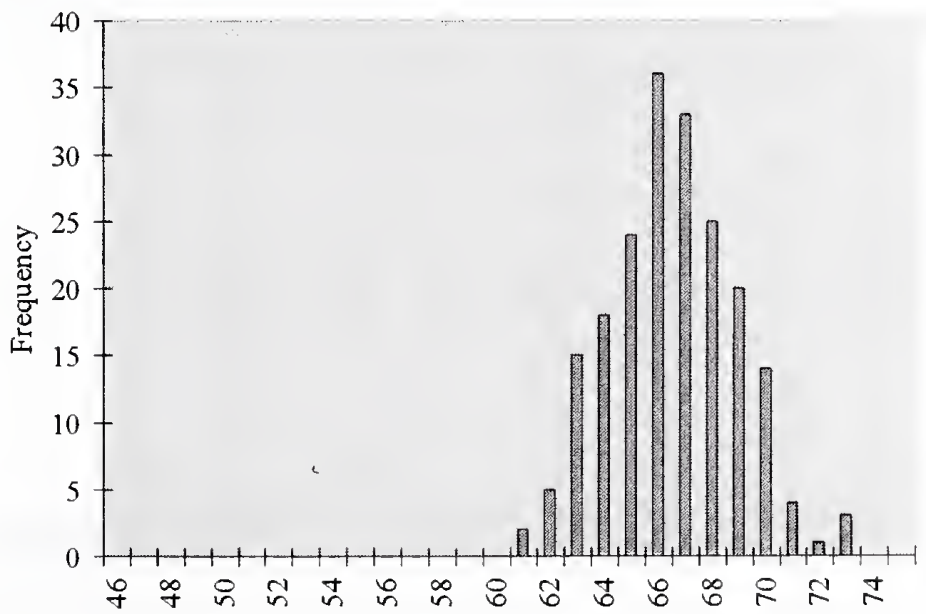

Contamination conversion factors $(\mathrm{kg} / \mathrm{kg} \rightarrow \mathrm{ppm})$

\begin{tabular}{|c|c|c|}
\hline Day & $\mathbf{C O}_{2}$ & $\mathbf{N H}_{\mathbf{3}}$ \\
\hline $\mathbf{1}$ & 785000 & 417 \\
\hline $\mathbf{2}$ & 785000 & 795 \\
\hline $\mathbf{3}$ & 785000 & 1225 \\
\hline $\mathbf{4}$ & 785000 & 2233 \\
\hline $\mathbf{5}$ & 785000 & 3694 \\
\hline $\mathbf{6}$ & 785000 & 5451 \\
\hline $\mathbf{7}$ & 785000 & 8136 \\
\hline $\mathbf{8}$ & 785000 & 10163 \\
\hline $\mathbf{9}$ & 785000 & 12281 \\
\hline $\mathbf{1 0}$ & 785000 & 11384 \\
\hline
\end{tabular}




\section{Casename Case $\mathbf{5 5}$}

\section{Description}

\begin{tabular}{|c|c|c|c|c|c|}
\hline Supply & Supply Discharge & Supply Discharge & Exhaust & Exhaust & Exhaust \\
\hline Configuration & Temperature $\left({ }^{\circ} \mathrm{C}\right)$ & $\mathbf{R H}$ & Configuration & Temperature $\left({ }^{\circ} \mathrm{C}\right)$ & RH \\
\hline Low Ind & 18.8 & $61 \%$ & Ceiling & 22 & $50 \%$ \\
\hline Change & Rack & Rack & Number of & Total mass of & Room \\
\hline Station & Orientation & Density & Mice in Room & Mice in Room & Pressurisation \\
\hline ON alt design & On wall & Single & 1050 & $21000 \mathrm{gr}$ & neg $100 \mathrm{cfm}$ \\
\hline
\end{tabular}

Room

$\mathrm{ACH}$

15

Cage

Condition

Top On

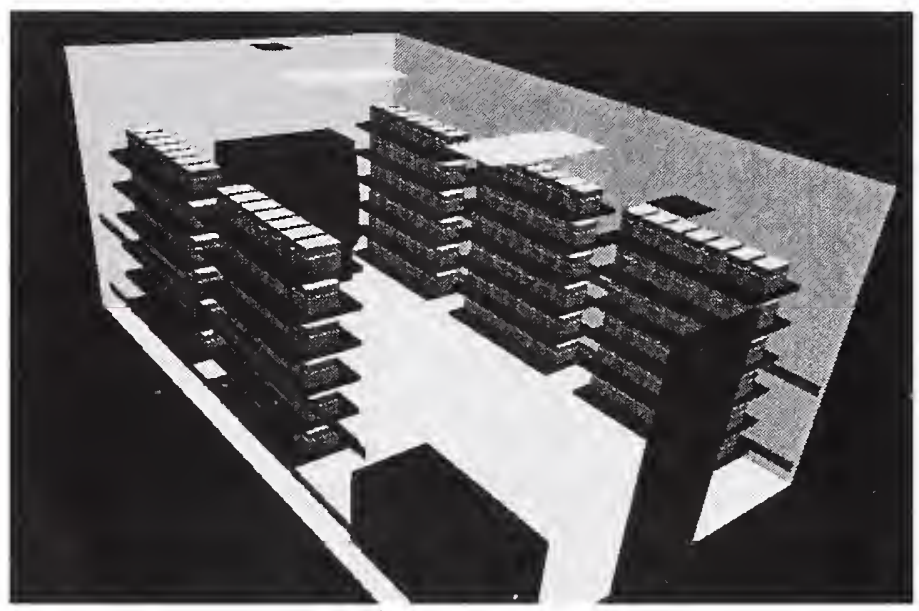

Analysis Results

Cage Occupied Zone

\begin{tabular}{|l|cc|c|c|}
\cline { 2 - 5 } \multicolumn{1}{c|}{} & \multicolumn{2}{c|}{ Temperature } & $\mathrm{CO}_{2}$ & RH \\
\cline { 2 - 5 } \multicolumn{1}{c|}{} & ${ }^{\circ} \mathrm{C}$ & ${ }^{\circ} \mathrm{F}$ & $(\mathrm{ppm})$ & \\
\hline Mean & 21.59 & 70.86 & 2039 & $67.77 \%$ \\
\hline S.D. & 0.15 & 0.27 & 307 & $2.34 \%$ \\
\hline Max. & 21.94 & 71.50 & 2624 & $71.91 \%$ \\
\hline
\end{tabular}

Cage Occupied Zone NH3 (ppm)

\begin{tabular}{|l|c|c|c|c|c|c|c|c|c|c|}
\hline Day & $\mathbf{1}$ & $\mathbf{2}$ & $\mathbf{3}$ & $\mathbf{4}$ & $\mathbf{5}$ & $\mathbf{6}$ & $\mathbf{7}$ & $\mathbf{8}$ & $\mathbf{9}$ & $\mathbf{1 0}$ \\
\hline Mean & 1.09 & 2.06 & 3.18 & 6.22 & 10.69 & 15.97 & 24.22 & 29.83 & 35.36 & 37.24 \\
\hline Max. & 1.40 & 2.66 & 4.09 & 8.00 & 13.75 & 20.55 & 31.16 & 38.38 & 45.49 & 47.92 \\
\hline
\end{tabular}

Room Breathing Zone

\begin{tabular}{|l|cc|c|c|}
\cline { 2 - 5 } \multicolumn{1}{c|}{} & \multicolumn{2}{c|}{ Temperature } & $\mathrm{CO}_{2}$ & RH \\
\cline { 2 - 5 } \multicolumn{1}{c|}{} & ${ }^{\circ} \mathrm{C}$ & ${ }^{\circ} \mathrm{F}$ & $(\mathrm{ppm})$ & \\
\hline Mean & 19.78 & 67.61 & 57 & $57.40 \%$ \\
\hline S.D. & 0.18 & 0.33 & 23 & \\
\hline Max. & 20.84 & 69.52 & 241 & \\
\hline
\end{tabular}

Room Breathing Zone NH3 (ppm)

\begin{tabular}{|l|c|c|c|c|c|c|c|c|c|c|}
\hline Day & $\mathbf{1}$ & $\mathbf{2}$ & $\mathbf{3}$ & $\mathbf{4}$ & $\mathbf{5}$ & $\mathbf{6}$ & $\mathbf{7}$ & $\mathbf{8}$ & $\mathbf{9}$ & $\mathbf{1 0}$ \\
\hline Mean & 0.03 & 0.06 & 0.09 & 0.17 & 0.30 & 0.45 & 0.68 & 0.84 & 0.99 & 1.04 \\
\hline Max. & 0.13 & 0.24 & 0.38 & 0.74 & 1.26 & 1.89 & 2.86 & 3.53 & 4.18 & 4.40 \\
\hline
\end{tabular}




\section{Histogram Distributions}

Cage occupied zone average temperature $\left({ }^{\circ} \mathrm{C}\right)$ distribution

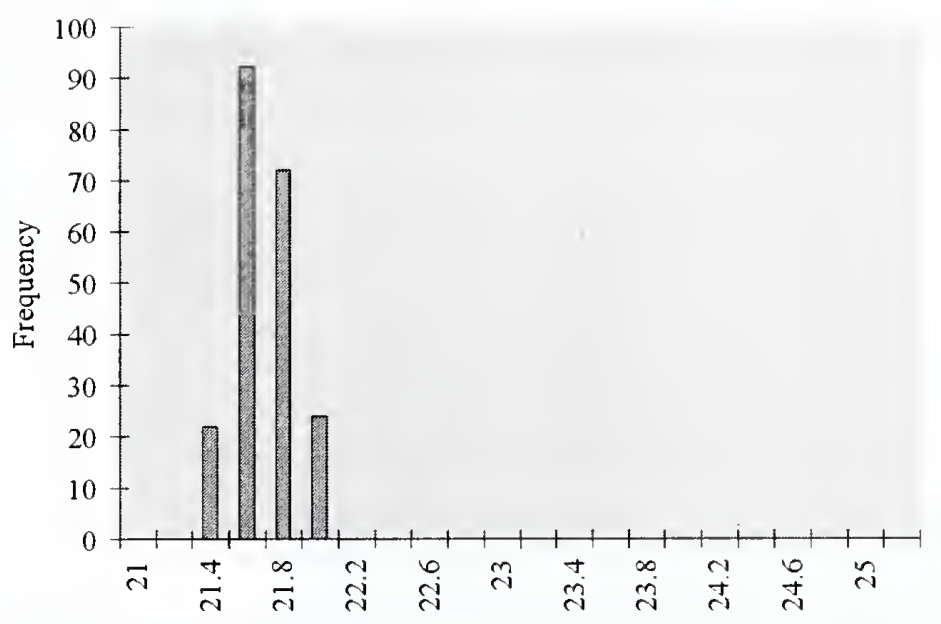

Cage occupied zone average contamination $(\mathrm{kg} / \mathrm{kg})$ distribution

Contamination conversion factors $(\mathrm{kg} / \mathrm{kg} \rightarrow \mathrm{ppm})$

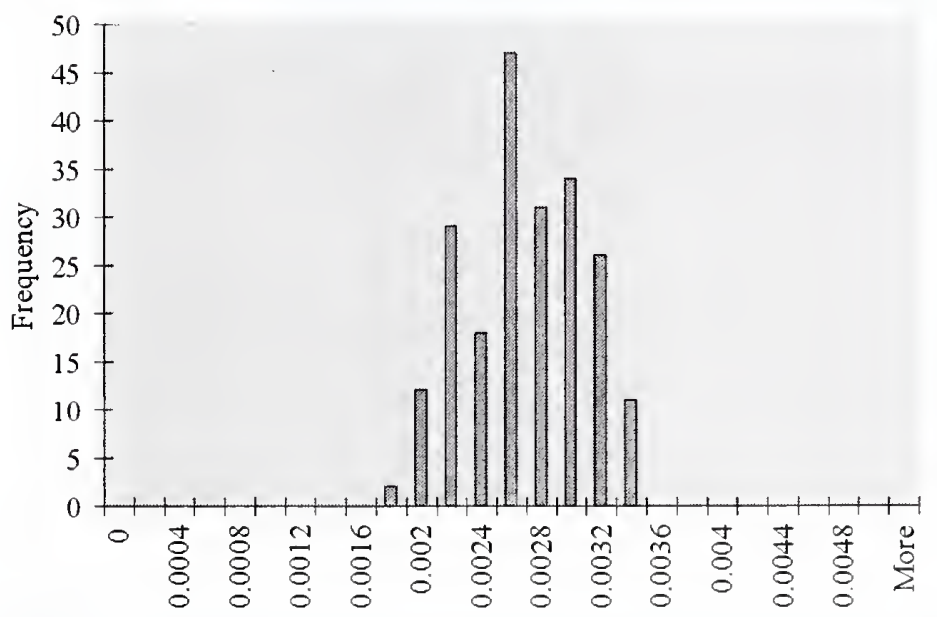

\begin{tabular}{|c|c|c|}
\hline Day & $\mathrm{CO}_{2}$ & $\mathrm{NH}_{\mathbf{3}}$ \\
\hline $\mathbf{1}$ & 785000 & 420 \\
\hline $\mathbf{2}$ & 785000 & 795 \\
\hline $\mathbf{3}$ & 785000 & 1225 \\
\hline $\mathbf{4}$ & 785000 & 2395 \\
\hline $\mathbf{5}$ & 785000 & 4115 \\
\hline $\mathbf{6}$ & 785000 & 6148 \\
\hline $\mathbf{7}$ & 785000 & 9324 \\
\hline $\mathbf{8}$ & 785000 & 11483 \\
\hline $\mathbf{9}$ & 785000 & 13612 \\
\hline $\mathbf{1 0}$ & 785000 & 11384 \\
\hline
\end{tabular}

Cage occupied zone average relative humidity (\%) distribution

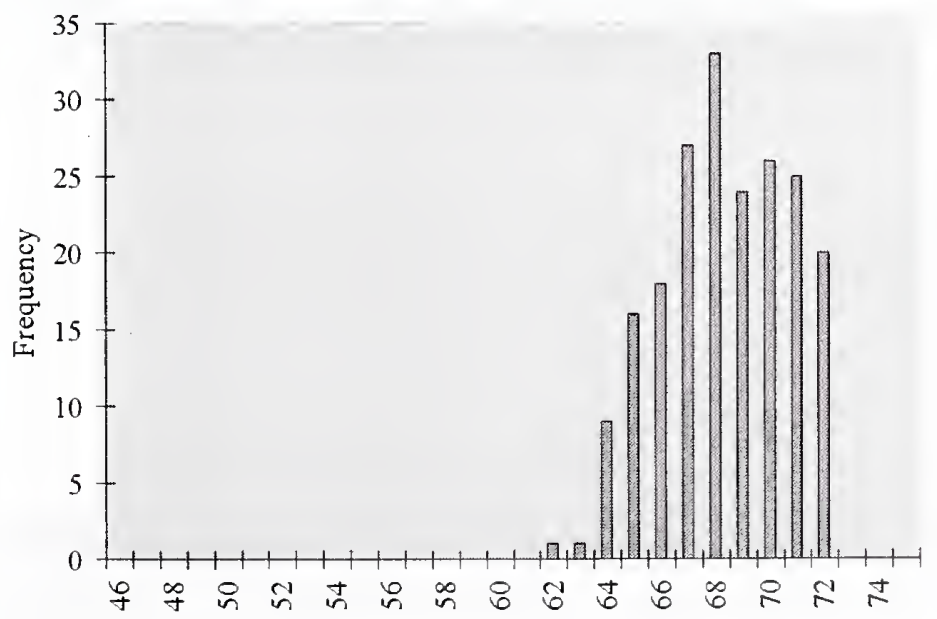


Casename

\section{Case 56}

\section{Description}

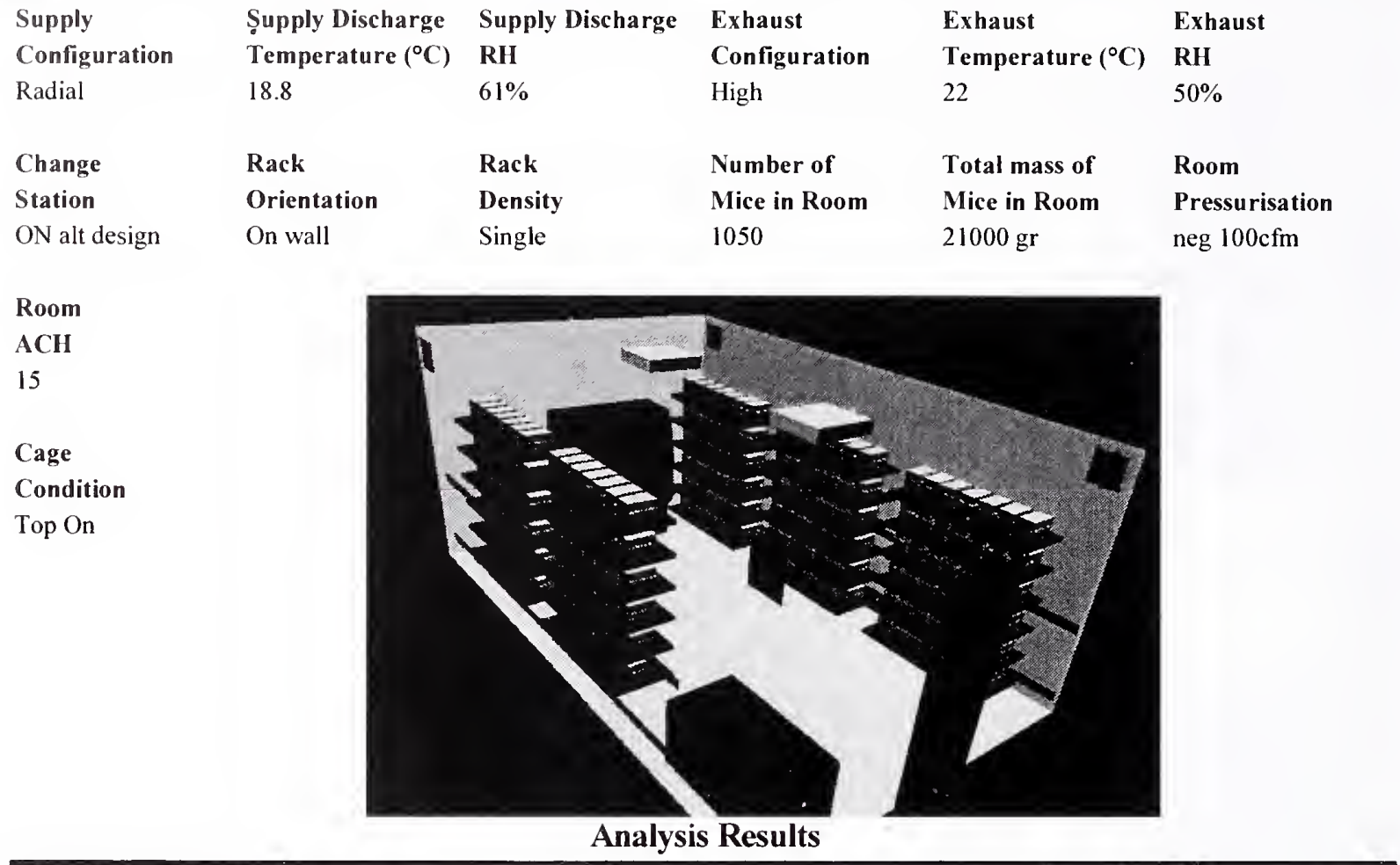

Cage Occupied Zone

\begin{tabular}{|l|cc|c|c|}
\cline { 2 - 5 } \multicolumn{1}{c|}{} & \multicolumn{2}{c|}{ Temperature } & $\mathbf{C O}_{2}$ & RH \\
\cline { 2 - 5 } \multicolumn{1}{c|}{} & ${ }^{\circ} \mathrm{C}$ & ${ }^{\circ}$ & $(\mathbf{p p m})$ & \\
\hline Mean & 22.09 & 71.76 & 1886 & $64.43 \%$ \\
\hline S.D. & 0.16 & 0.29 & 326 & $2.57 \%$ \\
\hline Max. & 22.43 & 72.37 & 2570 & $69.71 \%$ \\
\hline
\end{tabular}

Cage Occupied Zone NH3 (ppm)

\begin{tabular}{|l|c|c|c|c|c|c|c|c|c|c|}
\hline Day & $\mathbf{1}$ & $\mathbf{2}$ & $\mathbf{3}$ & $\mathbf{4}$ & $\mathbf{5}$ & $\mathbf{6}$ & $\mathbf{7}$ & $\mathbf{8}$ & $\mathbf{9}$ & $\mathbf{1 0}$ \\
\hline Mean & 1.00 & 1.91 & 2.94 & 5.01 & 7.95 & 11.56 & 16.93 & 21.50 & 26.57 & 30.53 \\
\hline Max. & 1.36 & 2.60 & 4.01 & 6.82 & 10.84 & 15.76 & 23.07 & 29.31 & 36.21 & 41.61 \\
\hline
\end{tabular}

Room Breathing Zone

\begin{tabular}{|l|cc|c|c|}
\cline { 2 - 5 } \multicolumn{1}{c|}{} & \multicolumn{2}{c|}{ Temperature } & $\mathrm{CO}_{2}$ & RH \\
\cline { 2 - 5 } \multicolumn{1}{c|}{} & ${ }^{\circ} \mathrm{C}$ & ${ }^{\circ} \mathrm{F}$ & $(\mathrm{ppm})$ & \\
\hline Mean & 20.34 & 68.61 & 69 & $55.51 \%$ \\
\hline S.D. & 0.18 & 0.33 & 25 & \\
\hline Max. & 21.07 & 69.93 & 157 & \\
\hline
\end{tabular}

Room Breathing Zone NH3 (ppm)

\begin{tabular}{|l|c|c|c|c|c|c|c|c|c|c|}
\hline Day & $\mathbf{I}$ & $\mathbf{2}$ & $\mathbf{3}$ & $\mathbf{4}$ & $\mathbf{5}$ & $\mathbf{6}$ & $\mathbf{7}$ & $\mathbf{8}$ & $\mathbf{9}$ & $\mathbf{1 0}$ \\
\hline Mean & 0.04 & 0.07 & 0.11 & 0.18 & 0.29 & 0.42 & 0.62 & 0.79 & 0.98 & 1.12 \\
\hline Max. & 0.08 & 0.16 & 0.25 & 0.42 & 0.66 & 0.96 & 1.41 & 1.79 & 2.21 & 2.54 \\
\hline
\end{tabular}




\section{Histogram Distributions}

Cage occupied zone average temperature $\left({ }^{\circ} \mathrm{C}\right)$ distribution

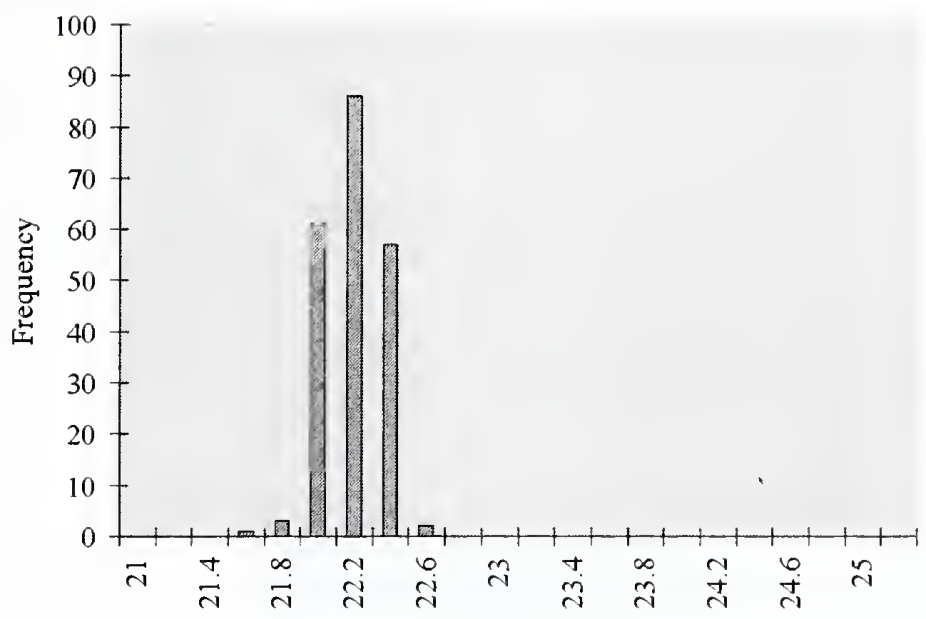

Cage occupied zone average contamination $(\mathrm{kg} / \mathrm{kg})$ distribution

Contamination conversion factors $(\mathrm{kg} / \mathrm{kg} \rightarrow \mathrm{ppm})$

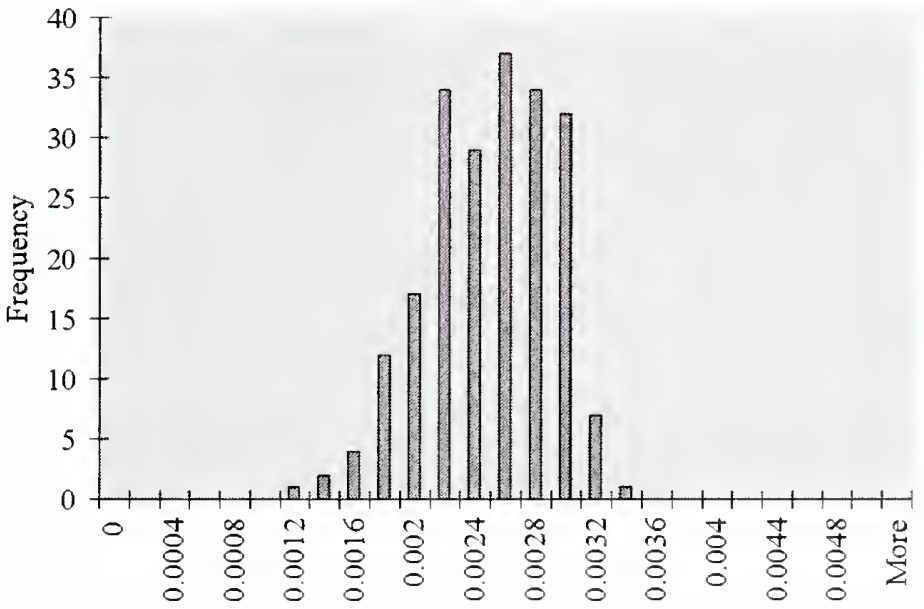

\begin{tabular}{|c|c|c|}
\hline Day & $\mathbf{C O}_{\mathbf{2}}$ & $\mathbf{N H}_{3}$ \\
\hline $\mathbf{1}$ & 785000 & 414 \\
\hline $\mathbf{2}$ & 785000 & 795 \\
\hline $\mathbf{3}$ & 785000 & 1225 \\
\hline $\mathbf{4}$ & 785000 & 2084 \\
\hline $\mathbf{5}$ & 785000 & 3309 \\
\hline $\mathbf{6}$ & 785000 & 4812 \\
\hline $\mathbf{7}$ & 785000 & 7047 \\
\hline $\mathbf{8}$ & 785000 & 8952 \\
\hline $\mathbf{9}$ & 785000 & 11059 \\
\hline $\mathbf{1 0}$ & 785000 & 11384 \\
\hline
\end{tabular}

Cage occupied zone average relative humidity (\%) distribution

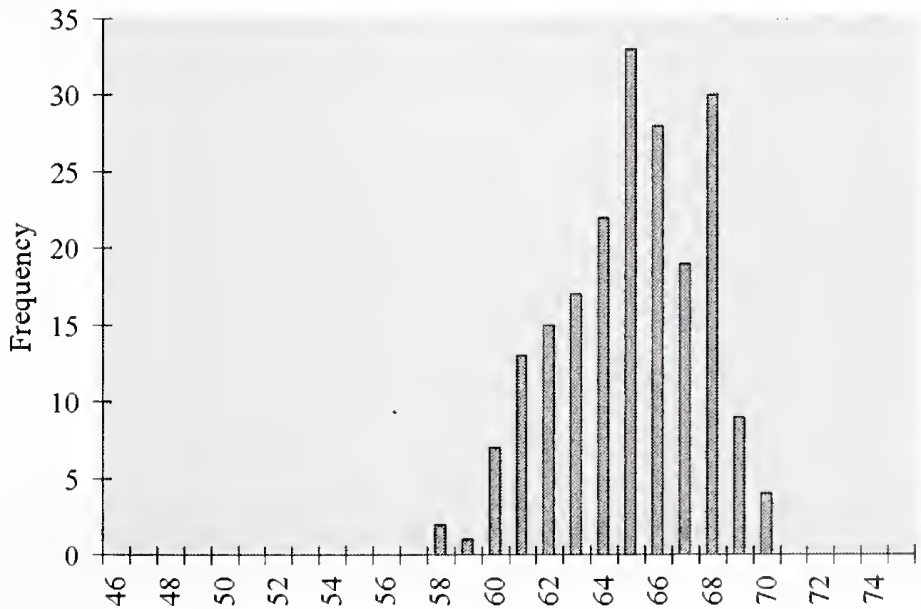


Casename Case 57

Description

\begin{tabular}{|c|c|c|c|c|c|}
\hline Supply & Supply Discharge & Supply Discharge & Exhaust & Exhaust & Exhaust \\
\hline Configuration & Temperature $\left({ }^{\circ} \mathrm{C}\right)$ & RH & Configuration & Temperature $\left({ }^{\circ} \mathrm{C}\right)$ & $\mathbf{R H}$ \\
\hline Radial & 18.8 & $61 \%$ & Low & 22 & $50 \%$ \\
\hline Change & Rack & Rack & Number of & Total mass of & Room \\
\hline Station & Orientation & Density & Mice in Room & Mice in Room & Pressurisation \\
\hline ON alt design & On wall & Single & 1050 & $21000 \mathrm{gr}$ & neg $100 \mathrm{cfm}$ \\
\hline
\end{tabular}

\section{Room}

$\mathrm{ACH}$

15

Cage

Condition

Top On

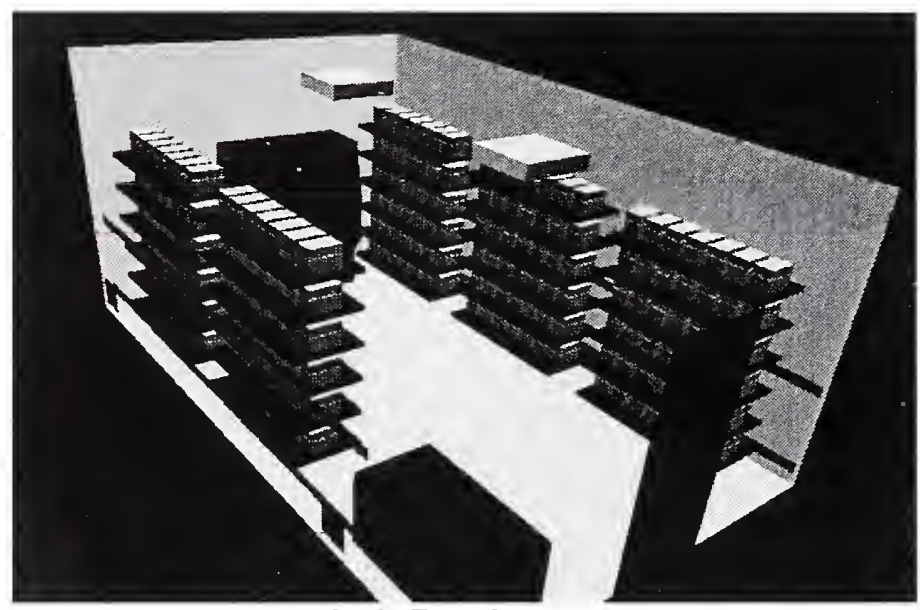

Analysis Results

Cage Occupied Zone

\begin{tabular}{|l|cc|c|c|}
\cline { 2 - 5 } \multicolumn{1}{c|}{} & \multicolumn{2}{c|}{ Temperature } & $\mathrm{CO}_{2}$ & RH \\
\cline { 2 - 5 } \multicolumn{1}{c|}{} & ${ }^{\mathrm{C}}$ & \multicolumn{1}{c}{$\mathrm{F}$} & $(\mathbf{p p m})$ & \\
\hline Mean & 23.00 & 73.40 & 1855 & $60.60 \%$ \\
\hline S.D. & 0.23 & 0.42 & 401 & $2.74 \%$ \\
\hline Max. & 23.50 & 74.31 & 2741 & $66.66 \%$ \\
\hline
\end{tabular}

Cage Occupied Zone NH3 (ppm)

\begin{tabular}{|l|c|c|c|c|c|c|c|c|c|c|}
\hline Day & $\mathbf{1}$ & $\mathbf{2}$ & $\mathbf{3}$ & $\mathbf{4}$ & $\mathbf{5}$ & $\mathbf{6}$ & $\mathbf{7}$ & $\mathbf{8}$ & $\mathbf{9}$ & $\mathbf{1 0}$ \\
\hline Mean & 0.96 & 1.88 & 2.89 & 4.17 & 5.87 & 8.14 & 11.13 & 15.02 & 19.95 & 26.08 \\
\hline Max. & 1.43 & 2.77 & 4.28 & 6.17 & 8.67 & 12.02 & 16.46 & 22.20 & 29.48 & 38.54 \\
\hline
\end{tabular}

Room Breathing Zone

\begin{tabular}{|l|cc|c|c|}
\cline { 2 - 5 } \multicolumn{1}{c|}{} & \multicolumn{2}{c|}{ Temperature } & $\mathbf{C O}_{2}$ & RH \\
\cline { 2 - 5 } \multicolumn{1}{c|}{} & ${ }^{\circ} \mathrm{C}$ & ${ }^{\circ} \mathrm{F}$ & $(\mathbf{p p m})$ & \\
\hline Mean & 21.73 & 71.12 & 57 & $50.72 \%$ \\
\hline S.D. & 0.28 & 0.50 & 32 & \\
\hline Max. & 22.54 & 72.56 & 279 & \\
\hline
\end{tabular}

Room Breathing Zone NH3 (ppm)

\begin{tabular}{|l|c|c|c|c|c|c|c|c|c|c|}
\hline Day & $\mathbf{I}$ & $\mathbf{2}$ & $\mathbf{3}$ & $\mathbf{4}$ & $\mathbf{5}$ & $\mathbf{6}$ & $\mathbf{7}$ & $\mathbf{8}$ & $\mathbf{9}$ & $\mathbf{1 0}$ \\
\hline Mean & 0.03 & 0.06 & 0.09 & 0.13 & 0.18 & 0.25 & 0.34 & 0.46 & 0.62 & 0.81 \\
\hline Max. & 0.15 & 0.28 & 0.44 & 0.63 & 0.88 & 1.22 & 1.68 & 2.26 & 3.00 & 3.92 \\
\hline
\end{tabular}




\section{Histogram Distributions}

Cage occupied zone average temperature $\left({ }^{\circ} \mathrm{C}\right)$ distribution

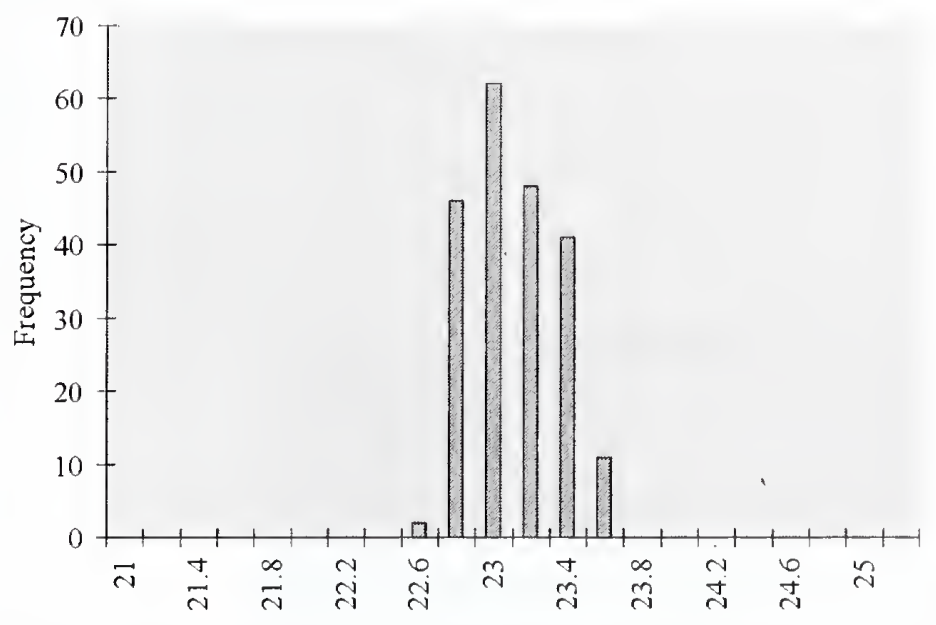

Cage occupied zone average contamination $(\mathrm{kg} / \mathrm{kg})$ distribution

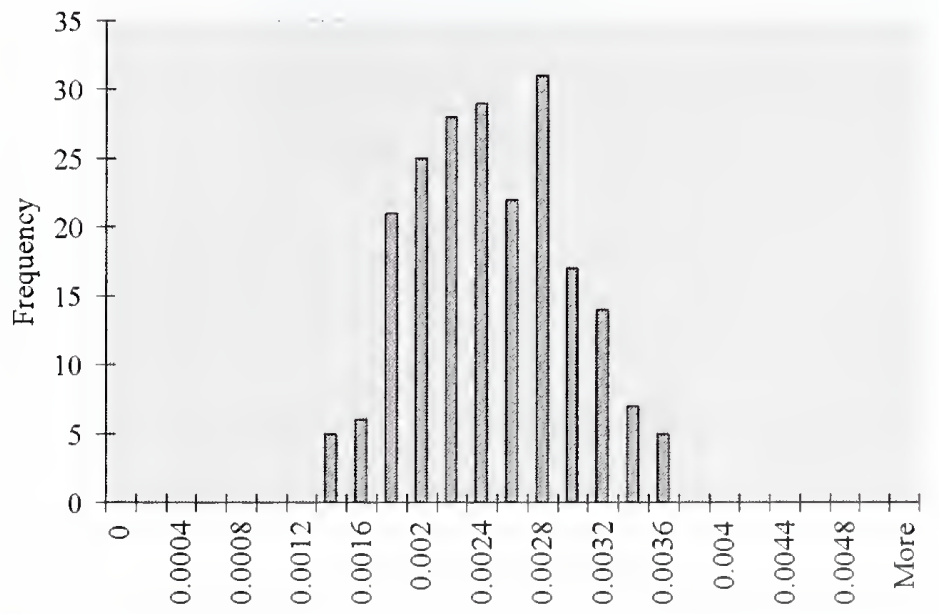

Cage occupied zone average relative humidity (\%) distribution

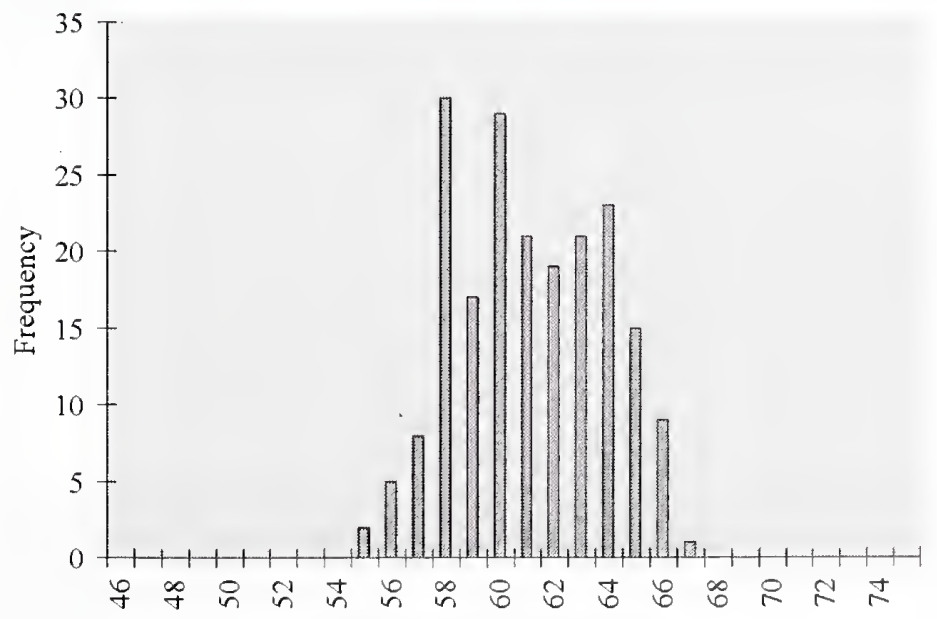

Contamination conversion factors $(\mathrm{kg} / \mathrm{kg} \rightarrow \mathrm{ppm})$

\begin{tabular}{|c|c|c|}
\hline Day & $\mathrm{CO}_{2}$ & $\mathrm{NH}_{3}$ \\
\hline $\mathbf{1}$ & 785000 & 408 \\
\hline $\mathbf{2}$ & 785000 & 795 \\
\hline $\mathbf{3}$ & 785000 & 1225 \\
\hline $\mathbf{4}$ & 785000 & 1766 \\
\hline $\mathbf{5}$ & 785000 & 2483 \\
\hline $\mathbf{6}$ & 785000 & 3443 \\
\hline $\mathbf{7}$ & 785000 & 4712 \\
\hline $\mathbf{8}$ & 785000 & 6356 \\
\hline $\mathbf{9}$ & 785000 & 8442 \\
\hline $\mathbf{1 0}$ & 785000 & 11384 \\
\hline
\end{tabular}


Casename

Case 58

Description

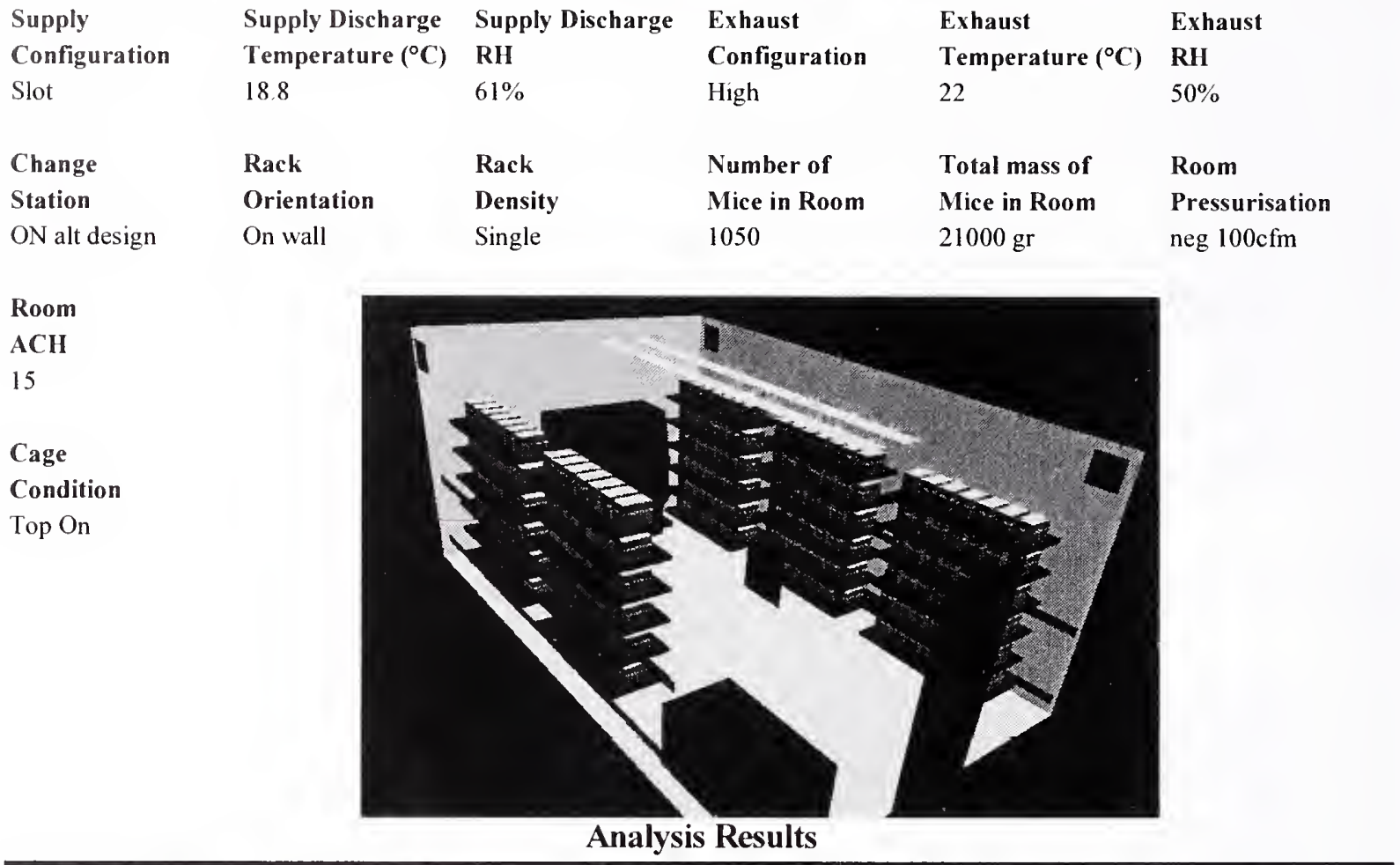

Cage Occupied Zone

\begin{tabular}{|l|cc|c|c|}
\cline { 2 - 5 } \multicolumn{1}{c|}{} & \multicolumn{2}{c|}{ Temperature } & $\mathbf{C O}_{2}$ & RH \\
\cline { 2 - 5 } \multicolumn{1}{c|}{} & ${ }^{\circ} \mathrm{C}$ & ${ }^{2} \mathrm{~F}$ & $(\mathbf{p p m})$ & \\
\hline Mean & 23.05 & 73.50 & 1990 & $61.43 \%$ \\
\hline S.D. & 0.25 & 0.45 & 426 & $2.90 \%$ \\
\hline Max. & 23.60 & 74.49 & 2869 & $67.54 \%$ \\
\hline
\end{tabular}

Cage Occupied Zone NH3 (ppm)

\begin{tabular}{|l|c|c|c|c|c|c|c|c|c|c|}
\hline Day & $\mathbf{1}$ & $\mathbf{2}$ & $\mathbf{3}$ & $\mathbf{4}$ & $\mathbf{5}$ & $\mathbf{6}$ & $\mathbf{7}$ & $\mathbf{8}$ & 9 & $\mathbf{1 0}$ \\
\hline Mean & $\mathbf{1 . 0 4}$ & 2.01 & $\mathbf{3 . 1 0}$ & 4.58 & 6.56 & 9.16 & 12.68 & 16.93 & 22.23 & 28.50 \\
\hline Max. & 1.50 & 2.90 & 4.48 & 6.60 & 9.45 & 13.21 & 18.29 & 24.42 & 32.05 & 41.10 \\
\hline
\end{tabular}

Room Breathing Zone

\begin{tabular}{|c|c|c|c|c|}
\hline & \multicolumn{2}{|c|}{ Temperature } & $\mathrm{CO}_{2}$ & $\mathbf{R H}$ \\
\hline & ${ }^{\circ} \mathrm{C}$ & ${ }^{\circ} \mathrm{F}$ & (ppm) & \\
\hline Mean & 21.78 & 71.20 & 68 & $50.65 \%$ \\
\hline S.D. & 0.28 & 0.50 & 28 & \\
\hline Max. & 22.52 & 72.54 & 254 & \\
\hline
\end{tabular}

Room Breathing Zone NH3 (ppm)

\begin{tabular}{|l|c|c|c|c|c|c|c|c|c|c|}
\hline Day & $\mathbf{I}$ & $\mathbf{2}$ & $\mathbf{3}$ & $\mathbf{4}$ & $\mathbf{5}$ & $\mathbf{6}$ & $\mathbf{7}$ & $\mathbf{8}$ & $\mathbf{9}$ & $\mathbf{1 0}$ \\
\hline Mean & 0.04 & 0.07 & 0.11 & 0.16 & 0.22 & 0.31 & 0.43 & 0.58 & 0.76 & 0.97 \\
\hline Max. & 0.13 & 0.26 & 0.40 & 0.58 & 0.84 & 1.17 & 1.62 & 2.16 & 2.83 & 3.63 \\
\hline
\end{tabular}




\section{Histogram Distributions}

Cage occupied zone average temperature $\left({ }^{\circ} \mathrm{C}\right)$ distribution

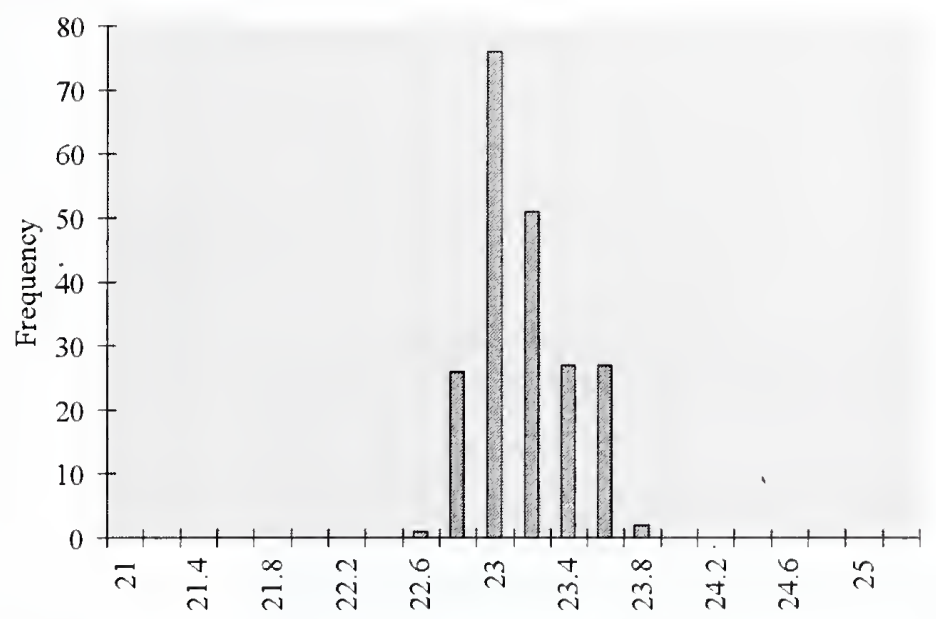

Cage occupied zone average contamination $(\mathrm{kg} / \mathrm{kg})$ distribution

Contamination conversion factors $(\mathrm{kg} / \mathrm{kg} \rightarrow \mathrm{ppm})$

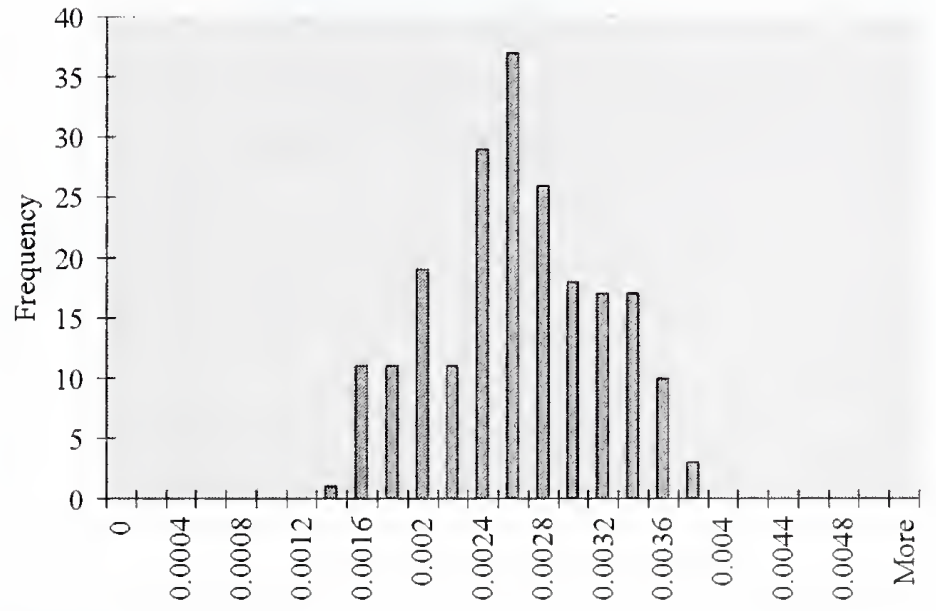

\begin{tabular}{|c|c|c|}
\hline Day & $\mathbf{C O}_{2}$ & $\mathbf{N H}_{3}$ \\
\hline $\mathbf{1}$ & 785000 & 409 \\
\hline $\mathbf{2}$ & 785000 & 795 \\
\hline $\mathbf{3}$ & 785000 & 1225 \\
\hline $\mathbf{4}$ & 785000 & 1806 \\
\hline $\mathbf{5}$ & 785000 & 2586 \\
\hline $\mathbf{6}$ & 785000 & 3614 \\
\hline $\mathbf{7}$ & 785000 & 5004 \\
\hline $\mathbf{8}$ & 785000 & 6681 \\
\hline $\mathbf{9}$ & 785000 & 8770 \\
\hline $\mathbf{1 0}$ & 785000 & 11384 \\
\hline
\end{tabular}

Cage occupied zone average relative humidity (\%) distribution

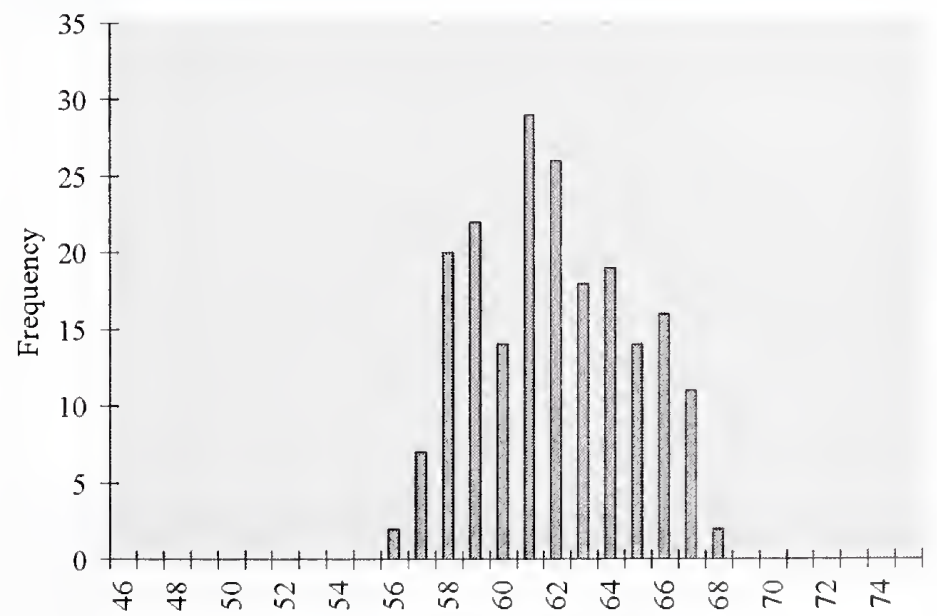


Casename Case 59

Description

\begin{tabular}{|c|c|c|c|c|c|}
\hline Supply & Supply Discharge & Supply Discharge & Exhaูust & Exhaust & Exhaust \\
\hline Configuration & Temperature $\left({ }^{\circ} \mathrm{C}\right)$ & RH & Configuration & Temperature $\left({ }^{\circ} \mathrm{C}\right)$ & RH \\
\hline Slot & 18.8 & $61 \%$ & Low & 22 & $50 \%$ \\
\hline $\begin{array}{l}\text { Change } \\
\text { Station }\end{array}$ & $\begin{array}{l}\text { Rack } \\
\text { Orientation }\end{array}$ & $\begin{array}{l}\text { Rack } \\
\text { Density }\end{array}$ & $\begin{array}{l}\text { Number of } \\
\text { Mice in Room }\end{array}$ & $\begin{array}{l}\text { Total mass of } \\
\text { Mice in Room }\end{array}$ & $\begin{array}{l}\text { Room } \\
\text { Pressurisation }\end{array}$ \\
\hline ON alt design & On wall & Single & 1050 & $21000 \mathrm{gr}$ & neg $100 \mathrm{cfm}$ \\
\hline
\end{tabular}

Room

$\mathrm{ACH}$

15

Cage

Condition

Top On

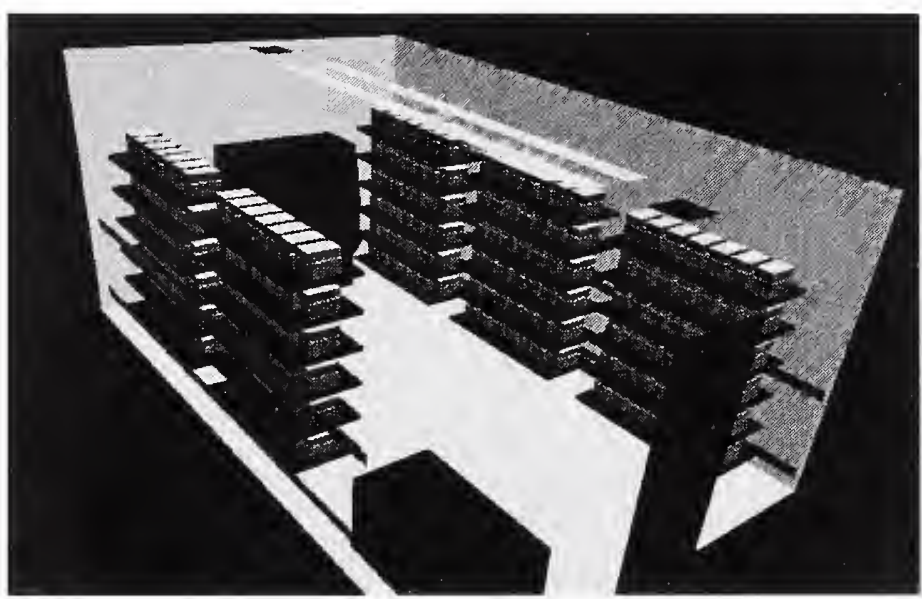

Analysis Results

Cage Occupied Zone

\begin{tabular}{|l|cc|c|c|}
\cline { 2 - 5 } \multicolumn{1}{c|}{} & \multicolumn{2}{c|}{ Temperature } & $\mathrm{CO}_{2}$ & RH \\
\cline { 2 - 5 } \multicolumn{1}{c|}{} & ${ }^{\circ} \mathrm{C}$ & ${ }^{\circ}$ & (ppm) & \\
\hline Mean & 22.02 & 71.64 & 2161 & $66.95 \%$ \\
\hline S.D. & 0.16 & 0.29 & 313 & $2.38 \%$ \\
\hline Max. & 22.30 & 72.15 & 2739 & $71.85 \%$ \\
\hline
\end{tabular}

Cage Occupied Zone NH3 (ppm)

\begin{tabular}{|l|c|c|c|c|c|c|c|c|c|c|}
\hline Day & $\mathbf{1}$ & $\mathbf{2}$ & $\mathbf{3}$ & $\mathbf{4}$ & $\mathbf{5}$ & $\mathbf{6}$ & $\mathbf{7}$ & $\mathbf{8}$ & $\mathbf{9}$ & $\mathbf{1 0}$ \\
\hline Mean & 1.15 & 2.19 & 3.37 & 6.38 & 10.78 & 16.02 & 24.13 & 29.90 & 35.75 & 38.37 \\
\hline Max. & 1.46 & 2.77 & 4.27 & 8.09 & 13.66 & 20.30 & 30.57 & 37.89 & 45.30 & 48.62 \\
\hline
\end{tabular}

Room Breathing Zone

\begin{tabular}{|l|cc|c|c|}
\cline { 2 - 5 } \multicolumn{1}{c|}{} & \multicolumn{2}{c|}{ Temperature } & $\mathrm{CO}_{2}$ & RH \\
\cline { 2 - 5 } \multicolumn{1}{c|}{} & ${ }^{\circ} \mathrm{C}$ & ${ }^{\circ}$ & $(\mathbf{p p m})$ & \\
\hline Mean & 20.34 & 68.62 & 15 & $54.99 \%$ \\
\hline S.D. & 0.17 & 0.31 & 9 & \\
\hline Max. & 21.22 & 70.20 & 90 & \\
\hline
\end{tabular}

Room Breathing Zone NH3 (ppm)

\begin{tabular}{|l|c|c|c|c|c|c|c|c|c|c|}
\hline Day & $\mathbf{1}$ & $\mathbf{2}$ & $\mathbf{3}$ & $\mathbf{4}$ & $\mathbf{5}$ & $\mathbf{6}$ & $\mathbf{7}$ & $\mathbf{8}$ & $\mathbf{9}$ & $\mathbf{1 0}$ \\
\hline Mean & 0.01 & 0.01 & 0.02 & 0.04 & 0.07 & 0.11 & 0.16 & 0.20 & 0.24 & 0.26 \\
\hline Max. & 0.05 & 0.09 & 0.14 & 0.27 & 0.45 & 0.67 & 1.01 & 1.25 & 1.49 & 1.60 \\
\hline
\end{tabular}




\section{Histogram Distributions}

Cage occupied zone average temperature $\left({ }^{\circ} \mathrm{C}\right)$ distribution

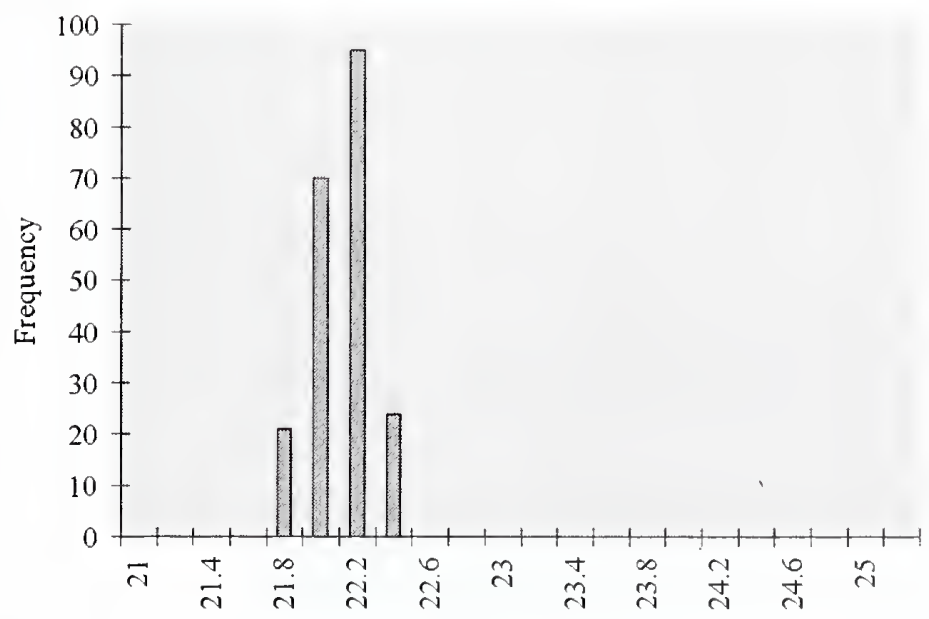

Cage occupied zone average contamination $(\mathrm{kg} / \mathrm{kg})$ distribution

Contamination conversion factors $(\mathrm{kg} / \mathrm{kg} \rightarrow \mathrm{ppm})$

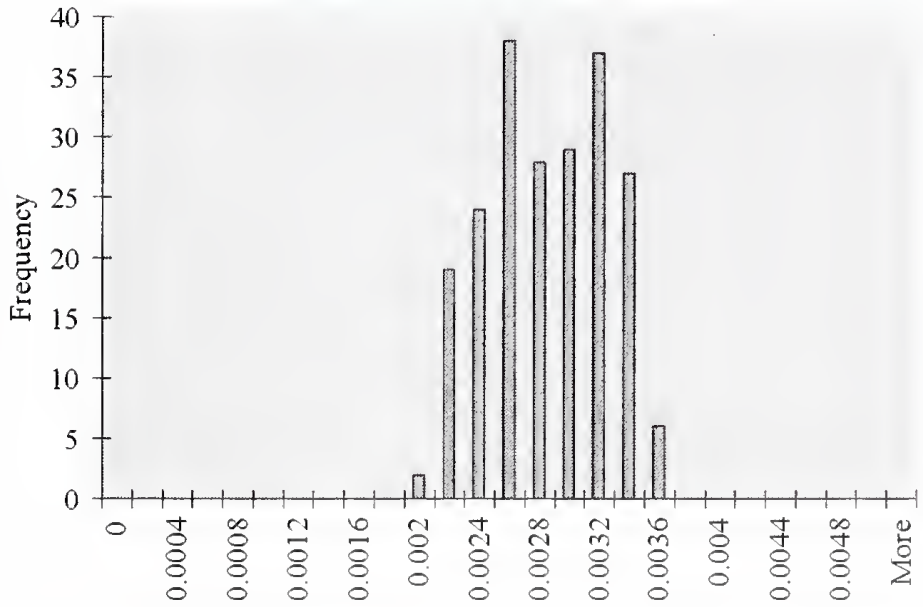

\begin{tabular}{|c|c|c|}
\hline Day & $\mathrm{CO}_{2}$ & $\mathrm{NH}_{3}$ \\
\hline $\mathbf{1}$ & 785000 & 419 \\
\hline $\mathbf{2}$ & 785000 & 795 \\
\hline $\mathbf{3}$ & 785000 & 1225 \\
\hline $\mathbf{4}$ & 785000 & 2318 \\
\hline $\mathbf{5}$ & 785000 & 3916 \\
\hline $\mathbf{6}$ & 785000 & 5819 \\
\hline $\mathbf{7}$ & 785000 & 8763 \\
\hline $\mathbf{8}$ & 785000 & 10860 \\
\hline $\mathbf{9}$ & 785000 & 12984 \\
\hline $\mathbf{1 0}$ & 785000 & 11384 \\
\hline
\end{tabular}

Cage occupied zone average relative humidity (\%) distribution

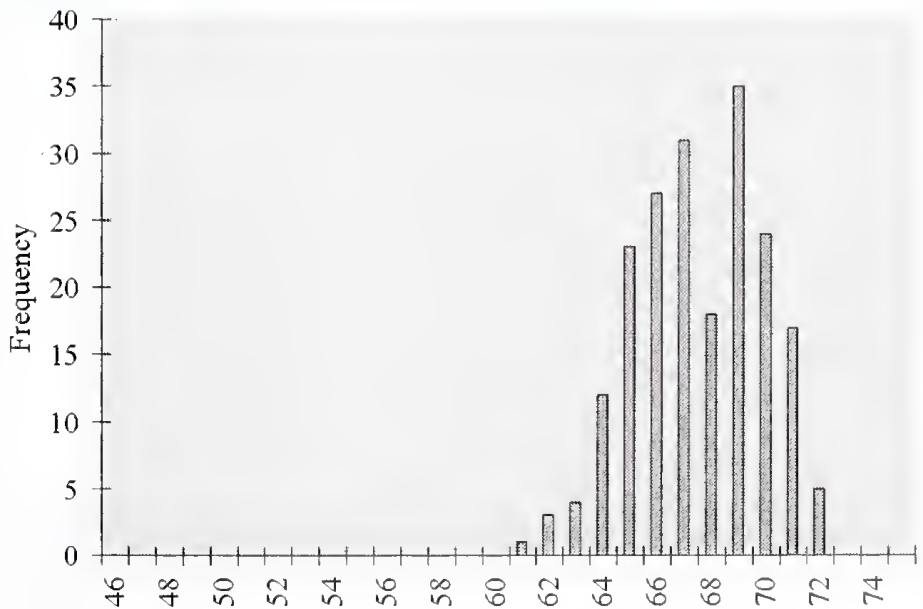


Casename

Case 60

Description

\begin{tabular}{|c|c|c|c|c|c|}
\hline Supply & Supply Discharge & Supply Discharge & Exhaust & Exhaust & Exhaust \\
\hline Configuration & Temperature $\left({ }^{\circ} \mathrm{C}\right)$ & RH & Configuration & Temperature $\left({ }^{\circ} \mathrm{C}\right)$ & RH \\
\hline Low Ind & 18.8 & $61 \%$ & High & 22 & $50 \%$ \\
\hline Change & Rack & Rack & Number of & Total mass of & Room \\
\hline Station & Orientation & Density & Mice in Room & Mice in Room & Pressurisation \\
\hline ON alt design & On wall & Single & 1050 & $21000 \mathrm{gr}$ & neg $100 \mathrm{cfm}$ \\
\hline
\end{tabular}

Room

$\mathrm{ACH}$

15

Cage

Condition

Top On

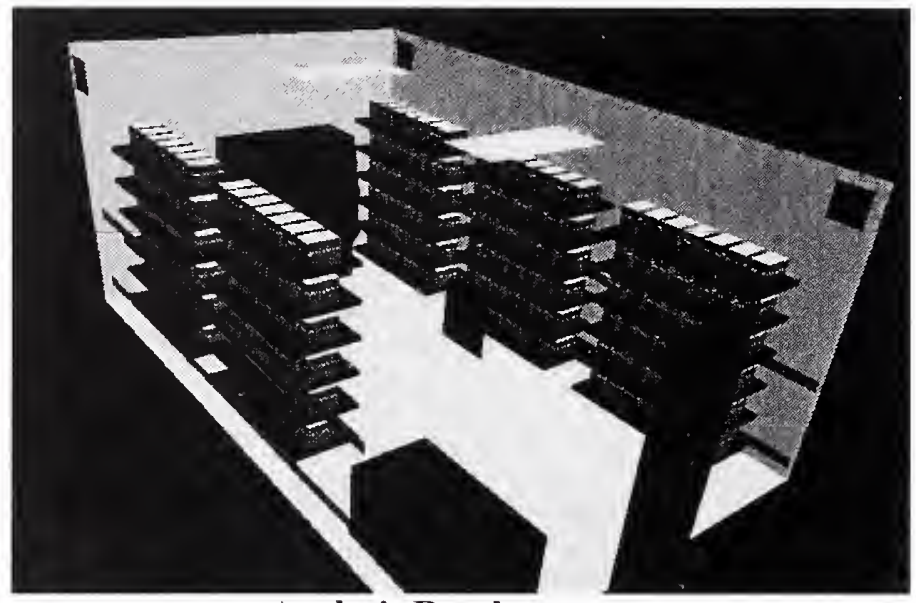

Analysis Results

Cage Occupied Zone

\begin{tabular}{|l|cc|c|c|}
\cline { 2 - 5 } \multicolumn{1}{c|}{} & \multicolumn{2}{c|}{ Temperature } & $\mathrm{CO}_{2}$ & RH \\
\cline { 2 - 5 } \multicolumn{1}{c|}{} & ${ }^{\circ} \mathrm{C}$ & ${ }^{\circ} \mathrm{F}$ & $(\mathrm{ppm})$ & \\
\hline Mean & 21.89 & 71.40 & 2354 & $69.12 \%$ \\
\hline S.D. & 0.27 & 0.48 & 236 & $2.57 \%$ \\
\hline Max. & 22.37 & 72.26 & 2929 & $74.25 \%$ \\
\hline
\end{tabular}

Cage Occupied Zone NH3 (ppm)

\begin{tabular}{|l|c|c|c|c|c|c|c|c|c|c|}
\hline Day & $\mathbf{1}$ & $\mathbf{2}$ & $\mathbf{3}$ & $\mathbf{4}$ & $\mathbf{5}$ & $\mathbf{6}$ & $\mathbf{7}$ & $\mathbf{8}$ & $\mathbf{9}$ & $\mathbf{1 0}$ \\
\hline Mean & 1.27 & 2.38 & 3.67 & 7.56 & 13.31 & 20.05 & 30.71 & 37.49 & 43.90 & 44.97 \\
\hline Max. & 1.58 & 2.96 & 4.57 & 9.40 & 16.57 & 24.95 & 38.22 & 46.66 & 54.63 & 55.95 \\
\hline
\end{tabular}

Room Breathing Zone

\begin{tabular}{|l|cc|c|c|}
\cline { 2 - 5 } \multicolumn{1}{c|}{} & \multicolumn{2}{c|}{ Temperature } & $\mathrm{CO}_{2}$ & RH \\
\cline { 2 - 5 } \multicolumn{1}{c|}{} & ${ }^{\circ} \mathrm{C}$ & ${ }^{\circ} \mathrm{F}$ & $(\mathrm{ppm})$ & \\
\hline Mean & 20.27 & 68.48 & 57 & $55.65 \%$ \\
\hline S.D. & 0.21 & 0.38 & 27 & \\
\hline Max. & 21.26 & 70.26 & 174 & \\
\hline
\end{tabular}

Room Breathing Zone NH3 (ppm)

\begin{tabular}{|l|c|c|c|c|c|c|c|c|c|c|}
\hline Day & $\mathbf{1}$ & $\mathbf{2}$ & $\mathbf{3}$ & $\mathbf{4}$ & $\mathbf{5}$ & $\mathbf{6}$ & $\mathbf{7}$ & $\mathbf{8}$ & $\mathbf{9}$ & $\mathbf{1 0}$ \\
\hline Mean & 0.03 & 0.06 & 0.09 & 0.18 & 0.32 & 0.48 & 0.74 & 0.90 & 1.05 & 1.08 \\
\hline Max. & 0.09 & 0.18 & 0.27 & 0.56 & 0.99 & 1.48 & 2.27 & 2.77 & 3.25 & 3.33 \\
\hline
\end{tabular}




\section{Histogram Distributions}

Cage occupied zone average temperature $\left({ }^{\circ} \mathrm{C}\right)$ distribution

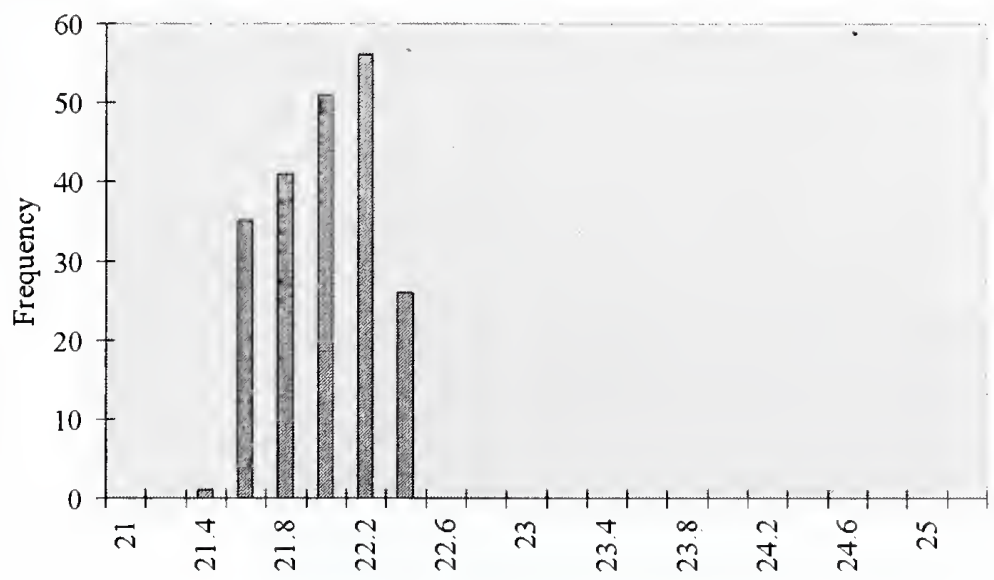

Cage occupied zone average contamination $(\mathrm{kg} / \mathrm{kg})$ distribution

Contamination conversion factors $(\mathrm{kg} / \mathrm{kg} \rightarrow \mathrm{ppm})$

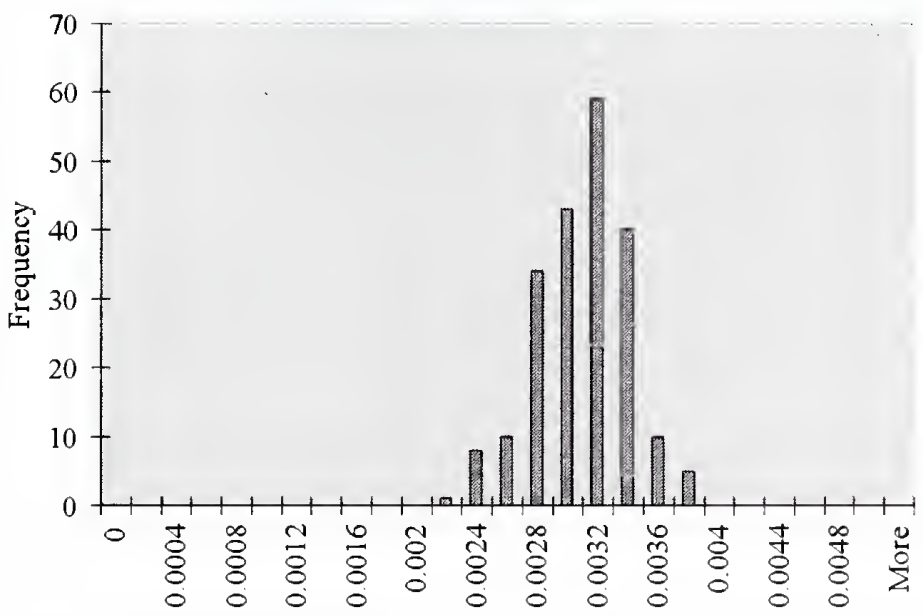

\begin{tabular}{|c|c|c|}
\hline Day & $\mathbf{C O}_{2}$ & $\mathrm{NH}_{\mathbf{3}}$ \\
\hline $\mathbf{1}$ & 785000 & 422 \\
\hline $\mathbf{2}$ & 785000 & 795 \\
\hline $\mathbf{3}$ & 785000 & 1225 \\
\hline $\mathbf{4}$ & 785000 & 2520 \\
\hline $\mathbf{5}$ & 785000 & 4439 \\
\hline $\mathbf{6}$ & 785000 & 6686 \\
\hline $\mathbf{7}$ & 785000 & 10242 \\
\hline $\mathbf{8}$ & 785000 & 12503 \\
\hline $\mathbf{9}$ & 785000 & 14641 \\
\hline $\mathbf{1 0}$ & 785000 & 11384 \\
\hline
\end{tabular}

Cage occupied zone average relative humidity (\%) distribution

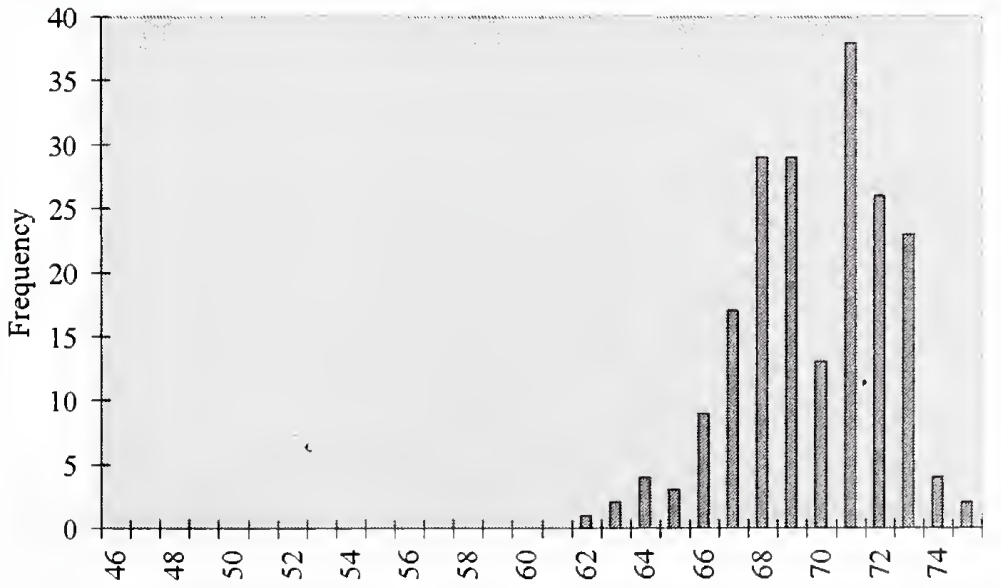


Casename

\section{Case 61}

\section{Description}

\begin{tabular}{|c|c|c|c|c|c|}
\hline Supply & Supply Discharge & Supply Discharge & Exhaust & Exhaust & Exhaust \\
\hline Configuration & Temperature $\left({ }^{\circ} \mathrm{C}\right)$ & RH & Configuration & Temperature $\left({ }^{\circ} \mathrm{C}\right)$ & $\mathbf{R H}$ \\
\hline Low Ind & 18.8 & $61 \%$ & Low & 22 & $50 \%$ \\
\hline Change & Rack & Rack & Number of & Total mass of & Room \\
\hline Station & Orientation & Density & Mice in Room & Mice in Room & Pressurisation \\
\hline ON alt design & On wall & Single & 1050 & $21000 \mathrm{gr}$ & neg $100 \mathrm{cfm}$ \\
\hline
\end{tabular}

\section{Room}

ACH

15

Cage

Condition

Top On

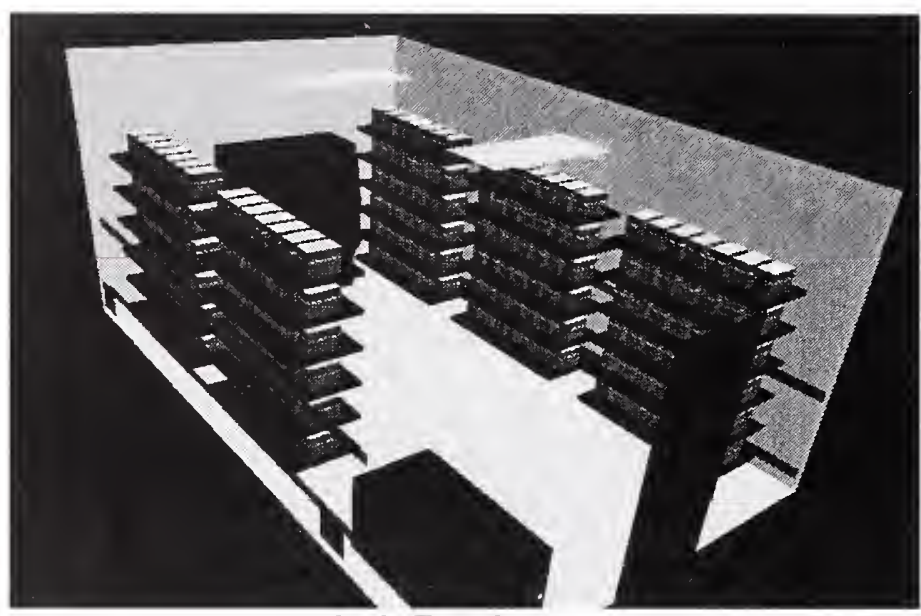

Analysis Results

\section{Cage Occupied Zone}

\begin{tabular}{|l|cc|c|c|}
\cline { 2 - 5 } \multicolumn{1}{c|}{} & \multicolumn{2}{c|}{ Temperature } & $\mathrm{CO}_{2}$ & RH \\
\cline { 2 - 5 } \multicolumn{1}{c|}{} & ${ }^{\circ} \mathrm{C}$ & ${ }^{\circ} \mathrm{F}$ & $(\mathrm{ppm})$ & \\
\hline Mean & 23.13 & 73.63 & 2042 & $61.56 \%$ \\
\hline S.D. & 0.40 & 0.73 & 410 & $2.86 \%$ \\
\hline Max. & 24.05 & 75.29 & 2824 & $66.70 \%$ \\
\hline
\end{tabular}

Cage Occupied Zone NH3 (ppm)

\begin{tabular}{|l|c|c|c|c|c|c|c|c|c|c|}
\hline Day & $\mathbf{1}$ & $\mathbf{2}$ & $\mathbf{3}$ & $\mathbf{4}$ & $\mathbf{5}$ & $\mathbf{6}$ & $\mathbf{7}$ & $\mathbf{8}$ & $\mathbf{9}$ & $\mathbf{1 0}$ \\
\hline Mean & 1.06 & 2.07 & 3.19 & 4.73 & 6.81 & 9.54 & 13.26 & 17.64 & 23.08 & 29.42 \\
\hline Max. & 1.47 & 2.86 & 4.41 & 6.54 & 9.42 & 13.20 & 18.33 & 24.40 & 31.92 & 40.69 \\
\hline
\end{tabular}

Room Breathing Zone

\begin{tabular}{|l|cc|c|c|}
\cline { 2 - 5 } \multicolumn{1}{c|}{} & \multicolumn{2}{c|}{ Temperature } & $\mathrm{CO}_{2}$ & RH \\
\cline { 2 - 5 } \multicolumn{1}{c|}{} & ${ }^{\circ} \mathrm{C}$ & ${ }^{\circ} \mathrm{F}$ & $(\mathrm{ppm})$ & \\
\hline Mean & 22.04 & 71.67 & 69 & $4983 \%$ \\
\hline S.D. & 0.49 & 0.88 & 26 & \\
\hline Max. & 23.21 & 73.77 & 310 & \\
\hline
\end{tabular}

Room Breathing Zone NH3 (ppm)

\begin{tabular}{|l|c|c|c|c|c|c|c|c|c|c|}
\hline Day & $\mathbf{1}$ & $\mathbf{2}$ & $\mathbf{3}$ & $\mathbf{4}$ & $\mathbf{5}$ & $\mathbf{6}$ & $\mathbf{7}$ & $\mathbf{8}$ & $\mathbf{9}$ & $\mathbf{1 0}$ \\
\hline Mean & 0.04 & 0.07 & 0.11 & 0.16 & 0.23 & 0.32 & 0.45 & 0.59 & 0.78 & 0.99 \\
\hline Max. & 0.16 & 0.31 & 0.48 & 0.72 & 1.03 & 1.45 & 2.01 & 2.68 & 3.50 & 4.47 \\
\hline
\end{tabular}




\section{Histogram Distributions}

Cage occupied zone average temperature $\left({ }^{\circ} \mathrm{C}\right)$ distribution

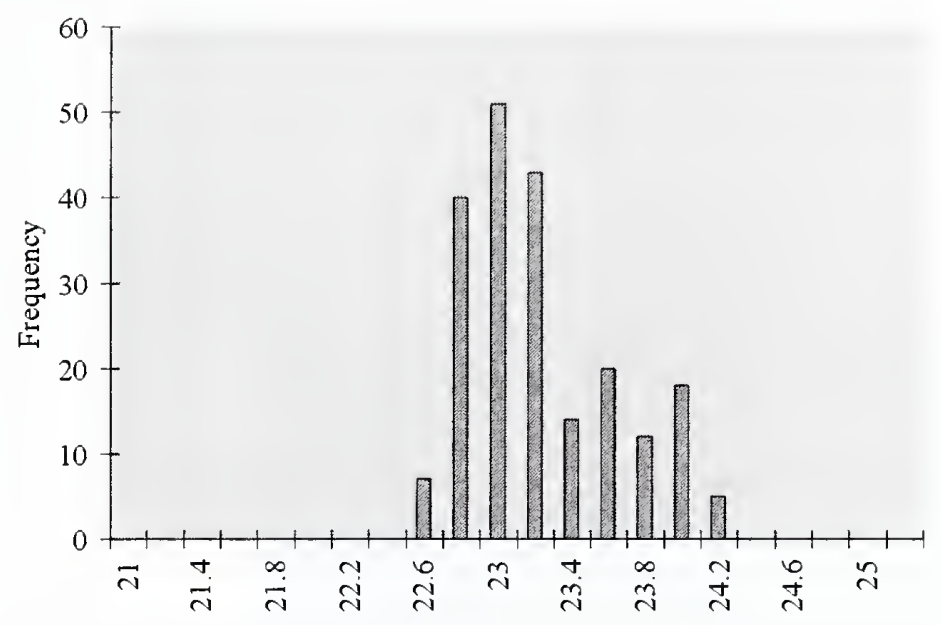

Cage occupied zone average contamination $(\mathrm{kg} / \mathrm{kg})$ distribution

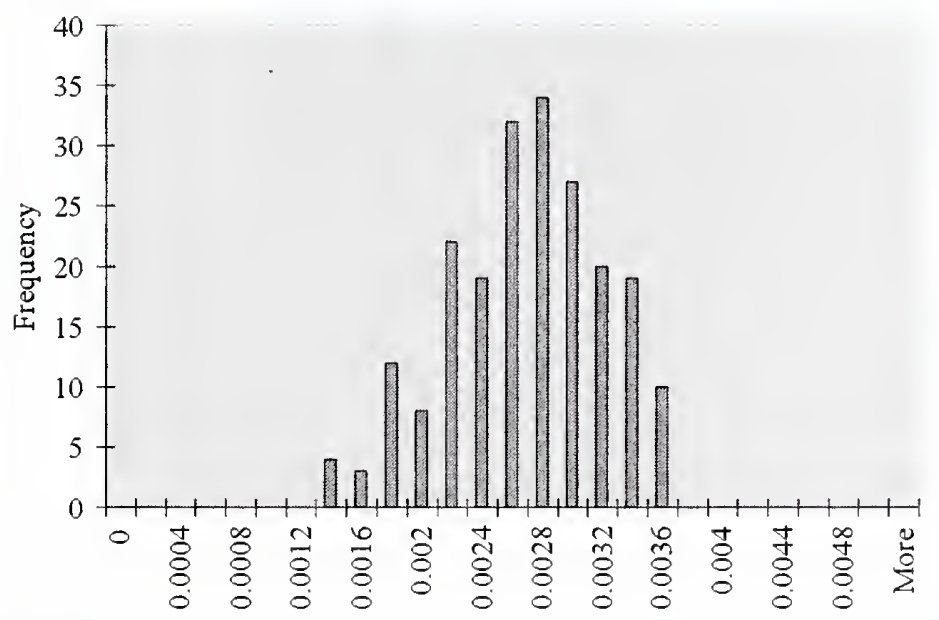

Cage occupied zone average relative humidity $(\%)$ distribution

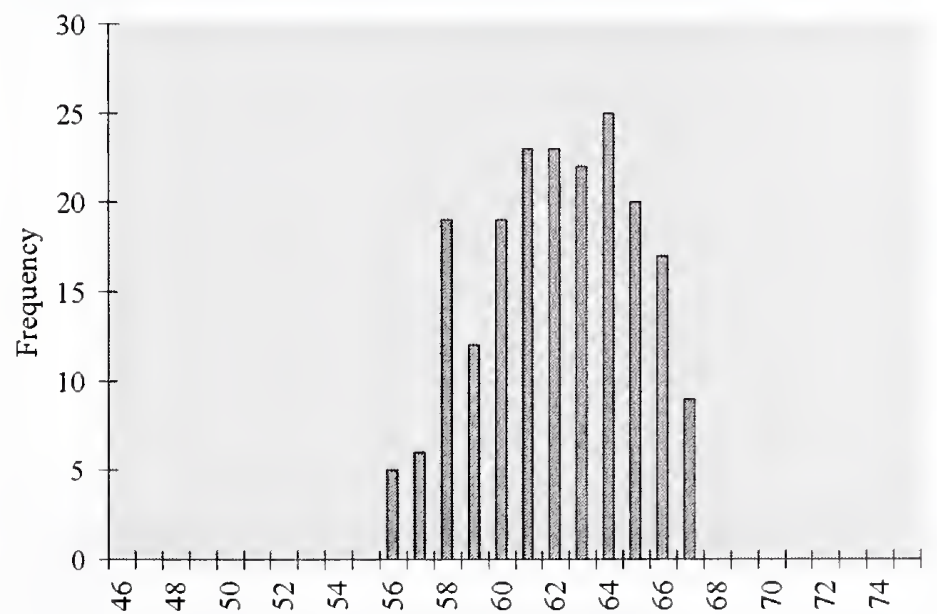

Contamination conversion factors $(\mathrm{kg} / \mathrm{kg} \rightarrow \mathrm{ppm})$

\begin{tabular}{|c|c|c|}
\hline Day & $\mathrm{CO}_{2}$ & $\mathrm{NH}_{3}$ \\
\hline $\mathbf{1}$ & 785000 & 409 \\
\hline $\mathbf{2}$ & 785000 & 795 \\
\hline $\mathbf{3}$ & 785000 & 1225 \\
\hline $\mathbf{4}$ & 785000 & 1818 \\
\hline $\mathbf{5}$ & 785000 & 2618 \\
\hline $\mathbf{6}$ & 785000 & 3668 \\
\hline $\mathbf{7}$ & 785000 & 5095 \\
\hline $\mathbf{8}$ & 785000 & 6782 \\
\hline $\mathbf{9}$ & 785000 & 8872 \\
\hline $\mathbf{1 0}$ & 785000 & 11384 \\
\hline
\end{tabular}




\section{Description}

\begin{tabular}{|c|c|c|c|c|c|}
\hline Supply & Supply Discharge & Supply Discharge & Exhaust & Exhaust & Exhaust \\
\hline Configuration & Temperature $\left({ }^{\circ} \mathrm{C}\right)$ & RH & Configuration & Temperature $\left({ }^{\circ} \mathrm{C}\right)$ & $\mathbf{R H}$ \\
\hline Low Ind & 6.6 & $89 \%$ & Low & 22 & $50 \%$ \\
\hline Change & Rack & Rack & Number of & Total mass of & Room \\
\hline Station & Orientation & Density & Mice in Room & Mice in Room & Pressurisation \\
\hline ON & Perp & Double & 2100 & $42000 \mathrm{gr}$ & neg $100 \mathrm{cfm}$ \\
\hline
\end{tabular}

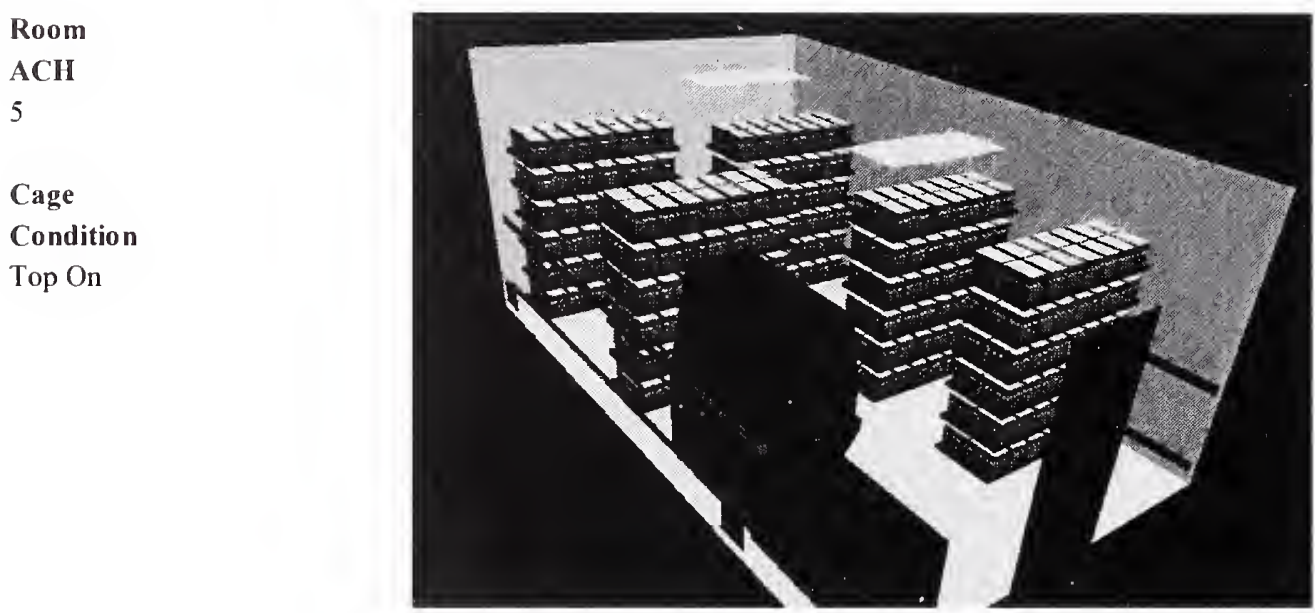

Analysis Results

Cage Occupied Zone

\begin{tabular}{|l|cc|c|c|}
\cline { 2 - 5 } \multicolumn{1}{c|}{} & \multicolumn{2}{c|}{ Temperature } & $\mathbf{C O}_{2}$ & RH \\
\cline { 2 - 5 } \multicolumn{1}{c|}{} & ${ }^{\circ} \mathrm{C}$ & ${ }^{\circ} \mathrm{F}$ & $(\mathbf{p p m})$ & \\
\hline Mean & 20.87 & 69.56 & 2325 & $52.77 \%$ \\
\hline S.D. & 0.77 & 1.38 & 520 & $4.06 \%$ \\
\hline Max. & 22.64 & 72.75 & 3389 & $59.89 \%$ \\
\hline
\end{tabular}

Cage Occupied Zone NH3 (ppm)

\begin{tabular}{|l|c|c|c|c|c|c|c|c|c|c|}
\hline Day & $\mathbf{1}$ & $\mathbf{2}$ & $\mathbf{3}$ & $\mathbf{4}$ & $\mathbf{5}$ & $\mathbf{6}$ & $\mathbf{7}$ & $\mathbf{8}$ & $\mathbf{9}$ & $\mathbf{1 0}$ \\
\hline Mean & 1.21 & 2.35 & 3.63 & 5.23 & 7.36 & 10.20 & 13.96 & 18.83 & 25.01 & 32.69 \\
\hline Max. & 1.76 & 3.43 & 5.29 & 7.62 & 10.72 & 14.86 & 20.34 & 27.44 & 36.44 & 47.64 \\
\hline
\end{tabular}

Room Breathing Zone

\begin{tabular}{|l|cc|c|c|}
\cline { 2 - 5 } \multicolumn{1}{c|}{} & \multicolumn{2}{|c|}{ Temperature } & $\mathrm{CO}_{2}$ & RH \\
\cline { 2 - 5 } \multicolumn{1}{c|}{} & ${ }^{\circ} \mathrm{C}$ & ${ }^{\circ}$ & $(\mathbf{p p m})$ & \\
\hline Mean & 19.15 & 66.47 & 342 & $62.56 \%$ \\
\hline S.D. & 0.95 & 1.71 & 216 & \\
\hline Max. & 25.27 & 77.49 & 897 & \\
\hline
\end{tabular}

Room Breathing Zone NH3 (ppm)

\begin{tabular}{|l|c|c|c|c|c|c|c|c|c|c|}
\hline Day & $\mathbf{1}$ & $\mathbf{2}$ & $\mathbf{3}$ & $\mathbf{4}$ & $\mathbf{5}$ & $\mathbf{6}$ & $\mathbf{7}$ & $\mathbf{8}$ & $\mathbf{9}$ & $\mathbf{1 0}$ \\
\hline Mean & 0.18 & 0.35 & 0.53 & 0.77 & 1.08 & 1.50 & 2.05 & 2.77 & 3.68 & 4.81 \\
\hline Max. & 0.47 & 0.91 & 1.40 & 2.02 & 2.84 & 3.94 & 5.39 & 7.27 & 9.65 & 12.61 \\
\hline
\end{tabular}




\section{Histogram Distributions}

Cage occupied zone average temperature $\left({ }^{\circ} \mathrm{C}\right)$ distribution

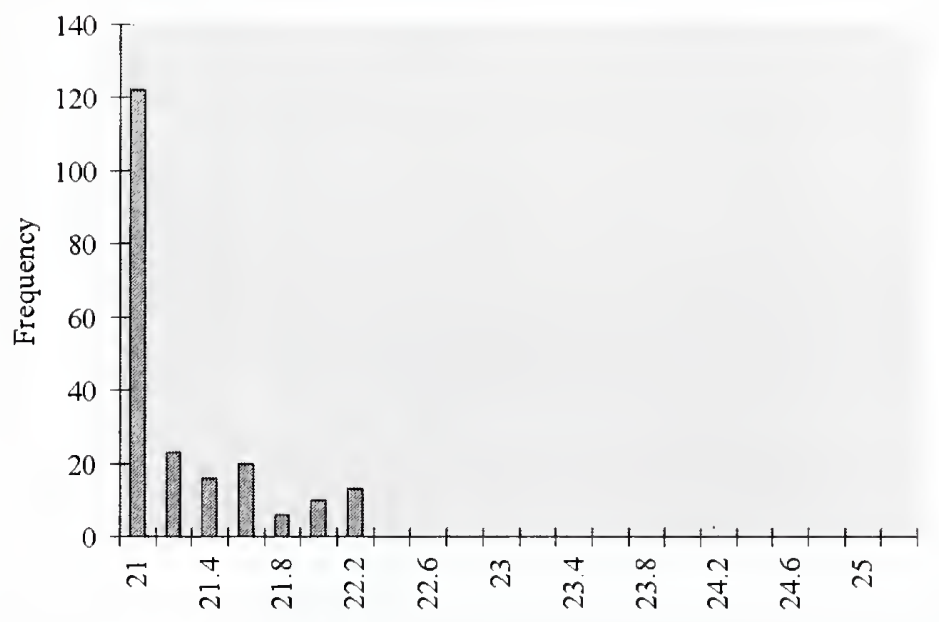

Cage occupied zone average contamination $(\mathrm{kg} / \mathrm{kg})$ distribution

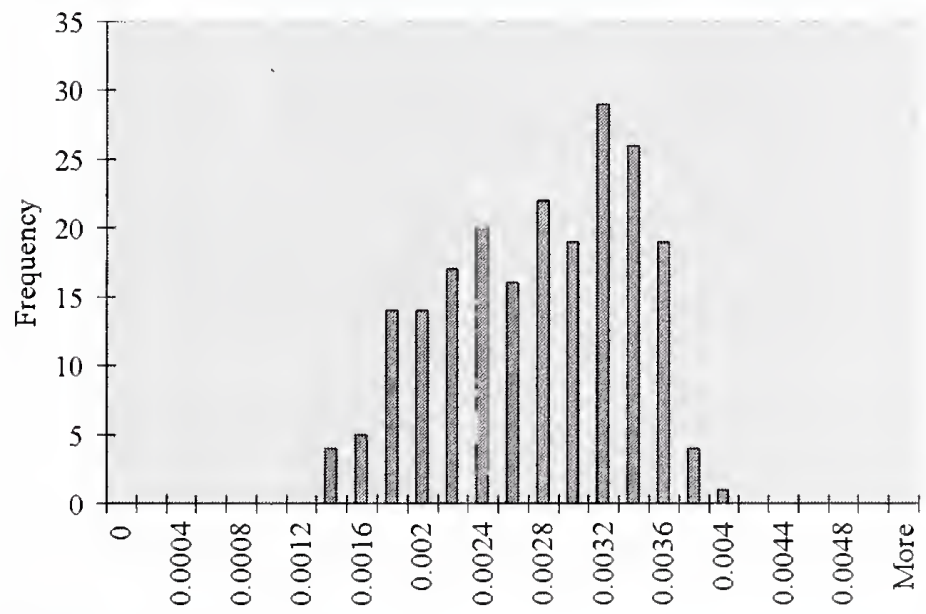

Cage occupied zone average relative humidity (\%) distribution

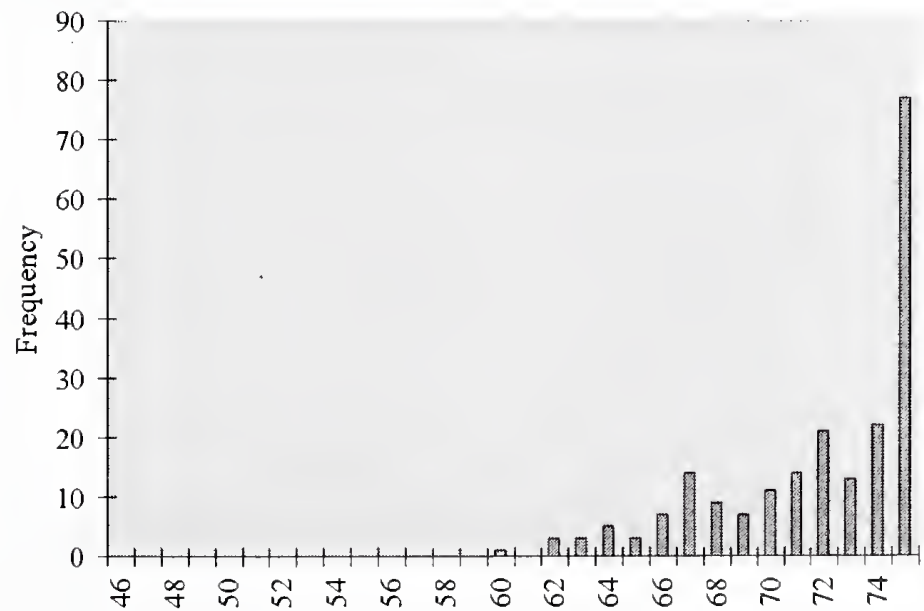

Contamination conversion factors $(\mathrm{kg} / \mathrm{kg} \rightarrow \mathrm{ppm})$

\begin{tabular}{|c|c|c|}
\hline $\mathbf{D a y}$ & $\mathrm{CO}_{2}$ & $\mathbf{N H}_{3}$ \\
\hline $\mathbf{1}$ & 785000 & 415 \\
\hline $\mathbf{2}$ & 785000 & 795 \\
\hline $\mathbf{3}$ & 785000 & 1225 \\
\hline $\mathbf{4}$ & 785000 & 2129 \\
\hline $\mathbf{5}$ & 785000 & 3425 \\
\hline $\mathbf{6}$ & 785000 & 5004 \\
\hline $\mathbf{7}$ & 785000 & 7374 \\
\hline $\mathbf{8}$ & 785000 & 9315 \\
\hline $\mathbf{9}$ & 785000 & 11426 \\
\hline $\mathbf{1 0}$ & 785000 & 11384 \\
\hline
\end{tabular}


Casename Case 63

Description

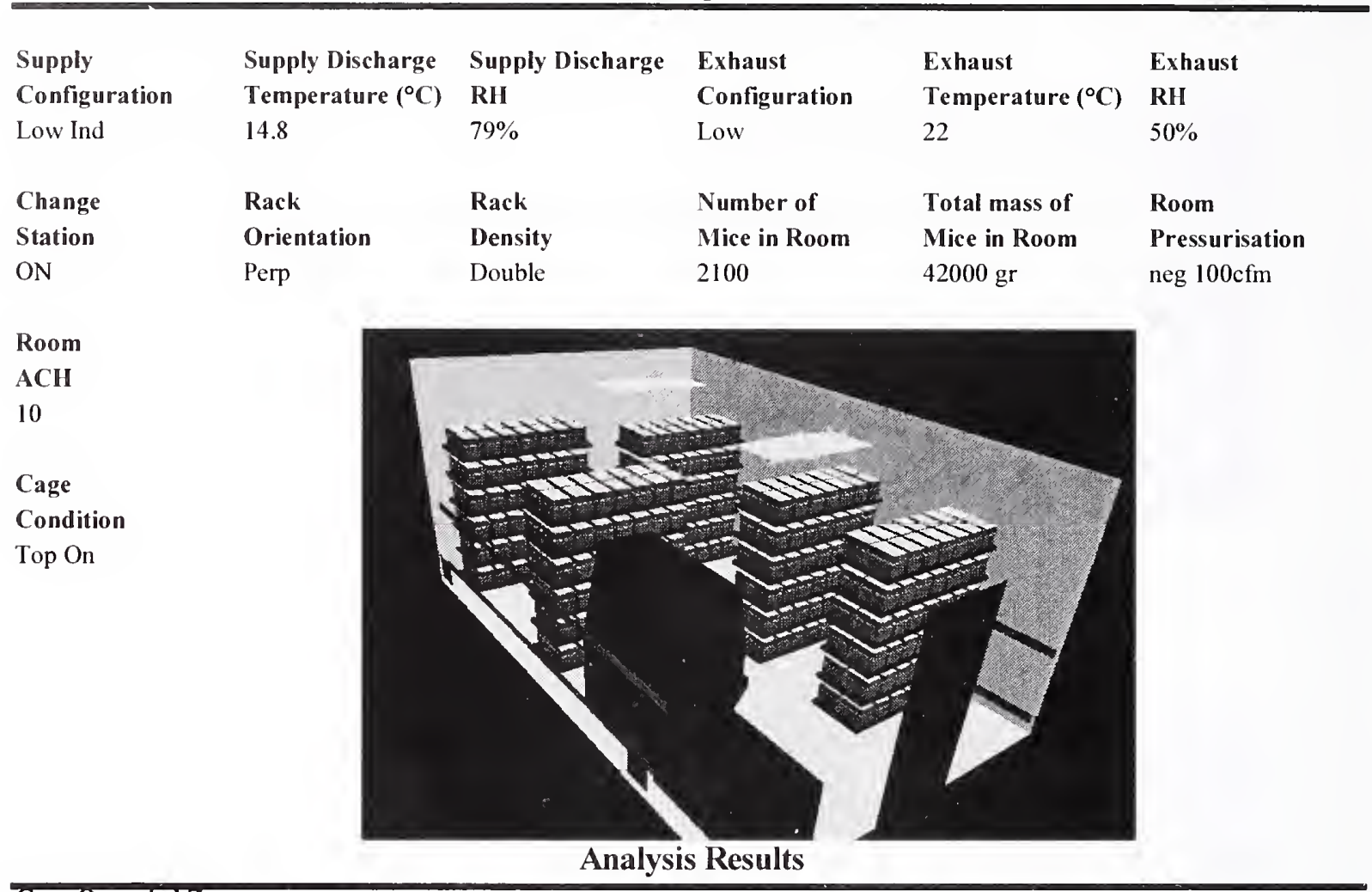

Cage Occupied Zone

\begin{tabular}{|l|cc|c|c|}
\cline { 2 - 5 } \multicolumn{1}{c|}{} & \multicolumn{2}{c|}{ Temperature } & $\mathrm{CO}_{2}$ & RH \\
\cline { 2 - 5 } \multicolumn{1}{c|}{} & ${ }^{\circ} \mathrm{C}$ & ${ }^{\circ} \mathrm{F}$ & $(\mathrm{ppm})$ & \\
\hline Mean & 21.91 & 71.44 & 2081 & $64.91 \%$ \\
\hline S.D. & 0.53 & 0.95 & 523 & $3.42 \%$ \\
\hline Max. & 23.10 & 73.57 & 3402 & $72.86 \%$ \\
\hline
\end{tabular}

Cage Occupied Zone NH3 (ppm)

\begin{tabular}{|l|c|c|c|c|c|c|c|c|c|c|}
\hline Day & $\mathbf{1}$ & $\mathbf{2}$ & $\mathbf{3}$ & $\mathbf{4}$ & $\mathbf{5}$ & $\mathbf{6}$ & $\mathbf{7}$ & $\mathbf{8}$ & $\mathbf{9}$ & $\mathbf{1 0}$ \\
\hline Mean & 1.10 & 2.11 & 3.25 & 5.64 & 9.08 & 13.26 & 19.55 & 24.69 & 30.29 & 34.30 \\
\hline Max. & 1.80 & 3.44 & 5.31 & 9.23 & 14.84 & 21.69 & 31.96 & 40.37 & 49.52 & 56.09 \\
\hline
\end{tabular}

Room Breathing Zone

\begin{tabular}{|l|cc|c|c|}
\cline { 2 - 5 } \multicolumn{1}{c|}{} & \multicolumn{2}{c|}{ Temperature } & $\mathrm{CO}_{2}$ & $\mathrm{RH}$ \\
\cline { 2 - 5 } \multicolumn{1}{c|}{} & ${ }^{\circ} \mathrm{C}$ & ${ }^{\circ} \mathrm{F}$ & $(\mathbf{p p m})$ & \\
\hline Mean & 20.34 & 68.62 & 215 & $56.81 \%$ \\
\hline S.D. & 0.81 & 1.46 & 109 & \\
\hline Max. & 26.82 & 80.28 & 650 & \\
\hline
\end{tabular}

Room Breathing Zone NH3 (ppm)

\begin{tabular}{|l|c|c|c|c|c|c|c|c|c|c|}
\hline Day & $\mathbf{1}$ & $\mathbf{2}$ & $\mathbf{3}$ & $\mathbf{4}$ & $\mathbf{5}$ & $\mathbf{6}$ & $\mathbf{7}$ & $\mathbf{8}$ & $\mathbf{9}$ & $\mathbf{1 0}$ \\
\hline Mean & 0.11 & 0.22 & 0.34 & 0.58 & 0.94 & 1.37 & 2.02 & 2.55 & 3.13 & 3.54 \\
\hline Max. & 0.34 & 0.66 & 1.01 & 1.76 & 2.83 & 4.14 & 6.10 & 7.71 & 9.46 & 10.71 \\
\hline
\end{tabular}




\section{Histogram Distributions}

Cage occupied zone average temperature $\left({ }^{\circ} \mathrm{C}\right)$ distribution

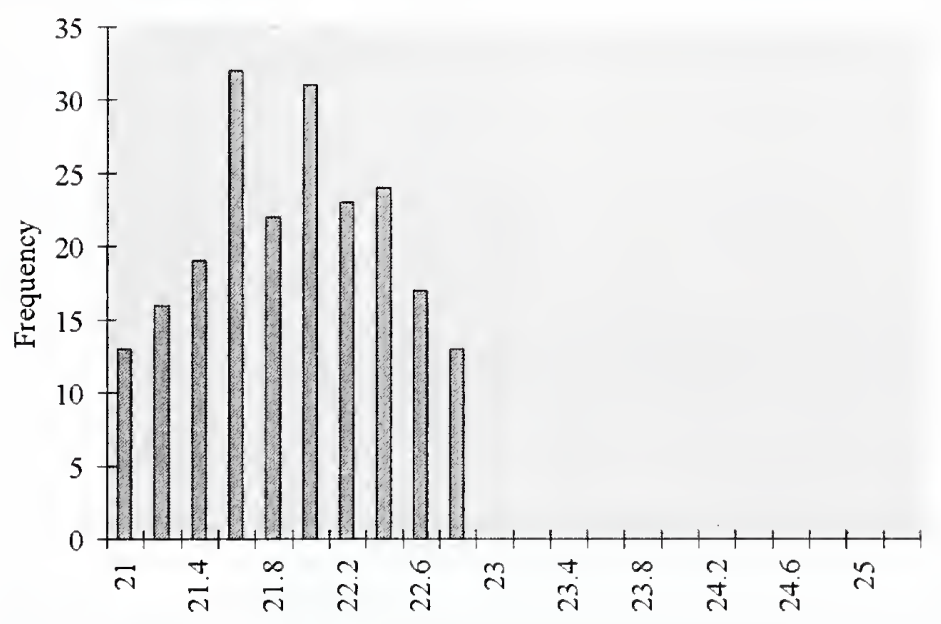

Cage occupied zone average contamination $(\mathrm{kg} / \mathrm{kg})$ distribution

Contamination conversion factors $(\mathrm{kg} / \mathrm{kg} \rightarrow \mathrm{ppm})$

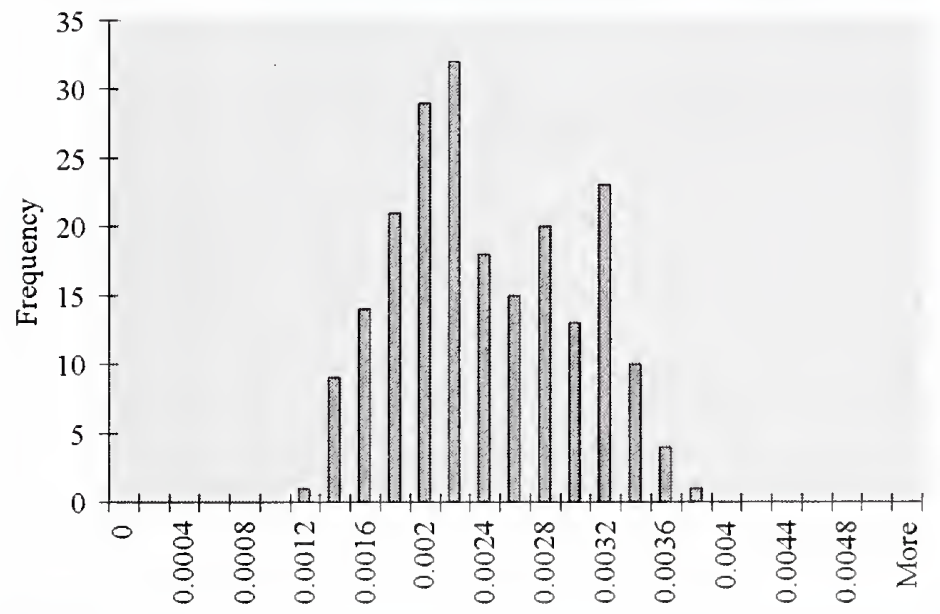

\begin{tabular}{|c|c|c|}
\hline Day & $\mathbf{C O}_{2}$ & $\mathbf{N H}_{3}$ \\
\hline $\mathbf{1}$ & 785000 & 415 \\
\hline $\mathbf{2}$ & 785000 & 795 \\
\hline $\mathbf{3}$ & 785000 & 1225 \\
\hline $\mathbf{4}$ & 785000 & 2129 \\
\hline $\mathbf{5}$ & 785000 & 3425 \\
\hline $\mathbf{6}$ & 785000 & 5004 \\
\hline $\mathbf{7}$ & 785000 & 7374 \\
\hline $\mathbf{8}$ & 785000 & 9315 \\
\hline $\mathbf{9}$ & 785000 & 11426 \\
\hline $\mathbf{1 0}$ & 785000 & 11384 \\
\hline
\end{tabular}

Cage occupied zone average relative humidity $(\%)$ distribution

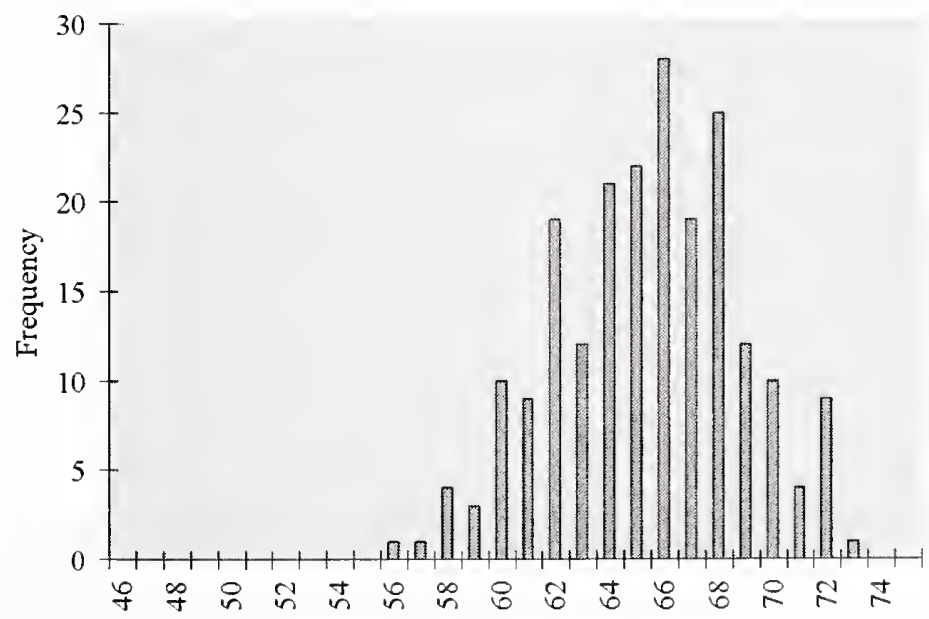


Casename

Case 64

Description

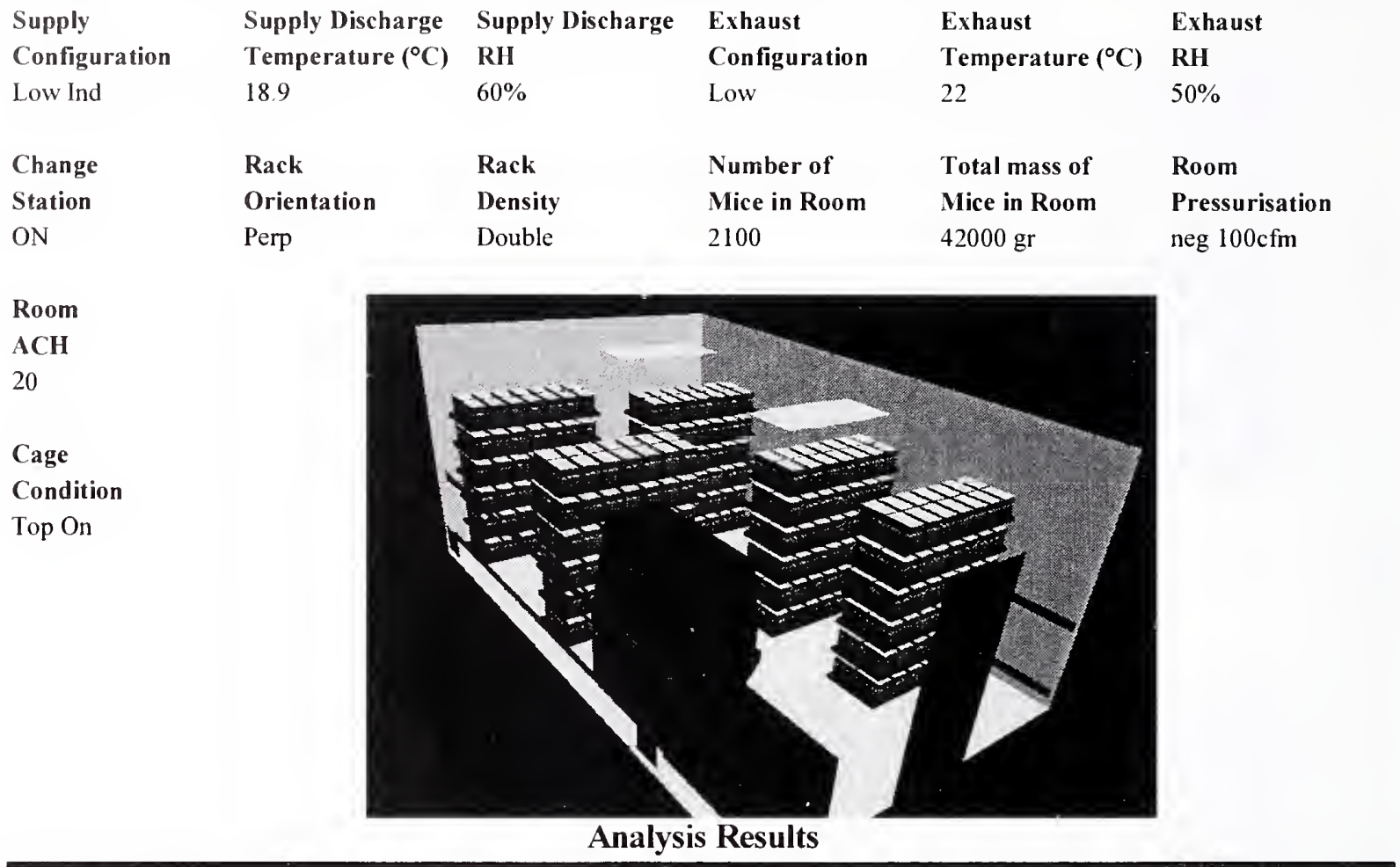

Cage Occupied Zone

\begin{tabular}{|l|cc|c|c|}
\cline { 2 - 5 } \multicolumn{1}{c|}{} & \multicolumn{2}{c|}{ Temperature } & $\mathbf{C O}_{2}$ & RH \\
\cline { 2 - 5 } \multicolumn{1}{c|}{} & ${ }^{\mathrm{C}}$ & ${ }_{\mathrm{F}}$ & $(\mathrm{ppm})$ & \\
\hline Mean & 22.66 & 72.79 & 1808 & $60.21 \%$ \\
\hline S.D. & 0.28 & 0.50 & 422 & $2.96 \%$ \\
\hline Max. & 23.34 & 74.02 & 2618 & $67.10 \%$ \\
\hline
\end{tabular}

Cage Occupied Zone NH3 (ppm)

\begin{tabular}{|l|c|c|c|c|c|c|c|c|c|c|}
\hline Day & $\mathbf{1}$ & $\mathbf{2}$ & $\mathbf{3}$ & $\mathbf{4}$ & $\mathbf{5}$ & $\mathbf{6}$ & $\mathbf{7}$ & $\mathbf{8}$ & $\mathbf{9}$ & $\mathbf{1 0}$ \\
\hline Mean & 0.94 & 1.83 & 2.82 & 4.07 & 5.72 & 7.93 & 10.85 & 14.64 & 19.44 & 25.42 \\
\hline Max. & 1.36 & 2.65 & 4.09 & 5.89 & 8.28 & 11.48 & 15.71 & 21.20 & 28.15 & 36.80 \\
\hline
\end{tabular}

Room Breathing Zone

\begin{tabular}{|l|cc|c|c|}
\cline { 2 - 5 } \multicolumn{1}{c|}{} & \multicolumn{2}{c|}{ Temperature } & $\mathbf{C O}_{2}$ & RH \\
\cline { 2 - 5 } \multicolumn{1}{c|}{} & ${ }^{\circ} \mathrm{C}$ & ${ }^{\circ} \mathrm{F}$ & $(\mathbf{p p m})$ & \\
\hline Mean & 21.17 & 70.10 & 93 & $52.87 \%$ \\
\hline S.D. & 0.71 & 1.27 & 47 & \\
\hline Max. & 27.72 & 81.89 & 324 & \\
\hline
\end{tabular}

Room Breathing Zone NH3 (ppm)

\begin{tabular}{|l|c|c|c|c|c|c|c|c|c|c|}
\hline Day & $\mathbf{1}$ & $\mathbf{2}$ & $\mathbf{3}$ & $\mathbf{4}$ & $\mathbf{5}$ & $\mathbf{6}$ & $\mathbf{7}$ & $\mathbf{8}$ & $\mathbf{9}$ & $\mathbf{1 0}$ \\
\hline Mean & 0.05 & 0.09 & 0.15 & 0.21 & 0.29 & 0.41 & 0.56 & 0.75 & 1.00 & 1.31 \\
\hline Max. & 0.17 & 0.33 & 0.51 & 0.73 & 1.02 & 1.42 & 1.94 & 2.62 & 3.48 & 4.55 \\
\hline
\end{tabular}




\section{Histogram Distributions}

Cage occupied zone average temperature $\left({ }^{\circ} \mathrm{C}\right)$ distribution

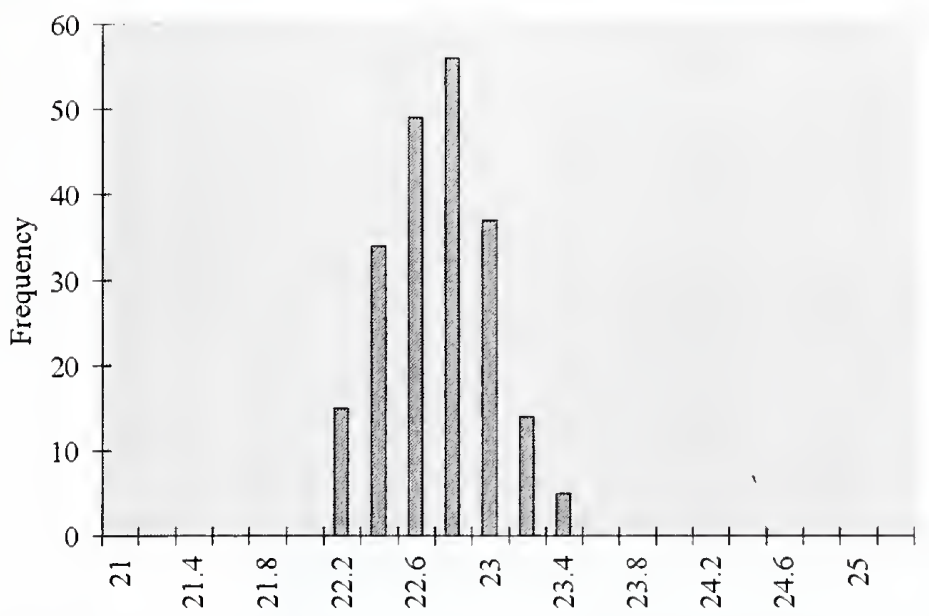

Cage occupied zone average contamination $(\mathrm{kg} / \mathrm{kg})$ distribution

Contamination conversion factors $(\mathrm{kg} / \mathrm{kg} \rightarrow \mathrm{ppm})$

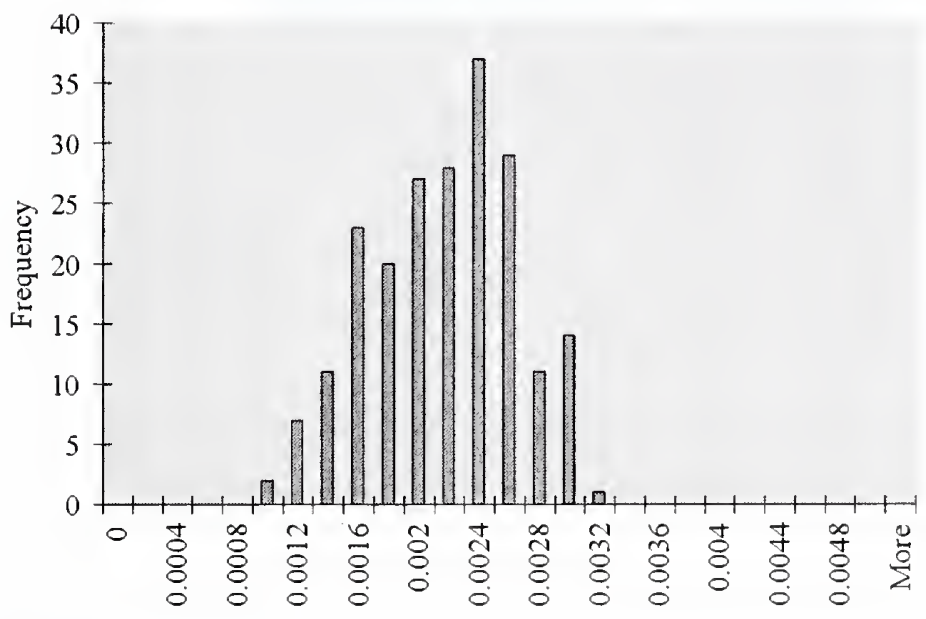

\begin{tabular}{|c|c|c|}
\hline Day & $\mathbf{C O}_{2}$ & $\mathbf{N H}_{3}$ \\
\hline $\mathbf{1}$ & 785000 & 408 \\
\hline $\mathbf{2}$ & 785000 & 795 \\
\hline $\mathbf{3}$ & 785000 & 1225 \\
\hline $\mathbf{4}$ & 785000 & 1766 \\
\hline $\mathbf{5}$ & 785000 & 2483 \\
\hline $\mathbf{6}$ & 785000 & 3443 \\
\hline $\mathbf{7}$ & 785000 & 4712 \\
\hline $\mathbf{8}$ & 785000 & 6356 \\
\hline $\mathbf{9}$ & 785000 & 8442 \\
\hline $\mathbf{1 0}$ & 785000 & 11384 \\
\hline
\end{tabular}

Cage occupied zone average relative humidity (\%) distribution

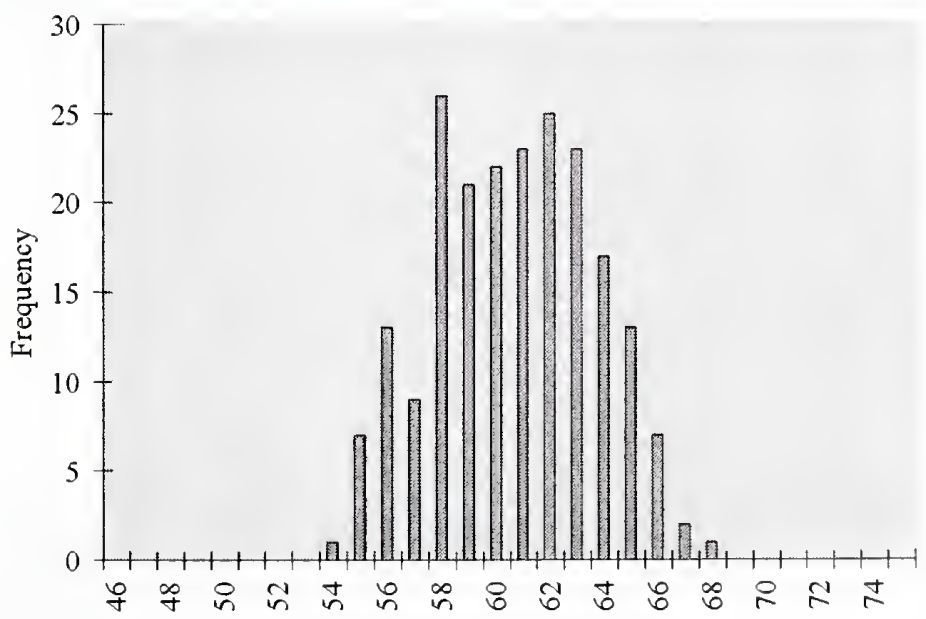


Casename

\section{Case 65}

\section{Description}

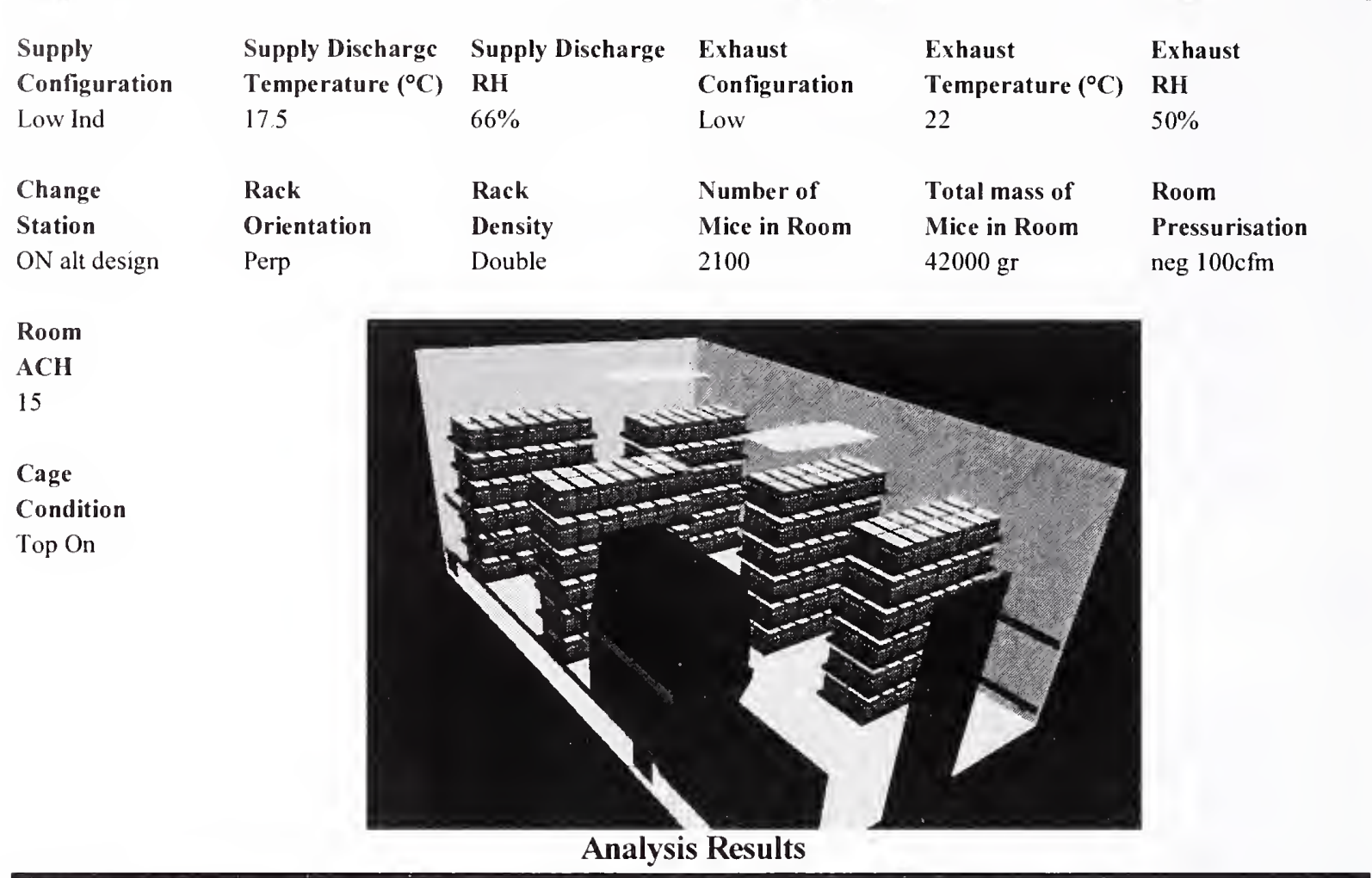

Cage Occupied Zone

\begin{tabular}{|l|cc|c|c|}
\cline { 2 - 5 } \multicolumn{1}{c|}{} & \multicolumn{2}{c|}{ Temperature } & $\mathrm{CO}_{2}$ & RH \\
\cline { 2 - 5 } \multicolumn{1}{c|}{} & ${ }^{\circ} \mathrm{C}$ & ${ }^{\circ} \mathrm{F}$ & (ppm) & \\
\hline Mean & 22.43 & 72.37 & 2002 & $62.67 \%$ \\
\hline S.D. & 0.29 & 0.52 & 461 & $2.88 \%$ \\
\hline Max. & 23.21 & 73.77 & 3148 & $69.19 \%$ \\
\hline
\end{tabular}

Cage Occupied Zone NH3 (ppm)

\begin{tabular}{|l|c|c|c|c|c|c|c|c|c|c|}
\hline Day & $\mathbf{1}$ & $\mathbf{2}$ & $\mathbf{3}$ & $\mathbf{4}$ & $\mathbf{5}$ & $\mathbf{6}$ & $\mathbf{7}$ & $\mathbf{8}$ & $\mathbf{9}$ & $\mathbf{1 0}$ \\
\hline Mean & 1.05 & 2.03 & 3.12 & 490 & 7.36 & 10.48 & 1492 & 19.44 & 24.79 & 30.23 \\
\hline Max. & 1.65 & 3.19 & 4.91 & 7.70 & 11.57 & 16.49 & 23.46 & 30.57 & 38.97 & 47.53 \\
\hline
\end{tabular}

Room Breathing Zone

\begin{tabular}{|l|cc|c|c|}
\cline { 2 - 5 } \multicolumn{1}{c|}{} & \multicolumn{2}{|c|}{ Temperature } & $\mathrm{CO}_{2}$ & $\mathrm{RH}$ \\
\cline { 2 - 5 } \multicolumn{1}{c|}{} & ${ }^{\circ} \mathrm{C}$ & ${ }^{\circ} \mathrm{F}$ & $(\mathrm{ppm})$ & \\
\hline Mean & 20.83 & 69.49 & 133 & $54.38 \%$ \\
\hline S.D. & 0.39 & 0.71 & 50 & \\
\hline Max. & 21.70 & 71.07 & 321 & \\
\hline
\end{tabular}

Room Breathing Zone NH3 (ppm)

\begin{tabular}{|l|c|c|c|c|c|c|c|c|c|c|}
\hline Day & $\mathbf{1}$ & $\mathbf{2}$ & $\mathbf{3}$ & $\mathbf{4}$ & $\mathbf{5}$ & $\mathbf{6}$ & $\mathbf{7}$ & $\mathbf{8}$ & $\mathbf{9}$ & $\mathbf{1 0}$ \\
\hline Mean & 0.07 & 0.14 & 0.21 & 0.33 & 0.49 & 0.70 & 0.99 & 1.30 & 1.65 & 2.01 \\
\hline Max. & 0.17 & 0.32 & 0.50 & 0.79 & 1.18 & 1.68 & 2.39 & 3.12 & 3.97 & 4.85 \\
\hline
\end{tabular}




\section{Histogram Distributions}

Cage occupied zone average temperature $\left({ }^{\circ} \mathrm{C}\right)$ distribution

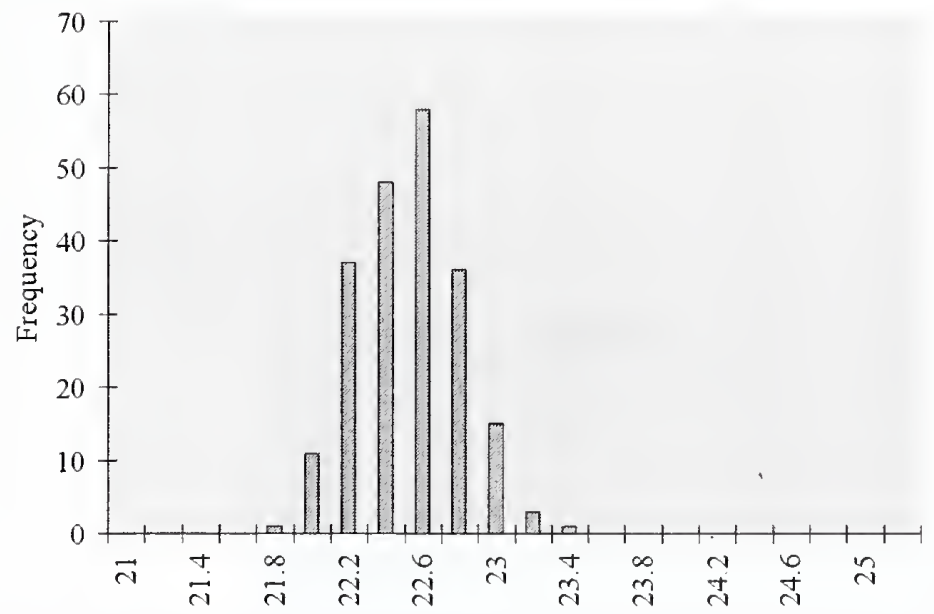

Cage occupied zone average contamination $(\mathrm{kg} / \mathrm{kg})$ distribution

Contamination conversion factors $(\mathrm{kg} / \mathrm{kg} \rightarrow \mathrm{ppm})$

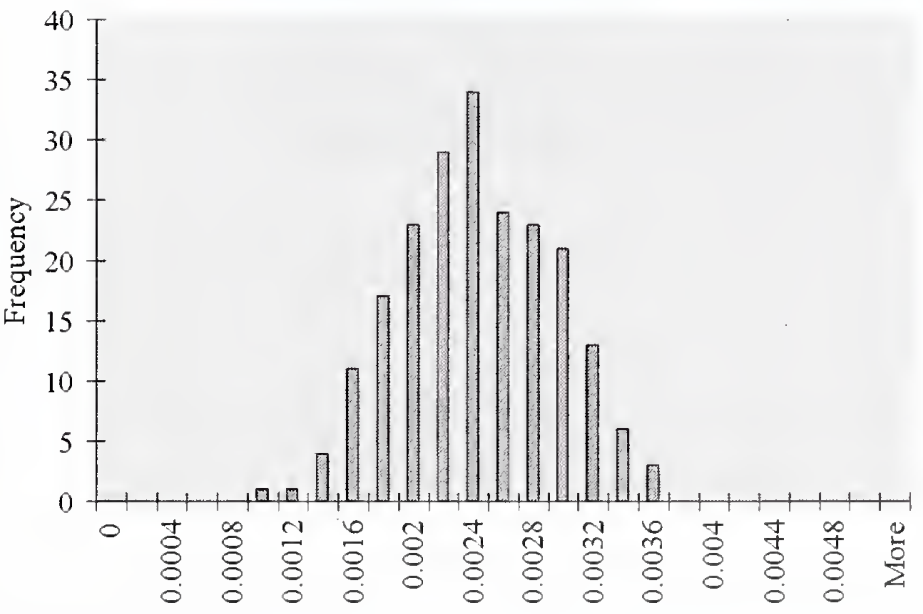

\begin{tabular}{|c|c|c|}
\hline Day & $\mathrm{CO}_{2}$ & $\mathrm{NH}_{3}$ \\
\hline $\mathbf{1}$ & 785000 & 411 \\
\hline $\mathbf{2}$ & 785000 & 795 \\
\hline $\mathbf{3}$ & 785000 & 1225 \\
\hline $\mathbf{4}$ & 785000 & 1921 \\
\hline $\mathbf{5}$ & 785000 & 2886 \\
\hline $\mathbf{6}$ & 785000 & 4111 \\
\hline $\mathbf{7}$ & 785000 & 5850 \\
\hline $\mathbf{8}$ & 785000 & 7622 \\
\hline $\mathbf{9}$ & 785000 & 9718 \\
\hline $\mathbf{1 0}$ & 785000 & 11384 \\
\hline
\end{tabular}

Cage occupied zone average relative humidity (\%) distribution

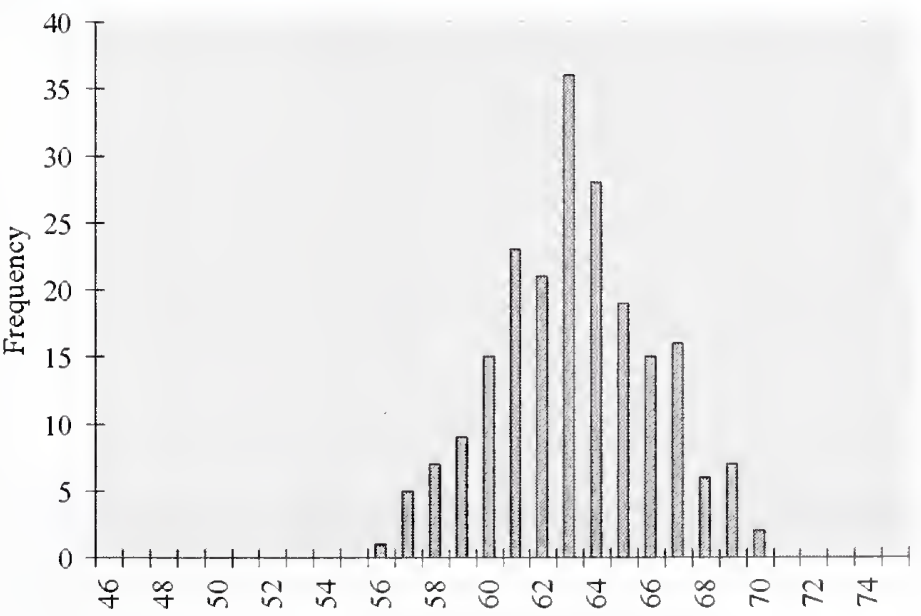


Casename Case 66

\section{Description}

Supply

Configuration

Low Ind

Change

Station

$\mathrm{ON}$ alt design

Room

$\mathrm{ACH}$

5

Cage

Condition

Top On

$\begin{array}{lllll}\begin{array}{l}\text { Supply Discharge } \\ \text { Temperature }\left({ }^{\circ} \mathrm{C}\right)\end{array} & \begin{array}{l}\text { Supply Discharge } \\ \text { RH }\end{array} & \begin{array}{l}\text { Exhaust } \\ \text { Configuration }\end{array} & \begin{array}{l}\text { Exhaust } \\ \text { Temperature }\left({ }^{\circ} \mathrm{C}\right)\end{array} & \begin{array}{l}\text { Exh } \\ \text { RH }\end{array} \\ & 89 \% & \text { Low } & 22 & 50 \% \\ \text { Rack } & \text { Rack } & \text { Number of } & \text { Total mass of } & \text { Room } \\ \text { Orientation } & \text { Density } & \text { Mice in Room } & \text { Mice in Room } & \text { Pressurisation } \\ \text { Perp } & \text { Double } & 2100 & 42000 \mathrm{gr} & \text { neg 100cfm }\end{array}$

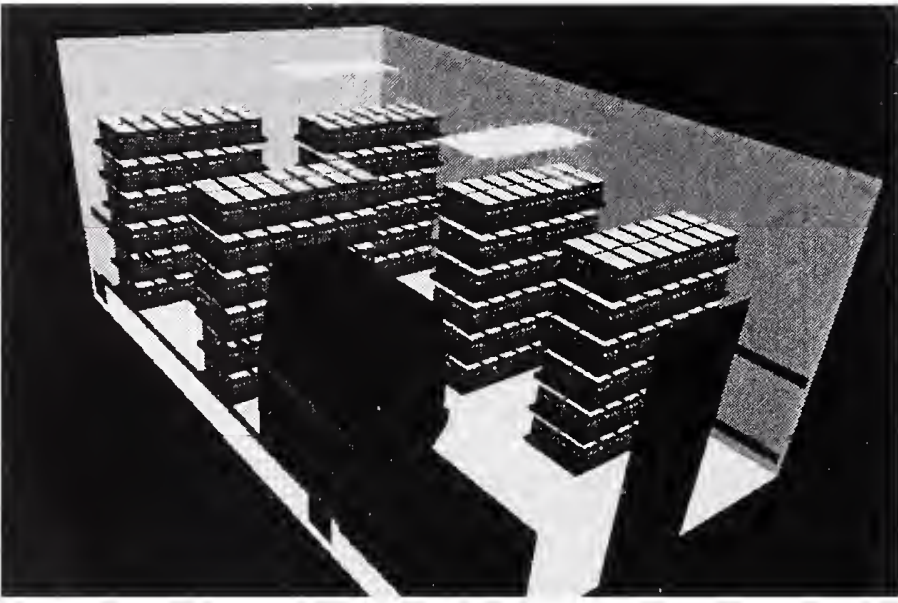

Analysis Results

Cage Occupied Zone

\begin{tabular}{|l|cc|c|c|}
\cline { 2 - 5 } \multicolumn{1}{c|}{} & \multicolumn{2}{c|}{ Temperature } & $\mathrm{CO}_{2}$ & RH \\
\cline { 2 - 5 } \multicolumn{1}{c|}{} & ${ }^{\circ} \mathrm{C}$ & ${ }^{\circ}$ & $(\mathbf{p p m})$ & \\
\hline Mean & 20.61 & 69.09 & 2318 & $52.96 \%$ \\
\hline S.D. & 0.61 & 1.09 & 529 & $4.37 \%$ \\
\hline Max. & 21.89 & 71.40 & 3468 & $61.72 \%$ \\
\hline
\end{tabular}

Cage Occupied Zone NH3 (ppm)

\begin{tabular}{|l|c|c|c|c|c|c|c|c|c|c|}
\hline Day & $\mathbf{1}$ & $\mathbf{2}$ & $\mathbf{3}$ & $\mathbf{4}$ & $\mathbf{5}$ & $\mathbf{6}$ & $\mathbf{7}$ & $\mathbf{8}$ & $\mathbf{9}$ & $\mathbf{1 0}$ \\
\hline Mean & 1.21 & 2.35 & 3.62 & 5.21 & 7.33 & 10.17 & 13.91 & 18.77 & 24.92 & 32.58 \\
\hline Max. & 1.80 & 3.51 & 5.41 & 7.80 & 10.97 & 15.21 & 20.82 & 28.08 & 37.30 & 48.76 \\
\hline
\end{tabular}

Room Breathing Zone

\begin{tabular}{|c|c|c|c|c|}
\hline & \multicolumn{2}{|c|}{ Temperature } & $\mathrm{CO}_{2}$ & RH \\
\hline & & ${ }^{\circ} \mathrm{F}$ & (ppm) & \\
\hline Mean & 18.65 & 65.56 & 318 & $64.38 \%$ \\
\hline S.D. & 0.68 & 1.22 & 117 & \\
\hline Max. & 20.41 & 68.75 & 819 & \\
\hline
\end{tabular}

Room Breathing Zone NH3 (ppm)

\begin{tabular}{|l|c|c|c|c|c|c|c|c|c|c|}
\hline Day & $\mathbf{1}$ & $\mathbf{2}$ & $\mathbf{3}$ & $\mathbf{4}$ & $\mathbf{5}$ & $\mathbf{6}$ & $\mathbf{7}$ & $\mathbf{8}$ & 9 & $\mathbf{1 0}$ \\
\hline Mean & 0.17 & 0.32 & 0.50 & 0.72 & 1.01 & 1.40 & 1.91 & 2.58 & 3.42 & 4.47 \\
\hline Max. & 0.43 & 0.83 & 1.28 & 1.84 & 2.59 & 3.59 & 4.91 & 6.63 & 8.81 & 11.51 \\
\hline
\end{tabular}




\section{Histogram Distributions}

Cage occupied zone average temperature $\left({ }^{\circ} \mathrm{C}\right)$ distribution

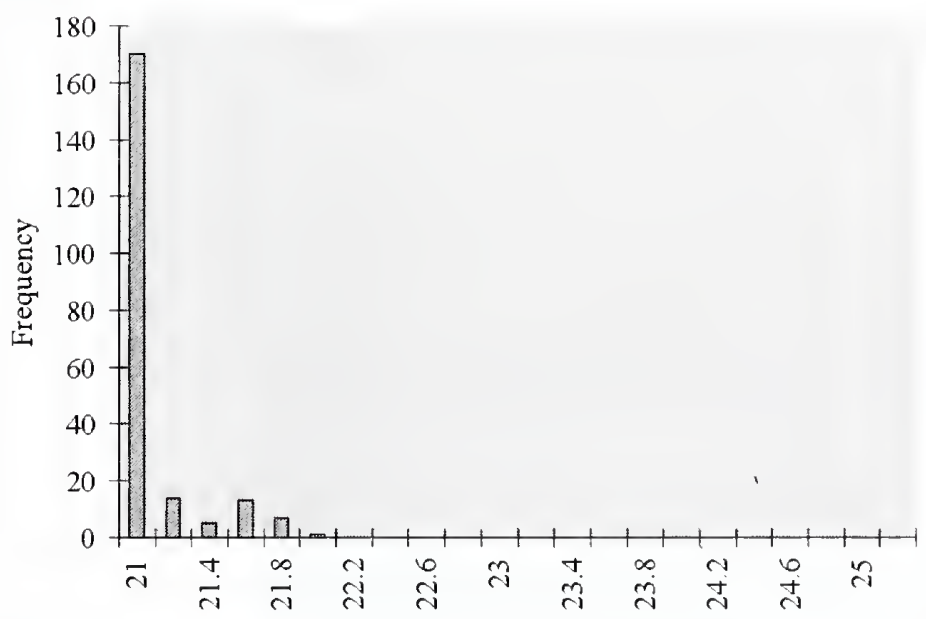

Cage occupied zone average contamination $(\mathrm{kg} / \mathrm{kg})$ distribution

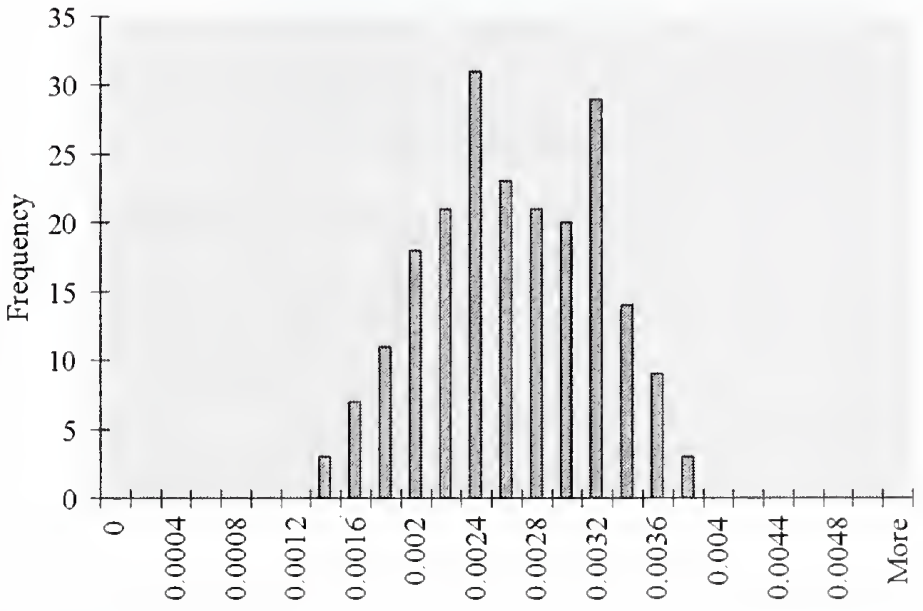

Cage occupied zone average relative humidity (\%) distribution

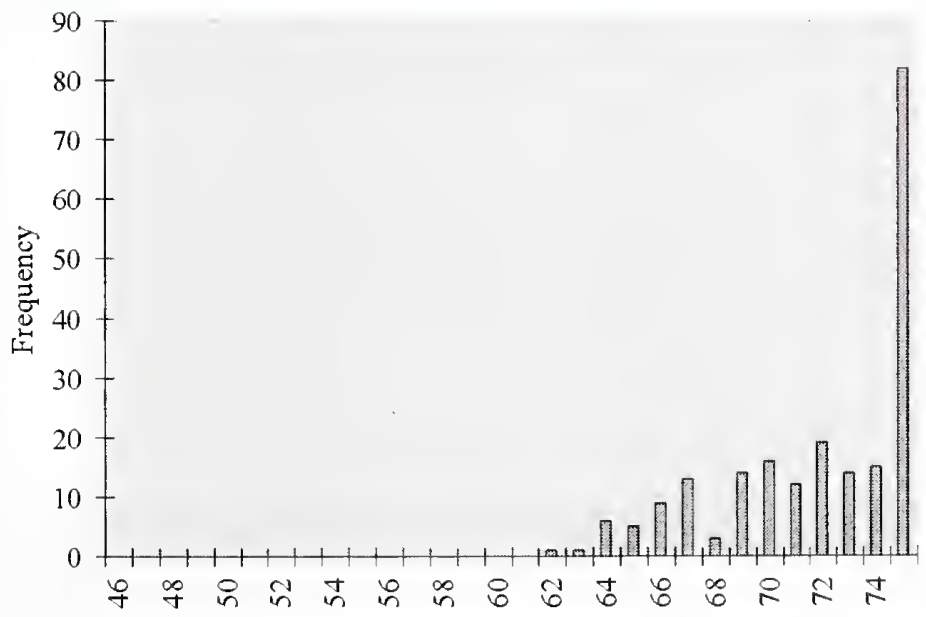

Contamination conversion factors $(\mathrm{kg} / \mathrm{kg} \rightarrow \mathrm{ppm})$

\begin{tabular}{|c|c|c|}
\hline Day & $\mathbf{C O}_{2}$ & $\mathrm{NH}_{3}$ \\
\hline $\mathbf{1}$ & 785000 & 408 \\
\hline $\mathbf{2}$ & 785000 & 795 \\
\hline $\mathbf{3}$ & 785000 & 1225 \\
\hline $\mathbf{4}$ & 785000 & 1766 \\
\hline $\mathbf{5}$ & 785000 & 2483 \\
\hline $\mathbf{6}$ & 785000 & 3443 \\
\hline $\mathbf{7}$ & 785000 & 4712 \\
\hline $\mathbf{8}$ & 785000 & 6356 \\
\hline $\mathbf{9}$ & 785000 & 8442 \\
\hline $\mathbf{1 0}$ & 785000 & 11384 \\
\hline
\end{tabular}


Casename

\section{Case 67}

\section{Description}

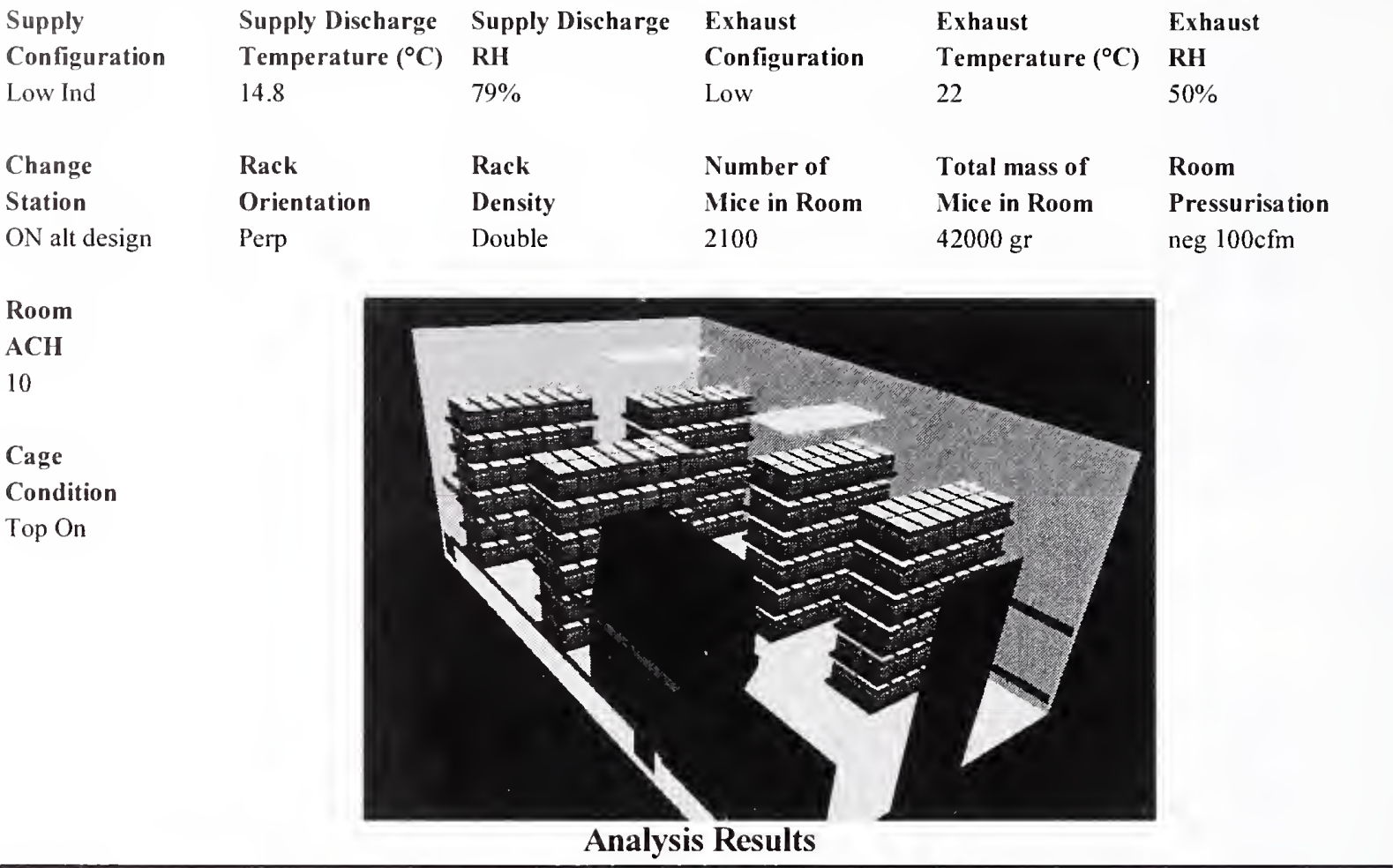

Cage Occupied Zone

\begin{tabular}{|l|cc|c|c|}
\cline { 2 - 5 } \multicolumn{1}{c|}{} & \multicolumn{2}{c|}{ Temperature } & $\mathrm{CO}_{2}$ & RH \\
\cline { 2 - 5 } \multicolumn{1}{c|}{} & ${ }^{\circ} \mathrm{C}$ & ${ }^{2} \mathrm{~F}$ & $(\mathrm{ppm})$ & \\
\hline Mean & 21.87 & 71.37 & 2144 & $65.77 \%$ \\
\hline S.D. & 0.45 & 0.80 & 509 & $3.79 \%$ \\
\hline Max. & 22.90 & 73.22 & 3404 & $72.36 \%$ \\
\hline
\end{tabular}

Cage Occupied Zone NH3 (ppm)

\begin{tabular}{|l|c|c|c|c|c|c|c|c|c|c|}
\hline Day & $\mathbf{1}$ & $\mathbf{2}$ & $\mathbf{3}$ & $\mathbf{4}$ & $\mathbf{5}$ & $\mathbf{6}$ & $\mathbf{7}$ & $\mathbf{8}$ & $\mathbf{9}$ & $\mathbf{1 0}$ \\
\hline Mean & 1.14 & 2.17 & 3.34 & 6.03 & 9.92 & 14.61 & 21.75 & 27.23 & 33.01 & 36.49 \\
\hline Max. & 1.81 & 3.45 & 5.31 & 9.58 & 15.76 & 23.20 & 34.53 & 43.24 & 52.41 & 57.95 \\
\hline
\end{tabular}

Room Breathing Zone

\begin{tabular}{|l|cc|c|c|}
\cline { 2 - 5 } \multicolumn{1}{c|}{} & \multicolumn{2}{c|}{ Temperature } & $\mathrm{CO}_{2}$ & RH \\
\cline { 2 - 5 } \multicolumn{1}{c|}{} & ${ }^{\circ} \mathrm{C}$ & ${ }^{\circ} \mathrm{F}$ & $(\mathbf{p p m})$ & \\
\hline Mean & 20.19 & 68.35 & 201 & $57.23 \%$ \\
\hline S.D. & 0.52 & 0.93 & 90 & \\
\hline Max. & 21.51 & 70.72 & 623 & \\
\hline
\end{tabular}

Room Breathing Zone NH3 (ppm)

\begin{tabular}{|l|c|c|c|c|c|c|c|c|c|c|}
\hline Day & $\mathbf{1}$ & $\mathbf{2}$ & $\mathbf{3}$ & $\mathbf{4}$ & $\mathbf{5}$ & $\mathbf{6}$ & $\mathbf{7}$ & $\mathbf{8}$ & $\mathbf{9}$ & $\mathbf{1 0}$ \\
\hline Mean & 0.11 & 0.20 & 0.31 & 0.57 & 0.93 & 1.37 & 2.04 & 2.55 & 3.10 & 3.42 \\
\hline Max. & 0.33 & 0.63 & 0.97 & 1.75 & 2.88 & 4.25 & 6.32 & 7.92 & 9.60 & 10.61 \\
\hline
\end{tabular}




\section{Histogram Distributions}

Cage occupied zone average temperature $\left({ }^{\circ} \mathrm{C}\right)$ distribution

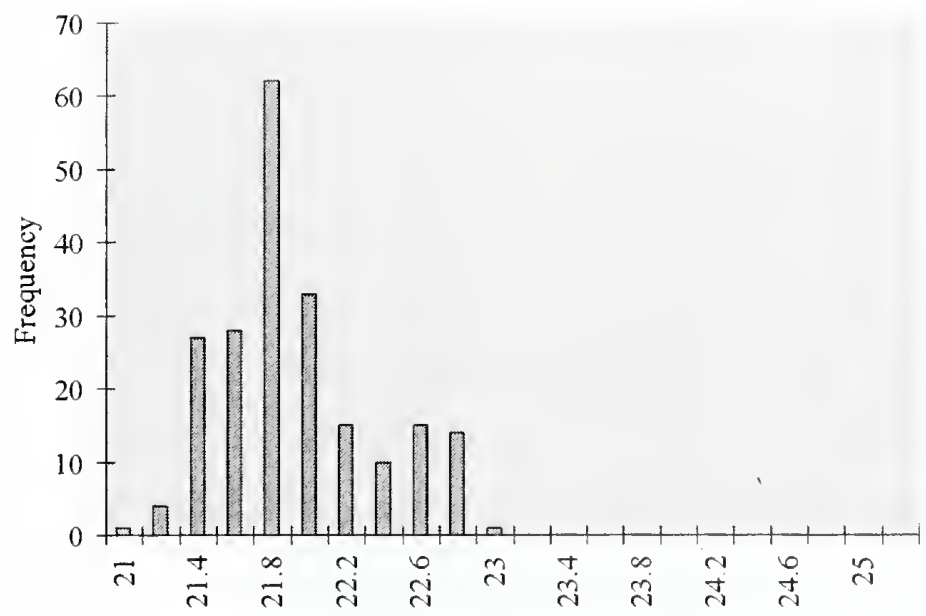

Cage occupied zone average contamination $(\mathrm{kg} / \mathrm{kg})$ distribution

Contamination conversion factors $(\mathrm{kg} / \mathrm{kg} \rightarrow \mathrm{ppm})$

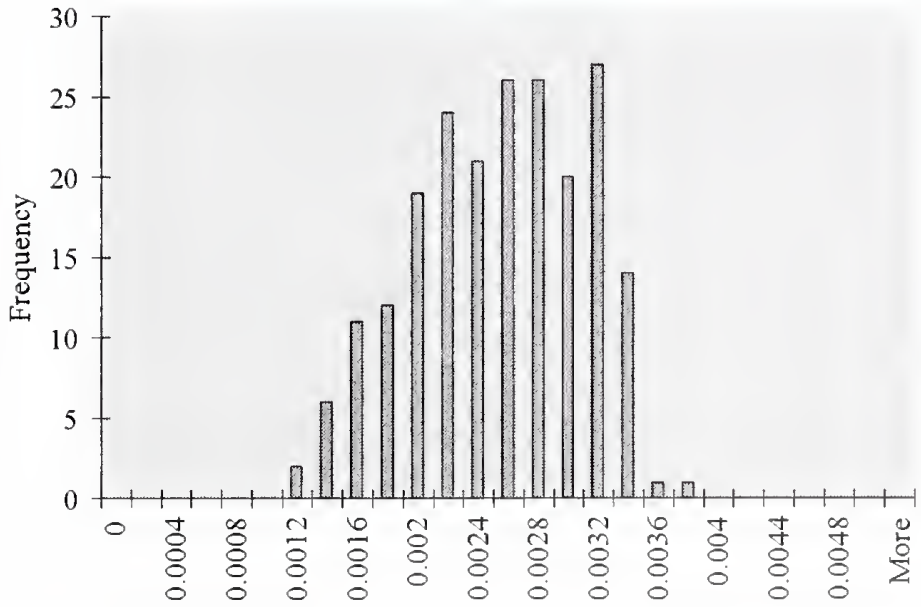

\begin{tabular}{|c|c|c|}
\hline Day & $\mathbf{C O}_{2}$ & $\mathbf{N H}_{3}$ \\
\hline $\mathbf{1}$ & 785000 & 417 \\
\hline $\mathbf{2}$ & 785000 & 795 \\
\hline $\mathbf{3}$ & 785000 & 1225 \\
\hline $\mathbf{4}$ & 785000 & 2209 \\
\hline $\mathbf{5}$ & 785000 & 3633 \\
\hline $\mathbf{6}$ & 785000 & 5350 \\
\hline $\mathbf{7}$ & 785000 & 7963 \\
\hline $\mathbf{8}$ & 785000 & 9970 \\
\hline $\mathbf{9}$ & 785000 & 12087 \\
\hline $\mathbf{1 0}$ & 785000 & 11384 \\
\hline
\end{tabular}

Cage occupied zone average relative humidity (\%) distribution

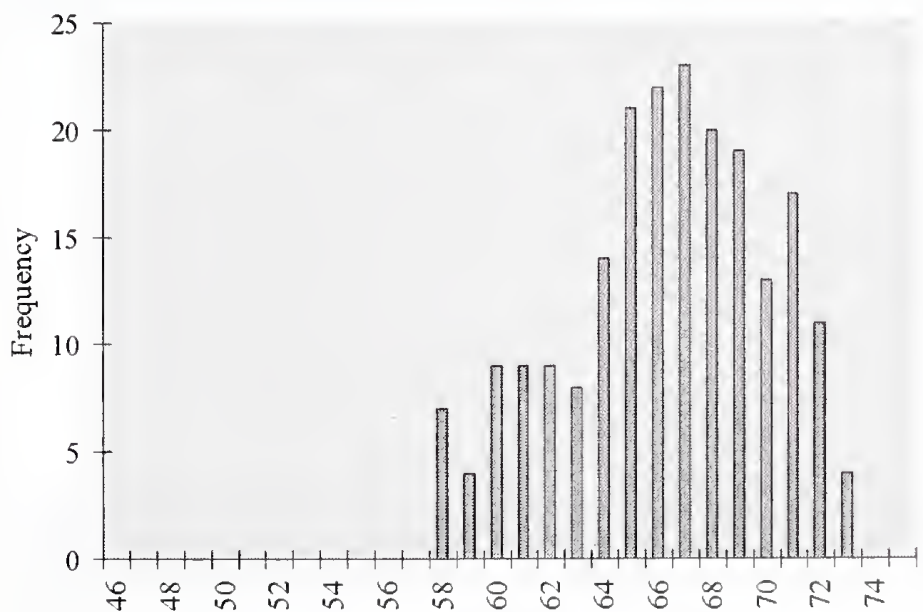


Casename

Case 68

Description

\begin{tabular}{|c|c|c|c|c|c|}
\hline $\begin{array}{l}\text { Supply } \\
\text { Configuration }\end{array}$ & $\begin{array}{l}\text { Supply Discharge } \\
\text { Temperature }\left({ }^{\circ} \mathrm{C}\right)\end{array}$ & $\begin{array}{l}\text { Supply Discharge } \\
\text { RH }\end{array}$ & $\begin{array}{l}\text { Exhaust } \\
\text { Configuration }\end{array}$ & $\begin{array}{l}\text { Exhaust } \\
\text { Temperature }\left({ }^{\circ} \mathrm{C}\right)\end{array}$ & $\begin{array}{l}\text { Exhaust } \\
\text { RH }\end{array}$ \\
\hline Low ind & 18.9 & $60 \%$ & Low & 22 & $50 \%$ \\
\hline $\begin{array}{l}\text { Change } \\
\text { Station } \\
\text { ON alt design }\end{array}$ & $\begin{array}{l}\text { Rack } \\
\text { Orientation } \\
\text { Perp }\end{array}$ & $\begin{array}{l}\text { Rack } \\
\text { Density } \\
\text { Double }\end{array}$ & $\begin{array}{l}\text { Number of } \\
\text { Mice in Room } \\
2100\end{array}$ & $\begin{array}{l}\text { Total mass of } \\
\text { Mice in Room } \\
42000 \mathrm{gr}\end{array}$ & $\begin{array}{l}\text { Room } \\
\text { Pressurisation } \\
\text { neg } 100 \mathrm{cfm}\end{array}$ \\
\hline
\end{tabular}

\section{Room}

$\mathrm{ACH}$

20

Cage

Condition

Top On

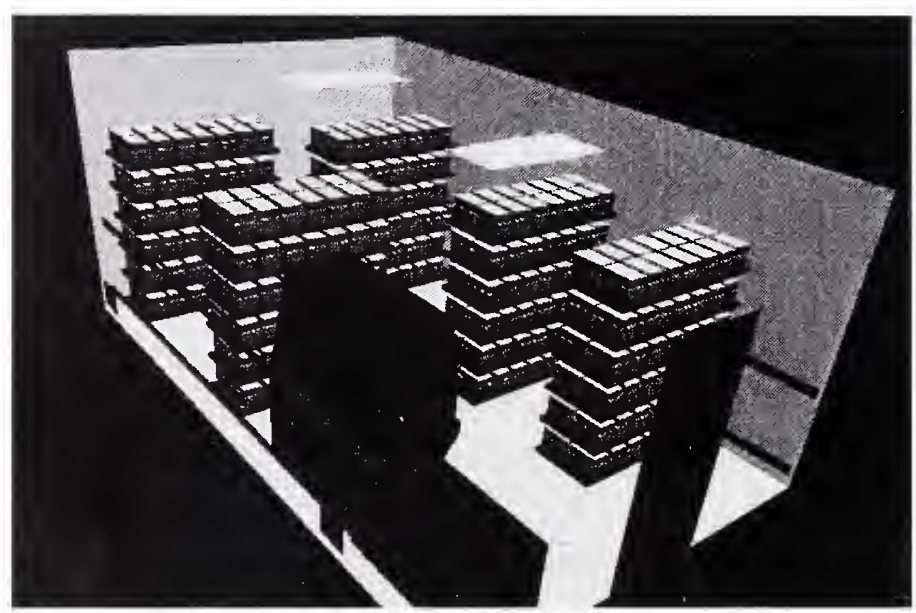

Analysis Results

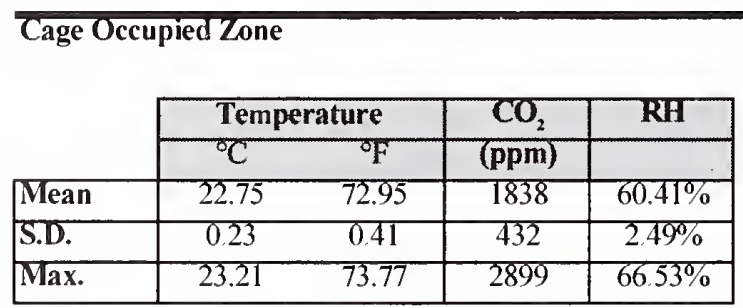

Cage Occupied Zone NH3 (ppm)

\begin{tabular}{|l|c|c|c|c|c|c|c|c|c|c|}
\hline Day & $\mathbf{1}$ & $\mathbf{2}$ & $\mathbf{3}$ & $\mathbf{4}$ & $\mathbf{5}$ & $\mathbf{6}$ & $\mathbf{7}$ & $\mathbf{8}$ & $\mathbf{9}$ & $\mathbf{1 0}$ \\
\hline Mean & 0.96 & 1.86 & 2.87 & 4.13 & 5.81 & 8.06 & 11.03 & 14.89 & 19.77 & 25.84 \\
\hline Max. & 1.51 & 2.93 & 4.52 & 6.52 & 9.17 & 12.71 & 17.40 & 23.47 & 31.17 & 40.75 \\
\hline
\end{tabular}

Room Breathing Zone

\begin{tabular}{|l|cc|c|c|}
\cline { 2 - 5 } \multicolumn{1}{c|}{} & \multicolumn{2}{c|}{ Temperature } & $\mathrm{CO}_{2}$ & $\mathrm{RH}$ \\
\cline { 2 - 5 } \multicolumn{1}{c|}{} & ${ }^{\circ} \mathrm{C}$ & ${ }^{\circ}$ & $(\mathrm{ppm})$ & \\
\hline Mean & 21.23 & 70.22 & 93 & $52.65 \%$ \\
\hline S.D. & 0.31 & 0.55 & 43 & \\
\hline Max. & 22.20 & 71.95 & 262 & \\
\hline
\end{tabular}

Room Breathing Zone NH3 (ppm)

\begin{tabular}{|l|c|c|c|c|c|c|c|c|c|c|}
\hline Day & 1 & 2 & 3 & $\mathbf{3}$ & $\mathbf{5}$ & $\mathbf{6}$ & $\mathbf{7}$ & $\mathbf{8}$ & $\mathbf{9}$ & $\mathbf{1 0}$ \\
\hline Mean & 0.05 & 0.09 & 0.15 & 0.21 & 0.29 & 0.41 & 0.56 & 0.75 & 1.00 & 1.31 \\
\hline Max. & 0.14 & 0.26 & 0.41 & 0.59 & 0.83 & 1.15 & 1.57 & 2.12 & 2.81 & 3.68 \\
\hline
\end{tabular}




\section{Histogram Distributions}

Cage occupied zone average temperature $\left({ }^{\circ} \mathrm{C}\right)$ distribution

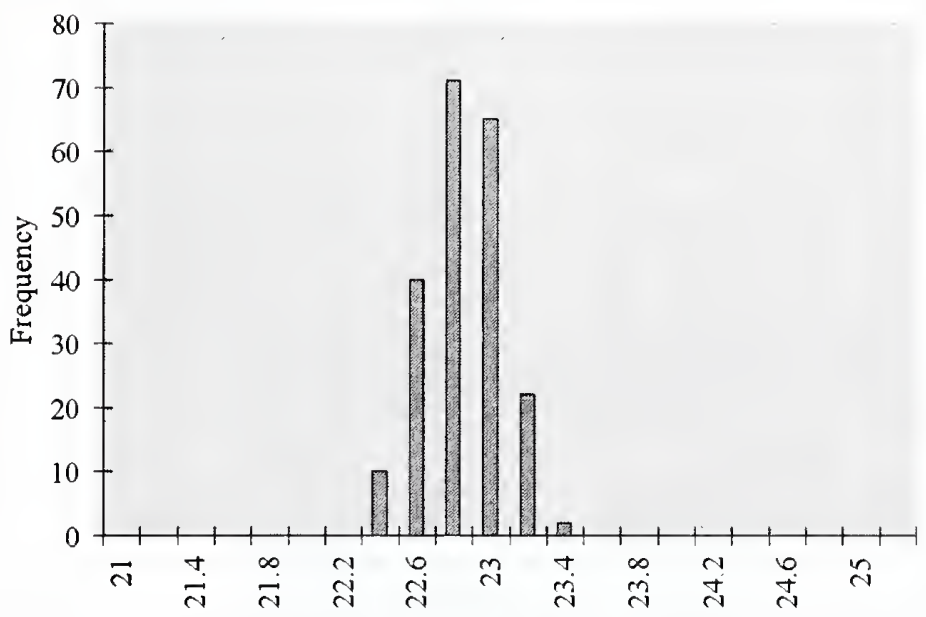

Cage occupied zone average contamination $(\mathrm{kg} / \mathrm{kg})$ distribution

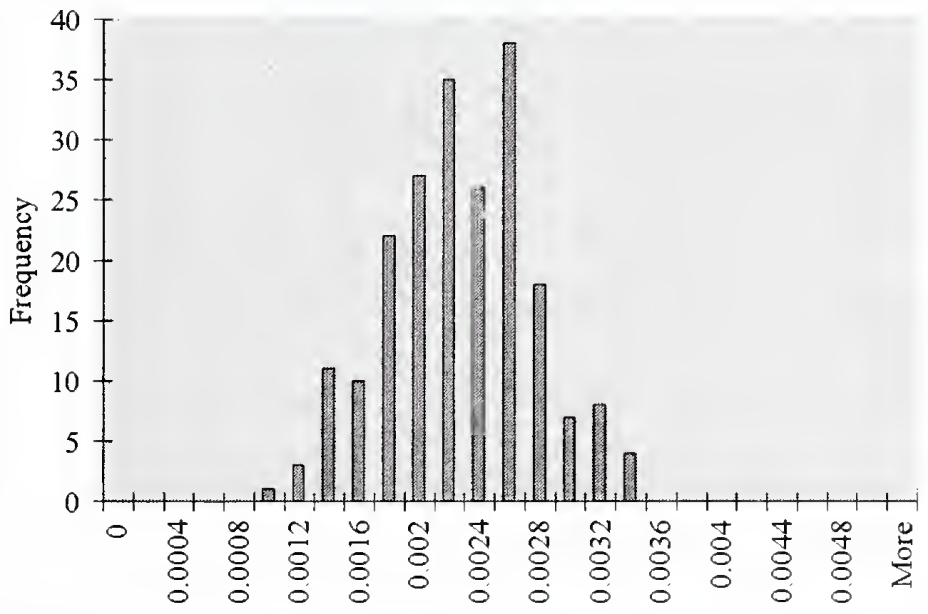

Cage occupied zone average relative humidity (\%) distribution

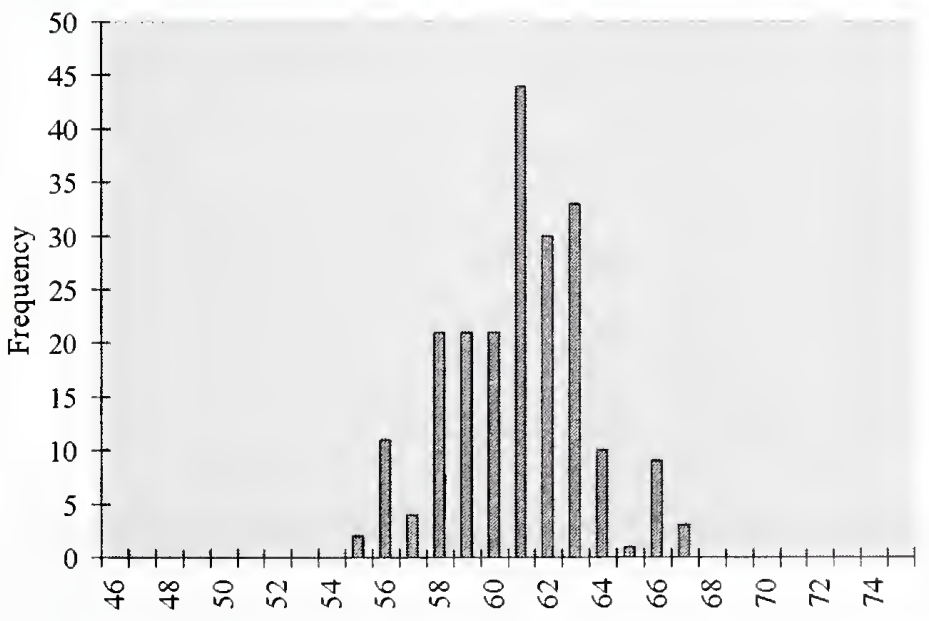

Contamination conversion factors $(\mathrm{kg} / \mathrm{kg} \rightarrow \mathrm{ppm})$

\begin{tabular}{|c|c|c|}
\hline Day & $\mathbf{C O}_{2}$ & $\mathbf{N H}_{3}$ \\
\hline $\mathbf{1}$ & 785000 & 408 \\
\hline $\mathbf{2}$ & 785000 & 795 \\
\hline $\mathbf{3}$ & 785000 & 1225 \\
\hline $\mathbf{4}$ & 785000 & 1766 \\
\hline $\mathbf{5}$ & 785000 & 2483 \\
\hline $\mathbf{6}$ & 785000 & 3443 \\
\hline $\mathbf{7}$ & 785000 & 4712 \\
\hline $\mathbf{8}$ & 785000 & 6356 \\
\hline $\mathbf{9}$ & 785000 & 8442 \\
\hline $\mathbf{1 0}$ & 785000 & 11384 \\
\hline
\end{tabular}


Casename

Case 69

Description

\begin{tabular}{|c|c|c|c|c|c|}
\hline Supply & Supply Discharge & Supply Discharge & Exhaust & Exhaust & Exhaust \\
\hline Configuration & Temperature $\left({ }^{\circ} \mathrm{C}\right)$ & RH & Configuration & Temperature $\left({ }^{\circ} \mathrm{C}\right)$ & RH \\
\hline Radial & 188 & $61 \%$ & High $\times 4$ / Low $\times 4$ & 22 & $50 \%$ \\
\hline Change & Rack & Rack & Number of & Total mass of & Room \\
\hline Station & Orientation & Density & Mice in Room & Mice in Room & Pressurisation \\
\hline ON & On wall & Single & 1050 & $21000 \mathrm{gr}$ & neg $100 \mathrm{cfm}$ \\
\hline
\end{tabular}

\section{Room}

$\mathrm{ACH}$

15

Cage

Condition

Top On

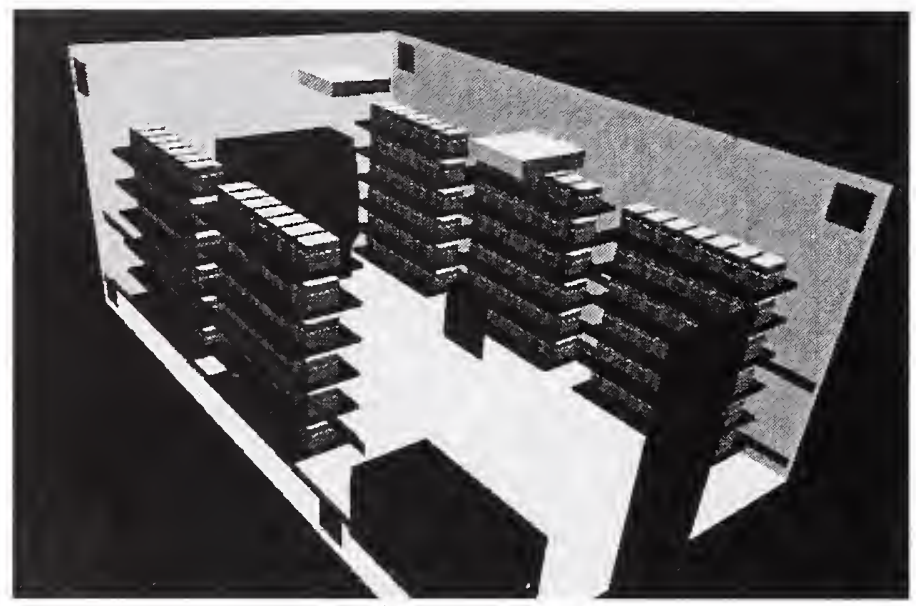

Analysis Results

Cage Occupied Zone

\begin{tabular}{|l|cc|c|c|}
\cline { 2 - 5 } \multicolumn{1}{c|}{} & \multicolumn{2}{c|}{ Temperature } & $\mathrm{CO}_{2}$ & RH \\
\cline { 2 - 5 } \multicolumn{1}{c|}{} & ${ }^{\circ} \mathrm{C}$ & ${ }^{\circ} \mathrm{F}$ & $(\mathbf{p p m})$ & \\
\hline Mean & 22.73 & 72.91 & 1750 & $60.88 \%$ \\
\hline S.D. & 0.37 & 0.67 & 328 & $2.88 \%$ \\
\hline Max. & 23.85 & 74.93 & 2582 & $66.91 \%$ \\
\hline
\end{tabular}

Cage Occupied Zone NH3 (ppm)

\begin{tabular}{|l|c|c|c|c|c|c|c|c|c|c|}
\hline Day & $\mathbf{1}$ & $\mathbf{2}$ & $\mathbf{3}$ & $\mathbf{4}$ & $\mathbf{5}$ & $\mathbf{6}$ & $\mathbf{7}$ & $\mathbf{8}$ & $\mathbf{9}$ & $\mathbf{1 0}$ \\
\hline Mean & 0.91 & $\mathbf{1 . 7 7}$ & 2.73 & 3.94 & 5.54 & 7.68 & 10.51 & 14.17 & 18.82 & 24.61 \\
\hline Max. & 1.34 & 2.61 & 4.03 & 5.81 & 8.17 & 11.33 & 15.50 & 20.91 & 27.77 & 36.30 \\
\hline
\end{tabular}

Room Breathing Zone

\begin{tabular}{|l|cc|c|c|}
\cline { 2 - 5 } \multicolumn{1}{c|}{} & \multicolumn{2}{c|}{ Temperature } & $\mathrm{CO}_{2}$ & RH \\
\cline { 2 - 5 } \multicolumn{1}{c|}{} & ${ }^{\circ} \mathrm{C}$ & ${ }^{\circ} \mathrm{F}$ & $(\mathrm{ppm})$ & \\
\hline Mean & 21.25 & 70.25 & 51 & $52.23 \%$ \\
\hline S.D. & 0.25 & 0.44 & 35 & \\
\hline Max. & 22.03 & 71.66 & 260 & \\
\hline
\end{tabular}

Room Breathing Zone NH3 (ppm)

\begin{tabular}{|l|c|c|c|c|c|c|c|c|c|c|}
\hline Day & 1 & 2 & 3 & 4 & 5 & 6 & 7 & 8 & 9 & 10 \\
\hline Mean & 0.03 & 0.05 & 0.08 & 0.12 & 0.16 & 0.22 & 0.31 & 0.41 & 0.55 & 0.72 \\
\hline Max. & 0.14 & 0.26 & 0.41 & 0.59 & 0.82 & 1.14 & 1.56 & 2.11 & 2.80 & 3.66 \\
\hline
\end{tabular}




\section{Histogram Distributions}

Cage occupied zone average temperature $\left({ }^{\circ} \mathrm{C}\right)$ distribution

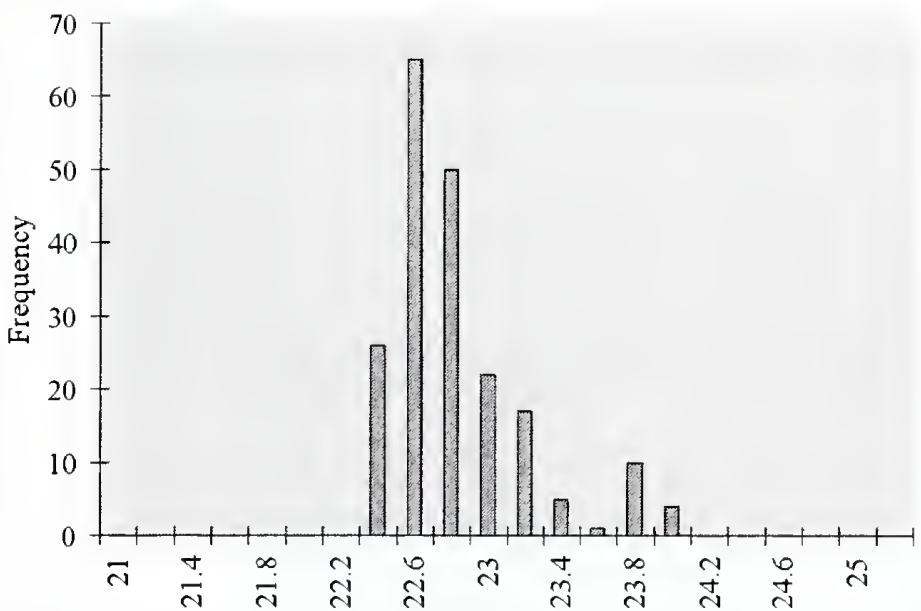

Cage occupied zone average contamination $(\mathrm{kg} / \mathrm{kg})$ distribution

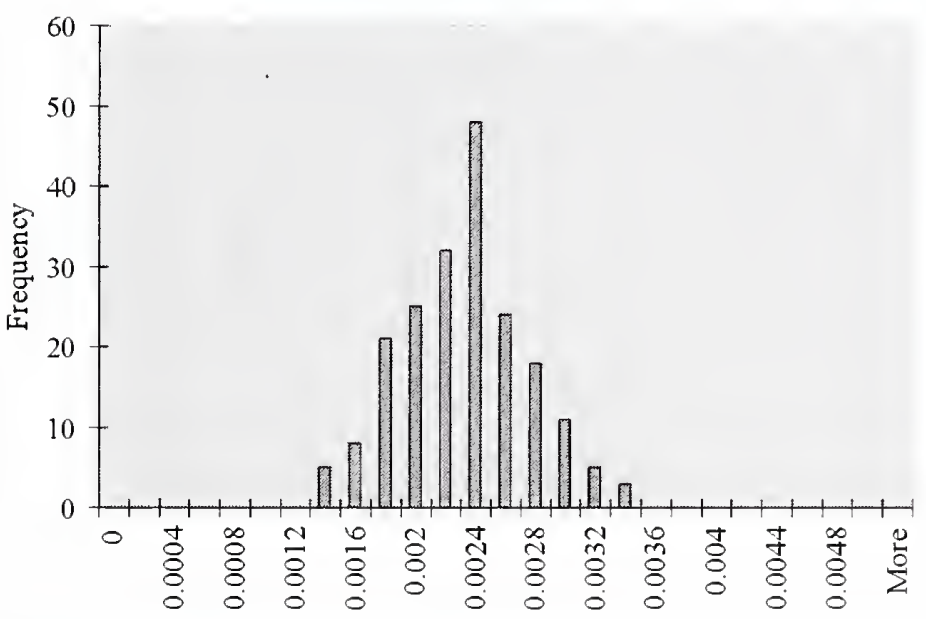

Cage occupied zone average relative humidity (\%) distribution

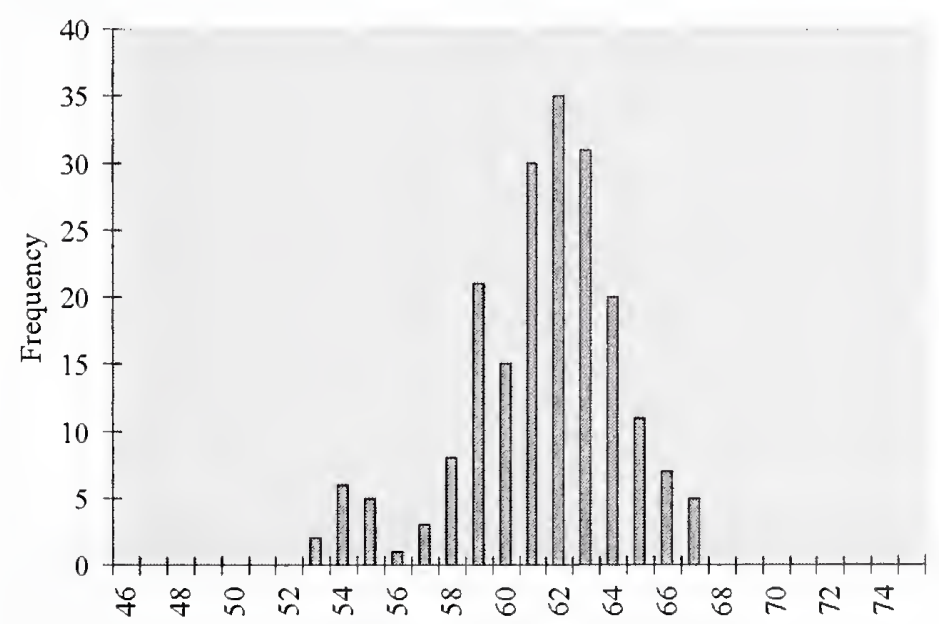

Contamination conversion factors $(\mathrm{kg} / \mathrm{kg} \rightarrow \mathrm{ppm})$

\begin{tabular}{|c|c|c|}
\hline Day & $\mathrm{CO}_{2}$ & $\mathrm{NH}_{\mathbf{3}}$ \\
\hline $\mathbf{1}$ & 785000 & 408 \\
\hline $\mathbf{2}$ & 785000 & 795 \\
\hline $\mathbf{3}$ & 785000 & 1225 \\
\hline $\mathbf{4}$ & 785000 & 1766 \\
\hline $\mathbf{5}$ & 785000 & 2483 \\
\hline $\mathbf{6}$ & 785000 & 3443 \\
\hline $\mathbf{7}$ & 785000 & 4712 \\
\hline $\mathbf{8}$ & 785000 & 6356 \\
\hline $\mathbf{9}$ & 785000 & 8442 \\
\hline $\mathbf{1 0}$ & 785000 & 11384 \\
\hline
\end{tabular}


Casename Case 70

Description

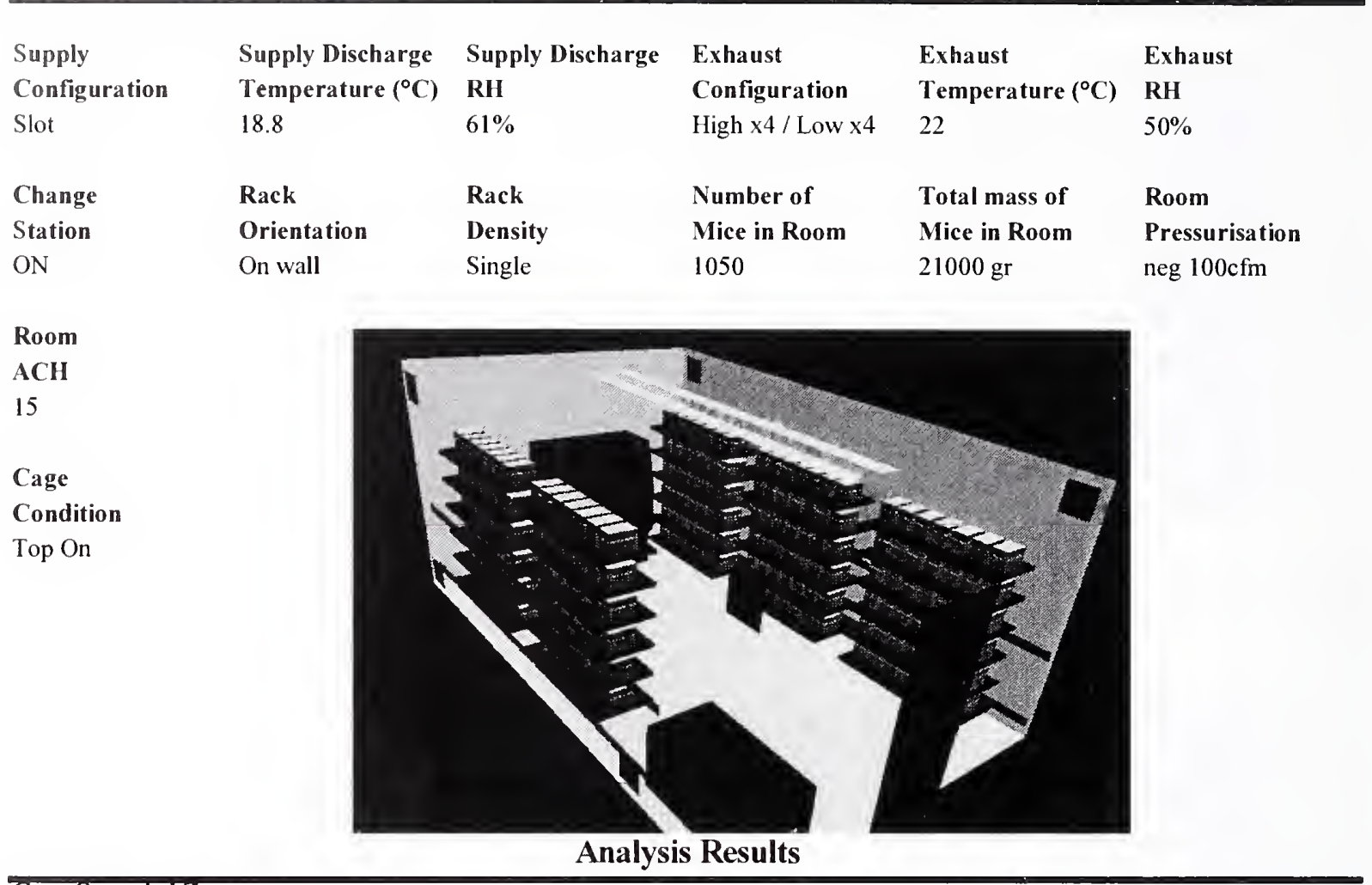

Cage Occupied Zone

\begin{tabular}{|l|cc|c|c|}
\cline { 2 - 5 } \multicolumn{1}{c|}{} & \multicolumn{2}{c|}{ Temperature } & $\mathrm{CO}_{2}$ & RH \\
\cline { 2 - 5 } \multicolumn{1}{c|}{} & ${ }^{\circ} \mathrm{C}$ & ${ }^{\circ} \mathrm{F}$ & $(\mathbf{p p m})$ & \\
\hline Mean & 22.89 & 73.20 & 1955 & $61.79 \%$ \\
\hline S.D. & 0.39 & 0.71 & 371 & $2.32 \%$ \\
\hline Max. & 23.83 & 74.90 & 2741 & $66.48 \%$ \\
\hline
\end{tabular}

Cage Occupied Zone NH3 (ppm)

\begin{tabular}{|l|c|c|c|c|c|c|c|c|c|c|}
\hline Day & $\mathbf{1}$ & $\mathbf{2}$ & $\mathbf{3}$ & $\mathbf{4}$ & $\mathbf{5}$ & $\mathbf{6}$ & $\mathbf{7}$ & $\mathbf{8}$ & $\mathbf{9}$ & $\mathbf{1 0}$ \\
\hline Mean & 1.02 & 1.98 & 3.05 & 4.58 & 6.66 & 9.36 & 13.08 & 17.32 & 22.53 & 28.44 \\
\hline Max. & 1.43 & 2.77 & 4.28 & 6.42 & 9.33 & 13.12 & 18.33 & 24.28 & 31.58 & 39.88 \\
\hline
\end{tabular}

Room Breathing Zone

\begin{tabular}{|l|cc|c|c|}
\cline { 2 - 5 } \multicolumn{1}{c|}{} & \multicolumn{2}{c|}{ Temperature } & $\mathrm{CO}_{2}$ & RH \\
\cline { 2 - 5 } \multicolumn{1}{c|}{} & ${ }^{\circ} \mathrm{C}$ & ${ }^{\circ} \mathrm{F}$ & $(\mathbf{p p m})$ & \\
\hline Mean & 21.45 & 70.60 & 24 & $51.36 \%$ \\
\hline S.D. & 0.37 & 0.67 & 14 & \\
\hline Max. & 22.53 & 72.55 & 122 & \\
\hline
\end{tabular}

Room Breathing Zone NH3 (ppm)

\begin{tabular}{|l|c|c|c|c|c|c|c|c|c|c|}
\hline Day & $\mathbf{1}$ & $\mathbf{2}$ & $\mathbf{3}$ & $\mathbf{4}$ & $\mathbf{5}$ & $\mathbf{6}$ & $\mathbf{7}$ & $\mathbf{8}$ & $\mathbf{9}$ & $\mathbf{1 0}$ \\
\hline Mean & 0.01 & 0.02 & 0.04 & 0.06 & 0.08 & 0.11 & 0.16 & 0.21 & 0.28 & 0.35 \\
\hline Max. & 0.06 & 0.12 & 0.19 & 0.29 & 0.42 & 0.58 & 0.82 & 1.08 & 1.41 & 1.78 \\
\hline
\end{tabular}




\section{Histogram Distributions}

Cage occupied zone average temperature $\left({ }^{\circ} \mathrm{C}\right)$ distribution

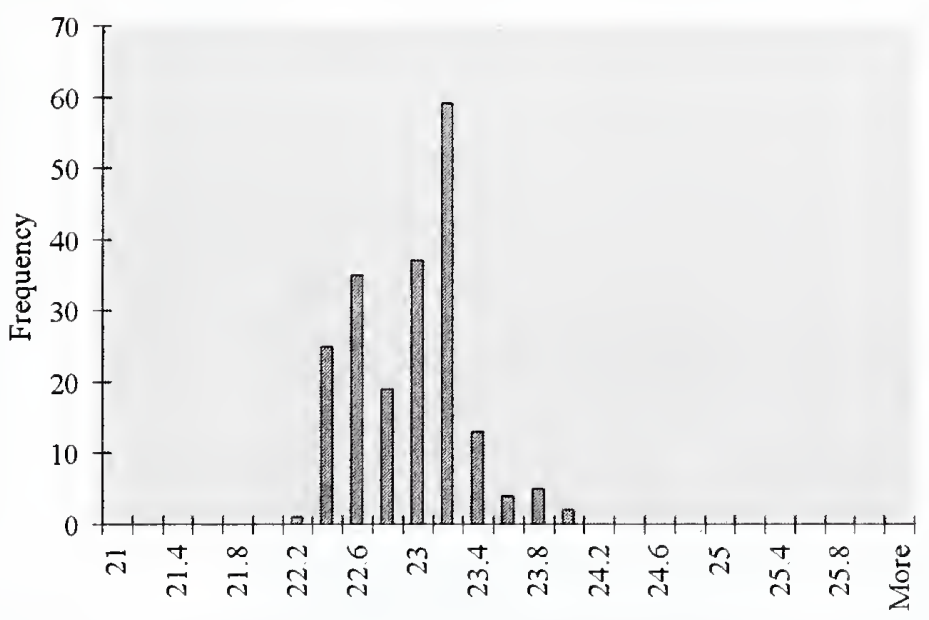

Cage occupied zone average contamination $(\mathrm{kg} / \mathrm{kg})$ distribution

Contamination conversion factors $(\mathrm{kg} / \mathrm{kg} \rightarrow \mathrm{ppm})$

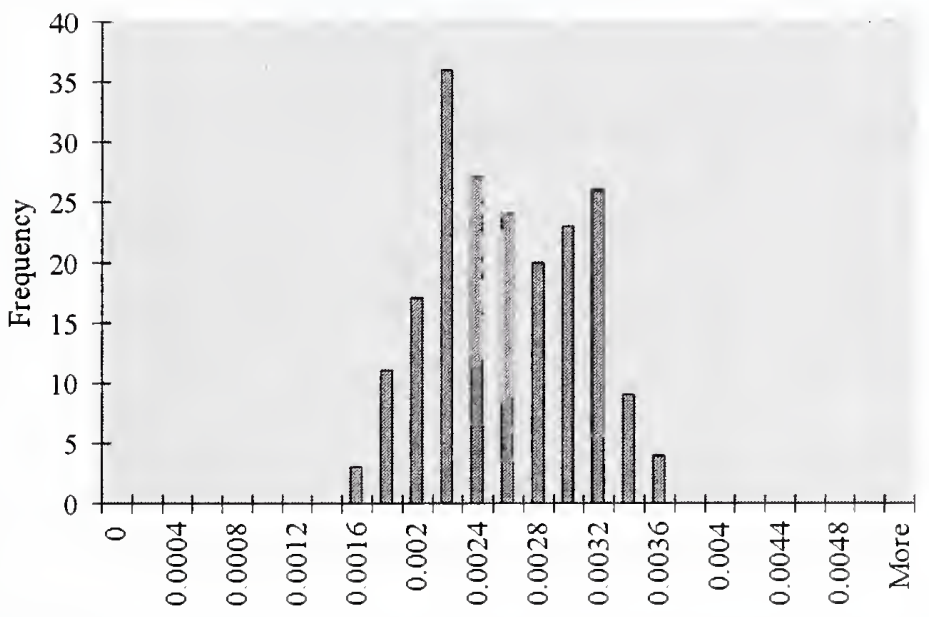

\begin{tabular}{|c|c|c|}
\hline Day & $\mathbf{C O}_{\mathbf{2}}$ & $\mathbf{N H}_{\mathbf{3}}$ \\
\hline $\mathbf{1}$ & 785000 & 410 \\
\hline $\mathbf{2}$ & 785000 & 795 \\
\hline $\mathbf{3}$ & 785000 & 1225 \\
\hline $\mathbf{4}$ & 785000 & 1839 \\
\hline $\mathbf{5}$ & 785000 & 2673 \\
\hline $\mathbf{6}$ & 785000 & 3758 \\
\hline $\mathbf{7}$ & 785000 & 5250 \\
\hline $\mathbf{8}$ & 785000 & 6954 \\
\hline $\mathbf{9}$ & 785000 & 9045 \\
\hline $\mathbf{1 0}$ & 785000 & 11384 \\
\hline
\end{tabular}

Cage occupied zone average relative humidity $(\%)$ distribution

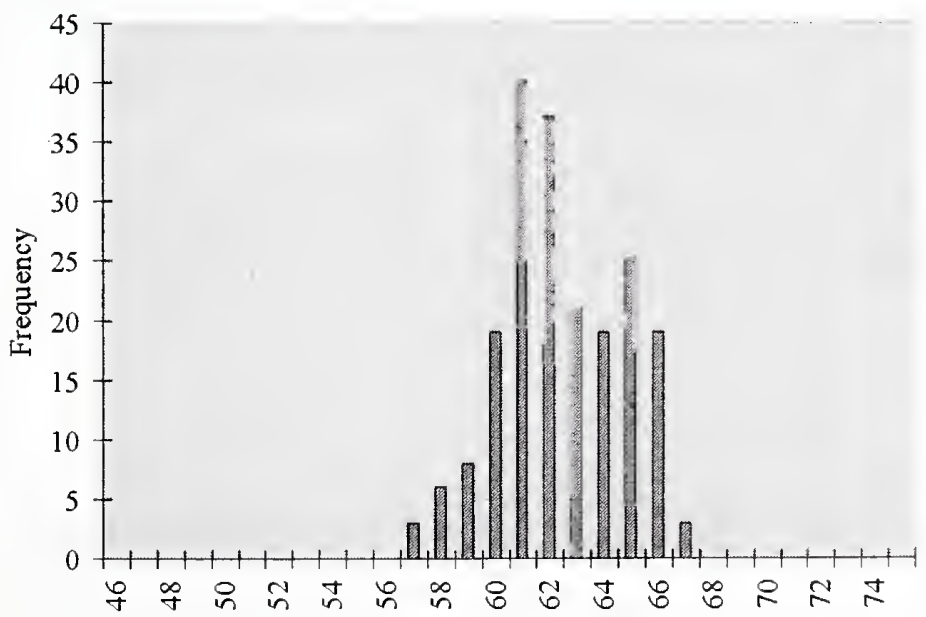




\section{Description}

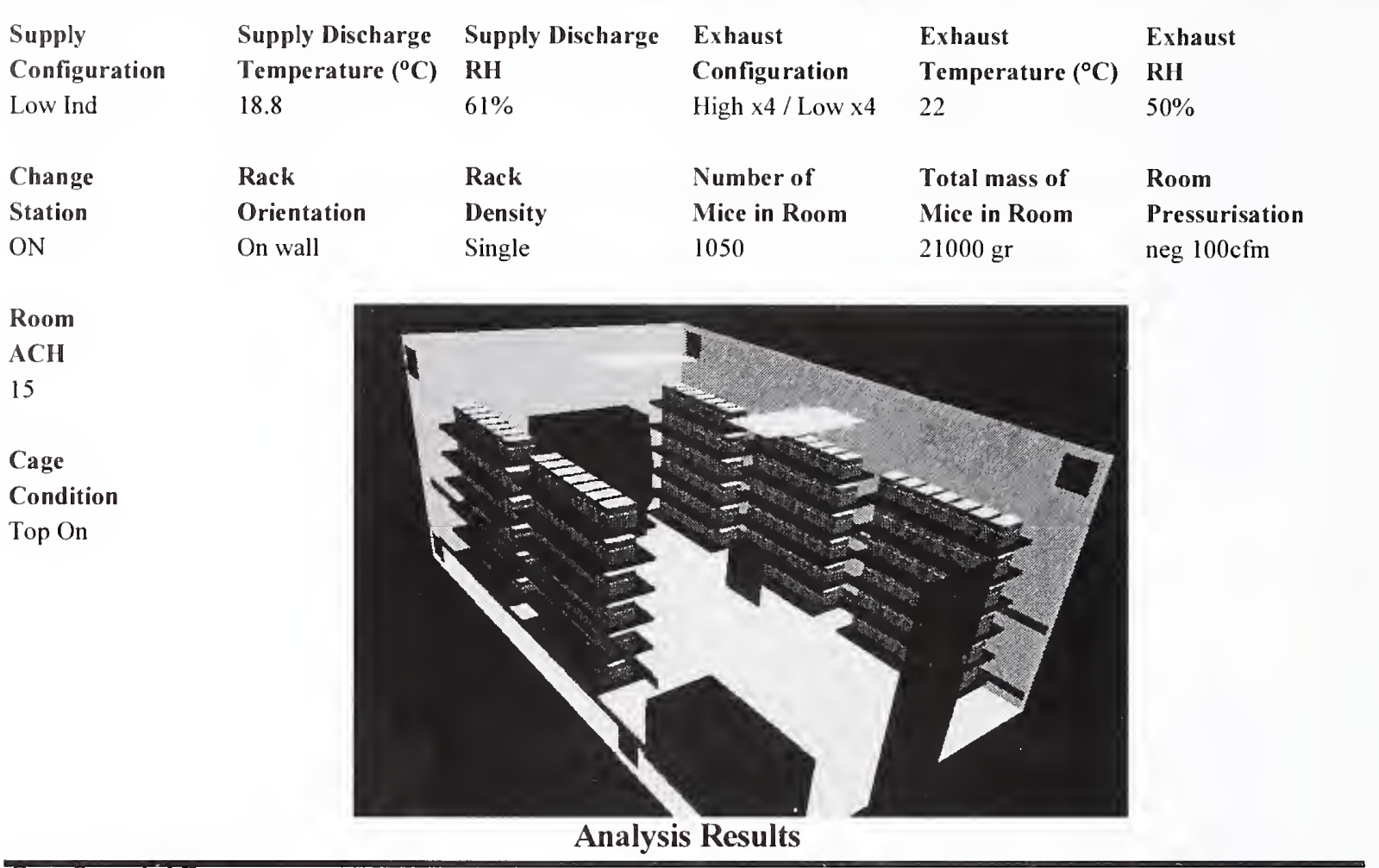

Cage Occupied Zone

\begin{tabular}{|l|cc|c|c|}
\cline { 2 - 5 } \multicolumn{1}{c|}{} & \multicolumn{2}{c|}{ Temperature } & $\mathrm{CO}_{2}$ & RH \\
\cline { 2 - 5 } \multicolumn{1}{c|}{} & ${ }^{\circ} \mathrm{C}$ & ${ }^{\circ} \mathrm{F}$ & $(\mathrm{ppm})$ & \\
\hline Mean & 22.97 & 73.35 & 1968 & $61.59 \%$ \\
\hline S.D. & 0.38 & 0.69 & 339 & $2.42 \%$ \\
\hline Max. & 23.95 & 75.10 & 2683 & $66.10 \%$ \\
\hline
\end{tabular}

Cage Occupied Zone NH3 (ppm)

\begin{tabular}{|l|c|c|c|c|c|c|c|c|c|c|}
\hline Day & $\mathbf{1}$ & $\mathbf{2}$ & $\mathbf{3}$ & $\mathbf{4}$ & $\mathbf{5}$ & $\mathbf{6}$ & $\mathbf{7}$ & $\mathbf{8}$ & $\mathbf{9}$ & $\mathbf{1 0}$ \\
\hline Mean & 1.03 & 1.99 & 3.07 & 4.56 & 6.58 & 9.22 & 12.82 & 17.06 & 22.30 & 28.39 \\
\hline Max. & 1.40 & 2.72 & 4.19 & 6.22 & 8.97 & 12.58 & 17.48 & 23.26 & 30.40 & 38.70 \\
\hline
\end{tabular}

Room Breathing Zone

\begin{tabular}{|l|cc|c|c|}
\cline { 2 - 5 } \multicolumn{1}{c|}{} & \multicolumn{2}{c|}{ Temperature } & $\mathrm{CO}_{2}$ & RH \\
\cline { 2 - 5 } \multicolumn{1}{c|}{} & ${ }^{\circ} \mathrm{C}$ & ${ }^{\circ} \mathrm{F}$ & $(\mathrm{ppm})$ & \\
\hline Mean & 21.56 & 70.81 & 51 & $51.22 \%$ \\
\hline S.D. & 0.33 & 0.59 & 25 & \\
\hline Max. & 22.41 & 72.35 & 305 & \\
\hline
\end{tabular}

Room Breathing Zone NH3 (ppm)

\begin{tabular}{|l|c|c|c|c|c|c|c|c|c|c|}
\hline Day & $\mathbf{1}$ & $\mathbf{2}$ & $\mathbf{3}$ & $\mathbf{4}$ & $\mathbf{5}$ & $\mathbf{6}$ & $\mathbf{7}$ & $\mathbf{8}$ & $\mathbf{9}$ & $\mathbf{1 0}$ \\
\hline Mean & 0.03 & 0.05 & 0.08 & 0.12 & 0.17 & 0.24 & 0.34 & 0.45 & 0.58 & 0.74 \\
\hline Max. & 0.16 & 0.31 & 0.48 & 0.71 & 1.02 & 1.43 & 1.99 & 2.65 & 3.46 & 4.40 \\
\hline
\end{tabular}




\section{Histogram Distributions}

Cage occupied zone average temperature $\left({ }^{\circ} \mathrm{C}\right)$ distribution

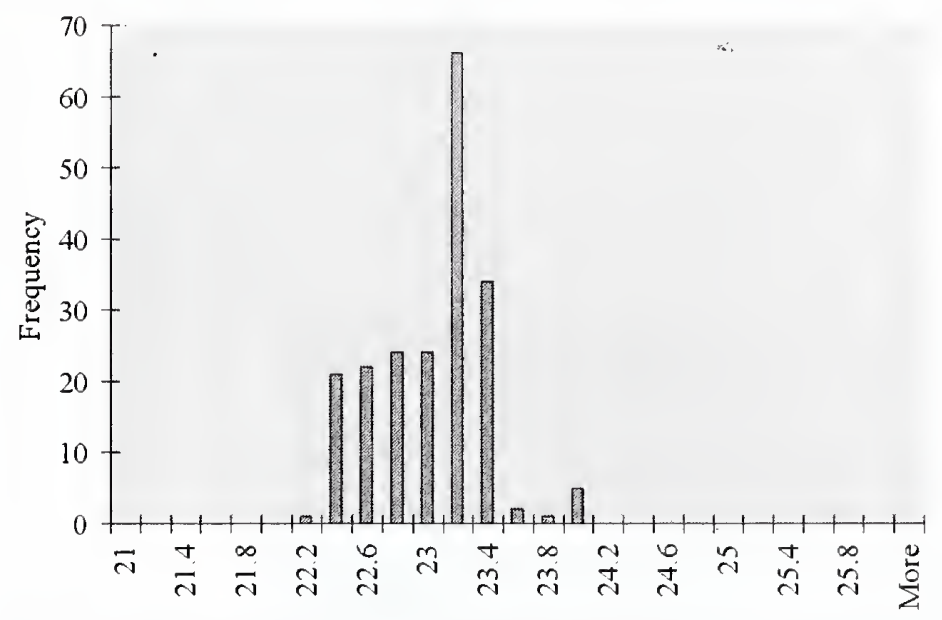

Cage occupied zone average contamination $(\mathrm{kg} / \mathrm{kg})$ distribution

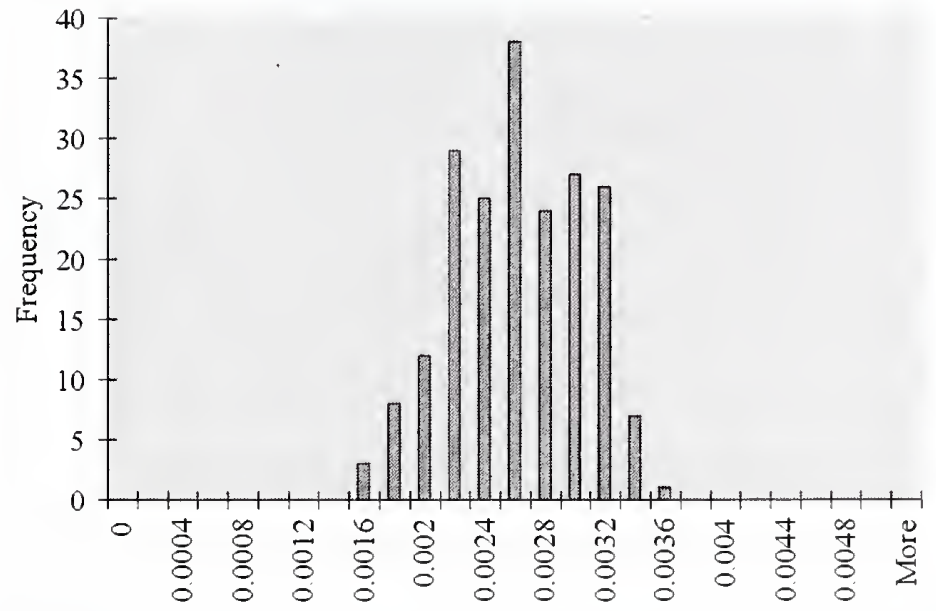

Cage occupied zone average relative humidity (\%) distribution

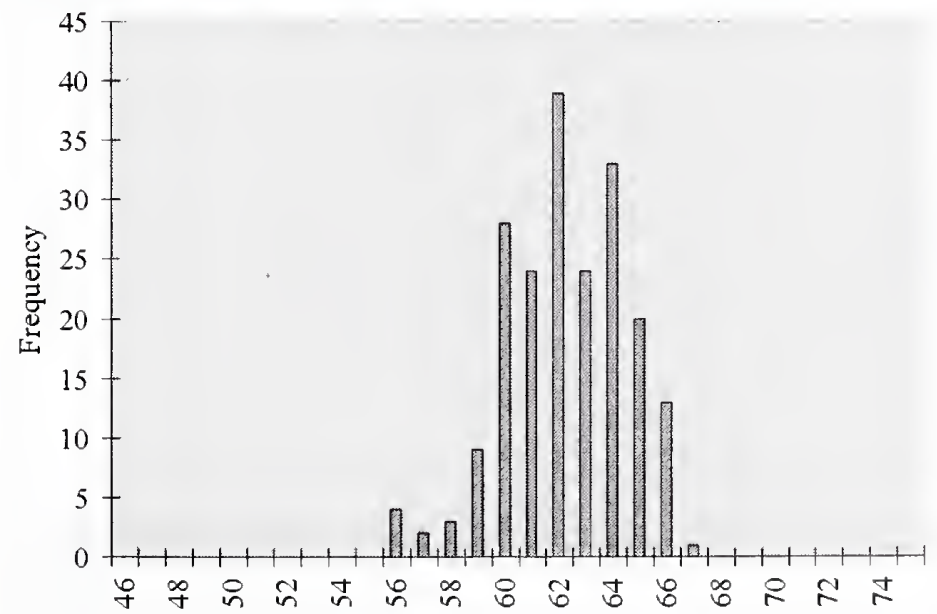

Contamination conversion factors $(\mathrm{kg} / \mathrm{kg} \rightarrow \mathrm{ppm})$

\begin{tabular}{|c|c|c|}
\hline Day & $\mathbf{C O}_{\mathbf{2}}$ & $\mathbf{N H}_{3}$ \\
\hline $\mathbf{1}$ & 785000 & 409 \\
\hline $\mathbf{2}$ & 785000 & 795 \\
\hline $\mathbf{3}$ & 785000 & 1225 \\
\hline $\mathbf{4}$ & 785000 & 1821 \\
\hline $\mathbf{5}$ & 785000 & 2626 \\
\hline $\mathbf{6}$ & 785000 & 3680 \\
\hline $\mathbf{7}$ & 785000 & 5116 \\
\hline $\mathbf{8}$ & 785000 & 6805 \\
\hline $\mathbf{9}$ & 785000 & 8894 \\
\hline $\mathbf{1 0}$ & 785000 & 11384 \\
\hline
\end{tabular}




\section{Description}

Supply

Configuration

Radial

Change

Station

$\mathrm{ON}$

Room

$\mathrm{ACH}$

15

Cage

Condition

Top On
Supply Discharge Supply Discharge

Temperature $\left({ }^{\circ} \mathrm{C}\right)$

18.8

RH

$61 \%$

Rack

Orientation

On wall
Exhaust

Configuration

High $x 4$ / Low $x 2$

Number of

Mice in Room 1050

$\begin{array}{ll}\text { Exhaust } & \text { Exhaust } \\ \text { Temperature }\left({ }^{\circ} \mathrm{C}\right) & \text { RH } \\ 22 & 50 \%\end{array}$

Total mass of Mice in Room $21000 \mathrm{gr}$
Room

Pressurisation neg $100 \mathrm{cfm}$

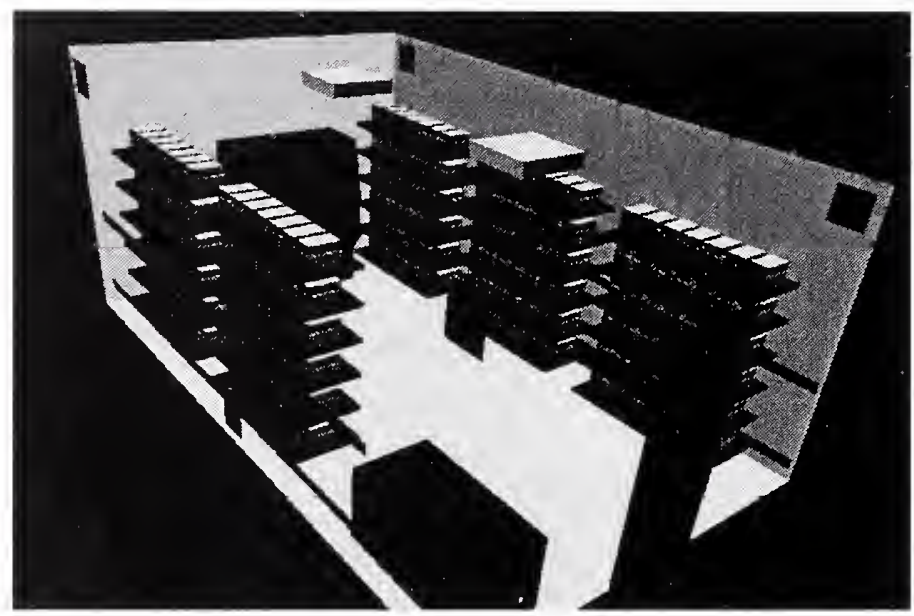

Analysis Results

Cage Occupied Zone

\begin{tabular}{|l|cc|c|c|}
\cline { 2 - 5 } \multicolumn{1}{c|}{} & \multicolumn{2}{c|}{ Temperature } & $\mathbf{C O}_{2}$ & RH \\
\cline { 2 - 5 } \multicolumn{1}{c|}{} & ${ }^{\circ} \mathrm{C}$ & ${ }^{\circ} \mathrm{F}$ & $(\mathbf{p p m})$ & \\
\hline Mean & 22.53 & 72.56 & 1790 & $61.90 \%$ \\
\hline S.D. & 0.31 & 0.55 & 355 & $2.67 \%$ \\
\hline Max. & 23.69 & 74.65 & 2663 & $67.80 \%$ \\
\hline
\end{tabular}

Cage Occupied Zone NH3 (ppm)

\begin{tabular}{|l|c|c|c|c|c|c|c|c|c|c|}
\hline Day & $\mathbf{1}$ & $\mathbf{2}$ & $\mathbf{3}$ & $\mathbf{4}$ & $\mathbf{5}$ & $\mathbf{6}$ & $\mathbf{7}$ & $\mathbf{8}$ & $\mathbf{9}$ & $\mathbf{1 0}$ \\
\hline Mean & 0.93 & $\mathbf{1 . 8 1}$ & 2.79 & 4.22 & 6.15 & 8.67 & 12.14 & 16.04 & 20.81 & 26.16 \\
\hline Max. & 1.39 & 2.69 & 4.15 & 6.27 & 9.16 & 12.90 & 18.06 & 23.87 & 30.96 & 38.92 \\
\hline
\end{tabular}

Room Breathing Zone

\begin{tabular}{|l|cc|c|c|}
\cline { 2 - 5 } \multicolumn{1}{c|}{} & \multicolumn{2}{c|}{ Temperature } & $\mathbf{C O}_{2}$ & RH \\
\cline { 2 - 5 } \multicolumn{1}{c|}{} & ${ }^{\circ} \mathrm{C}$ & \multicolumn{1}{c|}{} & $(\mathbf{p p m})$ & \\
\hline Mean & 21.06 & 69.91 & 48 & $52.83 \%$ \\
\hline S.D. & 0.23 & 0.42 & 29 & \\
\hline Max. & 21.79 & 71.23 & 253 & \\
\hline
\end{tabular}

Room Breathing Zone NH3 (ppm)

\begin{tabular}{|l|c|c|c|c|c|c|c|c|c|c|}
\hline Day & $\mathbf{1}$ & $\mathbf{2}$ & $\mathbf{3}$ & $\mathbf{4}$ & $\mathbf{5}$ & $\mathbf{6}$ & $\mathbf{7}$ & $\mathbf{8}$ & $\mathbf{9}$ & $\mathbf{1 0}$ \\
\hline Mean & 0.03 & 0.05 & 0.07 & 0.11 & 0.17 & 0.23 & 0.33 & 0.43 & 0.56 & 0.70 \\
\hline Max. & 0.13 & 0.26 & 0.40 & 0.60 & 0.87 & 1.23 & 1.72 & 2.27 & 2.95 & 3.70 \\
\hline
\end{tabular}




\section{Histogram Distributions}

Cage occupied zone average temperature $\left({ }^{\circ} \mathrm{C}\right)$ distribution

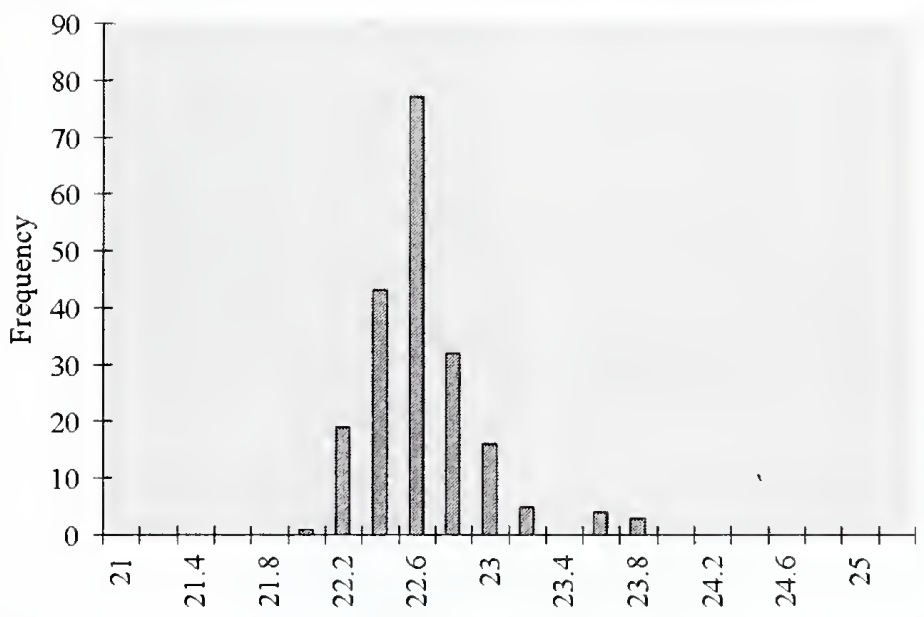

Cage occupied zone average contamination $(\mathrm{kg} / \mathrm{kg})$ distribution

Contamination conversion factors $(\mathrm{kg} / \mathrm{kg} \rightarrow \mathrm{ppm})$

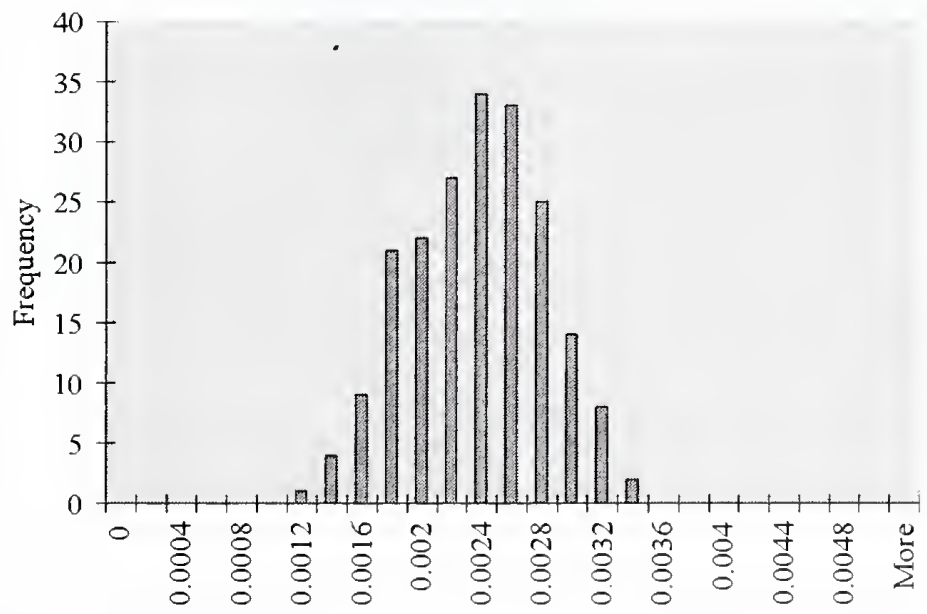

\begin{tabular}{|c|c|c|}
\hline Day & $\mathrm{CO}_{2}$ & $\mathrm{NH}_{3}$ \\
\hline $\mathbf{1}$ & 785000 & 418 \\
\hline $\mathbf{2}$ & 785000 & 795 \\
\hline $\mathbf{3}$ & 785000 & 1225 \\
\hline $\mathbf{4}$ & 785000 & 2303 \\
\hline $\mathbf{5}$ & 785000 & 3876 \\
\hline $\mathbf{6}$ & 785000 & 5752 \\
\hline 7 & 785000 & 8650 \\
\hline $\mathbf{8}$ & 785000 & 10734 \\
\hline $\mathbf{9}$ & 785000 & 12856 \\
\hline $\mathbf{1 0}$ & 785000 & 11384 \\
\hline
\end{tabular}

Cage occupied zone average relative humidity (\%) distribution

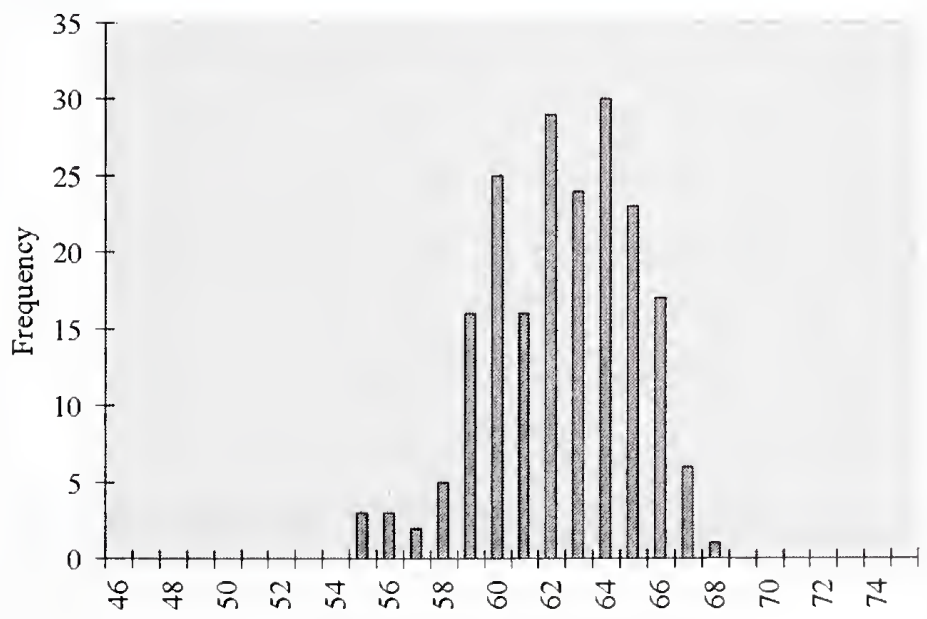


Casename Case 73

\section{Description}

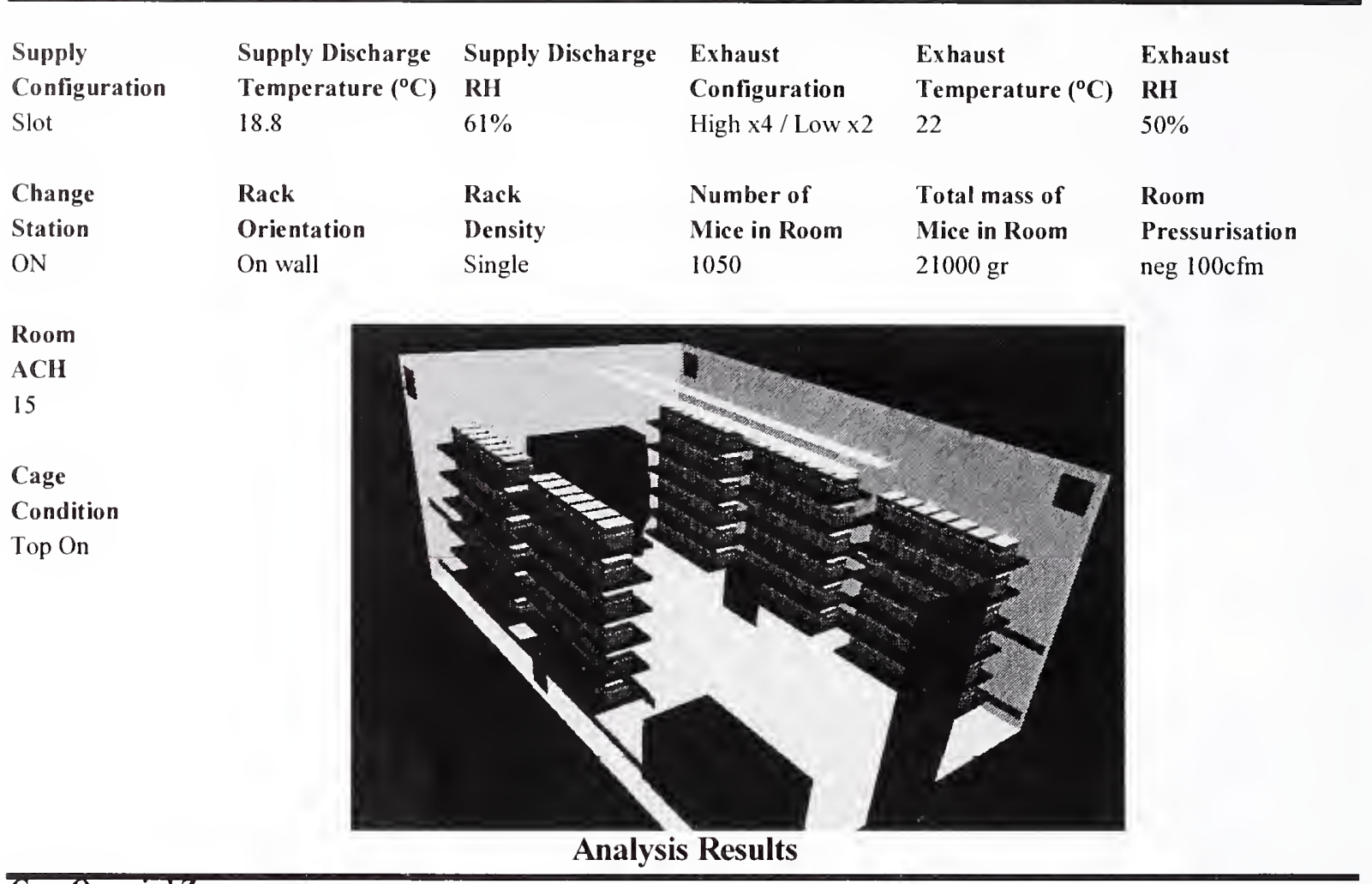

Cage Occupied Zone

\begin{tabular}{|l|cc|c|c|}
\cline { 2 - 5 } \multicolumn{1}{c|}{} & \multicolumn{2}{c|}{ Temperature } & $\mathrm{CO}_{2}$ & RH \\
\cline { 2 - 5 } \multicolumn{1}{c|}{} & ${ }^{\circ} \mathrm{C}$ & ${ }^{\circ} \mathrm{F}$ & $(\mathbf{p p m})$ & \\
\hline Mean & 22.62 & 72.71 & 1966 & $62.95 \%$ \\
\hline S.D. & 0.33 & 0.59 & 397 & $2.62 \%$ \\
\hline Max. & 23.53 & 74.35 & 2701 & $67.74 \%$ \\
\hline
\end{tabular}

Cage Occupied Zone NH3 (ppm)

\begin{tabular}{|l|c|c|c|c|c|c|c|c|c|c|}
\hline Day & $\mathbf{1}$ & $\mathbf{2}$ & $\mathbf{3}$ & $\mathbf{4}$ & $\mathbf{5}$ & $\mathbf{6}$ & $\mathbf{7}$ & $\mathbf{8}$ & $\mathbf{9}$ & $\mathbf{1 0}$ \\
\hline Mean & 1.03 & 1.99 & 3.07 & 4.87 & 7.39 & 10.57 & 15.12 & 19.61 & 24.86 & 30.01 \\
\hline Max. & 1.42 & 2.73 & 4.22 & 6.70 & 10.16 & 14.53 & 20.78 & 26.95 & 34.17 & 41.25 \\
\hline
\end{tabular}

Room Breathing Zone

\begin{tabular}{|l|cc|c|c|}
\cline { 2 - 5 } \multicolumn{1}{c|}{} & \multicolumn{2}{c|}{ Temperature } & $\mathrm{CO}_{2}$ & RH \\
\cline { 2 - 5 } \multicolumn{1}{c|}{} & ${ }^{\circ} \mathrm{C}$ & ${ }^{\circ} \mathrm{F}$ & $(\mathrm{ppm})$ & \\
\hline Mean & 21.11 & 69.99 & 37 & $52.59 \%$ \\
\hline S.D. & 0.30 & 0.54 & 17 & \\
\hline Max. & 22.05 & 71.69 & 146 & \\
\hline
\end{tabular}

Room Breathing Zone NH3 (ppm)

\begin{tabular}{|l|c|c|c|c|c|c|c|c|c|c|}
\hline Day & $\mathbf{1}$ & $\mathbf{2}$ & $\mathbf{3}$ & $\mathbf{4}$ & $\mathbf{5}$ & $\mathbf{6}$ & $\mathbf{7}$ & $\mathbf{8}$ & $\mathbf{9}$ & $\mathbf{1 0}$ \\
\hline Mean & 0.02 & 0.04 & 0.06 & 0.09 & 0.14 & 0.20 & 0.28 & 0.37 & 0.47 & 0.56 \\
\hline Max. & 0.08 & 0.15 & 0.23 & 0.36 & 0.55 & 0.78 & 1.12 & 1.45 & 1.84 & 2.22 \\
\hline
\end{tabular}




\section{Histogram Distributions}

Cage occupied zone average temperature $\left({ }^{\circ} \mathrm{C}\right)$ distribution

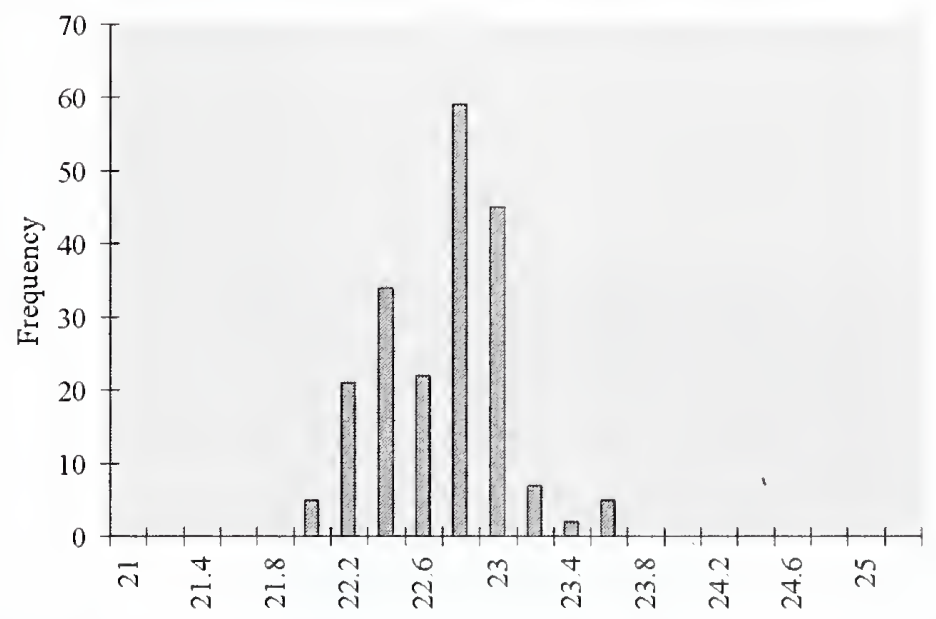

Cage occupied zone average contamination $(\mathrm{kg} / \mathrm{kg})$ distribution

Contamination conversion factors $(\mathrm{kg} / \mathrm{kg} \rightarrow \mathrm{ppm})$

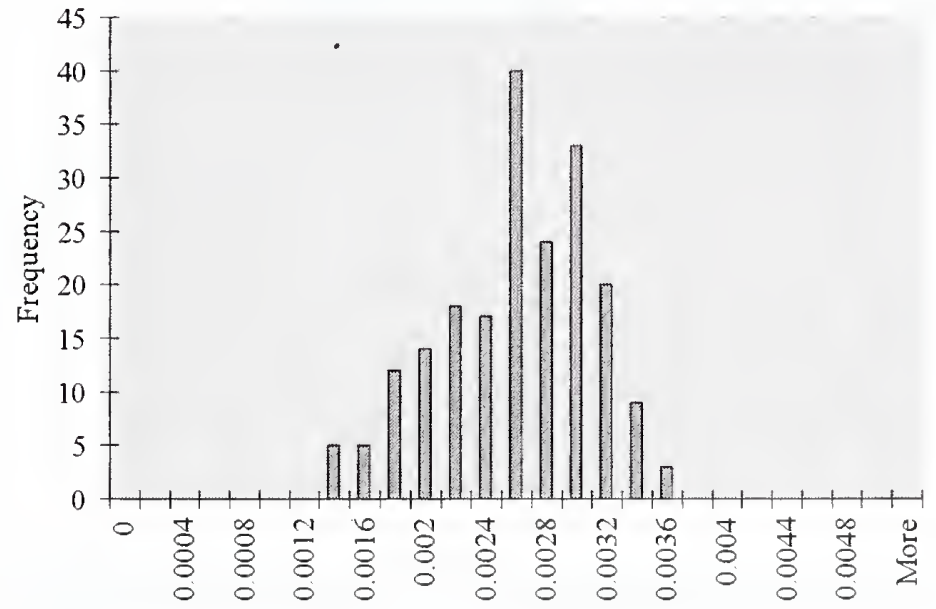

\begin{tabular}{|c|c|c|}
\hline Day & $\mathbf{C O}_{\mathbf{2}}$ & $\mathbf{N H}_{\mathbf{3}}$ \\
\hline $\mathbf{1}$ & 785000 & 412 \\
\hline $\mathbf{2}$ & 785000 & 795 \\
\hline $\mathbf{3}$ & 785000 & 1225 \\
\hline $\mathbf{4}$ & 785000 & 1947 \\
\hline $\mathbf{5}$ & 785000 & 2952 \\
\hline $\mathbf{6}$ & 785000 & 4221 \\
\hline $\mathbf{7}$ & 785000 & 6038 \\
\hline $\mathbf{8}$ & 785000 & 7831 \\
\hline $\mathbf{9}$ & 785000 & 9929 \\
\hline $\mathbf{1 0}$ & 785000 & 11384 \\
\hline
\end{tabular}

Cage occupied zone average relative humidity (\%) distribution

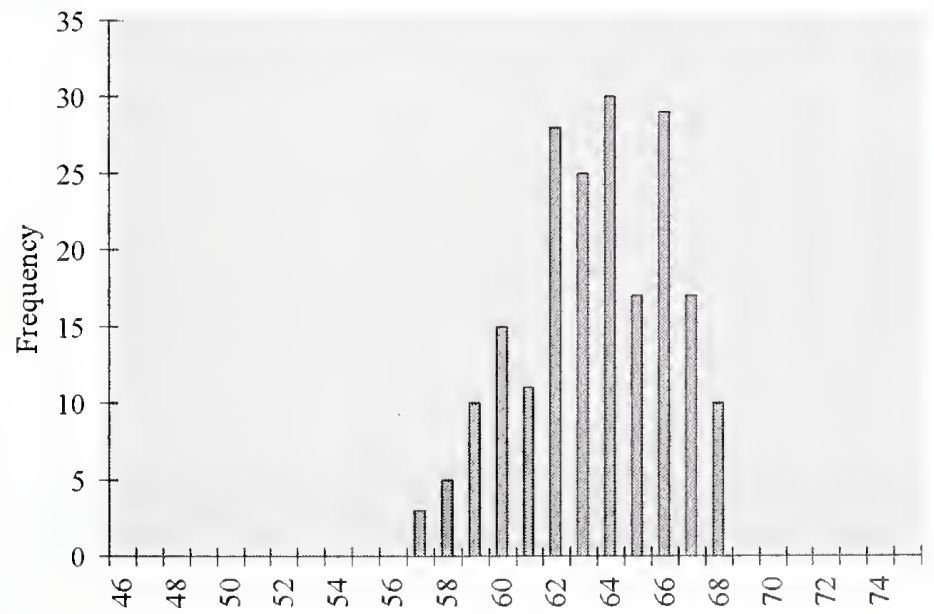


Casename Case 74

Description

Supply

Configuration

Low Ind

Change

Station

$\mathrm{ON}$

Room

$\mathrm{ACH}$

15

Cage

Condition

Top On

\begin{tabular}{|c|c|c|c|c|}
\hline Supply Discharge & Supply Discharge & Exhaust & Exhaust & Exhaust \\
\hline Temperature $\left({ }^{\circ} \mathrm{C}\right)$ & $\mathbf{R H}$ & Configuration & Temperature $\left({ }^{\circ} \mathrm{C}\right)$ & RH \\
\hline 18.8 & $61 \%$ & High $x 4$ / Low $x 2$ & 22 & $50 \%$ \\
\hline Rack & Rack & Number of & Total mass of & Room \\
\hline Orientation & Density & Mice in Room & Mice in Room & Pressurisation \\
\hline On wall & Single & 1050 & $21000 \mathrm{gr}$ & neg $100 \mathrm{cfm}$ \\
\hline
\end{tabular}

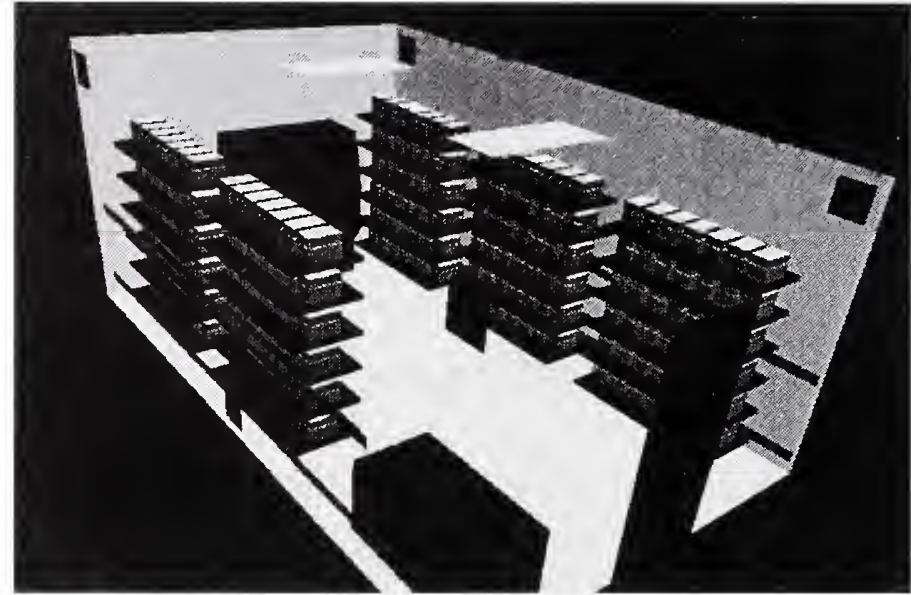

Analysis Results

Cage Occupied 7 one

\begin{tabular}{|l|cc|c|c|}
\cline { 2 - 5 } \multicolumn{1}{c|}{} & \multicolumn{2}{c|}{ Temperature } & $\mathrm{CO}_{2}$ & RH \\
\cline { 2 - 5 } \multicolumn{1}{c|}{} & ${ }^{\circ} \mathrm{C}$ & ${ }^{\circ} \mathrm{F}$ & $(\mathrm{ppm})$ & \\
\hline Mean & 22.62 & 72.71 & 1884 & $62.32 \%$ \\
\hline S.D. & 0.29 & 0.51 & 345 & $2.56 \%$ \\
\hline Max. & 23.56 & 74.41 & 2633 & $67.44 \%$ \\
\hline
\end{tabular}

Cage Occupied Zone NH3 (ppm)

\begin{tabular}{|l|c|c|c|c|c|c|c|c|c|c|}
\hline Day & $\mathbf{1}$ & $\mathbf{2}$ & $\mathbf{3}$ & $\mathbf{4}$ & $\mathbf{5}$ & $\mathbf{6}$ & $\mathbf{7}$ & $\mathbf{8}$ & $\mathbf{9}$ & $\mathbf{1 0}$ \\
\hline Mean & 0.99 & 1.91 & 2.94 & 4.53 & 6.72 & 9.53 & 13.46 & 17.65 & 22.68 & 28.03 \\
\hline Max. & 1.38 & 2.66 & 4.11 & 6.33 & 9.39 & 13.31 & 18.81 & 24.66 & 31.69 & 39.17 \\
\hline
\end{tabular}

Room Breathing Zone

\begin{tabular}{|l|cc|c|c|}
\cline { 2 - 5 } \multicolumn{1}{c|}{} & \multicolumn{2}{c|}{ Temperature } & $\mathbf{C O}_{2}$ & RH \\
\cline { 2 - 5 } \multicolumn{1}{c|}{} & ${ }^{\circ} \mathrm{C}$ & ${ }^{\circ} \mathrm{F}$ & $(\mathbf{p p m})$ & \\
\hline Mean & 21.09 & 69.96 & 48 & $52.74 \%$ \\
\hline S.D. & 0.31 & 0.56 & 22 & \\
\hline Max. & 22.37 & 72.26 & 247 & \\
\hline
\end{tabular}

Room Breathing Zone NH3 (ppm)

\begin{tabular}{|l|c|c|c|c|c|c|c|c|c|c|}
\hline Day & $\mathbf{1}$ & $\mathbf{2}$ & $\mathbf{3}$ & $\mathbf{4}$ & $\mathbf{5}$ & $\mathbf{6}$ & $\mathbf{7}$ & $\mathbf{8}$ & $\mathbf{9}$ & $\mathbf{1 0}$ \\
\hline Mean & 0.02 & 0.05 & 0.07 & 0.11 & 0.17 & 0.24 & 0.34 & 0.45 & 0.57 & 0.71 \\
\hline Max. & 0.13 & 0.25 & 0.39 & 0.59 & 0.88 & 1.25 & 1.77 & 2.32 & 2.98 & 3.68 \\
\hline
\end{tabular}




\section{Histogram Distributions}

Cage occupied zone average temperature $\left({ }^{\circ} \mathrm{C}\right)$ distribution

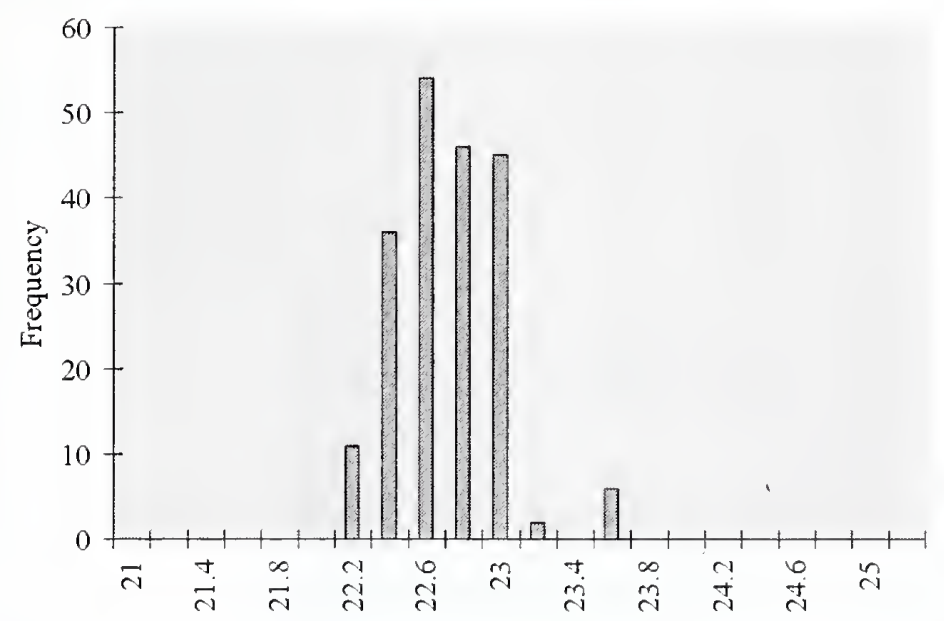

Cage occupied zone average contamination $(\mathrm{kg} / \mathrm{kg})$ distribution Contamination conversion factors $(\mathrm{kg} / \mathrm{kg} \rightarrow \mathrm{ppm})$

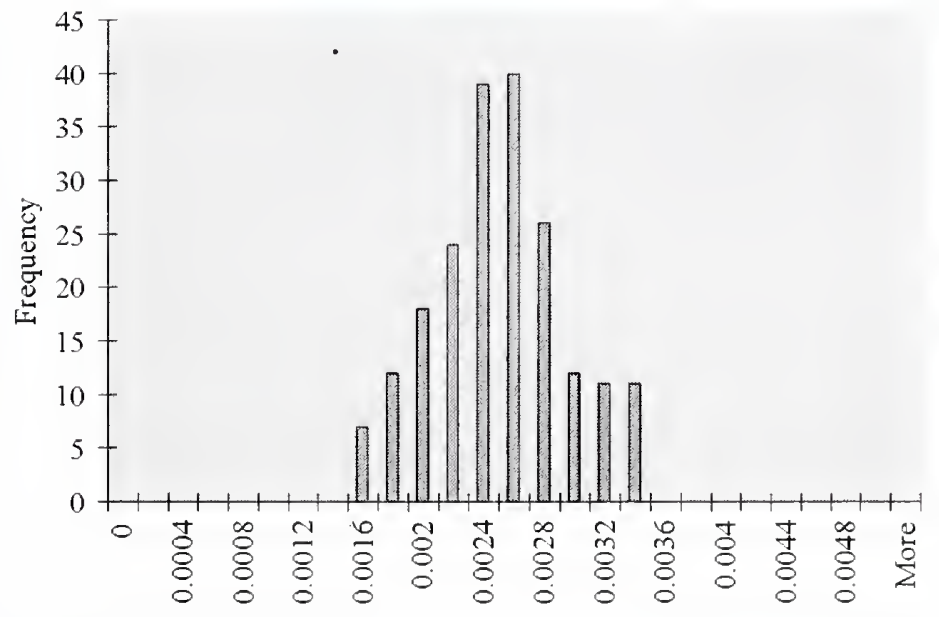

\begin{tabular}{|c|c|c|}
\hline Day & $\mathbf{C O}_{2}$ & $\mathbf{N H}_{3}$ \\
\hline $\mathbf{1}$ & 785000 & 411 \\
\hline $\mathbf{2}$ & 785000 & 795 \\
\hline $\mathbf{3}$ & 785000 & 1225 \\
\hline $\mathbf{4}$ & 785000 & 1888 \\
\hline $\mathbf{5}$ & 785000 & 2800 \\
\hline $\mathbf{6}$ & 785000 & 3969 \\
\hline 7 & 785000 & 5609 \\
\hline $\mathbf{8}$ & 785000 & 7353 \\
\hline $\mathbf{9}$ & 785000 & 9447 \\
\hline $\mathbf{1 0}$ & 785000 & 11384 \\
\hline
\end{tabular}

Cage occupied zone average relative humidity (\%) distribution

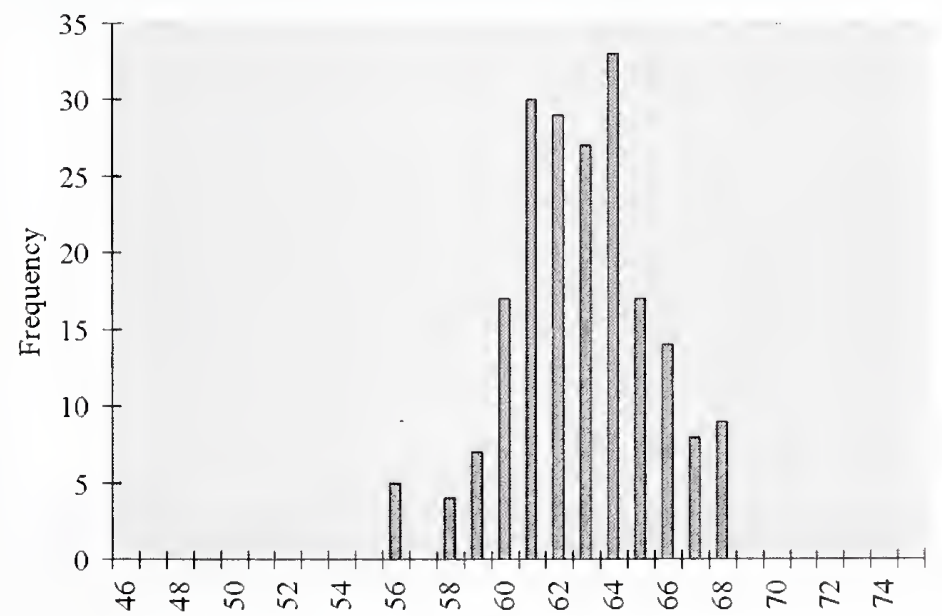


Casename

Case 75

Description

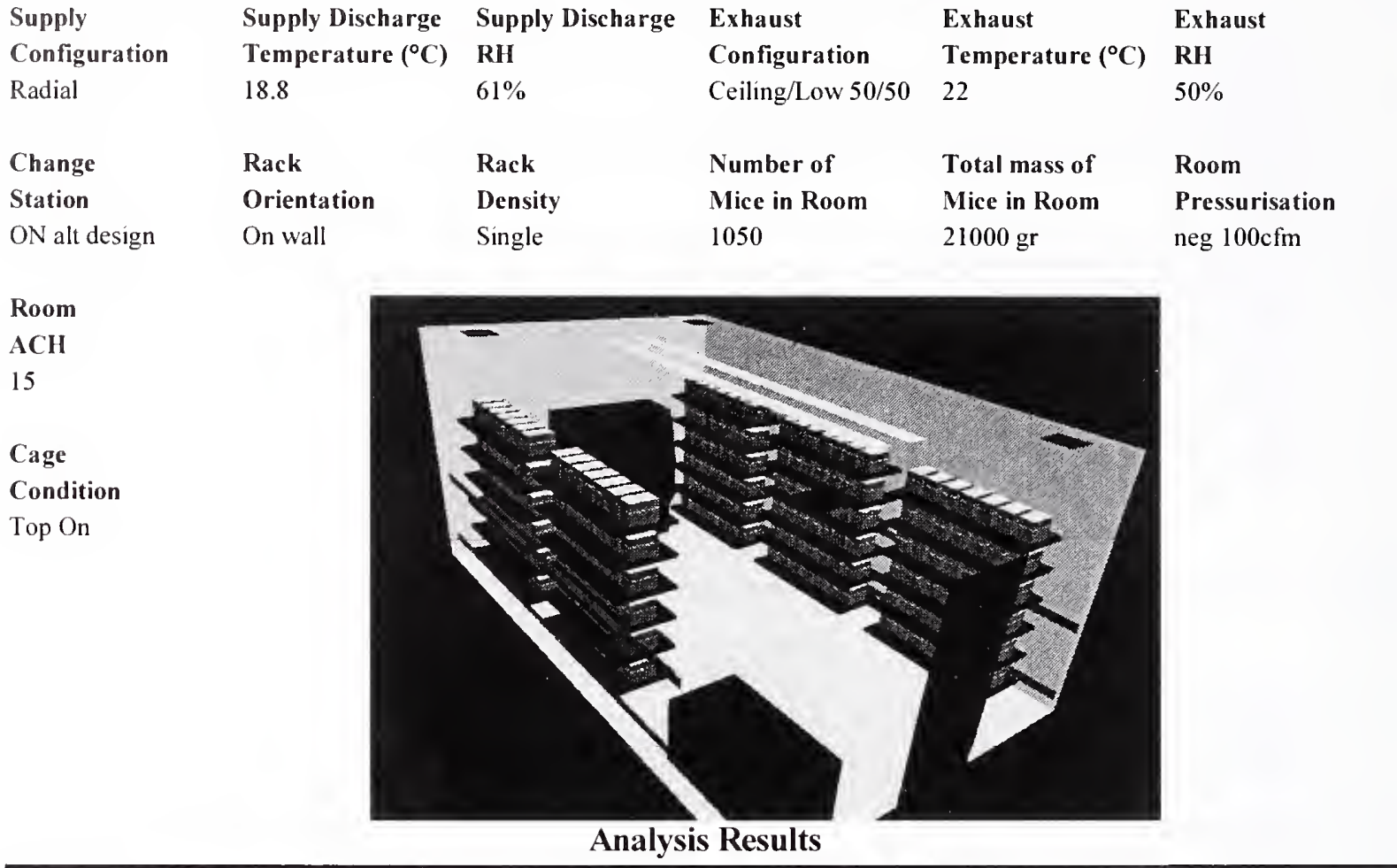

Cage Occupied Zone

\begin{tabular}{|l|cc|c|c|}
\cline { 2 - 5 } \multicolumn{1}{c|}{} & \multicolumn{2}{c|}{ Temperature } & $\mathbf{C O}_{2}$ & RH \\
\cline { 2 - 5 } \multicolumn{1}{c|}{} & ${ }^{\circ} \mathrm{C}$ & ${ }^{\circ} \mathrm{F}$ & $(\mathbf{p p m})$ & \\
\hline Mean & 21.88 & 71.38 & 1839 & $64.89 \%$ \\
\hline S.D. & 0.19 & 0.34 & 362 & $2.56 \%$ \\
\hline Max. & 22.31 & 72.15 & 2622 & $69.92 \%$ \\
\hline
\end{tabular}

Cage Occupied Zone NH3 (ppm)

\begin{tabular}{|l|c|c|c|c|c|c|c|c|c|c|}
\hline Day & $\mathbf{1}$ & $\mathbf{2}$ & $\mathbf{3}$ & $\mathbf{4}$ & $\mathbf{5}$ & $\mathbf{6}$ & $\mathbf{7}$ & $\mathbf{8}$ & $\mathbf{9}$ & $\mathbf{1 0}$ \\
\hline Mean & 0.97 & 1.86 & 2.87 & 4.98 & 8.01 & 11.70 & 17.24 & 21.79 & 26.73 & 30.30 \\
\hline Max. & 1.39 & 2.65 & 4.09 & 7.10 & 11.42 & 16.69 & 24.58 & 31.06 & 38.11 & 43.20 \\
\hline
\end{tabular}

Room Breathing Zone

\begin{tabular}{|l|cc|c|c|}
\cline { 2 - 5 } \multicolumn{1}{c|}{} & \multicolumn{2}{c|}{ Temperature } & $\mathbf{C O}_{2}$ & RH \\
\cline { 2 - 5 } \multicolumn{1}{c|}{} & \multicolumn{1}{c|}{$\mathrm{C}$} & ${ }^{\circ} \mathrm{F}$ & $(\mathbf{p p m})$ & \\
\hline Mean & 21.09 & 69.96 & 48 & $52.74 \%$ \\
\hline S.D. & 0.31 & 0.56 & 22 & \\
\hline Max. & 22.37 & 72.26 & 247 & \\
\hline
\end{tabular}

Room Breathing Zone NH3 (ppm)

\begin{tabular}{|l|c|c|c|c|c|c|c|c|c|c|}
\hline Day & $\mathbf{1}$ & $\mathbf{2}$ & $\mathbf{3}$ & $\mathbf{4}$ & $\mathbf{5}$ & $\mathbf{6}$ & $\mathbf{7}$ & $\mathbf{8}$ & $\mathbf{9}$ & $\mathbf{1 0}$ \\
\hline Mean & 0.03 & 0.05 & 0.07 & 0.13 & 0.21 & 0.30 & 0.45 & 0.57 & 0.69 & 0.79 \\
\hline Max. & 0.13 & 0.25 & 0.39 & 0.67 & 1.08 & 1.57 & 2.32 & 2.93 & 3.60 & 4.07 \\
\hline
\end{tabular}




\section{Histogram Distributions}

Cage occupied zone average temperature $\left({ }^{\circ} \mathrm{C}\right)$ distribution

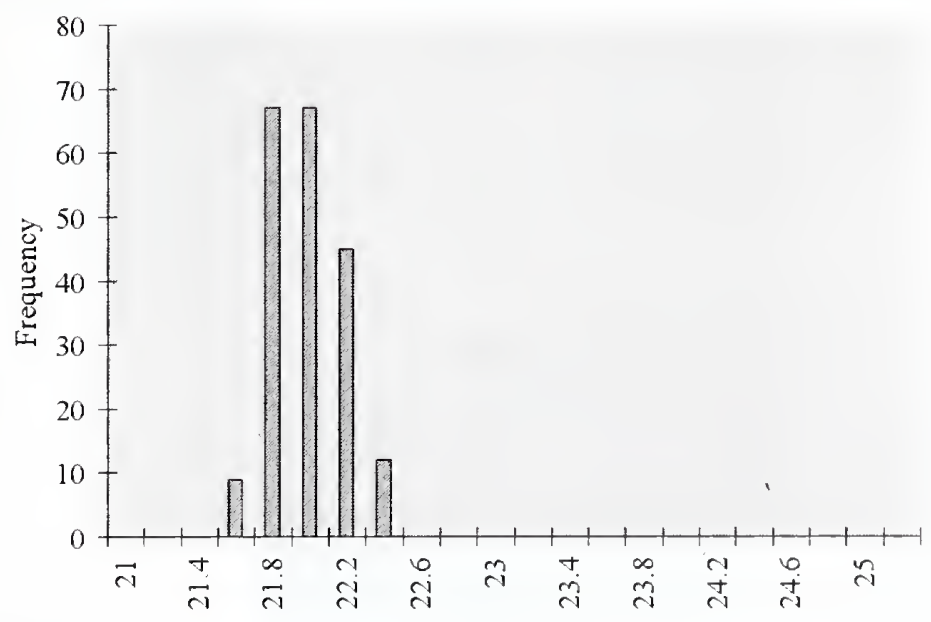

Cage occupied zone average contamination $(\mathrm{kg} / \mathrm{kg})$ distribution

Contamination conversion factors $(\mathrm{kg} / \mathrm{kg} \rightarrow \mathrm{ppm})$

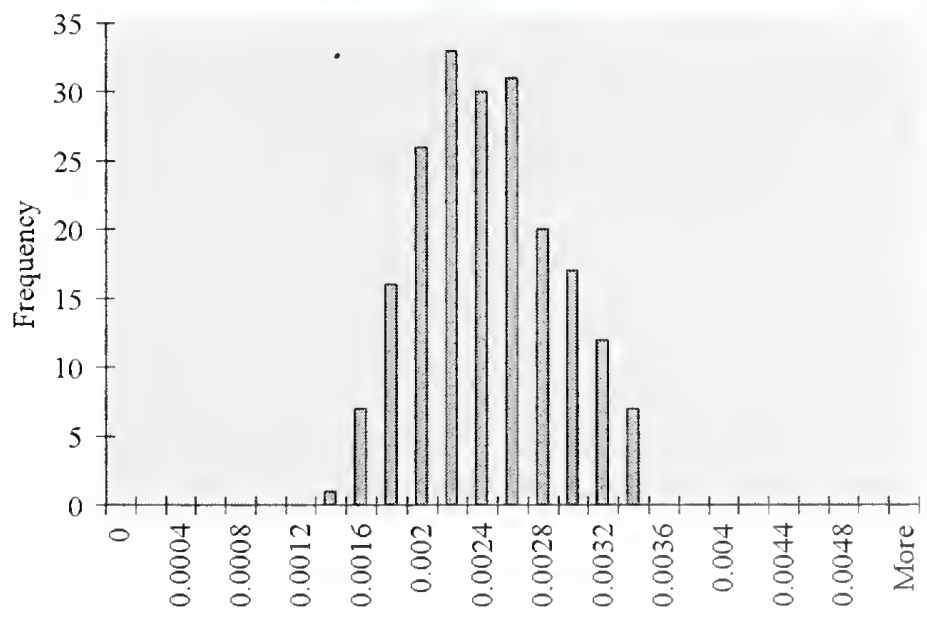

\begin{tabular}{|c|c|c|}
\hline Day & $\mathrm{CO}_{2}$ & $\mathrm{NH}_{3}$ \\
\hline $\mathbf{1}$ & 785000 & 415 \\
\hline 2 & 785000 & 795 \\
\hline 3 & 785000 & 1225 \\
\hline 4 & 785000 & 2127 \\
\hline 5 & 785000 & 3420 \\
\hline 6 & 785000 & 4996 \\
\hline 7 & 785000 & 7360 \\
\hline 8 & 785000 & 9300 \\
\hline 9 & 785000 & 11410 \\
\hline 10 & 785000 & 11384 \\
\hline
\end{tabular}

Cage occupied zone average relative humidity (\%) distribution

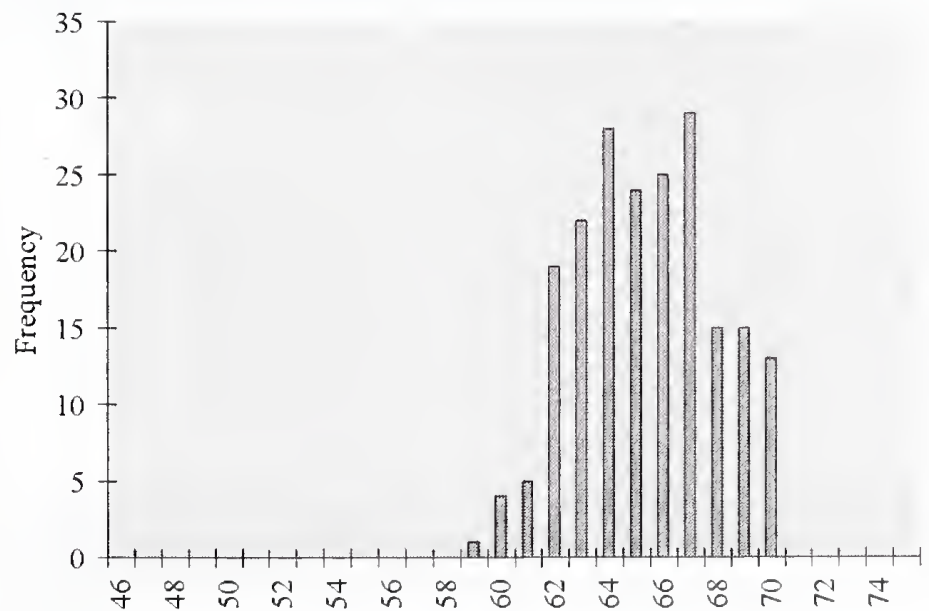


Casename Case 76

Description

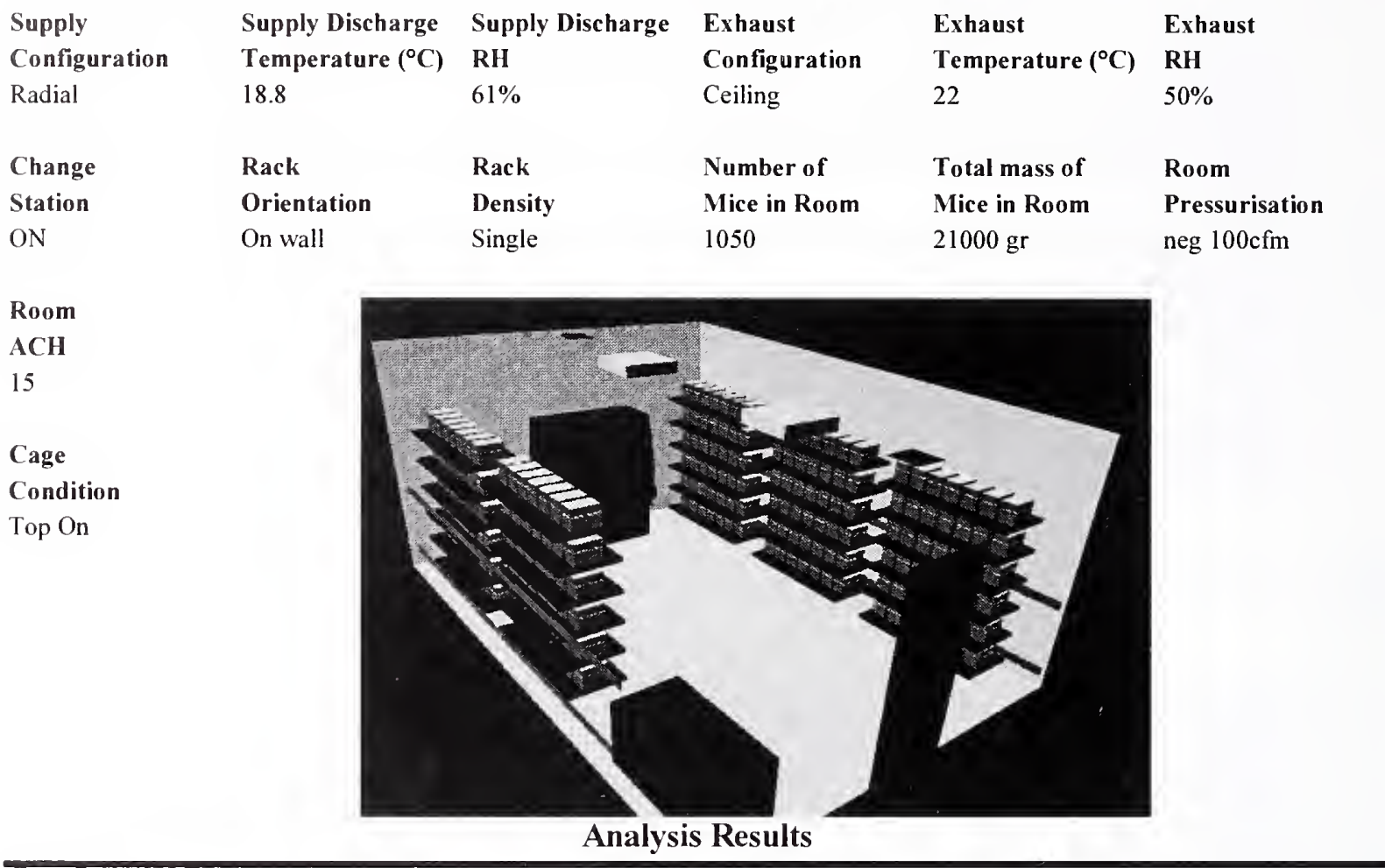

Cage Occupied Zone

\begin{tabular}{|l|cc|c|c|}
\cline { 2 - 5 } \multicolumn{1}{c|}{} & \multicolumn{2}{c|}{ Temperature } & $\mathrm{CO}_{2}$ & RH \\
\cline { 2 - 5 } \multicolumn{1}{c|}{} & ${ }^{\circ} \mathrm{C}$ & ${ }^{\circ} \mathrm{F}$ & $(\mathrm{ppm})$ & \\
\hline Mean & 22.13 & 71.83 & 1857 & $64.05 \%$ \\
\hline S.D. & 0.18 & 0.32 & 302 & $2.32 \%$ \\
\hline Max. & 22.89 & 73.21 & 2435 & $68.79 \%$ \\
\hline
\end{tabular}

Cage Occupied Zone NH3 (ppm)

\begin{tabular}{|l|c|c|c|c|c|c|c|c|c|c|}
\hline Day & $\mathbf{1}$ & $\mathbf{2}$ & $\mathbf{3}$ & $\mathbf{4}$ & $\mathbf{5}$ & $\mathbf{6}$ & $\mathbf{7}$ & $\mathbf{8}$ & $\mathbf{9}$ & $\mathbf{1 0}$ \\
\hline Mean & 0.98 & 1.88 & 2.90 & 4.85 & 7.61 & 11.03 & 16.07 & 20.51 & 25.49 & 29.63 \\
\hline Max. & 1.28 & 2.46 & 3.80 & 6.36 & 9.98 & 14.46 & 21.07 & 26.89 & 33.42 & 38.85 \\
\hline
\end{tabular}

Room Breathing Zone

\begin{tabular}{|l|cc|c|c|}
\cline { 2 - 5 } \multicolumn{1}{c|}{} & \multicolumn{2}{c|}{ Temperature } & $\mathrm{CO}_{2}$ & RH \\
\cline { 2 - 5 } \multicolumn{1}{c|}{} & ${ }^{\circ} \mathrm{C}$ & ${ }^{\circ} \mathrm{F}$ & $(\mathrm{ppm})$ & \\
\hline Mean & 20.39 & 68.71 & 55 & $55.19 \%$ \\
\hline S.D. & 0.19 & 0.35 & 27 & \\
\hline Max. & 21.23 & 70.21 & 236 & \\
\hline
\end{tabular}

Room Breathing Zone NH3 (ppm)

\begin{tabular}{|l|c|c|c|c|c|c|c|c|c|c|}
\hline Day & $\mathbf{1}$ & $\mathbf{2}$ & $\mathbf{3}$ & $\mathbf{4}$ & $\mathbf{5}$ & $\mathbf{6}$ & $\mathbf{7}$ & $\mathbf{8}$ & $\mathbf{9}$ & $\mathbf{1 0}$ \\
\hline Mean & 0.03 & 0.06 & 0.09 & 0.14 & 0.23 & 0.33 & 0.48 & 0.6 & 0.76 & 0.88 \\
\hline Max. & 0.12 & 0.24 & 0.37 & 0.62 & 0.97 & 1.40 & 2.04 & 2.61 & 3.24 & 3.77 \\
\hline
\end{tabular}




\section{Histogram Distributions}

Cage occupied zone average temperature $\left({ }^{\circ} \mathrm{C}\right)$ distribution

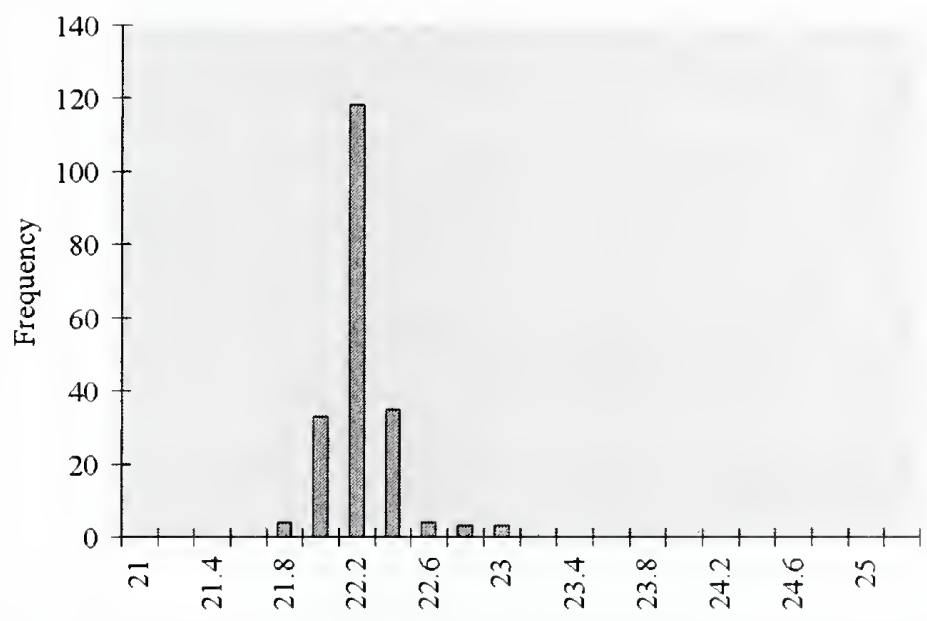

Cage occupied zone average contamination $(\mathrm{kg} / \mathrm{kg})$ distribution

Contamination conversion factors $(\mathrm{kg} / \mathrm{kg} \rightarrow \mathrm{ppm})$

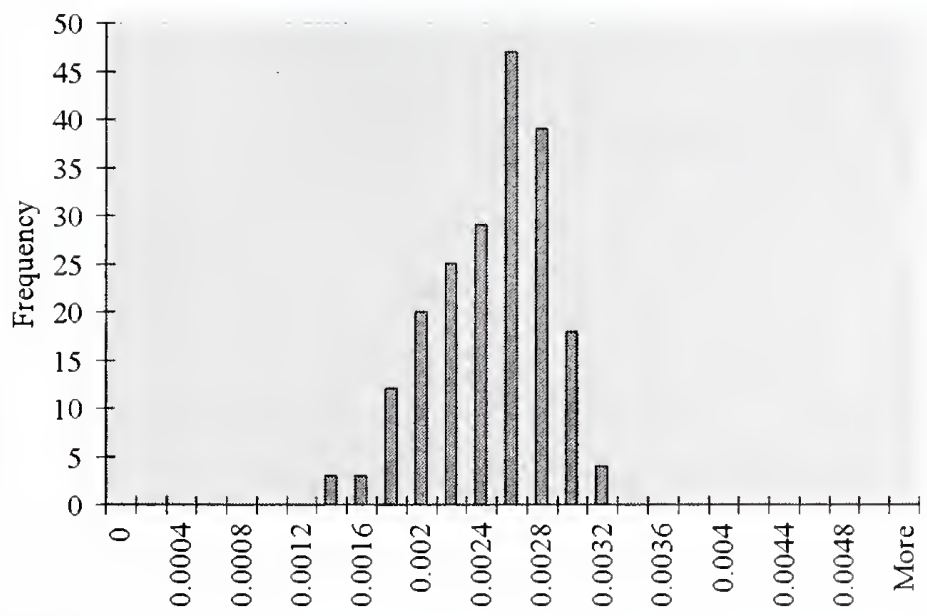

\begin{tabular}{|c|c|c|}
\hline Day & $\mathrm{CO}_{2}$ & $\mathrm{NH}_{3}$ \\
\hline $\mathbf{1}$ & 785000 & 414 \\
\hline $\mathbf{2}$ & 785000 & 795 \\
\hline $\mathbf{3}$ & 785000 & 1225 \\
\hline $\mathbf{4}$ & 785000 & 2049 \\
\hline $\mathbf{5}$ & 785000 & 3218 \\
\hline 6 & 785000 & 4662 \\
\hline 7 & 785000 & 6791 \\
\hline $\mathbf{8}$ & 785000 & 8667 \\
\hline 9 & 785000 & 10772 \\
\hline 10 & 785000 & 11384 \\
\hline
\end{tabular}

Cage occupied zone average relative humidity (\%) distribution

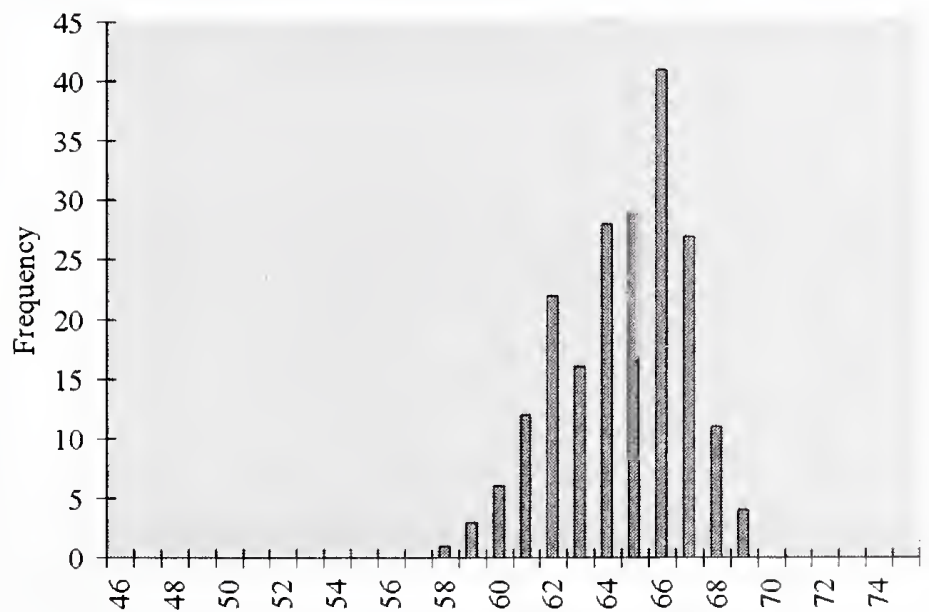




\section{Casename Case 77}

\section{Description}

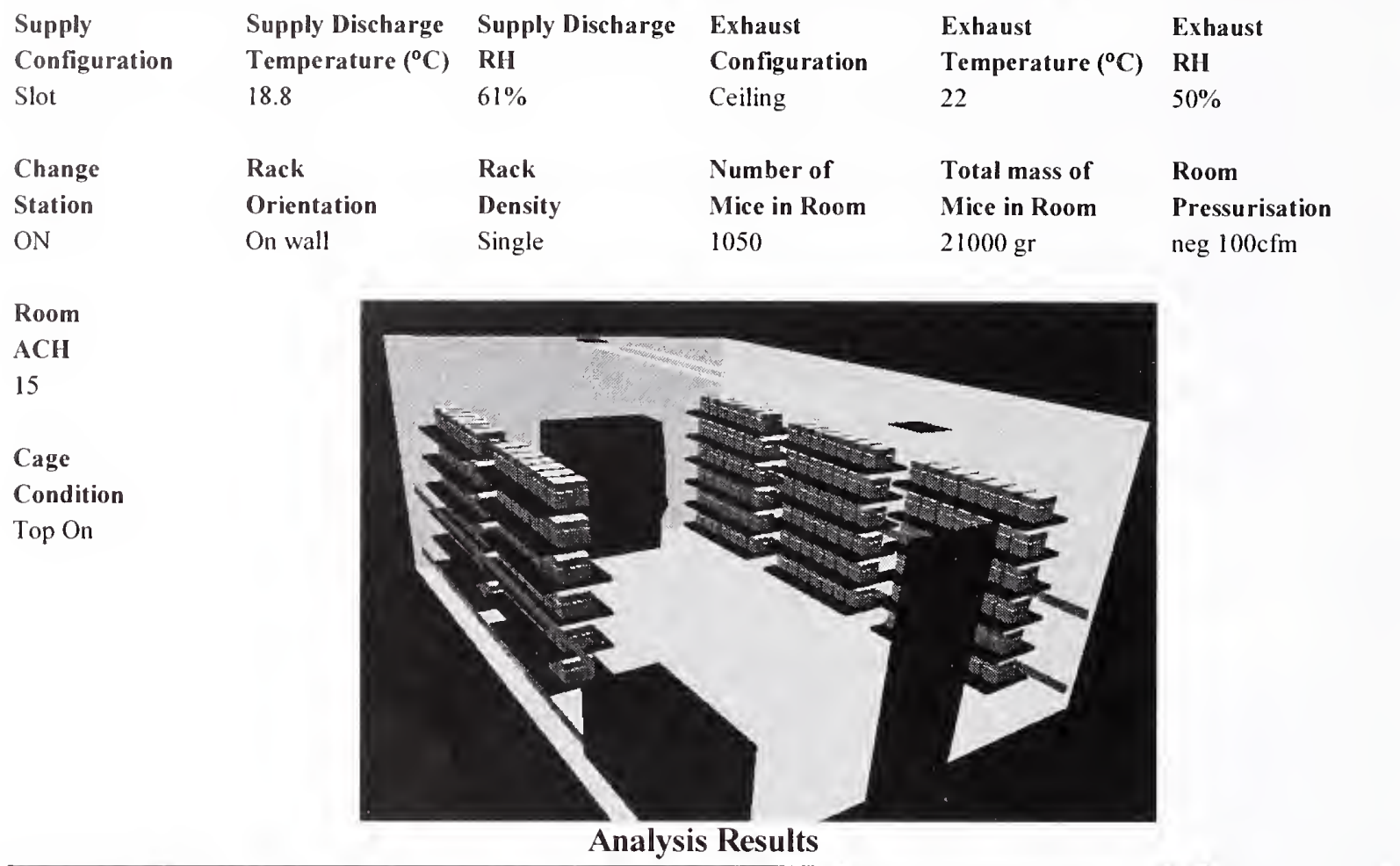

Cage Occupied Zone

\begin{tabular}{|l|cc|c|c|}
\cline { 2 - 5 } \multicolumn{1}{c|}{} & \multicolumn{2}{c|}{ Temperature } & $\mathrm{CO}_{2}$ & RH \\
\cline { 2 - 5 } \multicolumn{1}{c|}{} & ${ }^{\circ} \mathrm{C}$ & ${ }^{\circ} \mathrm{F}$ & $(\mathrm{ppm})$ & \\
\hline Mean & 22.14 & 71.86 & 1881 & $64.17 \%$ \\
\hline S.D. & 0.26 & 0.46 & 351 & $2.58 \%$ \\
\hline Max. & 22.90 & 73.22 & 2652 & $70.61 \%$ \\
\hline
\end{tabular}

Cage Occupied Zone NH3 (ppm)

\begin{tabular}{|l|c|c|c|c|c|c|c|c|c|c|}
\hline Day & $\mathbf{1}$ & $\mathbf{2}$ & $\mathbf{3}$ & $\mathbf{4}$ & $\mathbf{5}$ & $\mathbf{6}$ & $\mathbf{7}$ & $\mathbf{8}$ & $\mathbf{9}$ & $\mathbf{1 0}$ \\
\hline Mean & 0.99 & 1.90 & 2.93 & 4.94 & 7.78 & 11.29 & 16.47 & 20.98 & 26.03 & 30.15 \\
\hline Max. & 1.40 & 2.68 & 4.14 & 6.96 & 10.97 & 15.91 & 23.22 & 29.59 & 36.70 & 42.51 \\
\hline
\end{tabular}

Room Breathing Zone

\begin{tabular}{|l|cc|c|c|}
\cline { 2 - 5 } \multicolumn{1}{c|}{} & \multicolumn{2}{c|}{ Temperature } & $\mathrm{CO}_{2}$ & RH \\
\cline { 2 - 5 } \multicolumn{1}{c|}{} & ${ }^{\circ} \mathrm{C}$ & ${ }^{\circ} \mathrm{F}$ & $(\mathrm{ppm})$ & \\
\hline Mean & 20.39 & 68.70 & 50 & $55.16 \%$ \\
\hline S.D. & 0.23 & 0.42 & 21 & \\
\hline Max. & 21.44 & 70.59 & 191 & \\
\hline
\end{tabular}

Room Breathing Zone NH3 (ppm)

\begin{tabular}{|l|c|c|c|c|c|c|c|c|c|c|}
\hline Day & $\mathbf{1}$ & $\mathbf{2}$ & $\mathbf{3}$ & $\mathbf{4}$ & $\mathbf{5}$ & $\mathbf{6}$ & $\mathbf{7}$ & $\mathbf{8}$ & $\mathbf{9}$ & $\mathbf{1 0}$ \\
\hline Mean & 0.03 & 0.05 & 0.08 & 0.13 & 0.21 & 0.30 & 0.44 & 0.56 & 0.70 & 0.81 \\
\hline Max. & 0.10 & 0.19 & 0.30 & 0.50 & 0.79 & 1.14 & 1.67 & 2.13 & 2.64 & 3.06 \\
\hline
\end{tabular}




\section{Histogram Distributions}

Cage occupied zone average temperature $\left({ }^{\circ} \mathrm{C}\right)$ distribution

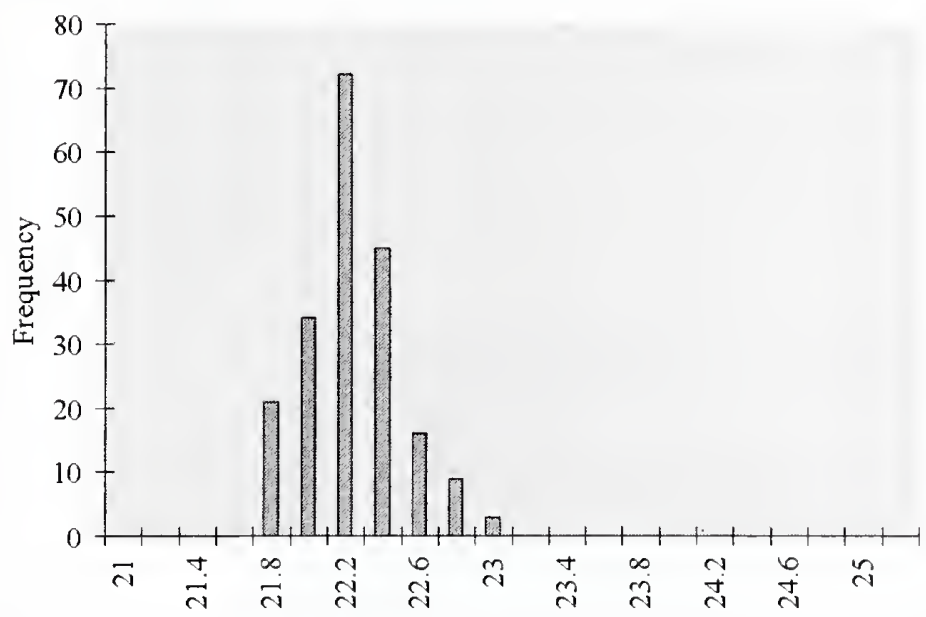

Cage occupied zone average contamination $(\mathrm{kg} / \mathrm{kg})$ distribution

Contamination conversion factors $(\mathrm{kg} / \mathrm{kg} \rightarrow \mathrm{ppm})$

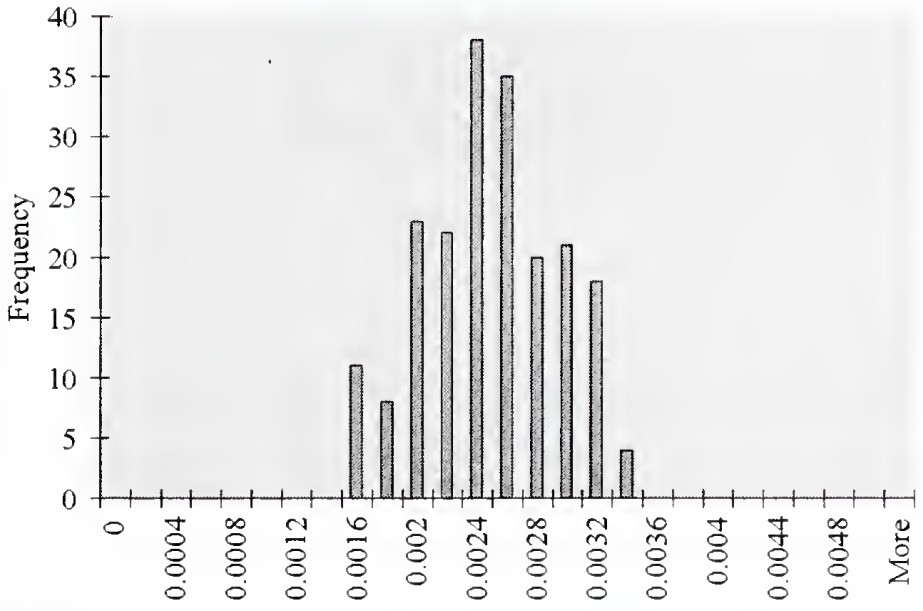

\begin{tabular}{|c|c|c|}
\hline Day & $\mathrm{CO}_{2}$ & $\mathrm{NH}_{3}$ \\
\hline $\mathbf{1}$ & 785000 & 414 \\
\hline $\mathbf{2}$ & 785000 & 795 \\
\hline $\mathbf{3}$ & 785000 & 1225 \\
\hline 4 & 785000 & 2060 \\
\hline $\mathbf{5}$ & 785000 & 3247 \\
\hline 6 & 785000 & 4710 \\
\hline 7 & 785000 & 6872 \\
\hline $\mathbf{8}$ & 785000 & 8758 \\
\hline 9 & 785000 & 10864 \\
\hline 10 & 785000 & 11384 \\
\hline
\end{tabular}

Cage occupied zone average relative humidity (\%) distribution

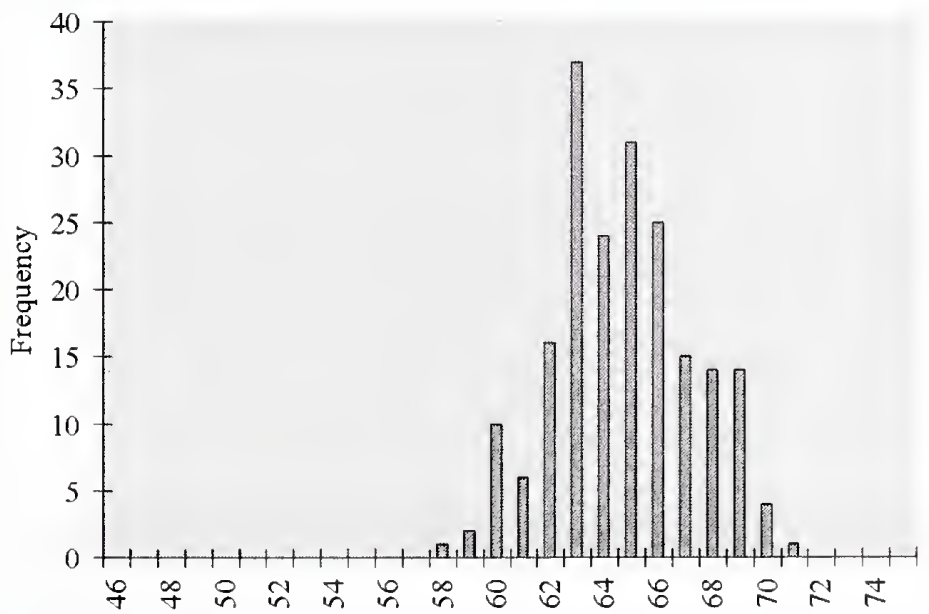




\section{$\begin{array}{ll}\text { Casename } & \text { Case } \mathbf{7 8}\end{array}$}

\section{Description}

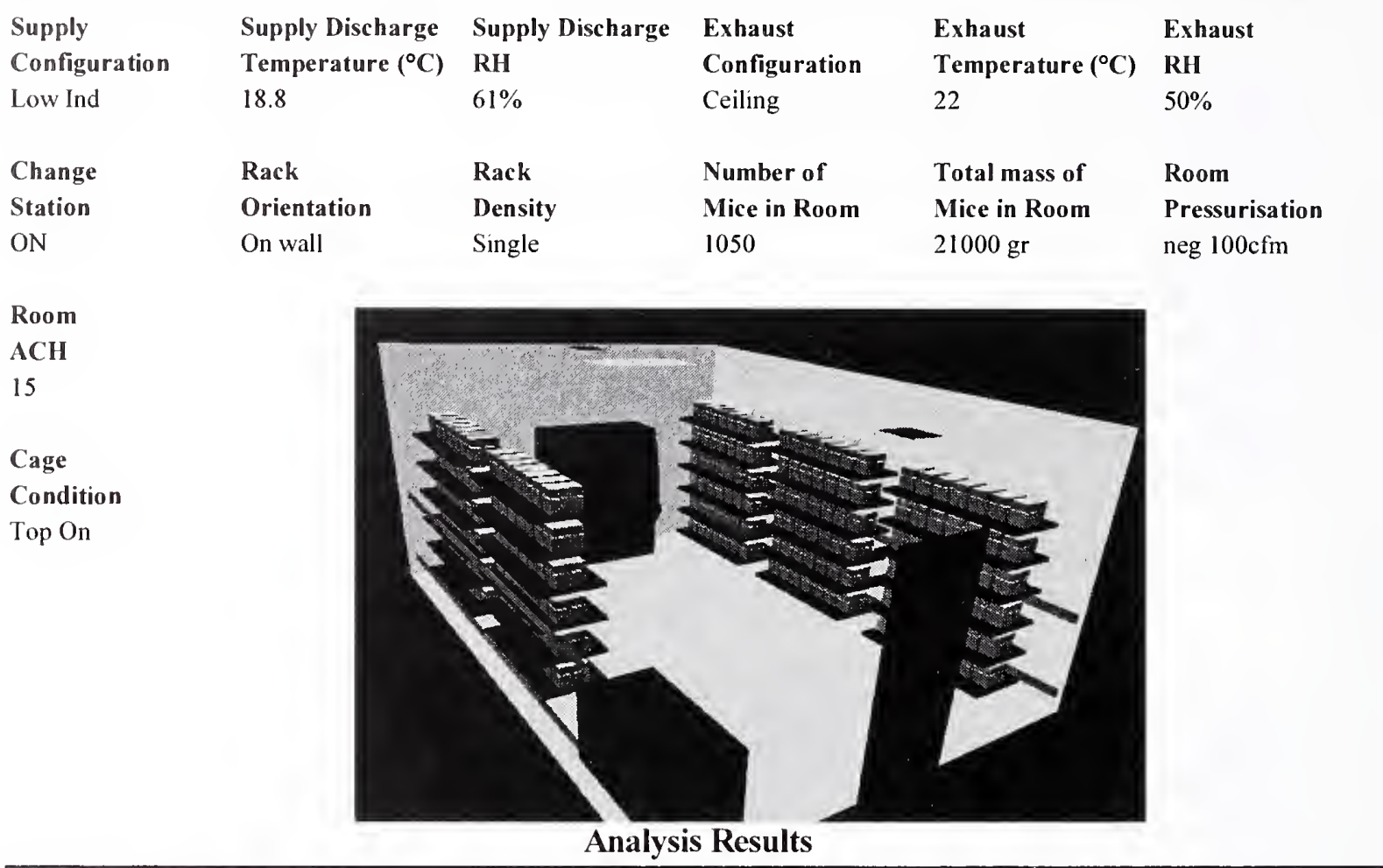

Cage Occupied Zone

\begin{tabular}{|l|cc|c|c|}
\cline { 2 - 5 } \multicolumn{1}{c|}{} & \multicolumn{2}{c|}{ Temperature } & $\mathbf{C O}_{2}$ & RH \\
\cline { 2 - 5 } \multicolumn{1}{c|}{} & ${ }^{\circ} \mathrm{C}$ & \multicolumn{1}{c|}{} & $(\mathbf{p p m})$ & \\
\hline Mean & 22.09 & 71.76 & 1919 & $64.69 \%$ \\
\hline S.D. & 0.22 & 0.40 & 362 & $2.65 \%$ \\
\hline Max. & 22.67 & 72.81 & 2625 & $70.05 \%$ \\
\hline
\end{tabular}

Cage Occupied Zone NH3 (ppm)

\begin{tabular}{|l|c|c|c|c|c|c|c|c|c|c|}
\hline Day & $\mathbf{1}$ & $\mathbf{2}$ & $\mathbf{3}$ & $\mathbf{4}$ & $\mathbf{5}$ & $\mathbf{6}$ & $\mathbf{7}$ & $\mathbf{8}$ & $\mathbf{9}$ & $\mathbf{1 0}$ \\
\hline Mean & 1.01 & 1.94 & 2.99 & 5.16 & 8.25 & 12.03 & 17.67 & 22.38 & 27.54 & 31.38 \\
\hline Max. & 1.39 & 2.66 & 4.10 & 7.05 & 11.28 & 16.45 & 24.17 & 30.61 & 37.66 & 42.92 \\
\hline
\end{tabular}

Room Breathing Zone

\begin{tabular}{|l|cc|c|c|}
\cline { 2 - 5 } \multicolumn{1}{c|}{} & \multicolumn{2}{c|}{ Temperature } & $\mathrm{CO}_{2}$ & RH \\
\cline { 2 - 5 } \multicolumn{1}{c|}{} & ${ }^{\circ} \mathrm{C}$ & ${ }^{\circ} \mathrm{F}$ & $(\mathbf{p p m})$ & \\
\hline Mean & 20.41 & 68.74 & 55 & $55.13 \%$ \\
\hline S.D. & 0.24 & 0.44 & 20 & \\
\hline Max. & 22.13 & 71.84 & 212 & \\
\hline
\end{tabular}

Room Breathing Zone NH3 (ppm)

\begin{tabular}{|l|c|c|c|c|c|c|c|c|c|c|}
\hline Day & $\mathbf{1}$ & $\mathbf{2}$ & $\mathbf{3}$ & $\mathbf{4}$ & $\mathbf{5}$ & $\mathbf{6}$ & $\mathbf{7}$ & $\mathbf{8}$ & $\mathbf{9}$ & $\mathbf{1 0}$ \\
\hline Mean & 0.03 & 0.06 & 0.09 & 0.15 & 0.24 & 0.35 & 0.51 & 0.64 & 0.79 & 0.90 \\
\hline Max. & 0.11 & 0.21 & 0.33 & 0.57 & 0.91 & 1.33 & 1.95 & 2.47 & 3.04 & 3.46 \\
\hline
\end{tabular}




\section{Histogram Distributions}

Cage occupied zone average temperature $\left({ }^{\circ} \mathrm{C}\right)$ distribution

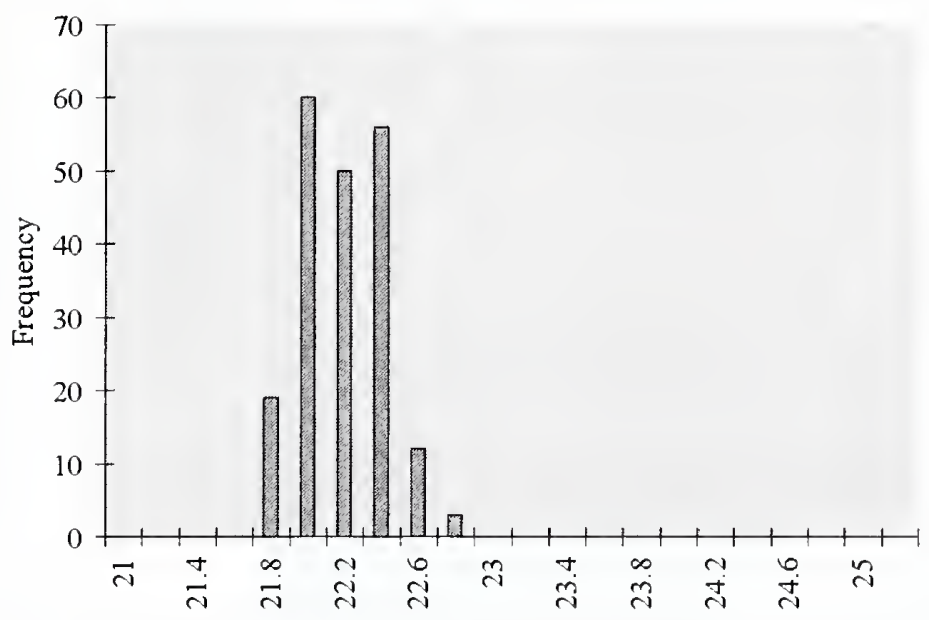

Cage occupied zone average contamination $(\mathrm{kg} / \mathrm{kg})$ distribution

Contamination conversion factors $(\mathrm{kg} / \mathrm{kg} \rightarrow \mathrm{ppm})$

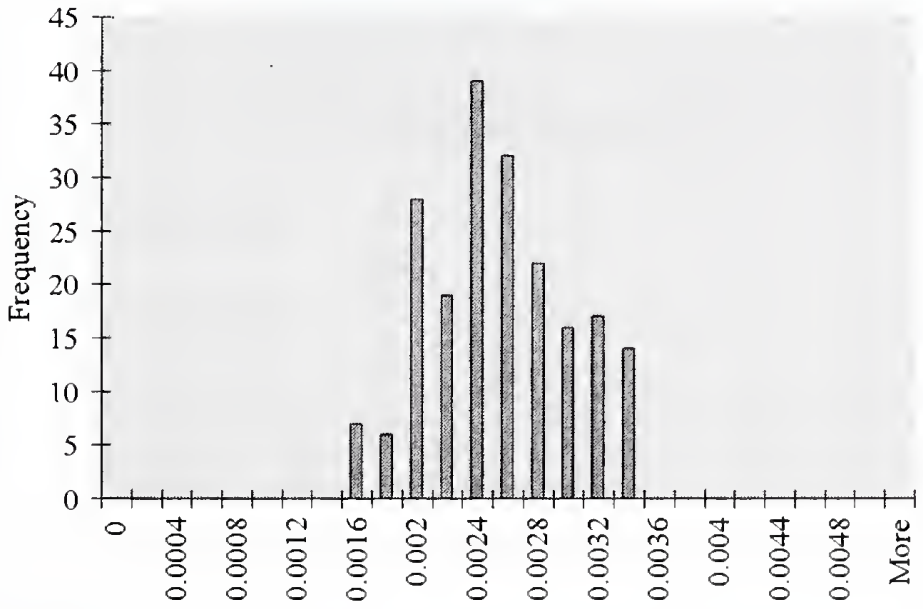

\begin{tabular}{|c|c|c|}
\hline Day & $\mathbf{C O}_{2}$ & $\mathbf{N H}_{3}$ \\
\hline $\mathbf{1}$ & 785000 & 415 \\
\hline $\mathbf{2}$ & 785000 & 795 \\
\hline $\mathbf{3}$ & 785000 & 1225 \\
\hline $\mathbf{4}$ & 785000 & 2109 \\
\hline $\mathbf{5}$ & 785000 & 3373 \\
\hline $\mathbf{6}$ & 785000 & 4919 \\
\hline 7 & 785000 & 7229 \\
\hline $\mathbf{8}$ & 785000 & 9154 \\
\hline $\mathbf{9}$ & 785000 & 11263 \\
\hline $\mathbf{1 0}$ & 785000 & 11384 \\
\hline
\end{tabular}

Cage occupied zone average relative humidity (\%) distribution

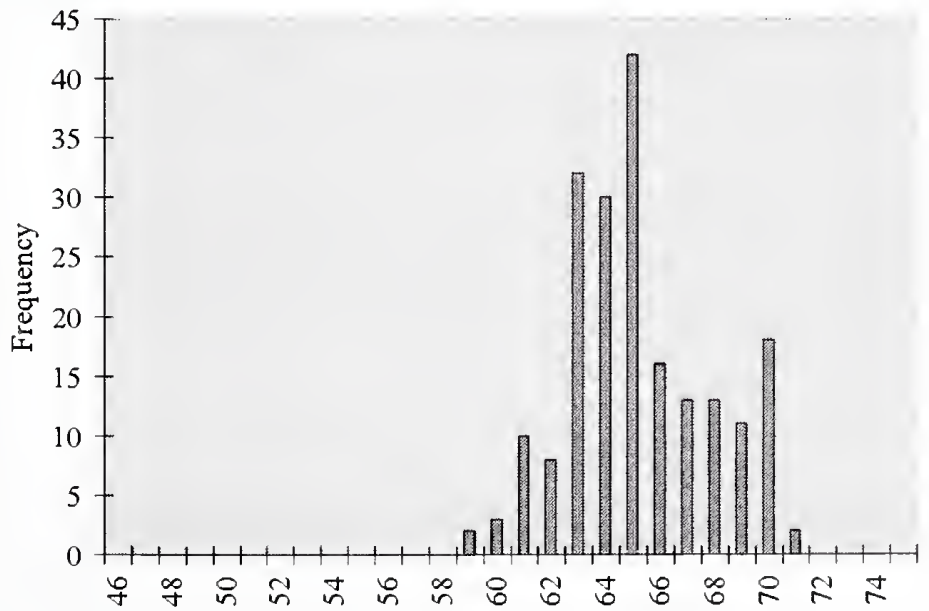


Casename Case 79

Description

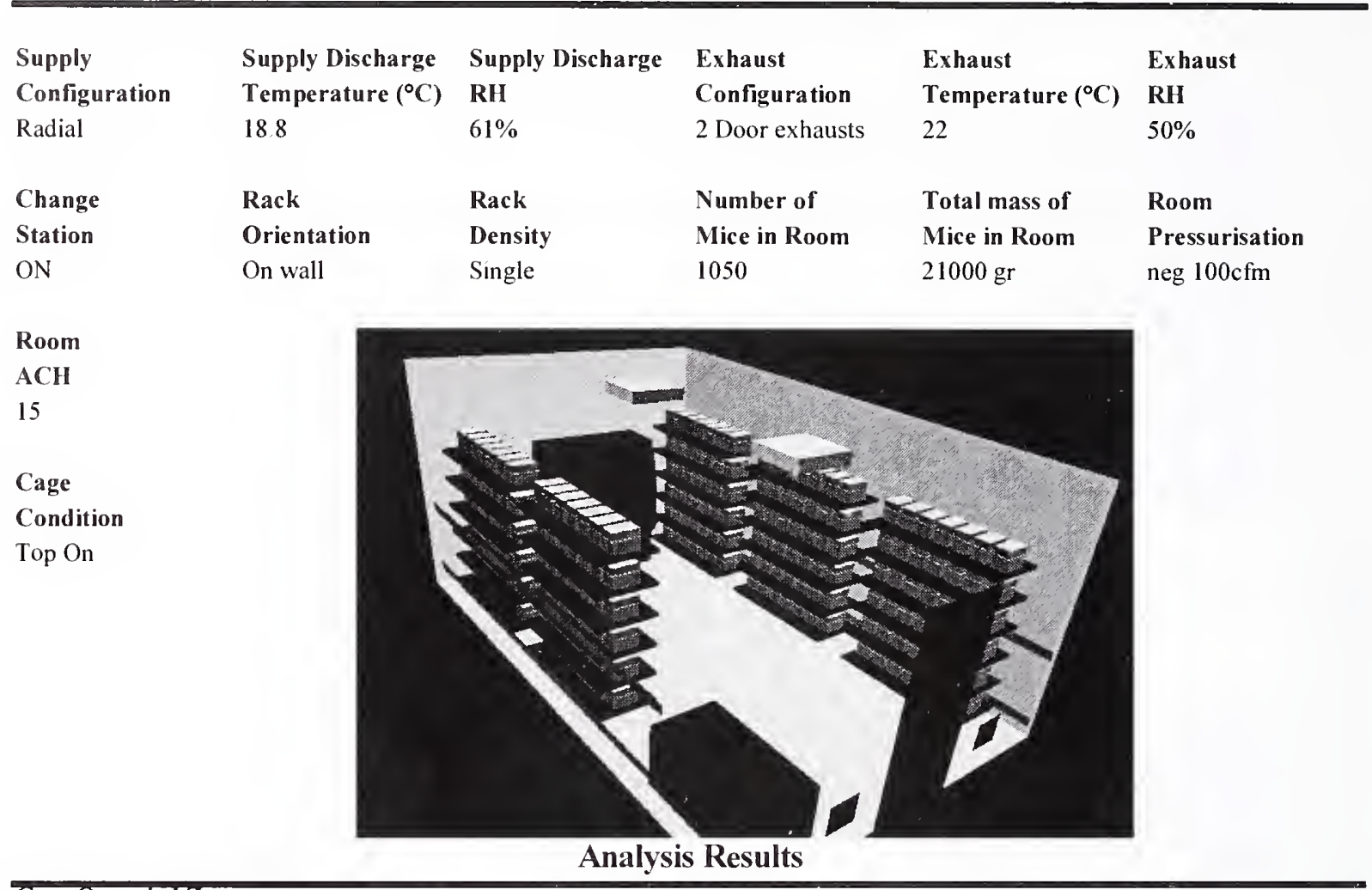

Cage Occupied Zone

\begin{tabular}{|l|cc|c|c|}
\cline { 2 - 5 } \multicolumn{1}{c|}{} & \multicolumn{2}{c|}{ Temperature } & $\mathrm{CO}_{2}$ & RH \\
\cline { 2 - 5 } \multicolumn{1}{c|}{} & ${ }^{\circ} \mathrm{C}$ & ${ }^{\circ} \mathrm{F}$ & $(\mathrm{ppm})$ & \\
\hline Mean & 23.12 & 73.62 & 1647 & $58.59 \%$ \\
\hline S.D. & 0.37 & 0.66 & 301 & $2.52 \%$ \\
\hline Max. & 24.34 & 75.81 & 2355 & $63.76 \%$ \\
\hline
\end{tabular}

Cage Occupied Zone NH3 (ppm)

\begin{tabular}{|l|c|c|c|c|c|c|c|c|c|c|}
\hline Day & $\mathbf{1}$ & $\mathbf{2}$ & $\mathbf{3}$ & $\mathbf{4}$ & $\mathbf{5} \cdot$ & $\mathbf{6}$ & $\mathbf{7}$ & $\mathbf{8}$ & $\mathbf{9}$ & $\mathbf{1 0}$ \\
\hline Mean & 0.86 & $\mathbf{1 . 6 7}$ & 2.57 & 3.70 & 5.21 & 7.22 & 9.88 & 13.33 & 17.71 & 23.15 \\
\hline Max. & 1.22 & 2.38 & 3.67 & 5.30 & 7.45 & 10.33 & 14.13 & 19.07 & 25.32 & 33.10 \\
\hline
\end{tabular}

Room Breathing Zone

\begin{tabular}{|l|cc|c|c|}
\cline { 2 - 5 } \multicolumn{1}{c|}{} & \multicolumn{2}{c|}{ Temperature } & $\mathrm{CO}_{2}$ & RH \\
\cline { 2 - 5 } \multicolumn{1}{c|}{} & ${ }^{\circ} \mathrm{C}$ & ${ }^{\circ} \mathrm{F}$ & $(\mathrm{ppm})$ & \\
\hline Mean & 21.94 & 71.49 & 81 & $50.26 \%$ \\
\hline S.D. & 0.36 & 0.64 & 26 & \\
\hline Max. & 22.89 & 73.20 & 195 & \\
\hline
\end{tabular}

Room Breathing Zone NH3 (ppm)

\begin{tabular}{|l|c|c|c|c|c|c|c|c|c|c|}
\hline Day & $\mathbf{1}$ & $\mathbf{2}$ & $\mathbf{3}$ & $\mathbf{4}$ & $\mathbf{5}$ & $\mathbf{6}$ & $\mathbf{7}$ & $\mathbf{8}$ & $\mathbf{9}$ & $\mathbf{1 0}$ \\
\hline Mean & 0.04 & 0.08 & 0.13 & 0.18 & 0.26 & 0.36 & 0.49 & 0.66 & 0.87 & 1.14 \\
\hline Max. & 0.10 & 0.20 & 0.30 & 0.44 & 0.62 & 0.86 & 1.17 & 1.58 & 2.10 & 2.74 \\
\hline
\end{tabular}




\section{Histogram Distributions}

Cage occupied zone average temperature $\left({ }^{\circ} \mathrm{C}\right)$ distribution

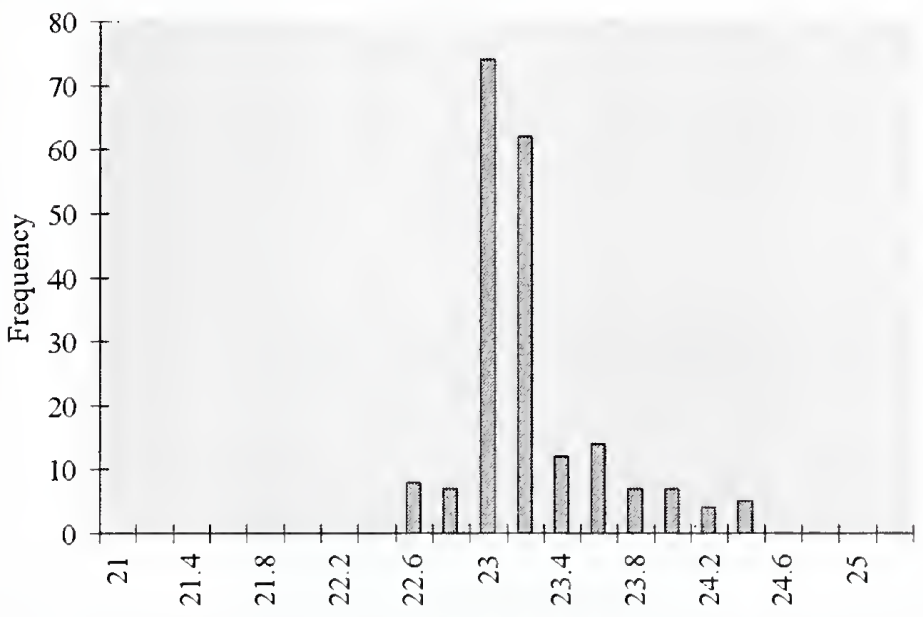

Cage occupied zone average contamination $(\mathrm{kg} / \mathrm{kg})$ distribution

Contamination conversion factors $(\mathrm{kg} / \mathrm{kg} \rightarrow \mathrm{ppm})$

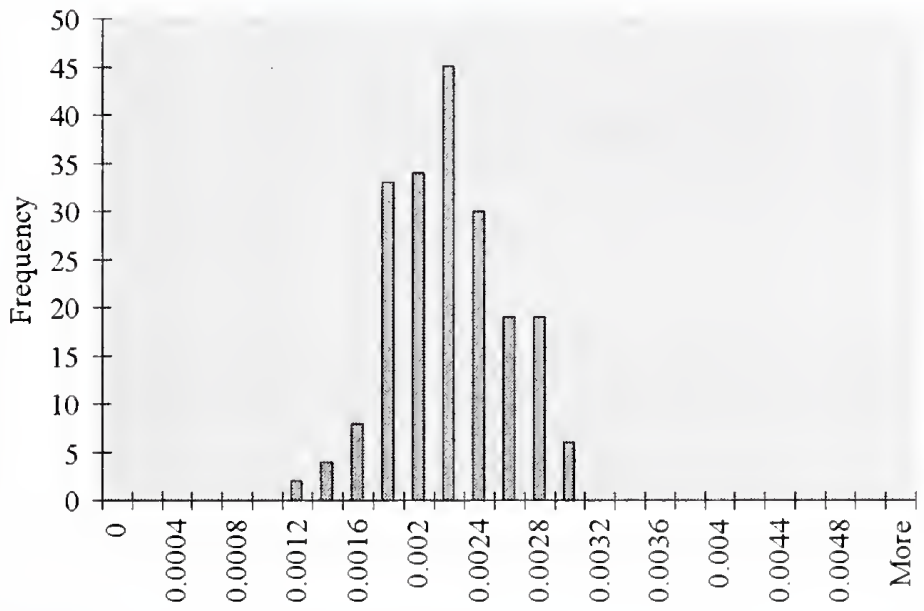

\begin{tabular}{|c|c|c|}
\hline Day & $\mathrm{CO}_{2}$ & $\mathrm{NH}_{3}$ \\
\hline 1 & 785000 & 408 \\
\hline 2 & 785000 & 795 \\
\hline 3 & 785000 & 1225 \\
\hline 4 & 785000 & 1766 \\
\hline 5 & 785000 & 2483 \\
\hline 6 & 785000 & 3443 \\
\hline 7 & 785000 & 4712 \\
\hline $\mathbf{8}$ & 785000 & 6356 \\
\hline 9 & 785000 & 8442 \\
\hline 10 & 785000 & 11384 \\
\hline
\end{tabular}

Cage occupied zone average relative humidity (\%) distribution

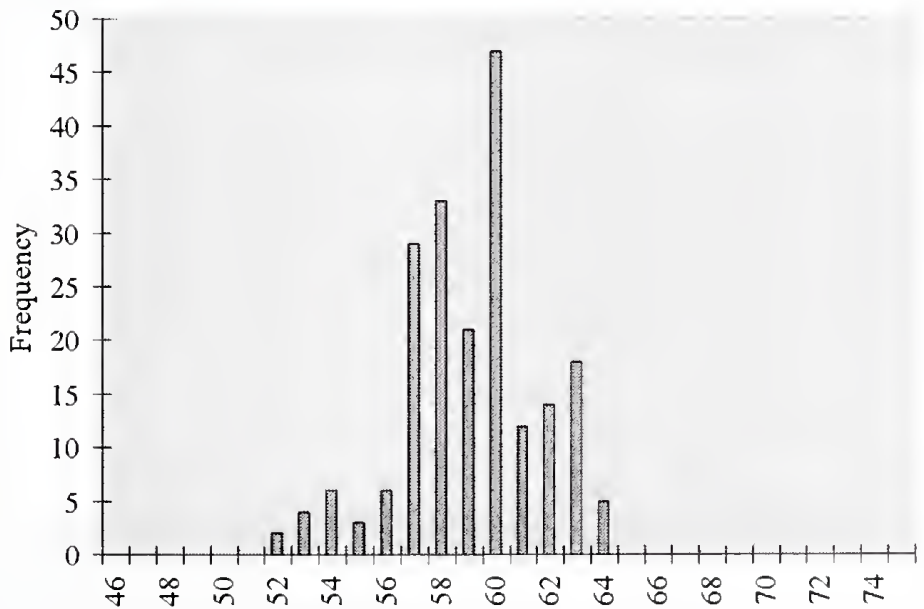


Casename Case 80

Description

\begin{tabular}{|c|c|c|c|c|c|}
\hline $\begin{array}{l}\text { Supply } \\
\text { Configuration }\end{array}$ & $\begin{array}{l}\text { Supply Discharge } \\
\text { Temperature }\left({ }^{\circ} \mathrm{C}\right)\end{array}$ & $\begin{array}{l}\text { Supply Discharge } \\
\text { RH }\end{array}$ & $\begin{array}{l}\text { Exhaust } \\
\text { Configuration }\end{array}$ & $\begin{array}{l}\text { Exhaust } \\
\text { Temperature }\left({ }^{\circ} \mathrm{C}\right)\end{array}$ & $\begin{array}{l}\text { Exhaust } \\
\text { RH }\end{array}$ \\
\hline Slot & 18.8 & $61 \%$ & 2 Door exhausts & 22 & $50 \%$ \\
\hline Change & Rack & Rack & Number of & Total mass of & $\begin{array}{l}\text { Room } \\
\text { Pressurisation }\end{array}$ \\
\hline $\begin{array}{l}\text { Station } \\
\text { ON }\end{array}$ & $\begin{array}{l}\text { Orientation } \\
\text { On wall }\end{array}$ & $\begin{array}{l}\text { Density } \\
\text { Single }\end{array}$ & $\begin{array}{l}\text { Mice in Room } \\
1050\end{array}$ & $\begin{array}{l}\text { Mice in Room } \\
21000 \mathrm{gr}\end{array}$ & $\begin{array}{l}\text { Pressurisation } \\
\text { neg } 100 \mathrm{cfm}\end{array}$ \\
\hline
\end{tabular}

Room

$\mathrm{ACH}$

15

Cage

Condition

Top On

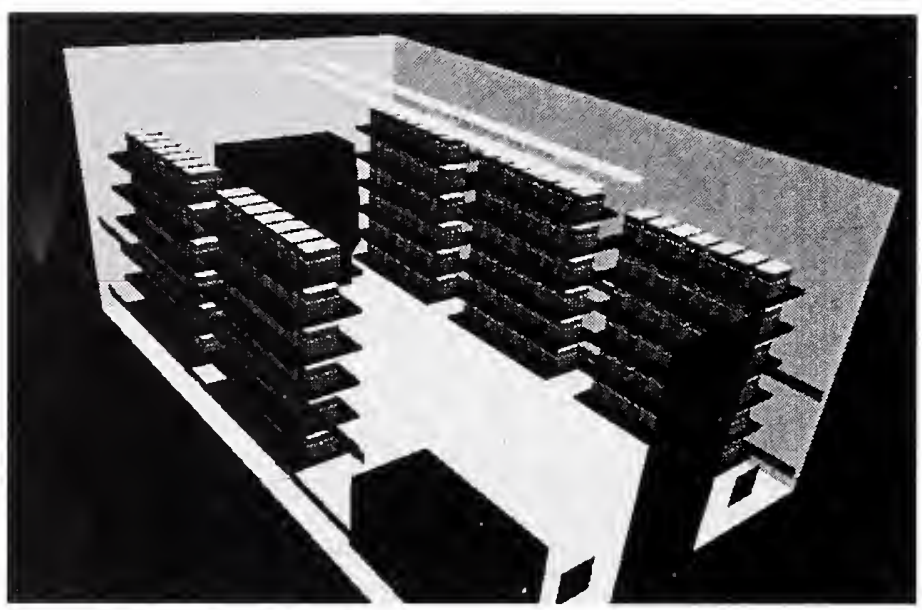

Analysis Results

Cage Occupied Zone

\begin{tabular}{|l|cc|c|c|}
\cline { 2 - 5 } \multicolumn{1}{c|}{} & \multicolumn{2}{c|}{ Temperature } & $\mathrm{CO}_{2}$ & RH \\
\cline { 2 - 5 } \multicolumn{1}{c|}{} & ${ }^{\circ} \mathrm{C}$ & ${ }^{\circ} \mathrm{F}$ & $(\mathrm{ppm})$ & \\
\hline Mean & 23.22 & 73.80 & 1707 & $58.68 \%$ \\
\hline S.D. & 0.44 & 0.79 & 311 & $2.60 \%$ \\
\hline Max. & 24.40 & 75.91 & 2688 & $64.90 \%$ \\
\hline
\end{tabular}

Cage Occupied Zone NH3 (ppm)

\begin{tabular}{|l|c|c|c|c|c|c|c|c|c|c|}
\hline Day & $\mathbf{1}$ & $\mathbf{2}$ & $\mathbf{3}$ & $\mathbf{4}$ & $\mathbf{5}$ & $\mathbf{6}$ & $\mathbf{7}$ & $\mathbf{8}$ & $\mathbf{9}$ & $\mathbf{1 0}$ \\
\hline Mean & 0.89 & 1.73 & 2.66 & 3.84 & 5.40 & 7.49 & 10.24 & 13.82 & 18.35 & 23.99 \\
\hline Max. & 1.40 & 2.72 & 4.19 & 6.05 & 8.50 & 11.79 & 16.14 & 21.77 & 28.91 & 37.79 \\
\hline
\end{tabular}

Room Breathing Zone

\begin{tabular}{|l|cc|c|c|}
\cline { 2 - 5 } \multicolumn{1}{c|}{} & \multicolumn{2}{c|}{ Temperature } & $\mathrm{CO}_{2}$ & RH \\
\cline { 2 - 5 } \multicolumn{1}{c|}{} & ${ }^{\circ} \mathrm{C}$ & ${ }^{\circ} \mathrm{F}$ & $(\mathbf{p p m})$ & \\
\hline Mean & 22.10 & 71.77 & 90 & $49.83 \%$ \\
\hline S.D. & 0.43 & 0.78 & 22 & \\
\hline Max. & 23.07 & 73.52 & 207 & \\
\hline
\end{tabular}

Room Breathing Zone NH3 (ppm)

\begin{tabular}{|l|c|c|c|c|c|c|c|c|c|c|}
\hline Day & $\mathbf{1}$ & $\mathbf{2}$ & $\mathbf{3}$ & $\mathbf{4}$ & $\mathbf{5}$ & $\mathbf{6}$ & $\mathbf{7}$ & $\mathbf{8}$ & $\mathbf{9}$ & $\mathbf{1 0}$ \\
\hline Mean & 0.05 & 0.09 & 0.14 & 0.20 & 0.29 & 0.40 & 0.54 & 0.73 & 0.97 & 1.27 \\
\hline Max. & 0.11 & 0.21 & 0.32 & 0.47 & 0.65 & 0.91 & 1.24 & 1.68 & 2.23 & 2.91 \\
\hline
\end{tabular}




\section{Histogram Distributions}

Cage occupied zone average temperature $\left({ }^{\circ} \mathrm{C}\right)$ distribution

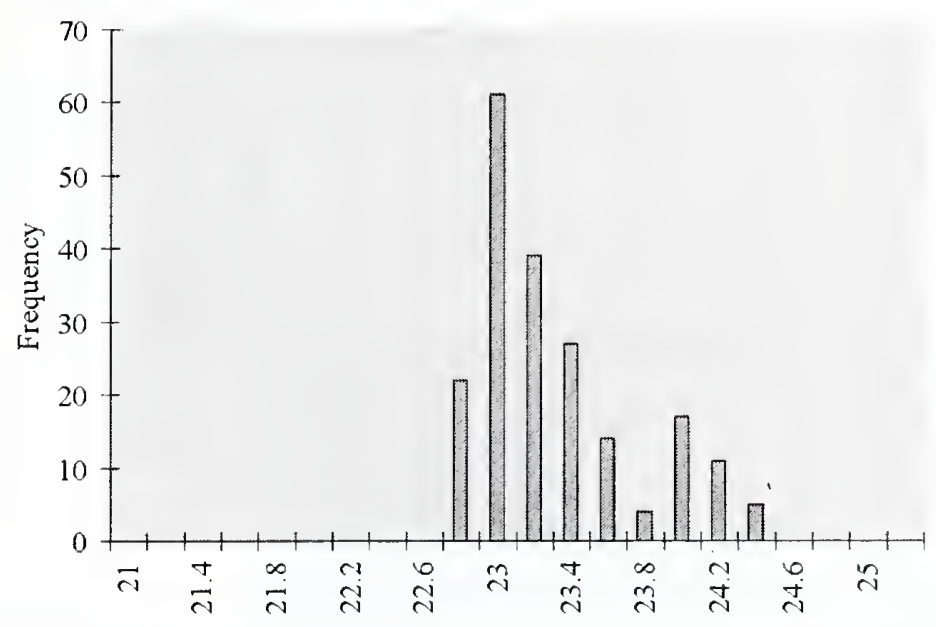

Cage occupied zone average contamination $(\mathrm{kg} / \mathrm{kg})$ distribution

Contamination conversion factors $(\mathrm{kg} / \mathrm{kg} \rightarrow \mathrm{ppm})$

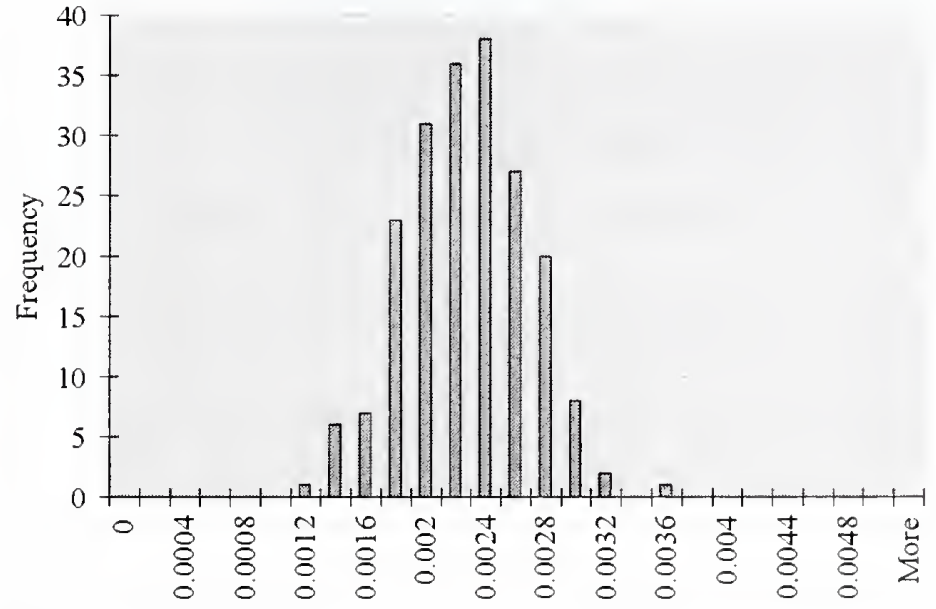

\begin{tabular}{|c|c|c|}
\hline Day & $\mathrm{CO}_{2}$ & $\mathrm{NH}_{3}$ \\
\hline $\mathbf{1}$ & 785000 & 408 \\
\hline $\mathbf{2}$ & 785000 & 795 \\
\hline $\mathbf{3}$ & 785000 & 1225 \\
\hline $\mathbf{4}$ & 785000 & 1766 \\
\hline $\mathbf{5}$ & 785000 & 2483 \\
\hline $\mathbf{6}$ & 785000 & 3443 \\
\hline 7 & 785000 & 4712 \\
\hline $\mathbf{8}$ & 785000 & 6356 \\
\hline $\mathbf{9}$ & 785000 & 8442 \\
\hline $\mathbf{1 0}$ & 785000 & 11384 \\
\hline
\end{tabular}

Cage occupied zone average relative humidity (\%) distribution

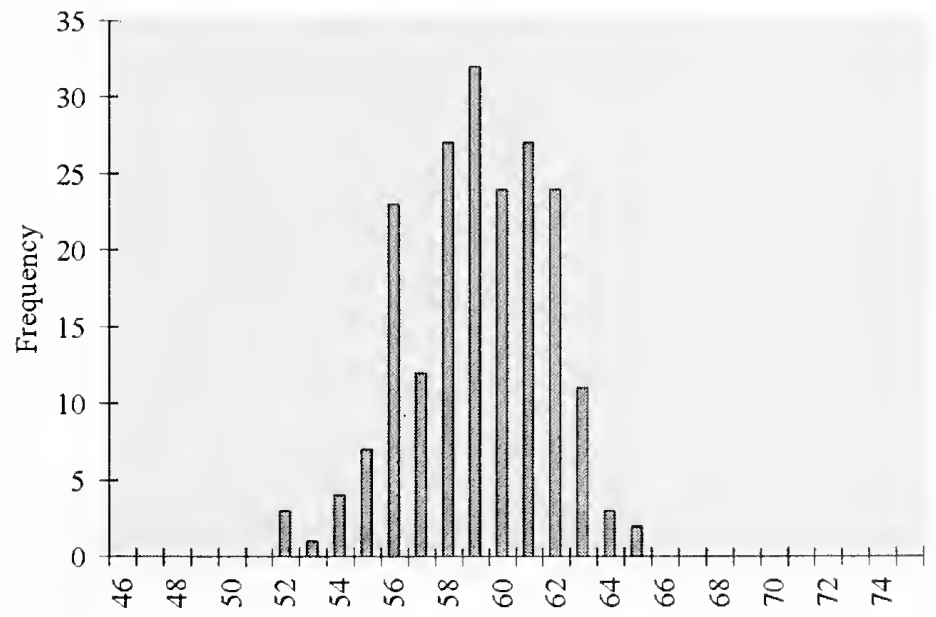


Casename

Case 81

Description

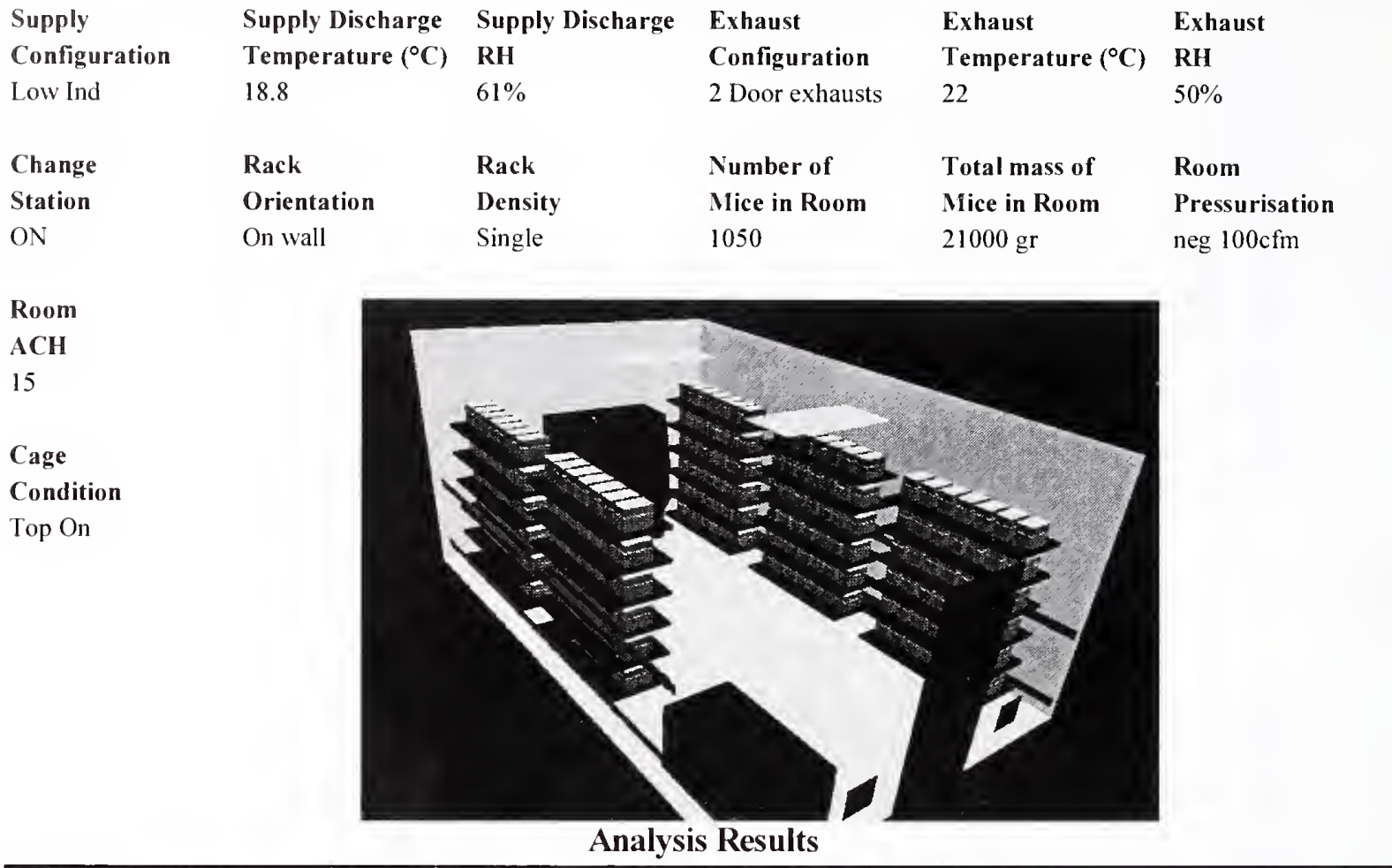

Cage Occupied Zone

\begin{tabular}{|l|cc|c|c|}
\cline { 2 - 5 } \multicolumn{1}{c|}{} & \multicolumn{2}{c|}{ Temperature } & $\mathrm{CO}_{2}$ & RH \\
\cline { 2 - 5 } \multicolumn{1}{c|}{} & ${ }^{\circ} \mathrm{C}$ & ${ }^{\circ} \mathrm{F}$ & $(\mathrm{ppm})$ & \\
\hline Mean & 23.40 & 74.13 & 1741 & $58.28 \%$ \\
\hline S.D. & 0.48 & 0.86 & 293 & $2.45 \%$ \\
\hline Max. & 24.45 & 76.00 & 2720 & $63.62 \%$ \\
\hline
\end{tabular}

Cage Occupied Zone NH3 (ppm)

\begin{tabular}{|l|c|c|c|c|c|c|c|c|c|c|}
\hline Day & $\mathbf{1}$ & $\mathbf{2}$ & $\mathbf{3}$ & $\mathbf{4}$ & $\mathbf{5}$ & $\mathbf{6}$ & $\mathbf{7}$ & $\mathbf{8}$ & $\mathbf{9}$ & $\mathbf{1 0}$ \\
\hline Mean & 0.91 & $\mathbf{1 . 7 6}$ & 2.72 & 3.92 & 5.51 & 7.64 & 10.45 & 14.10 & 18.72 & 24.47 \\
\hline Max. & 1.41 & 2.75 & 4.24 & 6.12 & 8.60 & 11.93 & 16.33 & 22.03 & 29.25 & 38.24 \\
\hline
\end{tabular}

Room Breathing Zone

\begin{tabular}{|l|cc|c|c|}
\cline { 2 - 5 } \multicolumn{1}{c|}{} & \multicolumn{2}{c|}{ Temperature } & $\mathrm{CO}_{2}$ & RH \\
\cline { 2 - 5 } \multicolumn{1}{c|}{} & ${ }^{\circ} \mathrm{C}$ & ${ }^{\circ} \mathrm{F}$ & $(\mathrm{ppm})$ & \\
\hline Mean & 22.45 & 72.41 & 89 & $48.73 \%$ \\
\hline S.D. & 0.55 & 0.99 & 30 & \\
\hline Max. & 23.36 & 74.05 & 278 & \\
\hline
\end{tabular}

Room Breathing Zone NH3 (ppm)

\begin{tabular}{|l|c|c|c|c|c|c|c|c|c|c|}
\hline Day & $\mathbf{1}$ & $\mathbf{2}$ & $\mathbf{3}$ & $\mathbf{4}$ & $\mathbf{5}$ & $\mathbf{6}$ & $\mathbf{7}$ & $\mathbf{8}$ & $\mathbf{9}$ & $\mathbf{1 0}$ \\
\hline Mean & 0.05 & 0.09 & 0.14 & 0.20 & 0.28 & 0.39 & 0.54 & 0.72 & 0.96 & 1.25 \\
\hline Max. & 0.14 & 0.28 & 0.43 & 0.63 & 0.88 & 1.22 & 1.67 & 2.25 & 2.99 & 3.91 \\
\hline
\end{tabular}




\section{Histogram Distributions}

Cage occupied zone average temperature $\left({ }^{\circ} \mathrm{C}\right)$ distribution

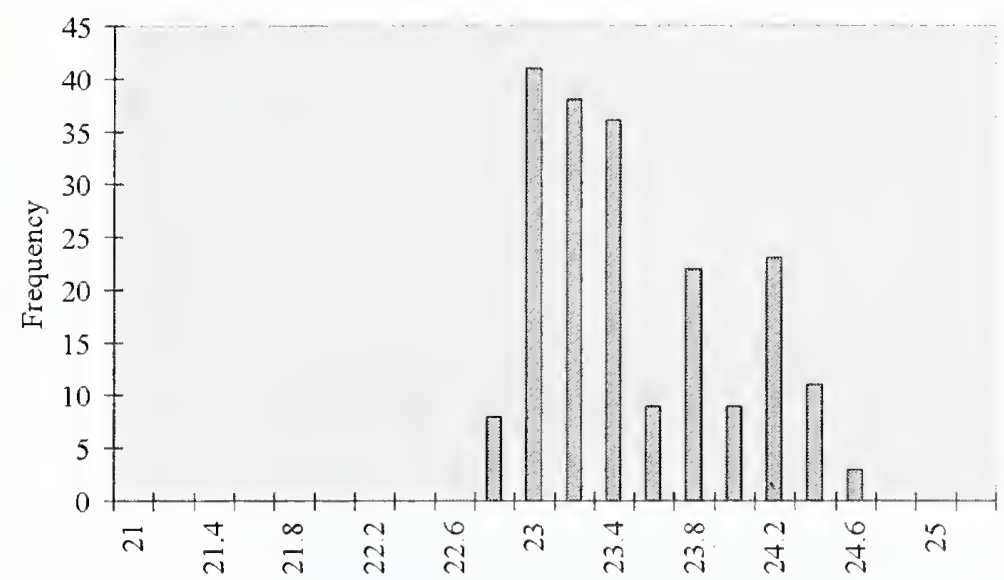

Cage occupied zone average contamination $(\mathrm{kg} / \mathrm{kg})$ distribution

Contamination conversion factors $(\mathrm{kg} / \mathrm{kg} \rightarrow \mathrm{ppm})$

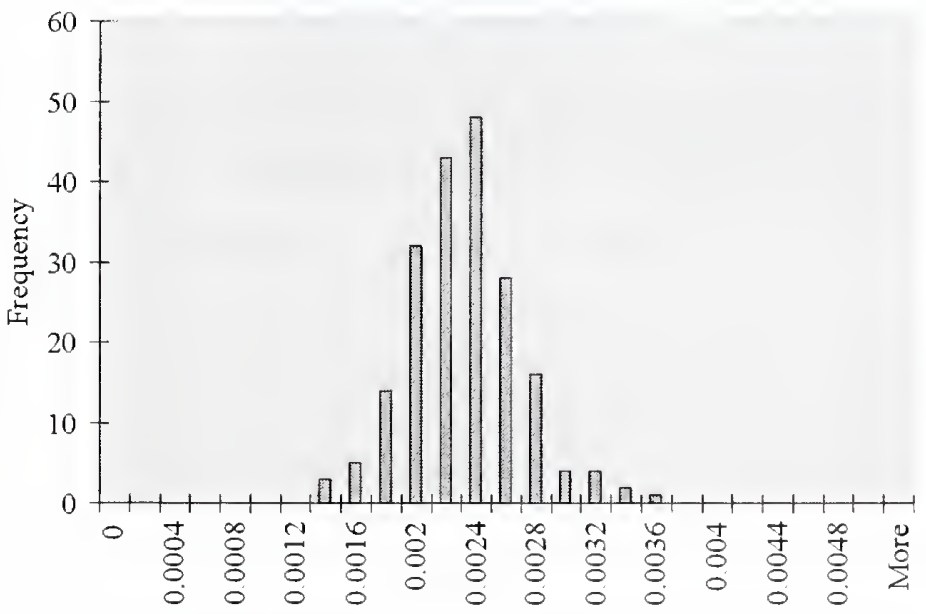

\begin{tabular}{|c|c|c|}
\hline Day & $\mathrm{CO}_{2}$ & $\mathrm{NH}_{3}$ \\
\hline 1 & 785000 & 408 \\
\hline 2 & 785000 & 795 \\
\hline 3 & 785000 & 1225 \\
\hline 4 & 785000 & 1766 \\
\hline 5 & 785000 & 2483 \\
\hline 6 & 785000 & 3443 \\
\hline 7 & 785000 & 4712 \\
\hline $\mathbf{8}$ & 785000 & 6356 \\
\hline 9 & 785000 & 8442 \\
\hline 10 & 785000 & 11384 \\
\hline
\end{tabular}

Cage occupied zone average relative humidity (\%) distribution

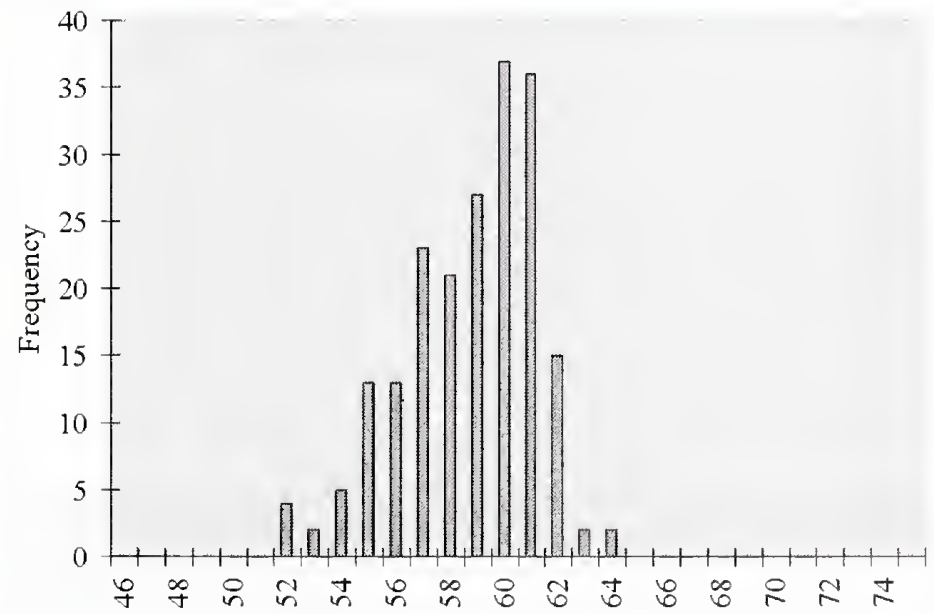


Casename

Case 82

Description

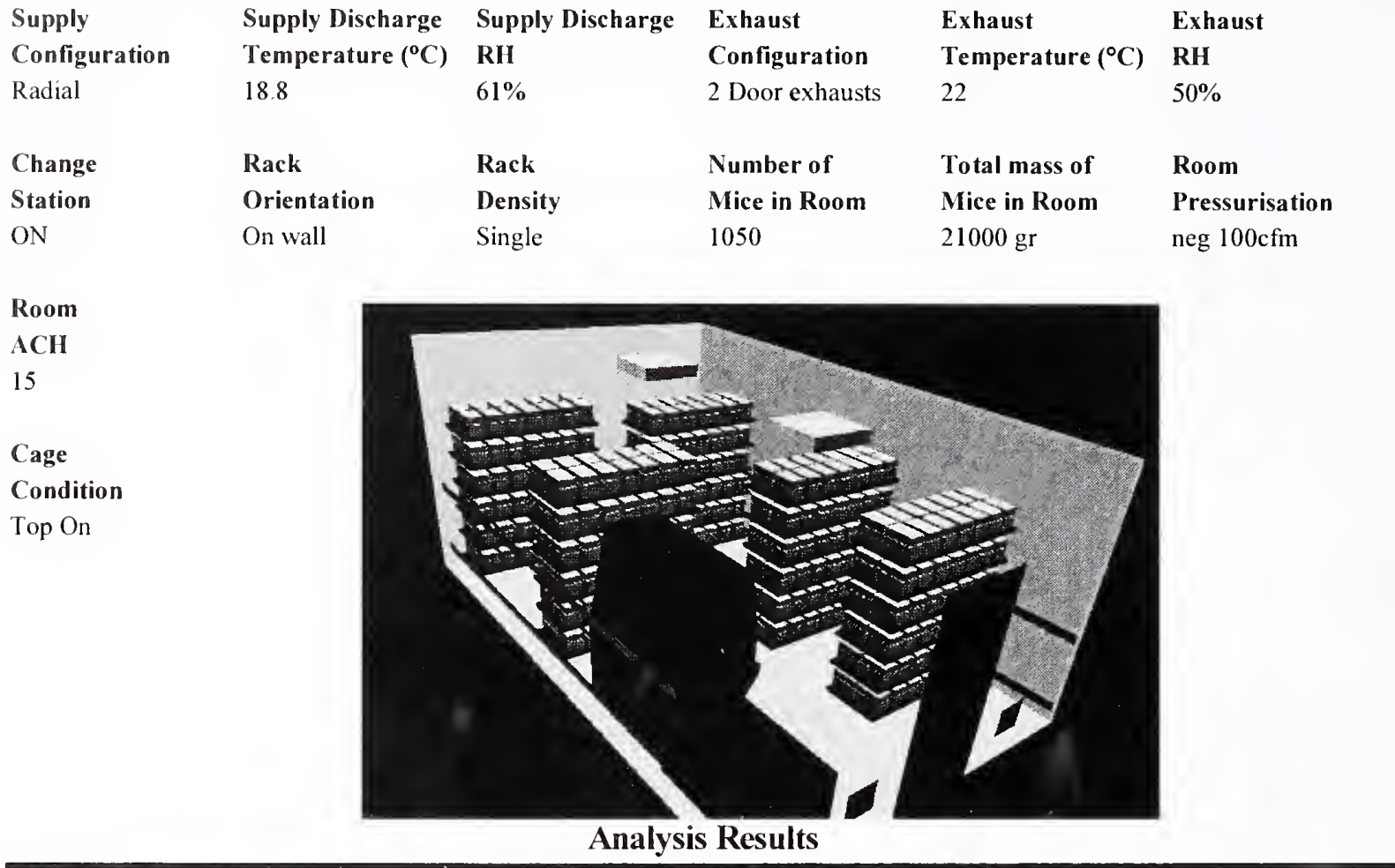

Cage Occupied Zone

\begin{tabular}{|l|cc|c|c|}
\cline { 2 - 5 } \multicolumn{1}{c|}{} & \multicolumn{2}{c|}{ Temperature } & $\mathbf{C O}_{2}$ & RH \\
\cline { 2 - 5 } \multicolumn{1}{c|}{} & ${ }^{\circ} \mathrm{C}$ & ${ }^{\circ} \mathrm{F}$ & $(\mathbf{p p m})$ & \\
\hline Mean & 22.29 & 72.13 & 1667 & $61.88 \%$ \\
\hline S.D. & 0.53 & 0.96 & 419 & $3.59 \%$ \\
\hline Max. & 23.63 & 74.53 & 2794 & $68.39 \%$ \\
\hline
\end{tabular}

Cage Occupied Zone NH3 (ppm)

\begin{tabular}{|l|c|c|c|c|c|c|c|c|c|c|}
\hline Day & $\mathbf{1}$ & $\mathbf{2}$ & $\mathbf{3}$ & $\mathbf{4}$ & $\mathbf{5}$ & $\mathbf{6}$ & $\mathbf{7}$ & $\mathbf{8}$ & $\mathbf{9}$ & $\mathbf{1 0}$ \\
\hline Mean & 0.87 & 1.69 & 2.60 & 3.92 & 5.72 & 8.06 & 11.28 & 14.91 & 19.35 & 24.34 \\
\hline Max. & 1.46 & 2.83 & 4.36 & 6.58 & 9.59 & 13.51 & 18.91 & 25.00 & 32.45 & 40.81 \\
\hline
\end{tabular}

Room Breathing Zone

\begin{tabular}{|l|cc|c|c|}
\cline { 2 - 5 } \multicolumn{1}{c|}{} & \multicolumn{2}{c|}{ Temperature } & $\mathrm{CO}_{2}$ & RH \\
\cline { 2 - 5 } \multicolumn{1}{c|}{} & ${ }^{\circ} \mathrm{C}$ & ${ }^{\circ} \mathrm{F}$ & $(\mathbf{p p m})$ & \\
\hline Mean & 21.26 & 70.27 & 116 & $52.75 \%$ \\
\hline S.D. & 0.91 & 1.64 & 53 & \\
\hline Max. & 27.19 & 80.94 & 332 & \\
\hline
\end{tabular}

Room Breathing Zone NH3 (ppm)

\begin{tabular}{|l|c|c|c|c|c|c|c|c|c|c|}
\hline Day & $\mathbf{1}$ & $\mathbf{2}$ & $\mathbf{3}$ & $\mathbf{4}$ & $\mathbf{5}$ & $\mathbf{6}$ & $\mathbf{7}$ & $\mathbf{8}$ & $\mathbf{9}$ & $\mathbf{1 0}$ \\
\hline Mean & 0.06 & 0.12 & 0.18 & 0.27 & 0.40 & 0.56 & 0.79 & 1.04 & 1.35 & 1.70 \\
\hline Max. & 0.17 & 0.34 & 0.52 & 0.78 & 1.14 & 1.61 & 2.25 & 2.97 & 3.86 & 4.86 \\
\hline
\end{tabular}




\section{Histogram Distributions}

Cage occupied zone average temperature $\left({ }^{\circ} \mathrm{C}\right)$ distribution

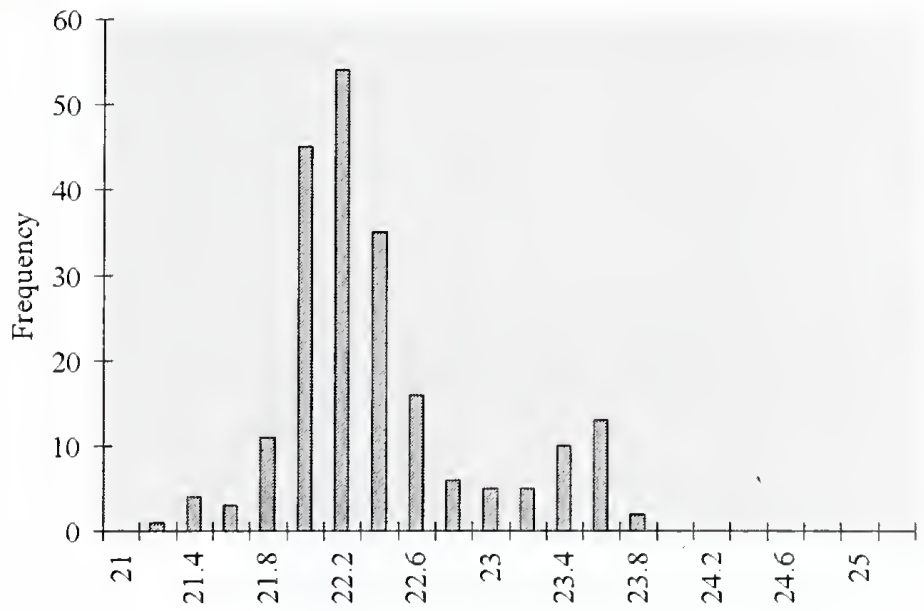

Cage occupied zone average contamination $(\mathrm{kg} / \mathrm{kg})$ distribution

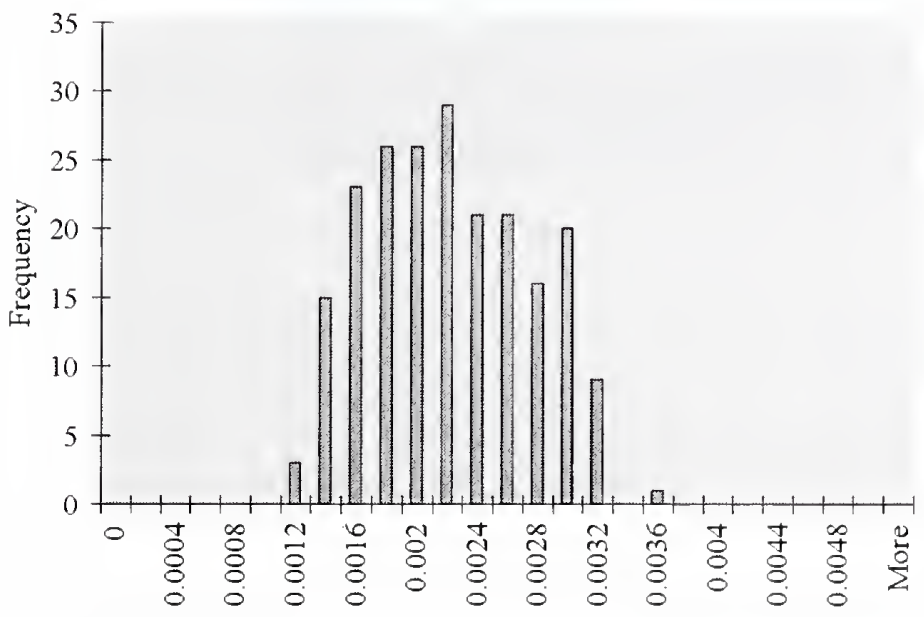

Cage occupied zone average relative humidity (\%) distribution

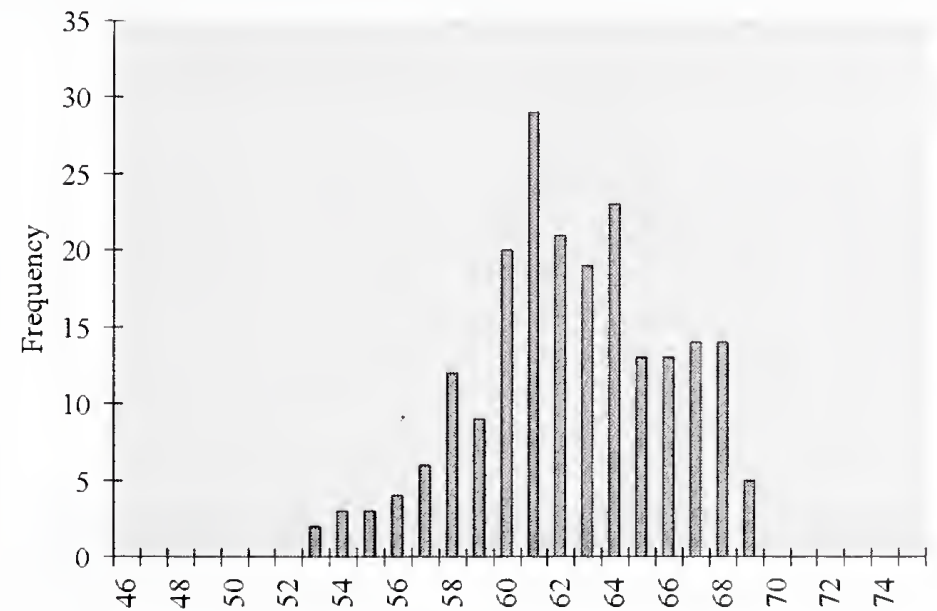

Contamination conversion factors $(\mathrm{kg} / \mathrm{kg} \rightarrow \mathrm{ppm})$

\begin{tabular}{|c|c|c|}
\hline Day & $\mathrm{CO}_{2}$ & $\mathrm{NH}_{3}$ \\
\hline $\mathbf{1}$ & 785000 & 410 \\
\hline 2 & 785000 & 795 \\
\hline 3 & 785000 & 1225 \\
\hline 4 & 785000 & 1848 \\
\hline $\mathbf{5}$ & 785000 & 2695 \\
\hline $\mathbf{6}$ & 785000 & 3795 \\
\hline 7 & 785000 & 5313 \\
\hline $\mathbf{8}$ & 785000 & 7024 \\
\hline 9 & 785000 & 9115 \\
\hline 10 & 785000 & 11384 \\
\hline
\end{tabular}


Casename

Case 83

Description

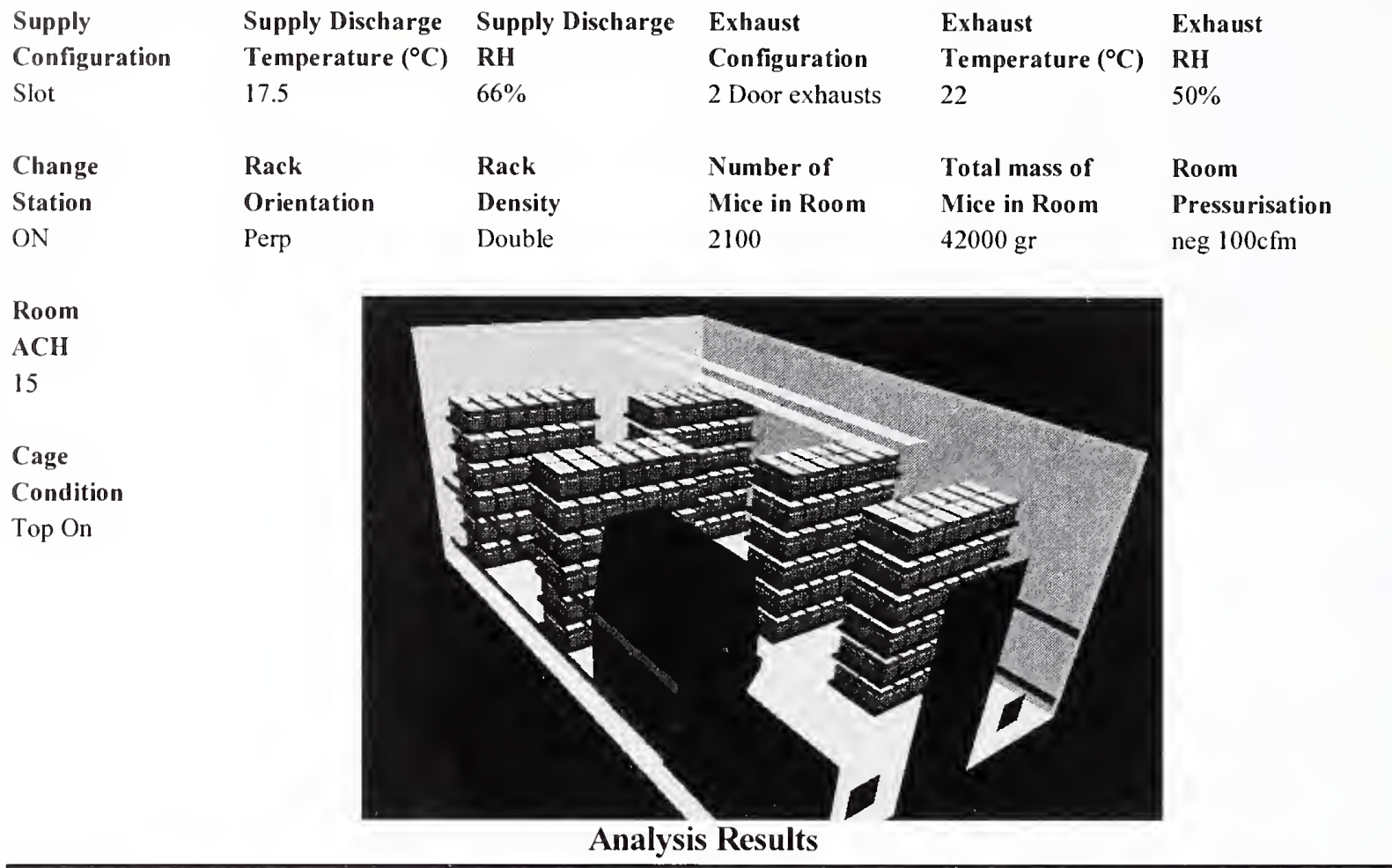

Cage Occupied Zone

\begin{tabular}{|l|cc|c|c|}
\cline { 2 - 5 } \multicolumn{1}{c|}{} & \multicolumn{2}{c|}{ Temperature } & $\mathbf{C O}_{2}$ & RH \\
\cline { 2 - 5 } \multicolumn{1}{c|}{} & ${ }^{\circ} \mathrm{C}$ & ${ }^{\circ} \mathrm{F}$ & $(\mathbf{p p m})$ & \\
\hline Mean & 22.20 & 71.97 & 1756 & $66.78 \%$ \\
\hline S.D. & 0.66 & 1.19 & 477 & $2.60 \%$ \\
\hline Max. & 23.55 & 74.39 & 3023 & $75.06 \%$ \\
\hline
\end{tabular}

Cage Occupied Zone NH3 (ppm)

\begin{tabular}{|l|c|c|c|c|c|c|c|c|c|c|}
\hline Day & $\mathbf{1}$ & $\mathbf{2}$ & $\mathbf{3}$ & $\mathbf{4}$ & $\mathbf{5}$ & $\mathbf{6}$ & $\mathbf{7}$ & $\mathbf{8}$ & $\mathbf{9}$ & $\mathbf{1 0}$ \\
\hline Mean & 0.94 & 1.78 & 2.74 & 5.15 & 8.67 & 12.87 & 19.34 & 24.01 & 28.75 & 30.99 \\
\hline Max. & 1.61 & 3.06 & 4.72 & 8.87 & 14.92 & 22.15 & 33.31 & 41.33 & 49.50 & 53.35 \\
\hline
\end{tabular}

Room Breathing Zone

\begin{tabular}{|l|cc|c|c|}
\cline { 2 - 5 } \multicolumn{1}{c|}{} & \multicolumn{2}{c|}{ Temperature } & $\mathrm{CO}_{2}$ & RH \\
\cline { 2 - 5 } \multicolumn{1}{c|}{} & ${ }^{\circ} \mathrm{C}$ & ${ }^{\circ} \mathrm{F}$ & $(\mathbf{p p m})$ & \\
\hline Mean & 21.44 & 70.58 & 167 & $52.60 \%$ \\
\hline S.D. & 0.81 & 1.46 & 42 & \\
\hline Max. & 26.98 & 80.56 & 403 & \\
\hline
\end{tabular}

Room Breathing Zone NH3 (ppm)

\begin{tabular}{|l|c|c|c|c|c|c|c|c|c|c|}
\hline Day & $\mathbf{1}$ & $\mathbf{2}$ & $\mathbf{3}$ & $\mathbf{4}$ & $\mathbf{5}$ & $\mathbf{6}$ & $\mathbf{7}$ & $\mathbf{8}$ & $\mathbf{9}$ & $\mathbf{1 0}$ \\
\hline Mean & 0.09 & 0.17 & 0.26 & 0.49 & 0.82 & 1.22 & 1.84 & 2.28 & 2.73 & 2.94 \\
\hline Max. & 0.21 & 0.41 & 0.63 & 1.18 & 1.99 & 2.95 & 4.44 & 5.51 & 6.60 & 7.11 \\
\hline
\end{tabular}




\section{Histogram Distributions}

Cage occupied zone average temperature $\left({ }^{\circ} \mathrm{C}\right)$ distribution

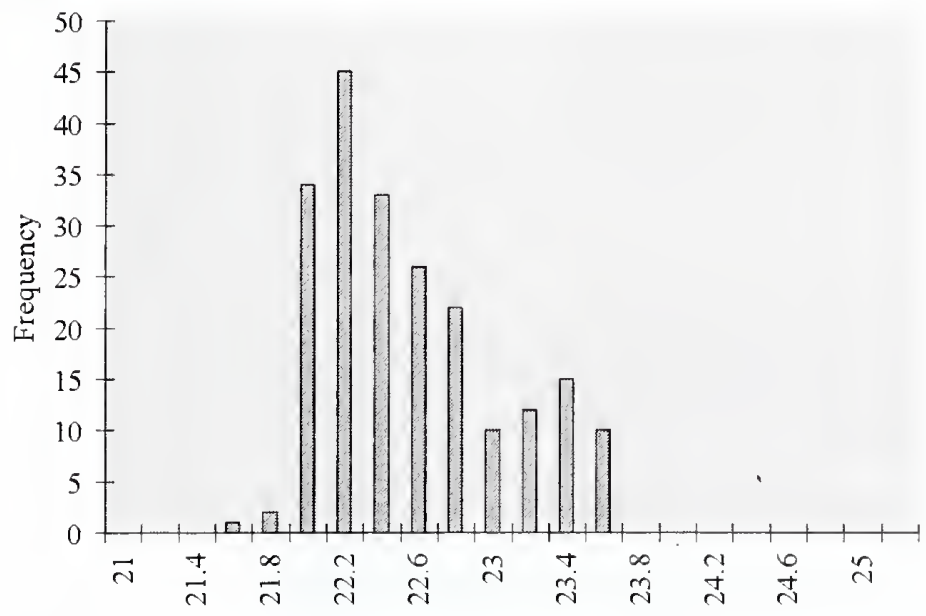

Cage occupied zone average contamination $(\mathrm{kg} / \mathrm{kg})$ distribution

Contamination conversion factors $(\mathrm{kg} / \mathrm{kg} \rightarrow \mathrm{ppm})$

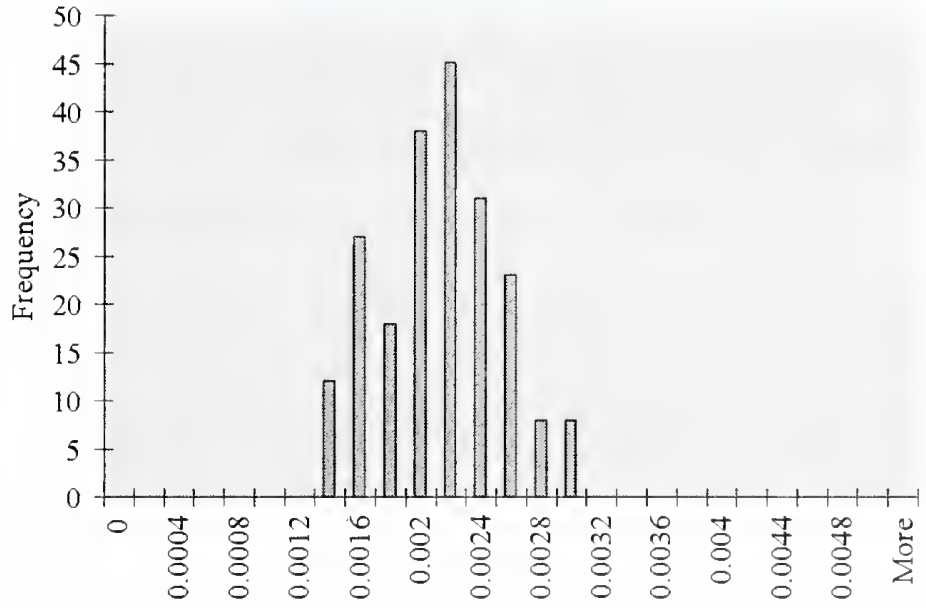

\begin{tabular}{|c|c|c|}
\hline Day & $\mathrm{CO}_{2}$ & $\mathrm{NH}_{3}$ \\
\hline $\mathbf{1}$ & 785000 & 418 \\
\hline $\mathbf{2}$ & 785000 & 795 \\
\hline $\mathbf{3}$ & 785000 & 1225 \\
\hline $\mathbf{4}$ & 785000 & 2303 \\
\hline $\mathbf{5}$ & 785000 & 3876 \\
\hline $\mathbf{6}$ & 785000 & 5752 \\
\hline 7 & 785000 & 8650 \\
\hline $\mathbf{8}$ & 785000 & 10734 \\
\hline $\mathbf{9}$ & 785000 & 12856 \\
\hline 10 & 785000 & 11384 \\
\hline
\end{tabular}

Cage occupied zone average relative humidity (\%) distribution

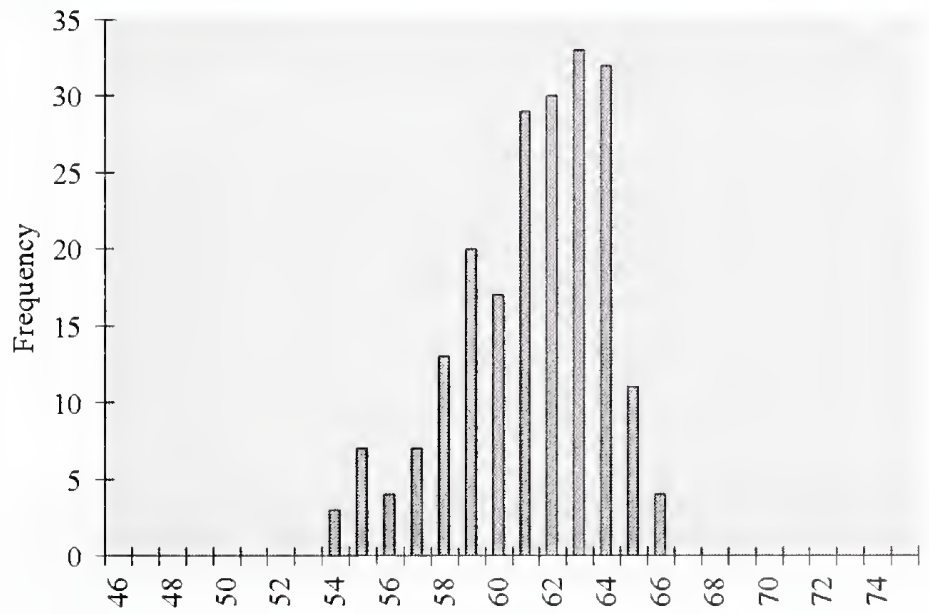


Casename $\quad$ Case 84

Description

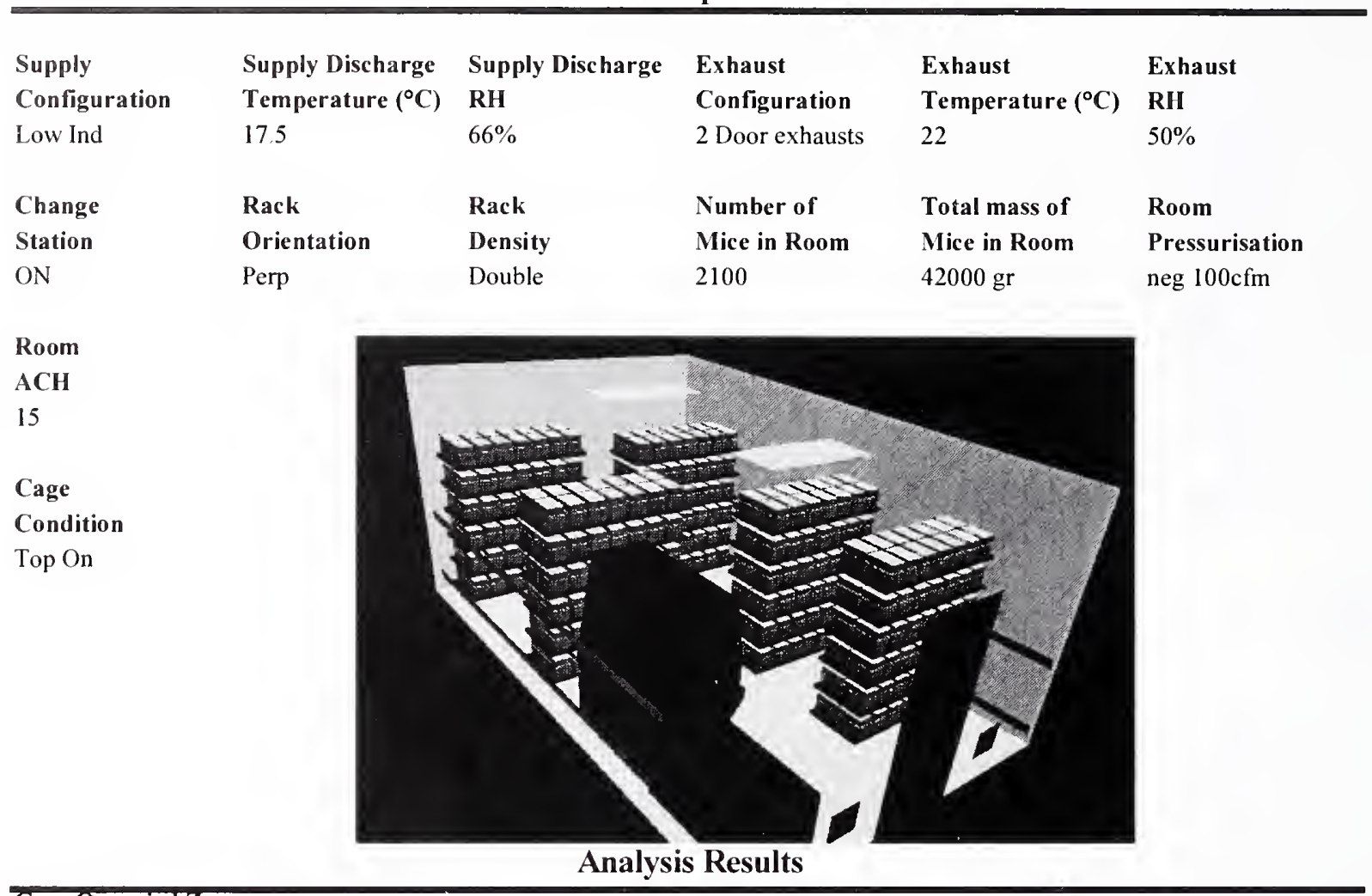

Cage Occupied Zone

\begin{tabular}{|l|cc|c|c|}
\cline { 2 - 5 } \multicolumn{1}{c|}{} & \multicolumn{2}{c|}{ Temperature } & $\mathrm{CO}_{2}$ & RH \\
\cline { 2 - 5 } \multicolumn{1}{c|}{} & ${ }^{\circ} \mathrm{C}$ & ${ }^{\circ} \mathrm{F}$ & $(\mathrm{ppm})$ & \\
\hline Mean & 22.48 & 72.46 & 1853 & $60.88 \%$ \\
\hline S.D. & 0.48 & 0.87 & 443 & $3.20 \%$ \\
\hline Max. & 23.73 & 74.71 & 2964 & $66.31 \%$ \\
\hline
\end{tabular}

Cage Occupied Zone NH3 (ppm)

\begin{tabular}{|l|c|c|c|c|c|c|c|c|c|c|}
\hline Day & $\mathbf{1}$ & $\mathbf{2}$ & $\mathbf{3}$ & $\mathbf{4}$ & $\mathbf{5}$ & $\mathbf{6}$ & $\mathbf{7}$ & $\mathbf{8}$ & 9 & $\mathbf{1 0}$ \\
\hline Mean & 0.96 & 1.88 & 2.89 & 4.17 & 5.86 & 8.13 & 11.12 & 15.00 & 19.93 & 26.05 \\
\hline Max. & 1.54 & 3.00 & 4.62 & 6.67 & 9.37 & 13.00 & 17.79 & 24.00 & 31.87 & 41.66 \\
\hline
\end{tabular}

Room Breathing Zone

\begin{tabular}{|l|cc|c|c|}
\cline { 2 - 5 } \multicolumn{1}{c|}{} & \multicolumn{2}{c|}{ Temperature } & $\mathrm{CO}_{2}$ & RH \\
\cline { 2 - 5 } \multicolumn{1}{c|}{} & ${ }^{\circ} \mathrm{C}$ & ${ }^{\circ} \mathrm{F}$ & $(\mathbf{p p m})$ & \\
\hline Mean & 21.48 & 70.67 & 152 & $52.33 \%$ \\
\hline S.D. & 0.89 & 1.60 & 47 & \\
\hline Max. & 26.19 & 79.14 & 380 & \\
\hline
\end{tabular}

Room Breathing Zone NH3 (ppm)

\begin{tabular}{|l|c|c|c|c|c|c|c|c|c|c|}
\hline Day & $\mathbf{1}$ & $\mathbf{2}$ & $\mathbf{3}$ & $\mathbf{4}$ & $\mathbf{5}$ & $\mathbf{6}$ & $\mathbf{7}$ & $\mathbf{8}$ & $\mathbf{9}$ & $\mathbf{1 0}$ \\
\hline Mean & 0.08 & 0.15 & 0.24 & 0.34 & 0.48 & 0.67 & 0.91 & 1.23 & 1.64 & 2.14 \\
\hline Max. & 0.20 & 0.38 & 0.59 & 0.85 & 1.20 & 1.67 & 2.28 & 3.07 & 4.08 & 5.34 \\
\hline
\end{tabular}




\section{Histogram Distributions}

Cage occupied zone average temperature $\left({ }^{\circ} \mathrm{C}\right)$ distribution

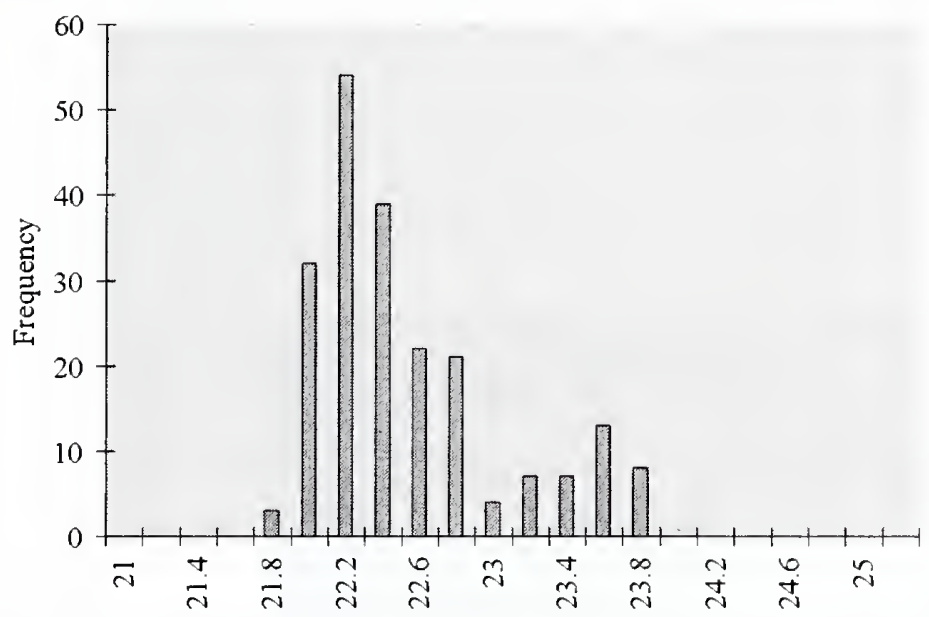

Cage occupied zone average contamination $(\mathrm{kg} / \mathrm{kg})$ distribution

Contamination conversion factors $(\mathrm{kg} / \mathrm{kg} \rightarrow \mathrm{ppm})$

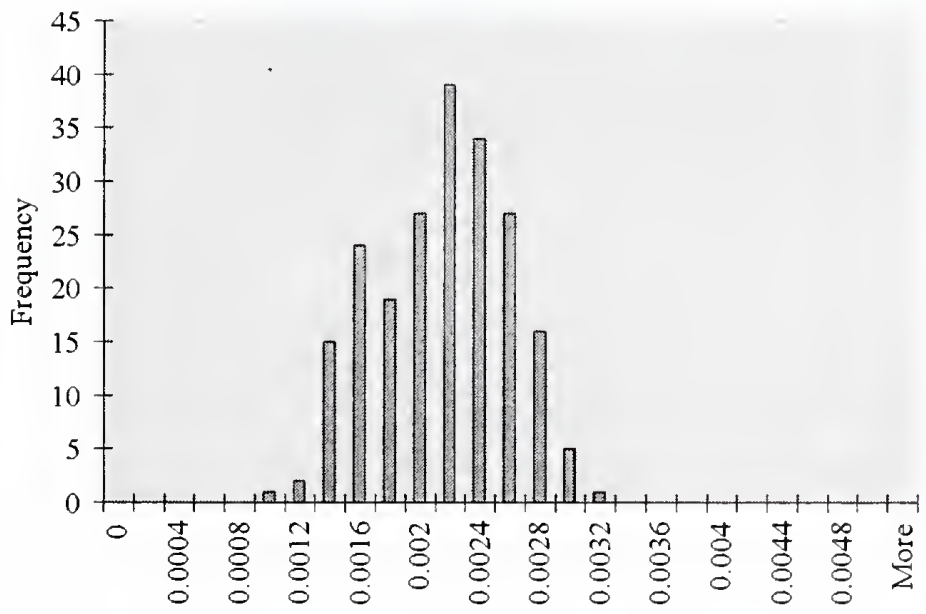

\begin{tabular}{|c|c|c|}
\hline Day & $\mathrm{CO}_{2}$ & $\mathrm{NH}_{3}$ \\
\hline $\mathbf{I}$ & 785000 & 408 \\
\hline $\mathbf{2}$ & 785000 & 795 \\
\hline $\mathbf{3}$ & 785000 & 1225 \\
\hline $\mathbf{4}$ & 785000 & 1766 \\
\hline $\mathbf{5}$ & 785000 & 2483 \\
\hline $\mathbf{6}$ & 785000 & 3443 \\
\hline 7 & 785000 & 4712 \\
\hline $\mathbf{8}$ & 785000 & 6356 \\
\hline $\mathbf{9}$ & 785000 & 8442 \\
\hline $\mathbf{1 0}$ & 785000 & 11384 \\
\hline
\end{tabular}

Cage occupied zone average relative humidity (\%) distribution

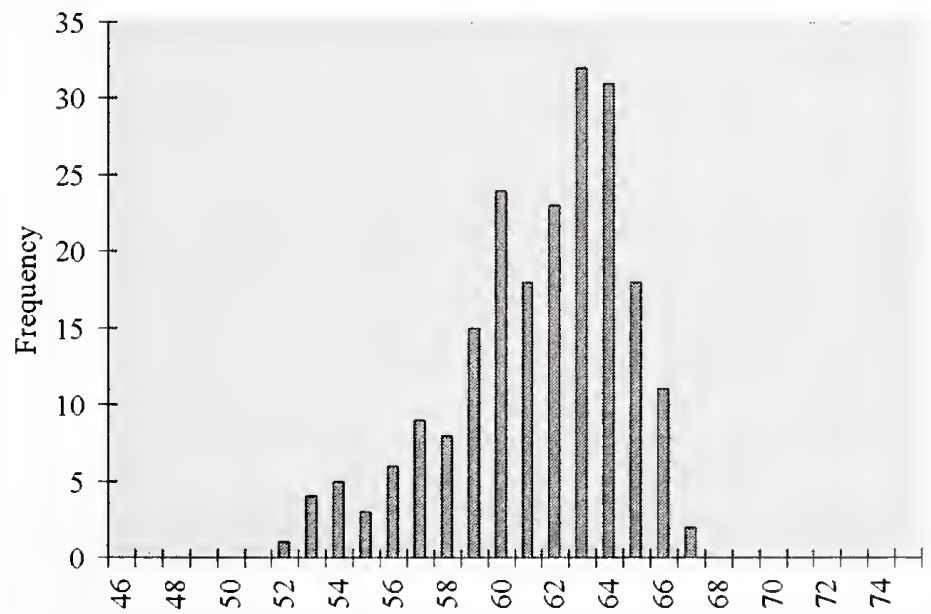


Cusename $\quad$ Case 85

Description

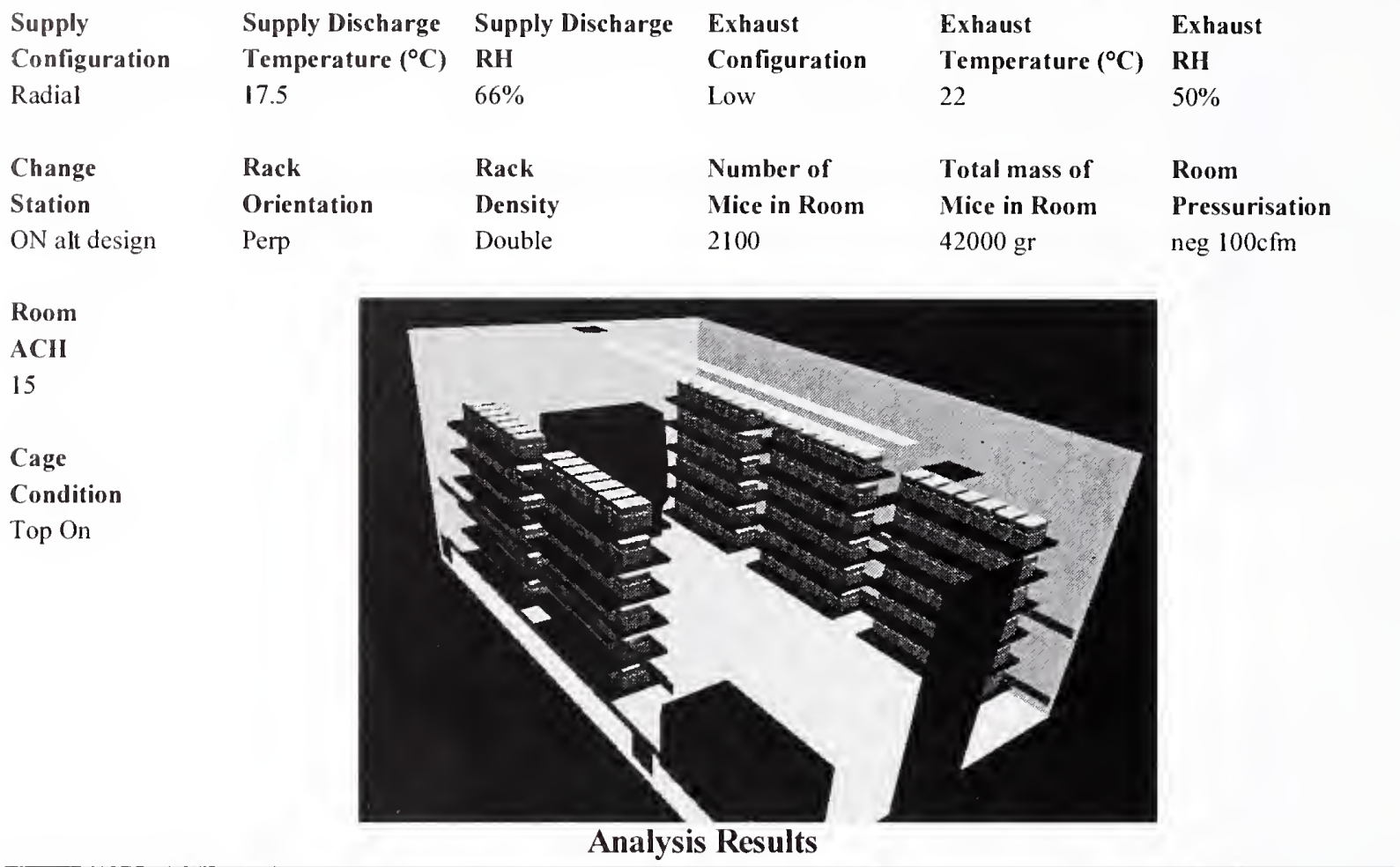

Cage Occupied Zone

\begin{tabular}{|l|cc|c|c|}
\cline { 2 - 5 } \multicolumn{1}{c|}{} & \multicolumn{2}{c|}{ Temperature } & $\mathbf{C O}_{2}$ & RH \\
\cline { 2 - 5 } \multicolumn{1}{c|}{} & ${ }^{\circ} \mathrm{C}$ & ${ }^{\circ} \mathrm{F}$ & $(\mathbf{p p m})$ & \\
\hline Mean & 22.37 & 72.27 & 1930 & $62.82 \%$ \\
\hline S.D. & 0.31 & 0.57 & 425 & $2.86 \%$ \\
\hline Max. & 23.02 & 73.44 & 2840 & $68.68 \%$ \\
\hline
\end{tabular}

Cage Occupied Zone NH3 (ppm)

\begin{tabular}{|l|c|c|c|c|c|c|c|c|c|c|}
\hline Day & $\mathbf{1}$ & $\mathbf{2}$ & $\mathbf{3}$ & $\mathbf{4}$ & $\mathbf{5}$ & $\mathbf{6}$ & $\mathbf{7}$ & $\mathbf{8}$ & $\mathbf{9}$ & $\mathbf{1 0}$ \\
\hline Mean & 1.01 & 1.95 & 3.01 & 4.76 & 7.19 & 10.26 & 14.64 & 19.02 & 24.18 & 29.32 \\
\hline Max. & 1.49 & 2.87 & 4.43 & 7.00 & 10.57 & 15.09 & 21.54 & 27.99 & 35.58 & 43.14 \\
\hline
\end{tabular}

Room Breathing Zone

\begin{tabular}{|l|cc|c|c|}
\cline { 2 - 5 } \multicolumn{1}{c|}{} & \multicolumn{2}{c|}{ Temperature } & $\mathrm{CO}_{2}$ & RH \\
\cline { 2 - 5 } \multicolumn{1}{c|}{} & ${ }^{\circ} \mathrm{C}$ & ${ }^{\circ} \mathrm{F}$ & $(\mathrm{ppm})$ & \\
\hline Mean & 20.74 & 69.33 & 132 & $54.66 \%$ \\
\hline S.D. & 0.36 & 0.65 & 53 & \\
\hline M.ax. & 21.49 & 70.68 & 445 & \\
\hline
\end{tabular}

Room Breathing Zone NH3 (ppm)

\begin{tabular}{|l|c|c|c|c|c|c|c|c|c|c|}
\hline Day & $\mathbf{1}$ & $\mathbf{2}$ & $\mathbf{3}$ & $\mathbf{4}$ & $\mathbf{5}$ & $\mathbf{6}$ & $\mathbf{7}$ & $\mathbf{8}$ & $\mathbf{9}$ & $\mathbf{1 0}$ \\
\hline Mean & 0.07 & 0.13 & 0.21 & 0.33 & 0.49 & 0.70 & 1.00 & 1.30 & 1.65 & 2.00 \\
\hline Max. & 0.23 & 0.45 & 0.69 & 1.10 & 1.66 & 2.36 & 3.37 & 4.38 & 5.57 & 6.75 \\
\hline
\end{tabular}




\section{Histogram Distributions}

Cage occupied zone average temperature $\left({ }^{\circ} \mathrm{C}\right)$ distribution

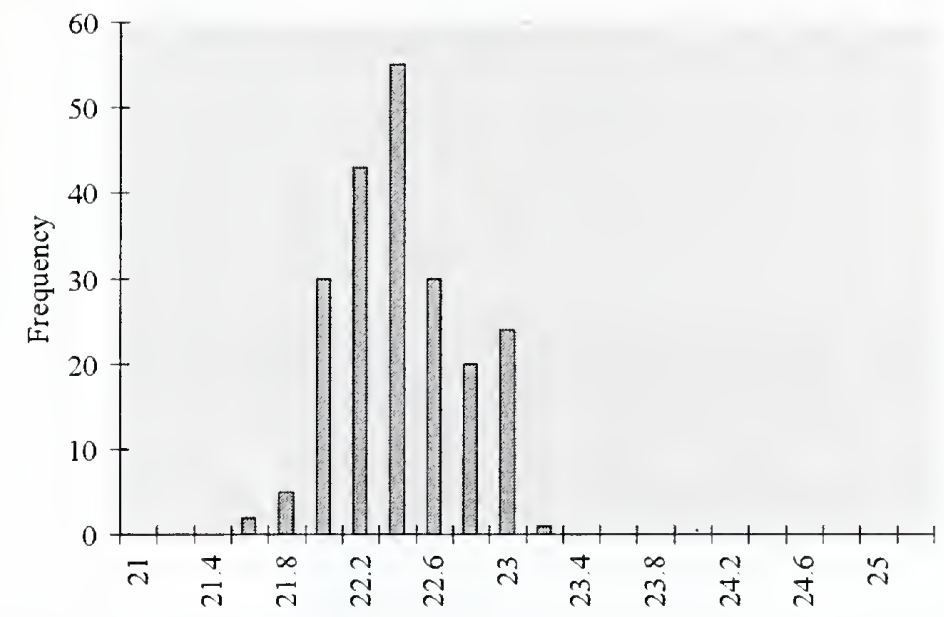

Cage occupied zone average contamination $(\mathrm{kg} / \mathrm{kg})$ distribution

Contamination conversion factors $(\mathrm{kg} / \mathrm{kg} \rightarrow \mathrm{ppm})$

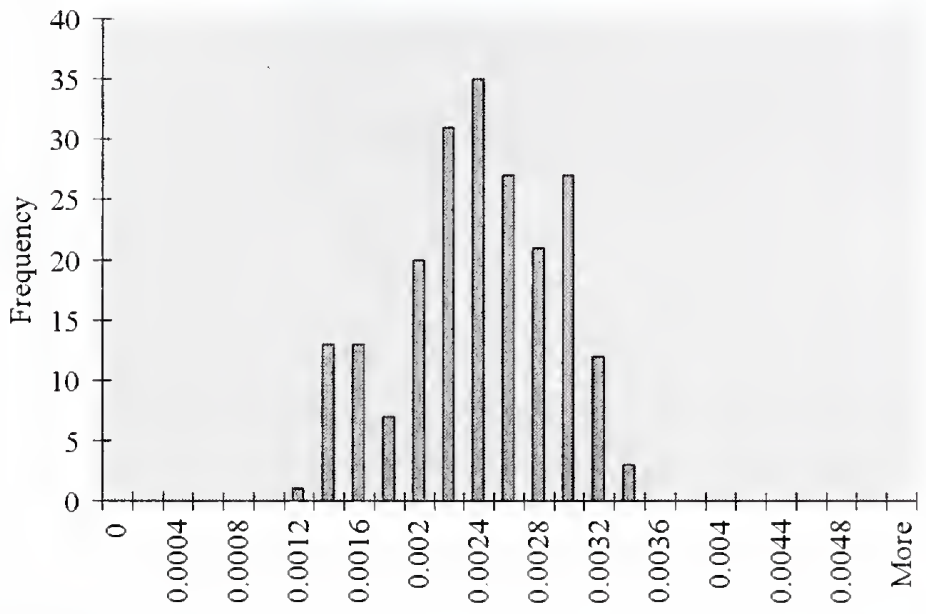

\begin{tabular}{|c|c|c|}
\hline Day & $\mathrm{CO}_{2}$ & $\mathrm{NH}_{3}$ \\
\hline $\mathbf{1}$ & 785000 & 411 \\
\hline $\mathbf{2}$ & 785000 & 795 \\
\hline $\mathbf{3}$ & 785000 & 1225 \\
\hline $\mathbf{4}$ & 785000 & 1935 \\
\hline $\mathbf{5}$ & 785000 & 2922 \\
\hline $\mathbf{6}$ & 785000 & 417 \\
\hline $\mathbf{7}$ & 785000 & 5953 \\
\hline $\mathbf{8}$ & 785000 & 7736 \\
\hline $\mathbf{9}$ & 785000 & 9833 \\
\hline $\mathbf{1 0}$ & 785000 & 11384 \\
\hline
\end{tabular}

Cage occupied zone average relative humidity (\%) distribution

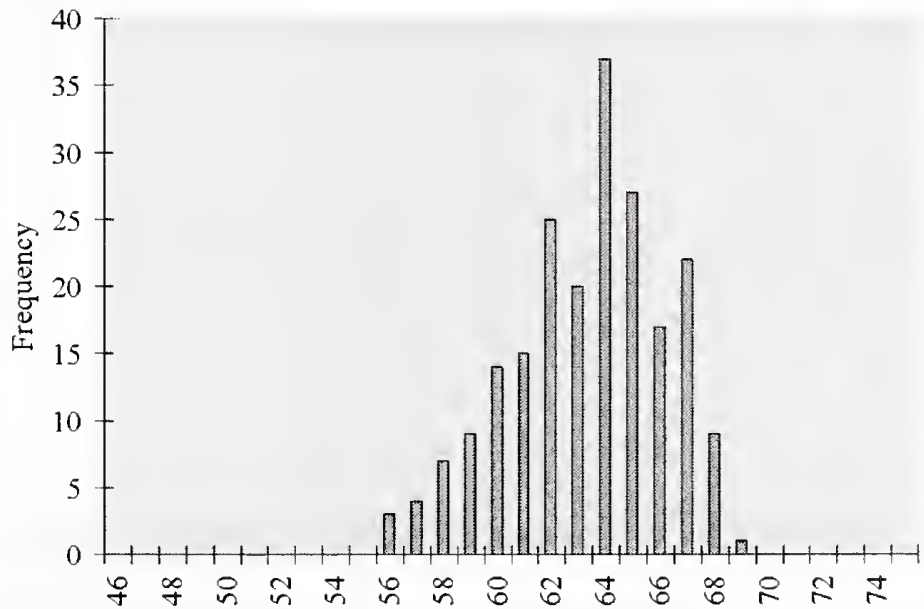




\section{Description}

Supply

Configuration

Slot

Change

Station

$\mathrm{ON}$ alt design

\section{Room \\ $\mathrm{ACH}$ \\ 15 \\ Cage \\ Condition \\ Top On}

Supply Discharge Supply Discharge

Temperature $\left({ }^{\circ} \mathrm{C}\right)$

RH

17.5

$66 \%$

Rack

Orientation

Perp

Rack

Density

Double
Exhaust

Configuration

Low

Number of

Mice in Room

2100
Exhaust

Temperature $\left({ }^{\circ} \mathrm{C}\right)$

22

Total mass of

Mice in Room

$42000 \mathrm{gr}$
Exhaust

RH

$50 \%$

Room

Pressurisation neg $100 \mathrm{cfm}$

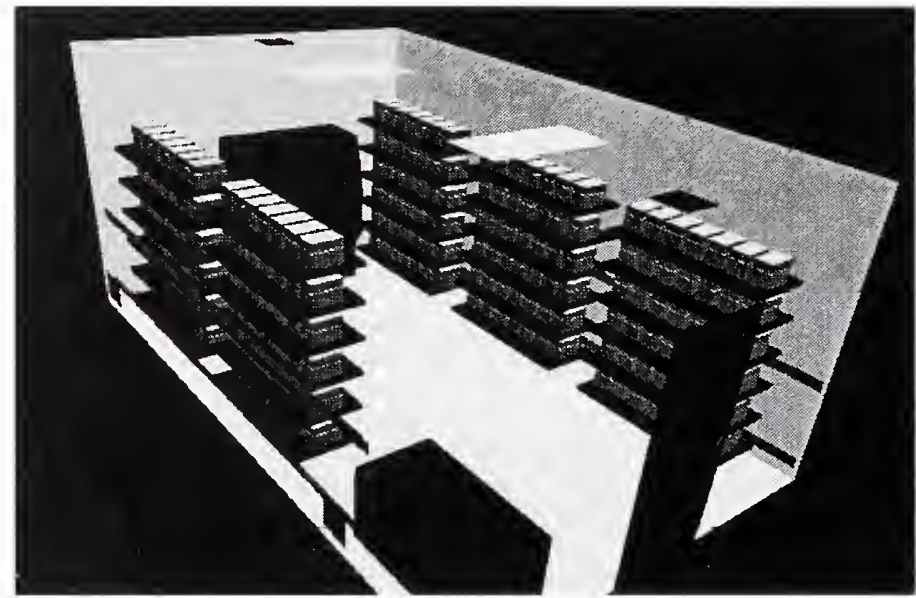

Analysis Results

Cage Occupied Zone

\begin{tabular}{|l|cc|c|c|}
\cline { 2 - 5 } \multicolumn{1}{c|}{} & \multicolumn{2}{c|}{ Temperature } & $\mathbf{C O}_{\mathbf{2}}$ & RH \\
\cline { 2 - 5 } \multicolumn{1}{c|}{} & ${ }^{\circ} \mathrm{C}$ & ${ }^{\circ} \mathrm{F}$ & $(\mathbf{p p m})$ & \\
\hline Mean & 22.30 & 72.13 & 1917 & $62.01 \%$ \\
\hline S.D. & 0.45 & 0.81 & 554 & $3.29 \%$ \\
\hline Max. & 23.25 & 73.84 & 3249 & $72.05 \%$ \\
\hline
\end{tabular}

Cage Occupied Zone NH3 (ppm)

\begin{tabular}{|l|c|c|c|c|c|c|c|c|c|c|}
\hline Day & $\mathbf{1}$ & $\mathbf{2}$ & $\mathbf{3}$ & $\mathbf{4}$ & $\mathbf{5}$ & $\mathbf{6}$ & $\mathbf{7}$ & $\mathbf{8}$ & $\mathbf{9}$ & $\mathbf{1 0}$ \\
\hline Mean & 1.00 & 1.94 & 2.99 & 4.54 & 6.66 & 9.39 & 13.19 & 17.39 & 22.50 & 28.15 \\
\hline Max. & 1.70 & 3.29 & 5.07 & 7.70 & 11.28 & 15.92 & 22.35 & 29.47 & 38.13 & 47.71 \\
\hline
\end{tabular}

Room Breathing Zone

\begin{tabular}{|l|cc|c|c|}
\cline { 2 - 5 } \multicolumn{1}{c|}{} & \multicolumn{2}{c|}{ Temperature } & $\mathrm{CO}_{2}$ & RH \\
\cline { 2 - 5 } \multicolumn{1}{c|}{} & ${ }^{\circ} \mathrm{C}$ & \multicolumn{1}{c|}{$\mathrm{F}$} & $(\mathrm{ppm})$ & \\
\hline Mean & 21.18 & 70.13 & 124 & $53.08 \%$ \\
\hline S.D. & 0.44 & 0.79 & 67 & \\
\hline Max. & 22.27 & 72.08 & 374 & \\
\hline
\end{tabular}

Room Breathing Zone NH3 (ppm)

\begin{tabular}{|l|c|c|c|c|c|c|c|c|c|c|}
\hline Day & $\mathbf{1}$ & $\mathbf{2}$ & $\mathbf{3}$ & $\mathbf{4}$ & $\mathbf{5}$ & $\mathbf{6}$ & $\mathbf{7}$ & $\mathbf{8}$ & 9 & $\mathbf{1 0}$ \\
\hline Mean & 0.06 & 0.13 & 0.19 & 0.29 & 0.43 & 0.61 & 0.85 & 1.13 & 1.46 & 1.82 \\
\hline Max. & 0.20 & 0.38 & 0.58 & 0.89 & 130 & 1.83 & 2.57 & 3.39 & 4.39 & 5.49 \\
\hline
\end{tabular}




\section{Histogram Distributions}

Cage occupied zone average temperature $\left({ }^{\circ} \mathrm{C}\right)$ distribution

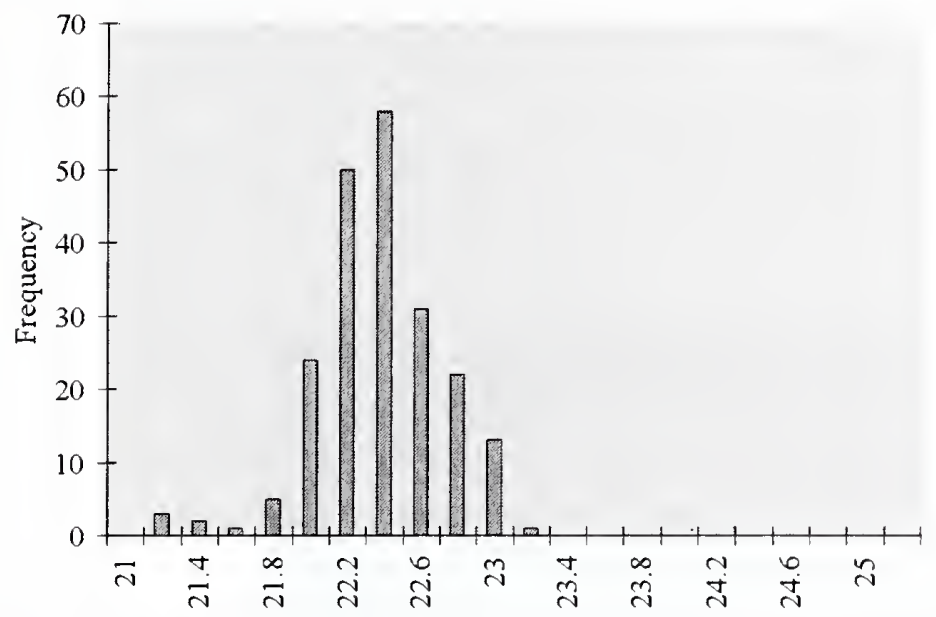

Cage occupied zone average contamination $(\mathrm{kg} / \mathrm{kg})$ distribution Contamination conversion factors $(\mathrm{kg} / \mathrm{kg} \rightarrow \mathrm{ppm})$

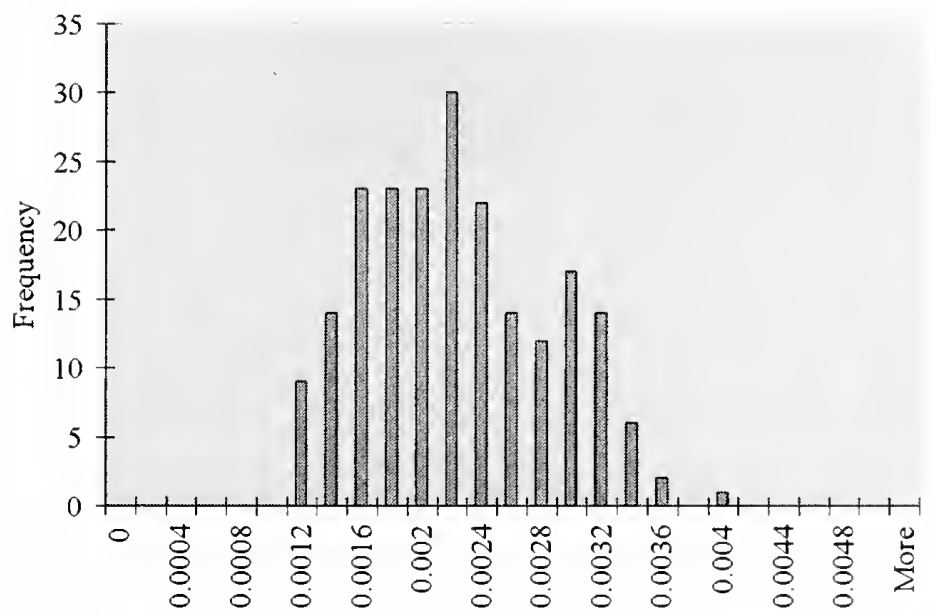

\begin{tabular}{|c|c|c|}
\hline Day & $\mathrm{CO}_{2}$ & $\mathbf{N H}_{3}$ \\
\hline $\mathbf{1}$ & 785000 & 410 \\
\hline $\mathbf{2}$ & 785000 & 795 \\
\hline $\mathbf{3}$ & 785000 & 1225 \\
\hline $\mathbf{4}$ & 785000 & 1859 \\
\hline $\mathbf{5}$ & 785000 & 2726 \\
\hline $\mathbf{6}$ & 785000 & 3846 \\
\hline $\mathbf{7}$ & 785000 & 5400 \\
\hline $\mathbf{8}$ & 785000 & 7121 \\
\hline $\mathbf{9}$ & 785000 & 9213 \\
\hline $\mathbf{1 0}$ & 785000 & 11384 \\
\hline
\end{tabular}

Cage occupied zone average relative humidity (\%) distribution

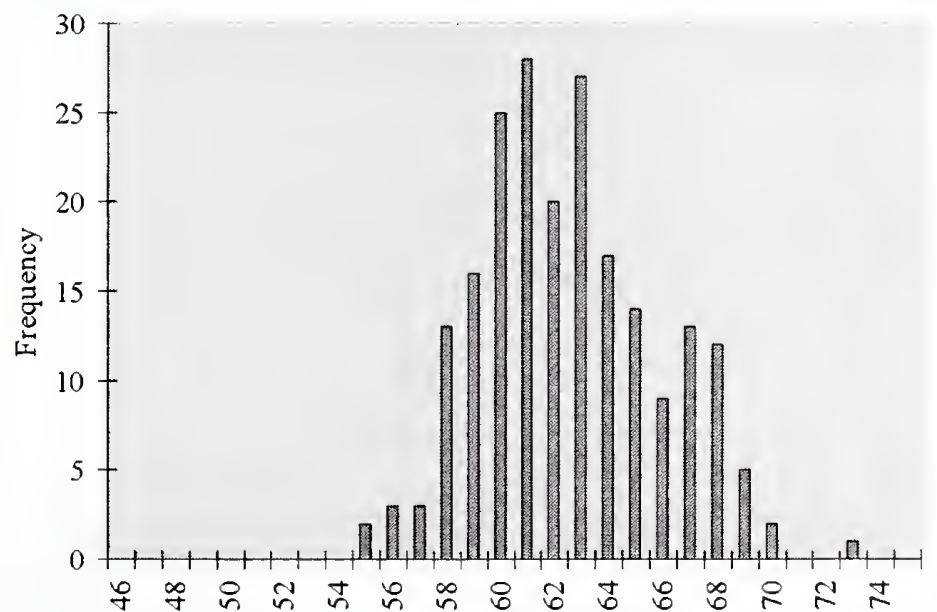




\section{Casename $\quad$ Case 87}

\section{Description}

\begin{tabular}{|c|c|c|c|c|c|}
\hline Supply & Supply Discharge & Supply Discharge & Exhaust & Exhaust & Exhaust \\
\hline Configuration & Temperature $\left({ }^{\circ} \mathrm{C}\right)$ & $\mathbf{R H}$ & Configuration & Temperature $\left({ }^{\circ} \mathrm{C}\right)$ & RH \\
\hline Radial & 19.2 & $59 \%$ & Low & 22 & $50 \%$ \\
\hline Change & Rack & Rack & Number of & Total mass of & Room \\
\hline Station & Orientation & Density & Mice in Room & Mice in Room & Pressurisation \\
\hline ON & On wall & Reduced & 700 & $14000 \mathrm{gr}$ & neg $100 \mathrm{cfm}$ \\
\hline
\end{tabular}

Room
ACH
15
Cage
Condition
Top On

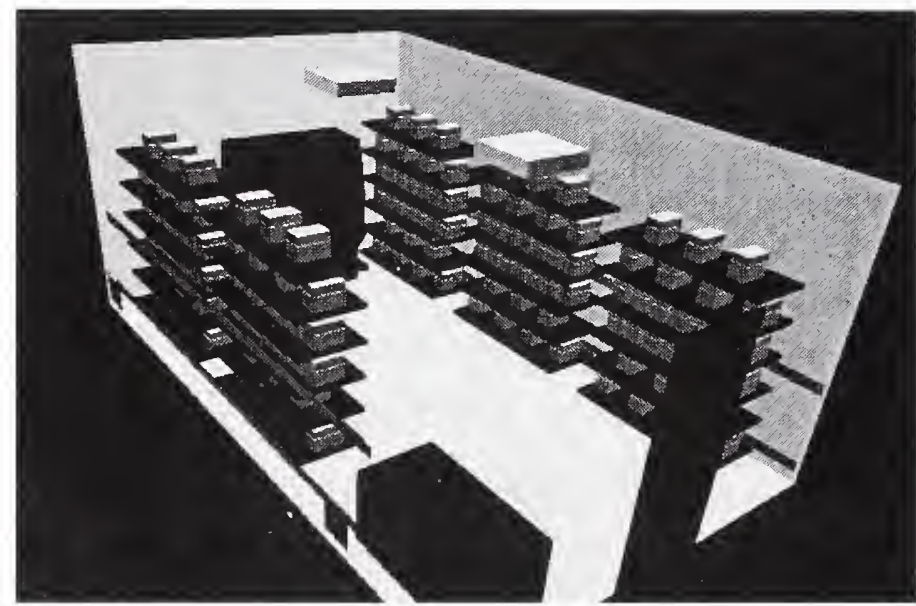

Analysis Results

Cage Occupied Zone

\begin{tabular}{|l|cc|c|c|}
\cline { 2 - 5 } \multicolumn{1}{c|}{} & \multicolumn{2}{c|}{ Temperature } & $\mathrm{CO}_{2}$ & RH \\
\cline { 2 - 5 } \multicolumn{1}{c|}{} & ${ }^{\circ} \mathrm{C}$ & ${ }^{\circ} \mathrm{F}$ & $(\mathbf{p p m})$ & \\
\hline Mean & 22.65 & 72.77 & 1391 & $55.84 \%$ \\
\hline S.D. & 4.31 & 7.76 & 455 & $3.11 \%$ \\
\hline Max. & 24.56 & 76.21 & 2638 & $64.18 \%$ \\
\hline
\end{tabular}

Cage Occupied Zone NH3 (ppm)

\begin{tabular}{|l|c|c|c|c|c|c|c|c|c|c|}
\hline Day & $\mathbf{1}$ & $\mathbf{2}$ & $\mathbf{3}$ & $\mathbf{4}$ & $\mathbf{5}$ & $\mathbf{6}$ & $\mathbf{7}$ & $\mathbf{8}$ & $\mathbf{9}$ & $\mathbf{1 0}$ \\
\hline Mean & 0.72 & 1.41 & 2.17 & 3.13 & 4.40 & 6.10 & 8.35 & 11.26 & 14.96 & 19.55 \\
\hline Max. & 1.37 & 2.67 & 4.12 & 5.93 & 8.34 & 11.57 & 15.84 & 21.36 & 28.37 & 37.09 \\
\hline
\end{tabular}

Room Breathing Zone

\begin{tabular}{|l|cc|c|c|}
\cline { 2 - 5 } \multicolumn{1}{c|}{} & \multicolumn{2}{c|}{ Temperature } & $\mathrm{CO}_{2}$ & RH \\
\cline { 2 - 5 } \multicolumn{1}{c|}{} & ${ }^{\circ} \mathrm{C}$ & ${ }^{\circ} \mathrm{F}$ & $(\mathrm{ppm})$ & \\
\hline Mean & 22.45 & 72.41 & 45 & $48.38 \%$ \\
\hline S.D. & 0.39 & 0.71 & 17 & \\
\hline Max. & 23.63 & 74.53 & 134 & \\
\hline
\end{tabular}

Room Breathing Zone NH3 (ppm)

\begin{tabular}{|l|c|c|c|c|c|c|c|c|c|c|}
\hline Day & $\mathbf{I}$ & $\mathbf{2}$ & $\mathbf{3}$ & $\mathbf{4}$ & $\mathbf{5}$ & $\mathbf{6}$ & $\mathbf{7}$ & $\mathbf{8}$ & $\mathbf{9}$ & $\mathbf{1 0}$ \\
\hline Mean & 0.02 & 0.05 & 0.07 & 0.10 & 0.14 & 0.20 & 0.27 & 0.36 & 0.48 & 0.63 \\
\hline Max. & 0.07 & 0.14 & 0.21 & 0.30 & 0.42 & 0.59 & 0.81 & 1.09 & 1.44 & 1.89 \\
\hline
\end{tabular}




\section{Histogram Distributions}

Cage occupied zone average temperature $\left({ }^{\circ} \mathrm{C}\right)$ distribution

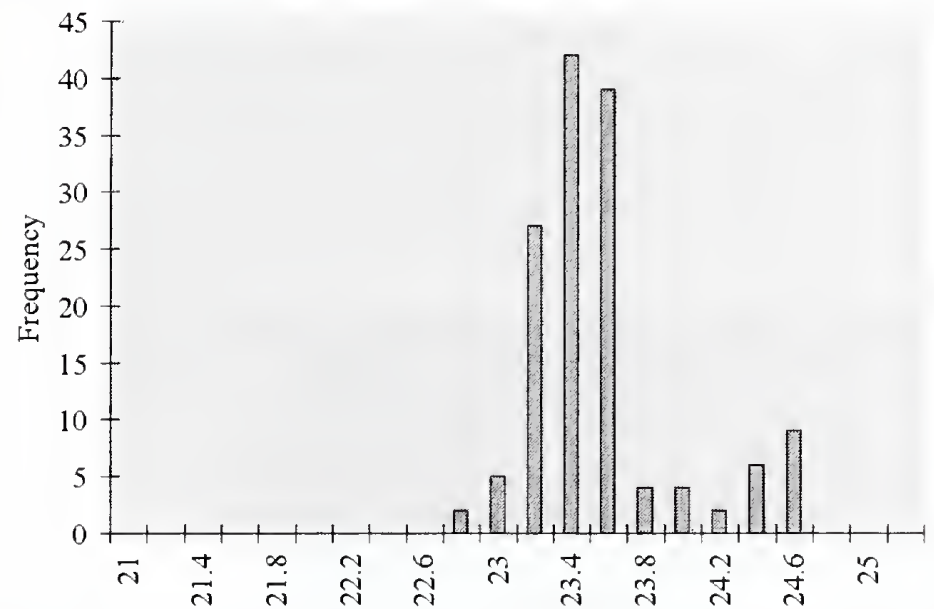

Cage occupied zone average contamination $(\mathrm{kg} / \mathrm{kg})$ distribution

Contamination conversion factors $(\mathrm{kg} / \mathrm{kg} \rightarrow \mathrm{ppm})$

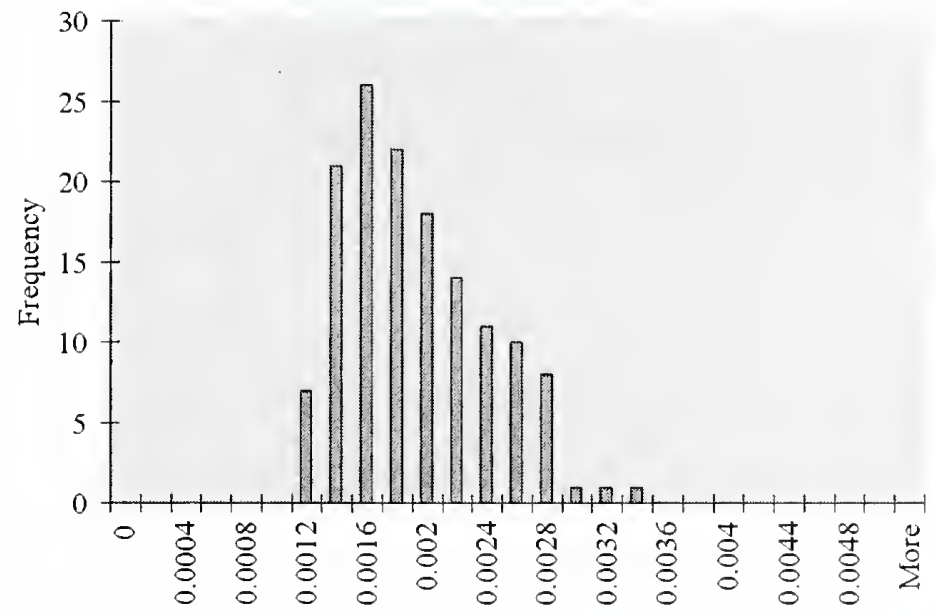

\begin{tabular}{|c|c|c|}
\hline Day & $\mathbf{C O}_{2}$ & $\mathrm{NH}_{3}$ \\
\hline $\mathbf{1}$ & 785000 & 408 \\
\hline $\mathbf{2}$ & 785000 & 795 \\
\hline $\mathbf{3}$ & 785000 & 1225 \\
\hline $\mathbf{4}$ & 785000 & 1766 \\
\hline $\mathbf{5}$ & 785000 & 2483 \\
\hline $\mathbf{6}$ & 785000 & 3443 \\
\hline $\mathbf{7}$ & 785000 & 4712 \\
\hline $\mathbf{8}$ & 785000 & 6356 \\
\hline $\mathbf{9}$ & 785000 & 8442 \\
\hline $\mathbf{1 0}$ & 785000 & 11384 \\
\hline
\end{tabular}

Cage occupied zone average relative humidity (\%) distribution

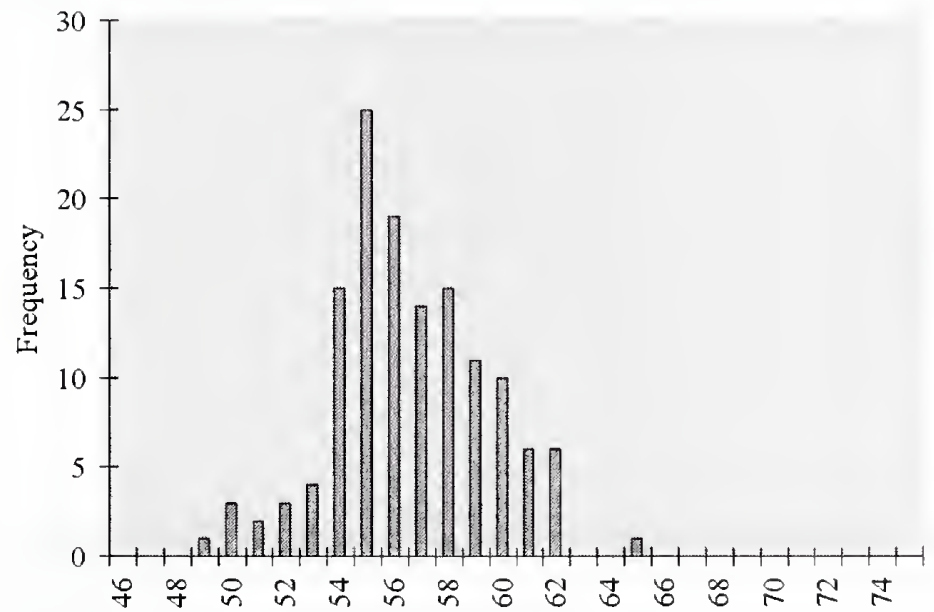


Casename $\quad$ Case 88

Description

$\begin{array}{llllll}\begin{array}{l}\text { Supply } \\ \text { Configuration }\end{array} & \begin{array}{l}\text { Supply Discharge } \\ \text { Temperature }\left({ }^{\circ} \mathrm{C}\right)\end{array} & \begin{array}{l}\text { Supply Discharge } \\ \text { RH }\end{array} & \begin{array}{l}\text { Exhaust } \\ \text { Configuration }\end{array} & \begin{array}{l}\text { Exhaust } \\ \text { Temperature }\left({ }^{\circ} \mathbf{C}\right)\end{array} & \begin{array}{l}\text { EH } \\ \text { Slot }\end{array} \\ 19.2 & 59 \% & \text { Low } & 22 & 50 \% \\ \text { Change } & \text { Rack } & \text { Rack } & \text { Number of } & \text { Total mass of } & \text { Room } \\ \text { Station } & \text { Orientation } & \text { Density } & \text { Mice in Room } & \text { Mice in Room } & \text { Pressurisation } \\ \text { ON } & \text { On wall } & \text { Reduced } & 700 & 14000 \mathrm{gr} & \text { neg } 100 \mathrm{cfm}\end{array}$

\section{Room}

$\mathrm{ACH}$

15

Cage

Condition

Top On

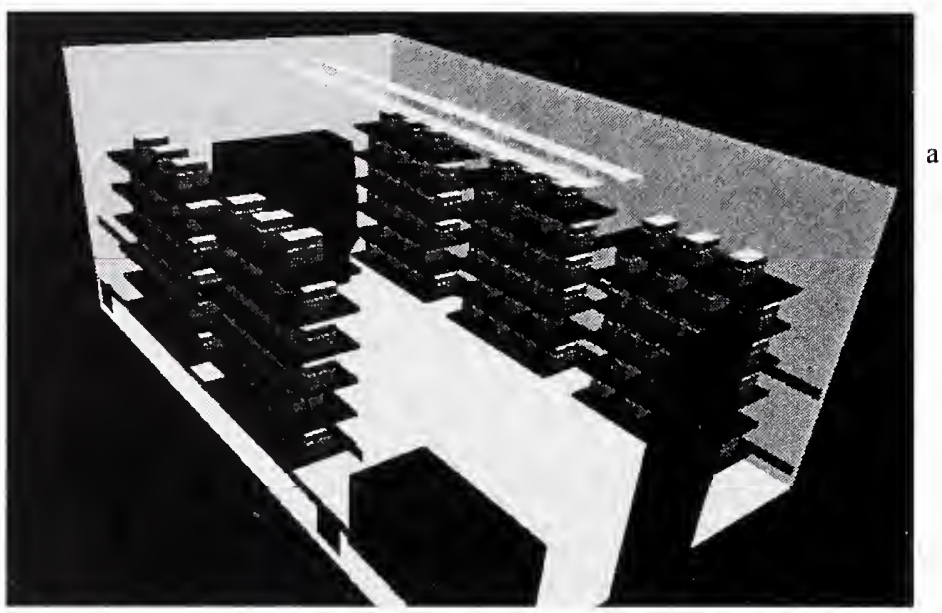

Analysis Results

Cage Occupied Zone

\begin{tabular}{|l|cc|c|c|}
\cline { 2 - 5 } \multicolumn{1}{c|}{} & \multicolumn{2}{c|}{ Temperature } & $\mathbf{C O}_{\mathbf{2}}$ & RH \\
\cline { 2 - 5 } \multicolumn{1}{c|}{} & ${ }^{\circ} \mathrm{C}$ & $\mathrm{F}$ & $(\mathbf{p p m})$ & \\
\hline Mean & 23.55 & 74.39 & 1550 & $56.31 \%$ \\
\hline S.D. & 0.39 & 0.70 & 378 & $2.71 \%$ \\
\hline Max. & 24.53 & 76.16 & 2516 & $63.30 \%$ \\
\hline
\end{tabular}

Cage Occupied Zone NH3 (ppm)

\begin{tabular}{|l|c|c|c|c|c|c|c|c|c|c|}
\hline Day & $\mathbf{1}$ & $\mathbf{2}$ & $\mathbf{3}$ & $\mathbf{4}$ & $\mathbf{5}$ & $\mathbf{6}$ & $\mathbf{7}$ & $\mathbf{8}$ & $\mathbf{9}$ & $\mathbf{1 0}$ \\
\hline Mean & 0.81 & 1.57 & 2.42 & 3.49 & 4.90 & 6.80 & 9.30 & 12.55 & 16.67 & 21.78 \\
\hline Max. & 1.31 & 2.55 & 3.93 & 5.66 & 7.96 & 11.04 & 15.10 & 20.37 & 27.06 & 35.37 \\
\hline
\end{tabular}

Room Breathing Zone

\begin{tabular}{|l|cc|c|c|}
\cline { 2 - 5 } \multicolumn{1}{c|}{} & \multicolumn{2}{c|}{ Temperature } & $\mathrm{CO}_{2}$ & RH \\
\cline { 2 - 5 } \multicolumn{1}{c|}{} & ${ }^{\circ} \mathrm{C}$ & ${ }^{\circ}$ & $(\mathbf{p p m})$ & \\
\hline Mean & 22.56 & 72.61 & 48 & $48.07 \%$ \\
\hline S.D. & 0.44 & 0.79 & 11 & \\
\hline Max. & 23.62 & 74.52 & 86 & \\
\hline
\end{tabular}

Room Breathing Zone NH3 (ppm)

\begin{tabular}{|l|c|c|c|c|c|c|c|c|c|c|}
\hline Day & $\mathbf{1}$ & $\mathbf{2}$ & $\mathbf{3}$ & $\mathbf{4}$ & $\mathbf{5}$ & $\mathbf{6}$ & $\mathbf{7}$ & $\mathbf{8}$ & $\mathbf{9}$ & $\mathbf{1 0}$ \\
\hline Mean & 0.02 & 0.05 & 0.07 & 0.11 & 0.15 & 0.21 & 0.29 & 0.39 & 0.51 & 0.67 \\
\hline Max. & 0.04 & 0.09 & 0.13 & 0.19 & 0.27 & 0.38 & 0.51 & 0.69 & 0.92 & 1.21 \\
\hline
\end{tabular}




\section{Histogram Distributions}

Cage occupied zone average temperature $\left({ }^{\circ} \mathrm{C}\right)$ distribution

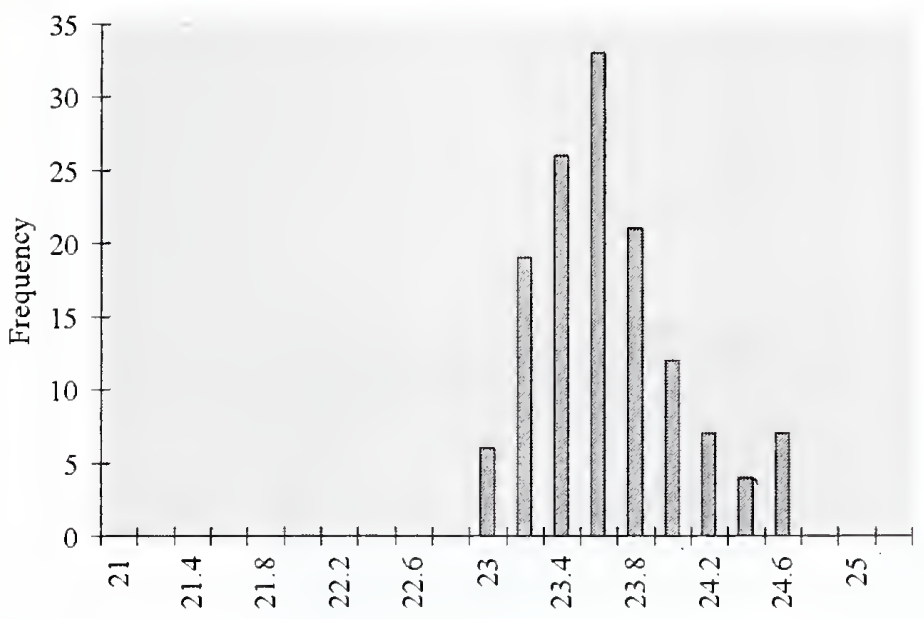

Cage occupied zone average contamination $(\mathrm{kg} / \mathrm{kg})$ distribution

Contamination conversion factors $(\mathrm{kg} / \mathrm{kg} \rightarrow \mathrm{ppm})$

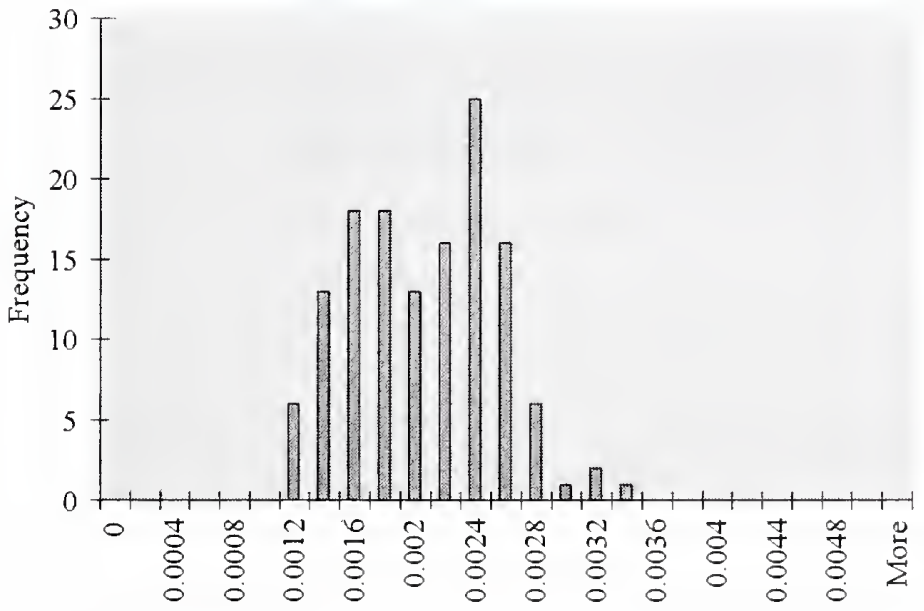

\begin{tabular}{|c|c|c|}
\hline Day & $\mathrm{CO}_{2}$ & $\mathrm{NH}_{3}$ \\
\hline 1 & 785000 & 408 \\
\hline 2 & 785000 & 795 \\
\hline 3 & 785000 & 1225 \\
\hline 4 & 785000 & 1766 \\
\hline 5 & 785000 & 2483 \\
\hline 6 & 785000 & 3443 \\
\hline 7 & 785000 & 4712 \\
\hline 8 & 785000 & 6356 \\
\hline 9 & 785000 & 8442 \\
\hline 10 & 785000 & 11384 \\
\hline
\end{tabular}

Cage occupied zone average relative humidity (\%) distribution

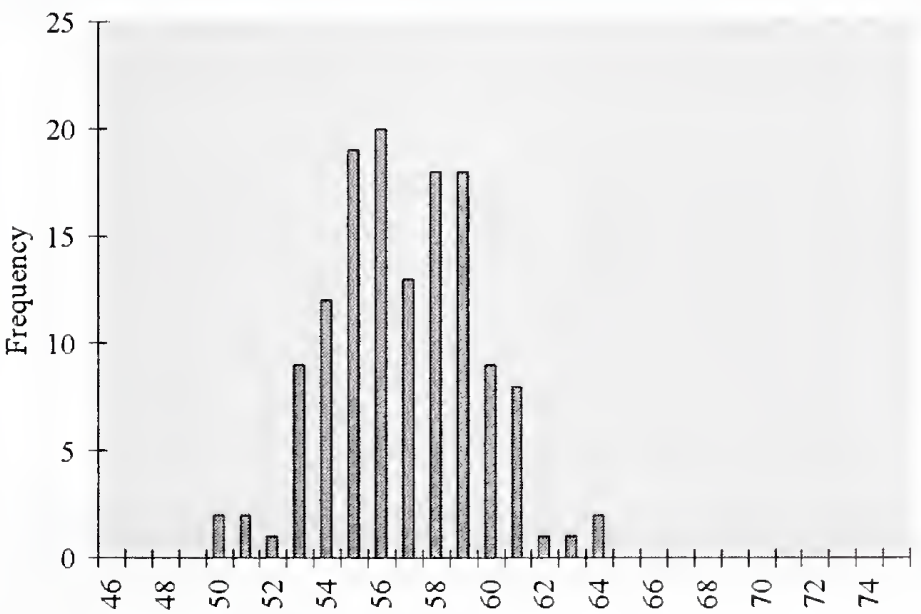


Casename

Case 89

\section{Description}

$\begin{array}{llllll}\begin{array}{l}\text { Supply } \\ \text { Configuration }\end{array} & \begin{array}{l}\text { Supply Discharge } \\ \text { Temperature }\left({ }^{\circ} \mathrm{C}\right)\end{array} & \begin{array}{l}\text { Supply Discharge } \\ \text { Row Ind }\end{array} & \begin{array}{l}\text { Exhaust } \\ \text { Configuration }\end{array} & \begin{array}{l}\text { Exhaust } \\ \text { Temperature }\left({ }^{\circ} \mathrm{C}\right)\end{array} & \begin{array}{l}\text { Exhaust } \\ \text { RH }\end{array} \\ \begin{array}{llll}\text { Change } \\ \text { Station }\end{array} & \text { Rack } & \text { Rack } & \text { Number of } & \text { Total mass of } & \text { Room } \\ \text { ON } & \text { Orientation } & \text { Density } & \text { Mice in Room } & \text { Mice in Room } & \text { Pressurisation } \\ & \text { Onwall } & \text { Reduced } & 700 & 14000 \mathrm{gr} & \text { neg } 100 \mathrm{cfm}\end{array}$

Room

$\mathrm{ACH}$

15

Cage

Condition

Top On

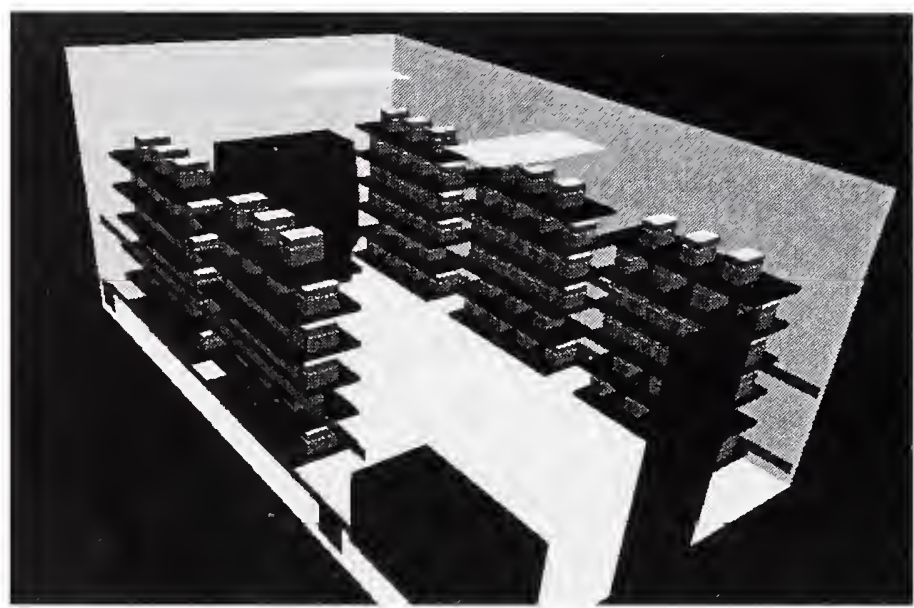

Analysis Results

Cage Occupied Zone

\begin{tabular}{|l|cc|c|c|}
\cline { 2 - 5 } \multicolumn{1}{c|}{} & \multicolumn{2}{c|}{ Temperature } & $\mathrm{CO}_{2}$ & RH \\
\cline { 2 - 5 } \multicolumn{1}{c|}{} & ${ }^{\circ} \mathrm{C}$ & $\mathrm{F}$ & $(\mathrm{ppm})$ & \\
\hline Mean & 23.68 & 74.62 & 1636 & $56.53 \%$ \\
\hline S.D. & 0.44 & 0.80 & 374 & $3.10 \%$ \\
\hline Max. & 24.92 & 76.86 & 2599 & $63.57 \%$ \\
\hline
\end{tabular}

Cage Occupied Zone NH3 (ppm)

\begin{tabular}{|l|c|c|c|c|c|c|c|c|c|c|}
\hline Day & $\mathbf{1}$ & $\mathbf{2}$ & $\mathbf{3}$ & $\mathbf{4}$ & $\mathbf{5}$ & $\mathbf{6}$ & $\mathbf{7}$ & $\mathbf{8}$ & $\mathbf{9}$ & $\mathbf{1 0}$ \\
\hline Mean & 0.85 & 1.66 & 2.55 & 3.68 & 5.17 & 7.17 & 9.82 & 13.25 & 17.59 & 23.00 \\
\hline Max. & 1.35 & 2.63 & 4.05 & 5.85 & 8.22 & 11.40 & 15.60 & 21.04 & 27.95 & 36.53 \\
\hline
\end{tabular}

Room Breathing Zone

\begin{tabular}{|l|cc|c|c|}
\cline { 2 - 5 } \multicolumn{1}{c|}{} & \multicolumn{2}{c|}{ Temperature } & $\mathrm{CO}_{2}$ & RH \\
\cline { 2 - 5 } \multicolumn{1}{c|}{} & ${ }^{\circ} \mathrm{C}$ & ${ }^{\circ}$ & $(\mathbf{p p m})$ & \\
\hline Mean & 22.92 & 73.26 & 62 & $47.10 \%$ \\
\hline S.D. & 0.55 & 1.00 & 21 & \\
\hline Max. & 24.09 & 75.36 & 217 & \\
\hline
\end{tabular}

Room Breathing Zone NH3 (ppm)

\begin{tabular}{|l|c|c|c|c|c|c|c|c|c|c|}
\hline Day & $\mathbf{1}$ & $\mathbf{2}$ & $\mathbf{3}$ & $\mathbf{4}$ & $\mathbf{5}$ & $\mathbf{6}$ & $\mathbf{7}$ & $\mathbf{8}$ & $\mathbf{9}$ & $\mathbf{1 0}$ \\
\hline Mean & 0.03 & 0.06 & 0.10 & 0.14 & 0.20 & 0.27 & 0.37 & 0.50 & 0.67 & 0.87 \\
\hline Max. & 0.11 & 0.22 & 0.34 & 0.49 & 0.69 & 0.95 & 1.30 & 1.75 & 2.33 & 3.04 \\
\hline
\end{tabular}




\section{Histogram Distributions}

Cage occupied zone average temperature $\left({ }^{\circ} \mathrm{C}\right)$ distribution

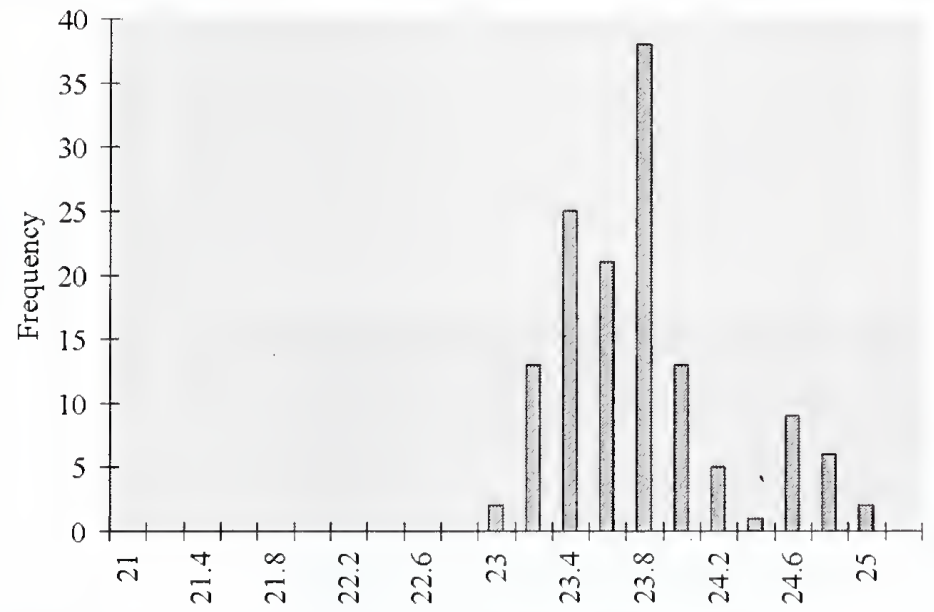

Cage occupied zone average contamination $(\mathrm{kg} / \mathrm{kg})$ distribution

Contamination conversion factors $(\mathrm{kg} / \mathrm{kg} \rightarrow \mathrm{ppm})$

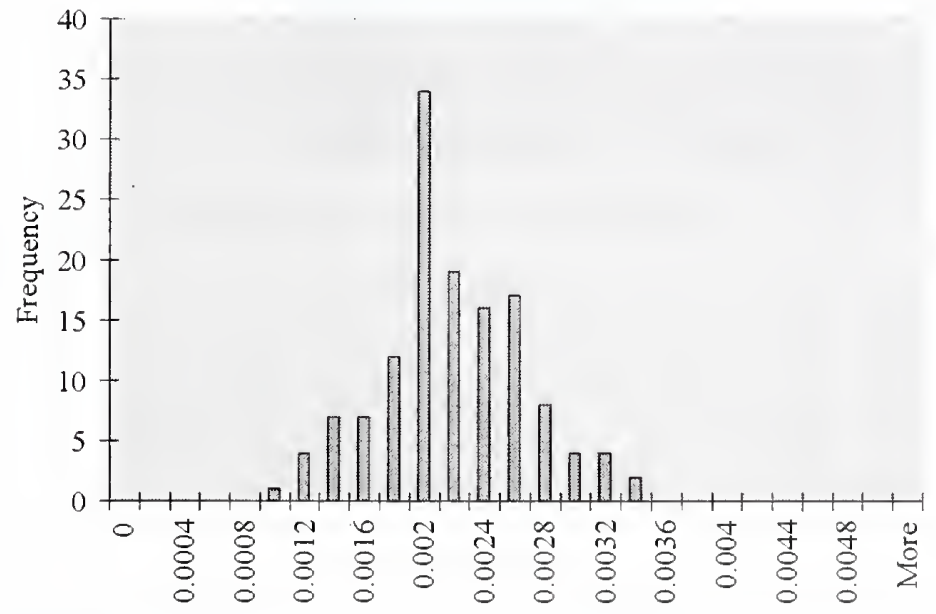

\begin{tabular}{|c|c|c|}
\hline Day & $\mathrm{CO}_{2}$ & $\mathrm{NH}_{3}$ \\
\hline 1 & 785000 & 408 \\
\hline 2 & 785000 & 795 \\
\hline 3 & 785000 & 1225 \\
\hline 4 & 785000 & 1766 \\
\hline 5 & 785000 & 2483 \\
\hline 6 & 785000 & 3443 \\
\hline 7 & 785000 & 4712 \\
\hline $\mathbf{8}$ & 785000 & 6356 \\
\hline 9 & 785000 & 8442 \\
\hline 10 & 785000 & 11384 \\
\hline
\end{tabular}

Cage occupied zone average relative humidity (\%) distribution

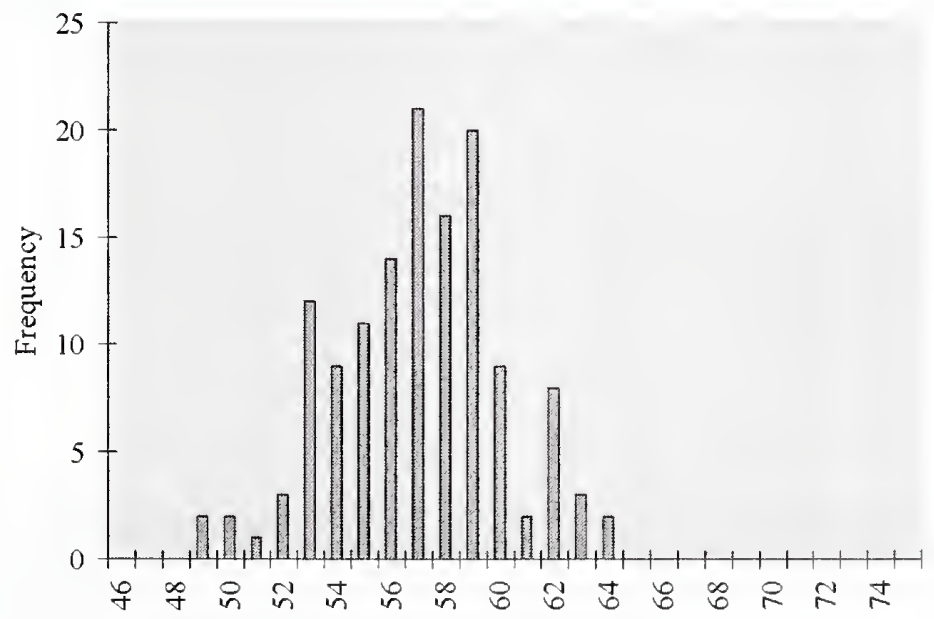


Casename

Case 90

\section{Description}

\begin{tabular}{|c|c|c|c|c|c|}
\hline Supply & Supply Discharge & Supply Discharge & Exhaust & Exhaust & Exhaust \\
\hline Configuration & Temperature $\left({ }^{\circ} \mathrm{C}\right)$ & RH & Configuration & Temperature $\left({ }^{\circ} \mathrm{C}\right)$ & RH \\
\hline Radial & 18.8 & $61 \%$ & Ceiling & 22 & $50 \%$ \\
\hline Change & Rack & Rack & Number of & Total mass of & Room \\
\hline Station & Orientation & Density & Mice in Room & Mice in Room & Pressurisation \\
\hline ON & Perp all 5 on 1 wall & Single & 1050 & $21000 \mathrm{gr}$ & neg $100 \mathrm{cfm}$ \\
\hline
\end{tabular}

Room

$\mathrm{ACH}$

15

Cage

Condition

Top On

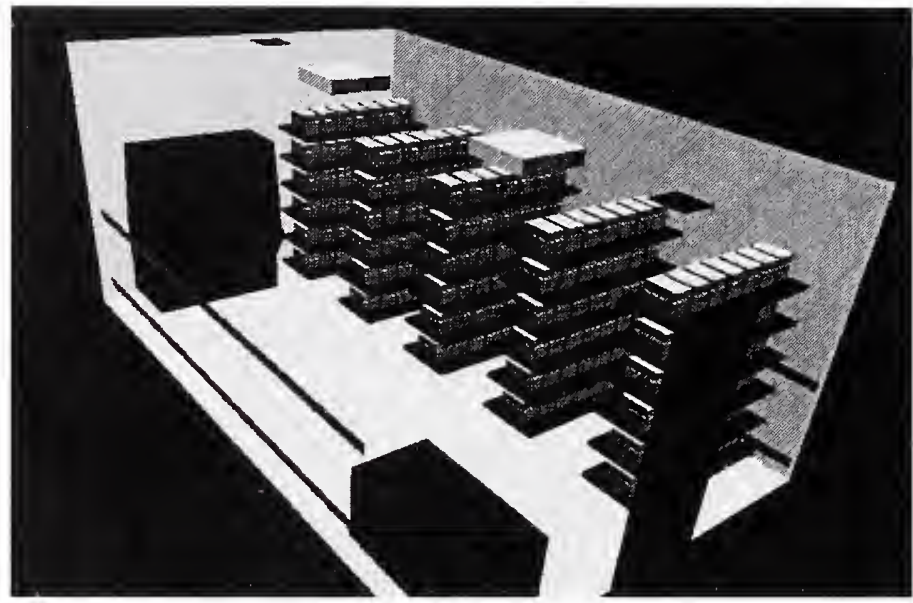

Analysis Results

Cage Occupied Zone

\begin{tabular}{|l|cc|c|c|}
\cline { 2 - 5 } \multicolumn{1}{c|}{} & \multicolumn{2}{c|}{ Temperature } & $\mathrm{CO}_{2}$ & RH \\
\cline { 2 - 5 } \multicolumn{1}{c|}{} & ${ }^{\circ} \mathrm{C}$ & ${ }^{\circ} \mathrm{F}$ & $(\mathrm{ppm})$ & \\
\hline Mean & 21.93 & 71.47 & 1673 & $63.32 \%$ \\
\hline S.D. & 0.29 & 0.52 & 385 & $2.63 \%$ \\
\hline Max. & 22.36 & 72.24 & 2511 & $69.19 \%$ \\
\hline
\end{tabular}

Cage Occupied Zone NH3 (ppm)

\begin{tabular}{|l|c|c|c|c|c|c|c|c|c|c|}
\hline Day & $\mathbf{1}$ & $\mathbf{2}$ & $\mathbf{3}$ & $\mathbf{4}$ & $\mathbf{5}$ & $\mathbf{6}$ & $\mathbf{7}$ & $\mathbf{8}$ & $\mathbf{9}$ & $\mathbf{1 0}$ \\
\hline Mean & 0.88 & 1.69 & 2.61 & 4.22 & 6.48 & 9.32 & 13.41 & 17.29 & 21.77 & 25.93 \\
\hline Max. & 1.32 & 2.54 & 3.92 & 6.34 & 9.73 & 13.98 & 20.13 & 25.96 & 32.68 & 38.92 \\
\hline
\end{tabular}

Room Breathing Zone

\begin{tabular}{|l|cc|c|c|}
\cline { 2 - 5 } \multicolumn{1}{c|}{} & \multicolumn{2}{c|}{ Temperature } & $\mathrm{CO}_{2}$ & RH \\
\cline { 2 - 5 } \multicolumn{1}{c|}{} & ${ }^{\circ} \mathrm{C}$ & ${ }^{\circ} \mathrm{F}$ & $(\mathrm{ppm})$ & \\
\hline Mean & 20.38 & 68.69 & 72 & $55.37 \%$ \\
\hline S.D. & 0.23 & 0.41 & 29 & \\
\hline Max. & 21.75 & 71.15 & 279 & \\
\hline
\end{tabular}

Room Breathing Zone NH3 (ppm)

\begin{tabular}{|l|c|c|c|c|c|c|c|c|c|c|}
\hline Day & $\mathbf{1}$ & $\mathbf{2}$ & $\mathbf{3}$ & $\mathbf{4}$ & $\mathbf{5}$ & $\mathbf{6}$ & $\mathbf{7}$ & $\mathbf{8}$ & $\mathbf{9}$ & $\mathbf{1 0}$ \\
\hline Mean & 0.04 & 0.07 & 0.11 & 0.18 & 0.28 & 0.40 & 0.57 & 0.74 & 0.93 & 1.11 \\
\hline Max. & 0.15 & 0.28 & 0.44 & 0.70 & 1.08 & 1.55 & 2.24 & 2.89 & 3.63 & 4.33 \\
\hline
\end{tabular}




\section{Histogram Distributions}

Cage occupied zone average temperature $\left({ }^{\circ} \mathrm{C}\right)$ distribution

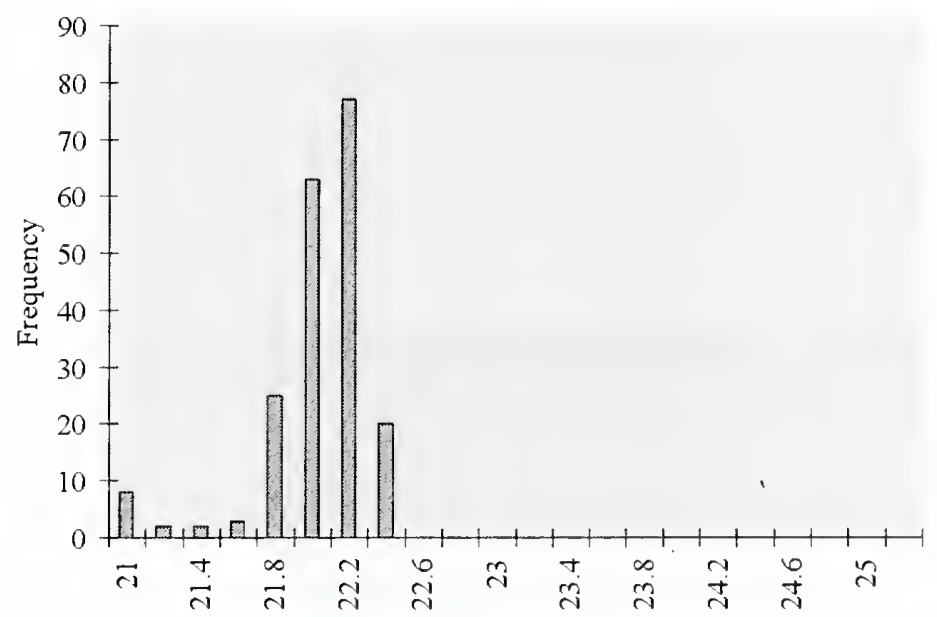

Cage occupied zone average contamination $(\mathrm{kg} / \mathrm{kg})$ distribution

Contamination conversion factors $(\mathrm{kg} / \mathrm{kg} \rightarrow \mathrm{ppm})$

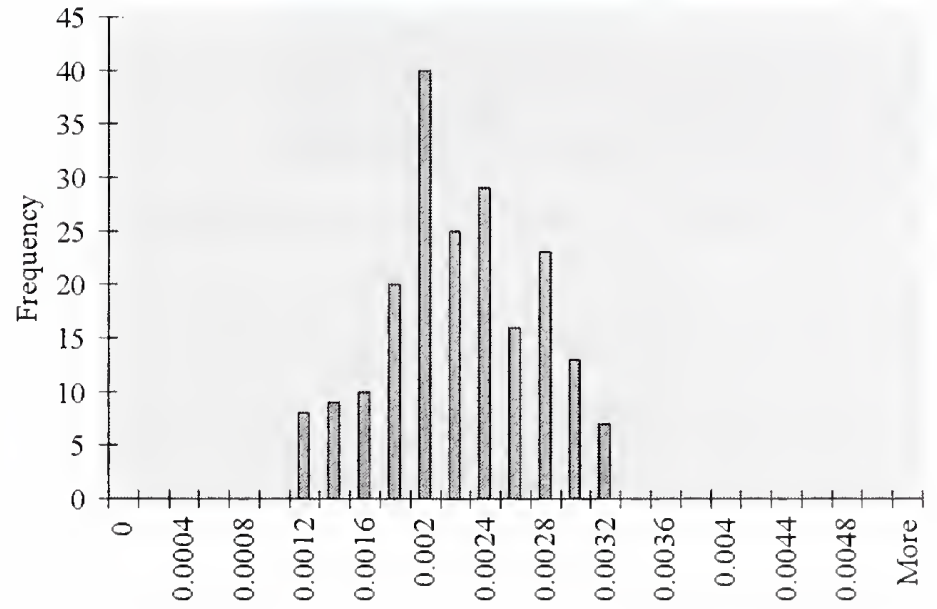

\begin{tabular}{|c|c|c|}
\hline Day & $\mathrm{CO}_{2}$ & $\mathbf{N H}_{3}$ \\
\hline $\mathbf{1}$ & 785000 & 412 \\
\hline 2 & 785000 & 795 \\
\hline $\mathbf{3}$ & 785000 & 1225 \\
\hline 4 & 785000 & 1982 \\
\hline 5 & 785000 & 3043 \\
\hline $\mathbf{6}$ & 785000 & 4371 \\
\hline 7 & 785000 & 6295 \\
\hline $\mathbf{8}$ & 785000 & 8116 \\
\hline 9 & 785000 & 10216 \\
\hline 10 & 785000 & 11384 \\
\hline
\end{tabular}

Cage occupied zone average relative humidity (\%) distribution

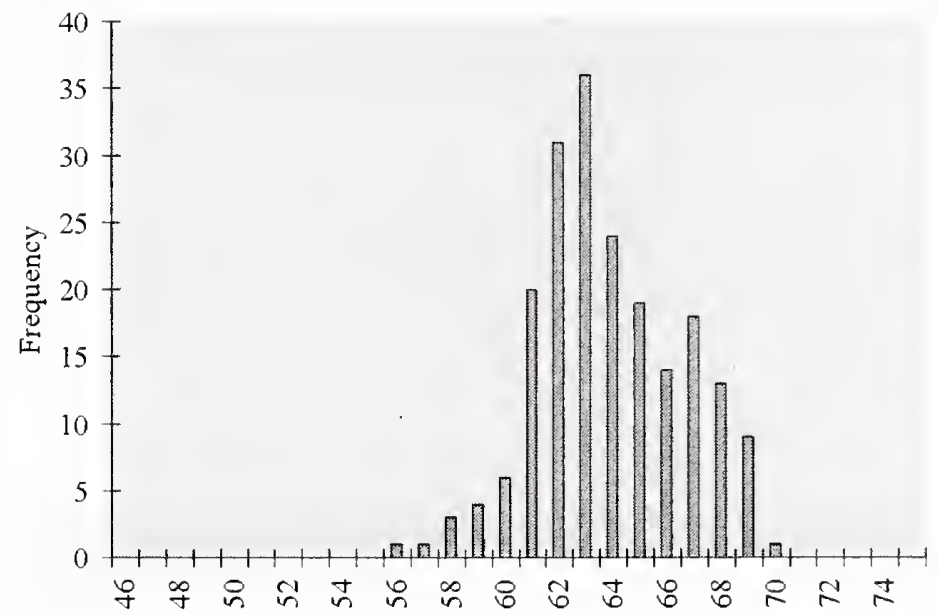


Casename

Case 91

Description

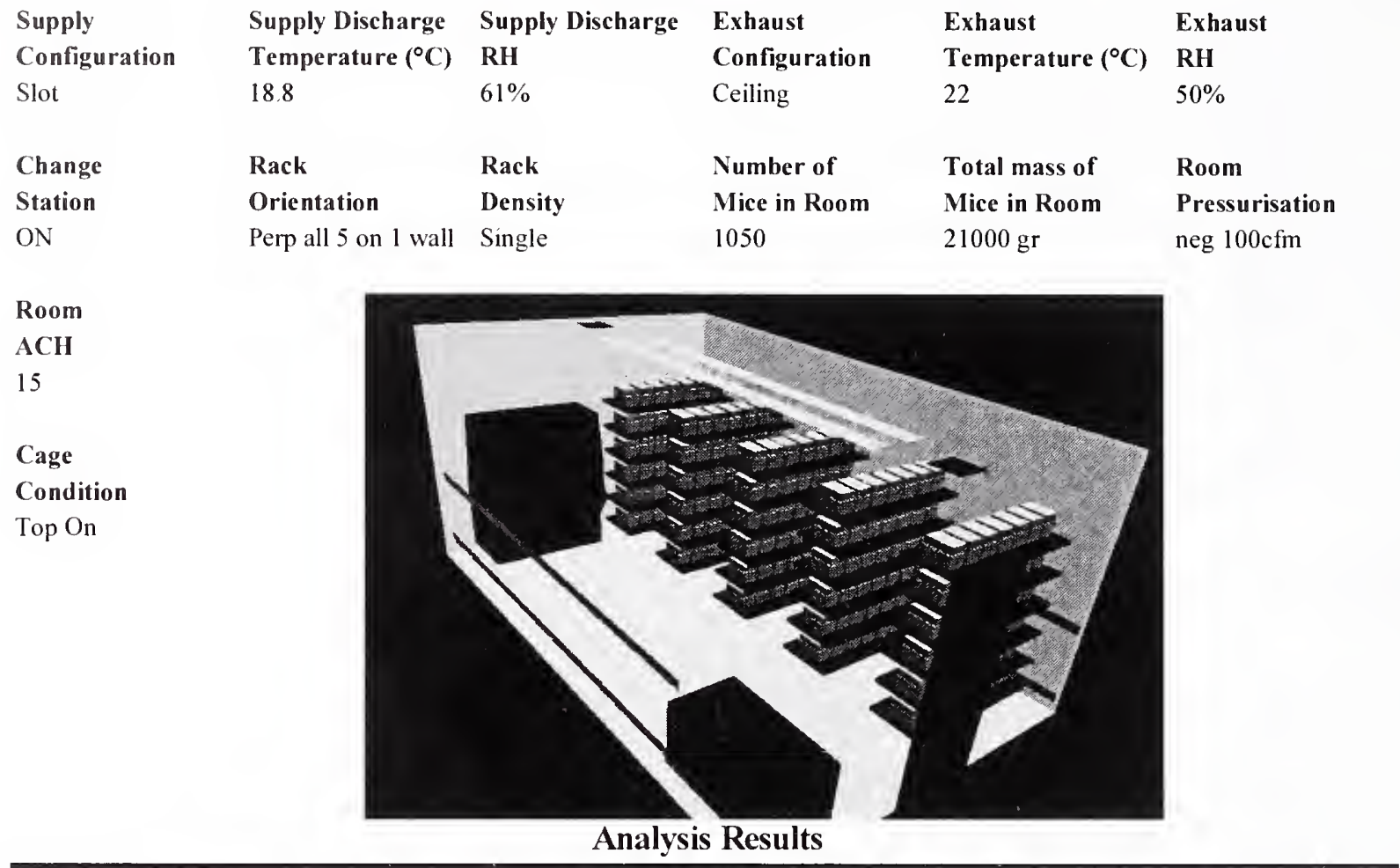

Cage Occupied Zone

\begin{tabular}{|l|cc|c|c|}
\cline { 2 - 5 } \multicolumn{1}{c|}{} & \multicolumn{2}{c|}{ Temperature } & $\mathbf{C O}_{2}$ & RH \\
\cline { 2 - 5 } \multicolumn{1}{c|}{} & ${ }^{\circ} \mathrm{C}$ & $\mathrm{F}$ & $(\mathbf{p p m})$ & \\
\hline Mean & 21.99 & 71.58 & 1709 & $63.38 \%$ \\
\hline S.D. & 0.30 & 0.54 & 381 & $2.49 \%$ \\
\hline Max. & 22.59 & 72.67 & 2643 & $69.86 \%$ \\
\hline
\end{tabular}

Cage Occupied Zone NH3 (ppm)

\begin{tabular}{|l|c|c|c|c|c|c|c|c|c|c|}
\hline Day & $\mathbf{1}$ & $\mathbf{2}$ & $\mathbf{3}$ & $\mathbf{4}$ & $\mathbf{5}$ & $\mathbf{6}$ & $\mathbf{7}$ & $\mathbf{8}$ & $\mathbf{9}$ & $\mathbf{1 0}$ \\
\hline Mean & 0.90 & 1.73 & 2.67 & 4.33 & 6.65 & 9.57 & 13.79 & 17.76 & 22.33 & 26.55 \\
\hline Max. & 1.39 & 2.68 & 4.12 & 6.69 & 10.29 & 14.79 & 21.32 & 27.47 & 34.54 & 41.07 \\
\hline
\end{tabular}

Room Breathing Zone

\begin{tabular}{|l|cc|c|c|}
\cline { 2 - 5 } \multicolumn{1}{c|}{} & \multicolumn{2}{c|}{ Temperature } & $\mathbf{C O}_{2}$ & RH \\
\cline { 2 - 5 } \multicolumn{1}{c|}{} & ${ }^{\circ} \mathrm{C}$ & ${ }^{\circ}$ & $(\mathbf{p p m})$ & \\
\hline Mean & 20.46 & 68.83 & 57 & $54.97 \%$ \\
\hline S.D. & 0.25 & 0.44 & 19 & \\
\hline Max. & 21.66 & 70.99 & 183 & \\
\hline
\end{tabular}

Room Breathing Zone NH3 (ppm)

\begin{tabular}{|l|c|c|c|c|c|c|c|c|c|c|}
\hline Day & $\mathbf{1}$ & $\mathbf{2}$ & $\mathbf{3}$ & $\mathbf{4}$ & $\mathbf{5}$ & $\mathbf{6}$ & $\mathbf{7}$ & $\mathbf{8}$ & $\mathbf{9}$ & $\mathbf{1 0}$ \\
\hline Mean & 0.03 & 0.06 & 0.09 & 0.14 & 0.22 & 0.32 & 0.46 & 0.59 & 0.75 & 0.89 \\
\hline Max. & 0.10 & 0.19 & 0.29 & 0.46 & 0.71 & 1.03 & 1.48 & 1.90 & 2.39 & 2.85 \\
\hline
\end{tabular}




\section{Histogram Distributions}

Cage occupied zone average temperature $\left({ }^{\circ} \mathrm{C}\right)$ distribution

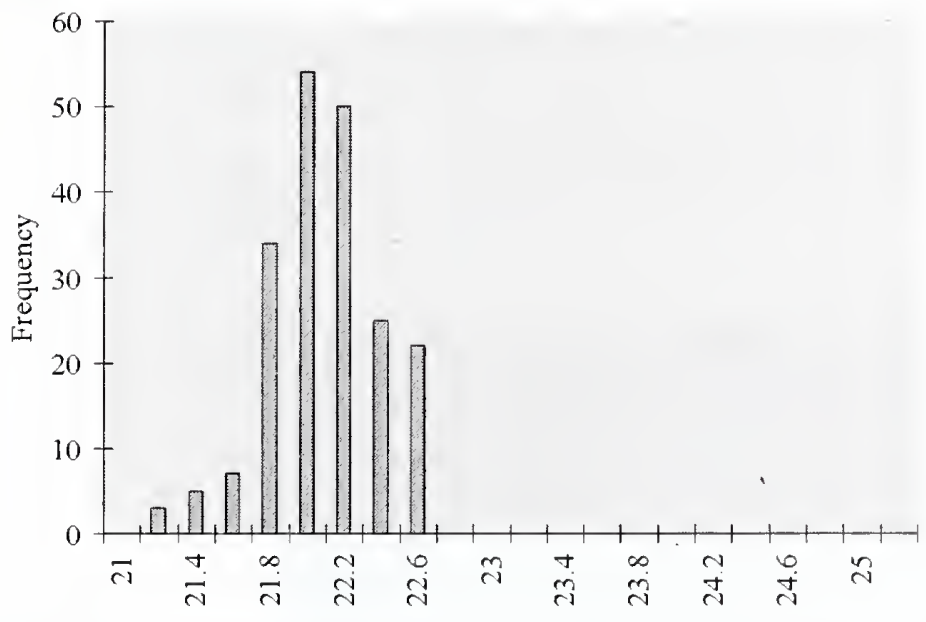

Cage occupied zone average contamination $(\mathrm{kg} / \mathrm{kg})$ distribution

Contamination conversion factors $(\mathrm{kg} / \mathrm{kg} \rightarrow \mathrm{ppm})$

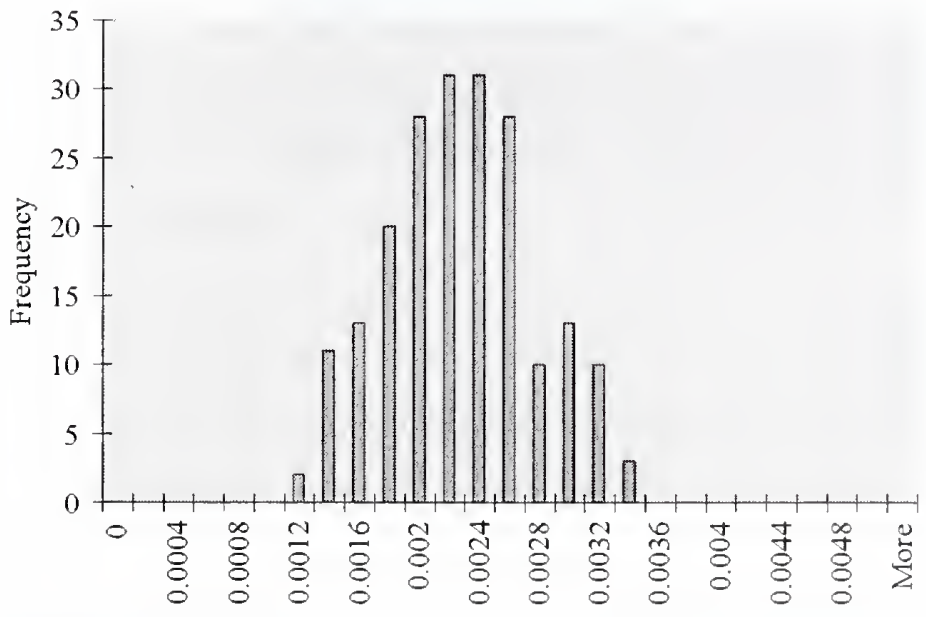

\begin{tabular}{|c|c|c|}
\hline Day & $\mathbf{C O}_{2}$ & $\mathbf{N H}_{3}$ \\
\hline $\mathbf{1}$ & 785000 & 412 \\
\hline $\mathbf{2}$ & 785000 & 795 \\
\hline $\mathbf{3}$ & 785000 & 1225 \\
\hline $\mathbf{4}$ & 785000 & 1987 \\
\hline $\mathbf{5}$ & 785000 & 3056 \\
\hline $\mathbf{6}$ & 785000 & 4394 \\
\hline 7 & 785000 & 6333 \\
\hline $\mathbf{8}$ & 785000 & 8158 \\
\hline $\mathbf{9}$ & 785000 & 10259 \\
\hline $\mathbf{1 0}$ & 785000 & 11384 \\
\hline
\end{tabular}

Cage occupied zone average relative humidity (\%) distribution

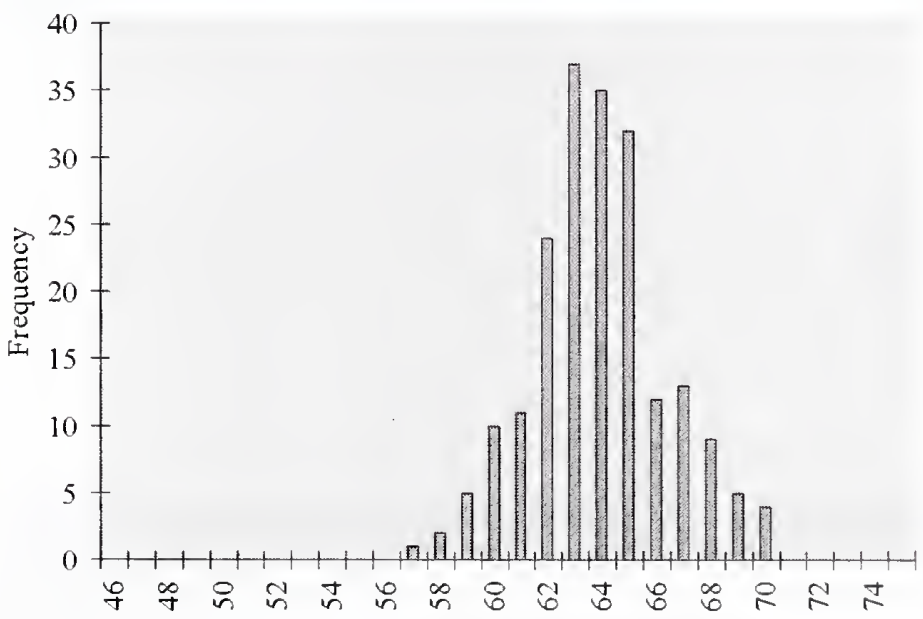


Casename

Case 92

Description

\begin{tabular}{|c|c|c|c|c|c|}
\hline Supply & Supply Discharge & Supply Discharge & Exhaust & Exhaust & Exhaust \\
\hline Configuration & Temperature $\left({ }^{\circ} \mathrm{C}\right)$ & RH & Configuration & Temperature $\left({ }^{\circ} \mathrm{C}\right)$ & RH \\
\hline Low Ind & 18.8 & $61 \%$ & Ceiling & 22 & $50 \%$ \\
\hline Change & Rack & Rack & Number of & Total mass of & Room \\
\hline Station & Orientation & Density & Mice in Room & Mice in Room & Pressurisation \\
\hline
\end{tabular}

\section{Room}

ACH

15

Cage

Condition

Top On

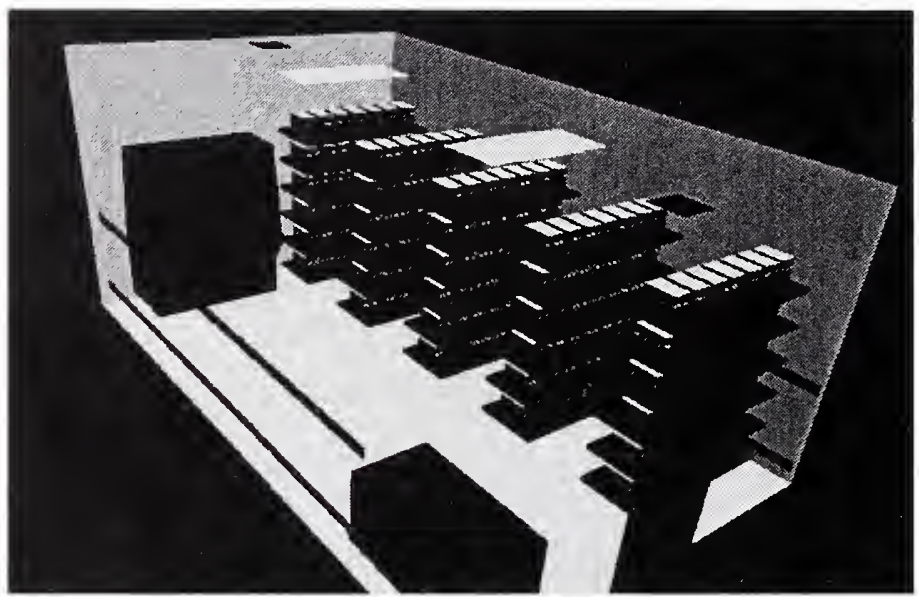

Analysis Results

Cage Occupied Zone

\begin{tabular}{|l|cc|c|c|}
\cline { 2 - 5 } \multicolumn{1}{c|}{} & \multicolumn{2}{c|}{ Temperature } & $\mathrm{CO}_{2}$ & RH \\
\cline { 2 - 5 } \multicolumn{1}{c|}{} & ${ }^{\circ} \mathrm{C}$ & ${ }^{\circ} \mathrm{F}$ & $(\mathrm{ppm})$ & \\
\hline Mean & 21.98 & 71.56 & 1631 & $62.80 \%$ \\
\hline S.D. & 0.31 & 0.56 & 314 & $2.29 \%$ \\
\hline Max. & 22.58 & 72.65 & 2404 & $69.73 \%$ \\
\hline
\end{tabular}

Cage Occupied Zone NH3 (ppm)

\begin{tabular}{|l|c|c|c|c|c|c|c|c|c|c|}
\hline Day & $\mathbf{1}$ & $\mathbf{2}$ & $\mathbf{3}$ & $\mathbf{4}$ & $\mathbf{5}$ & $\mathbf{6}$ & $\mathbf{7}$ & $\mathbf{8}$ & $\mathbf{9}$ & $\mathbf{1 0}$ \\
\hline Mean & 0.86 & 1.65 & 2.55 & 4.02 & 6.06 & 8.65 & 12.33 & 16.04 & 20.39 & 24.75 \\
\hline Max. & 1.26 & 2.43 & 3.75 & 5.92 & 8.93 & 12.74 & 18.18 & 23.63 & 30.06 & 36.48 \\
\hline
\end{tabular}

Room Breathing Zone

\begin{tabular}{|l|cc|c|c|}
\cline { 2 - 5 } \multicolumn{1}{c|}{} & \multicolumn{2}{c|}{ Temperature } & $\mathrm{CO}_{2}$ & RH \\
\cline { 2 - 5 } \multicolumn{1}{c|}{} & ${ }^{\circ} \mathrm{C}$ & ${ }^{\circ} \mathrm{F}$ & $(\mathrm{ppm})$ & \\
\hline Mean & 20.40 & 68.71 & 41 & $55.05 \%$ \\
\hline S.D. & 0.17 & 0.30 & 15 & \\
\hline Max. & 20.95 & 69.70 & 151 & \\
\hline
\end{tabular}

Room Breathing Zone NH3 (ppm)

\begin{tabular}{|l|c|c|c|c|c|c|c|c|c|c|}
\hline Day & 1 & 2 & 3 & 4 & 5 & 6 & 7 & 8 & 9 & 10 \\
\hline Mean & 0.02 & 0.04 & 0.06 & 0.10 & 0.15 & 0.22 & 0.31 & 0.41 & 0.52 & 0.63 \\
\hline Max. & 0.08 & 0.15 & 0.24 & 0.37 & 0.56 & 0.80 & 1.14 & 1.48 & 1.88 & 2.29 \\
\hline
\end{tabular}




\section{Histogram Distributions}

Cage occupied zone average temperature $\left({ }^{\circ} \mathrm{C}\right)$ distribution

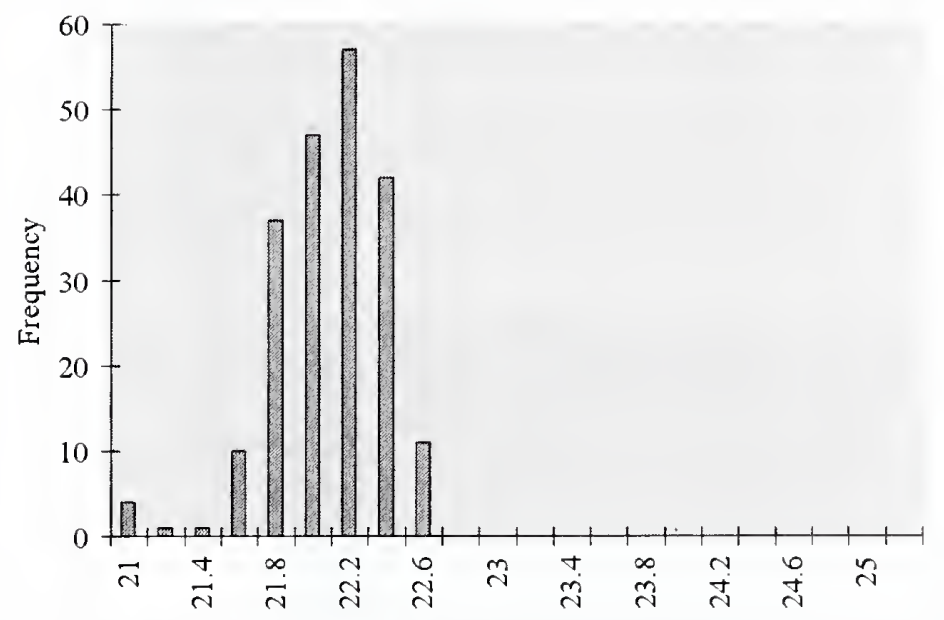

Cage occupied zone average contamination $(\mathrm{kg} / \mathrm{kg})$ distribution Contamination conversion factors $(\mathrm{kg} / \mathrm{kg} \rightarrow \mathrm{ppm})$

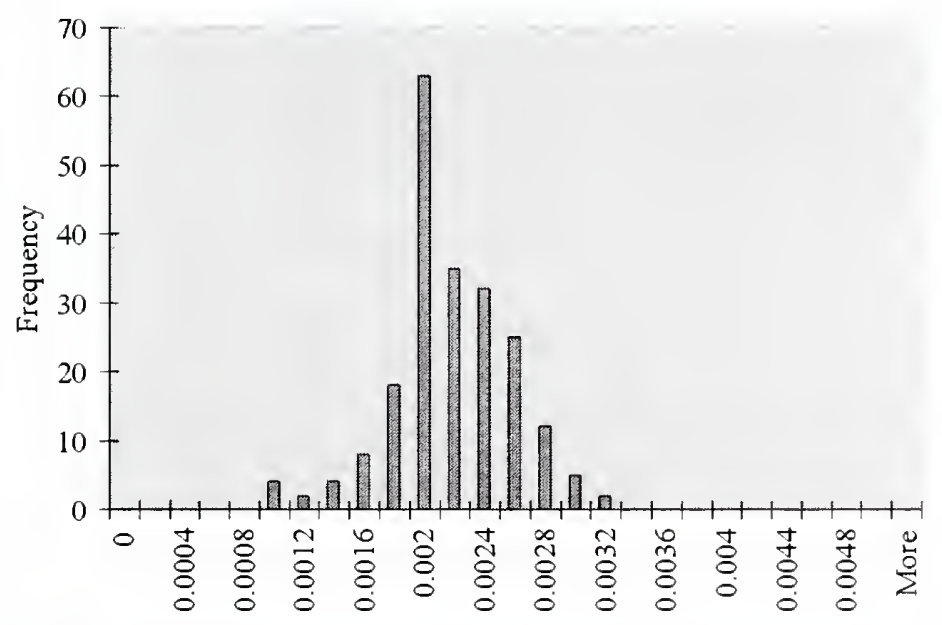

\begin{tabular}{|c|c|c|}
\hline Day & $\mathbf{C O}_{2}$ & $\mathbf{N H}_{3}$ \\
\hline $\mathbf{1}$ & 785000 & 411 \\
\hline $\mathbf{2}$ & 785000 & 795 \\
\hline $\mathbf{3}$ & 785000 & 1225 \\
\hline $\mathbf{4}$ & 785000 & 1933 \\
\hline $\mathbf{5}$ & 785000 & 2916 \\
\hline $\mathbf{6}$ & 785000 & 4161 \\
\hline $\mathbf{7}$ & 785000 & 5936 \\
\hline $\mathbf{8}$ & 785000 & 7716 \\
\hline $\mathbf{9}$ & 785000 & 9814 \\
\hline $\mathbf{1 0}$ & 785000 & 11384 \\
\hline
\end{tabular}

Cage occupied zone average relative humidity (\%) distribution

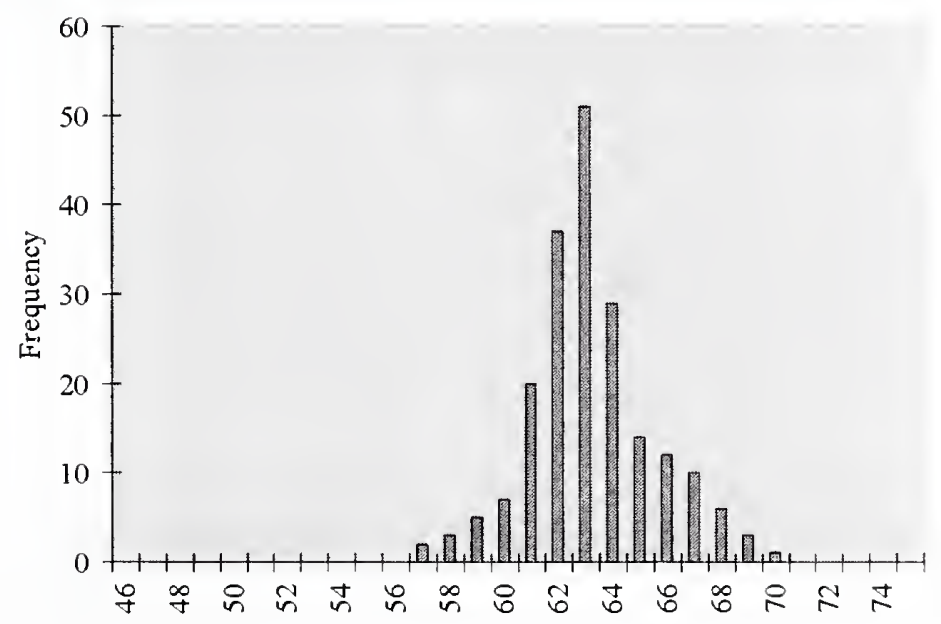




\section{Description}

\begin{tabular}{|c|c|c|c|c|c|}
\hline Supply & Supply Discharge & Supply Discharge & Exhaust & Exhaust & Exhaust \\
\hline Configuration & Temperature $\left({ }^{\circ} \mathrm{C}\right)$ & RH & Configuration & Temperature $\left({ }^{\circ} \mathrm{C}\right)$ & RH \\
\hline Radial & 17.5 & $66 \%$ & Ceiling & 22 & $50 \%$ \\
\hline Change & Rack & Rack & Number of & Total mass of & Room \\
\hline Station & Orientation & Density & Mice in Room & Mice in Room & Pressurisation \\
\hline $\mathrm{ON}$ & Perp all 5 on 1 wall & Double & 2100 & $42000 \mathrm{gr}$ & neg $100 \mathrm{cfm}$ \\
\hline
\end{tabular}

Room

$\mathrm{ACH}$

15

Cage

Condition

Top On

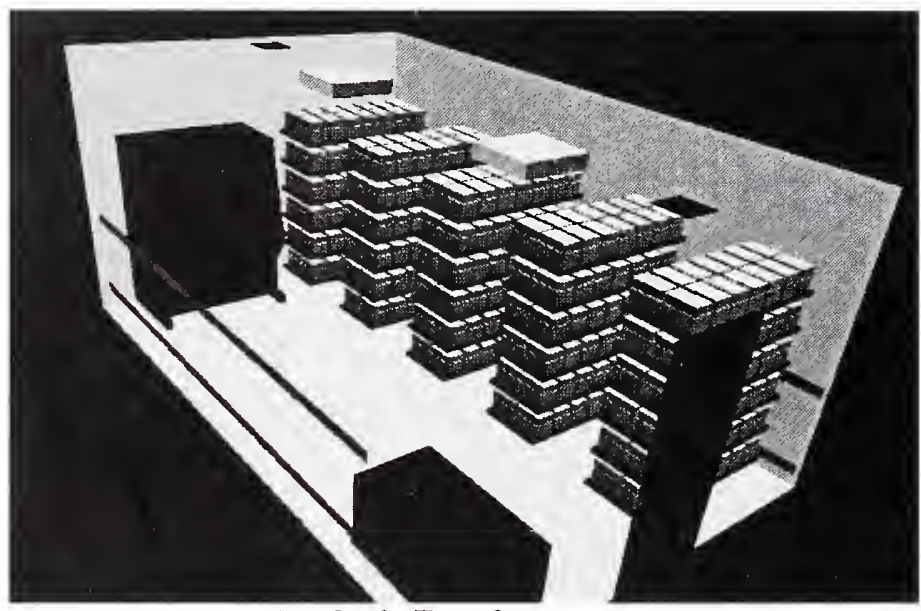

Analysis Results

Cage Occupied Zone

\begin{tabular}{|l|cc|c|c|}
\cline { 2 - 5 } \multicolumn{1}{c|}{} & \multicolumn{2}{c|}{ Temperature } & $\mathrm{CO}_{2}$ & RH \\
\cline { 2 - 5 } \multicolumn{1}{c|}{} & ${ }^{\circ} \mathrm{C}$ & \multicolumn{1}{c|}{$\mathrm{F}_{\mathrm{y}}$} & $(\mathrm{ppm})$ & \\
\hline Mean & 21.94 & 71.49 & 1822 & $65.04 \%$ \\
\hline S.D. & 0.37 & 0.66 & 402 & $2.43 \%$ \\
\hline Max. & 22.66 & 72.79 & 2780 & $69.72 \%$ \\
\hline
\end{tabular}

Cage Occupied Zone NH3 (ppm)

\begin{tabular}{|l|c|c|c|c|c|c|c|c|c|c|}
\hline Day & $\mathbf{1}$ & $\mathbf{2}$ & $\mathbf{3}$ & $\mathbf{4}$ & $\mathbf{5}$ & $\mathbf{6}$ & $\mathbf{7}$ & $\mathbf{8}$ & $\mathbf{9}$ & $\mathbf{1 0}$ \\
\hline Mean & 0.96 & 1.84 & 2.84 & 4.97 & 8.02 & 11.74 & $\mathbf{1 7 . 3 2}$ & 21.85 & 26.75 & 30.18 \\
\hline Max. & 1.47 & 2.81 & 4.34 & 7.58 & 12.24 & 17.91 & 26.44 & 33.35 & 40.83 & 46.07 \\
\hline
\end{tabular}

Room Breathing Zone

\begin{tabular}{|l|cc|c|c|}
\cline { 2 - 5 } \multicolumn{1}{c|}{} & \multicolumn{2}{c|}{ Temperature } & $\mathrm{CO}_{2}$ & RH \\
\cline { 2 - 5 } \multicolumn{1}{c|}{} & ${ }^{\circ} \mathrm{C}$ & ${ }^{\circ} \mathrm{F}$ & $(\mathrm{ppm})$ & \\
\hline Mean & 20.26 & 68.47 & 86 & $55.93 \%$ \\
\hline S.D. & 0.52 & 0.94 & 399 & \\
\hline Max. & 30.00 & 86.00 & 361 & \\
\hline
\end{tabular}

Room Breathing Zone NH3 (ppm)

\begin{tabular}{|l|c|c|c|c|c|c|c|c|c|c|}
\hline Day & $\mathbf{1}$ & $\mathbf{2}$ & $\mathbf{3}$ & $\mathbf{4}$ & $\mathbf{5}$ & $\mathbf{6}$ & $\mathbf{7}$ & $\mathbf{8}$ & $\mathbf{9}$ & $\mathbf{1 0}$ \\
\hline Mean & 0.05 & 0.09 & 0.13 & 0.24 & 0.38 & 0.56 & 0.82 & $\mathbf{1 . 0 4}$ & 1.27 & 1.43 \\
\hline Max. & 0.19 & 0.37 & 0.56 & 0.98 & 1.59 & 2.33 & 3.43 & 4.33 & 5.30 & 5.98 \\
\hline
\end{tabular}




\section{Histogram Distributions}

Cage occupied zone average temperature $\left({ }^{\circ} \mathrm{C}\right)$ distribution

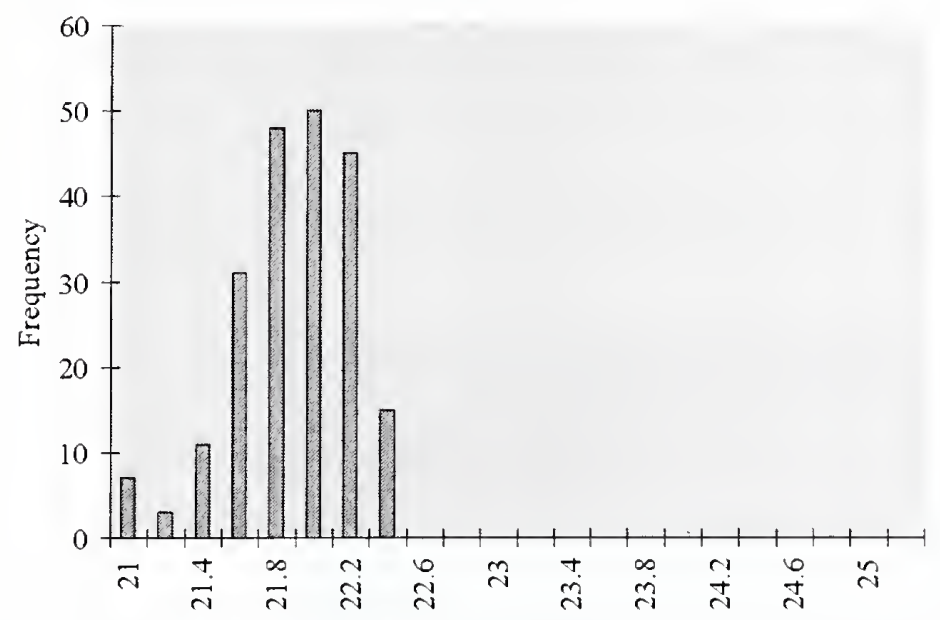

Cage occupied zone average contamination $(\mathrm{kg} / \mathrm{kg})$ distribution

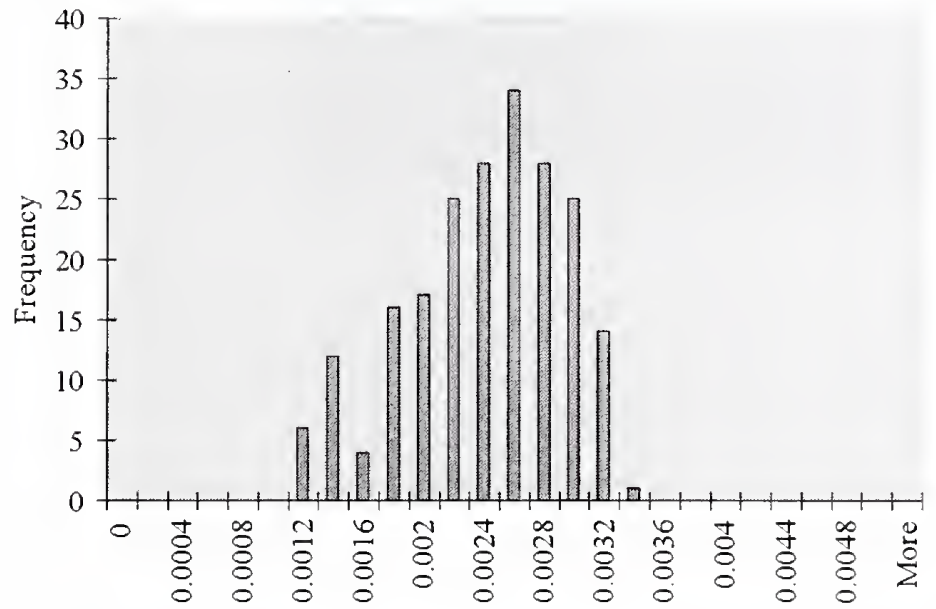

Cage occupied zone average relative humidity (\%) distribution

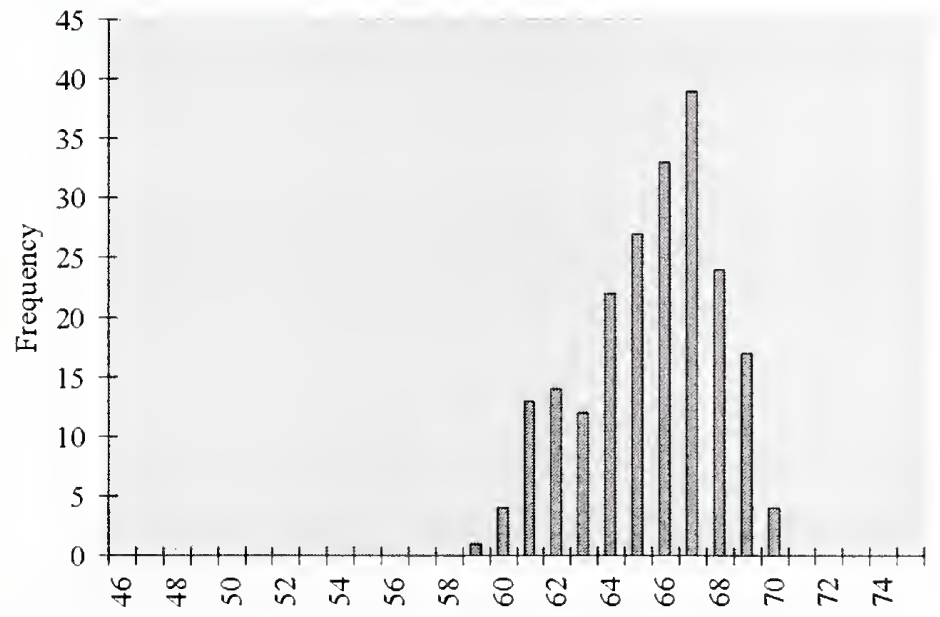

Contamination conversion factors $(\mathrm{kg} / \mathrm{kg} \rightarrow \mathrm{ppm})$

\begin{tabular}{|c|c|c|}
\hline Day & $\mathbf{C O}_{2}$ & $\mathbf{N H}_{3}$ \\
\hline $\mathbf{1}$ & 785000 & 415 \\
\hline $\mathbf{2}$ & 785000 & 795 \\
\hline $\mathbf{3}$ & 785000 & 1225 \\
\hline $\mathbf{4}$ & 785000 & 2141 \\
\hline $\mathbf{5}$ & 785000 & 3457 \\
\hline $\mathbf{6}$ & 785000 & 5058 \\
\hline 7 & 785000 & 7466 \\
\hline $\mathbf{8}$ & 785000 & 9418 \\
\hline $\mathbf{9}$ & 785000 & 11529 \\
\hline $\mathbf{1 0}$ & 785000 & 11384 \\
\hline
\end{tabular}




\section{Description}

$\begin{array}{llllll}\begin{array}{l}\text { Supply } \\ \text { Configuration }\end{array} & \begin{array}{l}\text { Supply Discharge } \\ \text { Temperature }\left({ }^{\circ} \mathrm{C}\right)\end{array} & \begin{array}{l}\text { Supply Discharge } \\ \text { Slot }\end{array} & \begin{array}{l}\text { Exhaust } \\ \text { Configuration }\end{array} & \begin{array}{l}\text { Exhaust } \\ \text { Temperature }\left({ }^{\circ} \mathrm{C}\right)\end{array} & \begin{array}{l}\text { Exhaust } \\ \text { RH }\end{array} \\ \text { Change } & \text { Rack } & \text { Rack } & \text { Number of } & \text { Total mass of } & \text { Room } \\ \text { Station } & \text { Orientation } & \text { Density } & \text { Mice in Room } & \text { Mice in Room } & \text { Pressurisation } \\ \text { ON } & \text { Perp all } 5 \text { on 1 wall } & \text { Double } & 2100 & 42000 \mathrm{gr} & \text { neg } 100 \mathrm{cfm}\end{array}$

Room
ACH
15
Cage
Condition
Top On

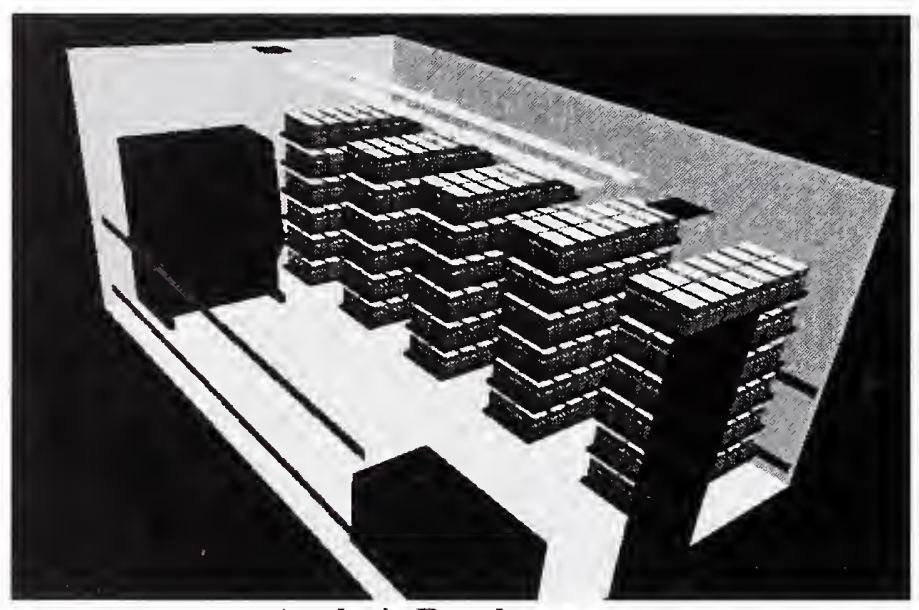

Analysis Results

Cage Occupied Zone

\begin{tabular}{|l|cc|c|c|}
\cline { 2 - 5 } \multicolumn{1}{c|}{} & \multicolumn{2}{c|}{ Temperature } & $\mathbf{C O}_{2}$ & RH \\
\cline { 2 - 5 } \multicolumn{1}{c|}{} & ${ }^{\circ} \mathrm{C}$ & $\mathrm{F}$ & \multicolumn{1}{c|}{$(\mathbf{p p m})$} & \\
\hline Mean & 21.85 & 71.34 & 1757 & $64.38 \%$ \\
\hline S.D. & 0.39 & 0.71 & 395 & $2.10 \%$ \\
\hline Max. & 22.72 & 72.89 & 2734 & $69.23 \%$ \\
\hline
\end{tabular}

Cage Occupied Zone NH3 (ppm)

\begin{tabular}{|l|c|c|c|c|c|c|c|c|c|c|}
\hline Day & $\mathbf{1}$ & $\mathbf{2}$ & $\mathbf{3}$ & $\mathbf{4}$ & $\mathbf{5}$ & $\mathbf{6}$ & $\mathbf{7}$ & $\mathbf{8}$ & $\mathbf{9}$ & $\mathbf{1 0}$ \\
\hline Mean & 0.93 & 1.78 & 2.74 & 4.66 & 7.38 & 10.73 & 15.71 & 19.97 & 24.68 & 28.40 \\
\hline Max. & 1.44 & 2.77 & 4.27 & 7.24 & 11.49 & 16.70 & 24.44 & 31.06 & 38.40 & 44.18 \\
\hline
\end{tabular}

Room Breathing 7one

\begin{tabular}{|l|cc|c|c|}
\cline { 2 - 5 } \multicolumn{1}{c|}{} & \multicolumn{2}{c|}{ Temperature } & $\mathrm{CO}_{2}$ & RH \\
\cline { 2 - 5 } \multicolumn{1}{c|}{} & ${ }^{\circ} \mathrm{C}$ & ${ }^{\circ} \mathrm{F}$ & $(\mathbf{p p m})$ & \\
\hline Mean & 20.23 & 68.41 & 117 & $56.34 \%$ \\
\hline S.D. & 0.26 & 0.46 & 36 & \\
\hline Max. & 20.69 & 69.24 & 328 & \\
\hline
\end{tabular}

Room Breathing Zone NH3 (ppm)

\begin{tabular}{|l|c|c|c|c|c|c|c|c|c|c|}
\hline Day & $\mathbf{1}$ & $\mathbf{2}$ & $\mathbf{3}$ & $\mathbf{4}$ & $\mathbf{5}$ & $\mathbf{6}$ & $\mathbf{7}$ & $\mathbf{8}$ & $\mathbf{9}$ & $\mathbf{1 0}$ \\
\hline Mean & 0.06 & 0.12 & 0.18 & 0.31 & 0.49 & 0.71 & 1.04 & 1.33 & 1.64 & 1.89 \\
\hline Max. & 0.17 & 0.33 & 0.51 & 0.87 & 1.38 & 2.01 & 2.94 & 3.73 & 4.61 & 5.31 \\
\hline
\end{tabular}




\section{Histogram Distributions}

Cage occupied zone average temperature $\left({ }^{\circ} \mathrm{C}\right)$ distribution

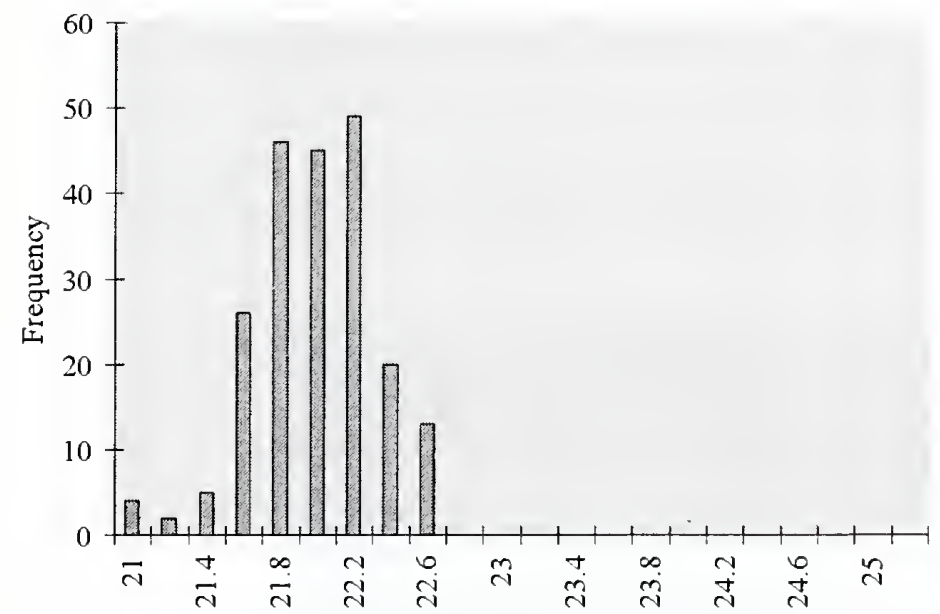

Cage occupied zone average contamination $(\mathrm{kg} / \mathrm{kg})$ distribution

Contamination conversion factors $(\mathrm{kg} / \mathrm{kg} \rightarrow \mathrm{ppm})$

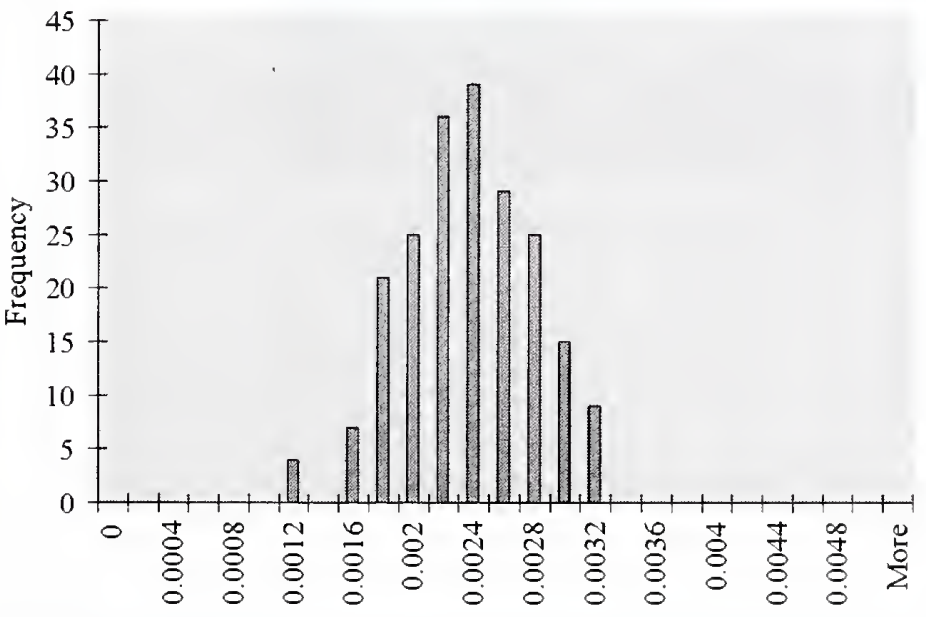

\begin{tabular}{|c|c|c|}
\hline Day & $\mathbf{C O}_{2}$ & $\mathbf{N H}_{3}$ \\
\hline $\mathbf{1}$ & 785000 & 414 \\
\hline $\mathbf{2}$ & 785000 & 795 \\
\hline $\mathbf{3}$ & 785000 & 1225 \\
\hline $\mathbf{4}$ & 785000 & 2080 \\
\hline $\mathbf{5}$ & 785000 & 3299 \\
\hline $\mathbf{6}$ & 785000 & 4795 \\
\hline $\mathbf{7}$ & 785000 & 7017 \\
\hline $\mathbf{8}$ & 785000 & 8919 \\
\hline $\mathbf{9}$ & 785000 & 11026 \\
\hline $\mathbf{1 0}$ & 785000 & 11384 \\
\hline
\end{tabular}

Cage occupied zone average relative humidity (\%) distribution

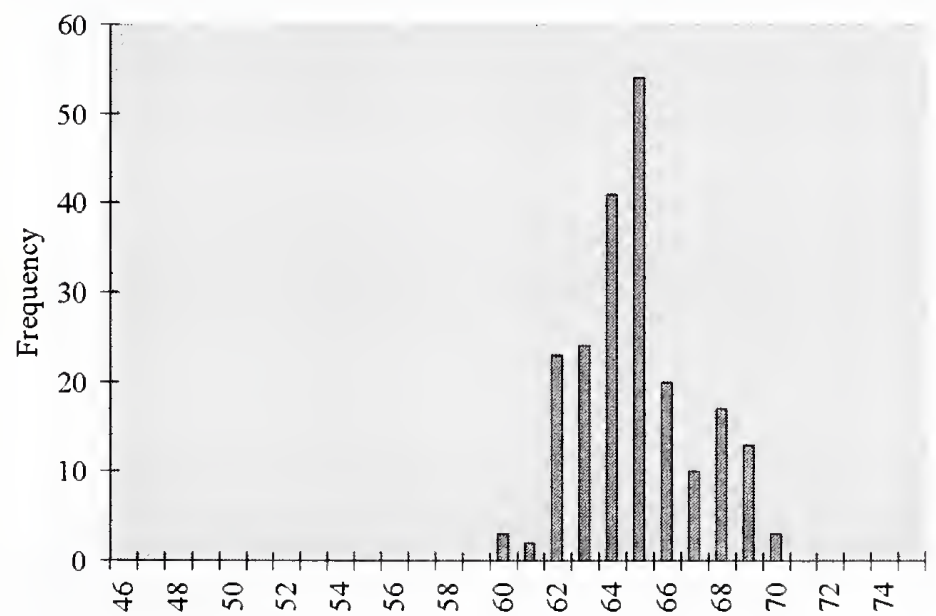




\section{Description}

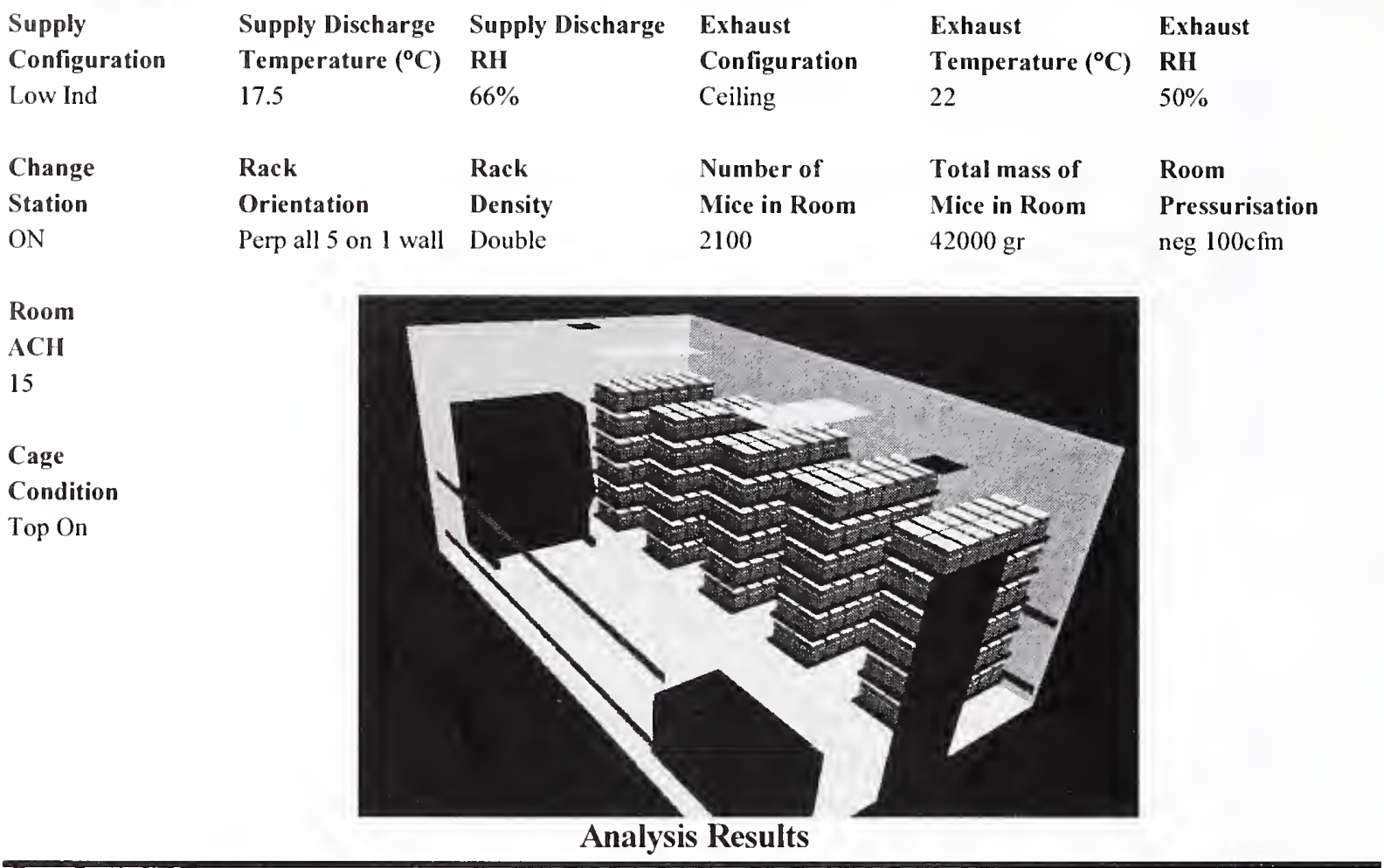

Cage Occupied Zone

\begin{tabular}{|l|cc|c|c|}
\cline { 2 - 5 } \multicolumn{1}{c|}{} & \multicolumn{2}{c|}{ Temperature } & $\mathrm{CO}_{2}$ & RH \\
\cline { 2 - 5 } \multicolumn{1}{c|}{} & ${ }^{\circ} \mathrm{C}$ & ${ }^{\circ} \mathrm{F}$ & $(\mathrm{ppm})$ & \\
\hline Mean & 21.94 & 71.49 & 1822 & $64.10 \%$ \\
\hline S.D. & 0.37 & 0.66 & 402 & $2.75 \%$ \\
\hline Max. & 22.66 & 72.79 & 2780 & $70.20 \%$ \\
\hline
\end{tabular}

Cage Occupied Zone NH3 (ppm)

\begin{tabular}{|l|c|c|c|c|c|c|c|c|c|c|}
\hline Day & $\mathbf{1}$ & $\mathbf{2}$ & $\mathbf{3}$ & $\mathbf{4}$ & $\mathbf{5}$ & $\mathbf{6}$ & $\mathbf{7}$ & $\mathbf{8}$ & $\mathbf{9}$ & $\mathbf{1 0}$ \\
\hline Mean & 0.96 & 1.84 & 2.84 & 4.77 & 7.50 & 10.87 & 15.84 & 20.21 & 25.09 & 29.12 \\
\hline Max. & 1.47 & 2.81 & 4.34 & 7.28 & 11.44 & 16.59 & 24.18 & 30.84 & 38.30 & 44.45 \\
\hline
\end{tabular}

Room Breathing Zone

\begin{tabular}{|l|cc|c|c|}
\cline { 2 - 5 } \multicolumn{1}{c|}{} & \multicolumn{2}{c|}{ Temperature } & $\mathrm{CO}_{2}$ & RH \\
\cline { 2 - 5 } \multicolumn{1}{c|}{} & ${ }^{\circ} \mathrm{C}$ & ${ }^{\circ} \mathrm{F}$ & $\mathbf{( p p m )}$ & \\
\hline Mean & 20.23 & 68.42 & 69 & $55.88 \%$ \\
\hline S.D. & 0.25 & 0.45 & 34 & \\
\hline Max. & 20.79 & 69.42 & 281 & \\
\hline
\end{tabular}

Room Breathing Zone NH3 (ppm)

\begin{tabular}{|l|c|c|c|c|c|c|c|c|c|c|}
\hline Day & 1 & 2 & 3 & 4 & 5 & 6 & 7 & 8 & 9 & 10 \\
\hline Mean & 0.04 & 0.07 & 0.11 & 0.18 & 0.29 & 0.41 & 0.60 & 0.77 & 0.96 & 1.11 \\
\hline Max. & 0.15 & 0.28 & 0.44 & 0.74 & 1.16 & 1.68 & 2.44 & 3.12 & 3.87 & 4.49 \\
\hline
\end{tabular}




\section{Histogram Distributions}

Cage occupied zone average temperature $\left({ }^{\circ} \mathrm{C}\right)$ distribution

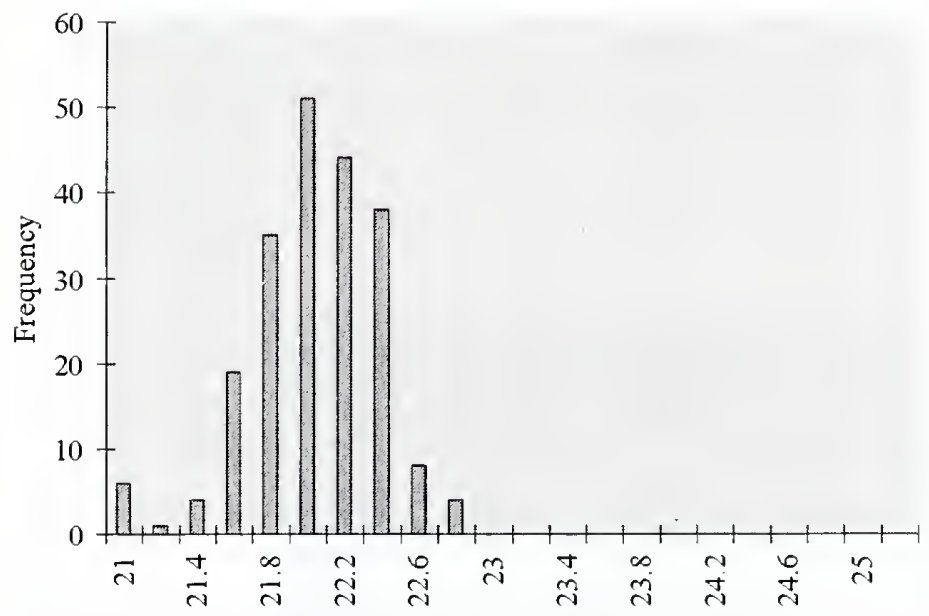

Cage occupied zone average contamination $(\mathrm{kg} / \mathrm{kg})$ distribution

Contamination conversion factors $(\mathrm{kg} / \mathrm{kg} \rightarrow \mathrm{ppm})$

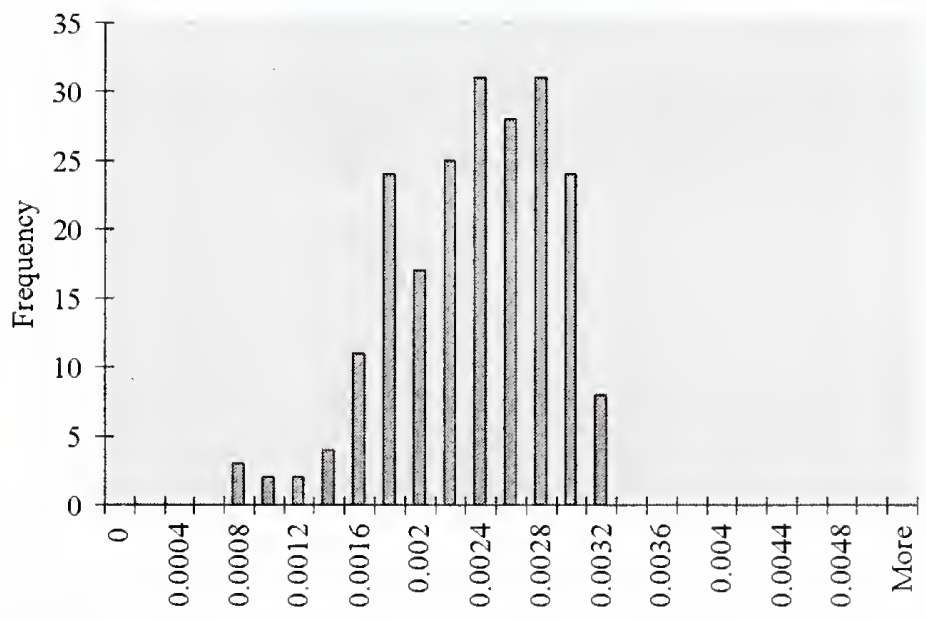

\begin{tabular}{|c|c|c|}
\hline Day & $\mathbf{C O}_{2}$ & $\mathbf{N H}_{3}$ \\
\hline $\mathbf{1}$ & 785000 & 414 \\
\hline $\mathbf{2}$ & 785000 & 795 \\
\hline $\mathbf{3}$ & 785000 & 1225 \\
\hline $\mathbf{4}$ & 785000 & 2054 \\
\hline $\mathbf{5}$ & 785000 & 3231 \\
\hline $\mathbf{6}$ & 785000 & 4684 \\
\hline $\mathbf{7}$ & 785000 & 6827 \\
\hline $\mathbf{8}$ & 785000 & 8708 \\
\hline $\mathbf{9}$ & 785000 & 10814 \\
\hline $\mathbf{1 0}$ & 785000 & 11384 \\
\hline
\end{tabular}

Cage occupied zone average relative humidity (\%) distribution

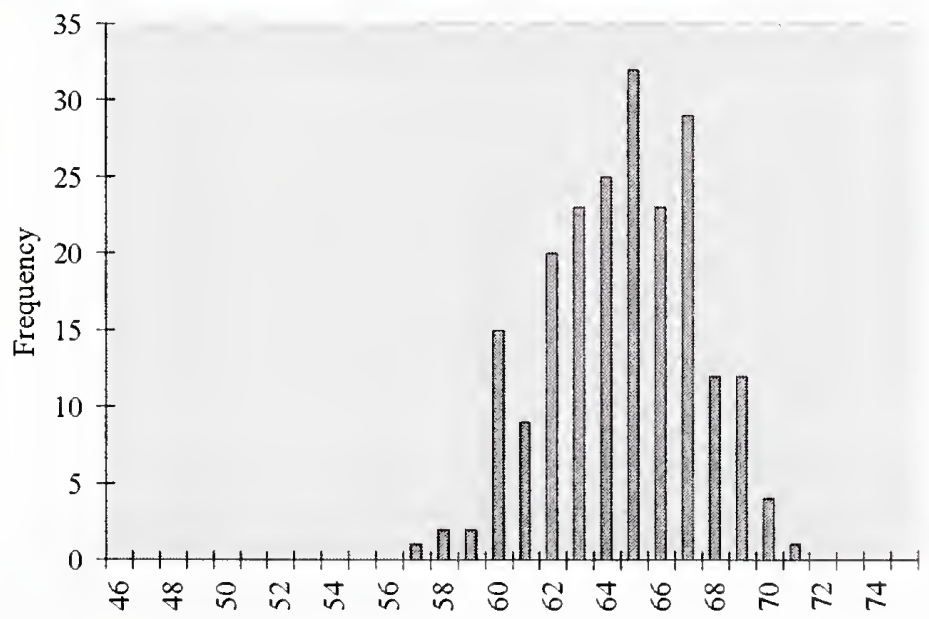


Casename

Case 96

Description

\begin{tabular}{|c|c|c|c|c|c|}
\hline Supply & Supply Discharge & Supply Discharge & Exhaust & Exhaust & Exhaust \\
\hline Configuration & Temperature $\left({ }^{\circ} \mathrm{C}\right)$ & RH & Configuration & Temperature $\left({ }^{\circ} \mathrm{C}\right)$ & RH \\
\hline Radial & 17.5 & $66 \%$ & Ceiling & 22 & $50 \%$ \\
\hline Change & Rack & Rack & Number of & Total mass of & Room \\
\hline Station & Orientation & Density & Mice in Room & Mice in Room & Pressurisation \\
\hline ON alt design & Perp all 5 on 1 wall & Double & 2100 & $42000 \mathrm{gr}$ & neg $100 \mathrm{cfm}$ \\
\hline
\end{tabular}

Room

$\mathrm{ACH}$

15

Cage

Condition

Top On

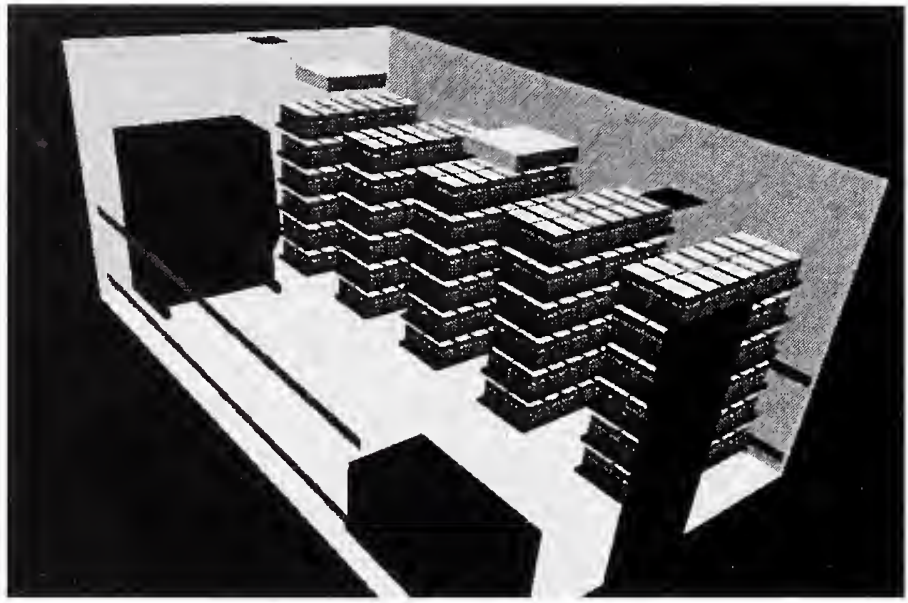

Analysis Results

Cage Occupied Zone

\begin{tabular}{|l|cc|c|c|}
\cline { 2 - 5 } \multicolumn{1}{c|}{} & \multicolumn{2}{c|}{ Temperature } & $\mathbf{C O}_{2}$ & RH \\
\cline { 2 - 5 } \multicolumn{1}{c|}{} & ${ }^{\circ} \mathrm{C}$ & ${ }^{\circ} \mathrm{F}$ & $(\mathbf{p p m})$ & \\
\hline Mean & 21.76 & 71.17 & 1957 & $65.79 \%$ \\
\hline S.D. & 0.34 & 0.61 & 459 & $2.90 \%$ \\
\hline Max. & 22.32 & 72.17 & 2763 & $71.27 \%$ \\
\hline
\end{tabular}

Cage Occupied Zone NH3 (ppm)

\begin{tabular}{|l|c|c|c|c|c|c|c|c|c|c|}
\hline Day & 1 & $\mathbf{2}$ & $\mathbf{3}$ & $\mathbf{4}$ & $\mathbf{5}$ & $\mathbf{6}$ & $\mathbf{7}$ & $\mathbf{8}$ & $\mathbf{9}$ & $\mathbf{1 0}$ \\
\hline Mean & 1.04 & 1.98 & 3.05 & 5.51 & 9.06 & 13.35 & 19.87 & 24.88 & 30.15 & 33.32 \\
\hline Max. & 1.47 & 2.80 & 4.31 & 7.78 & 12.80 & 18.85 & 28.06 & 35.12 & 42.57 & 47.05 \\
\hline
\end{tabular}

Room Breathing Zone

\begin{tabular}{|l|cc|c|c|}
\cline { 2 - 5 } \multicolumn{1}{c|}{} & \multicolumn{2}{c|}{ Temperature } & $\mathrm{CO}_{2}$ & RH \\
\cline { 2 - 5 } \multicolumn{1}{c|}{} & ${ }^{\circ} \mathrm{C}$ & ${ }^{\circ} \mathrm{F}$ & $(\mathbf{p p m})$ & \\
\hline Mean & 20.01 & 68.02 & 100 & $56.95 \%$ \\
\hline S.D. & 0.27 & 0.48 & 41 & \\
\hline Max. & 20.70 & 69.26 & 280 & \\
\hline
\end{tabular}

Room Breathing Zone NH3 (ppm)

\begin{tabular}{|l|c|c|c|c|c|c|c|c|c|c|}
\hline Day & $\mathbf{1}$ & $\mathbf{2}$ & $\mathbf{3}$ & $\mathbf{4}$ & $\mathbf{5}$ & $\mathbf{6}$ & $\mathbf{7}$ & $\mathbf{8}$ & $\mathbf{9}$ & $\mathbf{1 0}$ \\
\hline Mean & 0.05 & 0.10 & 0.16 & 0.28 & 0.46 & 0.68 & 1.01 & 1.27 & 1.54 & 1.70 \\
\hline Max. & 0.15 & 0.28 & 0.44 & 0.79 & 1.30 & 1.91 & 2.85 & 3.56 & 4.32 & 4.77 \\
\hline
\end{tabular}




\section{Histogram Distributions}

Cage occupied zone average temperature $\left({ }^{\circ} \mathrm{C}\right)$ distribution

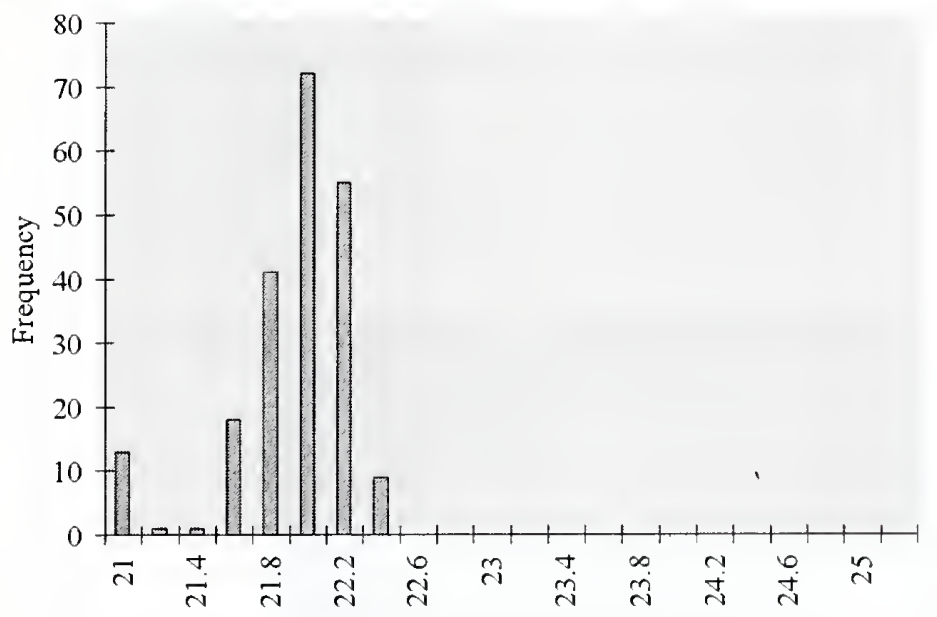

Cage occupied zone average contamination $(\mathrm{kg} / \mathrm{kg})$ distribution

Contamination conversion factors $(\mathrm{kg} / \mathrm{kg} \rightarrow \mathrm{ppm})$

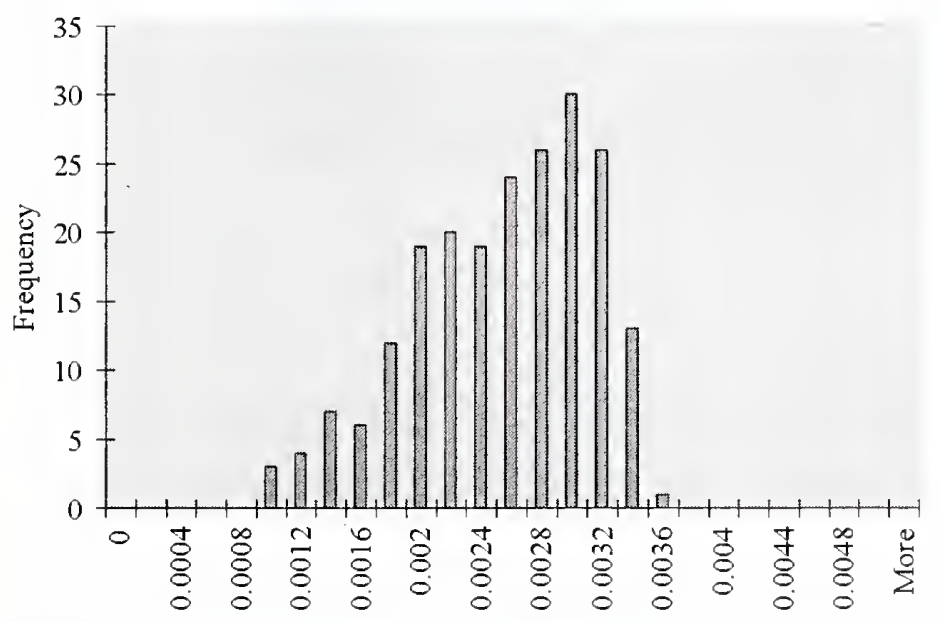

\begin{tabular}{|c|c|c|}
\hline Day & $\mathbf{C O}_{2}$ & $\mathbf{N H}_{3}$ \\
\hline $\mathbf{1}$ & 785000 & 417 \\
\hline $\mathbf{2}$ & 785000 & 795 \\
\hline $\mathbf{3}$ & 785000 & 1225 \\
\hline $\mathbf{4}$ & 785000 & 2210 \\
\hline $\mathbf{5}$ & 785000 & 3636 \\
\hline $\mathbf{6}$ & 785000 & 5355 \\
\hline 7 & 785000 & 7972 \\
\hline $\mathbf{8}$ & 785000 & 9981 \\
\hline $\mathbf{9}$ & 785000 & 12097 \\
\hline $\mathbf{1 0}$ & 785000 & 11384 \\
\hline
\end{tabular}

Cage occupied zone average relative humidity (\%) distribution

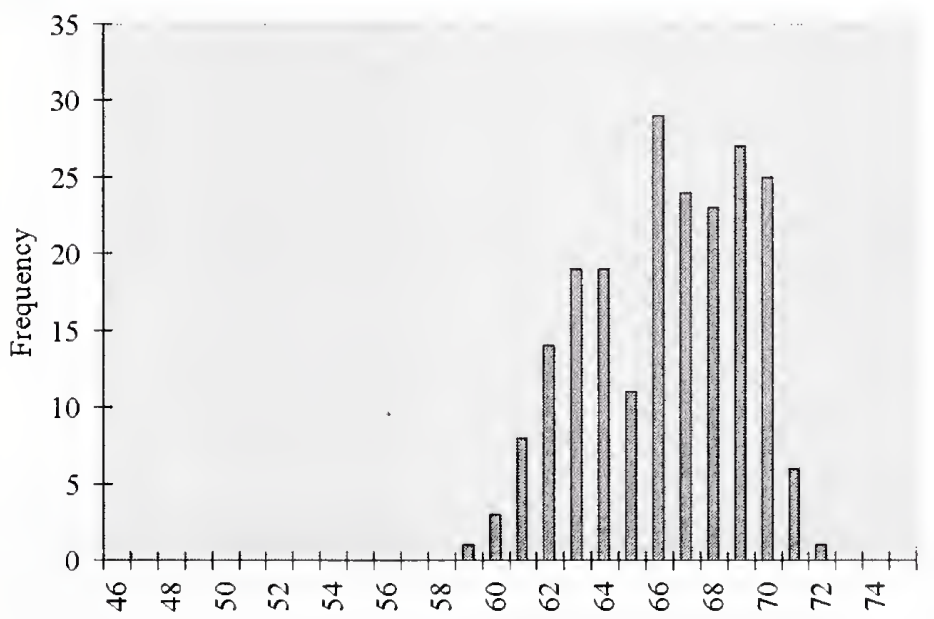


Casename

Case 97

Description

\begin{tabular}{|c|c|c|c|c|c|}
\hline Supply & Supply Discharge & Supply Discharge & Exhaust & Exhaust & Exhaust \\
\hline Configuration & Temperature $\left({ }^{\circ} \mathrm{C}\right)$ & RH & Configuration & Temperature $\left({ }^{\circ} \mathrm{C}\right)$ & RH \\
\hline Slot & 17.5 & $66 \%$ & Ceiling & 22 & $50 \%$ \\
\hline Change & Rack & Rack & Number of & Total mass of & Room \\
\hline Station & Orientation & Density & Mice in Room & Mice in Room & Pressurisation \\
\hline ON alt design & Perp all 5 on 1 wall & Double & 2100 & $42000 \mathrm{gr}$ & neg $100 \mathrm{cfm}$ \\
\hline
\end{tabular}

Room

ACH

15

Cage

Condition

Top On

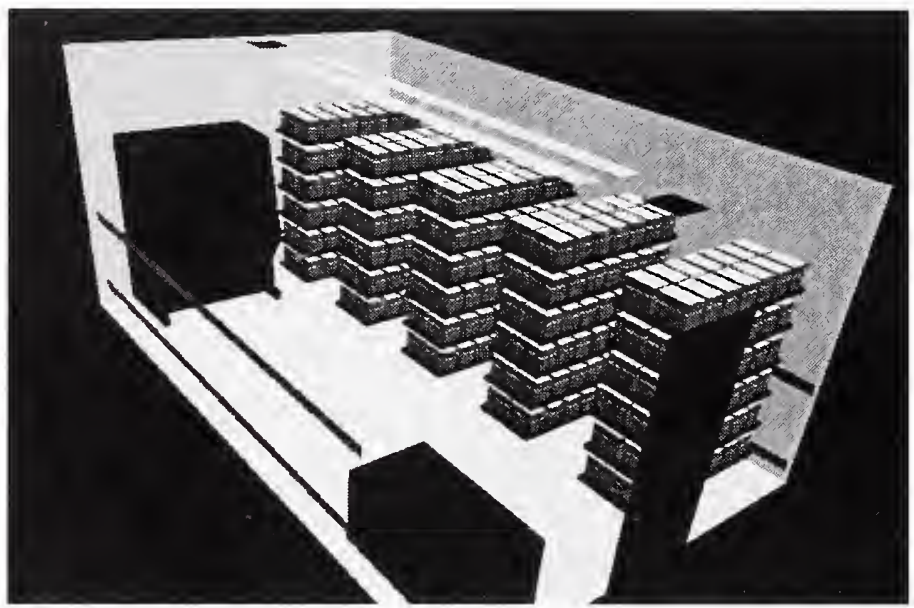

Analysis Results

Cage Occupied Zone

\begin{tabular}{|l|cc|c|c|}
\cline { 2 - 5 } \multicolumn{1}{c|}{} & \multicolumn{2}{c|}{ Temperature } & $\mathrm{CO}_{2}$ & RH \\
\cline { 2 - 5 } \multicolumn{1}{c|}{} & ${ }^{\circ} \mathrm{C}$ & ${ }^{\circ} \mathrm{F}$ & $(\mathrm{ppm})$ & \\
\hline Mean & 21.65 & 70.97 & 1725 & $64.22 \%$ \\
\hline S.D. & 0.32 & 0.58 & 427 & $2.16 \%$ \\
\hline Max. & 22.29 & 72.12 & 2885 & $69.99 \%$ \\
\hline
\end{tabular}

Cage Occupied Zone NH3 (ppm)

\begin{tabular}{|l|c|c|c|c|c|c|c|c|c|c|}
\hline Day & $\mathbf{1}$ & $\mathbf{2}$ & $\mathbf{3}$ & $\mathbf{4}$ & $\mathbf{5}$ & $\mathbf{6}$ & $\mathbf{7}$ & $\mathbf{8}$ & $\mathbf{9}$ & $\mathbf{1 0}$ \\
\hline Mean & 0.91 & 1.75 & 2.69 & 4.54 & 7.16 & 10.39 & 15.18 & 19.33 & 23.96 & 27.70 \\
\hline Max. & 1.52 & 2.92 & 4.50 & 7.59 & 11.98 & 17.39 & 25.38 & 32.33 & 40.07 & 46.34 \\
\hline
\end{tabular}

Room Breathing Zone

\begin{tabular}{|l|cc|c|c|}
\cline { 2 - 5 } \multicolumn{1}{c|}{} & \multicolumn{2}{c|}{ Temperature } & $\mathbf{C O}_{2}$ & RH \\
\cline { 2 - 5 } \multicolumn{1}{c|}{} & ${ }^{\circ} \mathrm{C}$ & ${ }^{\circ} \mathrm{F}$ & $(\mathrm{ppm})$ & \\
\hline Mean & 20.02 & 68.03 & 111 & $57.04 \%$ \\
\hline S.D. & 0.25 & 0.45 & 29 & \\
\hline Max. & 20.78 & 69.40 & 294 & \\
\hline
\end{tabular}

Room Breathing Zone NH3 (ppm)

\begin{tabular}{|l|c|c|c|c|c|c|c|c|c|c|}
\hline Day & $\mathbf{1}$ & $\mathbf{2}$ & $\mathbf{3}$ & $\mathbf{4}$ & $\mathbf{5}$ & $\mathbf{6}$ & $\mathbf{7}$ & $\mathbf{8}$ & $\mathbf{9}$ & $\mathbf{1 0}$ \\
\hline Mean & 0.06 & 0.11 & 0.17 & 0.29 & 0.46 & 0.67 & 0.98 & 1.25 & 1.54 & 1.79 \\
\hline Max. & 0.15 & 0.30 & 0.46 & 0.77 & 1.22 & 1.77 & 2.59 & 3.29 & 4.08 & 4.72 \\
\hline
\end{tabular}




\section{Histogram Distributions}

Cage occupied zone average temperature $\left({ }^{\circ} \mathrm{C}\right)$ distribution

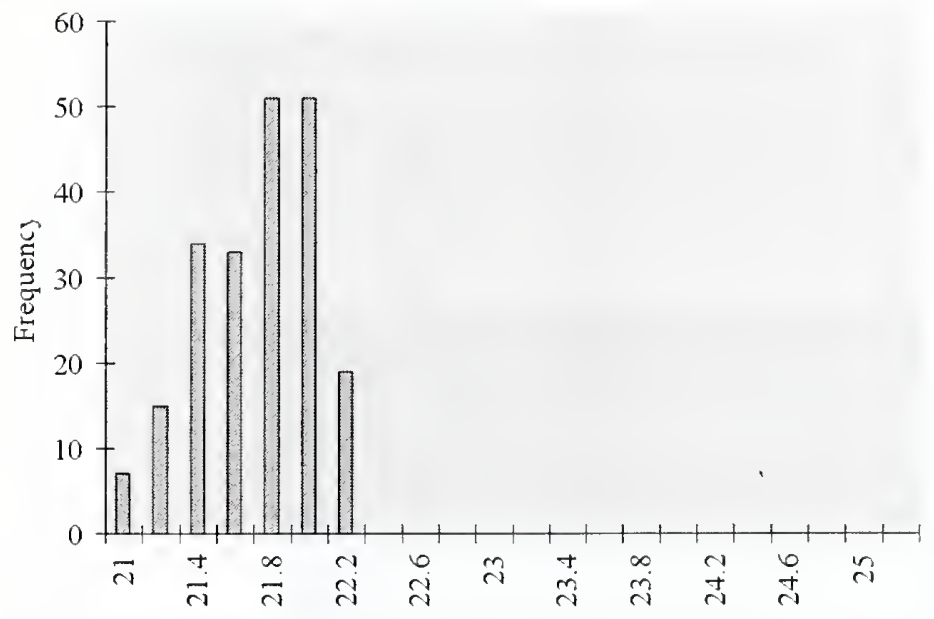

Cage occupied zone average contamination $(\mathrm{kg} / \mathrm{kg})$ distribution Contamination conversion factors $(\mathrm{kg} / \mathrm{kg} \rightarrow \mathrm{ppm})$

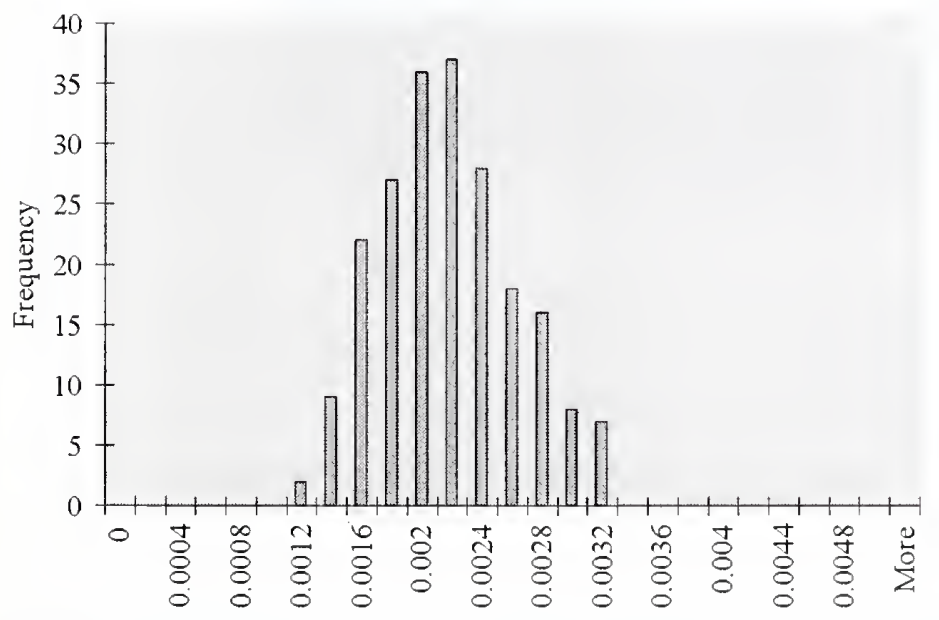

\begin{tabular}{|c|c|c|}
\hline Day & $\mathrm{CO}_{2}$ & $\mathrm{NH}_{3}$ \\
\hline $\mathbf{1}$ & 785000 & 414 \\
\hline $\mathbf{2}$ & 785000 & 795 \\
\hline $\mathbf{3}$ & 785000 & 1225 \\
\hline $\mathbf{4}$ & 785000 & 2065 \\
\hline $\mathbf{5}$ & 785000 & 3259 \\
\hline $\mathbf{6}$ & 785000 & 4730 \\
\hline 7 & 785000 & 6907 \\
\hline $\mathbf{8}$ & 785000 & 8796 \\
\hline $\mathbf{9}$ & 785000 & 10902 \\
\hline $\mathbf{1 0}$ & 785000 & 11384 \\
\hline
\end{tabular}

Cage occupied zone average relative humidity (\%) distribution

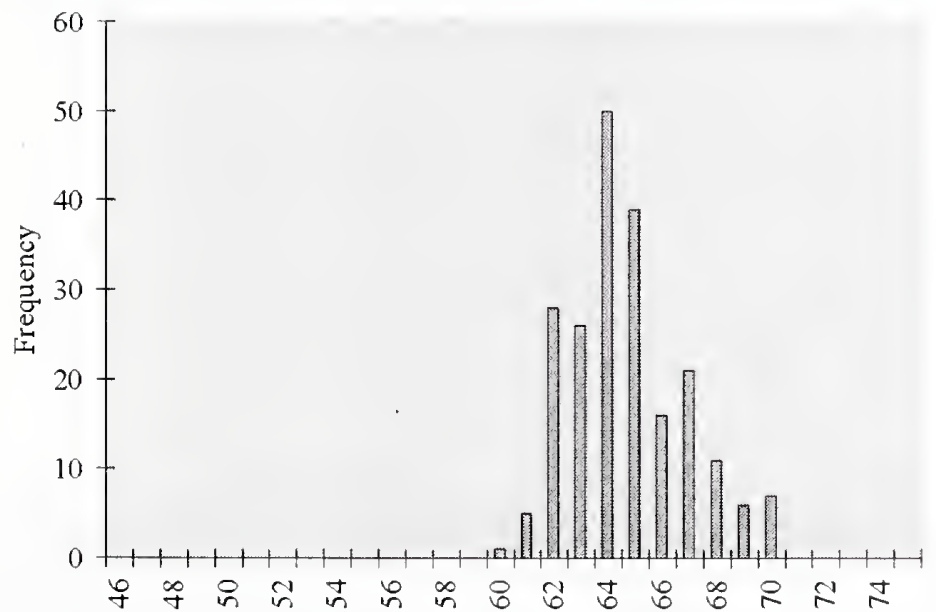




\section{Casename $\quad$ Case 98}

\section{Description}

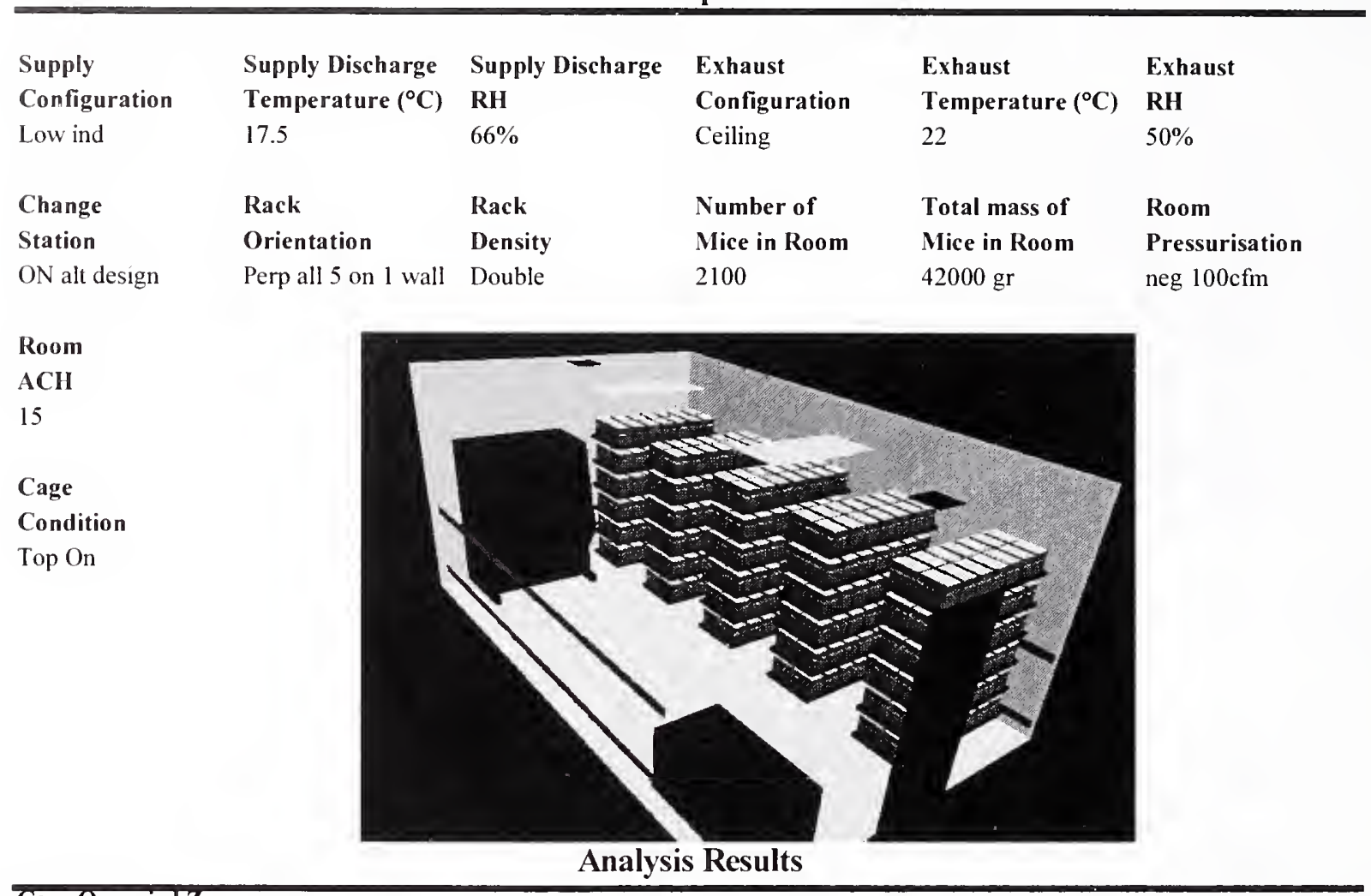

Cage Occupied Zone

\begin{tabular}{|l|cc|c|c|}
\cline { 2 - 5 } \multicolumn{1}{c|}{} & \multicolumn{2}{c|}{ Temperature } & $\mathrm{CO}_{2}$ & RH \\
\cline { 2 - 5 } \multicolumn{1}{c|}{} & ${ }^{\circ} \mathrm{C}$ & ${ }^{\circ} \mathrm{F}$ & $(\mathrm{ppm})$ & \\
\hline Mean & 21.70 & 71.07 & 1955 & $65.78 \%$ \\
\hline S.D. & 0.30 & 0.54 & 428 & $3.05 \%$ \\
\hline Max. & 22.30 & 72.13 & 2712 & $73.07 \%$ \\
\hline
\end{tabular}

Cage Occupied Zone NH3 (ppm)

\begin{tabular}{|l|c|c|c|c|c|c|c|c|c|c|}
\hline Day & $\mathbf{1}$ & $\mathbf{2}$ & $\mathbf{3}$ & $\mathbf{4}$ & $\mathbf{5}$ & $\mathbf{6}$ & $\mathbf{7}$ & $\mathbf{8}$ & $\mathbf{9}$ & $\mathbf{1 0}$ \\
\hline Mean & $\mathbf{1 0 4}$ & 1.98 & 3.05 & 5.50 & 9.05 & 13.33 & 19.85 & 24.85 & 30.12 & 33.29 \\
\hline Max. & 1.44 & 274 & 4.23 & 7.64 & 1256 & 18.50 & 27.54 & 34.48 & 41.79 & 46.19 \\
\hline
\end{tabular}

Room Breathing Zone

\begin{tabular}{|l|cc|c|c|}
\cline { 2 - 5 } \multicolumn{1}{c|}{} & \multicolumn{2}{c|}{ Temperature } & $\mathrm{CO}_{2}$ & RH \\
\cline { 2 - 5 } \multicolumn{1}{c|}{} & ${ }^{\circ} \mathrm{C}$ & ${ }^{\circ}$ & $(\mathrm{ppm})$ & \\
\hline Mean & 19.99 & 67.98 & 83 & $5688 \%$ \\
\hline S.D. & 0.20 & 0.35 & 47 & \\
\hline Max. & 20.65 & 69.17 & 334 & \\
\hline
\end{tabular}

Room Breathing Zone NH3 (ppm)

\begin{tabular}{|l|c|c|c|c|c|c|c|c|c|c|}
\hline Day & $\mathbf{1}$ & $\mathbf{2}$ & $\mathbf{3}$ & $\mathbf{4}$ & $\mathbf{5}$ & $\mathbf{6}$ & $\mathbf{7}$ & $\mathbf{8}$ & $\mathbf{9}$ & $\mathbf{1 0}$ \\
\hline Mean & 0.04 & 0.08 & 0.13 & 0.23 & 0.39 & 0.57 & 0.85 & 1.06 & 1.29 & 1.42 \\
\hline Max. & 0.18 & 0.34 & 0.52 & 0.94 & 1.54 & 2.28 & 3.39 & 4.24 & 5.14 & 5.68 \\
\hline
\end{tabular}




\section{Histogram Distributions}

Cage occupied zone average temperature $\left({ }^{\circ} \mathrm{C}\right)$ distribution

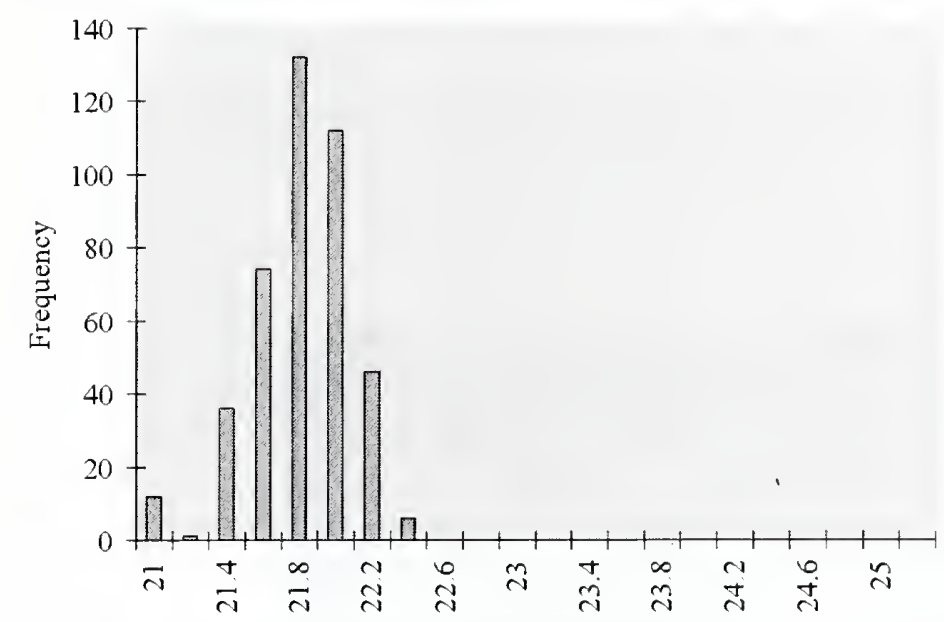

Cage occupied zone average contamination $(\mathrm{kg} / \mathrm{kg})$ distribution

Contamination conversion factors $(\mathrm{kg} / \mathrm{kg} \rightarrow \mathrm{ppm})$

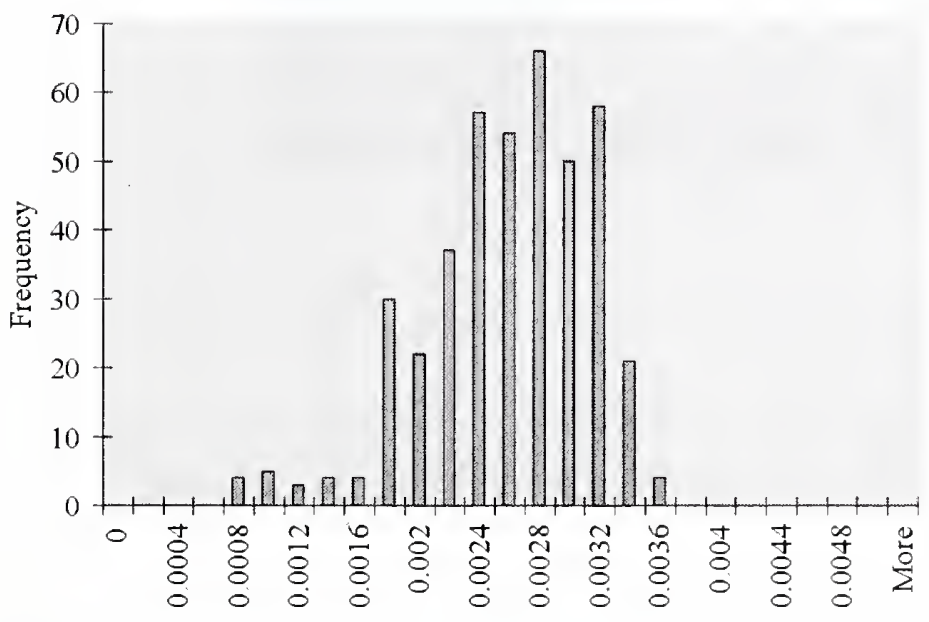

\begin{tabular}{|c|c|c|}
\hline Day & $\mathbf{C O}_{2}$ & $\mathrm{NH}_{3}$ \\
\hline $\mathbf{1}$ & 785000 & 417 \\
\hline $\mathbf{2}$ & 785000 & 795 \\
\hline $\mathbf{3}$ & 785000 & 1225 \\
\hline $\mathbf{4}$ & 785000 & 2210 \\
\hline $\mathbf{5}$ & 785000 & 3636 \\
\hline $\mathbf{6}$ & 785000 & 5355 \\
\hline $\mathbf{7}$ & 785000 & 7971 \\
\hline $\mathbf{8}$ & 785000 & 9979 \\
\hline $\mathbf{9}$ & 785000 & 12096 \\
\hline $\mathbf{1 0}$ & 785000 & 11384 \\
\hline
\end{tabular}

Cage occupied zone average relative humidity (\%) distribution

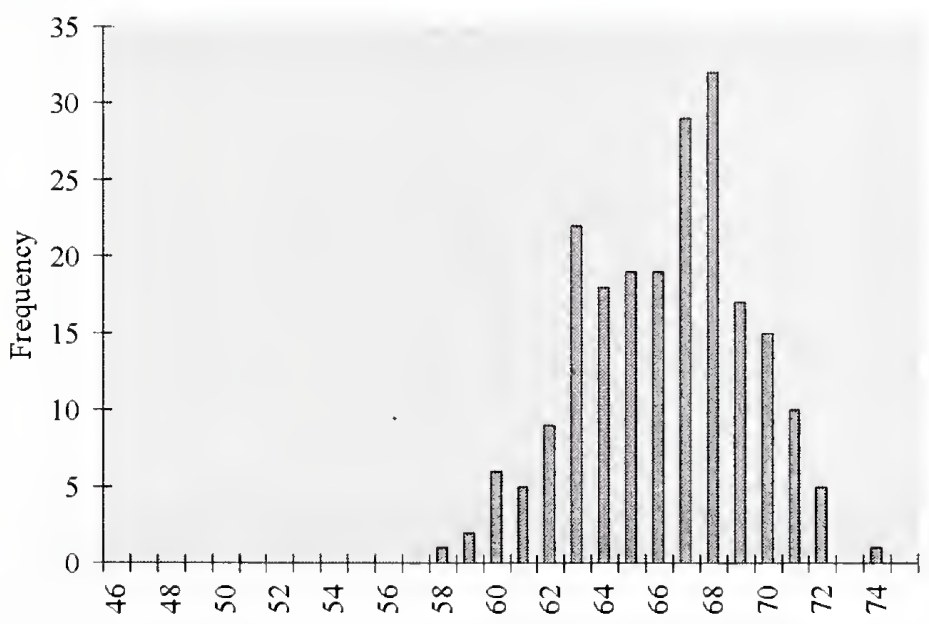


Casename $\quad$ Case 99

\section{Description}

\begin{tabular}{|c|c|c|c|c|c|}
\hline $\begin{array}{l}\text { Supply } \\
\text { Configuration }\end{array}$ & Supply Discharge & $\begin{array}{l}\text { Supply Discharge } \\
\text { RH }\end{array}$ & $\begin{array}{l}\text { Exhaust } \\
\text { Confiouration }\end{array}$ & $\begin{array}{l}\text { Exhaust } \\
\text { Temperature }\left(^{\circ} \mathrm{C}\right.\end{array}$ & Exhaust \\
\hline Radial & 22 & $49 \%$ & Ceiling & 26 & $50 \%$ \\
\hline $\begin{array}{l}\text { Change } \\
\text { Station } \\
\text { ON }\end{array}$ & $\begin{array}{l}\text { Rack } \\
\text { Orientation } \\
\text { On wall }\end{array}$ & $\begin{array}{l}\text { Rack } \\
\text { Density } \\
\text { Single }\end{array}$ & $\begin{array}{l}\text { Number of } \\
\text { Mice in Room } \\
1050\end{array}$ & $\begin{array}{l}\text { Total mass of } \\
\text { Mice in Room } \\
21000 \text { gr }\end{array}$ & $\begin{array}{l}\text { Room } \\
\text { Pressurisation } \\
\text { neg } 100 \mathrm{cfm}\end{array}$ \\
\hline
\end{tabular}

Room

$\mathrm{ACH}$

15

Cage

Condition

Top On

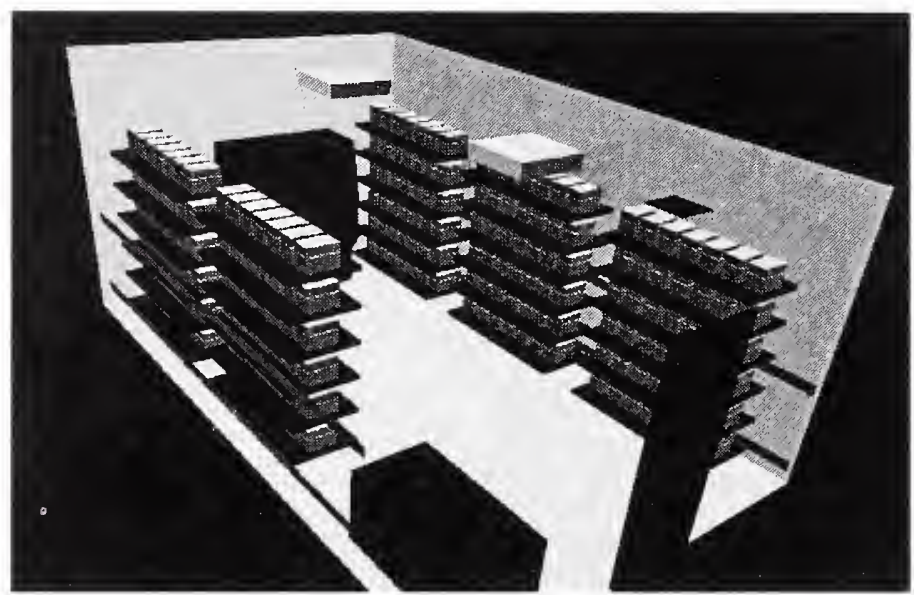

Analysis Results

Cage Occupied Zone

\begin{tabular}{|l|cc|c|c|}
\cline { 2 - 5 } \multicolumn{1}{c|}{} & \multicolumn{2}{c|}{ Temperature } & $\mathrm{CO}_{2}$ & RH \\
\cline { 2 - 5 } \multicolumn{1}{c|}{} & ${ }^{\circ} \mathrm{C}$ & ${ }^{\circ} \mathrm{F}$ & $(\mathrm{ppm})$ & \\
\hline Mean & 24.78 & 76.60 & 1984 & $58.69 \%$ \\
\hline S.D. & 0.32 & 0.58 & 357 & $1.55 \%$ \\
\hline Max. & 25.73 & 78.32 & 2959 & $61.50 \%$ \\
\hline
\end{tabular}

Cage Occupied Zone NH3 (ppm)

\begin{tabular}{|l|c|c|c|c|c|c|c|c|c|c|}
\hline Day & $\mathbf{1}$ & $\mathbf{2}$ & $\mathbf{3}$ & $\mathbf{4}$ & $\mathbf{5}$ & $\mathbf{6}$ & $\mathbf{7}$ & $\mathbf{8}$ & $\mathbf{9}$ & $\mathbf{1 0}$ \\
\hline Mean & 1.03 & 2.01 & 3.10 & 4.46 & 6.28 & 8.70 & 11.91 & 16.07 & 21.34 & 27.89 \\
\hline Max. & 1.54 & 3.00 & 4.62 & 6.66 & 9.36 & 12.98 & 17.76 & 23.96 & 31.82 & 41.60 \\
\hline
\end{tabular}

Room Breathing Zone

\begin{tabular}{|l|cc|c|c|}
\cline { 2 - 5 } \multicolumn{1}{c|}{} & \multicolumn{2}{c|}{ Temperature } & $\mathrm{CO}_{2}$ & RH \\
\cline { 2 - 5 } \multicolumn{1}{c|}{} & ${ }^{\circ} \mathrm{C}$ & ${ }^{\circ}$ & $(\mathrm{ppm})$ & \\
\hline Mean & 23.86 & 74.94 & 27 & $44.18 \%$ \\
\hline S.D. & 0.41 & 0.74 & 12 & \\
\hline Max. & 24.70 & 76.46 & 123 & \\
\hline
\end{tabular}

Room Breathing Zone NH3 (ppm)

\begin{tabular}{|l|c|c|c|c|c|c|c|c|c|c|}
\hline Day & $\mathbf{1}$ & $\mathbf{2}$ & $\mathbf{3}$ & $\mathbf{4}$ & $\mathbf{5}$ & $\mathbf{6}$ & $\mathbf{7}$ & $\mathbf{8}$ & $\mathbf{9}$ & $\mathbf{1 0}$ \\
\hline Mean & 0.01 & 0.03 & 0.04 & 0.06 & 0.09 & 0.12 & 0.16 & 0.22 & 0.29 & 0.38 \\
\hline Max. & 0.06 & 0.12 & 0.19 & 0.28 & 0.39 & 0.54 & 0.74 & 1.00 & 1.33 & 1.73 \\
\hline
\end{tabular}




\section{Histogram Distributions}

Cage occupied zone average temperature $\left({ }^{\circ} \mathrm{C}\right)$ distribution

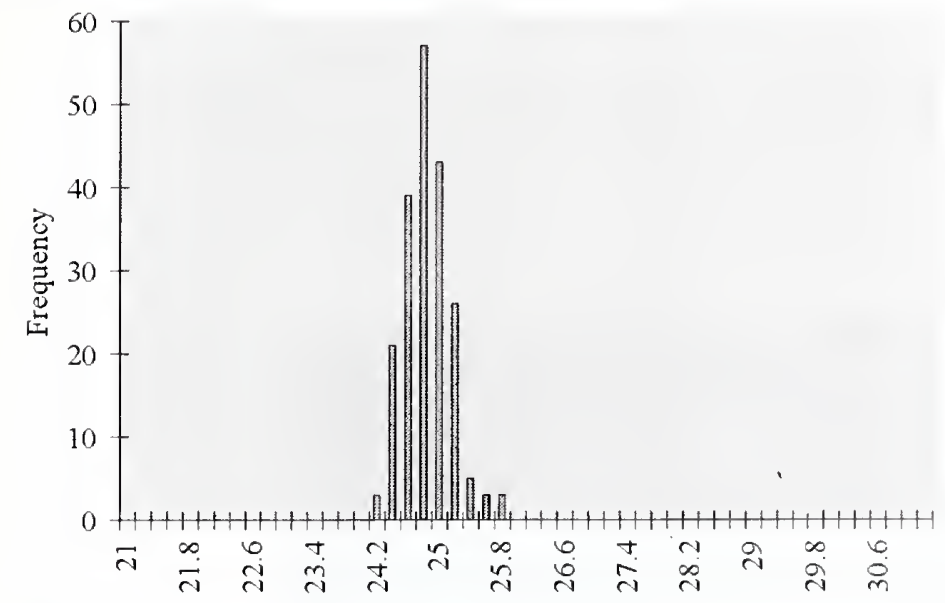

Cage occupied zone average contamination $(\mathrm{kg} / \mathrm{kg})$ distribution

Contamination conversion factors $(\mathrm{kg} / \mathrm{kg} \rightarrow \mathrm{ppm})$

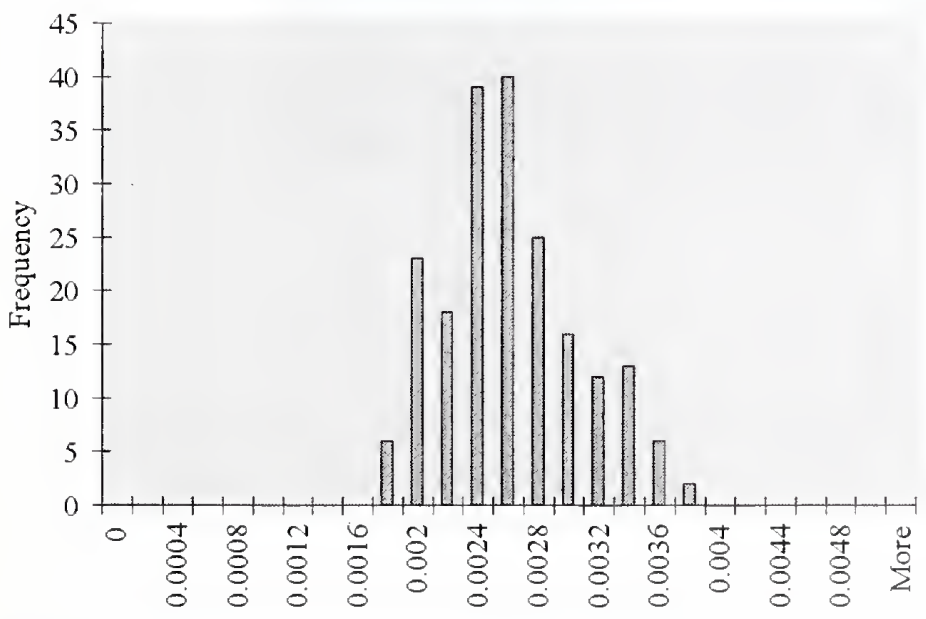

\begin{tabular}{|c|c|c|}
\hline Day & $\mathrm{CO}_{2}$ & $\mathrm{NH}_{3}$ \\
\hline $\mathbf{1}$ & 785000 & 408 \\
\hline 2 & 785000 & 795 \\
\hline $\mathbf{3}$ & 785000 & 1225 \\
\hline 4 & 785000 & 1766 \\
\hline 5 & 785000 & 2483 \\
\hline $\mathbf{6}$ & 785000 & 3443 \\
\hline 7 & 785000 & 4712 \\
\hline $\mathbf{8}$ & 785000 & 6356 \\
\hline 9 & 785000 & 8442 \\
\hline 10 & 785000 & 11384 \\
\hline
\end{tabular}

Cage occupied zone average relative humidity (\%) distribution

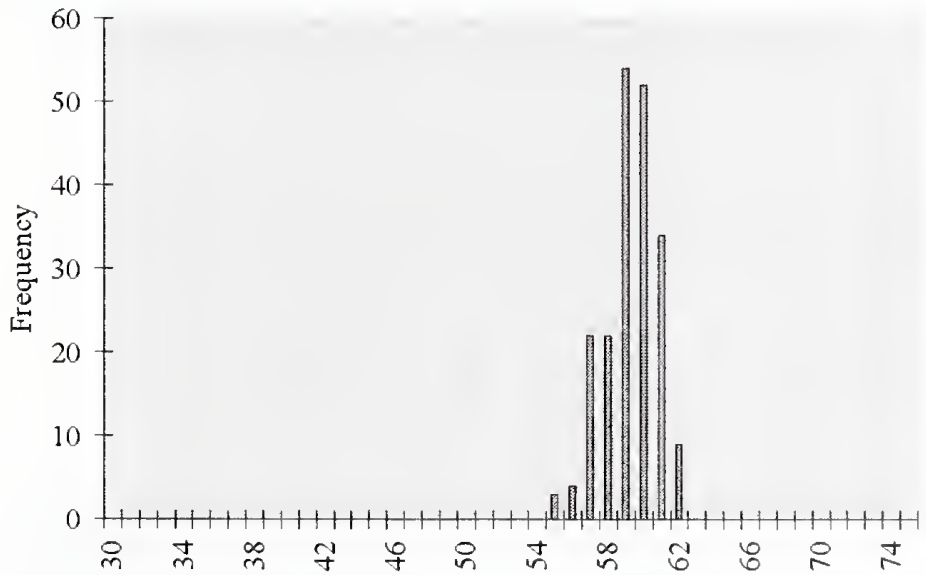


Casename Case 100

Description

\begin{tabular}{|c|c|c|c|c|c|}
\hline Supply & Supply Discharge & Supply Discharge & Exhaust & Exhaust & Exhaust \\
\hline Configuration & Temperature $\left({ }^{\circ} \mathrm{C}\right)$ & RH & Configuration & Temperature $\left({ }^{\circ} \mathrm{C}\right)$ & RH \\
\hline Radial & 22 & $49 \%$ & Low & 25 & $50 \%$ \\
\hline Change & Rack & Rack & Number of & Total mass of & Room \\
\hline $\begin{array}{l}\text { Station } \\
\text { ON }\end{array}$ & Orientation & $\begin{array}{l}\text { Density } \\
\text { Sinole }\end{array}$ & $\begin{array}{l}\text { Mice in Room } \\
1050\end{array}$ & Mice in Room & Pressurisation \\
\hline
\end{tabular}

Room

$\mathrm{ACH}$

15

Cage

Condition

Top On

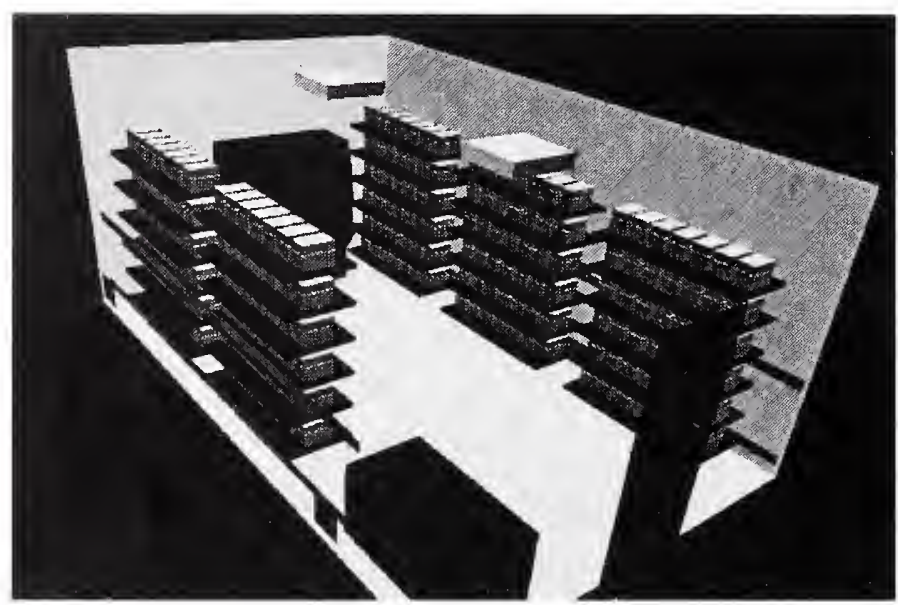

Analysis Results

Cage Occupied Zone

\begin{tabular}{|l|cc|c|c|}
\cline { 2 - 5 } \multicolumn{1}{c|}{} & \multicolumn{2}{c|}{ Temperature } & $\mathrm{CO}_{2}$ & RH \\
\cline { 2 - 5 } \multicolumn{1}{c|}{} & ${ }^{\circ} \mathrm{C}$ & ${ }^{\circ} \mathrm{F}$ & $(\mathbf{p p m})$ & \\
\hline Mean & 25.43 & 77.77 & 1730 & $55.39 \%$ \\
\hline S.D. & 0.47 & 0.85 & 428 & $1.89 \%$ \\
\hline Max. & 26.84 & 80.30 & 3305 & $60.49 \%$ \\
\hline
\end{tabular}

Cage Occupied Zone NH3 (ppm)

\begin{tabular}{|l|c|c|c|c|c|c|c|c|c|c|}
\hline Day & $\mathbf{1}$ & $\mathbf{2}$ & $\mathbf{3}$ & $\mathbf{4}$ & $\mathbf{5}$ & $\mathbf{6}$ & $\mathbf{7}$ & $\mathbf{8}$ & $\mathbf{9}$ & $\mathbf{1 0}$ \\
\hline Mean & 0.90 & 1.75 & 2.70 & 3.89 & 5.47 & 7.59 & 10.38 & 14.01 & 18.61 & 24.32 \\
\hline Max. & 1.72 & 3.35 & 5.16 & 7.43 & 10.46 & 14.50 & 19.84 & 26.77 & 35.55 & 46.47 \\
\hline
\end{tabular}

Room Breathing Zone

\begin{tabular}{|l|cc|c|c|}
\cline { 2 - 5 } \multicolumn{1}{c|}{} & \multicolumn{2}{c|}{ Temperature } & $\mathrm{CO}_{2}$ & RH \\
\cline { 2 - 5 } \multicolumn{1}{c|}{} & ${ }^{\circ} \mathrm{C}$ & ${ }^{\circ}$ & $(\mathbf{p p m})$ & \\
\hline Mean & 24.82 & 76.67 & 40 & $41.72 \%$ \\
\hline S.D. & 0.33 & 0.59 & 20 & \\
\hline Max. & 25.82 & 78.47 & 192 & \\
\hline
\end{tabular}

Room Breathing Zone NH3 (ppm)

\begin{tabular}{|l|c|c|c|c|c|c|c|c|c|c|}
\hline Day & $\mathbf{1}$ & $\mathbf{2}$ & $\mathbf{3}$ & $\mathbf{4}$ & $\mathbf{5}$ & $\mathbf{6}$ & $\mathbf{7}$ & $\mathbf{8}$ & $\mathbf{9}$ & $\mathbf{1 0}$ \\
\hline Mean & 0.02 & 0.04 & 0.06 & 0.09 & 0.13 & 0.18 & 0.24 & 0.32 & 0.43 & 0.56 \\
\hline Max. & 0.10 & 0.19 & 0.30 & 0.43 & 0.61 & 0.84 & 1.16 & 1.56 & 2.07 & 2.71 \\
\hline
\end{tabular}




\section{Histogram Distributions}

Cage occupied zone average temperature $\left({ }^{\circ} \mathrm{C}\right)$ distribution

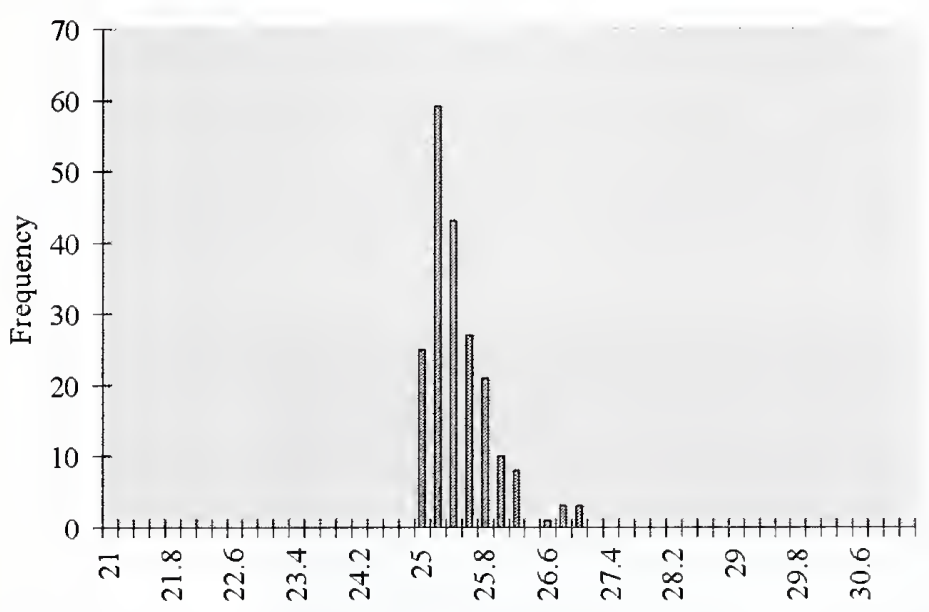

Cage occupied zone average contamination $(\mathrm{kg} / \mathrm{kg})$ distribution

Contamination conversion factors $(\mathrm{kg} / \mathrm{kg} \rightarrow \mathrm{ppm})$

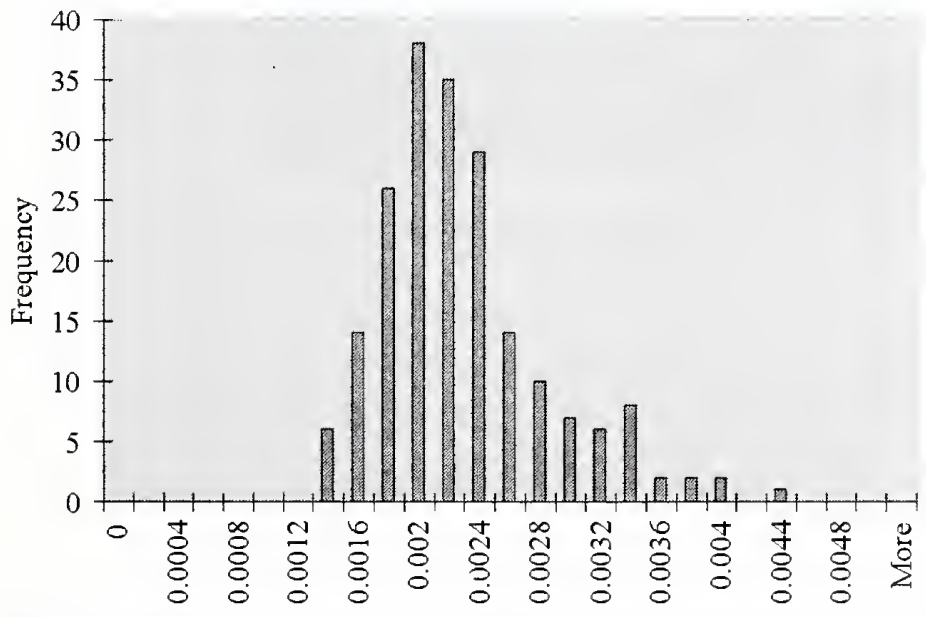

\begin{tabular}{|c|c|c|}
\hline Day & $\mathbf{C O}_{2}$ & $\mathbf{N H}_{3}$ \\
\hline $\mathbf{1}$ & 785000 & 408 \\
\hline $\mathbf{2}$ & 785000 & 795 \\
\hline $\mathbf{3}$ & 785000 & 1225 \\
\hline $\mathbf{4}$ & 785000 & 1766 \\
\hline $\mathbf{5}$ & 785000 & 2483 \\
\hline $\mathbf{6}$ & 785000 & 3443 \\
\hline 7 & 785000 & 4712 \\
\hline $\mathbf{8}$ & 785000 & 6356 \\
\hline $\mathbf{9}$ & 785000 & 8442 \\
\hline 10 & 785000 & 11384 \\
\hline
\end{tabular}

Cage occupied zone average relative humidity (\%) distribution

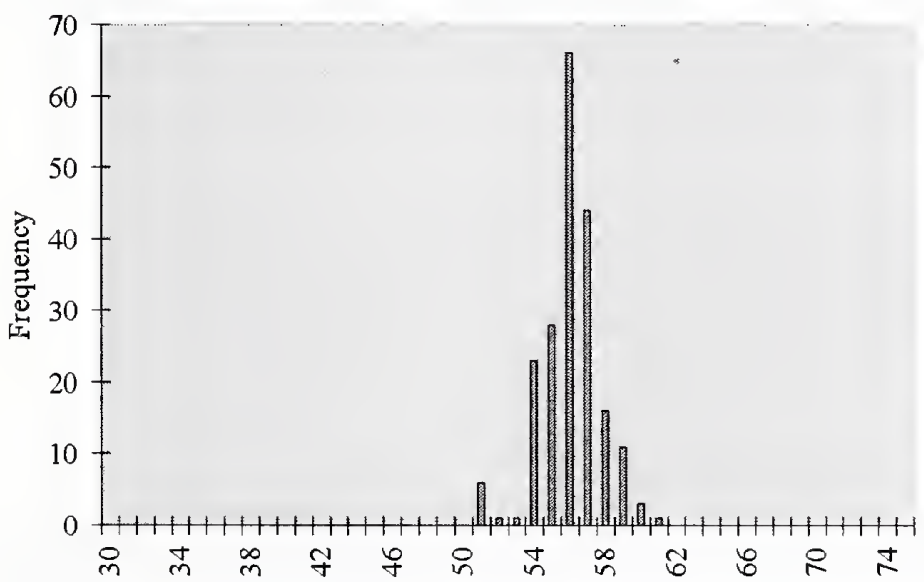




\section{Casename Case 101}

\section{Description}

Supply

Configuration

Low Ind

Change

Station

$\mathrm{ON}$ alt design

\section{Room}

$\mathrm{ACH}$

5

Cage

Condition

Top On
Supply Discharge Supply Discharge

Temperature $\left({ }^{\circ} \mathrm{C}\right.$ )

22

Rack

Orientation

Perp
Supply Discharge Exhaust

Configuration

Low

Number of

Mice in Room

1050

$\begin{array}{ll}\text { Exhaust } & \text { Exhaust } \\ \text { Temperature }\left({ }^{\circ} \mathrm{C}\right) & \text { RH } \\ 25 & 50 \%\end{array}$

Total mass of Mice in Room 21000 gr
Room

Pressurisation neg $100 \mathrm{cfm}$

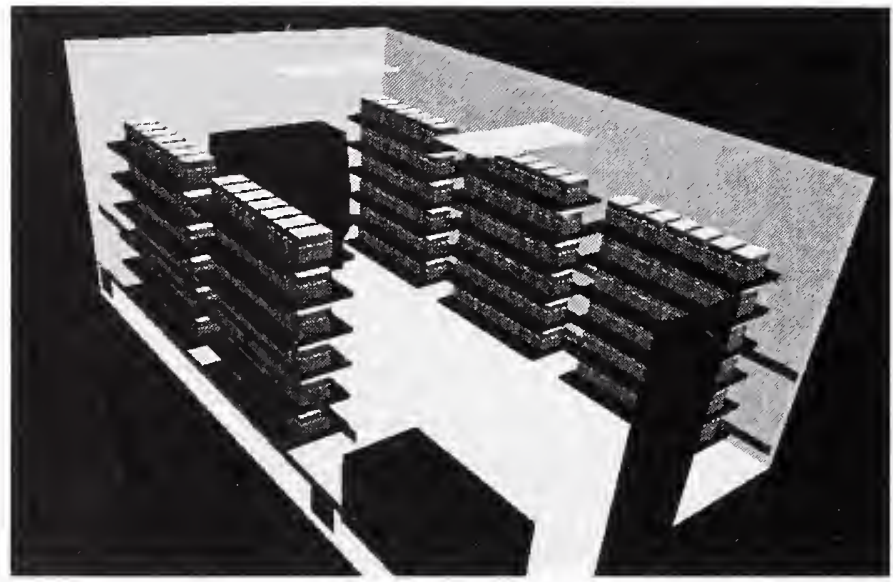

Analysis Results

Cage Occupied Zone

\begin{tabular}{|l|cc|c|c|}
\cline { 2 - 5 } \multicolumn{1}{c|}{} & \multicolumn{2}{c|}{ Temperature } & $\mathrm{CO}_{2}$ & RH \\
\cline { 2 - 5 } \multicolumn{1}{c|}{} & ${ }^{\circ} \mathrm{C}$ & ${ }^{\circ} \mathrm{F}$ & $(\mathbf{p p m})$ & \\
\hline Mean & 26.82 & 80.27 & 2400 & $55.39 \%$ \\
\hline S.D. & 0.69 & 1.23 & 530 & $1.89 \%$ \\
\hline Max. & 28.34 & 83.02 & 3522 & $60.49 \%$ \\
\hline
\end{tabular}

Cage Occupied Zone NH3 (ppm)

\begin{tabular}{|l|c|c|c|c|c|c|c|c|c|c|}
\hline Day & $\mathbf{1}$ & $\mathbf{2}$ & $\mathbf{3}$ & $\mathbf{4}$ & $\mathbf{5}$ & $\mathbf{6}$ & $\mathbf{7}$ & $\mathbf{8}$ & $\mathbf{9}$ & $\mathbf{1 0}$ \\
\hline Mean & 1.25 & 2.43 & 3.74 & 5.40 & 7.59 & 10.53 & 14.40 & 19.43 & 25.81 & 33.74 \\
\hline Max. & 1.83 & 3.56 & 5.50 & 7.92 & 11.14 & 15.45 & 21.14 & 28.51 & 37.87 & 49.50 \\
\hline
\end{tabular}

Room Breathing Zone

\begin{tabular}{|l|cc|c|c|}
\cline { 2 - 5 } \multicolumn{1}{c|}{} & \multicolumn{2}{|c|}{ Temperature } & $\mathrm{CO}_{2}$ & RH \\
\cline { 2 - 5 } \multicolumn{1}{c|}{} & ${ }^{\circ} \mathrm{C}$ & ${ }^{\circ} \mathrm{F}$ & $(\mathrm{ppm})$ & \\
\hline Mean & 26.24 & 79.22 & 284 & $39.78 \%$ \\
\hline S.D. & 1.52 & 2.74 & 138 & \\
\hline
\end{tabular}

Room Breathing Zone NH3 (ppm)

\begin{tabular}{|l|c|c|c|c|c|c|c|c|c|c|}
\hline Day & 1 & 2 & 3 & 4 & 5 & 6 & 7 & 8 & 9 & 10 \\
\hline Mean & 0.15 & 0.29 & 0.44 & 0.64 & 0.90 & 1.25 & 1.71 & 2.30 & 3.06 & 4.00 \\
\hline Max. & 0.41 & 0.80 & 1.24 & 1.78 & 2.51 & 3.48 & 4.76 & 6.42 & 8.53 & 11.15 \\
\hline
\end{tabular}




\section{Histogram Distributions}

Cage occupied zone average temperature $\left({ }^{\circ} \mathrm{C}\right)$ distribution

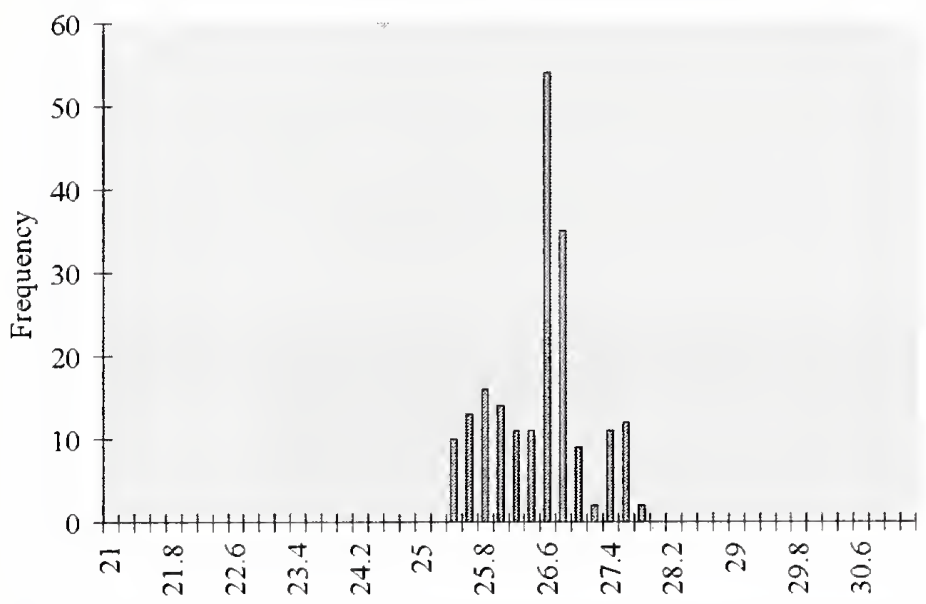

Cage occupied zone average contamination $(\mathrm{kg} / \mathrm{kg})$ distribution

Contamination conversion factors $(\mathrm{kg} / \mathrm{kg} \rightarrow \mathrm{ppm})$

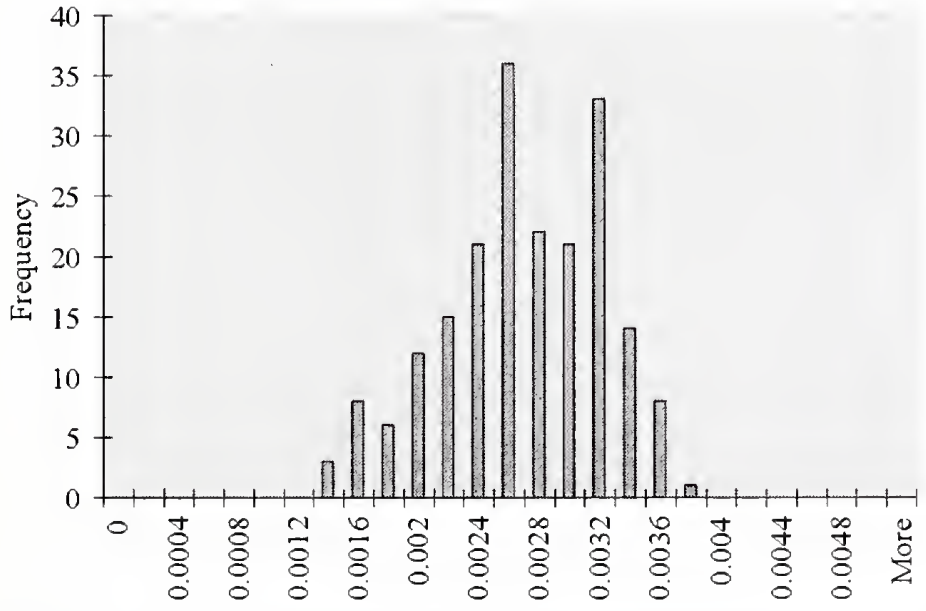

\begin{tabular}{|c|c|c|}
\hline Day & $\mathrm{CO}_{2}$ & $\mathrm{NH}_{3}$ \\
\hline $\mathbf{1}$ & 785000 & 408 \\
\hline 2 & 785000 & 795 \\
\hline 3 & 785000 & 1225 \\
\hline 4 & 785000 & 1766 \\
\hline $\mathbf{5}$ & 785000 & 2483 \\
\hline $\mathbf{6}$ & 785000 & 3443 \\
\hline 7 & 785000 & 4712 \\
\hline $\mathbf{8}$ & 785000 & 6356 \\
\hline $\mathbf{9}$ & 785000 & 8442 \\
\hline $\mathbf{1 0}$ & 785000 & 11384 \\
\hline
\end{tabular}

Cage occupied zone average relative humidity (\%) distribution

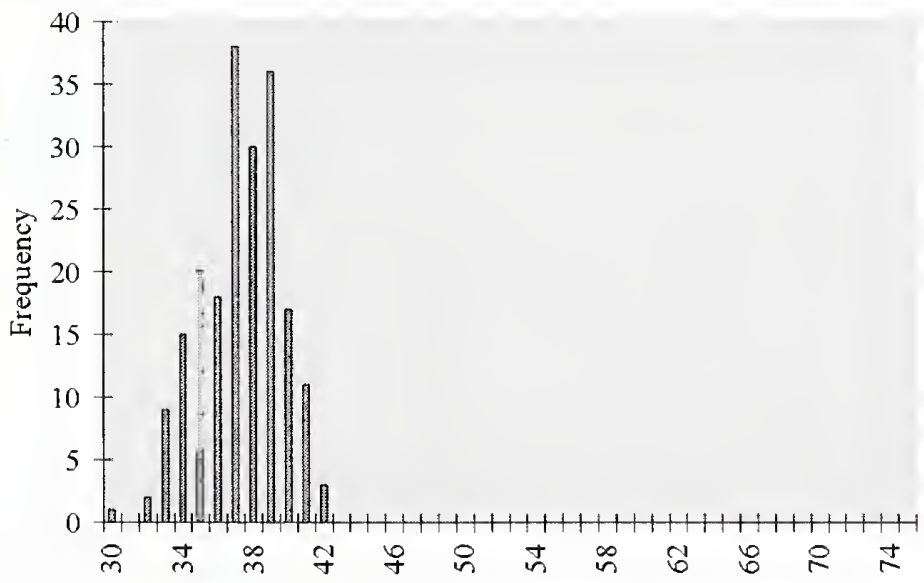





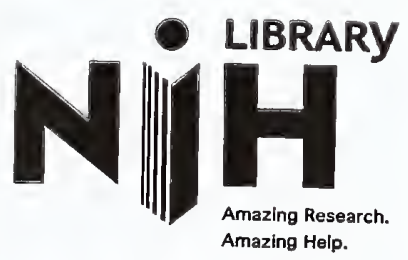

http://nihlibrary.nih.gov

10 Center Drive

Bethesda, MD 20892-1150

301-496-1080 

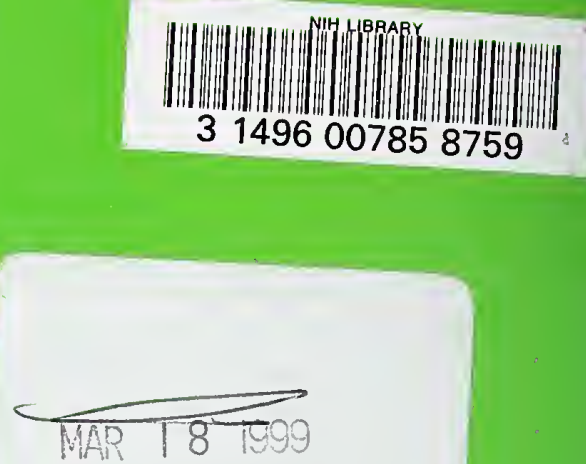